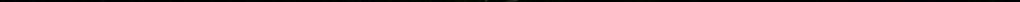







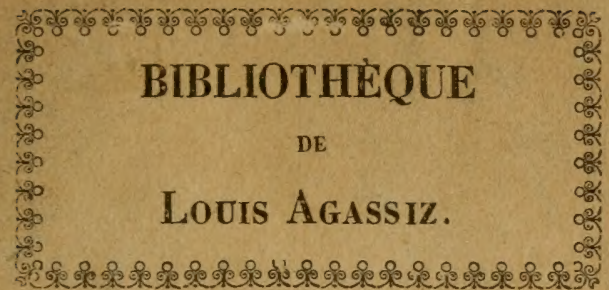




\title{
Beiträge
}

\author{
o $น \mathrm{r}$
}

\section{idhlefiphen $\$$}

$-\mathfrak{0} 0 \mathrm{n}$

\section{Dr. R. F. Robert Schnteiber,}

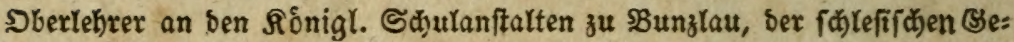

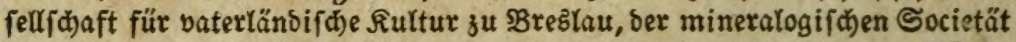

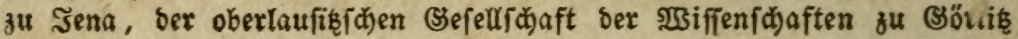
Eorrefponbirenbem Mtitgliebe.

1.

3ur fhlefif hen פPflanzengeographie.

a. Sur Sunbe ber ortlicten Berbåltniffe.

\section{促eretan,}

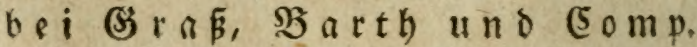

1838 . 


\section{Die \\ Hertheifung mo Herbertumg

\author{
Der
}

\section{Tdhlevithen \$flanzen,}

nachgewiejen in vierzehn (sebieten ber ichlefifchen flora.

Rebjt

einem 2 nhange

über bie $\mathfrak{B e r g l e i d s u n g}$ ber fohlefitidhen mit ber britifaten Flora.

Bon

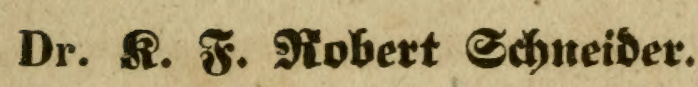

ఇebft einer botanifich=geognoftif

\section{政restau,}

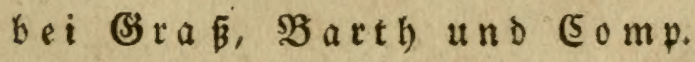

1838 . 



\title{
Seinen geliebten Freumben
}

\author{
Dem
}

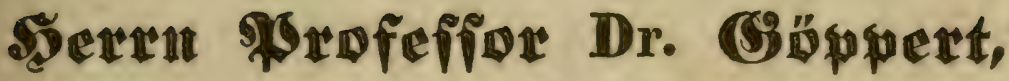

bem unetmitblidjen Forfther ber vorweltlichen Flora;

\section{bem

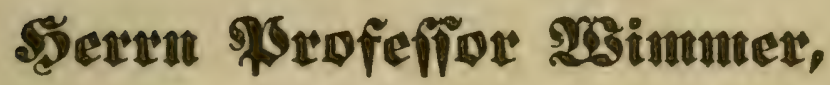

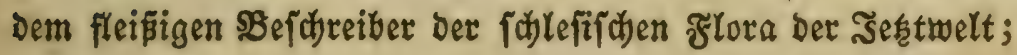

bem

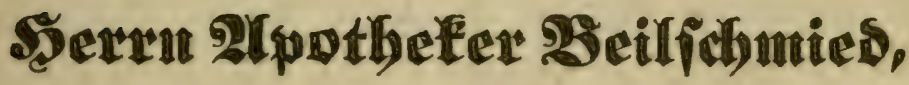

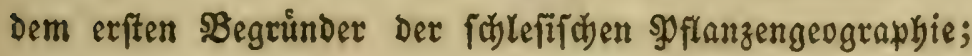

in Riebe uno Danfbarkeit

Der Berfaffer. 



\section{1 บ1 1 .}

Diefe sBeiträge enthalten bie erften \$erfuct)e zut einer genaueren Darlegung ber pflanzengeograpbifíten SBerbält=

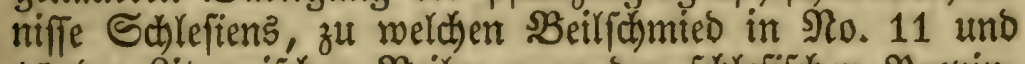

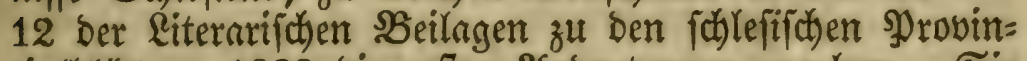
ziarblättern 1829 bie erften 2unbeutungen gegeber. Sie grünben fict auf bie Bergleictung Der \$oflanzenverthärtnifife

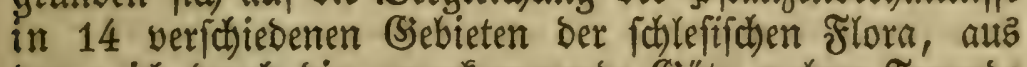

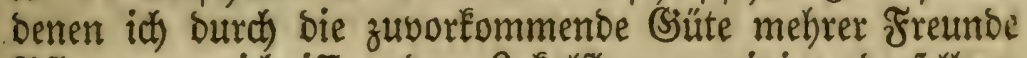

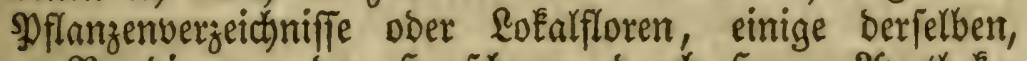

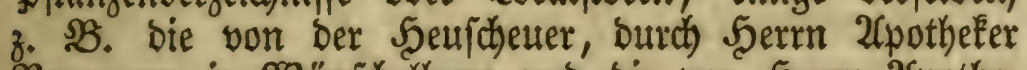
Neumann in $\Re$ Bünithelburg uno bie von 5errn 2(pothe=

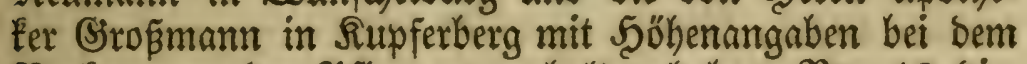
Sortemmen ber \$flanzen, erbalten babe. Sur 10 Dies

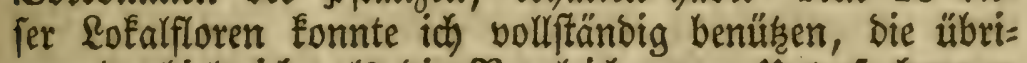

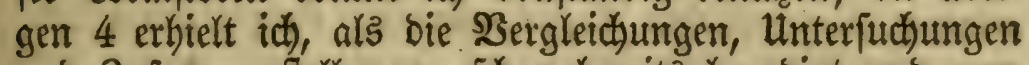
und 3ufammenffellungen fidon bereits beendigt und zum Iheil faton im Drude begriffen waren; bie Saluptreful= tate fint aber bennoch ben übrigen binzugefügt morben.

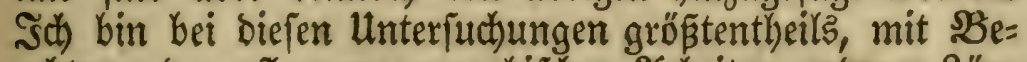

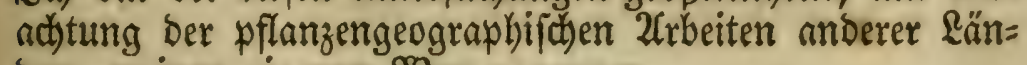

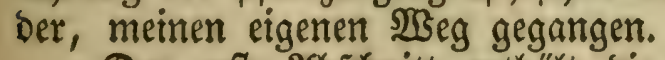

Der erfte 206rofnitt enthâlt Die Darlegung ber ört=

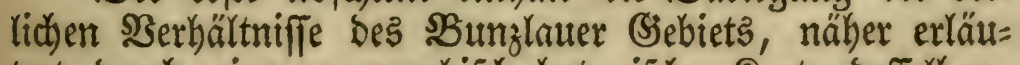

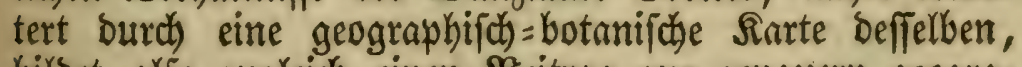
bilbet alfo zugleith einen seittrag zur genautern geogras

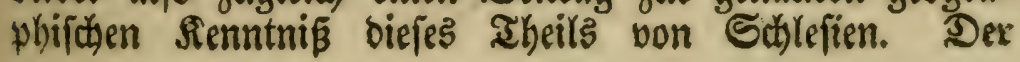




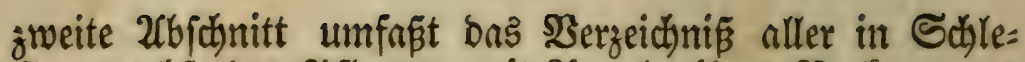

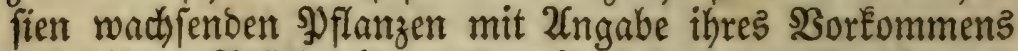
und ihrer SBlüthezeit, und mit bejonderer 2(ngabe Der

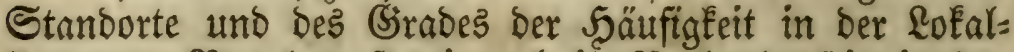

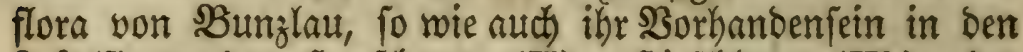

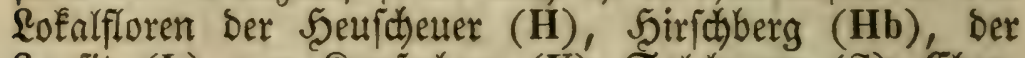

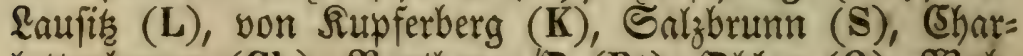

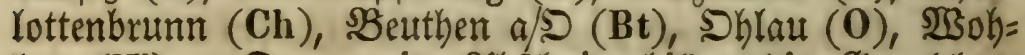

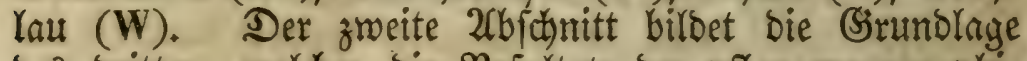
Des britten, welcter bie Siejultate ber pflanzengeographi=

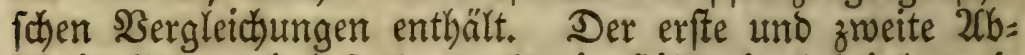
fanitt find auch befonders als ein Shilfsmittel bei botani= idjen Exfurfionen und zur 2inlegung und 2inregung von

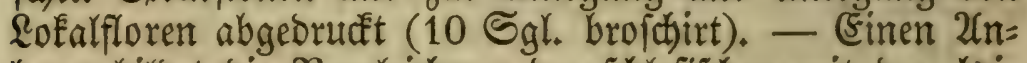

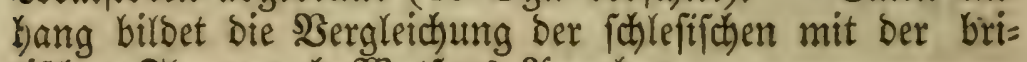

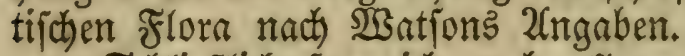

Schlię̧lich fage ich auch allen geebrten Freunden, bie mich burch Bufenoungen von $\mathfrak{L}_{\text {ofalfloren }}$ gütigit un= terftürgt haben, hiermit öffentlich meinen innigften Dane

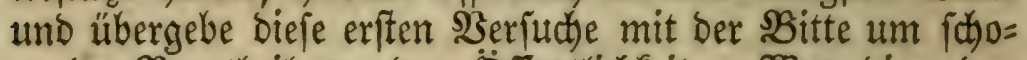

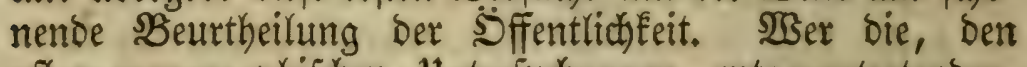
pflanzengeographift)en Unteriuct)ungen entgegentretenden,

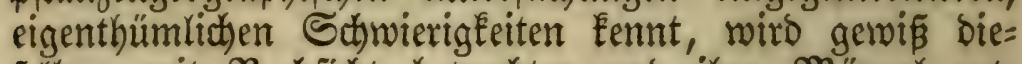
felben mit Nactjictet betracten uno ihre Mlangel ent= fichulbigen.

SBunglau ben 31. Sctober 1837.

Der Berfaffer. 


\section{Erriter $\mathfrak{A}$ bidnnitt.}

Die örtlichen Berb̨ăltniffe det Siunglmet Flora.

Die Flora eimer Begend ift von Den örtlid)en und Elimati= fchen $\mathfrak{B}$ erfältniffen berferben abhängig. Še mamnigfaltiger bie= felben befonders hinficttich) ber Seftandtheile des SBodens, nach

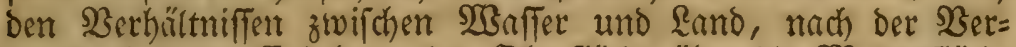
fchiedentsit Der Erhebung der Sberflïhe über die Meeresflïche fino, befto größer ift im 2f(lgemeinen ihr DDflnmzenreichthum, befto mammigfaltiger fino bie gegenfeitigen Berbältniffe oer

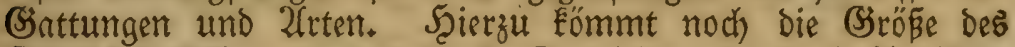
Sebiets berfelben, weil unter fonft gleichen oder boch "alhnlichen

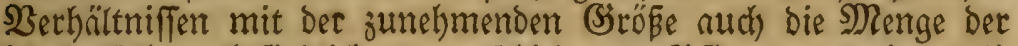
in berfelben befindlicken, verichiedenen Spflanzen zunimmt.*) Die Renntnis des (Sefiets einer Flora ift bemmach fut richti= gen SBeurtheilung derfelben nothwendig.

\section{Gebiet Der Flora von şuthlat.}

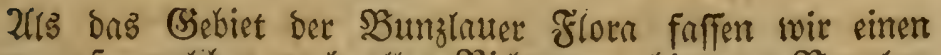

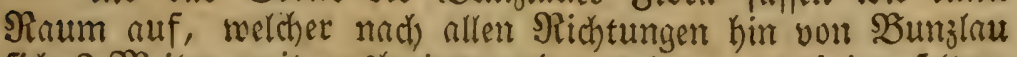
fich 3 Meilen weit nusbreitet, und aus dem unt einige, feltente SPflanzen enthaltende, Sunfte in weitere Entfermung himastre=

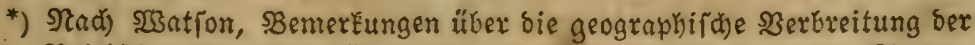

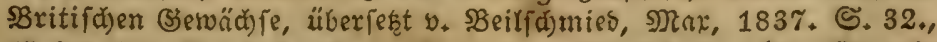
(d)eint im. Durd) Jälffte ber ganzen in sritannien gefunoenen Speziezanzall zu

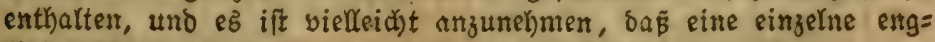
rifde Meire bie Scälfte ber Urten einer Brafidyaft enthalte. 
ten, boch aber butch cine halbtïgige 23 andoctung zu erreichen

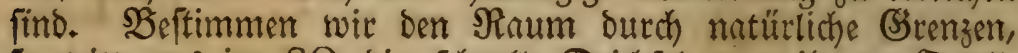
fo tritt uns im SO Die fothrelle Deichfet von ihrem Duell= punEt am Spifberg bei গ̧robfthann und ibr I Ihal in feinem

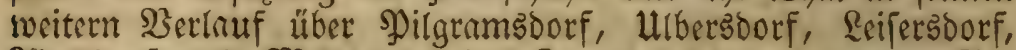
2 loelsbouf unt Modelsborf im $\mathrm{O}$ als folche entyegen. "Im NO begrenzen bie Suellarme und nördictsen 3uflüffe Des Schwarzwaffers uno die füblichen Quellarme Der Sprotte bei fiogennu. Son bier geht bie (Strenze obne Deutlidje Marfe nerch WV zum Sober und über ben Sueis zur ITfhirne, wel=

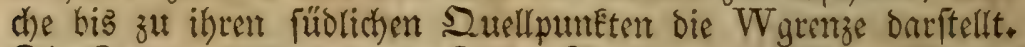
Die Sgrentze bildoet Der weftliche Theil bes fhlesitchen \$3orge= birges, welches von den uffern Der Rakbrach bei Goldberg in

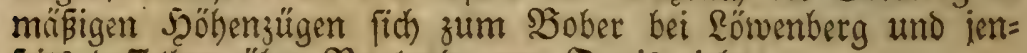
feits beffelben über গReuland zum Suteis zieht.

\section{Geftaltung Sev SGerfäc).}

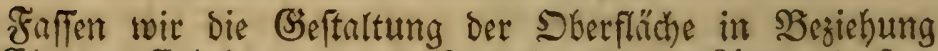
auf Ebenten, Erhebutngen und BBertifungen ins 2fuge, fo ftel= Len fich) nur wenige bedeutende \$errchiedenheitent entgegen. (5s trïgt Das ganje (Sibiet bas Geprïge simer von $S$ nach $N$ fich

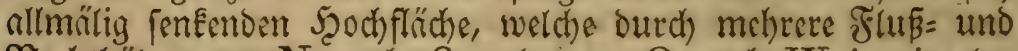
Bardththiler von $\mathrm{N}$ nad) $\mathrm{S}$ umb von $\mathrm{O}$ nach $\mathrm{W}$ in cimzelne Sarthien getremt wird, bie fidh als von $S$ nady W an Szöhe abnehmende Stufen oder Şöhenzüge darftellen und im $\mathrm{N}$ ins

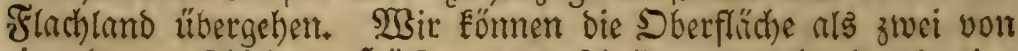
eimander ver[chiedene Şillften untericheiden, welche burch eime von ONO nach WSW Durd) Sumzlau gehenoe Rimie gettennt und als bie nörolidae ebene und füblid)e hüglige und wellenförmige am Sfaume von einzelnen J̧öhen, SZergen

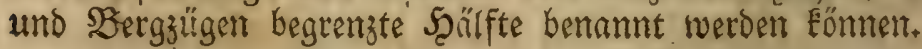

Erkebungen. Sie tringen mut am Sübfaume sinigermit= Ben dent Sergearneter uno bieten uns in den übrigen Thyeiten meht

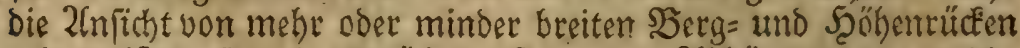
mit meift fanft uno allmälig anfteigenden 2(bh)intgen bar, welche 


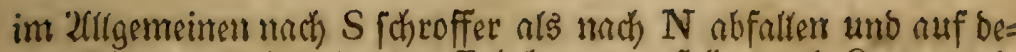
nen fich nut wenige einzelne Erthebungen, Shügel uno Ruppen zei= gen. Der Sübraum, Das WBeftende des fothefifichen 230 rgebirges, beginnt ats mákiger Shöbenzug am Sarbbachufer bei Goloberg, wiro bei \$ilgramsborf vom IL hale ber Deichfel unterbrod)en und

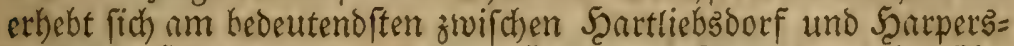
borf im Scainwald und Dem Sceiliger Spigberg bei 2 fr=

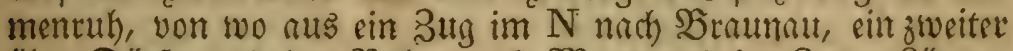

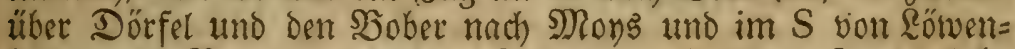
berg nach Reuland zieft. Z(us Dem nörolichen 3uge erhebt

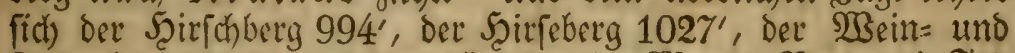

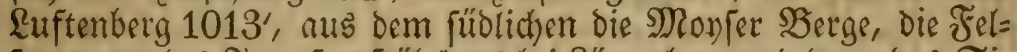
fentruppe des sutugfernftübchens bei عówenberg uno dann bas Si=

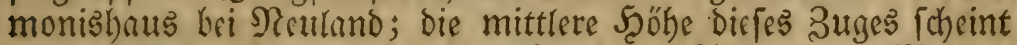

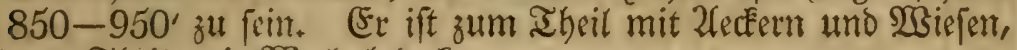
zum Iheil mit $\mathfrak{S B a l d}_{3}$ bedectit.

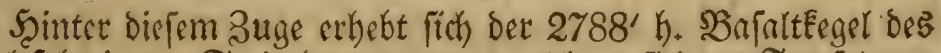

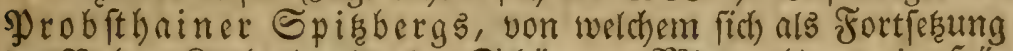

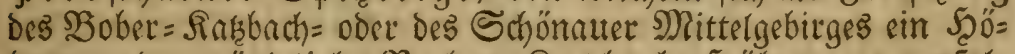

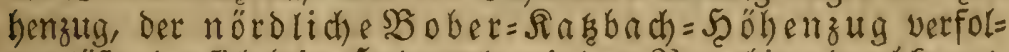

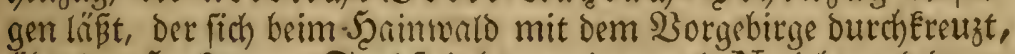
über den 5ocfennuer Sandffeinberg weiter nach $\mathrm{N}$ zieft und bann

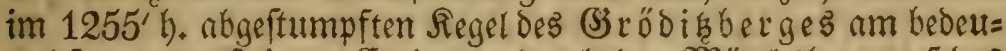
tenoffen emporfteigt. (Er Deutet Dutch) Den Mönch) 36 erg auf Das Booloberger 23orgebirge und zieft als beutlich) zu unterfheibender

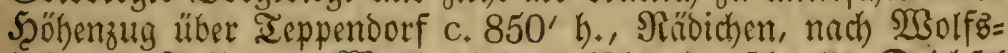

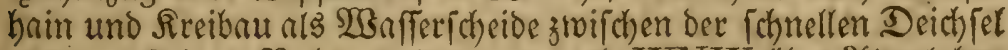

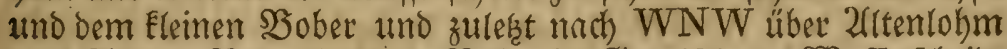

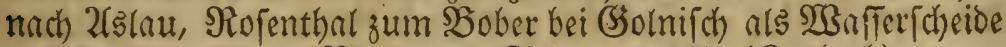
zwifhen Dem Elemen Bober und Sethanzzwarfer (

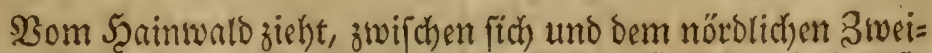

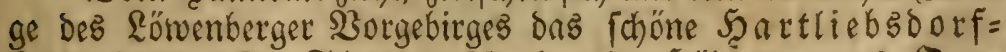

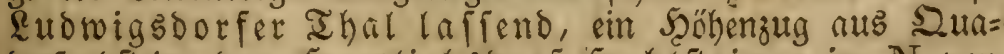
Derfanditein, Der Scartlieb3oorf = Scohliteiner im $\mathrm{N}$ won

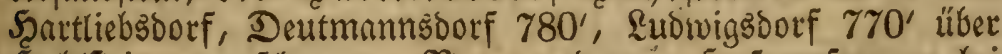
Şohliftein zum fotwarzen Serge uno zum Şufarenfprunge bei 


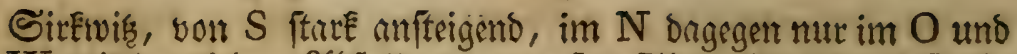

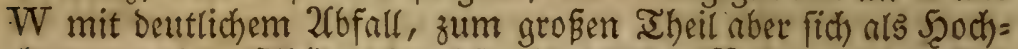

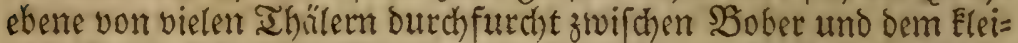

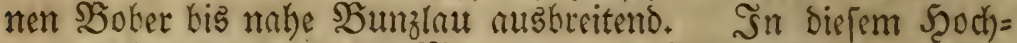
Iande zirht, ziemlich in ber Mitte zivirden $\mathfrak{B o b e r}$ und Eleitrem $\mathfrak{B}_{0}=$

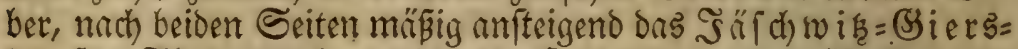
Dorfer Thal, burd) welches bas J Jochtano in einen öftlichen und weftlichen Theil gettennt wird. Der weftlid, Theil, welcher mit

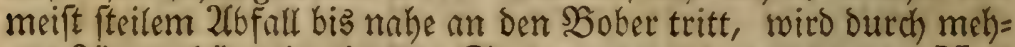
rere Riúngentbäler in einzelne Stufen gettennt, weld)e nach $\mathrm{N}$ zu

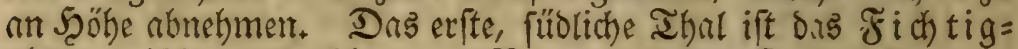
that zwichen bem fetwarzen Serge und dem Scufareniprunge;

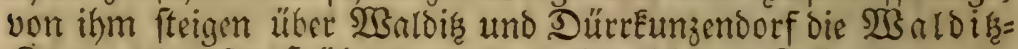
Sitnzendorfer J̧öhen an und fenEen fich ins D Dür rEunzen= Dorfer Ihal, burch weldes das Gsiersoorfer $\mathbb{M} a f$ fer von $\mathrm{O}$ nad) W aus bem gleid)namigen Ihale abfliest. Senfeits biefes

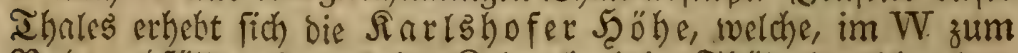
Bober abfïllt und audy im O burch) einige Thbäler butchbrochen,

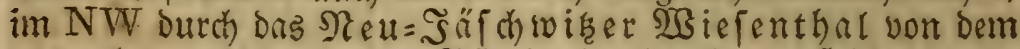

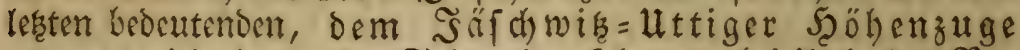
gettemt wird, ber in ber $\mathbb{S}$ dy wedenfchanze bei uttig das $\mathfrak{B}_{0}=$ berthal berührt und durch drs an (S) rö belv or wer É entipringende

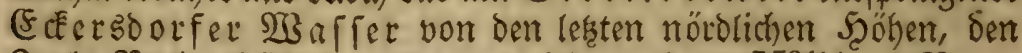

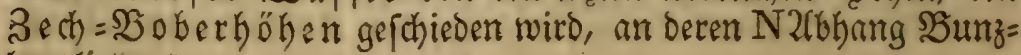
lau liegt.

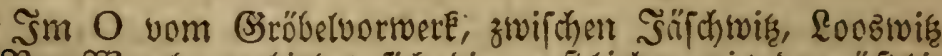

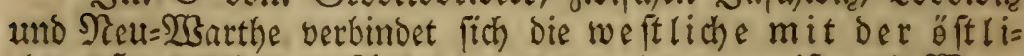

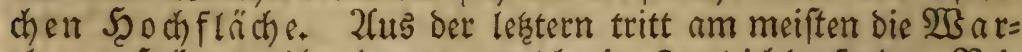
thaer Sulfgelreihe hervor, welche in 2 gleichlaufenden Rei= ben im SW von NBatthe und dem (Soldbachthale zum Net=2Rar= thace Steinbeud unt zur Sternmauer und von da in einem

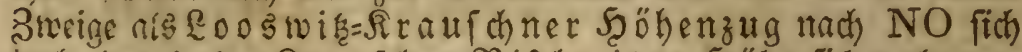
vertberitet, in ber $\Re \mathfrak{r a t}\{$ d) en=

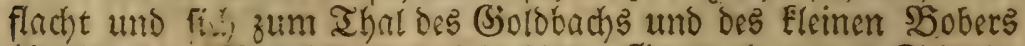
binabjenft, Szon bier aus zieht binter Ginabenberg und Schwie=

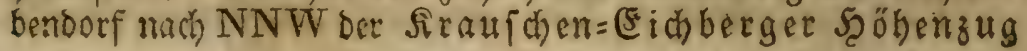


bis zum Sober bei (Fichberg, im füblichen Drittel vom Bnnobenbers ger $2 \mathbb{B}$ nfler burchbrod)en mit zum Theil fteilen 2(bhängen im W, nach NO allmälig als geneigte Fläche ins Thal bes Eleinen $20=$ bers abfallent. Ein anderer Sweig, ber Drübelhöhenzug zieht im W von Sooswif zur D $\mathrm{r}$ ü B̈elhöhe, welche ourch das Thal Des Förfterbachs von ber 3echhöhe getrent wird, im NW nach SBunlau, im NO nach Sinadenberg fich bimabjentet und im

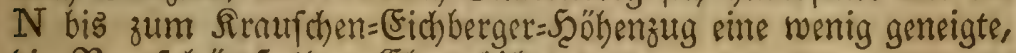

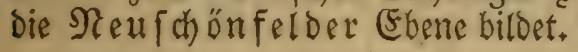

Das \&ano zwilchen Soker uno Sueis triggt noch mehr Den

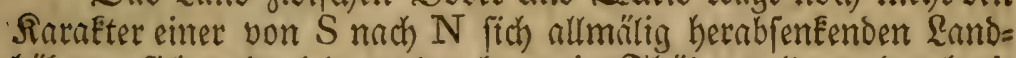
höhe an fich uno wiro nur burch wenige Ihäler und mur butch ei= nige Şügel unterbrochen; Doch erbält fich Der SiaraEter bes Şügel= und 2 Bellenlandes noch bis SBirEenbrück, von wo an bie Şaide=

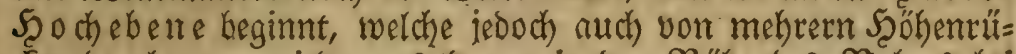
cFen burctzogen wiro, aus denen in Der Nähe bes SBobers bei

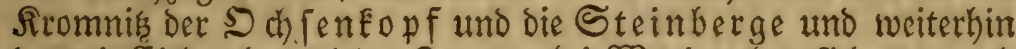

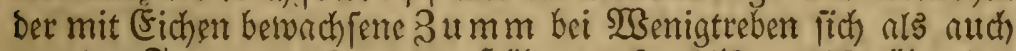
von Der Ferme zu erEemente ScöhenpunEte 150-200' über Den Boberpipiegel erbetien.

Senfeits des Sueis tritt noch mehr Der Farafter ber Esbene herbor, aus welcher in ber Nähe von STaumburg und $\mathbb{B}$ nalonu einige einzelme Scügel emporfteigen; im $N$ bavon zeigen fid nut

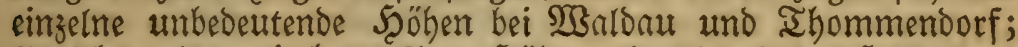
fie ziehen als wenig bemertbare Scöhe noch weiter in Der Scaibe fort.

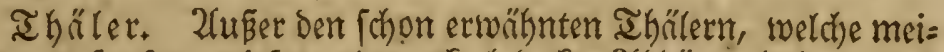
ftens nur fanfte, wiefen= oder adferbedecfte 2lbhänge baben, find

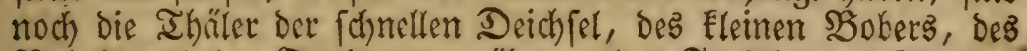
Sobers und des Sueis zu erwähnen; bns Flusthal Der ITchirne tritt faft nirgends beutlich als a Sal vor 2fugen und ift bir feimem Fortgang in ber Scaibe burch Sumpfwiejen und Mloorbrüche bezeidi)net:

Die Flubthäler ber \{chnellen Deich fel und Deb Eleinen Bo bers fino burch ifren rsichen 2(nbau aubgezeichnet, ein Dorf reiht fich in bemferben an bas andere; bas Deichfelthal mit feiner 
langen Gaffe begremgt nur. SIn Eleinen 230 berthale liegen Wilkelm5oorf, (Sroß = Şartmanuedorf, Mittlau, Ricbichau,

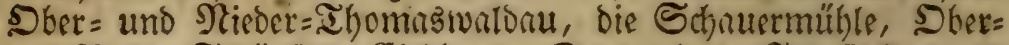
und Nieder=Schönferd, Eichberg. Das obere Flüthal bes

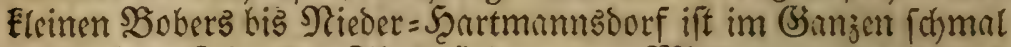
mit make anftsigenden Ihalwintoen; von Mittlau an ertweitert eక fich, bie Thaltwände treten mehr zurück, werden fanfter anfteigend, breite $2 B$ irfeniftrifen, von Bebulf hen hin und wieder unterbrochen, liegen zu beioen Seiten, bas mittlere $\mathfrak{B}$ öberlethal, weldhes fich bei Sber= Ihomastonldan wieder verengt, unterhalb aber als un= teres Flüthal an Sbreite zunimmt, häufiger mit (Sebüfeb be= fest ift und nod) tweniger aniteigenoe Ihaltwönde hat.

Das Flüthal des $\mathfrak{B}$ obers wiro bis Sumzlau von 100 - 150' hohen Ihalwänden gebildet, welche eimen buth fhnitt= lichen Neigungswintel von $20-30^{\circ}$ haben. 2(n cinigen Stellen

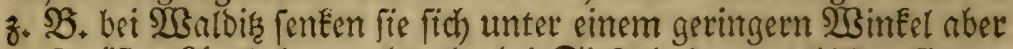
auf größere 2luzbehnumg herab, bei Sirtwiß̧ dagegen bilden fie am Scufarenfprunge 100' bohe Felfentwände, welche unmittelbar aus bem 30 beripiegel emporfteigen; bei Rackwis am jenreitigen ufer baben fie einen Neigungsivintel von $35-40^{\circ}$. Nur bei Sirk = wis und zwifochen Rroifchwis und Bunglau find fie betwaldet oder mit Sebüfhen berest, fonft meift mit 2leceern bebaut; an meh=

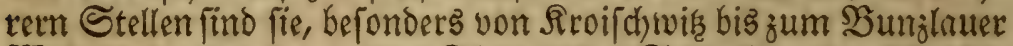
Webre von einzelnen, zum Theil tiefen Schludsten burchfurcht, von benen einige burch vertaffene Sandfteinbrïde entftenden find.

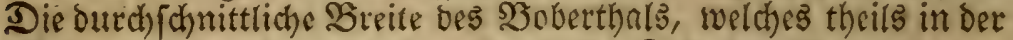
Mitte, theils mehr auf rechter oder linker Seite das 93 ober bet te bat, läßf fich auf 1,2-3000' nnmehmen; doch ertusitert es fich be=

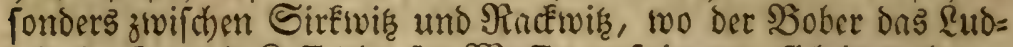

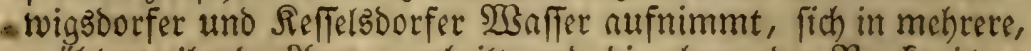
mühlentreibende 2frme zertheilt und bie obere oder $\Re$ a cétwifer Bobertu bildet; zwifden Siroifhwis uno sRothlich bagegen verengt fich bas Thal. Der Ifyalboden ift gröstentheils, be=

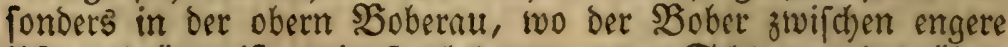
ufer gedrängt ift, mit fruchtbaren, vom Schlamm beš über= forwemmenten Sobers geoüngten SBiefen beocelt, auf benen im

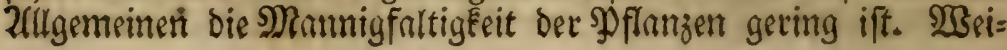




\section{7}

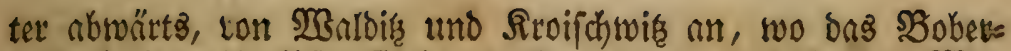
bette beriter, Lie Ulfer flacher werben, treten zwifchen ben $\mathscr{W}$ Bies

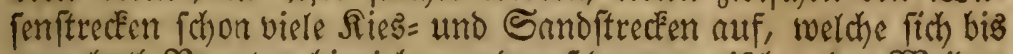

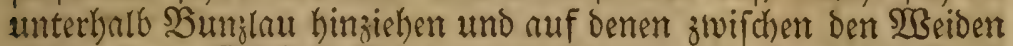
tnto inderm (Sebüifch (Rhamnus, Prunus Padus) vorzüglid Oenothera, Sarothamnus, Lathyrus sylvestris, Echium, Saponaria, Tanacetum in großer SRenge gruppenweis twach=

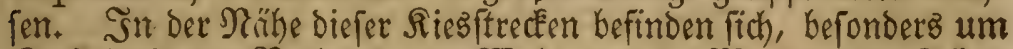
Iroifchwis uno Rothlach, am 2 Behre uno bei 23 iefaut viele Tüm= pel, Sobertilmpel, voll eines meift Elaten ober eifenbaltigen WBaffers von eigenthümlichem Seructh, weldhs, bie Heberrefte vom

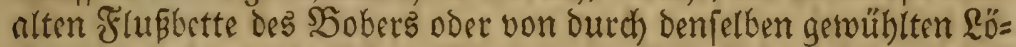
chern, mit hyorophytifchen গy flanzen angefüllt find, befonders mit Ceratophyllum, Myriophyllum, Hottonia, Isoêtes, Potamogeton, Nymphaea, Utricularia, Callitriche, Lemna, Ranunculus aquatilis, Acorus, Phellandrium, Scirpus radicans $\mathfrak{H}_{2} \mathfrak{m}_{0} a_{+}$, uno viele \$flanzennusbeute geben. Die

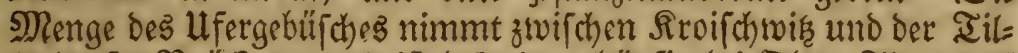
Ienborfer SBruicke zu uno ift bejonders häufig bei Sber = Iillendorf

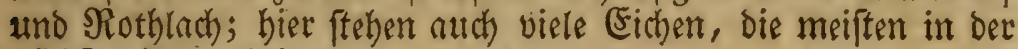

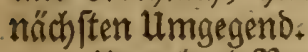

unterhalb Sumzlau wiro bra Boberthal weiter, bie untere Boberau; Die Ifhatwände werben niebriger, Eahter und ftellen fich weniger als Erthebungen und Scügel, Fonbern vielmehr als bie

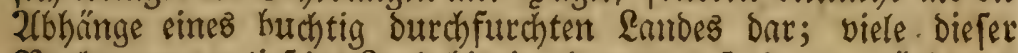
Buchten trefen tief inb \&and binein, Deuten auf einen veränberten und höhern Rauf Des Bobers in früheren Beiten. Statt Der $\mathbb{B}$ Bis= fen und Giebü che zeigen fich nut (jebüfchgruppen, MBefenftreifen und Der meifte 20 oden iff zum 2féerbau angewendet. Einzelne Eict)en und cinzerne $\mathfrak{R} a c h e n$ zeigen fich noch bin und wieder; unter= batb Eicthberg nehmen die Eichengruppen wieber zu. Die Ihal= woüroe fino grö̈stentheils von Sandrethen gebildet, fint 20-30 $-40^{\prime}$ boch und enden oben auf beiden Seiten in weite thonig=fan= bige oder rein fandige Ebenen; welche, meift mit Scaibe bedectet,

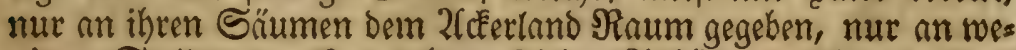
nigen Steflen von 'Eaum bemetélichen 2(nböben wellig unterbros chen werden. 


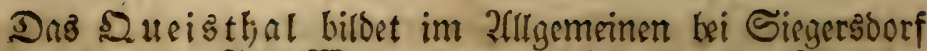

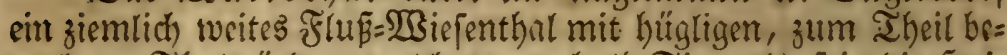
waldeten I Saltwänoen, weldes unterbalb Siegersborf in bie Şai= deregion eintritt, von ber fich, befonders auf ber red)ten Seite von Sirternbrlid her, fumpfige Iorfmoote bis ins Thal binnthiehen, (Andromeda, Erica Tetralix, Vaccinium Oxycoccos, uliginosum). Sei Ihommendorf nimmt es ben Sinrafter ber untern'Soberan an; von ben Şod)flichen Der Şaiben fenEen fich von beiden Seiten meift fandige, wenig betwach fene Iefhen binab

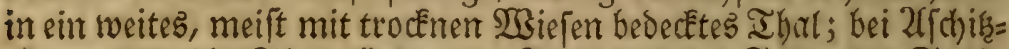
au werden bie Ihaltwände zum Theil von den Felien der Gand= fteinbrüche gebildet. (Viola tricolor mit großsen, fdoön blau gefïrb= ten BlumenEronen, Anagallis mit lillafarbner Rrone). SDerthalb WBehrau treten die Ihalwände näher zu[ammen, bas athal füllt

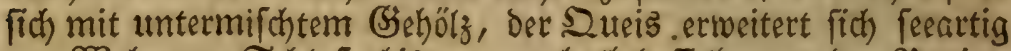

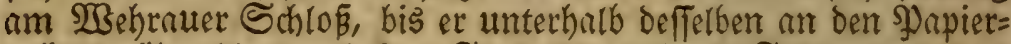
mühlen über die burch Den Flü ftch ziehende Fellenwand bes Ie ufels wehres (ein quarziger, fefter Sanditein) als 2 bafferfturz in feimer ganzen Breite fich ichüumeno himunterftürzt, fowohl beibe

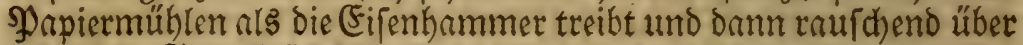
zahleciche Felienblöce fortfließst, fo daß man fich zu feiner groben

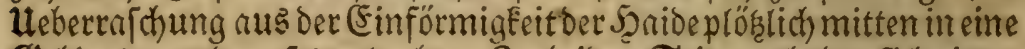
Esebirgsgegeno ver est glaubt. Su beioen Seiten erheben fich ziem= lich fteile, zum Iheil betwach fene Ihaltwände, welche fich, befonders am linken Utfer, Durch Sanofteinfelfen (Der RaffreÉrug) gebiloct, em= porbeben; bei Rlitfchoorf erweitert fich ons Shal uno bildet be= fonders am Schlofie einige weite, mulden=thalförmige Sathten, weldye von Eablen Sandbergen eingefd)loffen fint. Bon hier nus wiro bas ISt breiter und bie 2(nföhen verlieren fich.

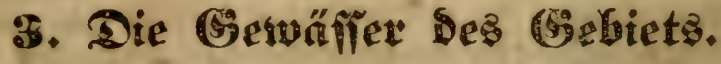

Das Giebict umfaßst Den unittlern Theil bes Mittellaufs des 23 obers (Rähn bis Eagnn) (Flora des mittlern Soberges biets) ganj; zum Iheil gehört es bem otern Rauf ber fch nellen

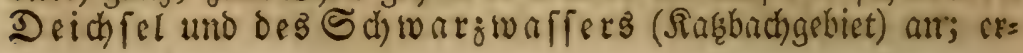


ftere berührt bie SIttgrenge, lef̧eres gebört nur in ihrem obern \&auf mit ifren Quellen Dem Sebiete an.

Der $\mathfrak{B}$ ober Gat nốroliche F̧auptrichtung, bildet in der obern

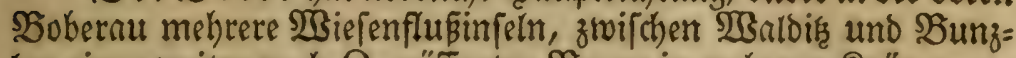
lau eimen weiten nach $O$ geöffneten $S_{0}$ ogen in mehtern Rrümmun= gen, fenbet am 2 Befe ber $\mathfrak{M}$ ühlgraben und biefer von ber Sd) (eube ben 2(bzugsgraben ab, welcher fid mit dem erftern am Scoipital wieder vereinigt und bie vom \$Bober und Mühlgra= ben gebildete Sinfel in einen weiftlichen unb öftlichen Iheil, $\mathfrak{B} u \mathrm{rg}=$ lehn und Sand genannt, theilt. $\mathfrak{B}$ on lesterem jieht ein mit

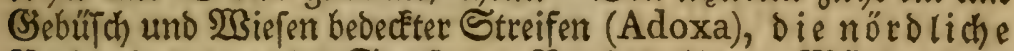
B oberinfel von ber Straß̉e am Sanuhofe bis zur Münoung des Mühlgrabens an Der Niedermüble; bewirffert von Dem nördlichen

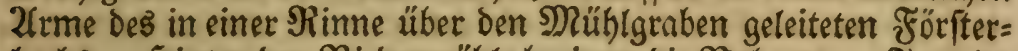
badh. 3. Şinter ber Niebermühle beginnt Die Bobernu. Senfeits

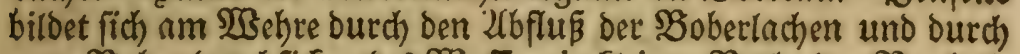

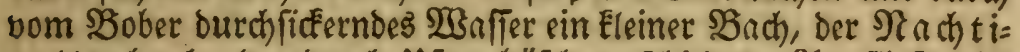

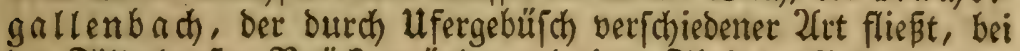
Der Iillendorfer Brücte mündet und einen Ihyeil Des Eiefigen, aber bewach fenen Soberbettes als Infel, we ftliche $\mathfrak{B}$ oberinfel im W begrengt.

Während feines Raufez burch bas Gebiet nimmt ber Bober auf ber rechten Seite bis solngwiąer=, Rubwigsborfer=, Dürtekun=

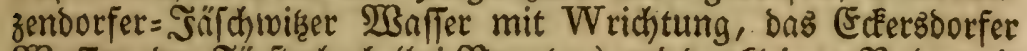
2Briffer, Den Förfferbad) (bei Sunżlau) und Den Eleinen Bober mit

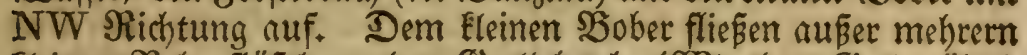

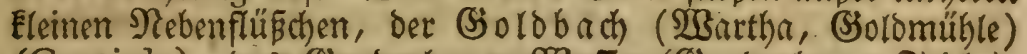
(Gratiola), Das" Ginndenberger $23 a f f e r($ (Snadenberger İeiche),

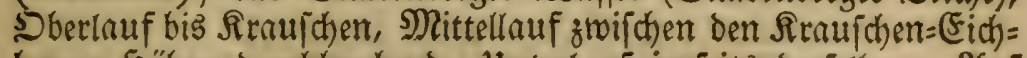
berger Şöhen Durchbrechend, Unterlauf jenfeits Derferben. 2luf Der linken Boberfeite ift Das Reffelsoorfer uno Das Dttendorfer 2Banfer.

Der $2 u e i s$, twelcher von Naumburg aus ziemlid) nad) $N$ fließ̧t uno mehtere Ŝnfeln bildet, bei Siegersoorf in mehrere 2(rme

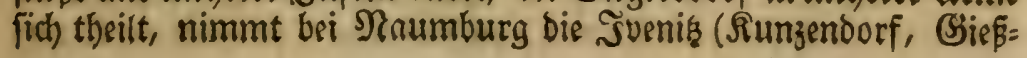




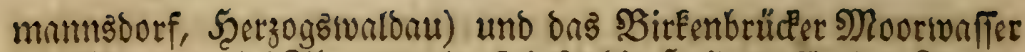

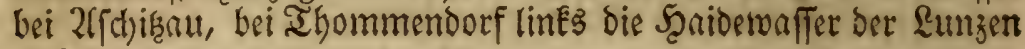
nuf.

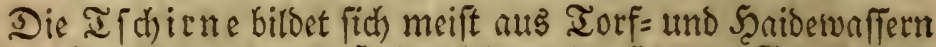

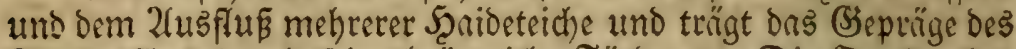
Iorfwaffers burd feine brïunliche Firtbung. Die Duellen bes Sch warzwaffers fint aud) Scaide und Iorfquellen.

Stehende Gewäfier giebtes im Simzen nut wenige und

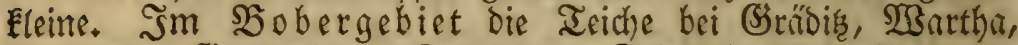

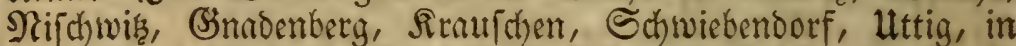

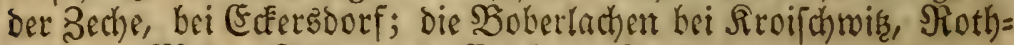

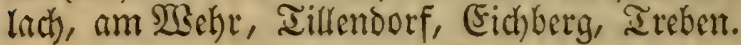

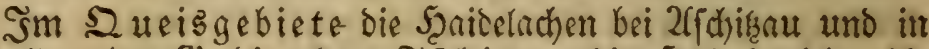

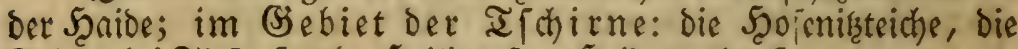
Ieid)e bei Tiefenfurth, Sceiligenfee, Sceibrgersoorf.

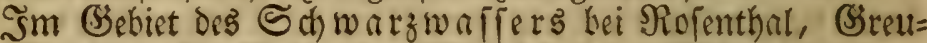
lich, Moblau; im Sebiet ber Deichfel bei MRattinstwaldau, Ireibau, Steinşor r.

Menge.

Sümpfe und Moore bagegen zeigen fich in groper

Sümpfe: in ber 3eche, am Förfterbach (Calla), am Siolo= bach (Epipactis palustris, Gladiolus, Valeriana dioeca),

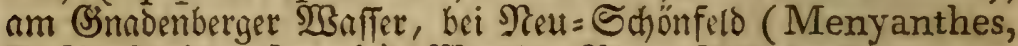

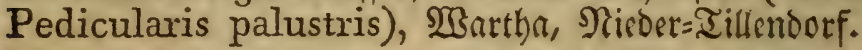

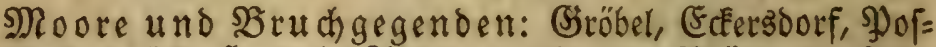

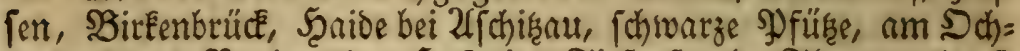

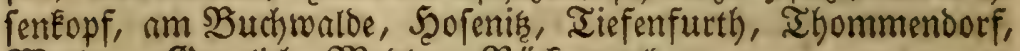

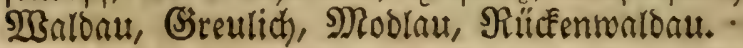

\section{unterlage uno umgébungen.}

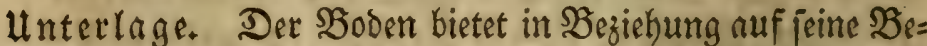
ftandtbeile nur geringe Berfchiedenheiten bor. Die Süobälfte deF=

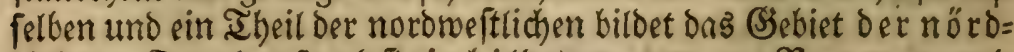
lichen 2 uaberfanditeinbiloung von v. Raumer und umfaßst mit 2utusnahme bes füboweftlichen ITheils das ganze Ëebiet

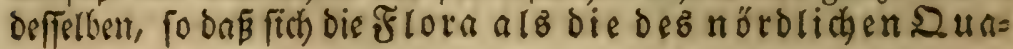




\section{1}

Derfandfteingebirges und der fildöftichen Şatben bezsichnen ließ̄e.

Der 2 underfandftein wirb im $S$ im Zfllgemeinen von bem Sorgebirge begrenzt, zieft fich aber von Biepmannsborf, wo

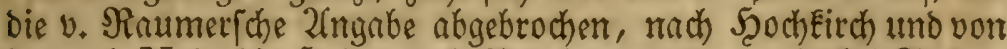

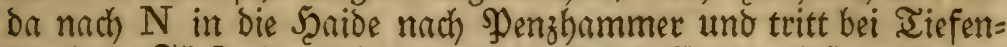
furth) am Förfferhauje (nusgegeichnet burch) Sicndeenblätter, andere

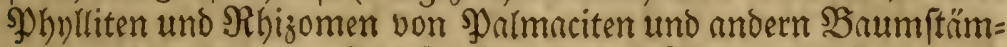
me) wohl als norbweftlichfter Sounft bes Suaderfandfteins auf,

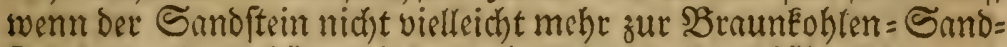
fteinformation gefört, mit ber er, befonders mit ber böhmif chen gro=

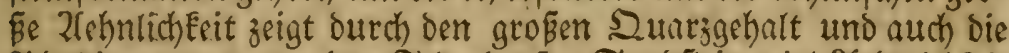

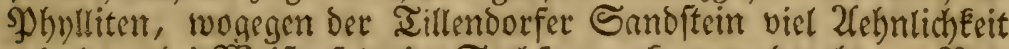

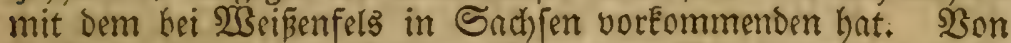

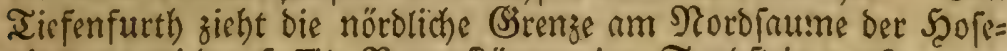
ntis, wo wieder foffile Baumftümme im Sanditein vorEonmen

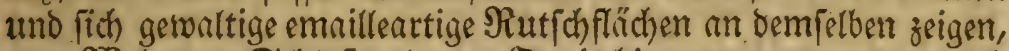
zum $2 \mathbb{B}$ chrnuer $\subseteq$ d)

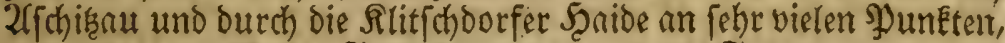

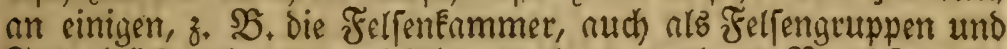

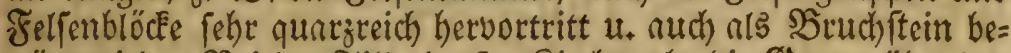
nükt wird. Sei Der Iillenoorfer Rirche geht Die Gsentze über Den Bober, hinter Dem Schieß̈haure weg zum Drüßel, und von ba über

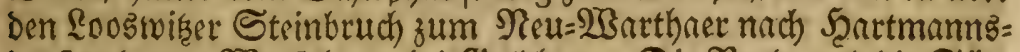

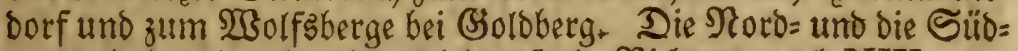
grenze haben eine ziemlich gleid) laufende ßichtung nach NW.

Der Qunderfanoftein felbft ift größptenttheils mit aufge= Fojwemmtem \&ande bebectet und tritt nur im 30 orgebirge und an wenigen Stellen ats Felfen hervor, woburch fich bie noboliche 2ua= Derfandfteinbildung yon Der [üblichen außer burch bie geringere (Er=

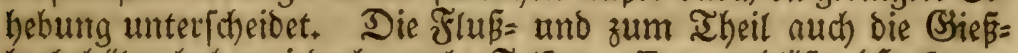

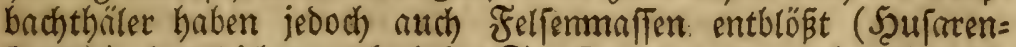
[prung), beşgleidjen aud einige Straßen, vor allem aber: bie zabl= reichent Sanofteinbrüche.

(Die ruid)tigften berferben find bei \$̧ockenau, Şartliebs: borf, Deutmannsorf, Mobs, Neutand, (sięmannbori, 


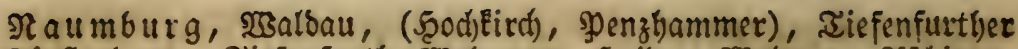

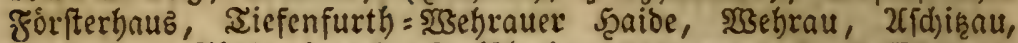
Dobrau, Tillenoorf, Rroifdwis, Sttendorf, Refleleborf,

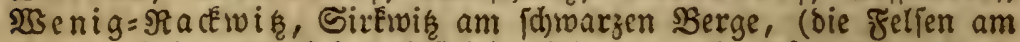
Sd)ottenfteine uno bei 5oblifein), (Biersoorf, Dirr=Sunzendorf,

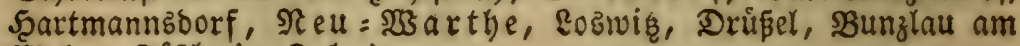

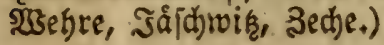

Betrachten wir ben aljo aufgedecften Sanditein, fo zeigt et fich unter mand)erlei Modifientionen. Ser füblide ift im 2ullge= meinen feinförniger, fefter, Elingenter, weiker, be ponoers gilt diefes

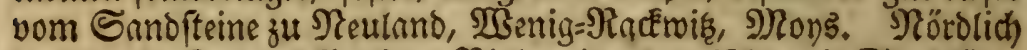
Dnvon tritt ftatt Des Eiezligen Sindemittels ztwif hen bie Sandeörm= d)en ein meht thoniges, wodurdh ztwar bie Frotbe noch tweiper, aber ber 3ufammenthang ber Theile geringer wirb, aud treten

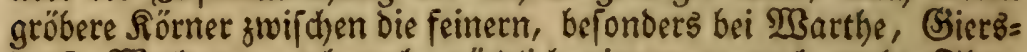
borf, MBridau; nod) mehr nördlid) nimmt er nod) mehr Ihon auf, verliert baburd am 3ufammentang der Itheile nod) mebr und ift burch bie reichen Ihonlager bei Iillenoorf, in ber Seche, bei Dttendorf uno $\mathfrak{W e b r a u ~ a u s g e g e i d n e t . ~ D e r ~ \Re o r d f a u m ~ b e s ~}$ Suaderfanditeins wiro von einem grobË̈rnigen, Eonglommeratnr=

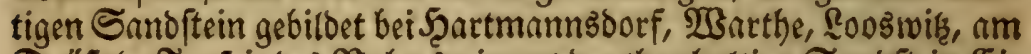

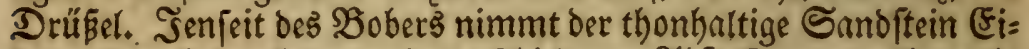
fenornd auf, burd) ben er in veridhiedenen 2(b6fufungen getb, roth), braun geförbt wiro, bejonbers zwijhen Iillendorf uno Dobrau und ber Scaide. Sn ber Şaibe tritt in ben Eonglommeratartigen Sanoftein ein Eiesliges $\mathfrak{B i n d e m i t t e l , ~ o u r d ) ~ w e l c h e s ~ b e r f e l b e ~ i n ~ e i n ~}$ feftes, quarzäbnlid)es (seftein verwandelt wiro, oas fich nod) wei= ter am Teufelswehr und in ber $\mathfrak{B}$ ehrau = Tiefenfurther Scaide zeigt uno burd) grobe emailleartige Rutich flächen ausgegeichnet ift. So läst fich ein füolicher, felter, cin mittlerer, thonhaltiger und ein nörolicher, Eonglommeratartiger Sanditein unter fbei= ben. Der fübliche Sanoftein enthält vorgüglich thierifhe Heber= refte, befonoers zweijchalige Mu[cheln uno (S)rnphiten, im mitt= lern treten, bejonders im weniger thonthaltigen, Fufoideen, vielleidht auch Calamites, entgegen, ber fehr thonbaltige zeigt filtner orga= nirche Utebrerefte, Der nördliche locfere, Eonglommeratartige ent= hält Muf̣cheln, oer mehr Eieslige im NW \&anbpflanzen=Ulebertefte, 


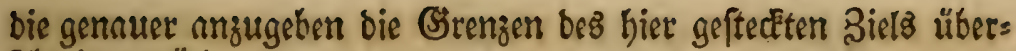
fchreiten würoen.

Süblich wirb biefer Suaberjandiftein burch ons nöroliche rothe Sanditeingebirge begrenzt, weldhes nur bei Neuland mit feinen Sinpslagern, bei जsieß̄mannsoorf, Seifersoorf und Runzendorf

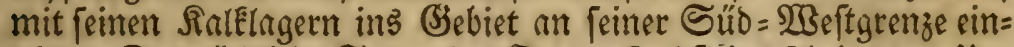
tritt. Der nöroliche Saum bes Suaberfandfteins focheint von $\mathfrak{u t}=$ fohiefer gebildet zu fein, welcher, jedoch meift von Schtwemm= lanb überdedt, nur an wenigen Stellen zu Tage Eommt und als Norbarm bes weitlichen Ulifhiefers, oder alsె nordweftlicher Urid)iefer zu betrachten ift. (Er tritt als (s) limmerf chiefer bei Ieppendorf, bei Sber = und Nieder=Thomastwaldau nuf beiben Seiten bez Eleinen SBobers, als (5) rün ftein mit Diftazit bei

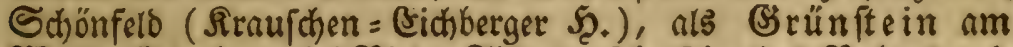
2Binomühlenberge bei Nieber=Tillendorf jenfeits bes SBobers unb

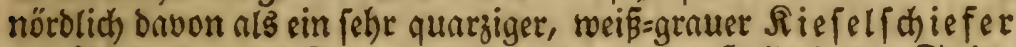

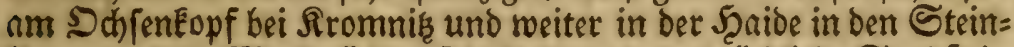
bergen auf. (פRerEwürbig ifts, baß auch ber nördliche Sanditein fo quarzreich ift). Die Scauptrichtung Diefer einzelnen SunEte ift nach NW, fo Das biefer 3ug mit bem weftlichen im fchlefifchen Dittelgebirge uno über \&auban uno (S̈örlis hinaus gehenden Schie= fergebirge eime nach NW geoffnete Mulde zu bilden id)eint, in welche fich ber rotbe und ber Suaberfandftein binein geligert haben.

3wifden bem Sunderfandftein und bem nordweftichen $\mathfrak{U t}=$

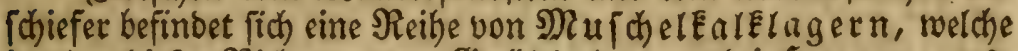

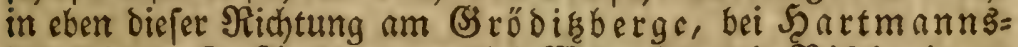
borf in groper 2usbehmung, bei $\mathfrak{W}$ a rthe und Nifd wif zu Tage liegen oder gebracht fino und nach langer unterbred) ung zu=

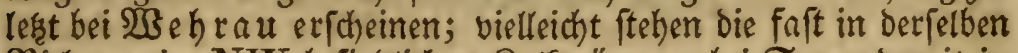
Richtung im NW befindtichen Raletrümmer bei Sornu bamit in Berbindung. Die Sdjichten biejer NalElager nehmen im 2allge=

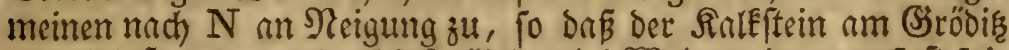
und bei Şartmanmboorf fait röhlig, bei 2 Behrau bagegen faft fai= ger ift. Sollte biefer Ralfeftein bem bes nördlichen, rothen Sand= iteingebirges entiprechent? Dabin beuten bie Ralebrüche bei \$a ajel 


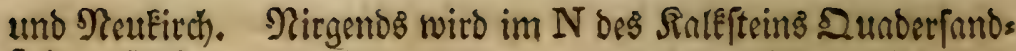
ftein gefunden.

2fus dem Qunderfandftein erhebt fich an mebreen গunEten

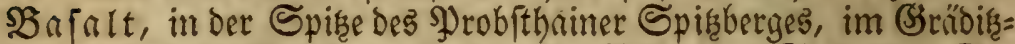

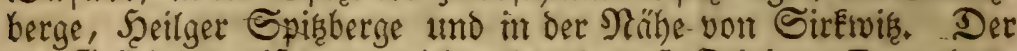

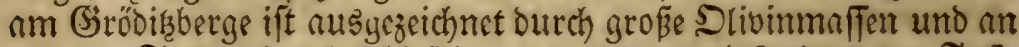
einigen Stellen burd) (d)iefrige, an andern, befonders nm Frupe

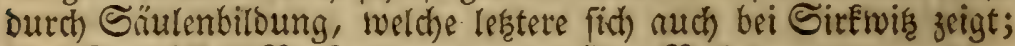
bier ift aud) bas SYorkommen von porófem Saafalt.

Die ben Dunderfanditein bedeckenden Maffen find im $S$ ein meift fruchtbarer, nut an wenigen Stellen mit Sand abrwech feln=

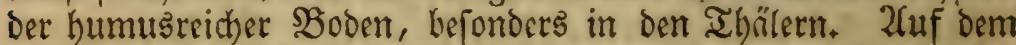
Davor liegentoen, burd)furchten Şochlande bagegen befinden fidh zum Theil fehr hohe Ragen von Eiefigem (S) ruß umo mebr oder minber grobent $\Re$ ollifteinen und Sserdhieben, welde be=

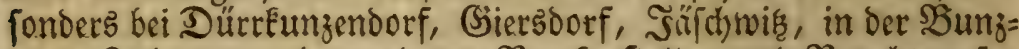
lauer Beche aus abgerundeten Bergernftallen und $\Re$ auchtopafen (2Banfiertopas) und 2(methyiten von mehr als Ei= biz Fraftgröß̈e, To wie aus 2lchaten, Falgebonen und saspis und einer groben Mentge weiser 2 unrzgerchiebe beftethen, aber mur wenig fruchtbare Eroe enthalten, fo on . Der Sooden faft überall sinen Dürten, trock" =

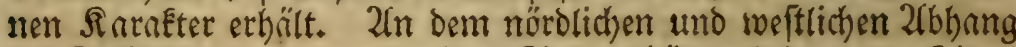
Des Drüßę Sand mit Braunéohlen= Rettentagen uno Sputent von BraunEoh= Ien und Thoneifenjtein, fo wie sin fehr eifenbaltiges, grobeiefeliges Qunizenglommerat, jeigen fich hin uno wieber Felfenblö de yon (Sranit mit fhroarzen (Simmer uno rothem Jeloipath; ber

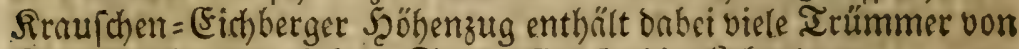
Sitefelfajiefer; an einigen Stellen find jandige Lechmlager.

Senfeits des Eleinrn Bobers tritt ein feinĒörniger Sano auf, welcher fidc) Dann in ber nördlichen Senibe weiter verbeeitet, wo er, wie nuch jenfeits bes SBobers, zwi d)en iffm uno dem 2 ueis und zur Tifhirne und weiterhin bie Scruptmaffe der DGerEtrume bildet und nur hin unt wieder von mehr ober minder ausgebreite=

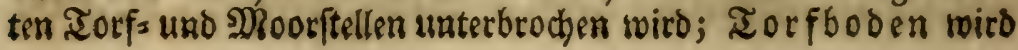


an vielen Drten gefunden, befonbers in ber Scaibe am \$uthroalbe

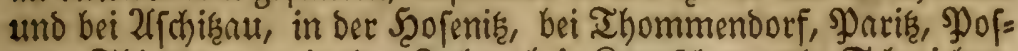
fen, Thiergarten, in Der Zeche, bei Araujchen und Schwiebens borf, Martinswaldau, Sreulich.

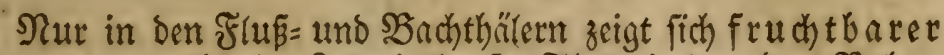
SBoben, namentlich im Rubwigboorfer Thal, in ber obern $\mathfrak{B}$ ober=

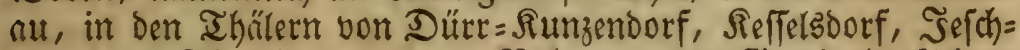
wiś, zum Iheil in Der untern SBoberau, am Soldbach, Eleinen Bober, am Groöbis und Spisberg, im 3umm und an einzelnen Stellen ber Şaide, doch zeigt firth an fehr vielen Stellen bie Şin= neigung zur Fauern Scumustiloung, bie Scirpus, Eriophorum, Drosera bejeichnen. Das $\mathfrak{Q}$ ueisthat und bie übrigen Thäler baben nur mittlern Boden, unterbrochen yon Sand uno Serölle.

Das eigentlid) Sand = ober Scaibegebiet beginnt jenreeits bes fleinen Sobers bei Sichtenwaldau, Mattinswalbau. 3rwichen Bober und 2 ueis beginnt es im S, wo ber eigentliche

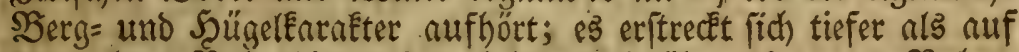

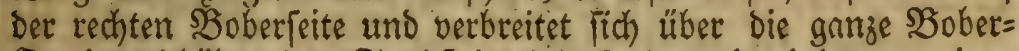
Sueislandhöhe, ben Sandfrein bebeckent, mit einigen wenigen fruchtbaren Strichen, (Bumm, feuchter Miefenftreifen vor ber [chwarzen Mf füke uno mit Torf= uno Moorgrünben wech je(nd).

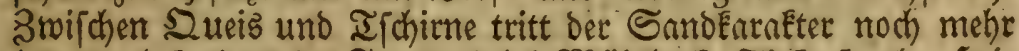
herbor, befonders ber Fllugfund bei MRüblbod, Tiefenfurth), Scei= ligemiee u. T. w.

umgebungen. Sie bieten eben fo menig grope Serrojie=

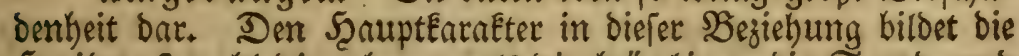
Şaibe, foroobl bie ebene, als bie hüglige, bie Sand =und Die Sand fteinhai be, in welcher lesetern fich nach und nach bie Iorfmoore verlieren. (Sróß̈tentheils wiro fie in ber Sanbhaibe von Siefern gebildet, nur an einzelnen Stellen untermircht mit Tannen und Fidyten, mit einzernen $\mathfrak{B} u$ chen und (Eidhen. Regtere nefymen in einzelnen Gegenden, bejonders in Dent fruchtba=

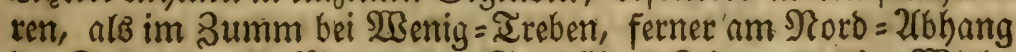
Der Steinberge an Menge zu. In frübern Beiten war eine $2 \mathbb{B} a l l=$

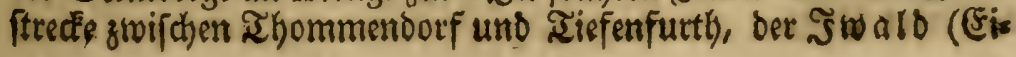


benbaum, Sbaum) reich an (Fibenbäumen (Taxus), weldje fich bis nuf wenige vertoren haben. Siele Scaibeffetlen, bejonders wo bas lcibige Streurechen ftattfindet, fino ganz bürr uno Eahl, ober

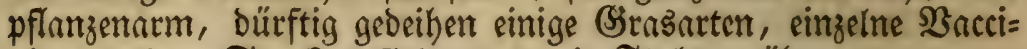
nien; andere Strecken fino ganz mit Flechten überzogen, unter Denen auch hin und wieder Cetraria islandica vorfómmt; weite Strecfen fint mit Calluna, mbere mit Vaccinium Myrtillus und Vibis Idaea, andere und zwar feuchte, torfige mit Ledum bedecft, welche mit Andromeda uno Vaccinium uliginosum untermifoht fint. Zuf trodfenen, lichten Stellen fteht Cytisus nigricans unt Genista germanica, pilosa uno tinctoria.

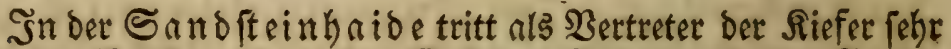

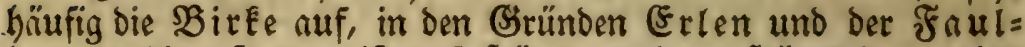
baum; Die erftern meift auf Şügeln und an Şügellehnen, Diefe zeigen wenig Pflanzenreichthum, fino wegen Der Sand fteinunterlage oder bes Gerölles meiftens zu trocken; an ben lekstern fteht Fehr Gäufig Oxalis Acetosella. Die Boberlehnen fint meift mit Raubholz betwachjen, bod) auch hier herticht Der trodfene Farafter yor, mangelt ber humusteidye $\mathfrak{B}_{0}$ dert. Nur in ber Nähe bes

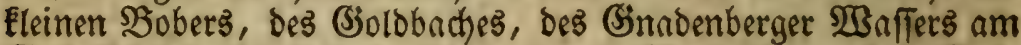
(siünfteinthügel, befonders aber am Norbabbang bes (Srö= Dik, am Spikberge, am Nhange bez fohwarzen Berges bei Sirt'=

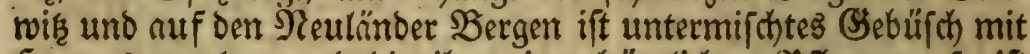
Scumusunterlage und bie ihm eigenthümlichent spflanzen, fo ift zum Theil auch Das Bobergebüfh zwifhen Sber=Iitlenborf und

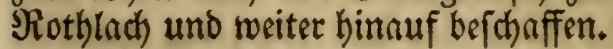

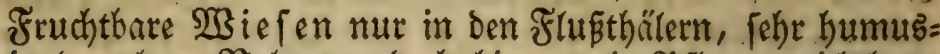
reich in Der obern $\mathfrak{B}_{0}$ oberau, boch hier wernig . Pflanzenreichthum

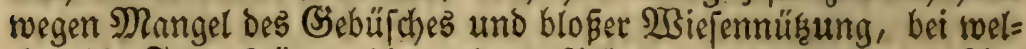
cher bie FutterEräuter bie andern SPflanzen verbrïngen. 2(m

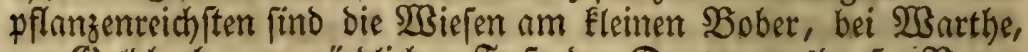

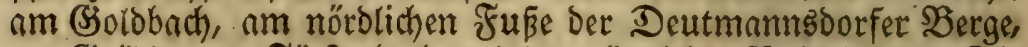

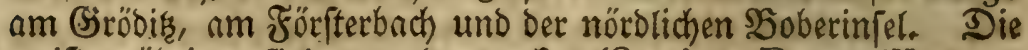
meiften übrigen fint entweder trodén. (Statice, Potentilla verna) ober torfig. 
Das Rlima. Ueber boffelbe fehlen gennue und butch jahts

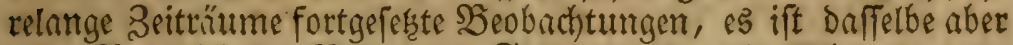

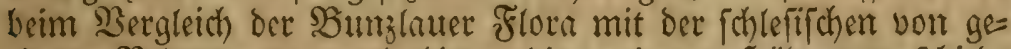

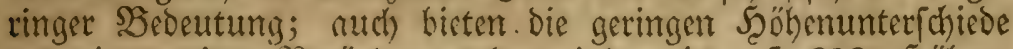
nur eine geringe S:crintoerung bar, indem ia crft 600' Shöhen=

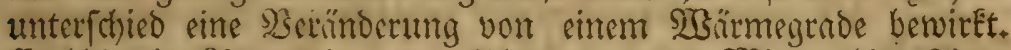
Es bitbet im 2fltgemeinen Den Ubergang vom MittelgebirģElima zu dem Der Ebene, worüber ppäter Siachric)t gegebent werden foll.

Faffen wir alle bicfe Berbältniffe zufammen, fo fehen wir

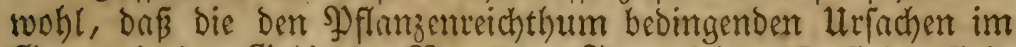
Ģanzen in Dem (jebiet ber 23 unzlater Flora nicht auf

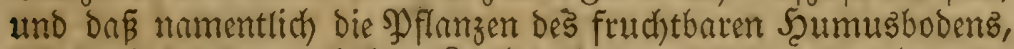
To wie Die ber untermifchten \&aubrvalbungen nut fpar [am bet uns vorfoommen fönnen, uno das cigentliche $\mathfrak{B}$ erggebiet nut an wenigen uno mehr ifolitten ŞunEten ein=, Dagegen bas ber Şaide am mirch)= tigftent nuftritt. Samb=, Şaibe= und Moorpflanzen bitbent ben überwiegenden und eigenthǘmlichen FaraEter Der Flora. 2(ts Die

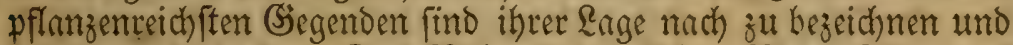

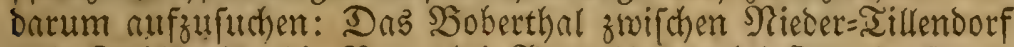

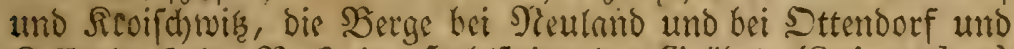

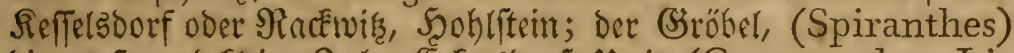
Die große und Eleime 3eche, Evckersborf, utttig (Centunculus, Li-

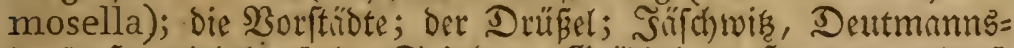

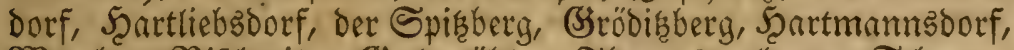
Wanthe, Niichwih, Golomühle, Ihomazwaldau; Schauter=

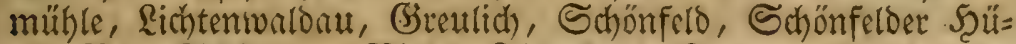
gel, Neut=Schönfeldo; Nieder = Iillentorf, Summ, Ritifchborfer

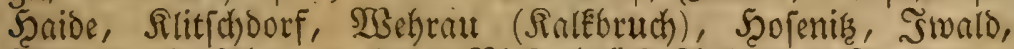

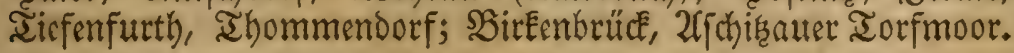

Șm Şanzen umfaßit Die Flora 881, alio gegen 900 offen= blühende Syflanzenarten in 376 Ssattungen; 445 2(rtent uno 92

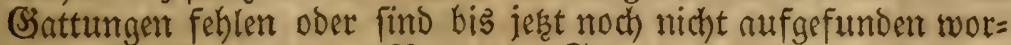
Dent. Die 2trtenzahl ber Bumzlauer Flora verhält fich Demmach zut

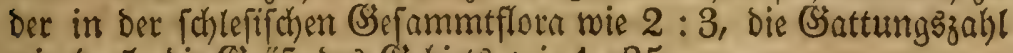

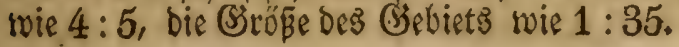




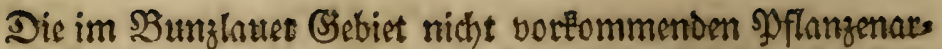
ten und Sattungen find burch (Sincüteren und cin * von ben bens ferben eigenen unterjchieden; beibe baben bejondere, fortlaufende Nummern.

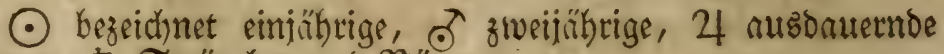

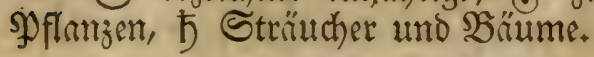

Die römifhen Biffern brücken den (S)rod Der Schüfigkeit nus: I. Fehr felten, II. Felten, IUI. zerftreut, IV. häufig, V. gemein.

Die nrabifken Biffem bie Blüthenjeit in ben verfobiedenen Monaten $2=F_{\text {ebruar, }} 3=$ März $\mathfrak{u}_{+}$T. $\mathfrak{w}_{+}$

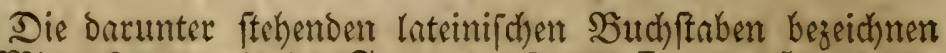

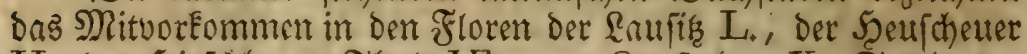

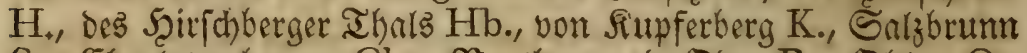
S., Eharlottenbrum Ch., Seuthen a. D. SDer B., Shlau O., WBohlau W., das $\mathfrak{E}$ af rung Ln.

Die 2lccente über ben Sylben bezeidynen deren SBetomung. 


\section{3weiter Abithitt.}

\section{Die sfrangen.}

\section{Errite Rlafie. Monándria. Énfabige.}

Crute Drsnung. Monogýnia. Cinftemplige.

1. Hippuris. Tannenwedel.

* 1. H. vulgaris, gemeiner $\mathfrak{I}$. Stillyt. und Iang=

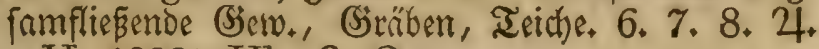
H. $1000^{\prime}$, Hb. S. O.

3weite Drsumy. Digýnia. Bueiftemplige. 1. Blítum. Ervbeeripinat.

1. Bl. virgatum, ruthiger oder feitenblütbiger $E_{\text {. }}$ Ģartemland, Schutthaufen. I. WBont m. verwildert als twild in ben $\mathfrak{B o r f t a ̈ b t e n ~ h i n ~ u n d ~ m i e d e r . ~ 5 , ~ 6 . ~ 7 . ~} \odot-W$.

2. Callitriche, Şafferftern.

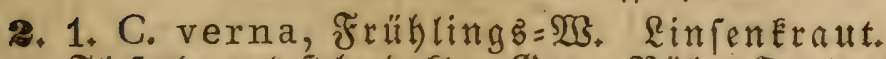

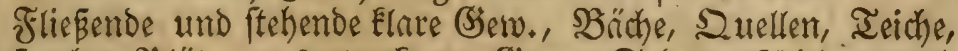

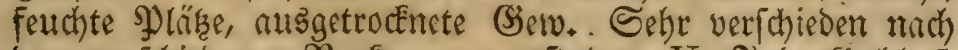
Dem verfchiedenten Sorfommen geftaltet. V. Subenfirdyhof,

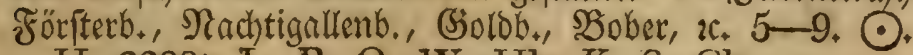

H. $2200^{\prime}$, L. B. O. W. Hb. K. S. Ch. 


\section{3weite Alaffe, Diándria. 3weifabige.}

Erite Sroumuty. Monogýnia. Cintitemplige.

\section{Lémna. গ̧saffertinfe.}

3. 1. L. trisulca, breifurchige 23 . Stehende ober lang= famfliesende Biewiffer. I. SBoberlachen am MBehre.

H. B. O. W. K. Ch.? Hb.

4. 2. L. minor, Eleiner 23 . Deşgleichen. TV. Faft auf allen IIİmpeln.

H. L. B. O. W. Hb. S. Ch.

ร. 3. L. gibba, hökrige 23 . Desgleicten. II. H. L. W. Hb.

6. 4. L. polyrrhiza, vielwurzlige. Hb. Ch. $\mathfrak{B}$. Deşgl. 2ulle blühen felten, fteigen an ber HI. bis 2200' boch, Doch Dam feltmer werdend. 4. Fráxinus. Eiche.

8. 1. Fr. excélsior, gemeine $F_{\text {. }} \mathscr{2}$ undholzbaum. Bergwaldungen, FluFufer, Dörfer. I. Tillendorf, Warthe. 4. 5. 5

H. $1100-2500^{\prime}$. L. O. W. Hb. K. 1480-1680'. S. Ch.

5. Ligústrum. SRainweibe, fpanifđe 230 eibe.

8. 1. L. vulgare, gemeine $\Re_{+}, \mathfrak{B}_{\text {eintolz }}$, 3atintie=

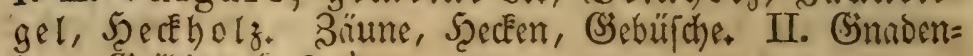
berg, Ëröoich. 5,$6 ; \hbar$.

H. $1000-1100^{\prime}+$ O. W. Hb. S. Ch.

6. Verónica. Egrenpreiz.

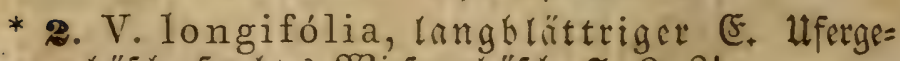

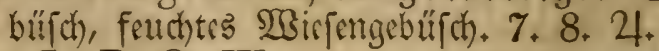
L. B. O. W.

* 3. V. spicáta, ähriger (ङ. Irodfene Şügel, Raime, SirÊn= uno Rirferbane. $\%, 8,2$.

L. O. W. 


\section{1}

9. 1. V, serpýllifolia, quenbelblättriger F. Feudthe

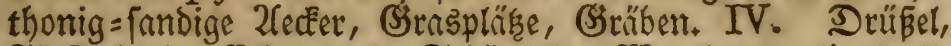
Förfterbach, (Eeferborf, Sdjönfeld, WBatthe 26. 5. 6. 7. 9. 10.4 .

H. $1500^{\prime}$ L. B. O. W. Hb. K. S. Ch.

* 4. V. alpína, $\mathfrak{A}$ tpen $=\mathbb{E}$. Sdmeetoppe, Eleiner Ieidh $\%, 8,4$.

* 5. V. bellidioídes, máliebenblätriger (E. Schneeloppe. 6.7.24.

* 6. V.Teúcrium, (B)mander=E. (Erd $=\mathfrak{B} a t h=$ engel. Trockene, bufchige Schügel, Şraslefhnen. 4. H. $1000^{\prime}$.

10. 2. V. Chamaédxys, $\mathscr{B} a l d=\mathbb{E}_{*}$. Gamanderlein, Bathengel, blatter $\mathfrak{B}$ iefen=E, Raine, SBiefen, Büt=

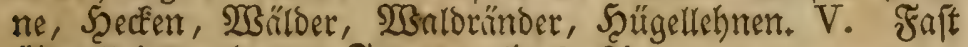
überall in mehreren Formen. 5. 6. 4 .

H. bis 2200'. L. Hb. K. S. Ch. B. O. W.

* \%. V. montána, $\mathfrak{B} e \mathfrak{r g}=$ E. $\mathfrak{B e r g}=$ und $\mathfrak{B}$ uthert= wăloer. 5, 6, 4 .

H. $2200^{\prime}$. S. Ch.

11. 3. V. officinális, arzmeilidyer, 2 potheker $=$ F,

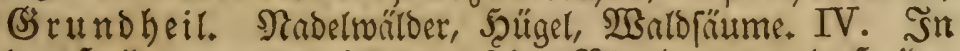
Der Scraide gruppenweis, wo Eeine SBlutbeeren und Sçaide;

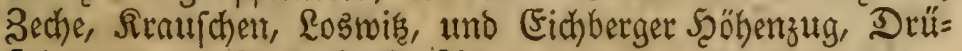
Eel $u+m_{*} \cdot a_{+} 6.7,8,9.4$.

H. $2000^{\prime}$. L. Hb. K. S. Ch. B. O. W.

12. 4. V. Anagallis, (S) auth heil = ober 2 Baffer $=\mathbb{E}$. Stefende Bew., Tümpel, Suellgatuto. III. Sehr häufig

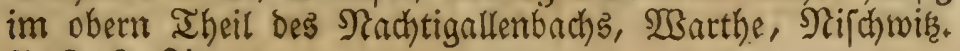
7. 8. 9. 4 .

H. $1100^{\prime}$. L. Hb. (jelten) K. S. Ch. B. O. W.

13. 5. V. Beccabúnga, $\mathfrak{B} a d \mathfrak{b}_{\mathfrak{u} g} \operatorname{en}=$ Doer $\mathfrak{Q} \mathfrak{u} \mathfrak{e}=$ $\mathfrak{l e n}=E_{.}$Quellengruno, Grabent = uno Teichrïnter. III.

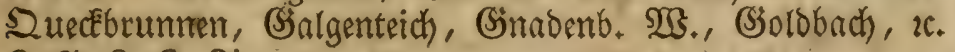
$6,7+8,9,4$

H. $\left(2200^{\prime}\right)$, L. Hb. K. S. Ch. B. O. W.

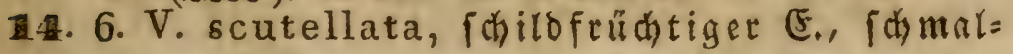




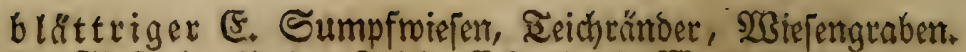

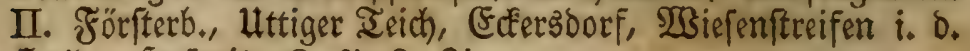

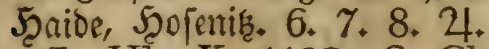

L. Hb. K. $1480^{\prime}$. S. Ch. B. O. W.

* 8. V. peregrina, frember E. 2uf 2lecfert.

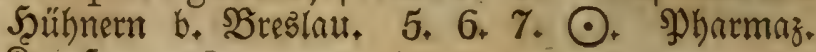
ภirnule.

15. 7. V. políta, geglitteter है.

H. $2200^{\prime}$. S. Ch.

16. 8. V. agréstis, $\mathfrak{A}$ cer $=\mathfrak{F}$.

H. 2200'. L. Hb. K. S. Ch. B. O. W.

18. 9. V. Buxbáumii, $\mathfrak{B} u x b a u m b=․$

2tle 3 auf loderem, bumusesid)em (Garten= uno 2ckerlant.

II. Rehrergarten, Sorftïbte, Scohlftein. 4-6, 9, 10. $\odot$.

H. $1500^{\prime}$. Hb. K. S. Ch, W.

18. 10. V. hederifólia, epheublätriger. (F. rother Meier. Gratenlano, 2läerbeste, Scecen, an SBaumitim= men, humusreicher Soden. V. Faft überall. 3. 4. 5. $\odot$.

H. $2000^{\prime}$. L. Hb. K. S. Ch. B. O. W.

19. 11. V. triphýlla, oreiblätriger (ङ., blaue 5ृüb)= nertaute. 2uff allen Zecfern und Srachen ber Umgegeno. V. 2. S. am Drü̈в̈l. 3. 4. 5. $\odot$.

H. $2000^{\prime}$. L. Hb. B. O. W.

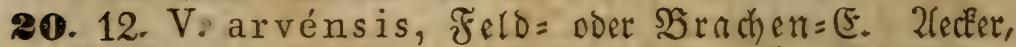

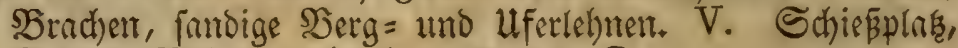
DrüBel, 30 ber 2 . $4.5 .6,9.10+\odot$.

H. $2000^{\prime}$, L. Hb. K. S. Ch. B. O. W.

21. 13. V. vérna, Frühlings= auf Der linken Soberfeite, bejonders bei Der Iillentorfer

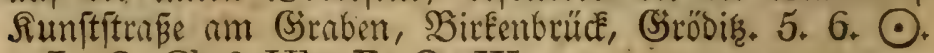
L. S. Ch.? Hb. B. O. W.

\section{Gratiola. Sinabenfraut.}

22. 1. Gr. officinális, arzneiliches (S). ग) urgir= Gi idfteraut, 2 urin. Feuthte Siefen, Sirabentinder, I. (Solbbach unterf. D. SRuthle. 7, 8, 2 .

Ln. B. O. W. 


\section{3 .}

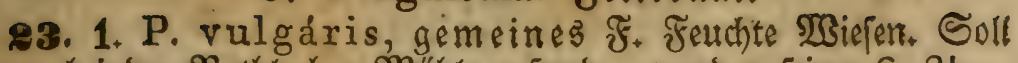

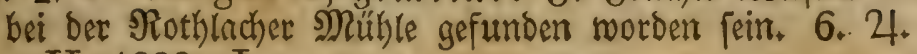

H. $1800^{\prime}$. L.

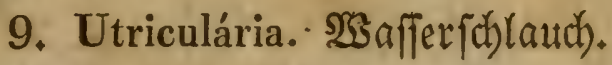

24. 1. U. vulgáris, gemeiner $\mathfrak{B}_{+}, \mathfrak{Z}$ afferhelm.

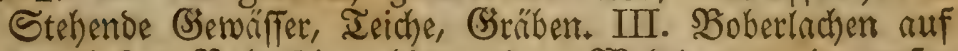

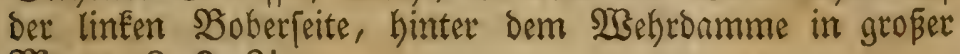
Menge. 8, 9, 24.

L. B. O. $\mathrm{Hb}$.

25. 2. U. intermédia, mittlerer 235 . Stehenbe Iorf=

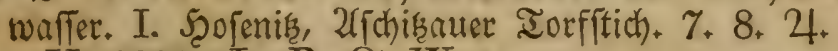

H. $2200^{\prime}$. L. B. O. W.

26. 3. U. minor, Eleiner 23 . Iorflachen Der Scofeni皇. 7. 8.4 .

L. B. $\mathrm{Hb}$.

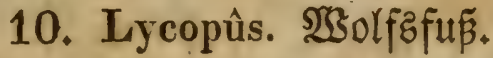

28. 1. L. europaéus, europäifcher $\mathfrak{W}$., $2 \mathfrak{B}$ affer=, Su mp $f=2(n$ oorn. Fenchte Stellen, Ufergebüiche, Ieich)= ränder. III. Banlgenteich, Förifterb., MBarthe, Nachtignallent.,

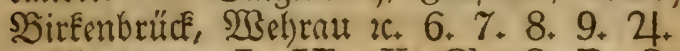

H. $1200^{\prime}$. L. Hb. K. Ch. S. B. O. W.

\section{Sálvia. Salbei.}

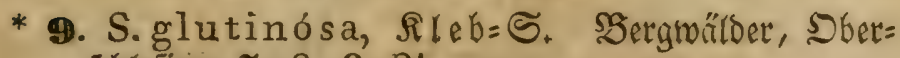
(क) lefien. 7. 8, 9. 4 .

* 10. S. praténsis, $\mathfrak{B}$ iefen $=S$. Irocene $\mathfrak{B} i=$

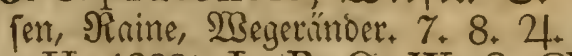
H. $1300^{\prime}$ L. B. O. W. S. Ch.

* 11. S. verticilláta, wirtelblüthige S. WBe= getünoer, Şügel. 6.7. 4 .

H. $1300^{\prime}$. L. S.

11. Circaéa. Şexentraut.

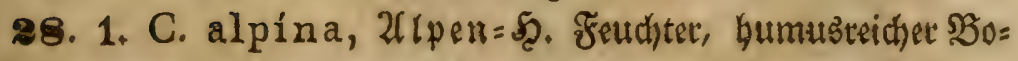




\section{- 24}

ben, bejonder's faute Baumftöcte. I. Waldquellen bei bet Solomühle. 7. 8. 4 .

H. v. $1500^{\prime}$ an, L. Hb. K. $1780^{\prime}$. S. Ch. B. O. 29. 2. C. intermédia, mittleres 5ृ. DumEle Wralos ftellen an Baumitïmmen und Bachrändern. I. Schwarz= wafferquellen bei Rofenthar. 7. 8. 2.

H. $1209^{\prime}$. L. O. W. S.

30. 3. C. lutetiána, gemeines Ş. Feuchte, dunťle

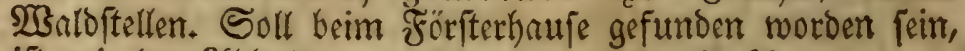
ift mit der 2cbholzung verichwumben. 6-8, 4 .

L. K. $1680^{\prime}$. S. Ch. B. O. W.

\section{Bweite Srounty. Digýnia. Biweiftemplige.}

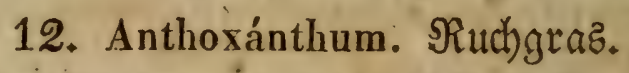

30. 1. A. odorátum, wohlriechendes, gelbes $R$.

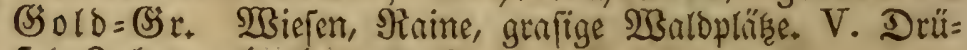
Bel, 3ecte . 2. 4. 5. 8, 9. 4 .

H. $2200^{\prime}$, L. Hb. K. S. Ch. B. O. W.

\section{Dritte Silaffe, Triándria. Dreifadige.}

\section{Erife Dronutg. Monogýnia. Eimftemplize.}

13. Valeriána. Şalbrian.

32. 1. V. officinális, arzmeilicher $\mathfrak{B}$, 赵任enturz.

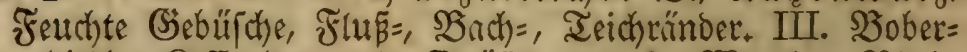
gebirfd), Refferthar y. D. Drüß̈elvorwere, Wanthe, SRifch=

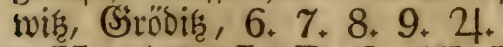

H. $1400^{\prime}$. L. B. O. W. Hb. K. S. Ch.

33. 2. V. samšucifólia, hollunderblättriger $\mathfrak{3}$.

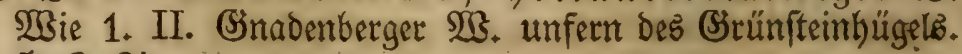
5. 6.4 .

H. 1700 , Hb. S. Ch. 
* 12. V. Trípteris, oreiblïtriger \$5. (5e= birgsbädye, serentee. 6.7. 4.

34. 3. V. dioéca, zweihn̈uliger, Eleiner, Sumpf= $\mathfrak{B}$. Sumpfwiefen. I. $\mathfrak{B}$. D. Soldomühle, dort Gäuftig. 5, 6. 4. H. $2200^{\prime}$. L. W. Hb. K. S. Ch.

* 12. V. Phu, gró̧er Baldrian. Feutchte Serg= fitllen. 6, 7, 24, S.?

14. Fédia. Febie. Felbrapunze.

35. 1. F. olitória, Rapunzel=, Salnt= Fedie. Heceer,

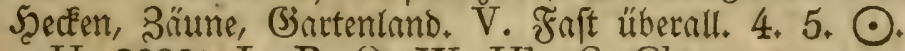

H. 2000'. L. B. O. W. Hb. S. Ch.

36. 2. F. dentáta, gez ühnte Fr. Gettribeñder. III. $\mathfrak{B}_{0}=$ berbette, Drüfel, Gnnodenberg. 7. $8, \odot$.

H. 1400' L: B. O. W. Hb. K: S. Ch.

3ə. 3. F. Aurícula, ohtrandige $\mathfrak{F}_{+} \mathfrak{W i e} 2$ ?

H. $1400^{\prime}$. K. L. S. Ch.

\section{Crócus. Saffran.}

* 14. C. vernus, Frühlingz=S. Iroppau. 3. 4. 2.

15. Gladiolus. WSiejenif wertel.

* 15. GI. commúnis, gemeiner $\mathfrak{W}$. Berg= twiefen. 7. 8. 2. Ch.?

38. 1. Gl imbricatus, Dichtblüthiger 2 B. Feuchte,

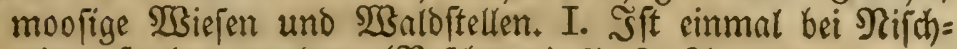
rwis gefunden worden. (Befdjerer.) 7. 8. 4.

H. $1400^{\prime}$. O. W. S. Ch.

\section{Iris. Schwertlilte.}

* I. gramínea, gragblättrige Sđ). Sierg= wiefen. Dober $=$ Schl. 5. 6. 4 .

* 1.. I. sibírica, $\mathfrak{B}$ iefen= üppige $\mathscr{B}$ iefen. 5, 6. 4 . H. $12-1400^{\prime}$. L. B. O.

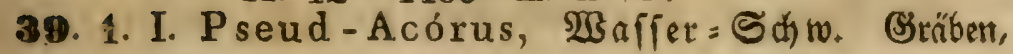




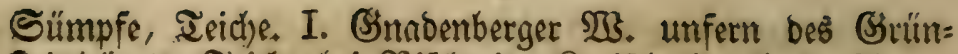

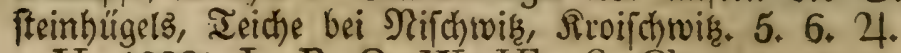

H. $1000^{\prime}$. L B. O. W. Hb. S. Ch.

* 18. I. nudicaúlis, matétenglige Sd) w. Laubrwälder. 5. 4. O.

17. Móntia. Montie, $\mathfrak{B s a j f e r f a l l a t . ~}$

40. 1. M. fontána, $\mathfrak{Z} u \in \mathfrak{l l e n}=\mathfrak{M}$., $\mathfrak{B}$ a fletbungel=

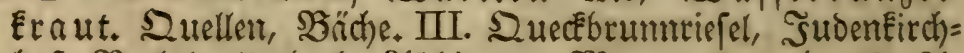
hof, Nachtigallenbach, 2(fchisauter Moorquellen. 4-9. 4.

H. $1000^{\prime}$. L. Hb. K. $1480^{\prime}$. S. Ch.

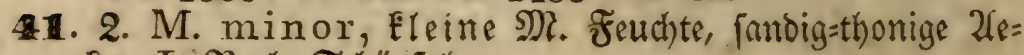
der. I. Nach Schönfeld.

W. S. Ch.

18. Polycnémum. Innorpelf́raut.

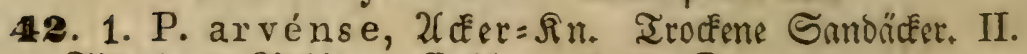
Tillendorf, 2lfaikau, Eichberg. 7. 8. $\odot$;

L. B. O. W.

19. Rhynchóspora. @dnabelriet. Rnopfôtaz.

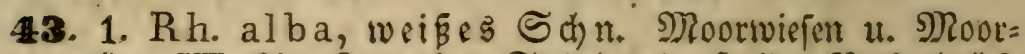

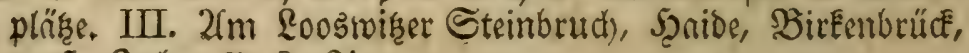
groß̄e 2eche. 7. 8. 4.

H. 2200 '. L。 O. W.

44. 2. Rh. fusca, braunes 5 (t) I. (Sreutich, Şofentis. 6.7. 4. L.

\section{Cládium. Sumpfgraz.}

* 19. Clad. germanicum, ocutiches S.

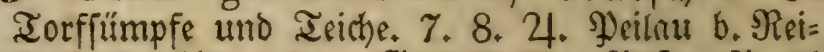

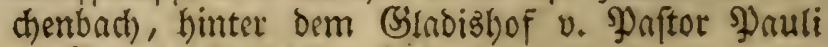
in Dttendorf 1828 sefundent.

\section{Scirpus. Binfe, Senoe.}

45: 1. Sc.palústris, $\subseteq u m p f=\mathfrak{B}$. Binfengrag. Sum=

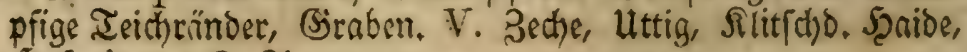
Şôenis 2 c. 6. 4 .

H. $2200^{\prime}$. L. H. O. W. Hb. K. $1480^{\prime}$. S. Ch. 
46. 2. Sc. uniglumis, einbalgige $\mathfrak{B}$. Moor $=$ unb

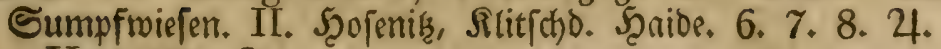
H. $1100^{\prime}$. O.

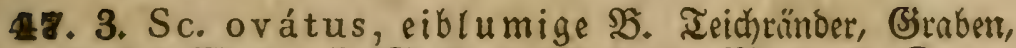

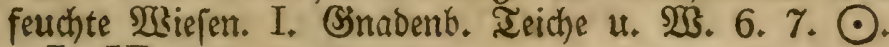
L. $\mathrm{Hb}$.

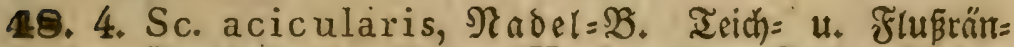

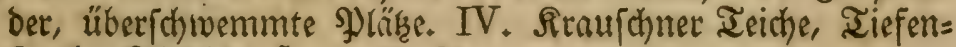
futth, sititfajo. 5. 6. 7. 4 .

L. Hb. S. Ch. B. O.

49. 5. Sc. Boeóthryon, Iorf= 2. Iorf= uno Moor= miefen, Siraben. III. Shofentis, (Streulich. 6. 7. 24.

H. $1400^{\prime}$. L. O. W. Hb. K. $1480^{\prime}$. Ch.?

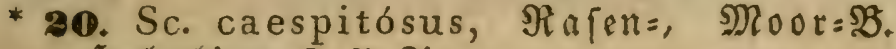
5ृodigebirge. 6. 7. 4.

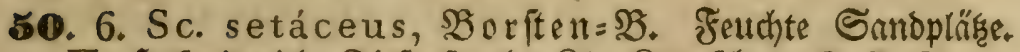

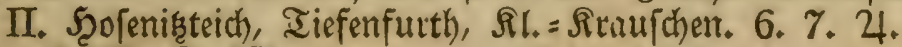
L. B. O. $\mathrm{Hb}$.

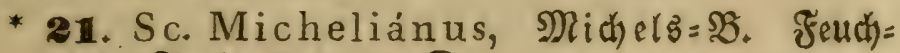
ter Sand. 7. 8. 9. $\odot$.

51. 7. Sc. lacústris, Teidy= III. Şofeniffteich, Bober, WBarthe. 6-9. 4.

H. $1100^{\prime}$. L. B. O. W. Hb.

* 22. Sc. Tabernaemontánus, wie 51.7.8.4

52. 8. Sc. marítimus, See=BB. Teichufer. 6, 7. 4. 3. 2libertint.

53. 9. Sc. sylvátic us, $\mathfrak{B} a \mathfrak{l}=\mathfrak{B}$. Graben=, $\mathfrak{F l u}=$ = und

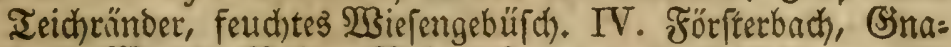
Denb. $\mathfrak{B B}_{\text {., }} \mathrm{El}$. Bober, Bober, 3ectse r. 6. 7. 4 .

H. 2000'. L. Hb. K. S. Ch. B. O. W.

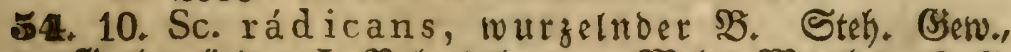
(Srabentänber. I. Bobertachent am Weht, Warthe. 6. 7. 8. 9. 24 . L. B. O. W. 


\section{8}

\section{Holoschoénus. ̊ugelriet.}

* 23. 1. H. filifórmis, fabenförmiges $\Omega$. Sumpfwiefen. 6.7. 24. W.

21. Blýsmus. Sueflentiet.

55. 1. Bl. compréssus, glnttes 2. Sumpfwiejent. I. Şofenif. 6.7.24.

H. $1100-2000^{\prime}$. L. W.

22. Erióphorum. $\mathfrak{S g o l l g r a z , ~} \mathfrak{E g}$ lltriet.

56. 1. E. vaginátum, fheibiges $\mathfrak{W}$. Moor $=\mathfrak{u}$. Torf $=$ wiefen. I. SBuchwalder, 2achisauer Iorfgrumb, 3eche. 4. 5. 9. 24 .

H. 2600' Ln. Hb. K. 1483'+ S. Ch. O. W.

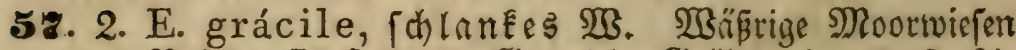

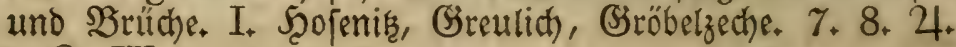
O. W.

58. 3. E. angustifólium, ft)malblättrige $\mathbb{T B H}_{+1}$ SBinfenjeide. Torfige Sumpfwiefen u. Sumpfitellem.

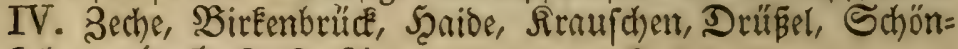
felo 2 , $4,5,8,9,24$.

H. $1150^{\prime}$, Ln. Hb. K. S. Ch. B. O. W.

59. 4. E. latifólium, breitblättrige $\mathfrak{B B}_{\text {. Torfige }}$ Sumpfwieren. III. Mit 3. 4.

H. 1200'. Lo. Hb. felten. K. S. Ch. B. O. W.

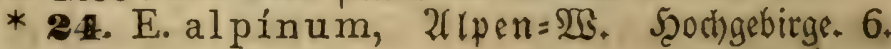
7. 4.

23 Cýperus. 3npergraz.

60. 1. C. flavéscens, gelbes 3. Gierwäferter Sand an Ieichent und Bräben. I. Seopmif. 7. 8. 9. $\odot$.

Ln. S. Ch. B. O. W.

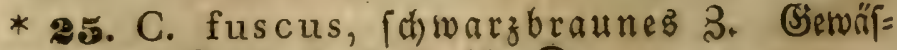
ferter Sand. 7. 8. 9. 10. $\odot$.

Ln. B. O. W. 


\section{9}

\section{Nárdus. Boorfítengraz.}

61. 1. N. stricta, fteifes $\mathfrak{B}$, Nät th. Feudte, torfige

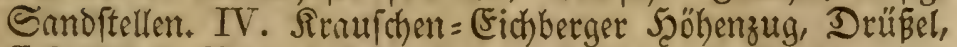

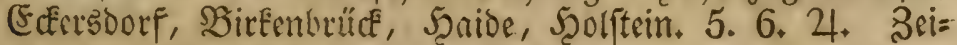
thent von fehe unfruchtbarem $\mathfrak{B}$ oben.

H. bis 2500'. L. Hb. S. Ch. B. O. W.

\section{Bweite Sronumg. Digýnia. Bweiftemplige.}

\section{Leérsia. Seerfita.}

62. 1. L. oryzoídes, reisăhnliche \&. Bewäfiferter Samb an Teich)=, Ġraben= $\mathfrak{u}$. Flußtinderm. Bif tini. 8, 9. 10. 4 .

L. B. $O$.

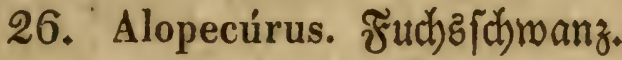

63. 1. A. praténsis, $\mathfrak{B}$ iefen $=\mathfrak{F}$. $\mathfrak{B}$ Biefen, befonders hu $\mathrm{u}=$ mustreche, grafige Rlecfert= nno Grabentänder, V. 5. 6. 8. 9. 10. 21. Faift übirall.

H. $10-2200$ '. L. Hb. K. S. Ch. B. O. W.

64. 2. A. geniculátus, gefnieter, $\mathfrak{Z} a f \int e r=\mathscr{F}$. Ģ:

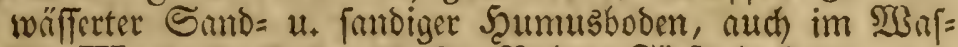

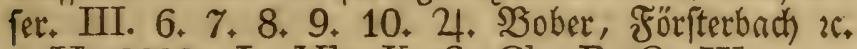
H. $2200^{\prime}$. L. Hb. K. S. Ch. B. O. W.

65. 3. A. fulvus, gelbrother doer $\subseteq u m p f=\mathfrak{F}$. Wie 2. wohl Gäufiger. $5-10.24$.

H. $1130^{\prime}$. L. Hb. K. S. Ch. O. W. B.?

* 26. A. agréstis, $\mathfrak{2} \mathfrak{e} \mathfrak{e} r=\mathfrak{F}$. Spppeln, leh̆mige 2lecter. 6. 7. $\odot$.

\section{Phléum. iejchgras.}

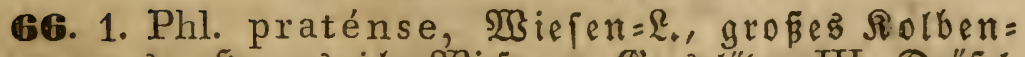

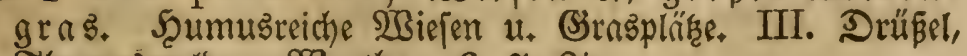
Thomašwaldau, 2Barthe. 6.7. 4 .

H. $10-2200^{\prime}$. L. Hb. K. Ch. B. O. W. 


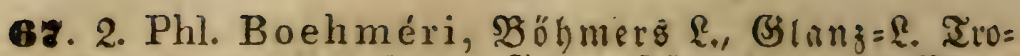
cFene, fandige ober fteinige Felder, Shlïgellehnen umo 2 Bege= rïnder. II. Tillendorf, 2ßehrau, Schönferto. 6. 7. 4.

H. $10-1100^{\circ}$. Ln. B. O. W.

* 22. Phl. alpínum, fipen=?. Şochgebirgg= wiejen. 7. 8. 4.

28. Phálaris. Ģlanzgraz.

69. 1. Ph. arundinácea, rohrartiges (3)., Schwer=

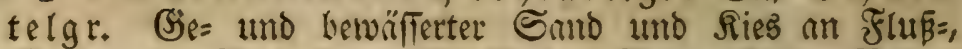

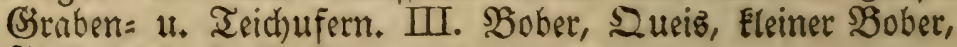
Förfterbach. 6. 7. 24.

H. $11-1200^{\prime}$. L. Hb. K. 1480'. S. Ch. B. O. W.

\section{Agróstis. Straū̄graz.}

69. 1. A. rubra, rothes Str. 2urf fonnigem uno bes (d) nttetem, trofenem $u$. feutchtem, leid)tem $u$. humushaltis

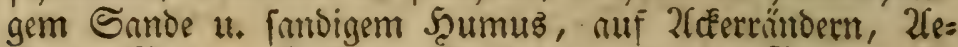

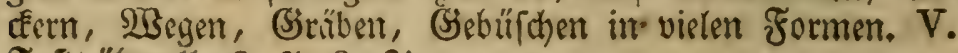
Fnift überall. 6. 7. 8. 24.

H. 2200' L. H.b. K. S. Ch. B. O. W.

89. 2. A. stolonifera, iproficndes Str. wie 1. Dod auth an signffertinndern. 7. 8. 2!.

H. $2200^{\prime}$. L. Hb. K. S. Ch. B. O. W.

81. 3. A. canína, Şunds= Str. Melhe auf fauter $\mathfrak{B B}_{\text {eide }}$ befonbers mit Samounterliage, als auf troctenem $\mathfrak{B}_{0 .}$., boch and auf trocfinem Sand 1t. Felfen, ändert banm ak. VI. Sanioe, SBirfentrüd, Jjofentis, Ihommendorf. 6. $\%$. 8. 4. H. 1400'. L. Hb. S. Ch. B. O. IV.

* 28. A. rupéstris, Felfen= St. Sinjenförmig,

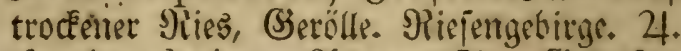

* 28 b. A. alpína, 2flpen= Str. Gejemfe. 7. 8. 4. 30. Anemagróstis. ふ3inthalm.

82. 1. A. spica Venti, gemeiner $\mathfrak{M}$. Sandig=thonige

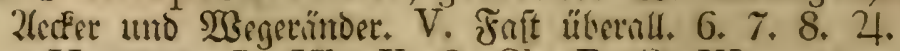

H. 2200' L. Hb. K. S. Ch. B. O. IV. 


\section{Calamagróstis. Rohrgraz.}

33. 1. C. I anceoláta, lanzettlid)es R. Bewäfferter torfig=fandiger unb- Scumusboden, auf SWBiefen, an Gra= benrínderm. II. Sobet, Ginndent. Wanfer. 7. 8. 24.

H. $1200^{\prime}$. L. Hb. S. Ch. B. O. W.

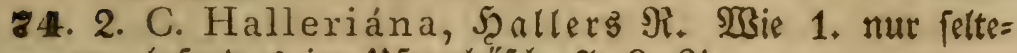

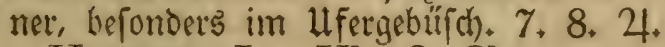

H. $2800^{\prime}$. Ln. Hb. S. Ch.

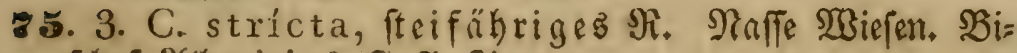
f(c) of 2(rbertini.? 6. 7. 24.

86. 4. C. Epigéios, $\mathfrak{L} a n d=\Re$. 2 \{uf trofenem, fanbigem

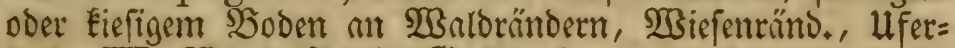

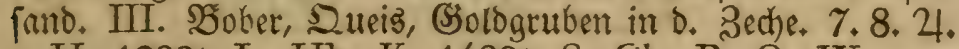

H. $1200^{\prime}$. L. Hb. K. $1480^{\prime}$. S. Ch. B. O. W.

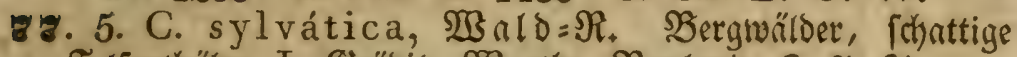

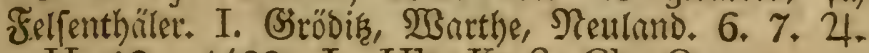

H. 12-1400'. L. Hb. K. S. Ch. O.

* 28. C. arenária, Sandrohr. Eofer frmgfamo.

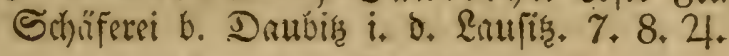

\section{Mílium. Scirfengraz.}

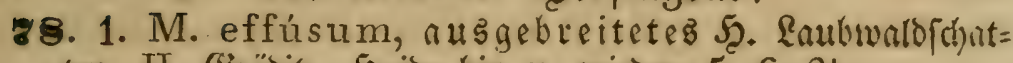
tent. II. Siröbis, Scaibe hin u. tvieder. 5. 6. 24.

H. 1500 '. L. Hb. S. Ch. O. W.

33. Digitária. Fingergrab.

89. 1. D. sanguinális, $\mathfrak{B} \mathfrak{l} \mathfrak{u t}=\widetilde{F}$. Şumuรămer, ange= bauter u. utunngrbauter, trockener Sano, Beete, $\mathfrak{B}$ ege. II. Sorttïbte, Tillemborf. 7. 8, $\odot$. Ln. B. W.

80. 2. D. humifusa, geftreffte马 $F_{*}$ Sandhirfe. wie

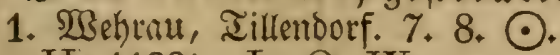

H. 1100' L. O. W.

34. Echinóchloa. Stachelgraz.

81. 1. E. Crus Galli, gemeines St. Feuhtes (sarten:

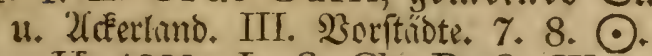

H. $1000^{\prime}$. L. S. Ch. B. O. W. 


\section{Setária. Fennid).}

82. 1. S. verticilláta, $\mathbb{R} l e b e=\mathscr{F}$. Gartenlano. II. M̉or ftî́ote. 7. 8. $\odot$ ?

H. $1000-1500$ '. B. O. W.

83. 2. S. glauca, grau=grüner $\varsubsetneqq$. Candiger Thonbo=

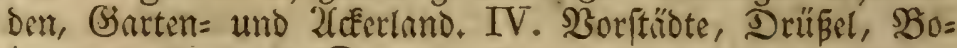
bernu 2 . $7,8,9 . \odot$.

H. 10-1500', L. Hb. K. S. Ch. B. O. W.

81. 3. S. viridis, grüner F. Desgleid)en, befonbers auf Fiartoffelfeldern. 7. 8, 9. $\odot$.

H. L. Hb. S. Ch. B. O. W.

\section{Phrágmites. Sdhilf.}

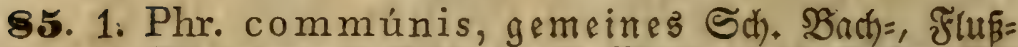
unt Ieichufer und Teiche, naffe WBiefen, muth 2(ecter. IV. Fait überall. Sober, NBarthe, Niichwis, 2ueis. 7. 8. 4 . H. 12-2200'. L. Hb. K. S. Ch. B. O. W.

\section{Arrhenathérum. (slattfafer.}

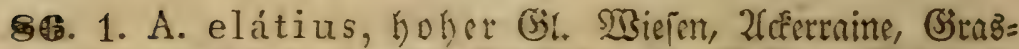

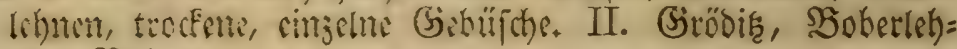
nen, SBoberau.

H. $1200^{\prime}$. L. Hb. S. Ch. B. O. W.

6. Hieróchloa. Darrgraz.

* 30. H. boreális, nöroliches D. Feuchte 93 iefen. 5. 6. 4.

H. $2200^{\prime}$. K. O.

38. Hólcus. Şoniggrab.

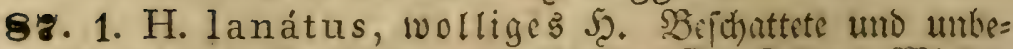

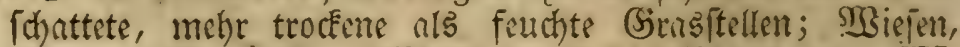

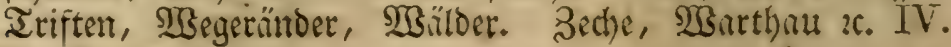
$6-9.24$.

H. $11-2200^{\prime}$. L. K. S. Ch. B. O. W. 88. 2. H. mollis, wriches 5 . WBie 1. minoer bin̈lfig, 
jeltner auf ख̧iefer. 7. 8. 9. 24.

H. 1100 ' L. K. S. Ch. B. O. W.

\section{Aíra. Sdbmiele.}

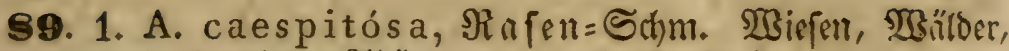
gemein in vielen 2lbänberungent. 6. 7. 8. 4.

H. L. Hb. K. S. Ch. B. O. W.

40. Corynéphorus. Reulengraz.

90. 1. C. canéscens, graues $\Re$. Trodfenter, lodêerer Sano u. Rię. IV. Sanogruben, Boberbette, Tillenoorf, T3ehrau, Tiefenfurth, 6. 7. 8. 24.

Ln. B. O. W.

\section{Avéna. Şafer.}

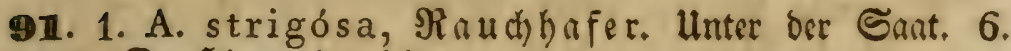
7. $\odot$. STin unto wieber.

H. $1200^{\prime}$. L.

D2. 2. A. fátua, Iaubhafer. Bie 1.

H. $1200^{\prime}$. L. Hb. K. Ch. B. W.

פ3. 3. A. pubéscens, weid)hanriger 5. Mehr troḱ=

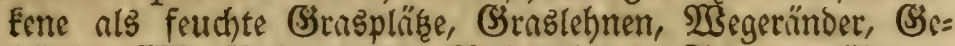
büfohe. III. Boberwiefen, Bobertehnen, Sthauermühle zc. 5. 6. 24 .

H. 11-1200'. L. Hb. S. Ch. B. O. W.

94. 4. A. flavéscens, gelblicher 5ૃ. WBięen, Triften,

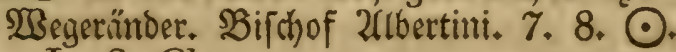

L. S. Ch.

* 31. A. planicúlmis, platthalmiger Ş. Feuchte Shochgebirgstwieren. 7. 8. 24.

95. 5. A. praténsis, $\mathfrak{B}$ iefẹn= 5 . Iroctene $\mathfrak{B}$. Iefen, I. Şartmannsborf, 2Barthe. 6. 7. 4 .

H. K. S. Ch.

96. 6. A. flexuósa, bogiger Ş. Flitterfđmiele.

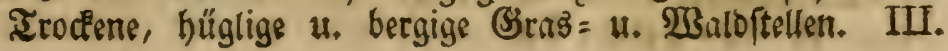




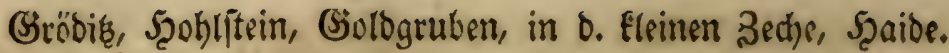
6. 7.24 .

H. 2800'. L. Hb. K. Ch. O.

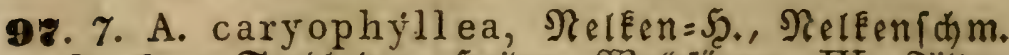

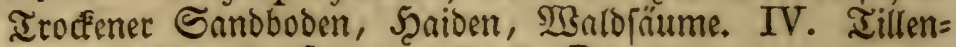
Dorf, Boberbette, Şaibe z. 6. 7. $\odot$.

H. $2200^{\prime}$. L. S. Ch. O.

98. 8. A. praecox, früher Ş., Eleine Sandidmiele.

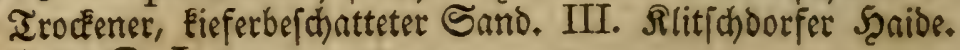
5. 6. $\odot$. Ln.

\section{Mélica. Şerlgraz.}

99. 1. M. nutans, hängende马 গু. Fruchtbater Boben, Gebüldhidhatten. II. Rleime Beche int Den Soldgruben, $\mathfrak{B}_{0}=$

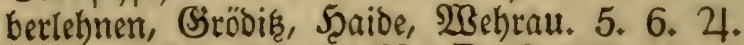

H. L. Hb. K. S. Ch. B. O. W.

100. 2. M. uniflora. 2Bie 1. mebr aber bei unterliegens Dem Felfengruno. I. Grö́die. 5. 6. 4.

H. L. S. Ch. O. W.

* 32. M. ciliáta, gefranztę, wolliges গু. Steinige u. trockeme, menig befd)attete Berglebnen Des Mittelgebirge3. 6. 7. 4.

H. $1000^{\prime}$. S. Ch.

\section{Beckmánnia. Secčmannie.}

* 33. B. erucaefórmis, taupenähnliche $\mathfrak{B}$.

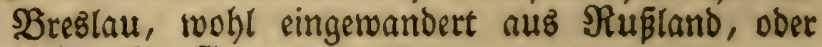
bier weftt. Gorenze. 6. 21.

\section{Koeléria. Rölerie.}

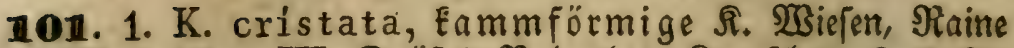
SBegeründer. III. Drǘkel, Boberthal, Firnuichen. 6. 7. 4. H. $10-1400$. L. K. S. Ch. B. O. W.

102. 2. K. glaúca, grau=grüne א. Irodêner Sand. I. Iiefenfurth, STaide. 6.7.24.

H. $12-1400$. Ln. 


\section{5}

\section{Molínia. Molinie.}

103. 1. M. coerúlea, blaue 刃․, blauez গูerlgraz. Feuchte 2 Biefen mit Torfarunde. V. Scaibe, 3ed)e, Drü=

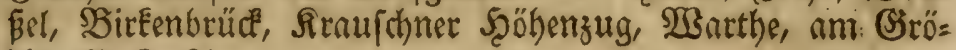
Dif. $7,8,4$.

H. L. Hb. S. Ch. B. O. W.

\section{Glycéria. Süīgras.}

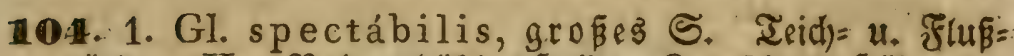

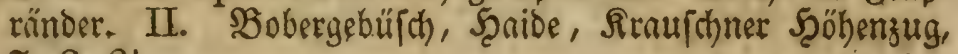
7. 8. 2 .

H. $10-1200^{\prime}$. L. Hb. B. O. W.

105. 2. Gl. flúitans, fhwimmendes S., Manna=

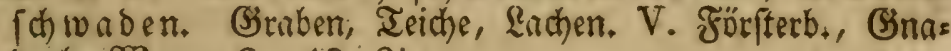
Dent. 20. 2 , 6-10. 4 .

H. 2250 , L. Hb. K. S. Ch. B. O. W.

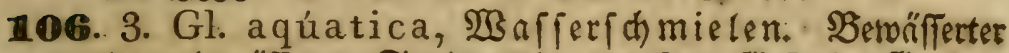
und unberwisfferter Sanogruno, langfam fließ̧ende Ģraben, ftet)endes $\mathfrak{M a}$ affer. I. Boher. 6. 7. 4.

H. 10-1200' Ln. B. O. W. Ch.? Hb.

* 34. Gl. distans, abitehendes S. Feuditer Boden burch Salziaffer, Miftiauche. 7. 8.9.24. 46. Poa. Rizpengraz.

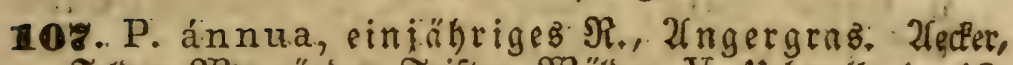

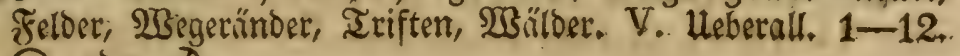
○. ober oे.

H. $2200^{\prime}$. L. Hb. K. S. Ch. B. O. W.

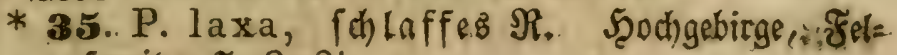
fenrifise. 7. 8. 2 .

* 36. P. áspera, $\mathfrak{r a u h e s} \Re$. Değgr.

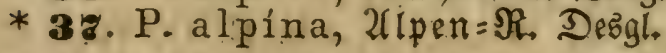

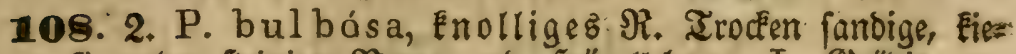
fige ober fteinige $\mathfrak{B} \mathrm{erg}=$ und J̧ügellehnen. I. Gröbik am: Sabhange. 6. 4 .

L. Hb. Ch. 


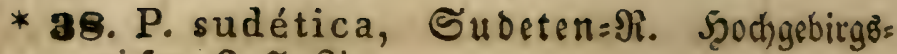
miefen. 6. 7. 4.

$$
\text { H. } 18-2000^{\circ} \text {. L. Ch.? }
$$

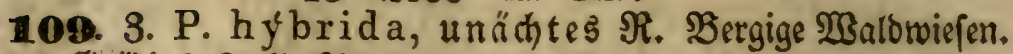
(3rö́biê? 6.7. 4 .

\section{H. $1700^{\prime}$. K. S. Ch.}

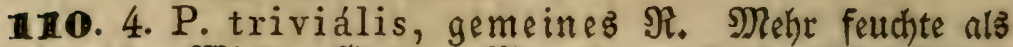

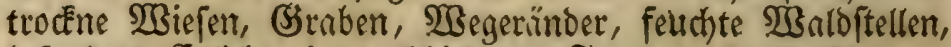
befonders. Erlicht, in ver/ojiebenen Formen. 6. 7. 24. H. 2000'. Hb. Ch. S. B. O. W.

111. 5. P. praténsis, $\mathfrak{W}$ ie $\lceil e n=\Re$. $\mathfrak{B}$ ie 4. in mehteren Formen hânfig.

H. 2000' L. Hb. K. S. Ch. B. O. W.

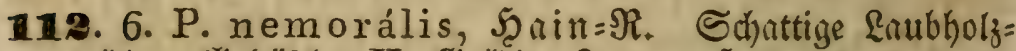

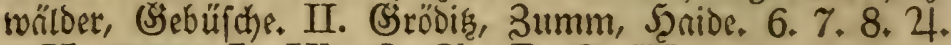
H. 2000' L. Hb. S. Ch. B. O. W.

113. 7. P. compréssa, zufammengedrü fétes গ. Irockene, fanbige unt fteinige Stellen, Matern. III. Ties fenfurt, $\mathfrak{3 a r t h e . ~ 5 . ~ 6 . ~} 4$.

H. 1500 ' L. Hb. K. S. Ch. B. O. W.

\section{Briza. Sittergraz.}

114. 1. Br. média, mittleres 3. Wiefen=3. Trodne

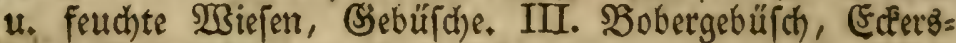
borf, sooberlefinen 26. 5. 6. 4 .

H. $1500^{\prime}$. L. Hb. K. S. Ch. B. O. W.

\section{Cynosúrus. Rammgraz.}

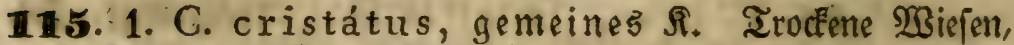

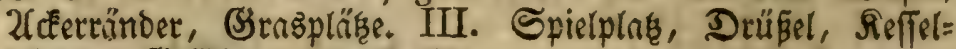

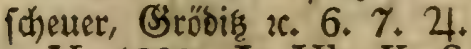

H. $1200^{\prime}$. L. Hb. K. S. Ch. B. O. W.

\section{Dáctylis. Inaulgraz.}

116. 1. D. glommeráta, gemeineg Sint, Şunbşs

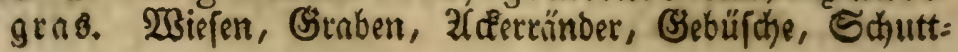




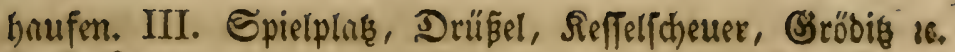
6. 7.24.

H. $1200^{\prime}$. L. Hb. K. S. Ch. B. O. W.

\section{Festúca. Sd)wingel.}

118. F. bromoídes, trespenartiger Shw. Feudter Sandboden. II. Tiefenfurt. \$Bifthof 2trbertini. 5. 6. ๑. L.

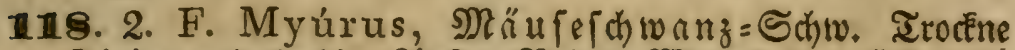

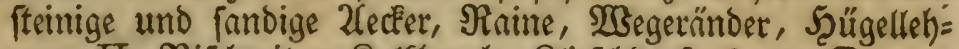

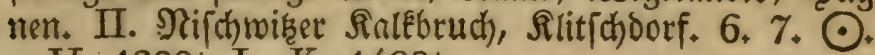

H. $1300^{\prime}$, L. K. $1480^{\prime}$.

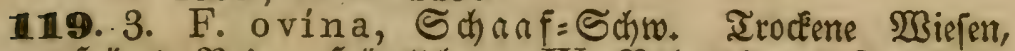

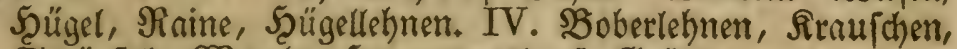

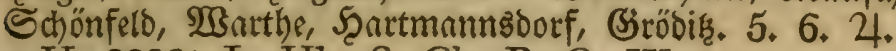

H. $2200^{\prime}$. L. Hb. S. Ch. B. O. W.

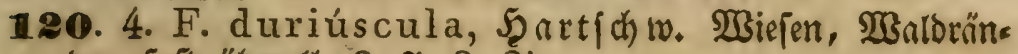
Der, faft überall. 6. 7. 8. 4 .

H. $2200^{\prime}$. L. Hb. S. Ch. B. O. W.

121. 5. F. rubra, rother Sdhiv. Şüglige Biefen, $23 a$ ab

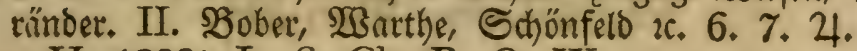

H. 1200'. L. S. Ch. B. O. W.

* 39. F. heterophylla, verfhiedenbr. Sdiv. Bergwaílder. 7. 8. 24. Hb.

* 40. F. varia, bunter Sd)w. Şodhgebirge, Rübeznhls Gantten. 7. 4 .

* 10. b. F. montána, şerg=Sdy. 6.7.21.Gn.

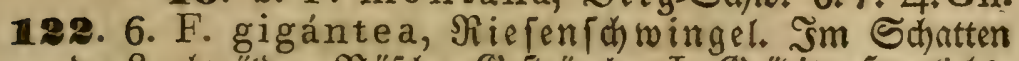

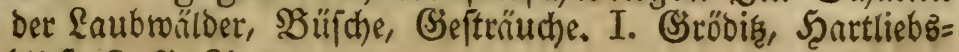
borf. 6.7. 4 .

H. $2000^{\prime}$. L. Hb. S. Ch. B. O.

123. 7. F. áspera, rauher Sđb. Trocêne, fteinige 23aldetehnen. II. Brröbis, Epiagberg. 6. 7. 24.

H. $1700^{\prime}$. L. Hb. K. $1680^{\prime}$. S. Ch. B.

124. 8. F. sylvática, $\mathfrak{Z} a l b=$ Sđjw. Bergwälber. I. Bifchof 2atbertini. 6. \%. 4.

H. $2000^{\circ}$ L. ${ }^{\circ}$. S. Ch. Hb. 
125. 9. F. elátior, hoher Sdjw. Fendtes 2Biefen= unb

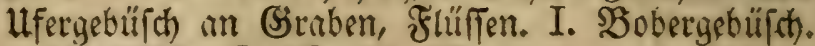
H. $1200^{\prime}$. S. Ch. O. W.

126. 10. F. praténsis, $\mathfrak{B}$ iefen= Sdw. Mebr auf

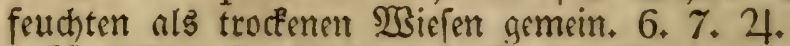

H. $2200^{\prime}$. L. Hb. K. S. B. O. W.

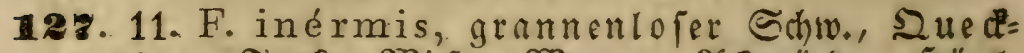

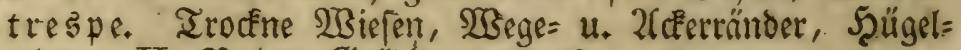
lebnen. II. SOober, Gröotí. 6. 7. 4.

L. B. O. W.

\section{Brachypódium. 3mectée.}

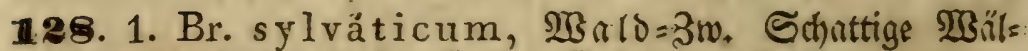
Der. II. Rleine 3eche, Şaibe, Ëröbis. 7. 8. 4.

H. 2000'. L. Hb. K. S. Ch. B. O. W.

129. 2. Br. pinnátum, gefiederte 3 w. Irocêne, fhat=

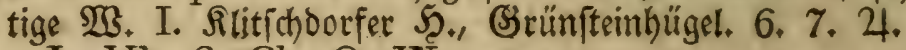

L. Hb. S. Ch. O. W.

\section{Triódia. Dreizabr.}

130. 1. Tr. decúmbens, liegenter Dr. Feuchte unb.

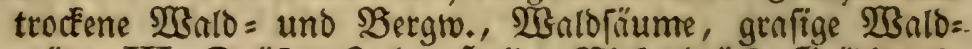

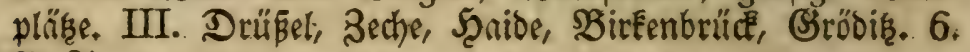
7. 2 .

H. 2000'. L. Hb. S. Ch. B. O. W.

\section{Brómus. İrespe.}

131. 1. Br. secalínus, Roggentr, Sommertr. Ule=

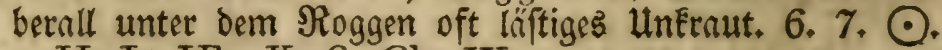

H. L. Hb. K. S. Ch. W.

* 41. Br. pratensis, Wiefen=IT. Fette Wies: - fert. 6. ๑. S.

132. 2. Br. mollis, weid)e oder $\mathfrak{F u t t e r}=\mathfrak{T r}$. Iroetne

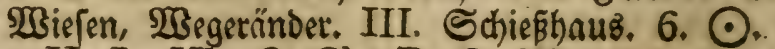

H. L. Ho. S. Ch. B. O. W. 


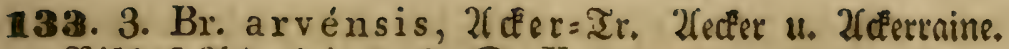
Bifch of Zltbertini. 7. 8. ๑. K.

* 2. Br. patulus, weitläuftige Tr. 6. ๑.

134. 4. Br. stérilis, taube Ir. $\mathfrak{Z}$ ege, Züune, Mauern, Schutthaufen, 2fecker, II. Soorftöbte, SBober, (Sröbick. 6. 7.8. ○.

H. 10-1100'. L. S. Ch. B. O. W. Hb.

135. 5. Br. tectórum. Sorếommen wie 1. bejonderş im Sande. II. Sinndenberg, Morftï̈te, Tiefenfurt. H. $1100^{\prime}$. L. K. S. Ch. B. O. W.

\section{Agropýrum. 2Cốermaizen.}

136. 1. A. repens, friechender $\mathfrak{x}_{*}$,

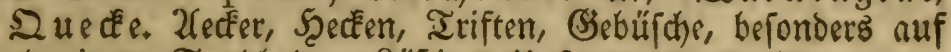
thonigem Sanoboden. Rä́ftiges unEraut. 6.7.24.

H. $2500^{\prime}$. L. Hb. K. S. Ch. B. O. W.

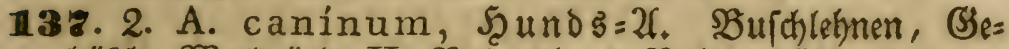

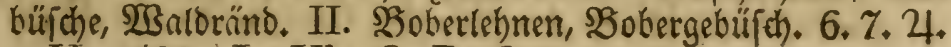
H. $1400^{\prime}$. L. Hb. S. B. O.

\section{Lólium. \&oldh.}

138. 1. L. perénne, $\Re$ a en $=\{$, englif̧es $\Re$ aigrab.

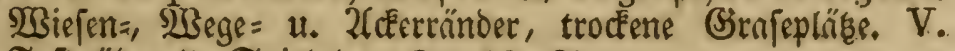
Fraft überall. Spielplag. 6-10. 24.

H. 2500' L. Hb. K. S. Ch. B. O. W.

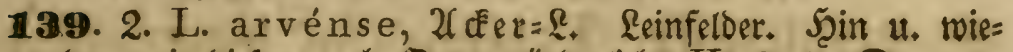
Der, mit biefen nach Sort verïnderticty. II. 6. 7. $\odot$.

H. L. K. S. Ch. O. W.

140. 3. L. temuléntum, Ia mel $=\mathfrak{R}$. UnEraut unter Dem Ëetraide, befonders unter Şafer. 6. 7. $\odot$.

* 43. L. multíflorum, vielblüthiger \&. Biejer. 6; 7. 24 .

8. Elymus. Şaargraz.

* 44. E. europaéus, europäifdes 5ृ. \$erg= wälloer. $6,7.24$.

H. $2200^{\prime}$. Lo. Hb. S. Ch. 
* 45. E. arenárius, Sand=5ุafer. Sandels Der, 7. 24. Ln.

\section{Hórdeum. Gierfíte.}

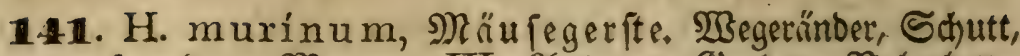
auf und an Mnarern. III. 2f nlngen, Graben, Boberbette, Boritiote $20.6-11 . \odot$.

H. 1000' L. Hb. K. S. Ch. B. O. W.

\section{Dritte Droutuy. Trigýnia. Dreiptemplige.}

57. Holósteum. Relfengras, Spurre.

142. 1. H. umbellátum, bolbenblüthiges . Trok=

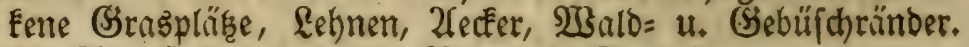

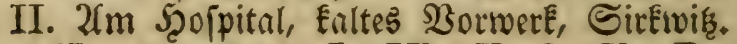

H. $11-1200^{\prime}$. L. Hb. K. S. Ch. B. O. W.

9. Polycárpon. Nageleraut.

* \#6. P. tetráphyllum, vierblättriges $\Re$.

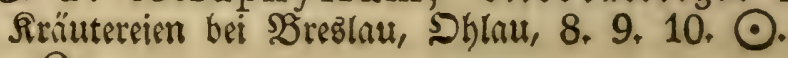
O.

\section{Sierte Rlaffe, Tetrándria. ßierfabige.}

Erite Dronung. Monogýnia. Cinftemplige.

10. Globulária. §uguelblume.

* 48. Gl. vulgáris, gemeine $\Omega$. Runbblu= me. Sonnige, fteinige Şügel, trodéene Wiefen. 6. 7.24 .

* 48. Gl. cordifólia, herzblättrige R. Şod)= gebirgentefen. Beibe in öftr. Sdylefien. 6. 2 . 
11. Dipsacus. Rarben, Iuthmadherbieftel.

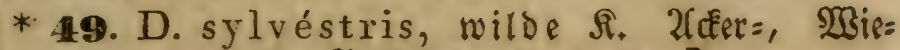
fen= u. kiefige Fluprinor. 7, 8, 9, 厄ै.

Ln. S. Ch. B. O. W.

* 50. D. laciniátus, 和liblätrige $\Omega$. Gandiges und fteiniges ufergebüf(h), feuchte Scecten น. 2Biejen, 6.7, $\sigma^{2}$.

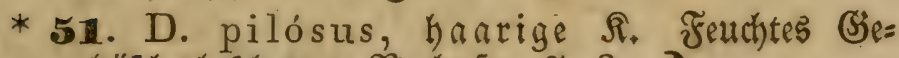
büfh, befdattete $\mathfrak{B a c h u f e r}+7,8$. ఠ

\section{Scabiósa. Skabioje.}

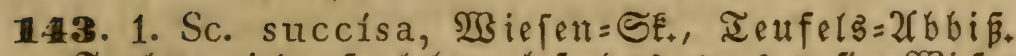
Fenchte, minder fruchtbare, befonders etwas torfige 2 Biejen

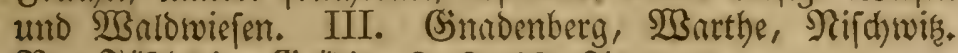

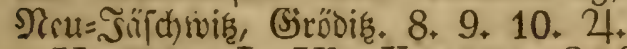

H. 2200'. L. Hb. K. 1680'. S. Ch. B. O. W.

14. 2. Sc. columbária, Iauben= SE. Felder, troteñ Şügel, Megerïnder. I. Şaide hinter dem Strä̈enEteticham. 6-10: 4 .

H. 10-1500'. L. BolEentain. S. Ch. B. O. W.

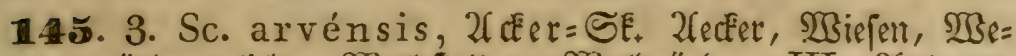
gerïnter, lichte Wralditellen, MBaldorïnder. III. 2Inlagen,

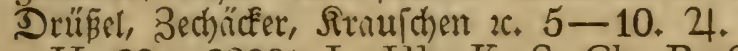

H. $20-2300^{\prime}$. L. Hb. K. S. Ch. B. O. W.

* 52. Sc. lúcida, glänzende SE. Şodgebirgg = wiefen. 7,8, 4 .

* 53. Sc. suavéolens, wohlriechende SE.

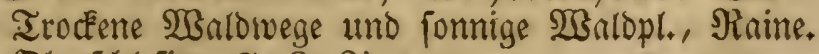
Sberidhlefien. 7. 8. 24.

59. Sherárdia. Sberarbie.

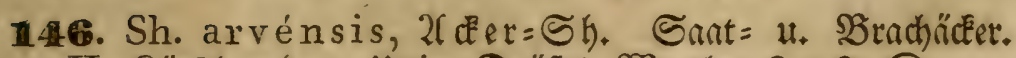

II. Rüridnerberg, Uttig, Dorübel, 2 Barthe, 6-9. $\odot$. H. $1500^{\prime}$. L. Hb. K. S. Ch. B. O. W. 
60. Aspérula. $\mathfrak{3 a l b m e i f t e r . ~}$

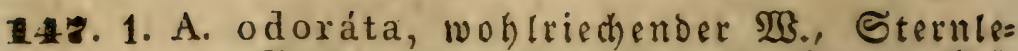

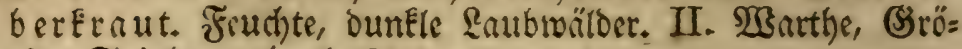
Dik, Spif̧berg. 4. 5. 24.

H. $2000^{\prime}$. L. Hb. K. S. Ch. B. O. IV.

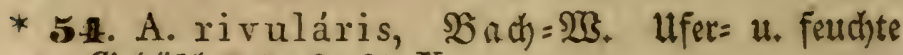
(sebürche. 7, 8, 24. K.

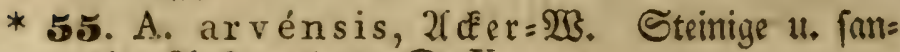
dige 2reçer. 5. 6. $\odot . \mathrm{K}$.

* 56. A. cynánchica, Şügel= $\mathfrak{B}$. Trodéene

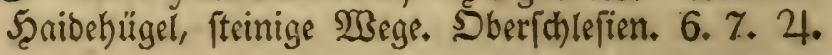

* 58. A. tinctória, Färber= $\mathbb{W}$., wilbe $\mathfrak{F}$ är= bertöthe. Trodene Şügel. 6, 7. 24.

H. $12-2200^{\prime}$.

\section{Gálium. abbraut.}

* 58. G. cruciáta, Écuzblättriges \&. Feuchte, fattige :Baloplike. 5. 6. 4. K. S. Ch.

* 59. G. Bauhíni, B̉ahins \&. Sdattige Bergwilloer. 5. 6. 24. H. $1700 \%$ K.

148. 1. G. A parine, $\mathfrak{R l e b}=\mathfrak{R}$, Bettlersläure. ŞeE=

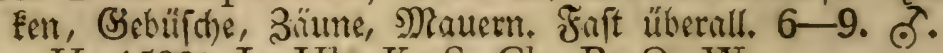

H. $1500^{\prime}$. L. Hb. K. S. Ch. B. O. W.

149. 1. G. uliginósum, Moor $=\mathfrak{R}$. Torf $=$ u. Sumpf $=$ wiefen, Ërnbentünder. IV. Föriterbach, Eckersoorf, Schö=

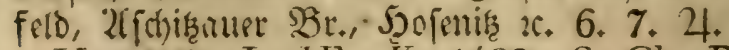

H. $2200^{\prime}$. L. Hb. K. $1480^{\prime}$. S. Ch. B. O. W.

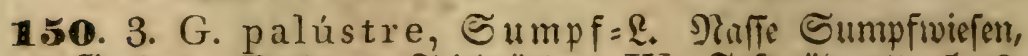

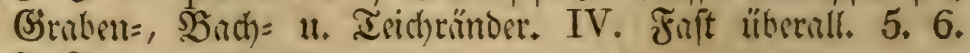
7. 24.

H. $1400^{\prime}$. L. Hb. K. 1480'. S. Ch. B. O. W. 
1511. 4. G. rotundifólium, runoblättriges @.

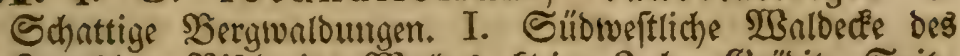

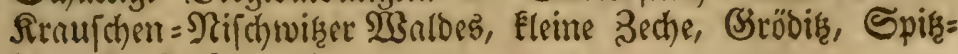
berg. 7. 8. 4 .

H. $1200^{\prime}$. L. Hb. K. S. Ch. W.

152. 5. G. boreále, nörbliches R. Fenchte WBiefen,

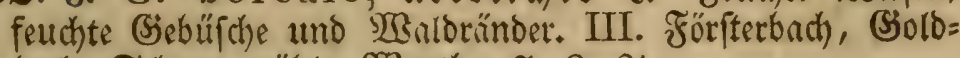
bach, Schauermühle, Warthe. 7. 8. 4.

H. 1500' \&amoshut. K. S. Ch.? B. O. W.

153. 6. G. Mollúgo, weí̈es \&., Butterftiel, wei=

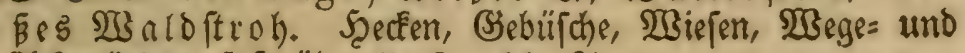
2feferrïnder; forf übernll. 6-11. 24 .

H. $1400^{\prime}$. L. Hb. K. S. Ch. B. O. W.

154. 7. G. verum, wahers \&., gelbes $\mathfrak{R}_{\text {., gelber }}$

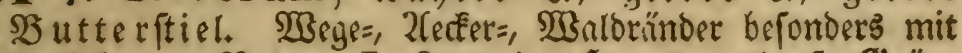

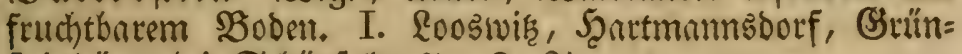
fteinhlighel bei Schonferto. 7-9. 4 .

H. $1400^{\prime}$. L. Hb. K. S. Ch. B. O. W.

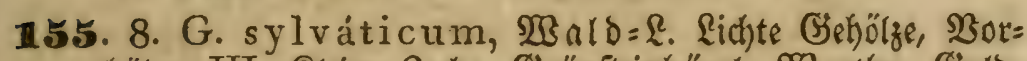

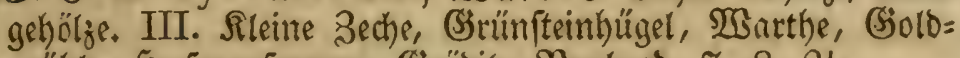

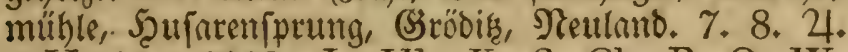

H. $18-2000^{\prime}$. L. Hb. K. S. Ch. B. O. W.

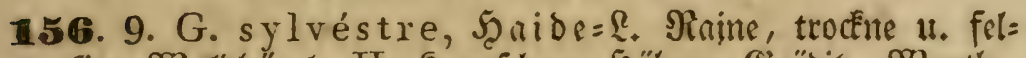

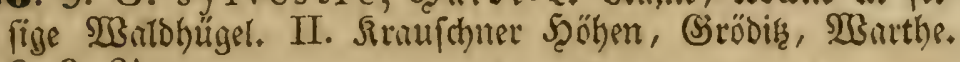
6. 8. 24 .

H. $1700^{\prime}$. L. Hb. K. S. Ch.

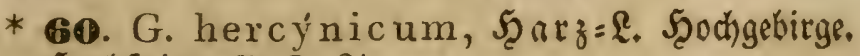
5̧ochjteine. 7. 8. 24.

62. Plantago. $\mathfrak{2 B e g e r i d}$.

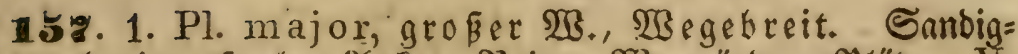

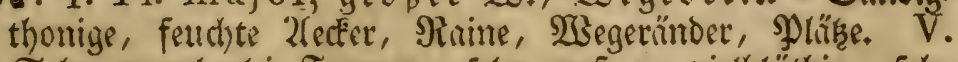
Eehr mand)ertci Formen, feht groß u. vielbhüthig, fehr flein 4t. wenigblüthig, 3. S. Ltttig, \&ooswik. 6-10. 21. H. 2200'. L. H Ib. K. S. Ch. B. O. W. 
158. 2. Pl. média, mittlerer, weifer 233 . Rnine,

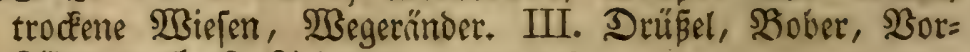
ftîbte $2,5,6,24$.

H. $2200^{\prime}$. L. Hb. K. S. Ch. B. O. W.

159. 3. Pl. lanceoláta, lanzett $=$ blättriger $\mathfrak{B B}_{\text {., }}$

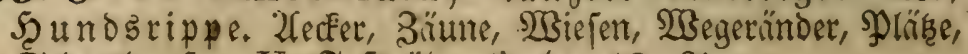
Echutthaufen. V. Fait ilberall. 4-10. 24.

H. 2200' L. Hb. K. S. Ch. B. O. W.

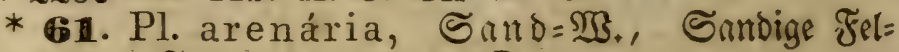
ber, Flǚfer. $6,7,8, \odot$.

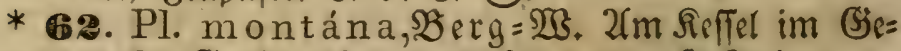

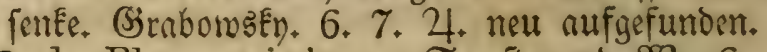

* 62. b. Pl. maritima, Seeftrano= $\mathfrak{B} .6-9$. 4. B)ubrau.

63. Centúnculus. Sleinling.

160. 1. C. mínimus, Eleinfter Sil. Feuchte Sandplähe, fanoig=thonige 2fecerer. I. Uttig über Dem Ieiche, Rroifch= wił, Wartbe, Wiefau. 7. 8. 9. ○. L. O.

64. Sanguisórba. RBiejenEnopf.

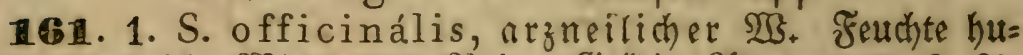

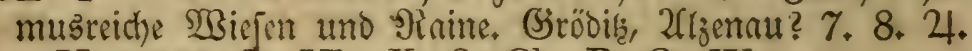
H. 2200' L. Hb. K. S. Ch. B. O. W. 65. Córnus. Sornftrauch.

162. C. sanguínea, rother $\mathfrak{S e}_{+1}$ Şartriegel. WBitloer, (siebüid)e. II. Boberau, Sröbis, Nieder = Ihomnstoaldau. $5-10.5$.

H. 2200'. L. Hb. K. S. Ch. B. O. W.

66. Majánthemum. Mai $=$, Gduatten=Rlume.

163. M. bifólium, gweiblättrige NR., Zweiblatt.

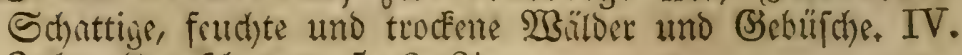

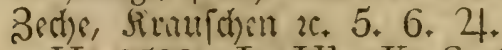

H. $2500^{\prime}$. L. Hb. K. S. Ch. B. O. W.

12. 'Trápa. $\mathfrak{B a \| f e r n u ́ ̃ . ~}$

* 63. Tr. natans, gemeine $\mathscr{B}$. Tiefe, ftehende (siewiffer. 6. 7. ○. L. 
13. Parietária. (S)labł́raut.

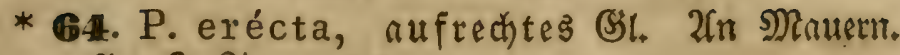
$7 \frac{9 .}{\text { L. O. }}$

67. Alchemílla. \&ömenfußן.

16月. 1. A. vulgáris, gemeiner $\mathfrak{L}_{+}$Frauenmantel, Sinnau. Feudite iefen, Raine, Srathenründer. III. Förfterbach, Driëßel, Schauermühle, Ihomaswaldau $2,5$. 6. 7. 9. 10. 24 .

H. 2500' L. Hb. K. S. Ch. B. O. W.

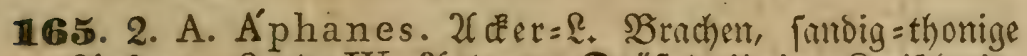

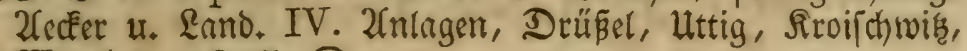
Warthe 26. 6. 7. $\odot$.

L. Hb. K. B. O. W.

- * 65. A. fissa, tiefipaltiger \&. Sueller im Riejengebirge. 7. 8. 24.

68. Potamógeton. aaidfraut.

166. 1. P. natans, fhroimmendes \&. Stehende (je= wäffer. V. Bobetlacten, Warthe, Srröbel, Srö̈bił ze. 6. 7. 8.24.

H. $900-1100^{\prime}$. L. Hb. S. Ch. B. O. W.

* 66. P. ruféscens, röthliches \&. Stehenoe Serwâffer. 6.7.8, 4. L.

* 68. P. heterophyllus, verfhiedenblät = riges \&. beģgl. L.

168. 2. P. lucens, fpiegetndes \&. Degght. II. Förfter= back. 6. 7, 4 .

L. Hb. B. O. W.

168. 3. P. crispus, Eraufes $\mathfrak{R}$. Stekende oder langfam

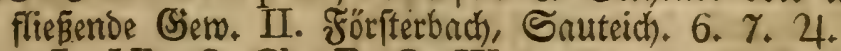

L. Hb. S. Ch. B. O. W. 
* 68. P. perfoliátus, burchwadyfenes Desgl. 6. 7. 24. B. O.

169. 4. P. compréssus, plattítengliches g. Enden,

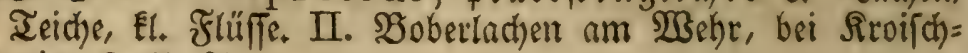
wis. 6. 7. 4.

L. O. W.

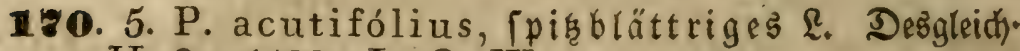
H. $9-1100^{\prime}$. L. O. W.

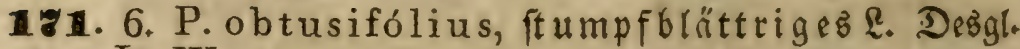
L. W.

12.7. 7. P. pusillus, Eleines … Bobertadten. 6.7.8.24. H. $9-1100$ ' L. O. W.

* 69. P. pectinátus, Eammförmiges \&. 6. \%. 2. L. W.

\section{Radíola. Smergfladbs.}

183. R. Millegrána, viefförniger 3iv. Feud)te, fho $=$

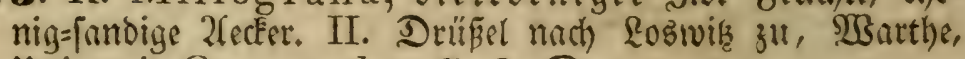
uttig mit Centunculus. 7. 8. $\odot$.

Ln. O. W.

\section{Fünfte Siafie. Pentándria. ร̌unffabige.}

Crîte Sroumin. Monogýnia. Eintemplige.

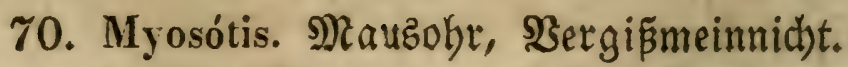

124. 1. M. palústris, Sumpf=M, Brtäben, Sümpf?, Sumpfwiefen, fohlammige Teich = und Errabentänber. V. Faft überall. 5, 6. 9, 10. 11. 4 .

H. 2200'. L. Hb. K. S. Ch. B. O. W. 
185. 2. M. caespitósa, rafenförmiges M. Wie 1 .

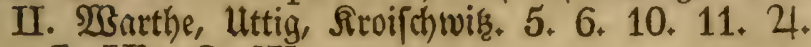
L. Hb. O. W.

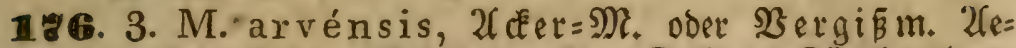

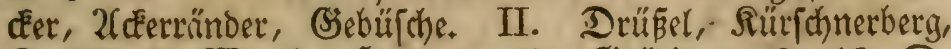

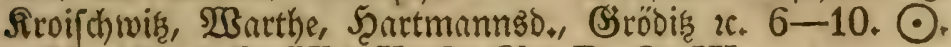
H. 1500'. L. Hb. K. S. Ch. B. O. W.

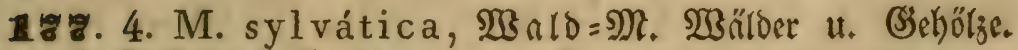

I. Sröbié, 4. 5.4 .

H. $12-2300^{\prime}$. L. Hb. K. S. Ch. O.

18. 5. M. versícolor, buntblumiges M. 2Recer,

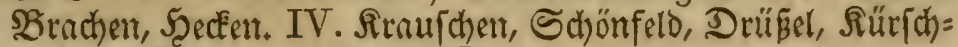
nerberg, SBoberbette. 5. 6. $\odot$.

H. 1200' L. Hb. K. S. Ch. O. W.

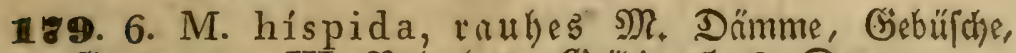
(3raregarten. III. Soberbette, ऊröbir. 5, 6. $\odot$.

H. 12-1700' L. Hb. K. O. W.

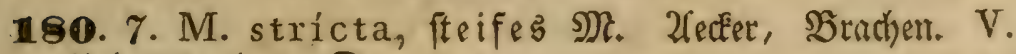
Ueberall. 5, 6. $\odot$.

H. $10-1200^{\prime}$. L. Hb. K. S. Ch. B. O. W.

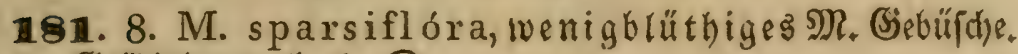
Sröbiaberg? 5. 6. $\odot$.

L. O. W.

\section{Echinospérmum. S̃gelfame.}

* 20. E. Láppulla, Elettenartiger S. 2uf Mauern, Echutt. 5. 6. 9. 10. $\odot$. H. $10-2200^{\prime}$. L. O.

* E. defléxum, herribgebogener Steinige 2lbbänge des Bsefentes. 6. $\odot$.

15. Lithospérmum. Steinfame.

* 82. L. officinále, arzneilicter St. Şü= geigebüfd)e. 5. 6. 4.

H. $10 \rightarrow 1100^{\prime}$. S. Ch. 


\section{Rhytispérmum. Rumzelfame.}

182. 1. Rh. larvénse, $\mathfrak{A}$ đer $=\Re$, $\mathfrak{B}$ auernid minke. Zleder, Brachen. V. Heberall oft in groper Menge. 4-6. 10. 11. $\odot$.

H. $10-2200^{\prime}$. L. Hb. K. S. Ch. B. O. W.

\section{Anchúsa. Sðhjenzunge.}

183. 1. A. officinális, arzneiliche \$. Wege, 2rêer=

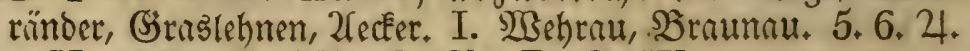
H. $1000^{\prime}$. L. Hb. S. Ch. B. O. W.

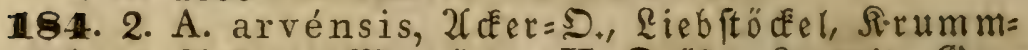
hals. 2lecker u. Megerïnder. II. Drüßjel, Roostwis, Sinn=

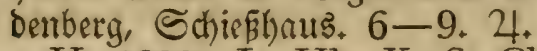

H. $1800^{\prime}$. L. Hb. K. S. Ch. B. O. W.

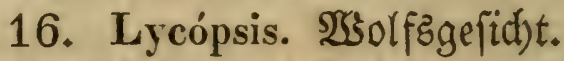

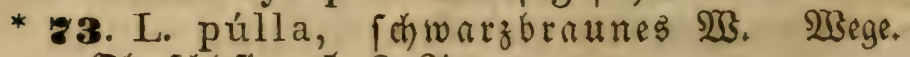
Dberichlefien. 5. 6. 4 .

73. Cynoglóssum. Şundezunge.

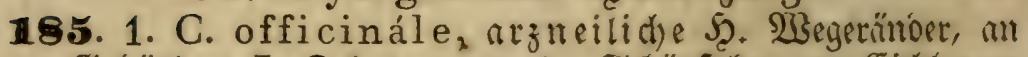
Sebäuden. I. Scheuern zw. D. Sdhönferder u. Sichberger Strape; jest fait veridhivumber. 5.6. ఠ’.

L. S. Ch. B. O. W.

74. Pulmonária. \&ungentraut.

186. 1. P. officinális, nrzneiliches $\mathfrak{R}$. Ridytes, trock nes (Set)ölz. I. Soberlefnen bei luttig u. Rothlad), Sirö=

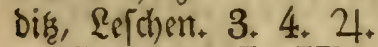

H. 1500 ' ' L. Hb. K. S. Ch. B. O. W.

* 4. P. angustifólia, 〔d)malblïtriges 2 Baldwiejen. 3, 4, 5. 24. K. $1380^{\prime}$. S. Ch.

\section{Omphalódes. Neabelfame.}

* 5. O. scorpioides, vergiß̈meinnid)tblätt= riger $\mathfrak{A}$. Sdhattige Rnubwälber. 4. 5. (. S. Ch. 
75. Sýmphitum. S̉einwurz.

18\%. 1. S. officinále, arzneilithe $\mathfrak{B}$., Şeinwelt,

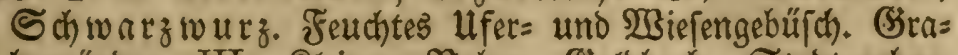
bentiönder. III. Rleimer Bober, Soldbach, Stadtgraben, Förifterbach), SBober, Sucig. 5. 6. 4.

H. 1100' L. Hb. K. S. Ch. B. O. W.

* \%. S. tuberósum, Enollige $\mathfrak{B}$. Wälber. 4. 5. 2. S. Ch.

18. Cerinthe. $\mathfrak{B a c h} \mathfrak{b}$ łume.

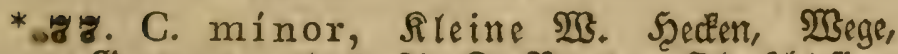
Grtasgatten. 5. 6. 24. O. Breslau, Dberidlefien.

76. Echium. Natterfopf.

188. 1. E. vulgáre, gemeiner $\Re$. Sonnige, trockene,

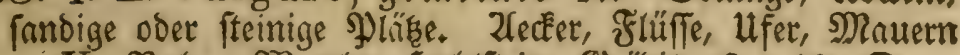

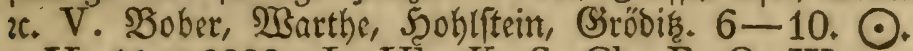

H. $11-2000^{\prime}$, L. Hb. K. S. Ch. B. O. W.

19. Asperúgo. Scharff́raut.

* 8. A. procúmbens, niederliegendes Sh.

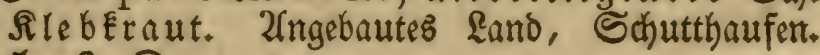
5-9. $\odot$.

H. $10-2000^{\prime}$. L. B. W.

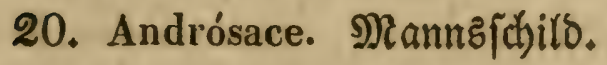

* ร. A. elongáta, verlängertes פ. Sand= ricfer. Iroppau. 5. $\odot$.

* 80. A. septentrionális, nörólides M. Sandiaffer. MBohlau. 6. \%. ○.

* 81. A. Chamaejásme, niedrigez M. Şod)= gebirgsfelien. 6. \%. 24. Л\%. Sdinneegrube.

\section{Prímula. Sprimel.}

189. 1. P. officinális, arzneilides गु $r_{+}$gelber

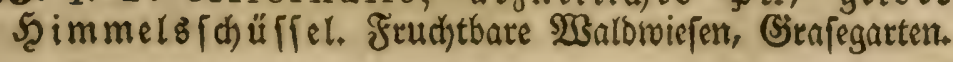




\section{0}

II. Schauermühle, Bandendorf, Schönfelder Şügel, Brö= bif. 4. 5. 4 .

H. $1000^{\prime}$. L. Hb. K. S. Ch. B. O. W.

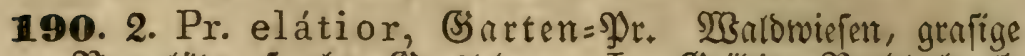

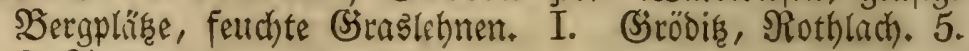
6. 24 .

H. 2200'. L. Hb. K. S. Ch.

* 82. Pr. mínima, Eleinftes $\mathfrak{~ r . ~ S R i e f e n g r b i r g e . ~}$ 5. 6. 4 .

21. Soldanélla. Âtpenglöckelein, Irnobelblume.

* 83. S. alpina, wahres 2 lpenglöcklêtir. Şa= bia Giota. 6. 4 .

78. Hottónia. $\mathfrak{B}$ afferfeder.

191. 1. H. palústris, $S_{u m p F}=\mathscr{2} 3$., gemeiner $\mathfrak{K}$. Iieferes, ftillftehendes 23 affer. II. S3oberlachen, beim $2 B e h r$,

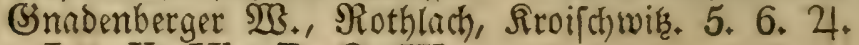

Ln. K. Hb. B. O. W.

\section{Lysimáchia. Injîmachie.}

192. 1. L. vulgáris, gemeine ‥, gelber $\mathfrak{Z B}_{\text {eidrich. }}$ Feuchters (Gebüifh, bejonders ufergebüf(h), an Grabetr. IV. Bober, Föriterbach, Ginadenberger 23 ., Goldbuch, Rosivis, Birkenbrücke, Siraufchen. 6.7. 24.

H. $1200^{\prime}$, L. Hb. K. $1480^{\prime}$. S. Ch. B. O. W.

193. 2. L. thyrsiflóra, f́trauß̧blüthige \&. Sumpfige

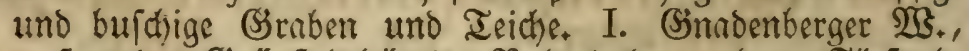

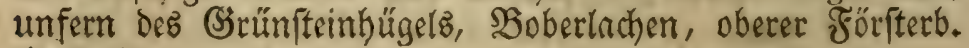

5. 6.24 .

L. B. O. W. Hb.

198. 3. L. nummulária, Eriechende ․, গPfenniggs=

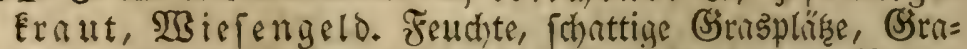
ben u. Ssrabenrïnder. IV. Untere Föriterbachwięé, SBober= infel, Balgenteich, হuecebrummen u. v. S. 6. 7. 4.

H. $1500^{\prime}$. L. Hb. K. S. Ch. B. O. W. 
195. 4. L. némorum, Shain=

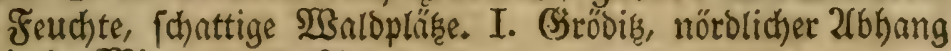
i. D. Mitte. 6. 7. 24.

H. 2000'. L. Hb. K. S. Ch.

\section{Anagállis. (3̧aucb)heil.}

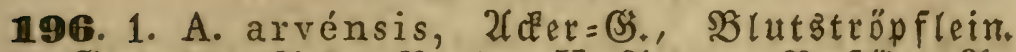

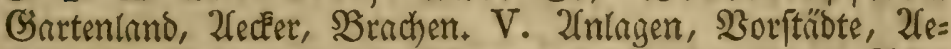
deet um Ulttig $x$. bei Thommenoorf auf einem 2teker

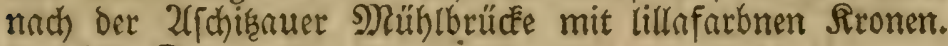
$6-10 . \odot$.

H. $2000^{\prime}$. L. Hb. K. S. Ch. B. O. W.

22. Villársia. Silllarfie.

* 84. V. nymphoides, feerofenartige $\mathfrak{B}$. $\mathfrak{Z}$ ei=

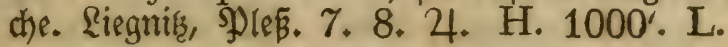
81. Menyánthes. Zottenblume.

198. 1. M. trifoliáta, oreiblättrige 3., Bitter=, Fieber= Ilee. Naffe Sdlamm = uno Mrootwiefen $u_{\text {. }}$ be=

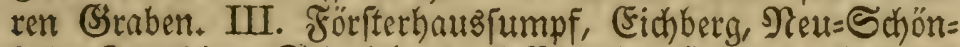

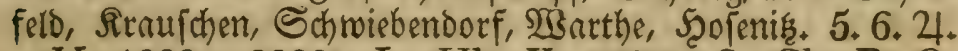
H. $1000-2200^{\prime}$. L. Hb. K. $1280^{\prime}$, S. Ch. B. O. W.

\section{Convólvulus. ŞSinde.}

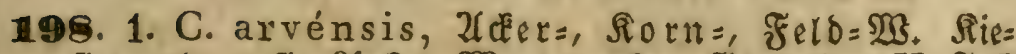

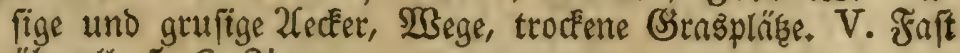
überall. 5, 6. 24 .

H. 2200' L. Hb. K. S. Ch. B. O. W.

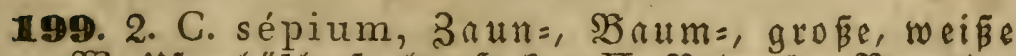

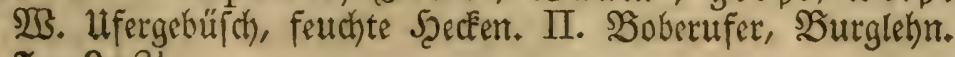
7-9. 24.

H. $1500^{\prime}$, L. B. O. W. Hb. Felten. S. Ch.? 83. Jasione. Sáfinto.

300. 1. J. montána, Berg= Ș, Schanfleabiofe, $3 *$ 


\section{2}

Shnafrapunzel. Trockente und fonmige Sandplizke, $2(e=$ ceer, uffertehmen. IV. Drüßscl, Sober, Tillenoorf, Roosivis, Warthe, Froifatwis, Sttenoorf. 6-10. 24.

H. $2200^{\prime}$. L. Hb. K. S. Ch. B. O. W.

84. Phyteúma. Rapwurzel.

201. 1. Ph. spicátum, ährenblüthige $\Re$., $\mathfrak{Z}$ alo=

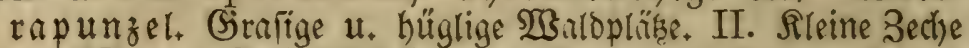

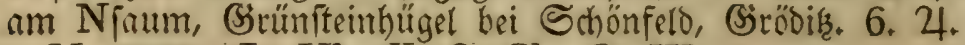

H. 2200'. L. Hb. K. S. Ch. O. W.

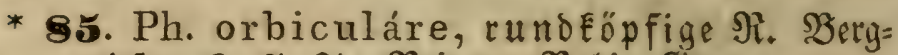

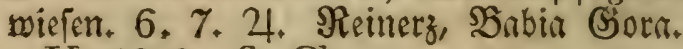

H. $1800^{\prime}$ : S. Ch.

\section{Campánula. Şlodéenblume.}

202. 1. C. rotundifólia, runoblättrige (3), Mil (t)=

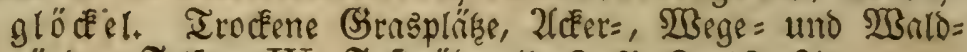
ränder, Forlfen. IV. Fraft überall. 6. 7. 9. 10. 4.

H. $1200-2200^{\prime}$. L. Hb. K. S. Ch. B. O. W.

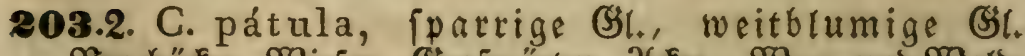

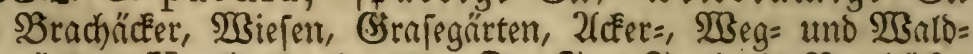

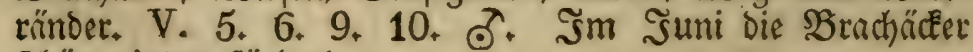
foion violett fïrbento.

H. $2200^{\prime}$. L. Hb. K. S. Ch. B. O. W.

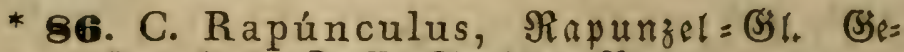
bölze. 5, 6. ఠ) K. Strehiner Serge.

204. 3. C. persicifólia, pfirfichblättrige B̆l,

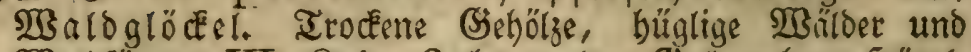

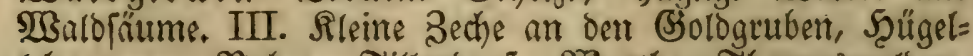
lehnen am Sober, Tillentorf, SBarthe, Thomastwalbau, WBehrau, Gröobis. 5. 6. 24.

H. 10-1800' L. Hb. K. S. Ch. O. W.

* 82. C. liliifólia, lilienblättrige (3). ßerg= twältoer. 6. 7. 4. Eeierbberg. Strehtner Berge. 


\section{3}

* 88. C. latifólia, breitblïttrige (5). Berg= wấlber. ? ? 6. 7. 24 .

$$
\text { L. Hb. K. S. Ch. }
$$

205. 4. C. Trachélium, rauke G\%. 2Biefengebüfche,

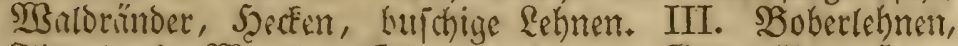
Dillenoorf, MBartbe, Thomasmaldau, Golomilhte, Seart= mannsoorf, Gröbig, Sabliftein. 7.8. 4 .

H. 2000', L. Hb. K. S. Ch. B. O. W.

206. 5. C. rapunculoídes, Eriechende (5) $\mathfrak{l}_{+}, \mathfrak{A}$ CEer=

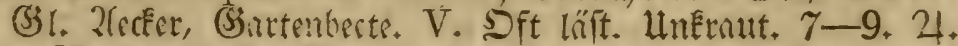
H. 2000' L. Hb. K. S. Ch. B. O. W.

* 93. C. bononiénsis, Bolognefer = \$\%) Trof=

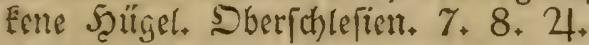

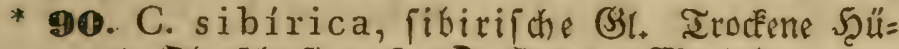
gel. Sberfhlefien. 6. ఠౌ. Sppeln, Morighb.

* D1. C. barbáta, bärtige (5) J. Şochgebirge,

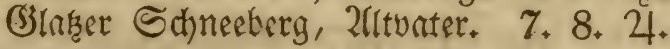

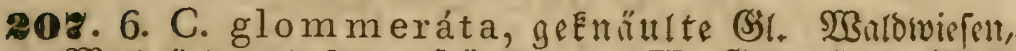

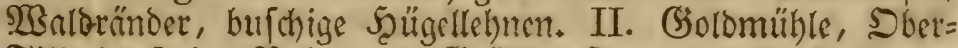
Tillenoorf, im Bobergeb., Giröbia, Sartmannso. 7. 8. 21. H. 1200' L. Hb. S. Ch. B. O. W.

208. 7. C. cervicária, natterkopfblätrige (5), wie

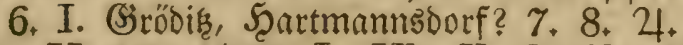

H. $10-1400^{\prime}$. L. Hb. K. S..Ch. W.

\section{Verbáscum. WSolrêraut.}

209. 1. V. Thapsus, bla

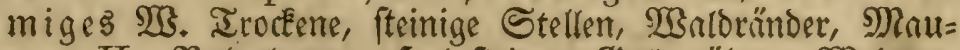
ern. II. Boberbette, Scohlftein, Gsolomühle, Wẹthrau,

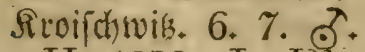

H. $1200^{\prime}$. L. Hib. K. S. Ch.

210. 2. V. thapsifórme, groß̈blumigeb $2 B ., \quad \Re_{0}=$

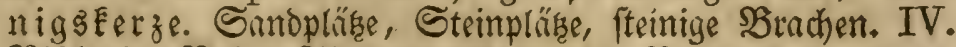

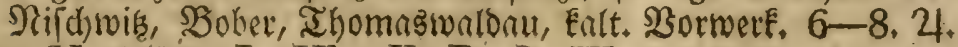
H. $1500^{\prime}-$ L. Hb. K. B. O. W. 


\section{4}

211. 3. V. phlomoldes, $\mathfrak{B}$ indblumen= 3 . Wie 2. $6-8.24$.

H. $1500^{\prime}$, L. Hb. S. Ch. B. O. W.

212. 4. V. Lýchnitis, $\mathfrak{A} e r z e n=2 B$. Trocene Şügel Mauern, Wegeränder. III. Tillendorf, Thomaşwalon Behrau. 6-8. ఠ?

H. $1200^{\prime}$. L. Hb. K. S. Ch. B. O. W.

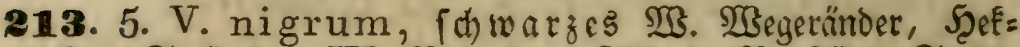
Een, Sträuther. III. Boberbette, Tillendo, Borftiobte, Stadt= graben, Filit [choorf $2,7,8.0 \%$.

H. 1400' L. Hb. K. S. Ch. B. O. W.

214. 6. V. Blattária, Motten= $\mathfrak{B}_{\text {., }}$ Schaben

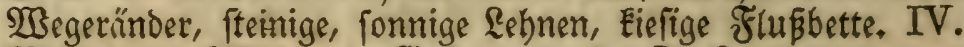
Boberbette, Iillenoorf, Sroöoi

* 92. V. phoeníceum, violettes $\mathfrak{W}$. Wege=,

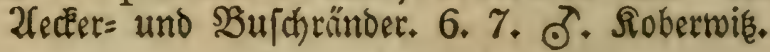

\section{Datúra. Stechapfel.}

215. 1. D. Stramónium, gemeiner St. Gartenland,

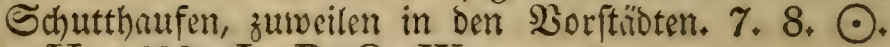
H. $1600^{\prime}$. L. B. O. W.

88. Hyoscýamus. Billenêraut.

216. 1. H. nigex, fth warzes $\mathfrak{B}$. Sd)utthaufen, ungebru=

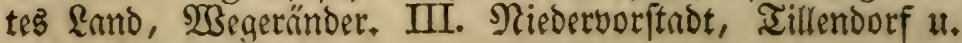
a. 5. 6-8. $\odot$.

H. 1400'. L. B. O. W. Hb. pelten. K. S. Ch.

23. Atropa. IolfÉiridoe.

* 93. A. Belladónna, gemcine $\mathfrak{I} ., \mathfrak{B}_{0}$ olf Ėir đe. Bergmálder. 6. 7. 24.

H. 1500'. Scţönna, S. Ch. Eute. 3obten. 80. Solánum. Nachtichatten.

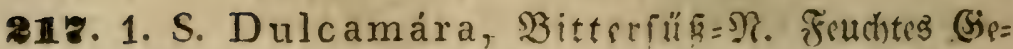


büf(h), Ufergeb. III. Mähtgraben, Bober, Gorolbbad), Eleiner Boter, Sinnoentertaer $2 \mathbb{3} a f f e r, 6-8.24$.

H. $1200^{\prime}$. L. Hb. K. S. Ch. B. O. W.

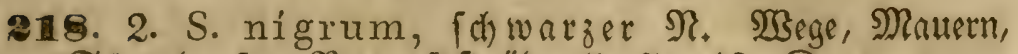
Schutthaufon, Seste, faift überall. $7-10, \odot$.

L. Hb. S. Ch. B. O. W.

\section{Erythráea. Ernthree.}

219. 1. E. Centaúrium, Taufendgulden=ङ. Trockene

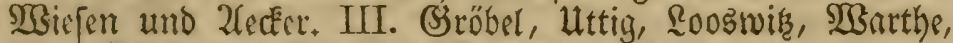

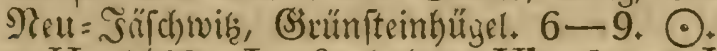

H. 1400'. L. Eanoshut. Hb. Feiter, K. S. Ch. B. O. W.

220. 2. E. ramosíssima, äfige, niedrige (E. Fentute

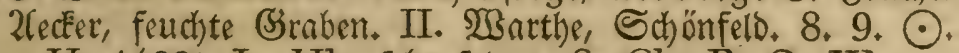
H. 1400'. L. Hb. fehr felten. S. Ch. B. O. W.

\section{Gentiána. Enzzian.}

* 94. G. punctáta, punktirter E., Şochgebirgg=

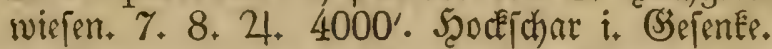

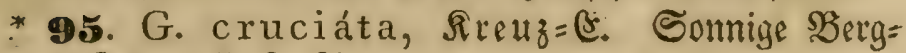
Ttellen+7,8, 4 .

H. $11-1200^{\prime}$. L. Hb. S. Ch.

* 96. G. asclepiadéa, for walbentwurzartiger (E. Scochgebirgatwaldung. 8, 9, 24, Echreiberzhau. H. $1200^{\prime}$, L.

221. 1. G. Pneumonánthe, $\mathfrak{B}$ iefen=ぼ, (Bebüfd auf

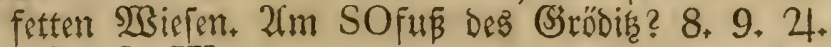

L. O. W.

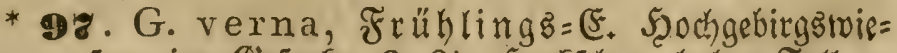

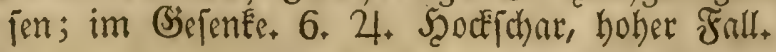

* 98. G. ciliáta, gefranzter E. Gebirgstwiefen,

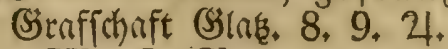
Hb. S. Ch. 


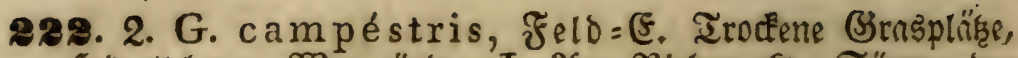
Shügellehnen, $\mathfrak{B}$ egeränder. I. 2(m $\mathfrak{B i e h m a r E t , ~ S i ̆ u m e ~ b e r ~}$ Sandgruben. 8, 9, $\odot$.

H. $12-1500^{\prime}$, L. Hb. K. $1680^{\prime}$. S. Ch.

223. 3. G. Amarélla, SBitteréraut $=$ F. Feuchte Sumpfwiefen, 5eofenilis? 8, 9. $\odot$.

H. $2200^{\prime}$. Hb. K. 1680', S. Ch.

*: Đ9. G. germánica, Deutfder E. Fette Berg= wiêen. 7. 8. $\odot$.

H. $12-1500^{\prime}$. Hb. K. $1730^{\prime}$, S. Ch.

\section{Swértia. Smertie.}

* 100. Sw. perénnis, mebriăhrige ङw., blauer ãant. Fendhte humusteiche Stellen Des Şochgebirges. 7. 8. 24. Schneegruben.

\section{Lonicera. ScectenEirif de.}

224. 1. L. Xylósteum, hartholzige Fૃ. Fenchte,

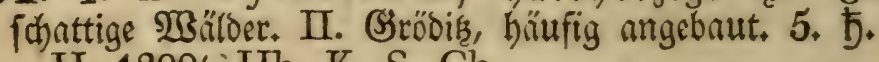

H. $1300^{\prime}, \mathrm{Hb}$. K. S. Ch.

225. 2. L. Periclymenum, beut (⿹勹巳) Ş., gemeines

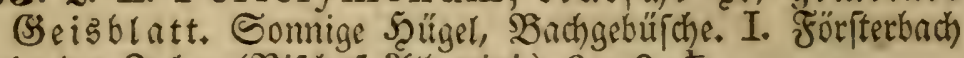
in ber Becthe, (Bijchof 2ulbertini.) 6-9. ந.

* 101. L. nigra, fdwarze 5ૃ. Esebirge 4. Şod)= gebirge. 5. 6. 5 .

H. $15-2500^{\prime}$, Lo. Hb. S. Ch.

- 93. Rhámnus. গ̇Gegoorn.

226. 1. Rh. cathártica, purgirender 203. 24ckerrän=

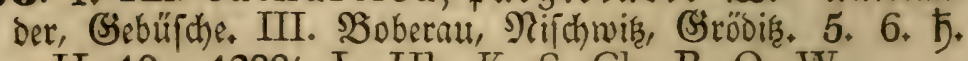
. H. 10-1200' L. Hb. K. S. Ch. B. O. W.

228. 2. Rh. saxátilis, FelfeñB. Steinige uno ponnige

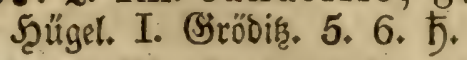

228. 3. Rh. Frángula, glatter $\mathfrak{3}$. Faulbaum. Bis=

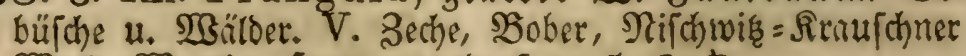
2Bald, 2Barthe, 5rattmannsborf 3. 5. 6. 5.

H. $2200^{\prime}$, L. Hb. K. S. Ch. B. O. W. 
94. Evónymus. Spindelbaum.

229. 1. E. europaéus, gemeiner Sp. \$faffenröfel.

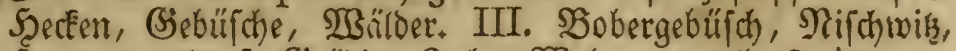

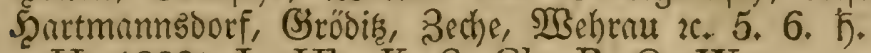

H. 1800' L. Hb. K. S. Ch. B. O. W.

* 102. E. verrucosus, warziger Sp. Wälder. 5. 6. 5. Trebnik.

\section{Ribes. Jobannizbeere.}

230. 1. R. rubrum, to the S. WBitlor, Seedfert, Băune. Mehr angebaut als twild. Sirfivifs im Fichtichthale. 4.

5. 5.

H. 1109-1200', L. Hb. K. S. Ch. B. O. W.

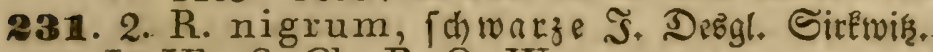
L. Hb. S. Ch. B.. O. W.

232. 3. R. grossulária, Stachel= . Deşgleichen. H. $1500^{\prime}$. L. Hb. K. S. Ch.

* 103. R. petraéum, F̌lfen= న. Şochgebirge. 5. 6.5 .

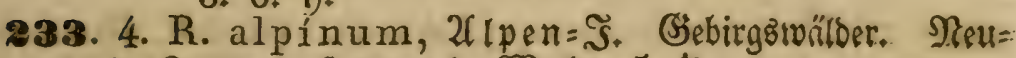
Lano, Runzenoorf unter D. Şalde. 5. ந.

L. S. Ch. Hb.

\section{Hédera. Ephyeu.}

234. 1. H. Helix, wahrer E. Sahattiye Wrälder. III.

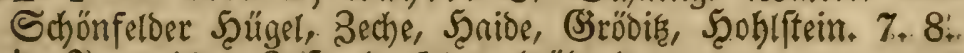
5. जm wilden Zufftande Felten blühend. H. $1500^{\prime}$, L. Hb. K. $1680^{\prime}$, S. Ch. B. O. W.

\section{Illécebrum. Inorpelblume.}

235. 1. I. verticillátum, quirlige $\mathfrak{I n}_{\text {. }}$ Nagel= Eraut. Feuchte, fandige Zfecéer, Şaibes, WSegränder. III. Müllbode, Iiefenfurth. 6-8. $\odot$. Ln.

\section{Glaux. Mild) fraut.}

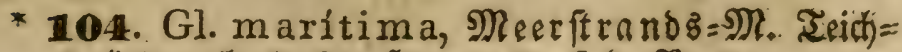
ränder. 5. 6. 24. Şermanzborf b. B̉eglau. 


\section{8}

\section{Thésium. T̃befüum.}

* 105. 1. Th. montánum, Berg= Ih. Irodne Sृüget. 7, 8, 4 .

K. $1730^{\circ}$, S. Ch.

* 106. 2. Th. ebracteátum, dectolattlofer

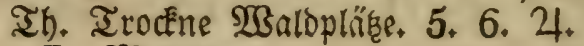

L. Malnpane.

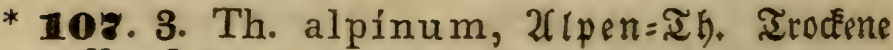
Bergítellen. 6. 7. 4.

Hb. K. $1880^{\prime}$.

\section{Vinca. Sinngrün.}

236. 1. V. minor, Eleines S. (Sebuliche. II. Sleine 3ెe= che, Soberinfel beim Seospital, SHiprsoorf. 5. 6. 21. (Nie blühend gefunden.)

H. 2100', L. O. S. Ch.

99. Impátiens. Saalfamine.

23\%. 1. I. Nolitángere, gelbe $\mathfrak{B}$. Feuthte, fchattige

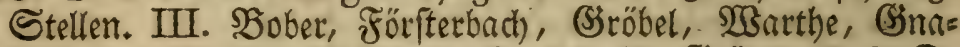

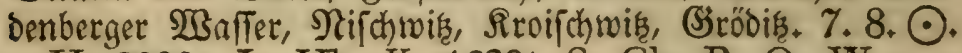

H. $2000^{\prime}$. L. Hb. K. 1639', S. Ch. B. O. W.

\section{Vióla. Seilchen.}

238. 1. V. palústris, Sump $=\mathfrak{B}$. Moor $=$ uno $\mathfrak{T}$ orf $=$ wiejen, oft zwijchen Torfmoos. V. 3eche, Drüßel, Schön= felder Sümpfe, Sirufchen, Şaibe, Birkenbr., Sröbiš. 4. 5. 24 .

H. 2200' L. Hb. S. Ch. B. O. W.

* 108. V. uliginósa, Moor = $\mathfrak{B}$. Torfgruno. 3. 4. 24. ? L. SDppeln.

239. 2. V. hirta, Eurzhaariges $\mathfrak{B}$. Šebüfche, 3ె̆une, Raine, trodene $\mathfrak{B S i e f e n . ~ I I I . ~ I i l l e n d o r f , ~ D r u ̈ b e l , ~ B ̉ o b e r , ~}$ ลecte. 3. 4. 24.

H. $2200^{\prime}$. L. K. S. Ch. B. O. W. 


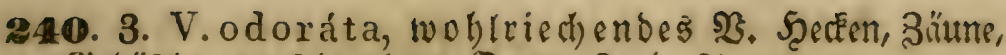
Gebüfthe, an feht visten Srten. 3* 4. 4.

H. L. Hb. K. S. Ch. B. O. W.

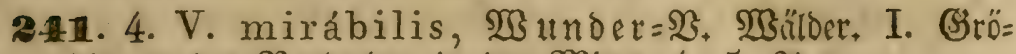
Dis an Der Norblehme in Der Mitte. 4. 5. 4.

H. $1200^{\prime}$, S. Ch. B. O.

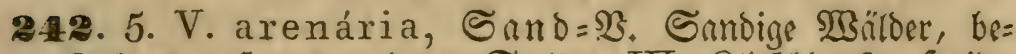
fonbers auf aubgerodeten Stellen. III. Siltiţchoorfer Scaide, bei Tillenoorf, Sect)e. 3, 4. 24.

W. $\mathrm{Hb}$.

243. 6. V. canín a, Şund

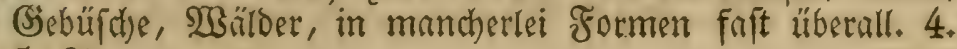
5. 24.

H. $2200^{\prime}$. L. Hb. K. S. Ch. B. O. W.

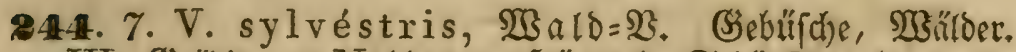
III. Ströbis am Nabhange, Sçügel b. Schönfeld. 4-6.24. H. 2200', L. Hb. K. S. Ch. B. O. W.

245. 8. V. Riviniána. Riving $=\mathfrak{B}$, wie 7. fettner. $\mathrm{Hb}$.

246. 9. V. persicifólia, pfirfichblättriges $\mathfrak{B}$.

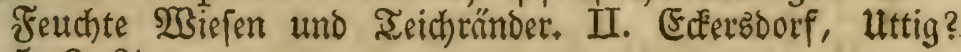
5. 6. 24.

B. O. W.

241. 10. V. praténsis, $\mathfrak{B}$ iefen $=\mathfrak{B}$. Feuthte $\mathfrak{W}$ iefen Gebüfhse. III. 2(m Gstünfteingügel bei Schönfelo, Thho= mastwalbau, Gröobiá, 5. 6. 24.

H. $1400^{\prime}$, O. W. Hb. Ch.

* 109. V. biflora, zmeiblüthiges $\mathfrak{B}$. Bebirgz=: maffertïnder. 5. 6. 4.

H. 15-2000', L. Hb. S. Ch.

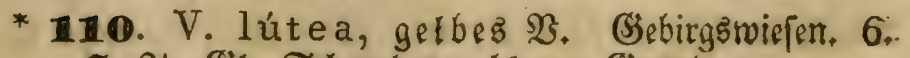
7. 24. (3). Sd)neeberg; blauer Grand. S? Gépenép. 


\section{0}

248. 11. V. tricolor, oreifarbiges $\mathfrak{B}$., $\mathfrak{A}$ de $\mathfrak{e}=\mathfrak{B}$, , Stiefmütterden. SBrachen, 2lecker, Sartentano, F́lußs= ufer, Scaibe. Faft überall, zutweilen in großer Menge, bie Srachen gelb färbend; viele Formen; eine grö̈blumige $m$. 2 bis 5 röthlichblauen SGlütbenblättern, bef. am Sueis; fait burchs ganze Sahr. $\odot$. auch in Friebeberg a. Sueis. H. L. Hb. K. S. Ch. B. O. W.

\section{3weite Sronung. Digýnia. Bweiptentplige.}

\section{Cynánchum. Şłnożwürget.}

249. 1. C. Vincetóxicum, gemeiner $\mathfrak{C}_{.}$, Schrort= benwurz. Raubwaldungen, Sebufd)e. I. Sipfel des Spik= kerges 6 . Probithain. 6-8. 24.

L. Hb. K. 1680'. S. Ch. O. W.

102. Herniária. Brutchkraut.

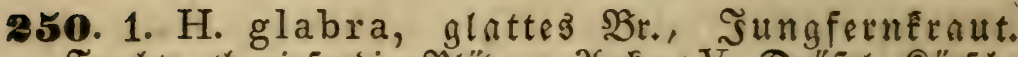

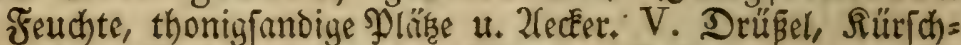
nerberg, Sober, Iillendorf, SBarthe, Gröbic u. a. v. D. 6-10: $\odot$.

H. $15-2100^{\prime}$. L. B. O. W. Hb.

* 111. H. hirsúta, baariges $\mathfrak{B r}$. Irockne Sando

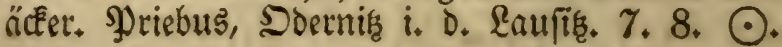

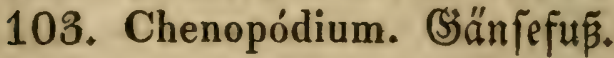

251. 1. Ch. Bonus Henricus, Dorf $=$ (S). Şumusrei= der $\mathfrak{B}$ oden an $\mathfrak{B}$ egen und Mauern, befonders in Dörfern und Sorftiotent. III. Borftíbte, befonders NiÉlnivorftadt. 4-9. 24 .

H. $1200^{\prime}$. L. Hb. K. S. Ch. B. O. W.

252. 2. Ch. hýbridum, $B a$ ftar $\delta=\mathbb{G}$. WBie 1. befon= bers nuf Schutthaufen. 7-9. $\odot$. aber feltener.

L. Hb. K. S. B. O. 


\section{1}

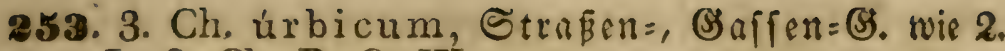
L. S. Ch. B. O. W.

254. 4. Ch. rubrum, rother (5), Neunipifen. Ș

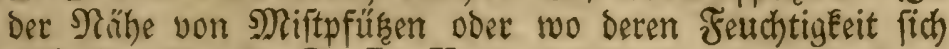
verbreitet. 7-9. $\odot$. L. K.

255. 5. Ch. murále, Mauer=G5. Maum, Schutt, $\mathfrak{Z} E=$ Eertano, 3üume. IV. '2́nftalt, Siraben 2 . 7-9. $\odot$.

L. Hb. K. S. Ch. B. O. W.

256. 6. Ch. album, wei Ber (5). 2Rfere u. Esnttentant, Rartoffelíçer $x$. V. Fafít überall. 6-9. $\odot$.

L. Hb. K. S. Ch. B. O. W.

* 112. Ch. opulifólium, bachyolderblättri=

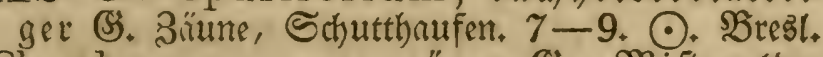
25\%. 7. Ch. glaucum, graugrüner (3), Mift melde.

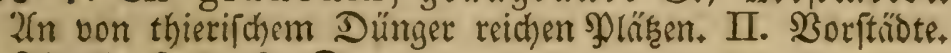
Iillendorf. 7-9. $\odot$.

L. S. Ch. B. O. W.

258. 8. Ch. polyspérmum, vielfamiger (5), Fif

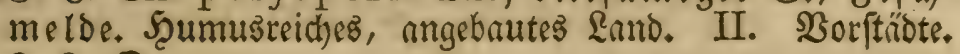
8. $9 . \odot$.

259. 9. Ch. ólidum, ftinÉender (5)., Sootésmelde. ZIn Manern. IV. 2Um Sauteich, in Der Mieber=2orftabt. Sceüchit wioriger (seruth. 7-10. $\odot$.

L. S. B. O. W.

* 113. Ch. ficifólium, feigenblättriger (B). Zlecêer. 7-9. ○. B̉restau.

* 114. Ch. Botrys, traubiger (3), Bñume, 2ReE=

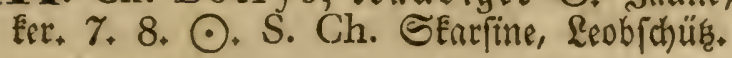

\section{Atriplex. פRelde.}

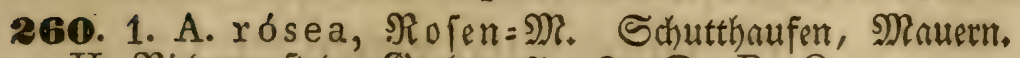
II. Niederborftadt, Graben, 7-9. ○. B. O.

261. 2. A. nitens, glänzende $\mathfrak{R}$. Schutthaufen, angs= bautes Land. ?? Niedervorftadt? 7. 8. ○. B. W.

262. 3. A. latifólia, breitblättrige M. Deşgleidyen.

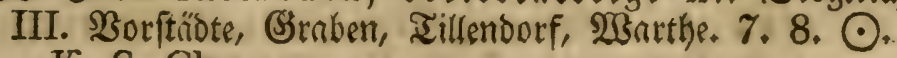

K. S. Ch. 
263. 4. A. pátula, auzgebreitete M. Deb̊gleichen. III. Niedervorftrdt $2.7 .8 . \odot$.

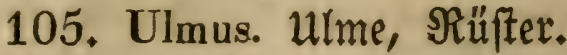

264. 1. U. cámpestris, $\mathscr{F} e l d=\Re$. SBälder, (Bebürd)e. II. Boberthal, SBröbis, Thomaswaldau. 4. ந.

H. 2500'. L. Hb. S. Ch. B. O. W.

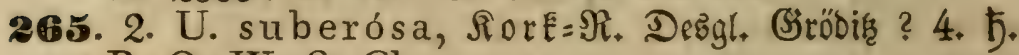
B. O. W. S. Ch.

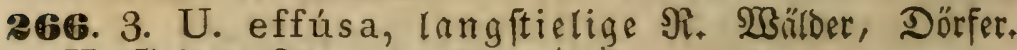

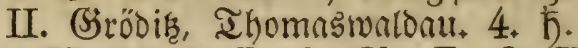

HI. $2500^{\prime}$ L. S. Ch. B. O. W.

106. Cuscúta. F́lachşęeide.

26z. 1. C. europaéa, gemeine F̂t., Neffelfeide. Şel=

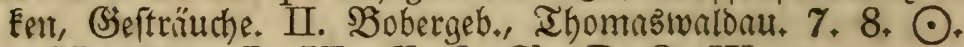
H. $1000^{\prime}$. L. Hb. K. S. Ch. B. O. W.

* 115. C. epithýmum, \uendel = ₹l. Şnibe=

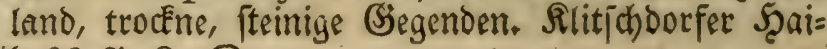
be? $7.8 . \odot$.

S. Ch.

268. 2. C. Epilinum, wahre Fl., Leinfeibe. \&eins felber. III. STin und rieder, mit biefen an andern SDten wech felnd. 7. 8. $\odot$.

H. $2000^{\prime}$, L. K. S. Ch. O. W.

269. 3. C. monógyna, einftemplige frl. Feuchte ufer= gebüfche. I. Bobergebüfhe am $\mathfrak{B}$ ehr. 6. 7. ๑. B. S. Ch.

\section{Dolbenträger ober fchirmblüthige $\$$}

107. Laserpitium. LajerEraut.

* 116. L. latifólium, breitblättriges $\mathfrak{R}$ : $\mathfrak{B} u=$ fobige $23 i e$ en und Sergnbhänge. Sobten, Strie= gau. 7. 8. 4.

* 118. L. Archangélica, angeliéablättriges

\&. Şod)gebirge. 7. 8. 24. 2(It)ater. 


\section{3}

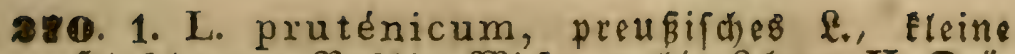

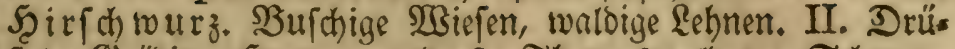
Bel, Sröbis, Şartmanngoorf, Ihhomastwaldau, Schauev mülte. 7. 8. 2 .

H. $1400^{\prime}$, L. Hb. S. Ch. O. W.

\section{Heracléum. Şeilfraut.}

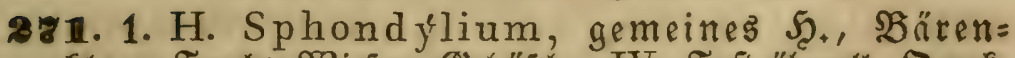

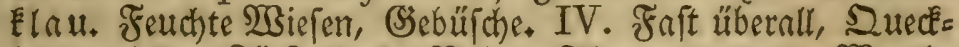
brunnwiejen, Trörfterbach, Bober, Thomastwalbau, $\mathfrak{B a r t h e}$ 2. $6-10.24$.

H. 2200'. L. Hb. K. S. Ch. B. O. W.

\section{Pastínaca. ŞaftinaÉ.}

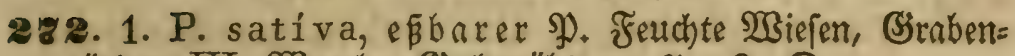

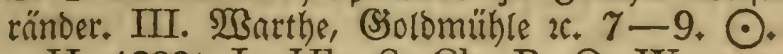

H. $1200^{\prime}$. L. Hb. S. Ch. B. O. W.

\section{Peucédanum. Scaarftrang.}

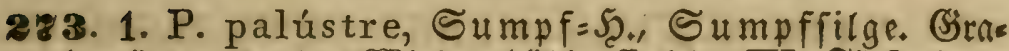

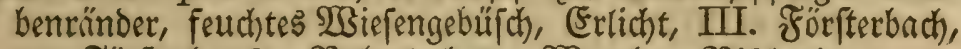

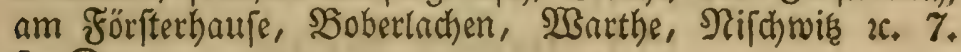
8. $\odot$.

L. Hb. K. $1430^{\prime}$, B. O. W.

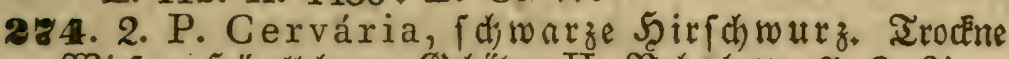
2Biefen, Şügellehnen, Eschölze. II. Soberbette. 7. 8. 4. O. W.

285. 3. P. Oreoselínum, Berg=

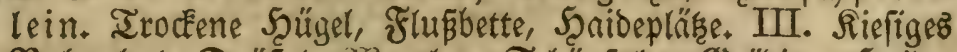

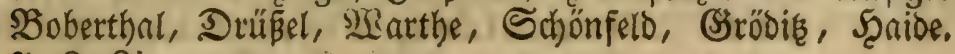
7. 8.24 .

L. B. O. W. S. Ch.

27. Imperatória. Reiftermurz.

* 118. I. Ostrútium, $\mathfrak{B} \mathfrak{e r g}=\mathfrak{M}$. Befdjattete Bebirgabüche. 7. 8. 24. 3acken= und Rodjelfall. H. 2200 ' L. Hb. 


\section{Archangélica. Engelmurz.}

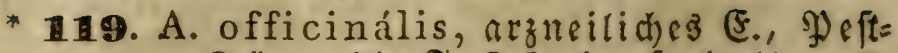

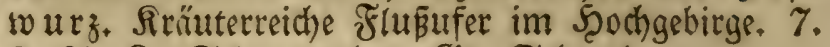
8. 2. S. Sdmeegruben, (si. Sdneeberg.

\section{Angélica. 2̂ngelifła.}

296. A. sylvéstris, wilbe 22. Wisfen, Grnabentünoer,

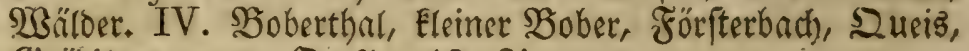
(sröbif u. v. a, D. 7-10. 2 .

H. 2200'. L. Hb. K. S. Ch. B. O. W.

\section{Selínum. Silge.}

28z. S. carvifólia, kümmelblättrige S. Bufchige

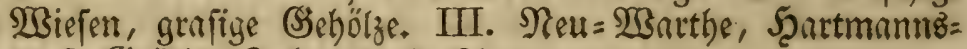
Dorf, Briöbiá, 3eche. 7. 8. 4.

H. $1200^{\prime}$. L. Hb. K. S. Ch. B. O. W.

29. Conioselínum. Esraujilge.

* 120. C. Fischeri, Fif thers Grr. Gefenke. 7. 8. 4.

113. Daucus. Möbre,

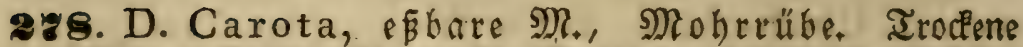

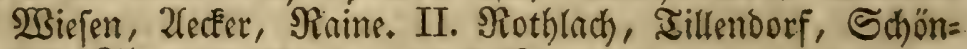
feld, Thomnstualdau $x .6 .7 .24$.

H. $1400^{\prime}$. L. Hb. S. Ch. B. O. W.

\section{Caúcalis. Şaftoolde.}

* 121. C. daucoides, möhrenartige 5ூ. UeE= Eet nuf Ralleboden. 7, 8. ○. Sppeln, Tejhen.

\section{Tórilis. Sorftoolde.}

289. T. Anthríscus, Shecken=\{B. Rlettentörbel. SheE=

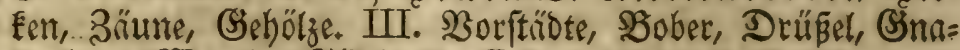

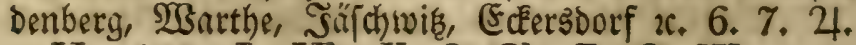

H. $1000^{\prime}$. L. Hb. K. S. Ch. B. O. W. 


\section{5}

\section{Meum. Särenwurg.}

* 12. M. Athamánticum, hanrblättrige 5. SBergiviejen. 7. 8. 24. L. Schmieobberg.

* 123. M. Mutéllina, Gebirgs= B. Bergnvié, Schneeberg, Gsejene. 7. 8. 24. S5\%. Schneeberg.

\section{Silaus. Silau.}

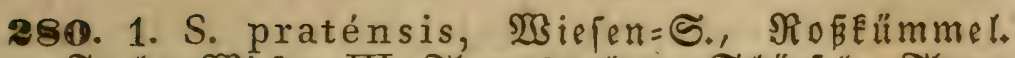

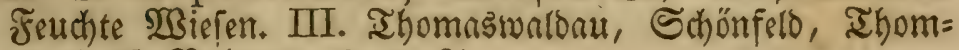
menoorf, Boberau. 6.7. 4 .

K. S. Ch. B. O. W.

\section{Cnidium. Srennoolbe.}

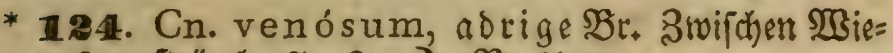
fengeftrïuch. 7-9. oे. SBrestau.

$\mathrm{Hb} . \mathrm{O}$.

\section{Séseli. Sejel.}

2811. S. ánnuum, ftarrer S. Trodfene $\mathfrak{B}$ iefen, grafige

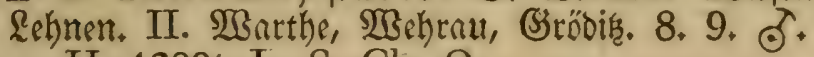

H. 1200'. L. S. Ch. O.

* 125. S. Libanótis, Ereuzblättriger S. Son= nige $\mathfrak{l}$ efhnen. 7. 8. 24. H. $12-1400^{\prime}$. Hb. S. Ch.

\section{Oenánthe. Rebendoldoe.}

282. 1. O. fistulósa, töhrige Rebeno., Iropfruurz. WBaffergraben, jumpfige Teichrinder. I. Soldomühle, Ginn= Denberger Teiche. $6,7,4$. Ln. B. O. W.

283. 2. O. Phellándrium, fenchelfamiger $\Re_{\text {, }}$

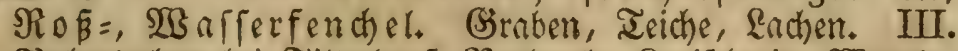

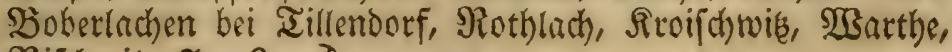

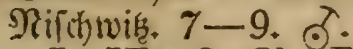

L. Hb. S. Ch. B. O. W. 


\section{6}

\section{Aethúsa. (S)leip̃.}

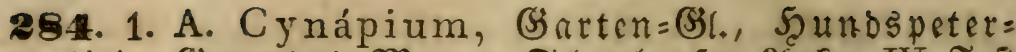
filie. Santenland, Mnuern, Sdhutthaufen, 2lecker. IV. Faft liberall in Den 230 ritiöten 2 . 6-9. $\odot$.

H. 2200'. L. Hb. K. S. Ch. B. O. W.

33. Bupleúrum. \$̧afenoht.

* 26. B. falcátum, fichelblättriges 5ூ. Steinige 2frtyöhen uno 2lecker. 8-10. 24. L.

* 122. B. longifólium, langblättriges $\mathfrak{H}$. Irockene, fteinige uno grafige Ssebulifhe, bejonders nuf MatEboden. 7. 24. Siejengruno.

* B28. B. rotundifólium, runoblättriges 5ृ. Santfelder, Sberichlefien. 6.7. ఠ)

119. Sium. $\mathfrak{S a f f e r m e r k ́ . ~}$

285. 1. S. latifólium, breitblättriger $\mathfrak{B}$. Siraben, Sümpfe, Ieichränder. UI. Nachtigallenbach, Soberlachen, IJanthe. 7, 8, 4.

L. K. $1430^{\prime}$. B. O. W.

\section{Bérula. B̊erle.}

286. 1. B. angustifólia, fómalblättrige B. (Sra= ben, Isichrintorr. I. Warthe, Siolomilhle. 7, 8. 24.

L. B. O. W.

\section{Pimpinélla. Bibermetl.}

28z. 1. P. Saxifraga, Stein= B. Wiefen, Dïmme,

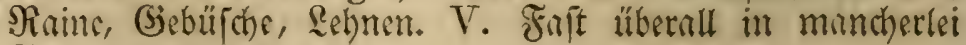
Formen. 7-11. 4.

H. 2200'. L. Hb. K. S. Ch. B. O. W.

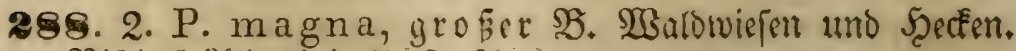
Sifrch of arbertimi. 7. 8, 4 .

H. $10-1100^{\prime}$. L. Hb. S. Ch. B. O. W. 


\section{Carum. Rarbe.}

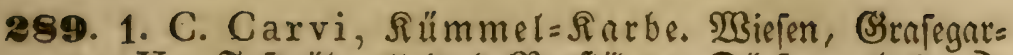
ten. V. Furft überall in D. Borftiöten, Dörferm. 5, 6. ఠे. 123. Aegopódium. (Sierifh.

29ه. 1. A. Podográria, gemeiner $\mathfrak{G}$. Sofftgärten,

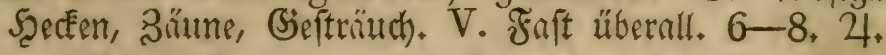

34. Crítamus. Sicheloolde.

* 129. Cr. agréstis, 2laker $=\mathbb{S}$. 2fecker beg $\mathfrak{B o r}=$ gebirgez. 7. 8. 24. L. $\mathrm{Hb} . \mathrm{B}$.

\section{Cicúta. Şafferjhierling.}

291. 1. C. virósa, giftger 23 . Sdhlammige Graben,

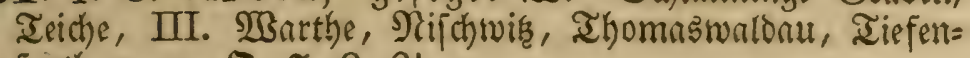
furth $\mathfrak{u}_{+}, \mathfrak{m}_{+}, 7,7,8,24$

L. $\mathrm{Hb}$. O. W.

\section{Chaerophýllum. Rälberfopf.}

292. 1. Ch. hirsútum, rauchhariger $\Omega_{\text {. Feudjte }}$

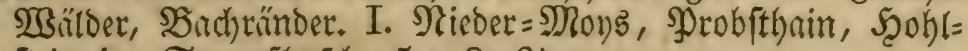
ftein im Sumpfoufth. 5-8. 24.

H. L. Hb. K. S. Ch. O.

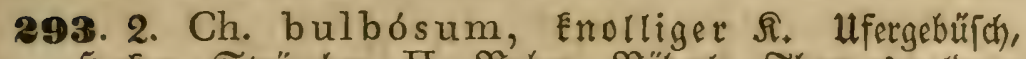
Syecten, Strüucher. II. Bober, Böberte, IThomaswaldau, Iillenoorf, Bröbis. 6. 7. बे.

H. 1200'. L. Hb. S. Ch. B. O. W.

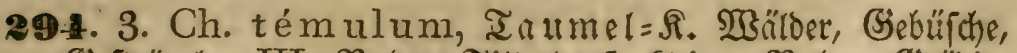

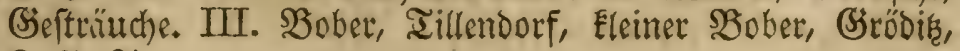
6. 7.24 .

L. Hb. S. Ch. B. O. W.

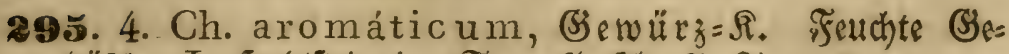

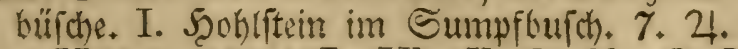

H. 10-2200' L. Hh. K. S. Ch. O. W. 


\section{8}

* 130. Ch. auréum, gelbfrüchtiger $\Re$. Şodit) gebirgswiefen. 7. 24.

K. S. Ch.

126. Anthriscus. Rlettenförbel.

296. 1. A. sylvéstris, grỗer R̂l. Wiejengebüf(h), Şef=

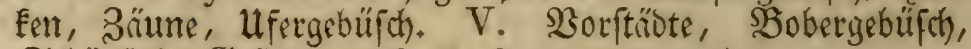

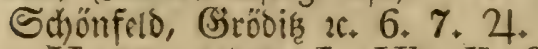

H. $10-1500^{\prime}$. L. Hb. K. S. Ch. B. O. W.

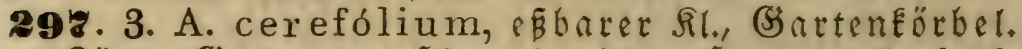
Bäume, Sirajegarten. Şin uno mieder, Şartmammborf. 6 . 7. बे.

L. S. Ch.

298. 2. A. vulgáris, gemeiner Sil, Efelstörbel. Säune, Sdutthaufen, Mauern. I. Şin uno wieder. B̉or= ftiote, Sdönfeld, 6. $\odot$.

H. 10-1500' L. K. B. O. W.

35. Mýrrhis. Süsoolde.

* 131. M. odorata, woblriechende S. Scobe Bergniejen. 6. 7. 4.

H. $12-2230^{\prime}, \mathrm{Hb} . \mathrm{K}$. S. Ch.

127. Cónium. Sdbierling.

299. 1. C. maculátum, gefledéter Sch. 2rn Mantern,

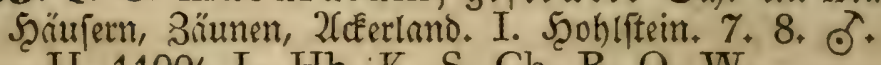

H. 1100'. L. Hb. K. S. Ch. B. O. W.

36. Pleurospérmum. SRibbenfame.

* 132. P1. aústriacum, öftreidifder $\Re$. Flup̣thäler auf hohen Bergrwiefen. 7. 8. 24. $\mathrm{Hb}$. K. S. Ch.

37. Astrántia. 2ffrtrantie.

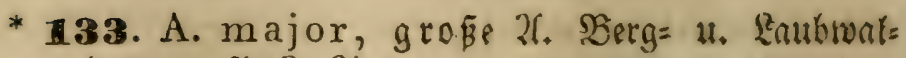
bungen. 7. 8. 2 .

H. $1700^{\prime}$. L. Hb. S. Ch. B. O. W. 


\section{Sanícula. Samikel.}

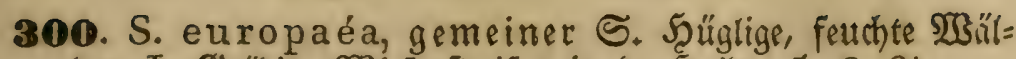
Der. I. (Sröbik, Wiejenftreifen in D. Scnibe. 5. 6. 4 .

H. 11-2300' L. Hb. K. S. Ch. W.

\section{Dóndia. Dondie.}

* 134. D. Epipáctis, grüue D. ßerg= uno

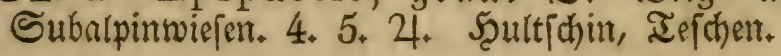
39. Erýngium. Nannztreu.

* 135. E. planum, fladblättrige M. Trode ne Felder. 6.7.24.

H. $1250-1300^{\prime}$. B. O. W.

* 163. E. campéstre, Feld = M. Trockene F̃el= ber, 23 egeränder. 6. 7. 24. L. SBrestau.

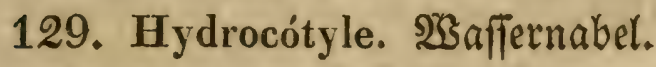

301. H. vulgáris, gemeiner $\mathfrak{Z}$. Iorfwiefen, İeichrän= Der. V. Forrtterbach, Şaide, (Snndenberger İeiche, Edefers= Dorf, Tiefenfurth 2 2. 7.8 .24$.

Ln. B. O. W.

\section{Dritte Drsumu. Trigýnia. Dreiftemplige.}

\section{Vibúrnum. Sdjneeball.}

302. V. Opulus, gemeiner Sthnt, $23 a f f e r b o l d e r$, Ralinénbeeren. Ufergebüfक. IV. Sober, Eleiner Sober

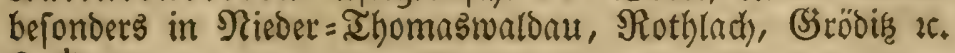
6. $\hbar$.

H. $10-1500^{\prime}$. L. Hb. K. S. Ch. B. O. W.

131. Sambúcus. Şolbet.

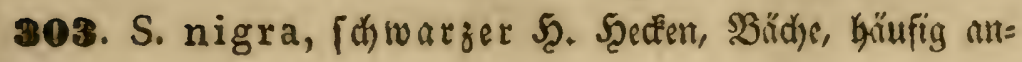


" gebnut. Förfterbach), Iillenoorf, Sothlach), Thomašwaldon x. 5. 6. 5 .

H. $1300^{\prime}$. L. Hb. K. S. Ch. B. O. W.

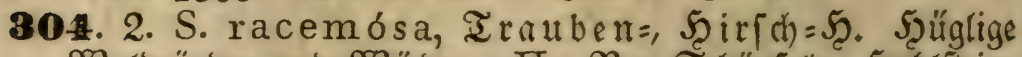

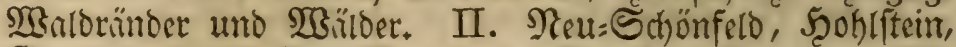

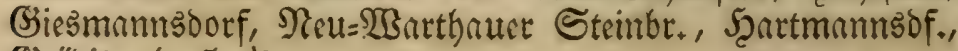
(Sröbif. 4. 5. 5 .

H. $14-2500^{\prime}$. L. W. Hb. K. S. Ch.

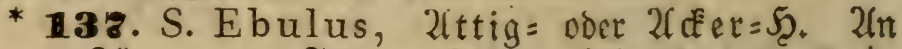

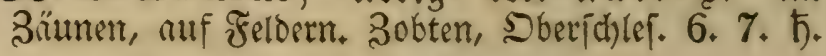

40. Staphyláea. Blajennū̃.

* 138. St. pínnata, federbl. Ş. গুimpernu. Wïlder. 6. 5. Reobichür.

41. Támarix. Zamarisłe.

* 130. T. germánica, beutiche I. Steinige înüufer. Deftreichifich Schlefien a. Der Weichjel uno Delfa. 7. 万.

132. Corrigióla. Stranding.

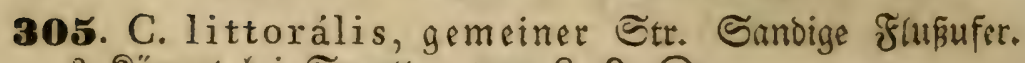
? Römmt bei Sprottau vor. 8. 9. $\odot$.

\section{Bierte Sronutg. Tetragynia. Sierfitemplige.}

\section{Parnássia. \$ुarnaffiete.}

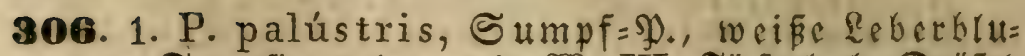

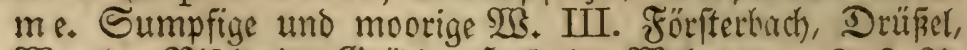

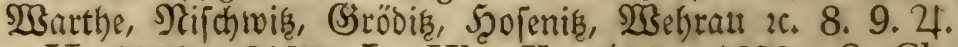
H. $1000-2100^{\prime}$. L. Hb. K. $1480-1680^{\prime}$. S. Ch. B. O. W. 


\section{1}

\section{Füufte Sronutug. Pentagýnia. Finnfitemplige.}

134. Arméria. (3ragnelke.

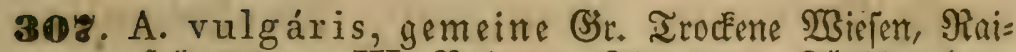

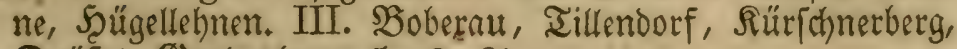
Dorǘpel, Sincidenterg. 5-9. 24.

H. $1780^{\prime}$. L. Hb. K. S. B. O. W.

\section{Línum. \&ein.}

308. 1. L. cathárticum, शurgiv = \&. Fenchte, fette

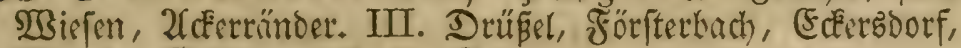

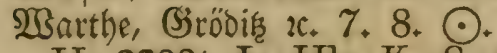

H. 2200' L. Hb. K. S. Ch. B. O. W.

\section{Drósera. Sonnenthaut.}

309. 1. Dr. rotundifólia, runoblättriger $\mathfrak{S}_{\text {. Torf }}$

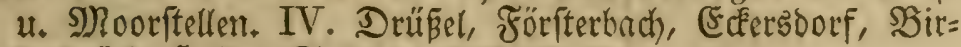
Eenbrück, Şaide, Seitenoorf. 7. 8. 4.

H. $12-2000^{\prime}$. L. Hb. K. $1380-1730^{\prime}$. S. Ch. B. O. W.

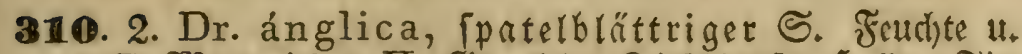
naffe Moorwiefert. II. Streulich, Sititf(hoorfer Scribe, Tie= fenfurth $x_{0}, 7,8,24$.

H. $2000^{\prime}$. L.

311. 3. Dr. intermédia, Eleiner S. Feurhte u. neffe

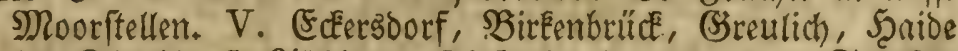

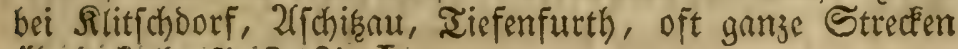
überbecefend. $7,8,4 . \mathrm{L}$. 


\section{Sechste filafie. Hexándria. Sechefabige.}

Crrfte Sorsunty. Monogýnia. Bueiftemplige. 42. Galánthus. Scd)nerglöcécher.

* 140. G. nivális, gemeines Sch. Raubholzge=

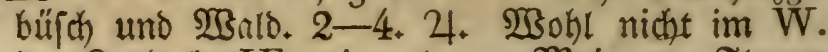
Der Ranfbach. Hb. eimmal vom Major v. Flototw gefunden. .

H. 1200'. S. Ch. O. W.

43. Narcissus. Narziffe.

* 141. N. P s eudo-N arcíssus, Märzbeder. Bergige 2 aldowiefen. 4. 5. 4.

H. $1100^{\prime}$. Ch.

137. Leucójum. Rnotenblume.

312. L. vernum, ซrühlingš= In. Sumpfige Biefen u. BBalopläge. III. Scohlftein, Sumpfbuich unter b. Sd)lö. 2-4. 4 .

H. $12-2200^{\prime}$. L. Hb. K. S. Ch. B. W.

\section{Allium. Raud).}

313. A. senéscens, graulicher $\mathfrak{R}$. Irodeme, fonnige SEerge. I. \$probfthainer Spif̧berg. 7. 8. 4.

314. 2. A. arenarium, $\widetilde{S}_{a n d}=\mathbb{R}$. Sandige Şügel und 2ecker.? Fiömmt bei Sprottau vor. 6. 7. 24. W.

* 142. A. urs inum, Băren= . $\mathfrak{B u}$ d)ige $\mathfrak{B e r g}=$ wiefen. 4-6, 4 .

K. S. Ch.

* 143. A. victorialis, Sieg=̊. Scohe Bergrwie= fert. 7. 8, 4 .

H. $12-2200^{\prime}$. L. S. Ch. O. 
* 140. A. acutángulum, đharfEantiger \&. Fenchte WBiefen. 7. 8. 4.

H. $1144^{\prime}$. B. O. W.

* 145. A. carinát um, Berg=?. Ģebulfché. 7. 8. 2. H. 1500'.

* 146. A. Scorodoprásum, Roccambole. WBaldränder. 6.7. 4 .

L. H. K. $1480^{\prime}$. B. O. W.

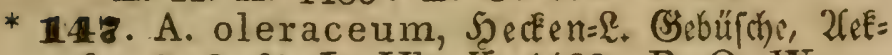
Eer. 7. 8. 24. L. Hb. K. 1480'. B. O. W.

* 148. A. Schoenoprasum, Schnittlauth. Ģebirgäwie

\section{Lílium. Rilie.}

315. L. Martagon, Soldlilie, Bolbwurg, Türên=

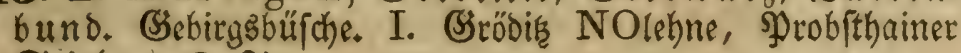
Spisberg. 6. 24.

H. 12-2235' L. Hb. K. S. Ch. O. W.

\section{Túlipa. ז̃ulpe.}

* 149. T. sylvéstris, $\mathscr{B} a l d=\mathfrak{I}$. SOfftgatten. 5. 24.

140. Ornithógalum. Nildbitern.

316. 1. O. stenopétalum, fdhmalblättriger $\mathfrak{M}$.

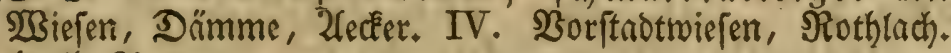
4. 5. 24 .

H. $1200^{\prime}$. L. Hb. K. 1380-1430'. S. Ch. B. O. W.

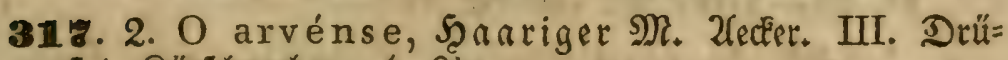
Eel, Rürrjonnerberg. 4. 2 .

$$
\text { L. W. S. Ch. Hb. }
$$

318. 3. O. minimum, Eleinfter Ml, \$bftgärten,

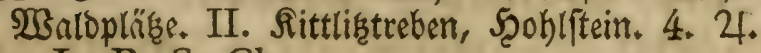
L. B. S. Ch.

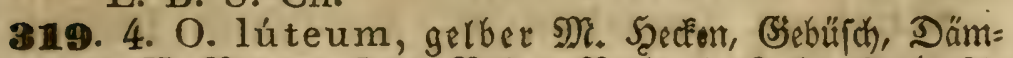
me. V. Riederborftadt, Sober, Rothlach, 3ed)e. 3. 4. 24. H. $12-1400^{\prime}$. L. Hb. K. $1430^{\prime}$. S. Ch. B. O. W. 


\section{4}

320. 5. O. umbellátum, fhirmtragender $\mathfrak{M}$. Uecéer an ber Sctönfeelder Straße, Pechüdter. 5. 6. 24. L. Hb. W.

321. 6. O. nutans, hängender M. Siraggarten. I. Barthe, Scoblitein. 5. 24. W.

* 150. O. spathaceum, fdheidiger M. Feudte Géebüifdere. 4. L.

* 151. O. bohémicum, böhmifcher M. Uet= Eer. 4. 24. SDberichlefien. Ultrow.

\section{Scilla. Reerzmiebel.}

* 152. Sc. a moéna, fdöne, blaue $\mathfrak{R}$., Stern= blume. 24. Dbitgntten. Singernoorf.

* 153. Sc. bifólia, zmeiblättrige M. 4. 5. 4. 46. Agráphis. Sternbyacinthe.

* 154. A. nutans, nickende St. 3. 4. 24. Sit vom Baton v. Stillftieo 1836 in Srasgañrten zu \&eipe bei Sauer aufgefunden worden, foll auth im פRochenwalde bei Schönau mit Cypripedium Calceolus vorkommen.

\section{Muscari. Nuß̨âai.}

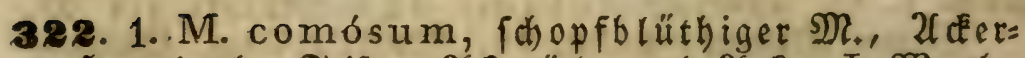

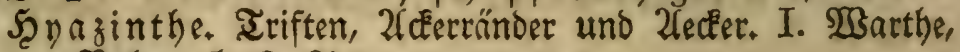
am $\mathfrak{B o b e r} .5,6.4$.

H. 12-1500'. L. Hb. B. W.

* 155. M. racemósum, Iraubiger $\mathfrak{R}$., Irau= ben = 5̧yazinthe. WBeinberge. 4, 5, 24. L.

142. Convallária. Thallitie.

ass. 1. C. majális, wohlriectende $\mathfrak{Z} h$., Springauf, Daiblume. Raubröltber. II. Siteine Seche, Scoblftein, 3umm. 4. 5. 4 .

H. $1500^{\prime}$. L. K. S. Ch. B. O. W. 


\section{5}

143. Polygonátum. W̉eiß̄murz.

324. 1. P. anceps, wohltiechende 233 ., Salomons= fiegel. Feuchte, f(h)attige WBaldiftllen. I. Scohliftein. 5. 6. 24

H. L. K. S. Ch. B. O. W.

325. 2. P. multiflorum, vielblumige 23 . Ģehölzs,

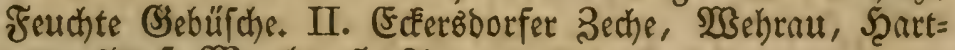
mannsoorf, פBatthe. 5. 4.

H. 1500' L. Hb. K. S. Ch. B. O. W.

* 156. P. verticillatum, quirlftindiger 23 .

Biefitgstwaldung. 5. 6. 4 .

H. 2518'. L. Hb. K. S. Ch.

47. Streptópus. Sinotenfū.

* 15\%. Str. amplexifólius, ftengelumfaf=

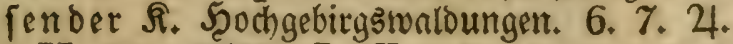

H. 20-2500'. L. K.

144. Anthéricum. 3aunblume.

326. 1. A. ramósum, äftige 3. Steinige Şügel, lichte Wálber. I. 3m. Richtenwaldaa u. Schönferd. 6. 7. 4.

L. B. O. W.

48. Aspáragus. Spargel.

* 58. A. officinalis, arzmeilidter Sp. 7. 8. 4. in Ssîrten gezogen.

S. Ch.

145. Acórus. Ralmus.

328. 1. A. Calamus, gemeiner $\mathcal{R}_{\text {. Steheno }}$ u. flie= Bende Esewäffer. III. Enabenberger Teiche und 2 Baffer, Barthe, El. Sober, Boberlachen, F̧örlterbad), Sctjönfeld. 6. 7. 24 .

H. $10-1200^{\prime}$, L. Hb. S. Ch. B. O. W. 


\section{6 \\ 146. Júncus. B̊infe.}

328. 1. J. conglommerátus, geknäulte S. Gruben,

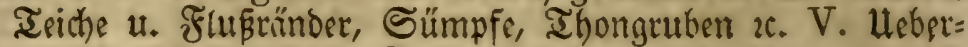
all [eht häufig. 7. 8. Smmer gruppenweis. 24.

H. $10-2200^{\prime}$, L. Hb. K. S. Ch. B. O. W.

329. 2. J. effúsus, Flatter $=\mathfrak{B}$. W̉ie 1. wie es focheint, am bî́ufigifter.

H. L. Hb. S. Ch. B. O. W.

330. 3. J. glaucus, graue $\mathfrak{b}$. Wie 1 u. 2. Docil min= Der bäufig. 7. 8. 4 .

L. Hb. S. Ch. B. O. W.

* 159. J. filiformis, $\mathfrak{F}_{a}$ enn= B. Scobgebirg moore. 6. 7. 24.

H. $12-2400^{\prime}$. L. Hb. K. $1310^{\prime}$.

* 160. J. trifidus, Dreifpaltige $\mathfrak{B}$. Deşgleidy. Roppenplan. 7. 8. 24.

331. 4. J. squarrósus, fparrige $\mathfrak{B}$. Unfruchtbare Iorf=

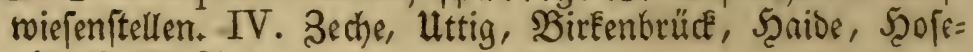
nig. 6. 7. 4 .

H. $2200^{\prime}$. L. Hb. S. Ch. O. W.

332. 5. J. bulbósus, Enollige $\mathfrak{B}$. Feuchte 2 Biefen, ber.

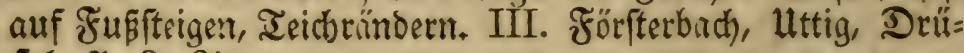
Fel. 7. 8. 2 .

H. $1400^{\prime}$. L. Hb. S. Ch. B. O. W.

* 161. J. Te nagéia, iăhrige $\mathfrak{S}_{\text {. }}$ Uuf über=

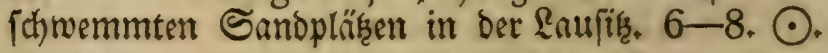

333. 6. J. bufónius, Riröten= B. Fentute 2lecter, Sar= tenbeete, Fufíteige, Giraben, Sanopläbe überntl. 6-9. $\odot$. H. $12-2235^{\prime}$. L. Hb. K. S. Ch. B. O. W.

* 162. J. capitatus, R̂pf $=\mathfrak{B}$. Feuchte Sand= plä̧̧⿻ 7. 8. 24. L.

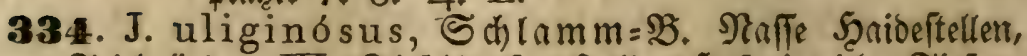

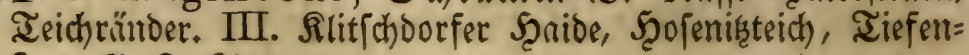
furt. $7,8,24$.

L. K. $1430^{\prime}$. Hb. O. W. 
335. 8. J. fuscoáter, fa) warzbraune B. Sumpfwie=

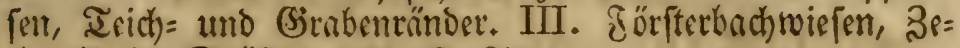
d)e, llttig, Drüßel x.7-9, 24.

H. $1100^{\prime}$. L. W.

336. 8. J. la mprocárpus, glanzfrüchtige B. Fetuchte Sandpläbe, Sümpfe, Grï̈bcn. IV. Förfterbach, uttig $x$. $7-9.24$.

H. $12-2235^{\prime}$. L. Hb. K. 1480' S. Ch. B. O. W.

33\%. 9. J. acutiflórus, fpiéblüthige $\mathfrak{B}$. Iorfwiefm.

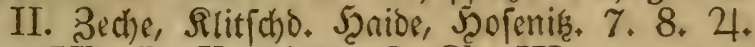
Hb. L. K. $1480^{\prime}$, S. Ch. W:

* 163. J. melananthos, fdiwarzblumige $\mathfrak{2}$.

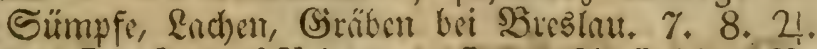

* 164. J. obtusiflórus, ftumpfblütbige-SB. Torfiviejen. $7,8,21$. BSnadenfeld.

147. Lúzula. Şainfimie.

338. 1. L. pilósa, $\mathfrak{F}$ rühlingg=

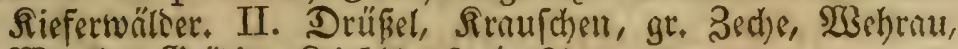
WBarthe, Broöois, Sititchoorf. 4. 24.

H. L. Hb. K.

* 165. L. máxima, größ̧te Sૃ. Ģebirgânälder. 6. 7.24 .

K. $1680^{\prime}$. H. $2500^{\prime}$.

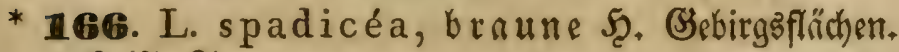
6. 7.24 .

339. 2. L. álbida, weíßliche $\mathfrak{T}$. Irockene $\mathfrak{B a l b f t e l l e n . ~}$

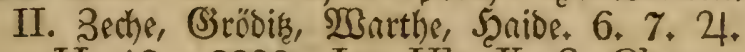
H. 12-2200'. Lo. Hb. K. S. Ch.

340. 3. L. campéstris, gemeine $\mathfrak{F}_{\text {., }}$ Şafenbrodt.

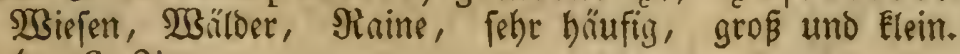
$4-6.24$.

H. $13-1500^{\prime}$. L. Hb. K. S. Ch. B. O. W.

* 16\%. L. spicáta, n̈hrige 5̧. Soppe, Gelfen= rił̧e. 7. 24. 


\section{Bérberis. Berberife.}

341. 1. B. vulgáris, gemeine B., Effigoorn. Seế= Een, (sebüche. III. SBobergeb. im N. von ber Scospital= brüde, an Secten $2 x, 5,6.5$.

H. 1180 '. L. Hb. B. O. W.

\section{Peplis. 3ipfelfraut.}

342. 1. P. Pórtula, gemeines 3. Teichriöner, (Sräben, feuchte 2ecfer. IV. 2(m Sauteich, Drüßel, SBarthe, Uttig u. m. 5. 6-9. $\odot$.

L. K. B. O. W.

Dritte Sxomung. Trigýnia. Dreiptemplige. 150. Rúmex. 2ُmpfer.

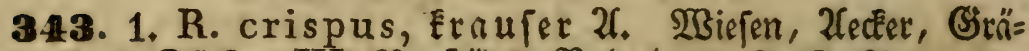
ben, Dörfer. III. Borttiote, Boberbette. 6-8. 4.

H. $11-1200^{\prime}$. L. Hb. K. S. Ch B. O. W.

39-2. R. obtusifólius, f́tumpfblättriger $\mathfrak{X}$. WBe ber vorige. IV. Sorftióte. 6-8. 4 .

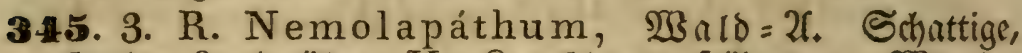

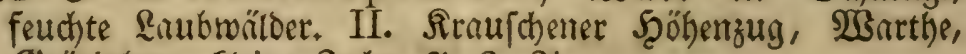

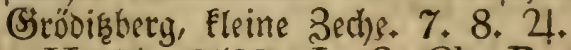

H. $14-2500$ '. L. S. Ch. B. O. W.

346. 4. R. conglommerátus, geênảulter 2 . WBăt=

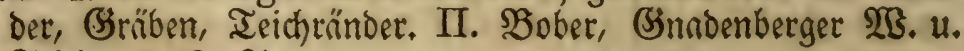
Ieiche, 7. 8. 24.

H. $11-1400$ '. L. K. B. O. W.

348. 5. R. maritimus, Neer=2A., gologelber $\mathfrak{A}$. Sumpfige, feuchte Stellen uno Brib̈brn. ? 7. 8. 21. L. B. O. W.

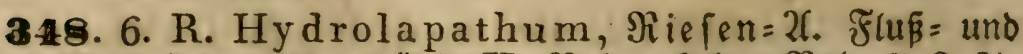
Teidhränber. Ufergebüfoh. II. Bober, Eleiter Bob. 7. 8. 4. L. B. O. W. 
349. 7. R. a quáticus, $\mathfrak{B}$ affer $=\mathfrak{2}$. $\mathfrak{B a d})=$ und $\mathfrak{T}$ eid $=$ riñoer. I. S3oberbette, 6, 7, 24.

H. 11-2200'. L. Hb. S. Ch.

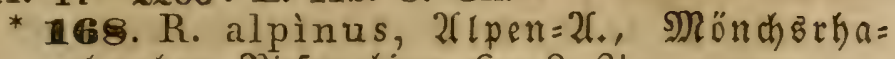
barber. Niefengebirye. 6-8. 24.

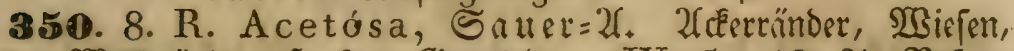
Balbränber, Sectên, Gitastehnen. IV. 5-10. 24. Bejon=

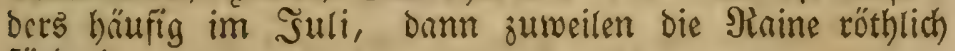
färtent.

H. $2800^{\prime}$. L. Hb. K. S. Ch. B. O. W.

351. 9. R. Acetosélla, fleiner Sauer=2r. SrachäÉ

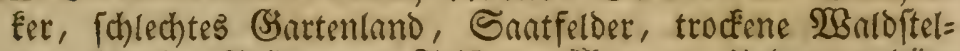
len, fonnige গglihbe uno Iriften, syinuern. Heberaus biüu=

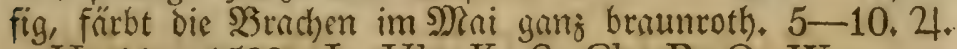
H. $11-1500$ '. L. Hb. K. S. Ch. B. O. W.

\section{Tofiéldia. Tofieloie.}

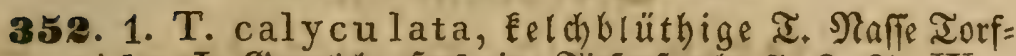
wiejen. I. Brreutich, Scofenik, Iiefenfurth. 7, 8, 24. W.

\section{Verátrum. Sermer.}

* 169. V. lobeliánum, Lobels (5). Scohe (5) birgstwiefen. 7, 8. 24.

Lo. H. 12-2300'. K. hier nicht blühend.

152. Scheuchzéria. Sdjeuchzerie.

353. 1. Sch. palustris, $\widetilde{S u m p}_{=}=S_{d y}$. Naffe Iorfmoore.

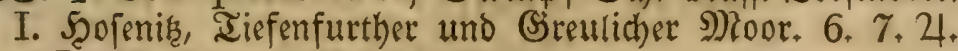
L. H. $20-2200^{\prime}$.

\section{Triglóchin. . Dreizace.}

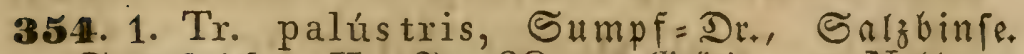
Sumpfwiefen. II. Sm SO des (jiöoin, am Nabhange

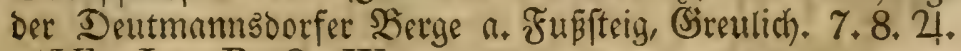
Hb. Ln. B. O. W.

* 120. Tr. maritimum, Seeftranbz=Dr. Feuchte $\mathfrak{B i s f e n . ~ 7 . ~ 8 . ~ 2 4 . ~ H . ~ 1 6 0 0 ' . ~}$ 
50. Cólchicum. 3eitlof̣e.

* 121. C. autumnale, Şerbft=3. Ssebirggtrie= โen. 7. 8. 4 .

$\therefore$ H. bis $1500^{\prime}$, Lo. Hb. K. 1430', S. Ch. O.

Sierte Subunty. Polygýnia. Bielitemplige.

154. Alísma. Froj̋löffel.

355. A. Plantảgo, gemeiner $\mathfrak{r}$. Serwäfiferte Thongru= ben, Graben, feidhte Genewiffer, IV. Fafit itberall. 7. 8. 24. H. $1500^{\prime}$. L. Hb. K. S. Ch. B. O. W.

\section{Giebente Rlafie, Heptåndria. Giebenfadige.}

155. Trientális. Sternblümdben.

356. 1. Tr. europaéa, europlíf ches St, Sieben=

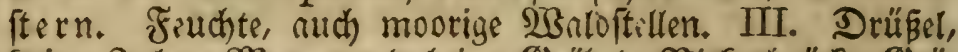

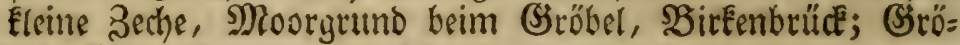
Dis. 5. 6.24 .

H. 12-2518'. L. Hb. S. Ch. O.

\section{2labte Slaffe, Octándria. 2ld $\mathfrak{t} \mathfrak{i} \mathfrak{a b i g e}+$}

Erpte Orbuuty. Monogýnia. Cinftemplige.

156. Daphne. Seibelbaft.

352. 1. D. Mezeréum, gemeiner S., Rellerhalb.

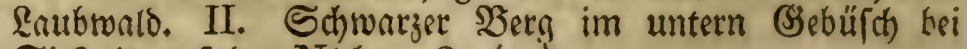
SirEwis auf Der Nlehne. 3, 4, 5 .

H. $10-2300$. L. Hb. K. S. Ch. B. O. 


\section{Passerina. গুafferine.}

* 182. P. ánnua, einjührige Sparzenzunge. Trodene 2lecter in Soberfhlefien. $7.8, \odot$.

\section{7. Érica. Şaibe.}

358. 1. E. Tetrálix, Meer=

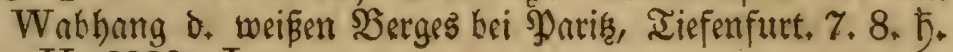
H. $2200^{\prime}$. Ln.

\section{Callúna. Şaibefraut.}

359. 1. C. vulgáris, gemeines 5ु. Ridyte Staibeftellen, troderene Berglehnen, biefe Ende 2luguft und 2lnfang Sep= tember auf weite Strecfen fojön lilla färbento, hin und wieder auch weís blüberto. 5 .

H. bis $2810^{\prime}$. L. Hb. K. S. Ch. B. O. W.

\section{Vaccínium. Sceibelbeere.}

360. 1. V. Myrtíllus, gemeine $\mathfrak{S T}_{+,} \mathfrak{B l a u b e e r e . ~}$ Fruchtbare Staibeftellen, gefellig, oft weite Striche bedekE= Eendo. 4. 5. 10, 万.

H. $2300^{\prime}$. L. B. O. W. Hb.

361. 2. V. uliginósum, Sumpf $=S_{\text {, }}$ Raufhbeere. Moorige Scribeftellen, gruppenweis und vereinzelt. IV.

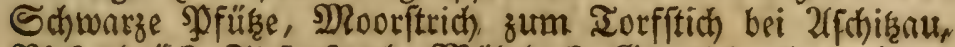

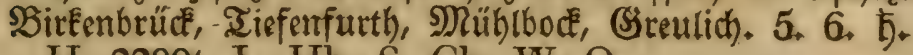
H. 2200'. L. Hb. S. Ch. W. O.

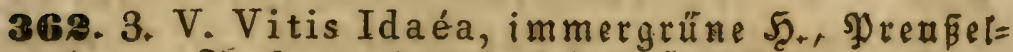
beere. Trodéne und unfruchtbare Saibeftellen, gruppen= weis u. gefellig. V. 3eche, Rlitffhoorfer Șaibe, allein ober mit 1. gemengt. 5. 6. aud 9, wieder. 5.

H. $2800^{\prime}$. L. Hb. K. S. Ch. B. O. W.

363. 4. V. Oxycóccus, Iorf = J̦, Mroşbeere,

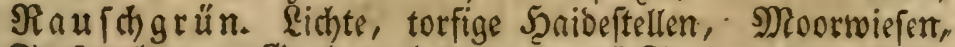
Iorfgruben u. Graben, befonders auf Torfmooz, Daffelte 


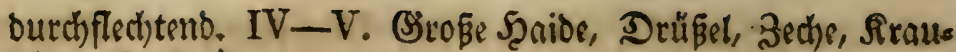
foren. 6.7. 5 .

H. $2200^{\prime}$. L. Hb. K. $1430^{\prime}$. S. Ch. O. W.

\section{Acer. 2fhorn.}

36t. 1. A. c ampéstre, $\mathfrak{F} e l d=\mathfrak{U}_{0}, \mathfrak{R} a f$ feller. $\mathfrak{W a ̈ l}$ er.

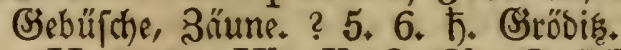

H. $1700^{\prime}$. Hb. K. S. Ch. O. W. B.

365. 2. A. Pseudo-Platánus, פ゚latanen=21. Bery= wailder, bei uns in Baumgängen angebaut, hin und wies Der. 5 . 5 .

H. bie $2500^{\prime}$. L. Hb. K. S. Ch. B. W.

366. 3. A. platanoides, fpibblättriger 2 . Feuchte

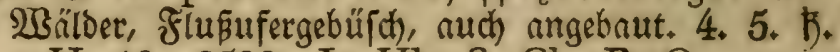

H. $10-2500^{\prime}$. L. Hb. S. Ch. B. O.

\section{Oenothéra. Nachtłerze.}

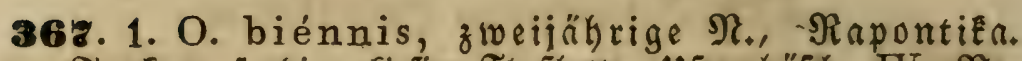

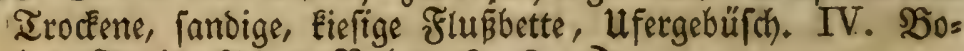
ber, Queis, Eleiner Sober. 6-8. oे.

H. $10-1200^{\prime}$. L. Hb. felten. K. 1480'. S. Ch. B. O. W.

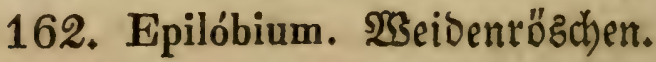

* 183. E. angustíssimum seu Dodonaei, lis nienblättr. 2 . Steinige F̆luß̧นfer. 7. 24. SDbers fitlefien.

368. 1. E. angustifólium, đđmalblättriges $\mathfrak{B}$.

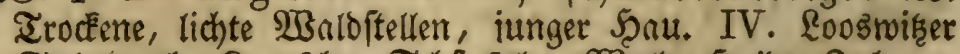

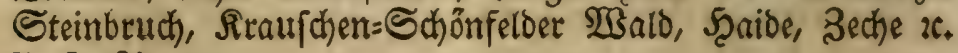
7. 8. 4 .

H. $10-2800^{\prime}$. L. Hb. K. S. Ch. B. O. W.

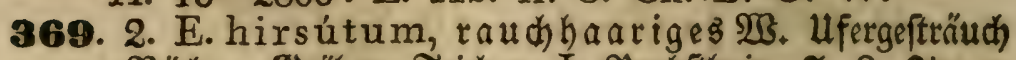

an $\mathfrak{B a ̈ c h e n , ~ S r r a ̈ b e r n , ~ I e i c h e n . ~ I . ~ P r o b i t h a i n . ~ 7 . ~ 8 . ~} 21$. H. $10-1200^{\prime}$. L. B. O. W.

380. 3. E. mólle, weich haariges 23 . Sumpfriefen, 


\section{3}

Sumpfitellen, Grräben. II. Zuellgrund am Seibelifhen Bortwerk, Sutecébrumngraben, STofenif. 7. 8. 21.

H. $1000^{\prime}$. L. Hb. K. B. O. W.

3\%1. 4. E. montánum, $\mathfrak{B} e \mathfrak{r g}=\mathfrak{B}$. Steinige Mläße,

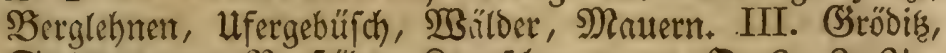
Strotmuntern, Borftiobte, Rraufhen u. m. 5. 6-8. 24.

H. bis 2200'. L. Hb. K. S. Ch. B. O. W.

* 183. E. trigónum, oreiłantiges 23 . (B)e birgsticthe. 7. 8. 24. S. Ch.

* 184. E. virgátum, ruthiges $\mathfrak{W}$. Breglau. 7. 8.24 .

382. 5. F. roséum, fleir harbenes $\mathfrak{B}$. Feuchte 2 Băl=

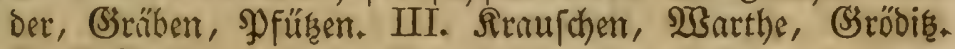
7. 8,24 .

H. $1200^{\prime}$. L. Hb. K. S. Ch. B. O. W.

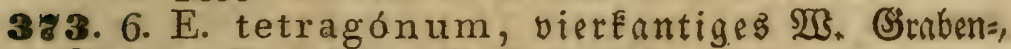
$\mathscr{F}(u \xi=u$. Teichränder. II. $7,8,4$.

H. $1200^{\prime}$. L. Hb. S. Ch. W.

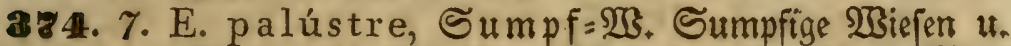

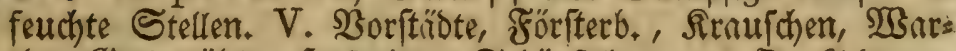
the, Solotomüble, STofenils, Schönfeld u. v. S. Eleine u. grobe, jehr verfhiedene Formen. 7-9. 4 .

H. L. Hb. K. S. Ch. O. W.

* 185. E. origanifólium, boftenblätriges

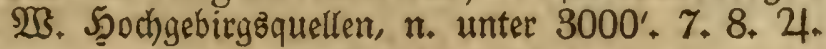

\section{Dritte Srsuming. Trigýnia. Dreif́templige.}

\section{Polýgonum. Innötrig.}

385. 1. P. Bistórta, đhlangentourzlicher $\Omega_{n}, 5 t=$ terwarz. I. Feuchte swiefen. Der Borftíbte hin u. wie= Der, ief̧t felten, früber felbft auf den Suecébrummiefen, Thommenoorf, Tirfenfurth. $5,6,4$.

H. 11-2200'. L. Hb. K. S. Ch. B. O. W.

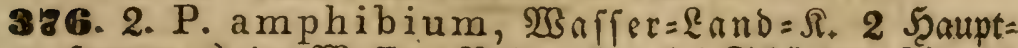
formen a) im $\mathfrak{W a f f e r : ~} \mathfrak{B o b e r l a c h e n ~ b e i ~ S d j o ̈ n f e l d . ~ b ) ~ a u f ~}$ 
bem Sanbe: an feuchten Stellen f. B. Quellengrund am Seibelfichen $\mathfrak{B o r t w e r E}$. 6. 7. 4.

H. $10-2200^{\prime}$. L. Hb. S. Ch. B. O. W.

3z 8. 3. P. lapathifólium, amp ferblättriger Iñ.,

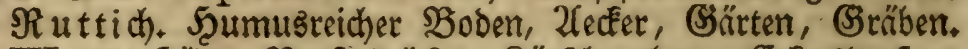

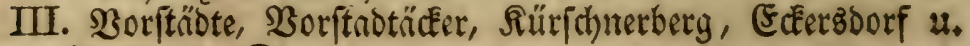
v. 5. 7-9. $\odot$.

H. $1000^{\prime}$ L. Hb. K. S. Ch. B. O. W.

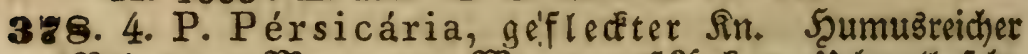
Boben, an Mauern, an $23 e g e n$, auf 2Geckern. Uleberall fehr băufig. $6-8+\odot$.

H. L. Hb. K. S. Ch. B. O. W.

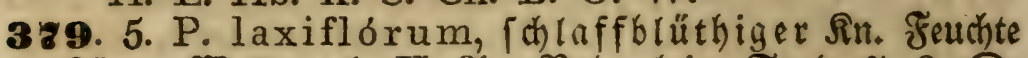

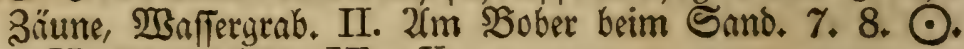
H. $10-1500^{\prime}, \mathrm{Hb}$. K.

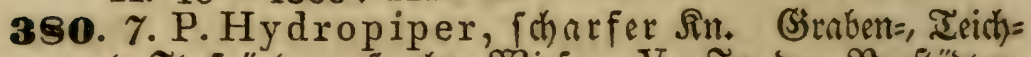

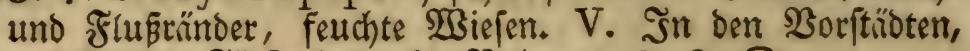

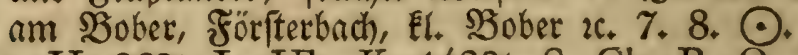

H. $960^{\prime}$. L. Hb. K. 1430 '. S. Ch. B. O. W.

381. 7. P. minus, Eleiner Sin. Feudhte Wiefen, Gras=

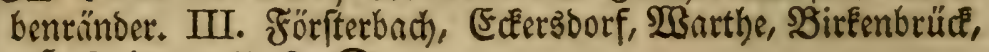

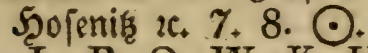

L. B. O. W. K. Hb.

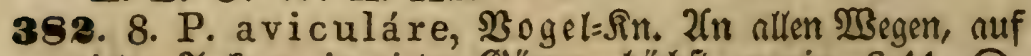
vielen 2lectern, in vielen Gsärten, höchit gemein. 6-11. $\odot$. H. L. Hb. K. S. Ch. B. O. W.

393. 9. P. Convólvulus, $\mathfrak{B}$ inden $=\Omega \mathfrak{R}$. Betraides und

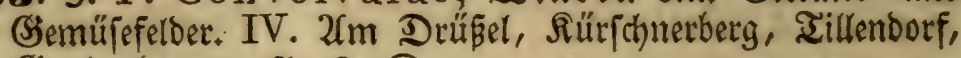
Sinndenberg 2 . 7-9. $\odot$.

H. $10-1500^{\prime}$. L. Hb. K. S. Ch. B. O. W.

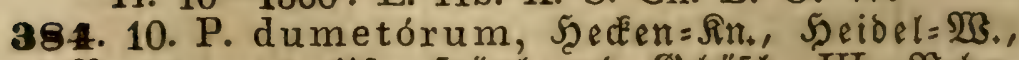
Bogelzunge. Ulfergefträuch) und Gebüló. III. Bober, Eleimer SBober. 7. 8. $\odot$.

H. $2000^{\prime}$. L. Hb. S. Ch. B. O. W. 
Bierte Sroutung. Tetragýnia. Sierfiemplige.

164. Adóxa. SBifaméraut.

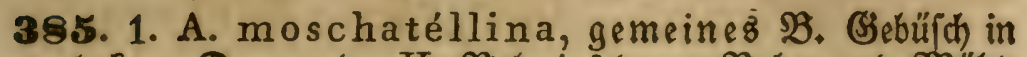
lockerer Dammerbe. II. Boberinfel z'w. Bober und Mübl=

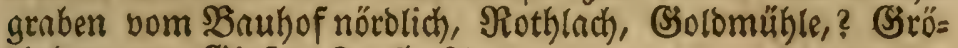
Difiberg am Gipfel. $3-5,24$.

H. biz 2200'. L. Hb. S. Ch. B. O. W.

165. Páris. Einbeere.

386. 1. P. quadrifólia, vierblättrige $F$. Sđanttiger, feuchter $\mathfrak{a n u b i v a l b . ~ I . ~ S u m p f i g e r ~ B u f ( h ) ~ u n t e r m ~ S d ) l o ß s = ~}$ garten zu Szohlítein. 5. 24 .

H. 11-2300'. L. Hb. K. S. Ch. B. O. W.

166. Elatíne. Iaumel.

388. 1. E. Hydropiper, fdarfer $\mathfrak{X}$. Teidyränder. I. Uttiger $\mathfrak{E a d h e . ~ 6 - 8 . ~ \odot . ~ L . ~}$

388. 2. E. Alsinastrum, Wirtel=

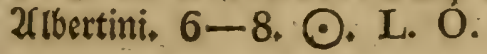

\section{NReunte Rlaife. Enneåndria. sReunfadige.}

167. Bútomus. Błumenbinje.

389. B. umbellátus, boldige $\mathfrak{B l .}$, $\mathfrak{T}$ ameelheu.

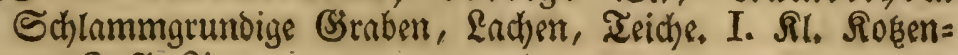
au. 6. 7. 24.

L. B. O. W. 
86

\section{3ebnte Rlaffe. Decándria. Żebnfabige.}

Erifte Dronuty. Monogýnia. Cintemplige.

168. Arctostáphylus. Bärentraube.

390. A. officinális, arzmeiliche $\mathfrak{B}$. T̉n Der Şaibe. I. Sn Der Rlitjchborfer auf Dem $2 B e g e$ zur Steintammer im erften Sechstheil des Warbes. 5. 6. 5. (geftrecft.)

H. $1170-1800^{\prime}$. L.

169. Andrómeda. 'Anoromede.

391. A. polifólia, Poleiblättrige $\mathfrak{Z}_{+}$Torfhaibe. Iorfitellen ber Scaide. III. 2ún febr vielen Stellen, fatwarze Pfühe uno weftlicher Shooritreifen von berfelben, Dhlen=

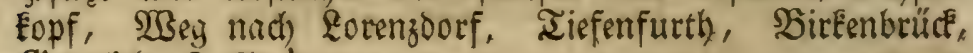
(Gireulich. 6. 7, 5.

H. 2200' Ln. O. Hb.

170. Lédum. \$gorfit.

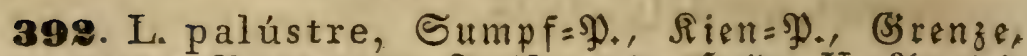

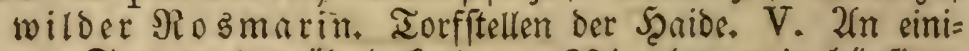
gen Stellen alles übcrbeckend m. 391 aber weit hiufiger,

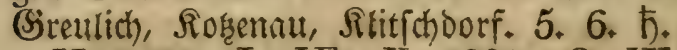

H. $2200^{\prime}$. L. Hb. K. $1330^{\prime}$. O. W.

171. Pýrola. 23sintergrün.

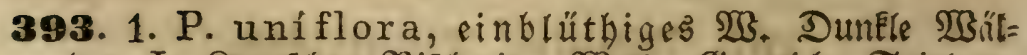

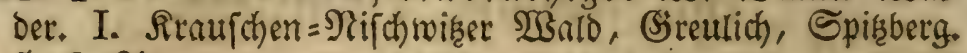
5. 6. 24.

. H. bis 2000'. L. Hb. K. S. Ch. O. W.

394. 2. P. secúnda, einfeitwendiges $\mathfrak{B B}_{*}$, Birn=

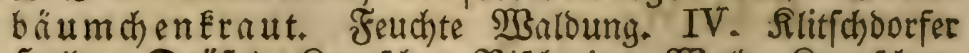

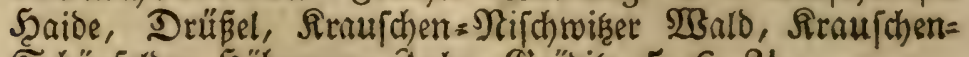

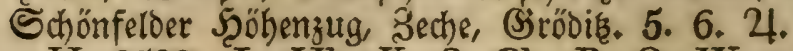

H. 2500' L. Hb. K. S. Ch. B. O. W. 


\section{7}

395. 3. P. minor, Eleineres $\mathfrak{B}$. Nabetwälber. III.

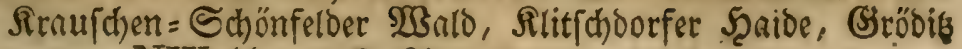
unterer NWabhang. 6. 2.

L. Hb. K. S. Ch. B. O. W.

396. 4. P. média, mittleres 20 . Nndel= und gemifote

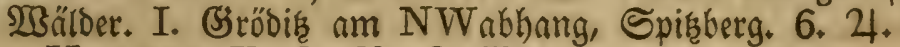

H. $1700^{\prime}$. K. S. Ch. O. Hb.

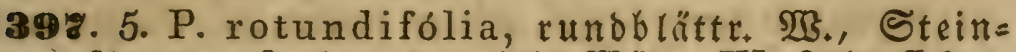

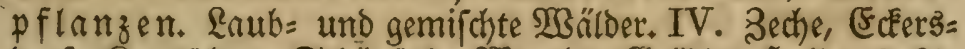

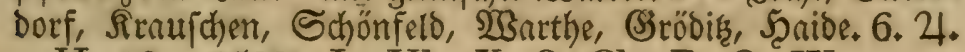

H. 12-1500' L. Hb. K. S. Ch. B. O. W.

398. 6. P. chlorántha, grünblumiges $\mathfrak{B}$. Nabel=

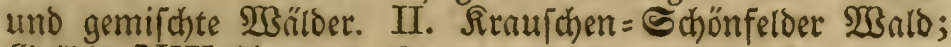
Bricois NWabhang 6. 4.

H. $1400^{\prime}$. Hb. S. Ch. W.

\section{Chimóphila. Ebimophile.}

399. 1. Ch. umbelláta, fđirmförmiges MBinter=

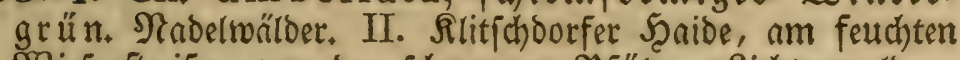

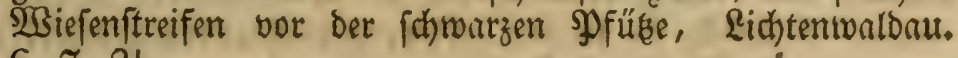
6. 7.24 .

H. $1400^{\prime}$. L. S. Ch. B. O. W.

\section{Monótropa. Fichtenipargel.}

400. 1. M. Hypópitys, gemeiner $\mathfrak{F}$. Troféne Nabels

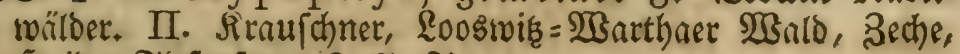
Scaibe, Iiefenfurt. 6. 7.24.

H. L. Hb. K. S. Ch.

\section{Dictámnus. Diptam.}

* 186. D. albus, roeißjer D. Bergwälbet, 5. 6. 4. SDeridhlefien. Iefchen. 
88

\section{Bweite Sronung. Digýnia. Sweiftemplige.}

\section{Saxifraga. Steinbrech.}

* 18z. S. umbrósa, fornttenliebender St. Feuchte Felfen. Bsefenté. 7. 24.

401. 1. S. granuláta, zwiebelểrniger St. Wegerän=

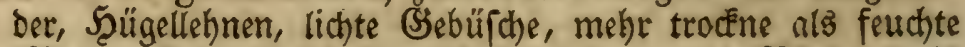

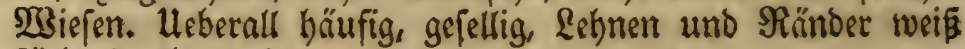
färbent. 5. 6. 4 .

H. bis 2300 '. L. Hb. K. S. Ch. B. O. W.

* 188. S. Hírculus, Bad $=$ St. Sumpfwiefen. Sberjhlefient. 7.

* 129. S. bryoídes, Eapletmoosartiger St. Felien, El. Schneegruben. 7. 4.

* 180. S. nivális, Sthnee= St. besgl.

* 181. S. Aízoon, nizoonartiger St. Scodhge= birge. 6. 7. 4.

* 182. S. oppositifolia, rotbblauer St. Deşgl. 5. 4 .

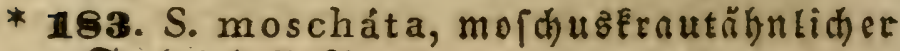
St. besgl. 7. 4.

* 184. S. sponhémica, fponbeimifoter St. H. 16-1800\%. Moospolffer auf einigen großen Steinen bildend, bei DarniEau. Mittelgebirgsfel= โem. 5, 6. 4 .

402. 3. S. tridactýlites, breifingriger St. Keefer,

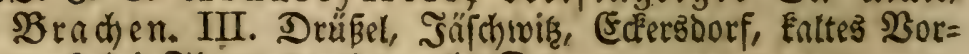
werk bei Thomasmaldau. 5 . $\odot$.

H. $1300^{\prime}$. L. Hb. B. O. W.

\section{Chrysosplénium. Ģolden= Milzerraut.}

403. 1. Chr. alternifólium, wedffelblättriges M. Badhrïnber, Errenbüfche. I. Şoblftein, Mtoiz, Sinadenber= ger 203 fifer, Schönfelder Sümpfe. 3. 4. 24.

H. bis $2235^{\prime}$. L. Hb. K. S. Ch. B. O. W. 
404. 2. Chr. oppositifólium, gegenblättrigez આR. Mnnloige Suellftellen. I. Suellen im N. Der Solbmüble, 23 arthe bei ber Sternmaner. 6. 24. L. $\mathrm{Hb}$.

\section{Gypsóphila. (Sinpzfraut.}

405. 1. G. murális, Mnauer=(3). FluÉrïnder, feuchte, thonig=Fandige Recker, Mege, Mnuern. IV. DrüB̈rl, Eckers: borf, Tilleno., 21;chisau, $\mathfrak{B a r t h e , ~ S c h o ̈ n f e l d ~ x c . ~ 7 - 1 1 . ~} \odot$. L. Hb. K. S. Ch. B. O. W.

* 185. G. Vaccária, 2 déer $=$ G5., (Erbtweid). Uecker. 6. 7. ○. Sppeln.

* 186. G. fastigiáta, gipfelblumiges (S). Sanoige Rieferwillber. 6. 7. 24. L. W.

177. Saponária. Seifenfraut.

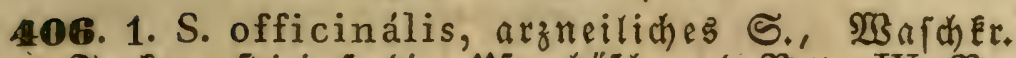

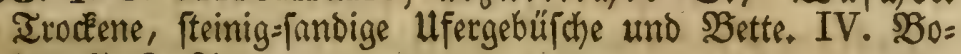
ber, 7, 8. 24.

L. B. O. W. Hb.

178. Diánthus. Nelke.

40\%. 1. D. Carthusianórum, Rarthäu ner $=\Re$. Trockne Şügel, Şügel= und uferlehnen. I. İhom= mendorf, 6-10. 24 .

L. S. B. O. W.

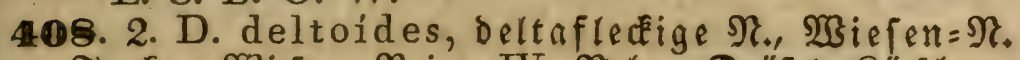
Irodfene $\mathfrak{B i e f e n , ~ \Re n i m e . ~ I V . ~ B ̉ o b e r , ~ D r u ̈ b e l , ~ R u ̈ r i d n e r = ~}$ berg, 2 Barthe 2c. 6-11. 24.

H. bis $1500^{\prime}$. L. B. O. W. Hb. K. S. Ch.

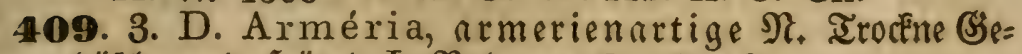
büide und Schigel. I. Bobernt. 6-10. 24.

L. Ch. S. B. O. W.

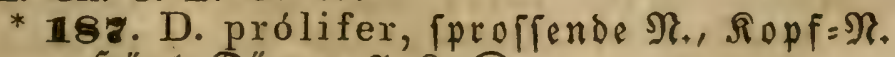
Sุügel, Dönmme. 7, 8. $\odot$.

L. W. S. Ch. 


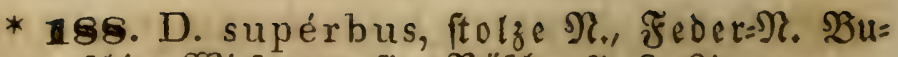
faige $\mathfrak{B b i e f e n , ~ g r u f i g e ~ B u ̈ f h e . ~ 7 . ~ 8 . ~} 4$.

K. $1680^{\prime}$. S. Ch.

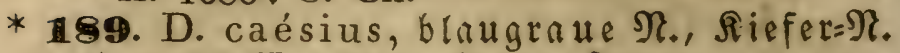
5. 6. 24. H. 1200'. Sulau, Sppeln.

* 1D0. D. arenárius, $S_{a n d}=\mathfrak{N}$. Samoftrecken. I. Grünberg, im Rieferwalde. SBom Phbarma. Schmïre nufgefunden. 7-9.24.

179. Scleránthus. Sinörich.

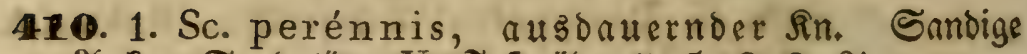
2lecter, Sambplähe. V. Janft überall. 5. 6. 9. 24.

H. $1500^{\prime}$. L. Hb. K. S. Ch. B. O. W.

411. 2. Sc. ánnuus, jähriger $\Omega_{n}, \mathfrak{X}$ cker $=\mathfrak{\Omega} n$. Wie 1 und mit demielben zujammen.

'H. $10-2200^{\prime}$. L. Hb. K. S. Ch. B. O. W.

Dritte Sorounut. Trigýnia. Dreiftemplige. 180. Stellária. SternÉtaut.

412. 1. St. aquática, $\mathscr{B} a f\left\lceil e r=S_{t}\right.$, $\mathbb{R}$ uellenmiere. Suellen u. SDäd)e. IV. Subentirdhofquelle, Gnadenberger Bach, Iorfquellenitellen. 5. 6. 9.

H. $1200^{\prime}$. L. Hb. K. S. Ch. B. O. W.

413. 2. St. gramínea, grajartiges St., $\mathfrak{B}$ iefenmie=

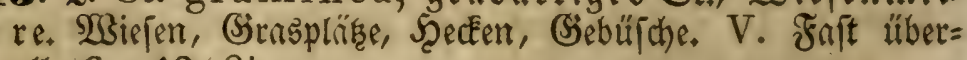
all. $6-10.4$.

H. bis $1500^{\prime}$. L. Hb. K. S. Ch. B. O. W.

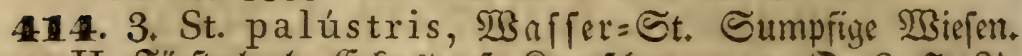

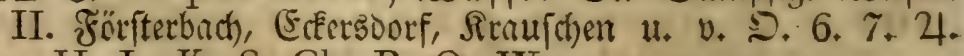
H. L. K. S. Ch. B. O. W.

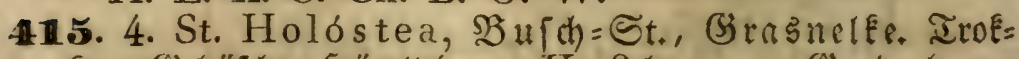

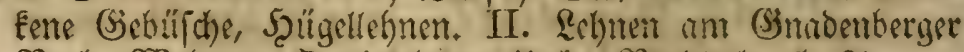

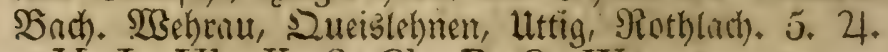

H. I. Hb. K. S. Ch. B. O. W. 
416. 5. St. média, gemeines St, $\mathfrak{B o g e l m e i e r . ~ S ̧ u = ~}$

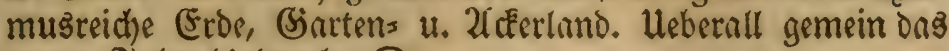
ganze Sabr himburch. $\odot$.

H. bis über $1500^{\prime}$. L. Hb. K. S. Ch. B. O. W. 41. 6. St. némorum, Şain= હt., $\mathfrak{B a l b m e i e r . ~}$

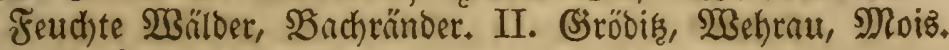
6. 7. 2 .

H. L. Hb. K. S. Ch.

\section{Arenária. Sanof́raut.}

418. 1. A. trinérvia, oreinerviges $\mathbb{S}$. Gebulifh, Mau= erm, hohle Bäume. IV. Boberinfel, Ginndenberger 2 Baffer,

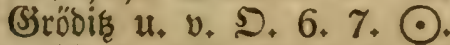

H. L. H.b. K. S. Ch. B. O. W.

419. 2. A. serpyllifólia, quendelblättrigez S.

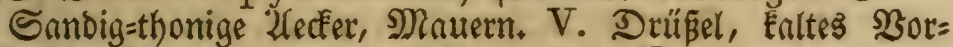

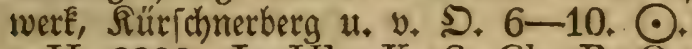

H. $2200^{\prime}$. L. Hb. K. S. Ch. B. O. W.

420. 3. A. rubra, rothblumigez S. Sambige 2fecter, Sanopläbe, Teidhrïnber, IV. Sandgruben, Drüß̈el, Sina=

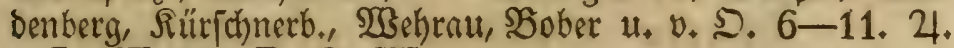
L. Hb. K. B. O. W.

421. 4. A. viscídula, Elebriges S. Sanbige Bracthen.

6. 7. ○. L. W. SBifch of 2ftbertini.

* 1191. A. verna, Frählings = S. Scochgebirge.

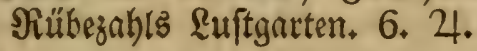

182. Cucúbalus. Taubenfopf.

422. 1. C. baccíferus, beerentragende

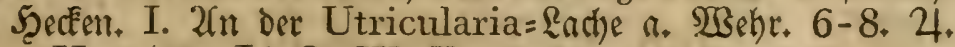
H. $1500^{\prime}$. L. O. W. K.

\section{Siléne. Reimf́raut.}

423. 1. S. nutans, hängendes \&. Warbränder, Şü= getief)nen. II. 2(m Gimabenberyer 20 affer im 23 alde, Ufer=

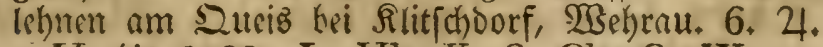

H. hiz 2200' L. Hb. K. S. Ch. O. W. 


\section{2}

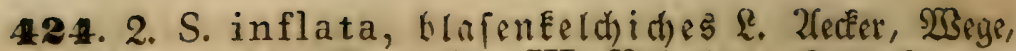

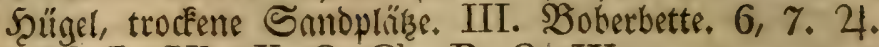

H. L. Hb. K. S. Ch. B. O. W.

* 92. S. cerastoides, hornErautartiges

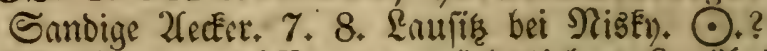

* 193. S. noctíflora, nüchtlides \&. Ufecfer. 6. 7. $\odot$.

Ln. O. W. Hb. S. Ch.

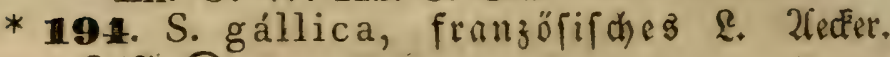
6. $7 . \odot$.

H. $1100^{\prime}$. O. W. S. Ch.

* 195. S. Otítes, ohrblättriges \&. Trodéne Sanohügel. $6.7 . \odot$.

H. $1200^{\prime}$. B. O. W. S. Ch.

* 196. S. chlorántha, grünblumiges Sandige Rieferwältber. 7. 24. W.

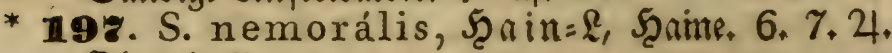
Sberfdilefien.

\section{Füufte Sronutg. Pentagýnia. Fünfitemplige.}

184. Maláchium. $\mathfrak{B}$ eidjÉraut.

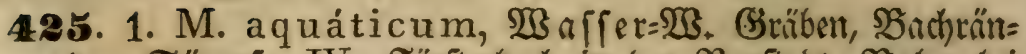
Der, Sümpfe. IV. Förfferbach in ber $\mathfrak{B}$ orftabt, $\mathfrak{B}$ ober bei Uttig, Dueilen am 2 ueis bei $\mathfrak{B}$ ehrau. 6-11. 4.

H. $1200^{\prime}$. L. Hb. K. S. Ch. B. O. W.

185. Cerástium. Şornêraut.

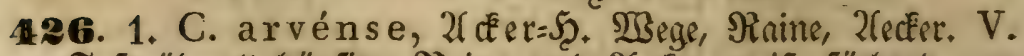

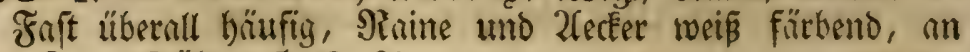
erfteren früher. 5. 6. 24.

H. bis $2235^{\prime}$. L. Hb. K. S. Ch. B. O. W.

* 198. C. alpínum, 2U1pen $=52$. Babia Borr. 2(uf Fellen. 7. 4. 


\section{3}

* 199. C. a nómalum, breigriffriges 5ૃ. Irif= ten uno $\mathfrak{2 B i e f e r t . ~ 5 . ~} \odot$.? ?

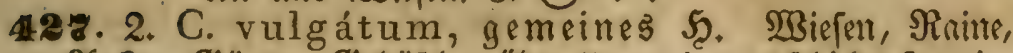

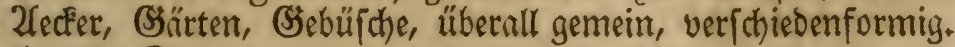
$5-9 . \odot$.

H. $11-1200^{\prime}$. L. Hb. K. S. Ch. B. O. W.

428. 3. C. ovále, eirunoblätriges 5̧. Fenchte Weefer, Schutthaufen. II. Schönfeld, Warthe 6+7. $\odot$.

L. Hb. K. S. Ch. B. O.

* 200. C. brachypétalum, grauhaariges burchige 2(déerrïnoer. $5 . \odot$.

429. 4. C. glutinósum, klebendes $\mathfrak{H}_{\text {. Sandige, }}$ feuchte Triften, Smopläbe, 2ctfertinnder. III. Boberau, Iillenborf, Sinndenbera. 4. 5. ๑.

H. $1200^{\prime}$. L. B. O. W.

430. 5. C. semidecándrum, fünffäbiges 5. Trok=

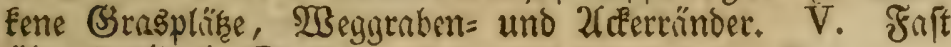
überall. 4, 5, $\odot$.

H. $1200^{\prime}$. L. Hb. S. Ch. B. O. W.

\section{Lýchnis. Ridytblume.}

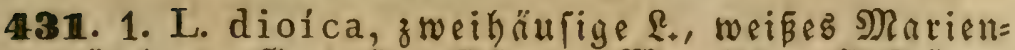

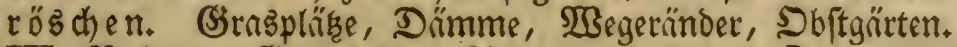
III. Soberau, Sinadenberg, 2unlagen $2 c_{*} 6.7 .0$.

H. $1500^{\prime}$. L. Hb. K. S. Ch. B. O. W.

432. 2. L. sylvéstris, $\mathfrak{B}_{\mathfrak{B}}$ ald $=\mathfrak{L}$, r 0 thes $\mathfrak{M}$. Feuchteb ufergebüfh. I. Sober bei Siroifthwis. 5. 6. 4 .

H. $10-1200^{\prime}$. L. Hb. K. S. Ch. B. O.

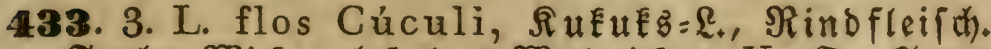

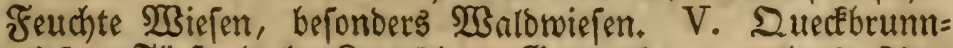

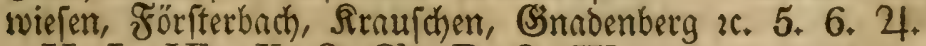
H. L. Hb. K. S. Ch. B. O. W.

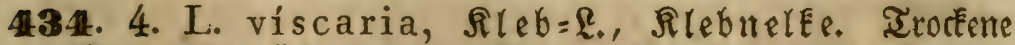

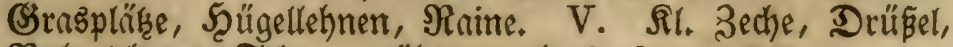
Bobertethnen, Schauermilhle 26. 5. 6. 24. H. $11-1300^{\prime}$. L. Hb. K. S. Ch. B. O. W. 


\section{4}

187. Agrostémma. Rorntathe.

435. 1. A. Githágo, gemeine న. Unter bem Stetraibe. IV. 6. \%. $\odot$.

H. L. Hb. K. S. Ch. B. O. W.

\section{Spérgula. Spark.}

436. 1. Sp. procúmbens, niederliegender Sp.,

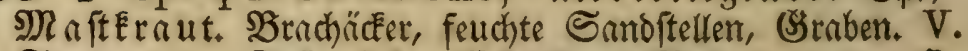
Sanogruben, Drüßelicterer, Eckerşoorf, Sober u. a. v. D. $6-11 . \odot$.

H. L. Hb. K. S. Ch. B. O. W.

438. 2. Sp. apétala, Eronenblattlofer Sp. Feuthte,

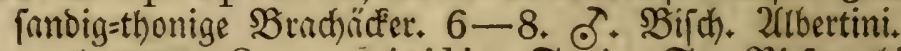

* 201. Sp. saxátilis, Stein= Sp. Riefengebirge. Riefentoppe. B̈ejenter. 7. 8. $\odot$.

* 202. Sp. subuláta, pfriemblättriger Sp. Brachäcer. 7. $\odot$.

438. 3. Sp. nodósa, Enotiger Sp. Sumpfwiejen. II. Sd)auermühle, $\mathfrak{B a r t b e , ~ F o ̈ r i t e r b a c h , ~ S c o i e n i s . ~ 7 - 9 . ~} \odot$. L. K. S. Ch. B. O. W.

439. 4. Sp. pentándra, fünffidiger Sp. Smoige

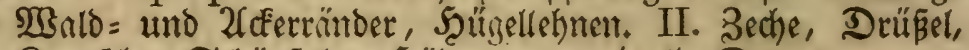

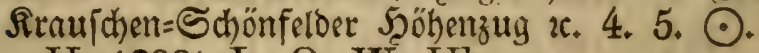

H. 1200'. L. O. W. Hb.

410. 5. Sp. sativa, Futteripare. Sandige und thonig=

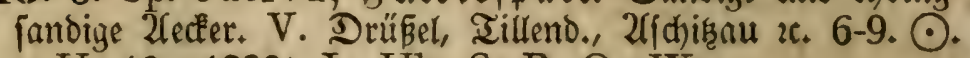
H. $10-1200^{\prime}$. L. Hb. S. B. O. W.

44. 6. Sp. vulgáris, gemeiner Sp. Sandige 2lecker,

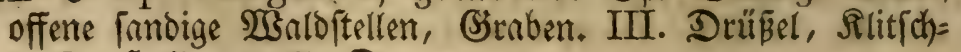
borfer Şaide. 6. 7. $\odot$.

H. Hb. K. S. Ch.

* 203. Sp. máxima, größter Sp. 2lecter. H. 1500'. O. Şirjhberg, Sdjmiedeberg. 


\section{5}

\section{Oxalis. Sauerklee.}

44. 1. O. Acetosélla, wahrer, weiser S. Fethte,

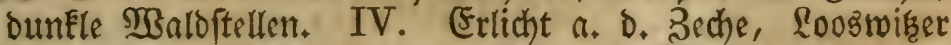

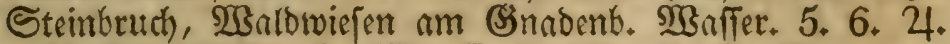
H. 26-2800'. L. Hb. K. S. Ch. B. O. W.

443. 2. O. strícta, fteifer S. Sinrtenland, fruchttare Uecfer. UnÉrnut. IV. Sobernu, Nifolaivorftadt, Wefrau น. a. v. 5., 6-10, $\odot$.

L. Hb. K. $1780^{\prime}$. S. Ch. B. O. W.

\section{Sedum. Mauerpfeffer.}

44. 1. S. Teléphium, eiblättrige श्., fette Şen= ne. Scecfen, trockene Şügel, Mauern, Schobendïcher. WI. Frift überall. 7-9. 24.

H. bis 2300'. L. Hb. K. S. Ch. B. O. W.

* 204. S. purpureum, purpurblüthiges M. Babia Sora. 7. 8.' 4.

* 205. S. Rhadíola, rofenduftiger M. Şoch= gebirge $6,7,24$.

445. 2. S. collinum, Sૃügel= M. Irodêne Sanohügel. II. Rnabengarten, Boberlehnen, (Eichberg. 6. 7. 4. L. B. O. W.

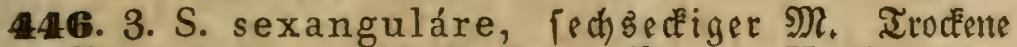
Raine, fandige und fiefige Plähe, Mauern. V. Boberbette

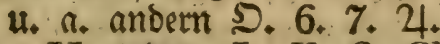
H. $1500^{\prime}$. L. K. S. Ch. B. O. W.

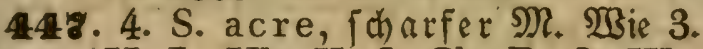
H. L. Hb. K. S. Ch. B. O. W.

* 206. S. rubens, röthlicher M. Şodhgebirge. 7. 24. bis Rrummbübel herabiteigent.

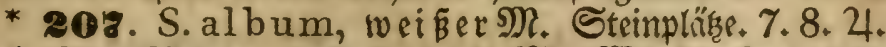
418. 5. S. villósum, haariger MA., WBefenEnorpel. Sumpfwiejen uno Suellgruno. I. Scofenis am untern Biertel ienfeitz ber Suerftraße fehr häufig. 6. 4. H. 12-2235'. L. Hb. K. Ch. 


\section{6}

\section{Erlfte Silafie. Dodecándria. 3wölffabige.}

- Crite Drsumug. Monogýnia. Eimftemplige. 191. Ásarum. Şajelmurz.

419. 1. A. europaéum, europäifde Ş. DunEle \&aub=

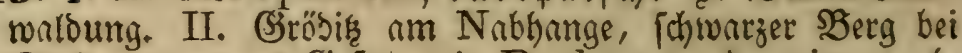
Sirfwich, unteres Gejtr. mit Daphne u. Aconitum. 4. 5. 24.

H. $11-2000^{\prime}$. L. Hb. K. S. Ch. B. O. W.

53. Portuláca. গুortulak̂.

* 208. P. olerácea, wilber গู. Gemülebette, Felfen. 7-10. $\odot$.

Şrestau. K.

192. Lýthrum. W̉eiberid).

450. 1. L. Salicária, $\mathfrak{2}$ ehren $=\mathbb{B}$., $\mathfrak{B l u t}=\mathfrak{W}$. Feuchte জesbüfdye u. WBaffertinnoer. V. Boker, Förfterbach, (Sna= Denberger İeiche u. MBaffer, Boberle, (Golbbach. 7. 8. 4. H. L. Hb. K. 1430-1680'. S. Ch. B. O. W.

451. 2. L. hyssopifólia, yjopblättriger $\mathfrak{B}$. Birn= benränder, feuchte Steillen, befonders in Dörferm. II.

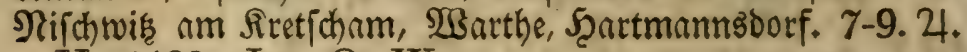
H. $1180^{\prime}$. Ln. O. W.

3weite Droutuy. Digýnia. Bweiptentplige. 193. Agrimónia. Soermennig.

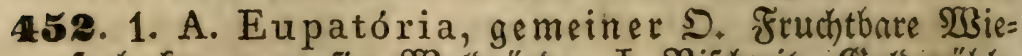

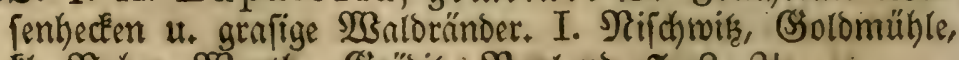

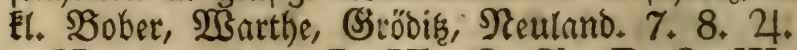
H. 10-2000' L. Hb. S. Ch. B. O. W. 
Dritte Svsunng. Trigýnia. Dreiftemulige. 194. Reséda. Rejeda.

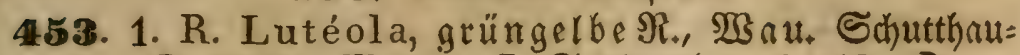
fen, Dorfpläbe, Mauern. I. Sambgruben. 6-10. ठै.

L. B.' Bres̆lau.

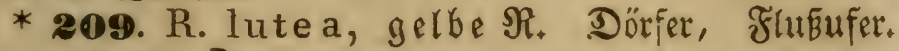
7. 8. बे.

\section{Siebente Sronung. Dodecagýnia. 3wölfitemulige.}

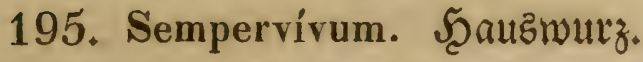

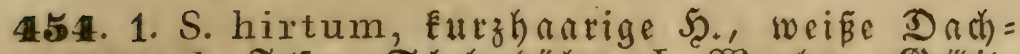
wo urzel. Fellen, Edhobendöcher. I. 'Barthau, Gröbis. 7. 8. 4 .

H. $1180^{\prime}$. L. Hb. S. Ch. B. O. W.

455. 2. S. tectórum, gemeine 5 . Mehr angebaut als wilo bin, uno wieder, auf Rirchböfen. 7. 8. 4 .

H. $2200^{\prime}$. L. K. Ch. O. W.

* 210. S. montánum, Berg= 5 . Scohe Ģebir= ge. 7. 8. 24, Babia Gsora.

\section{3wölfte Rlafie, Isocándria. Relchitänoig= Sielfabige.}

Crite Drbumug. Monogýnia. Cinftemplige. 196. Prunus. গুflaume.

456. 1. Pr. spinósa, Dornempfl., Schlefoorm.

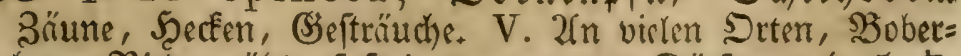
bette, Niedermühle, fofft in $\mathfrak{u}_{\text {. an allest Dörfertt. 4. 5. }}$. H. biz $2000^{\prime}$. L. Hb. K. S. Ch. B. O. W. 


\section{8}

457. 2. Pr. Ávium, Bogelfirfđbaum. MBälder, ŞeÉ= Een, Baumgänge, wohl vertwilbert. Beche, Siraufher $u_{*} a_{\text {. }}$ פ. 4.5 .5 .

H. L. Hb. K. $1530^{\prime}$. S. Ch. B. O. W.

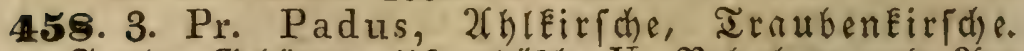

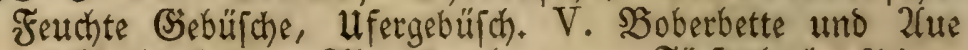
bei SRothlach, am 2rbzugsgraben, am Föriterbach, Eleimen Bober, Dürt=Sunzenoorf, Scohlftein. 4. 5. h.

H. $11-1200^{\prime}$. L. Hb. K. $1430^{\prime}$. S. Ch. B. O. W,

\section{3weite Oroumug. Di-Pentagýnia. Bwei bis Fünfitemplige.}

197. Spiraéa. Spierftautoe.

459. 1. Sp. salicifólia, weibenblättrige Sp. Seet= Een uno Ufergebüfó in Dörfernt, mehr angebaut ats wilo, Sraufchen, Ihomastwaldom, Tiefenfurth $\mathfrak{u}_{+} a_{+}$D. $, 6,7+\hbar$. L. Hb. K. S. Ch. B. O.

460. 2. Sp. Filipéndula, Enollwurzlige Sp, $\mathfrak{S}_{a} a \mathfrak{r}=$

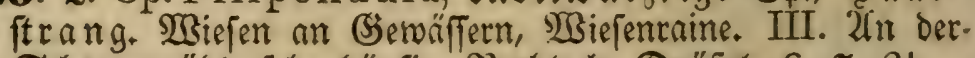

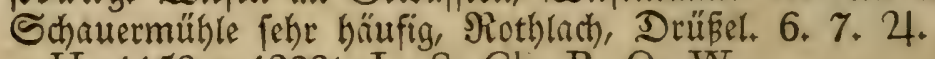
H. $1150-1300^{\prime}$. L. S. Ch. B. O. W.

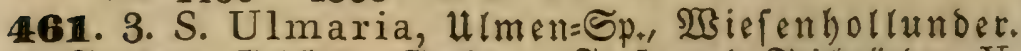

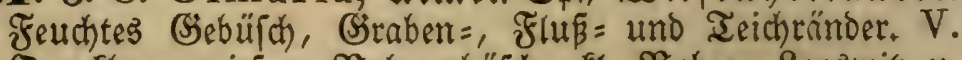

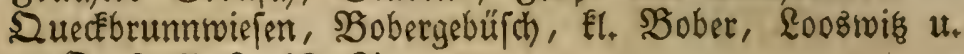
v. 5. 6. 7. 9. 10. 24.

H. $12-2200^{\prime}$. L. Hb. K. S. Ch. B. O. W.

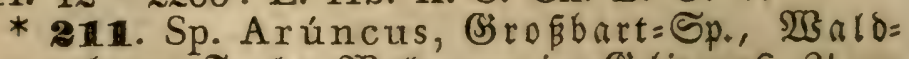
burt. Feudte $23 a r$ oungen im Sebirge. 6. 4. H. 1800'. L. Hb. K. S. Ch.

\section{Méspilus. Mi̇pel.}

462. 1. M. Cotonéster, 3 werg= $\mathbb{M}_{\text {., }} \mathfrak{B}$ ergquitte. Steimige, fonnige Berge. I. Sipfel bes Spişbergs bei Probfthain. 5, 6. 5.

L. Răhnb) 
463. 2. M. Oxyacántha, Dornen= M., Mefrbee=

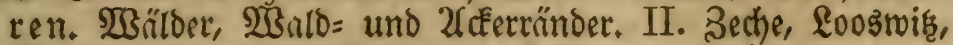
WBarthe, Jirnuichen = (Fichberger Şöhengug. 5. 5.

H. 2000'. L. Hb. K. 1430'. S. Gh. B. O. W.

\section{Pyrus. Bime.}

464. 1. P. commúnis, gemeine Waldbirne. S̃n खु̈lloctn, um Dörfer hin uno roieder, 5. $\hbar$.

H. $1300^{\prime}$. L. Hb. K. $1530^{\prime}$. S. Ch. B. O. W.

465. 2. P. Malus, wilder 'Up felbaum. Desgl.

466. 2. P. Aucupária, Ebereiche, Bogelbeeren. Millorr. III. Bect)e, Staide, Jraufthen, Watthe. 5. 5.

H. 2800'. L. Hb. S. Ch. B. O. W.

* 212. P. torminális, Љuhrbirme. Berge. 4. 5. 5.

* 213. P. Aria, tother Mehlbaum. Sebirge. 5. 6. 5. 2upen= uno Eltogruno.

\section{Dritte Dromung. Polygýnia. SBielfitemplige.}

200. Rubus. Srombeere.

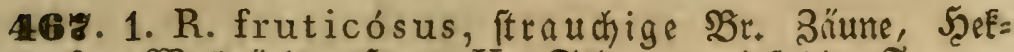
Een, $\mathscr{B a l}$ drïnoer, Şaue. V. Sehr mannigfaltige Formen, ja wohl auch mehrere zu unteriheidende 2urten, bie jedoch fo viele Uleberganggformen zrigen, Das fie fohrwer zu unter= fheiden find, befonders von Unföngern. Şon Rodh wieder vereinigt. 6. 7. aud 9. uno 10.5 .

H. 2000' L. Hb. K. S. Ch. B. O. W.

469. 2. R. caésius, graugrüne $\mathfrak{B r}_{+}, \mathfrak{R} \mathfrak{h}$ mbeere. Scecken, Sïune, feuchte (siebuifdse. V. Dod) nimber bäufig als 1. Borftübte, Seibels Borwerk, Rirch bof $2 x, 6-9.5$.

H. L. K. S. Ch. B. O. W.

469. 3. R. Idaéus, wohlfdh medende $\mathfrak{b}$, Şimbeere.

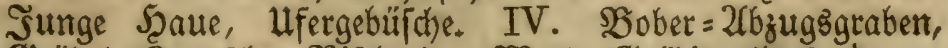

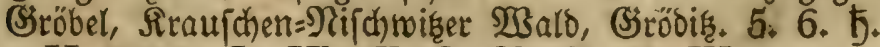

H. $2800^{\prime}$. L. Hb. K. S. Ch. B. O. W.

$$
5^{*}
$$




\section{0}

470. 4. R. saxátilis, Stein= $\mathfrak{B r}$. Steinige, trodêne $\mathfrak{B a ̈ l}=$

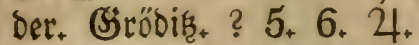

H. 12-1700'. L. K. S. Ch. B. O. W.

\section{Fragária. Errobeece.}

48 1. 1. Fr. vesca, $\mathfrak{B}$ ald $=\mathbb{E}$. $\mathfrak{B B i l d}$ er, Şaine, fdnattige Eehnen. V. 2f vielen Drten. 5, 6, 9. 4 .

H. L. Hb. K. S. Ch. B. O. W.

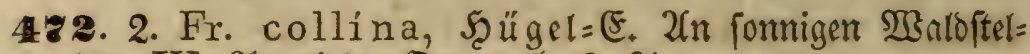
Ien. IV. 2ln vielen Stten. 5. 6. 4 .

H. L. K. S. Ch. B. O. W.

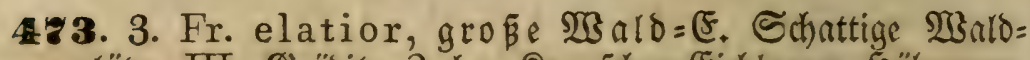

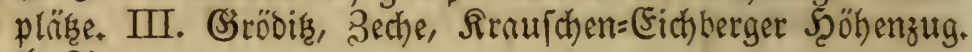
5. 4 .

H. biz 2800'. L. Hb. S. Ch. O. W.

\section{Potentílla. Fingerkraut.}

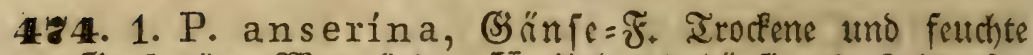

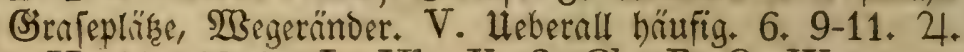
H. $11-2000^{\prime}$. L. Hb. K. S. Ch. B. O. W.

425. 2. P. reptans, Ériechendeis F. Dämme, Şecken, Schutthaufen, $\mathfrak{B i e j e n . ~ V . ~ G r a l g e n t e i c h , ~ B o b e r ~ u . ~ y . ~ a . ~}$ D. $6-8,4$.

H. L. Hb. K. S. Ch. B. O. W.

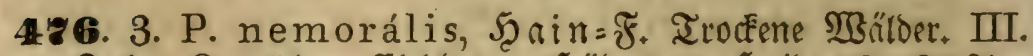

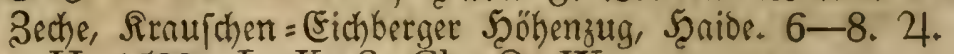
H. $1500^{\prime}$. L. K. S. Ch. O. W.

4\%. 4. P. Tormentílla, Tormentill=ף. Wiefen,

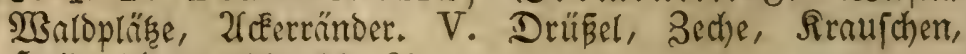
Sุaibe. $5-7.10,11.4$.

H. $2200^{\prime}$. L. Hb. K. S. Ch. B. O. W.

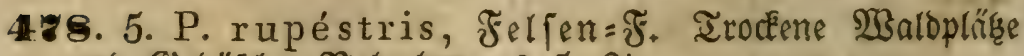
uno Bebuif che. Boberbette.? 5. 4.

L. W. 
489. 6. P. supina, überliegendes F. Schutthaufen, Mauern, Teichränber. I. SDber=Morftadt, am (salgenteiche, - Schlopteiche. 8. 9. 4.
L. O. W.

480. 7. P. norvégica, norwegif hes F. Teict)= und (3itabentïnder. I. Sil. Rog̨enat. 6-9. 21.

$\mathrm{Hb}$.

* 211. P. recta, aufredtes $\mathfrak{F}$. Şuchige Şü= gel. $6.7,21$. L. O. W.

* 2115. P. canéscens, graufilzigez F. Somi= ge STügel. 6. 7. 4 .

H. $2000^{\prime}$.

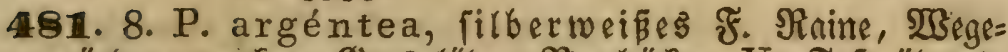

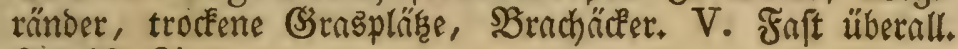
$6-10.24$.

H. $12-1500^{\prime}$, L. B. O. W. Hb. K. S.

482. 9. P. Guenthéri, (s) ünthers F̆. Dämme, Aecter, (3rabentïnder. I. Şin und wieder mit 8. 5. 4.

B. O. K. S.

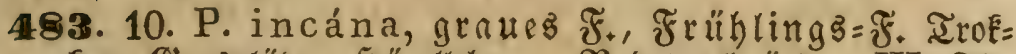

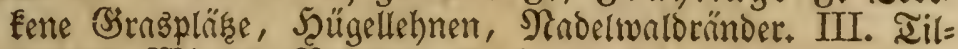
lenborf, SBiefau, Boberau. 3. 4. 24. S.

484. 11. P. verna, $\mathfrak{F}$ rühlingg $=\mathfrak{F}$. 3 Bie 10. befonderg

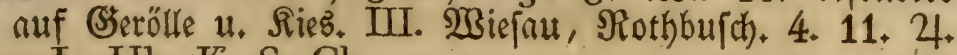
L. Hb. K. S. Ch.

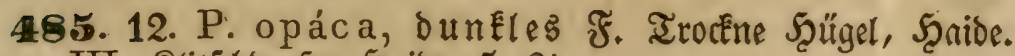
III. Flitifchoorfer Şaibe. 5, 4.

H. $1200^{\prime}$. Hb. S. Ch. B. O. W.

* 216. P. salisburgénsis, falzburgirches $\mathfrak{F}$. SBabia Gsora. 7. 4 .

* 218. P. auréa, gologelbes $\mathfrak{~ F . ~ B ̈ e b i r g s w i e f e n , ~}$ bis Snlberg herabfteigend. 6. 7. 2 .

* 218. P. alba, weí̄es F. Trocéne \&aubmälber. 5. 4. Berslau. O. Eeobjøük. 
203. Cómarum. Błutaugen.

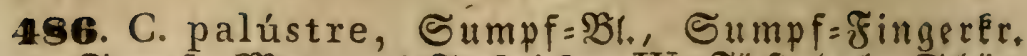
Sumpf $=$ Mloor $=$ und Torfwieien. IV. Förfterbnd), Sd)ön=

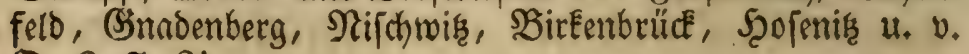
5. 6. 7. 4 .

H. $2200^{\prime}$. L. B. O. W. Hb. K. 1430'.

204. Géum. Benediftenmurz.

482. 1. G. rivále, $\mathscr{B}$ affer $=\mathfrak{B}$. Sumpfwiefen, Frlen= Srüche. I. Şohlftein, Sumpfbuifh unterm Sd)lögarten. 4. 5. 2 .

H. $10-2200^{\prime}$. L. Hb. K. S. Ch. B. O. W.

488. 2. G. urbánum, wahre $\mathfrak{B}$, Nelkenwur子. B̧e=

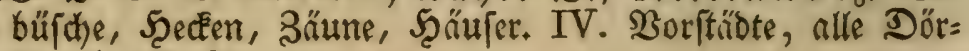
fer. 5-7. 4 .

H. 10-1500' L. Hb. K. S. Ch. B. O. W.

* 219. G. montánum, $\mathfrak{B}$ erg= $\mathfrak{B}$. Şochgebirge. 5-7. 2 .

* 220. G. pyrenafcum, pyrenäifbe. Desgl. 6. 24.

* 221. G. intermédium, mittlere $\mathfrak{B}$. $3 u=$ weilen mit 1.

\section{Rosa. Roje.}

489. 1. R. canína, 5ृundşrofe, 5ृagebutte. B̈̈ume,

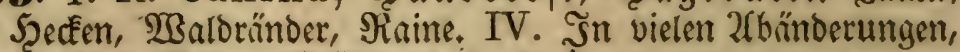

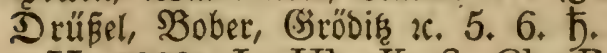

H. $2000^{\prime}$. L. Hb. K. S. Ch. B. O. W.

490. 2. R. villósa, zottige, graufilgige $\mathfrak{~}$. $\mathfrak{W}_{\text {ie } 1 .}$ nur feltener. Drüßel, Barthe, Esröbis, Bober 26.

L. W. Hb. Ch.

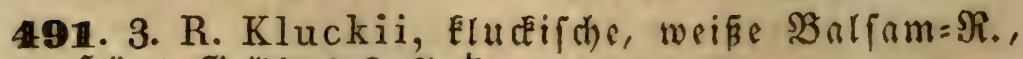

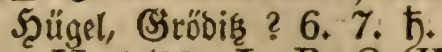

H. $1500^{\prime}$. L. B. O.'W. 


\section{3}

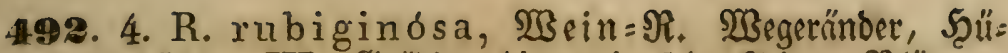

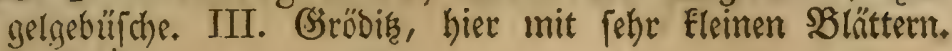
6. 7.5 .

H. 1500' L. O. W. S.

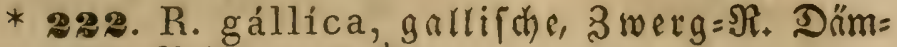

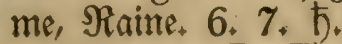

H. $1500^{\prime}$. L, B. O. W. Hb. K.

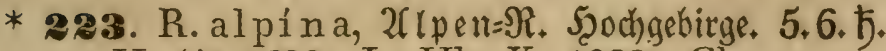
H. bis 2600', L. Hb. K. 1980'. Ch.

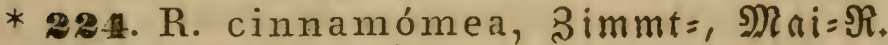
2Cckerränder. 5. 6. ந.

L. B, W. Hb.

\section{Dreizebnte Silafie Polyándria. Frutbtbobenftändig = Sielfabige .}

Crite Sromung. Monogýnia. Cinftemplige. 206. Papáver. Rohn.

493. 1. P. Argemóne, Reulen=, Sand= R. 2fecker, Srachen. V. Fant überall, 7. 8. $\odot$.

H. L. Hb. K. S. Ch. B. O. W.

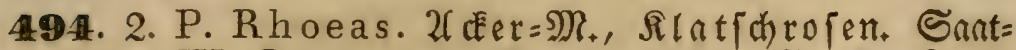
felder. IV. Desgl, m. Der Sant wechferno. WBatthe, Arau= ichen, Ihomastwaldau. 6, 7. $\odot$.

H. $1100^{\prime}$. L. Hb. S. B. O. W.

495. 3. P. dúbium, zweiferhafter $\mathfrak{M}$. Santfelder. 6. $7 . \odot$.

L. Hb. B. O. 


\section{4}

\section{Chelidónium. Sđföltéraut.}

496. 1. Ch. majus, gemeines Sth. Mauem, Sdutt= haufen, V. Faft überall. $6-9$. $\odot$.

H. bis $2200^{\prime}$. L. Hb. K. S. Ch. B. O. W.

208. Actáea. Schnalzmurz, Sriftopljzḱraut.

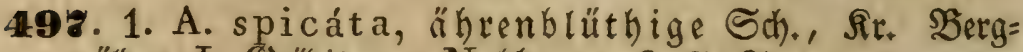
waillber. I. Sroöbif am Nabhange. 6. 7. 24.

H. 11-2300'. L. Hb. K. 1690'. S. Ch. O. o. i. auf bem Rummeläberge hei Strehlen.

\section{Tília. Rinbe.}

498. 1. T. parvifólia, Eleinblättrige \&., $\mathfrak{B}$ inter= linde.

H. bis 2235'. L.? Hb. K. S. Ch. B. O. W.

499. 2. T. vulgaris, groß̧blättrige linbe.

$\mathrm{Hb} . \mathrm{S}$.

500. 3. T. pauciflóra, wenigblumige o. weiche \&.

H. $10-2300^{\prime}$. W. Hb S. Ch.

2(lke 3 2(rten fommen untermifht in Straßenbaumgän=

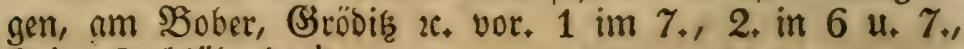
3 im 6. blühend. 5.

54. Heliánthemum. Sonmentöżchen.

* 225. H. vulgáre, gemeines S. Scohe Irif= ten. 6.5 .

H. $12-1500^{\prime}$. L. S. Ch B. O. W.

210. Nympháea. Seprofe.

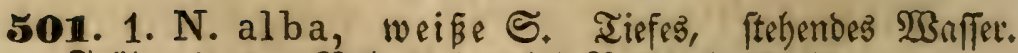

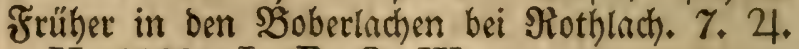

H. $1000^{\prime}$. L. B. O. W.

\section{Nuphar. Seekandel.}

502. 1. N. lúteum, gelfe S., Butterfar. Stillfte= 
hende Germäffer. I. Boberlachen bei uttig. 6. 7. 4 .

H. $1000^{\prime}$. Ln. B. O. W.

* 226. N. minimum, Eleinfte Seck. Teiche. 6. 7.24 .

\section{Dritte Stonung Trigýnia. Dreiftentplige.}

\section{Delphínium. Ritterfporn.}

503. 1. D. Consólida, $\mathfrak{F} e l d=\Re$. Ģetraibefelder. III.

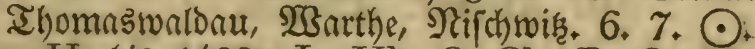

H. bis $1400^{\prime}$. L. Hb. S. Ch. B. O. W.

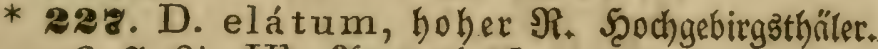

6. 7. 4. Hb. 21gnetenoorf.

213. Acónitum. (Eifenfut.

504. 1. A. variegátum, bunticheckiger. (F. Die fohlanke Form, meift niederliegeno mit umgekehrten Shel= men. Schwarjer Berg bei Sirtwis, Nlehne im untern (̧e= büf mit Daphne, Asarum. 7. 8. 4.

L. Hb. K. $1480^{\prime}$. S. Ch.

* 228. A. variabile, veränderlider E. Şö= here Gebirgstheile. 7. 8.24 . H. $2000^{\prime}$.

* 229. A. Cammarum, arzneilicher (E. Değgl. O. S.

* 230. A. Lycoctonum, getber E. Bergmäl= Der. 7.4 .

\section{Füufte Sronung. Pentagýnia. Fün fitentplige.}

- 214. Aquilégia. 2refelei.

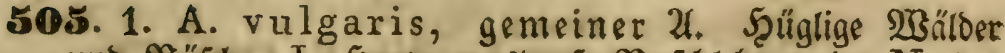
uno Büf(d)e. I, Sçartmannboorf, Bufdileknen im N. Dez 
Fatebruth8, vielleidht verwiloert. (Lïhn.) 5, 6. 4 .

H. 11-1200' Hb. K. S. Ch.

55. Nigélla. Sđhrarzzümmel.

* 231. N. arvénsis, wilber Schw. Uecter. $\odot$. S. W.

\section{Siebente Sromung. Polygýnia.}

\section{Sielftemplige.}

56. Adónis. 210oniz.

* 232. A. aestivális, Sommer=2l. Setraibef. 5. 6. $\odot$.

B. O. W.

215. Ranúnculus. Şałmenfuี.

506. 1. R. aquátilis, $\mathfrak{B}$ a f Die Scauptform in langam fliependen Graben, ftehendent Gienv. IV. Förfterbadh, SSoberlachen, Thongruben, Schön= feld. 5, 6. 9, 4 .

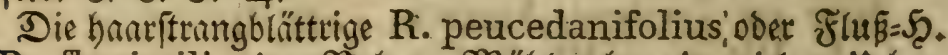
R. fluviatilis im $\mathfrak{B}_{0 \text { ber, }}$ Müblgraben in vielen Ueber=

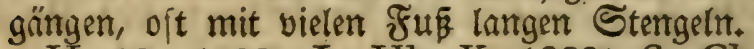

H. $10-1100^{\prime}$. L. Hb. K. $1380^{\prime}$. S. Ch. B. O. W.

* 233. R. illýricus, illyrifcher 5ृ. Irodente, fandige 2fecter, Ђ̦ügel. 6.7. 4 .

50\%. 2. R. Lingua, zungenblättriger ફ̧. Girnfige u.

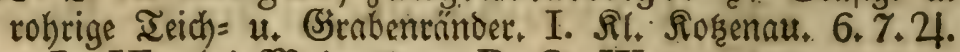
L. Hb. bei Maimaldau. B. O. W.

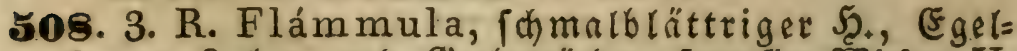
Eraut. Eadjen = uno Grabentänder, fumpfige 2Biefen. V. Faff überall. Förfferbach, Sitaufchen, Scaiberwiejen $x$. 6-9. 24.

H. $1300^{\prime}$. L. Hb. K. S. Ch. B. O. W.

* 234. R. a conitifólius, eifenhutblättr. 5ૃ. 5̧ochgebirge. $6-8.24$.

H. über 1200'. Lo. Hb. K. 1730'. Ch. 
509. 4. R. a urícomus, golbgelber 5. Feudhte Wirs=

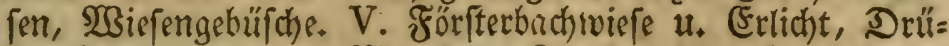

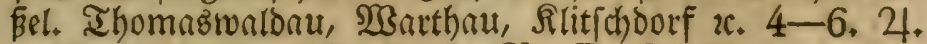
H. 1100' L. Hb. K. S. Ch. B. O. W.

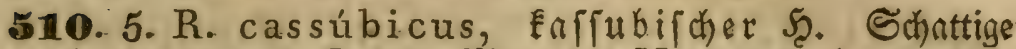
Eaubwillber. I. Sroöbiá, Mitte des Nabyangs, 4-6. 24. S. O.

511. 6. R. scelerátus, Bsift=5ૃ., Sifftblume. Süm=

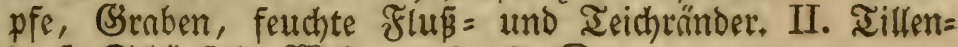
oorf, Schönfeld, 2Bsehrau. 6-9, $\odot$.

H. bis 1200 \% L. B. O. W.

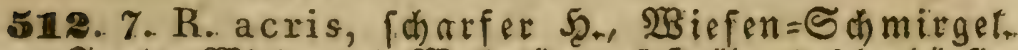

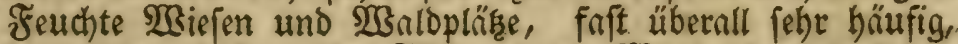
Die zweite, hobe gelbe Förbung. Der 2 Biefen beivirfent. 5 . 6. 9-11. 4 .

H. $1200 \%$ L. Hb. K. S. Ch. B. O. W.

513. 8. R. Polyánthem os, vielblumiger 5 . Ţald: und bufdige 2 Biefen, Bsebüfche, fohnttige Strakengraben,

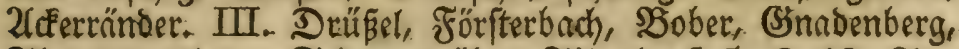
Ihomaswalbau, Schauermühle, Tillenoorf $5,6,10,24$. H. biz 2200'. L. Hb. S. Ch. B. O. W.

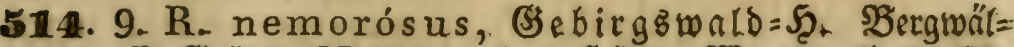

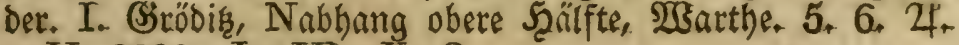
H. $2200^{\prime}$. L. Hb. K. S.

515. 10. R. lanuginósus, wolliger, Birk̂́ahmemf. Feuchte, fohattige MBälder. ? Bröbif ? 5. 6. 4.

H. bis $2200^{\prime}$. L. Hb. K. S. Ch. B. O. W.

516. 11. R. repens, friedender Ş, Sumpfidmir= gel. Faft überall auf feudstem Garten = und 2rdeerland", Fandigen $2 B i e\lceil e n$, Erräber. V. 5. 6. 9. 24.

H. 12-2200'. L. Hb. K. S. Ch. B. O. W.

518. 12. R. bul bósurs, Enolliger F̧. Trodéne Wiefen:

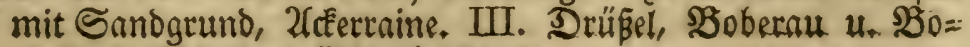
berinfel, Sd)nuermühle. 4. 5. 2 .

H. $1200^{\prime}$. L. O. W. Hb. 


\section{8}

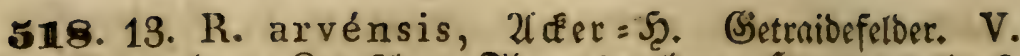
Ginnbenberg, Fraufchen, Thomaకwalonu, Scartmanmborf. 6. 7.24.

H. $10-2200^{\prime}$. L. Hb. K. S. Ch. B. O. W.

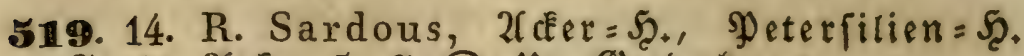

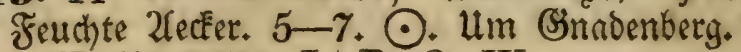

H. bis 2200'. L. B. O. W.

216. Ficária. Scharbodkzkraut.

520. 1. F. ranunculoídes, gemeines S(j). Sebülche,

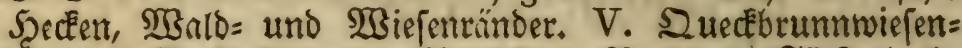

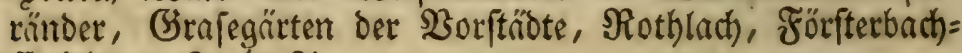
Erticht $2,3.4 .24$.

H. bis 2200'. L. Hb. K. S. Ch. B. O. W.

217. Tróllius. Iugetramunfel.

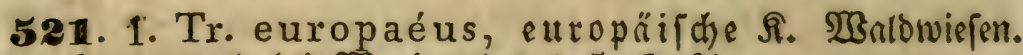
Summmald bei $\mathfrak{B}$ enigtreben ? 5.6. 4.

H. $12-2200^{\prime}$. O. W. S. Ch.

218. Myosúrus. Räujefdmanz.

522. 1. M. minimus, Eleinfter M. Feuthte 2lecter.

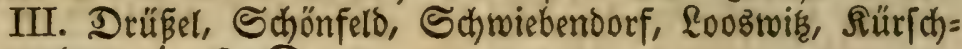
nerberg. $4-6, \bigodot_{\text {. }}$

L. B. O. W. Hb.

57. Isopýrum. Doltocke.

* 235. I. thalictroides, gemeine D. Raub= wäloer. 3. 4. 24. O.

58. Helléborus. NRiejetwurz.

* 236. 1. H. niger, đd warze গ. Sergwälber. 2. 3.24 .

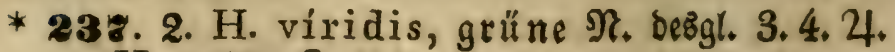
H. $1100^{\prime}$. S. 


\section{Caltha. Sdymirgel.}

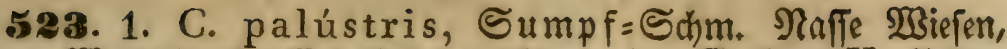

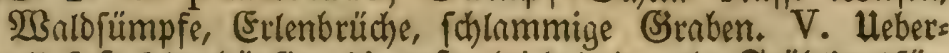
all foff fehr bäufig, bie erfte (niedrige) gelbe Frtüblingsfört= bung ber 2 Biefen. 4. 5. 24.

H. 10-1100' L. Hb. K. S. Ch. B. O. W. 220. Hepática. \&eberblume.

524. 1. H. tríloba, breilappige \&. Raubwälber. II. Groöbis, 3umm b. WBenigtreben, Rititchoorfer Scaibe, (Fid)= berg. 3. 4. 24. L. Hb. K. S. Ch. O.

\section{Anemúne. $23 i n b b t u m e$.}

525. 1. A. vernális, Frühlinģ=2B, tothe $\mathfrak{B}$ ald $=$

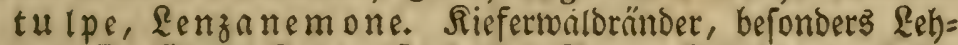

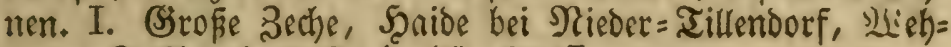
raure Ralebruch.? 3. 4. (5), 24. L.

* 238. A. patens, offene $\mathfrak{B}$. Moofige Riefer= u. (Eid)enwalbung. 4. 24. Sulau. Malapant.

* 239. A. Pulsatílla, arzneiliche $\mathfrak{B}_{\text {., }}$ wahre

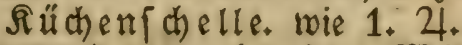

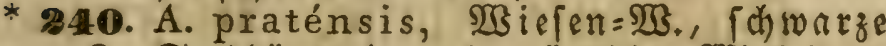
ת. Sanohügel. 4. nach mündlicher Mittheilung beim $\mathfrak{2 B e h r a u e r ~}$ RalEbetuch; wohl aber 1.

B. O. W.

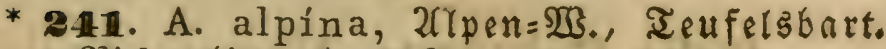
Riefengebirge. $5-7.24$.

* 241 b. A. sylvéstris, $\mathfrak{B}$ ald 22.4 .5 .4$.

526. 2. A. ne morósa, Şain= $\mathbb{B}$., weíce D fterblu=

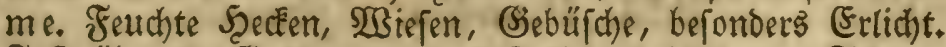
Faft überall. Grouppentweis, faft liegengebliebenten Sdinnee= baufen von fern ähnlich) fehend. 3. 4. 5. 24.

H. 2300' L. Hb. K. S. Ch. B. O. W.

528. 3. A. ranunculoídes, gelbe $\mathfrak{B}$. Feuthtę $\mathfrak{R} a u b=$

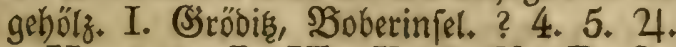

H. $1000^{\prime}$. L. Hb. K. S. Ch. B. O. W. 
* 242. A. narcissiflóra, $\mathfrak{R} a r z i f f e n=\mathfrak{B}$., Berghänd en. Scochgebirge. 5. 6. 4.

\section{Thalictrum. W3iefentaute.}

528. 1. Th. minus, Eleime 23 . Iroctme Şügel, 2tâer=

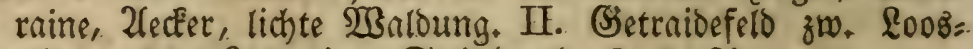
wis u. Dem \&oosivişer Steinbruch. 6, 7. 4 .

Hb. S. Ch. O. W.

* 243. Th. aquilegifólium, acéeleiblättri=

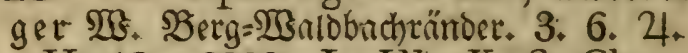

H. $12-2200^{\prime}$. L. Hb. K. S. Ch.

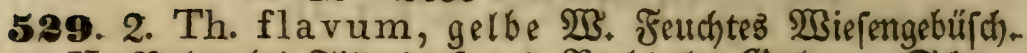
II. Bober bei Tillenborf unt Rothlnch), Gold= $u_{+}$Shau= ermühle. 6. 7. 4 .

H. 13-2000'. W. S.

530. 3. Th. angustifólium, rđa malblättrige $\mathfrak{W}$.

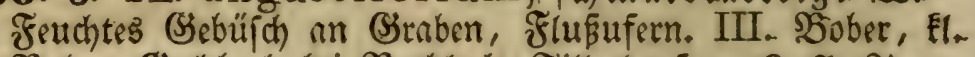

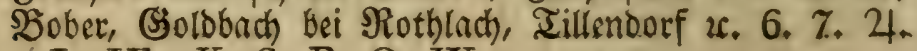
L. Hb. K. S. B. O. W.

\section{Bierzefnte Rlafie, Didynámia. 3wei und 3weifabige.}

\section{Crifte Droututy. Gymnospérmia. כreifamige.}

\section{Prunélla. Braunelle.}

531. 1. Pr. vulgáxis, gemeine $\mathfrak{B r}$. Bracher, B̉ierent.

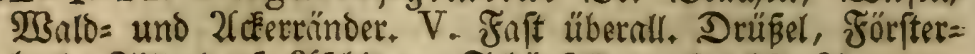

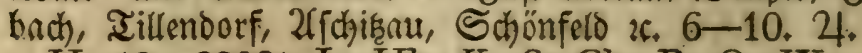

H. 10-2200' L. Hb. K. S. Ch. B. O. W.

* 244. Pr. grandiflóra, grobblumige $\mathfrak{B r}$. Biefen. $5,6,4 . S$. 


\section{4. 'Thýmus. Suendel.}

532. 1. Th. Serpyllum, wilber 2 . Uhuf 2lefertainen,

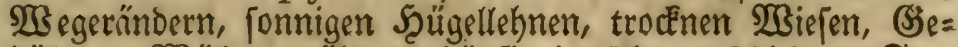

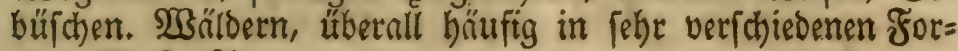
men. 7. 8. 24.

H. L. Hb. K. S. Ch. B. O. W.

533. 2. Th. angustifólius, fọmalblättriger 2 .

Sanoboden, Şügel, Rieferwalloung. III. Seche, Scaibe, \&id)= tentwaldau. 7.8 .24$.

H. 1500 ', S. Ch. B. O. W.

* 245. Th. lanuginósus, wollharager 2 . 23ege. Scabelfctwert. 5. 6. 24.

534. 3. Ph. Acinos, Stein= 2., blauer Bergthy=

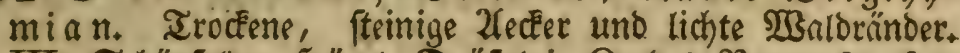

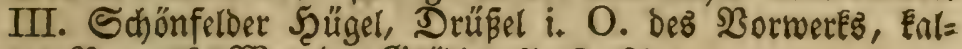

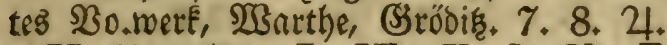

H. bis $1500^{\prime}$. L. Hb. K. S. Ch. B. O. W.

\section{Clinopódium. WBirbeldoften.}

535. 1. Cl. vulgáre, gemeiner $\mathfrak{B}$, Bettfuß. SheE=

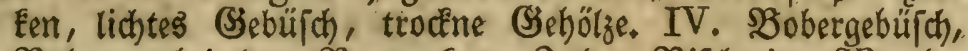

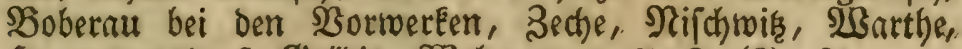
Scartmannsborf, BStöbí, -2Beh rau. 2.. 7. 8. (9). 24.

H. $1500^{\prime}$. L. Hb. K. S. Ch. B. O. W.

226. Melittis. Bienenfraut.

536. 1. M. Melissophýllum, Meliffen= $\mathfrak{B}$. $\mathfrak{B} \mathfrak{E}=$

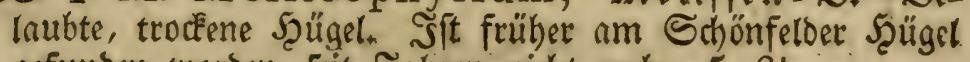
gefunben worden, peit Sahten nid)t mehr. 5. 24.

\section{Scutellária. Scetmeraut.}

53\%. 1. Sc. galexiculáta, gemeines $\mathfrak{F}^{\text {. Teich) }}$ unb

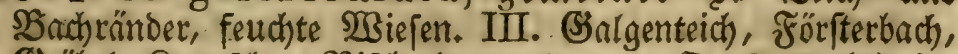

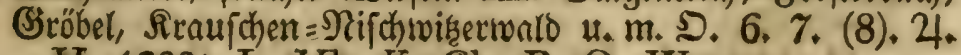
H. 1200\% L. Hb. K. Ch. B. O. W. 


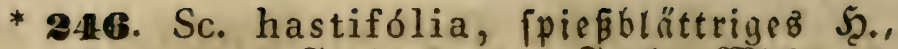

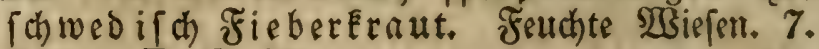
8. 24. B. O. W.

\section{Ajúga. জ̋̋̋njel.}

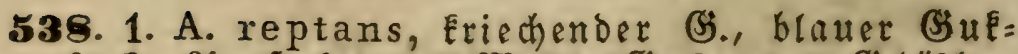

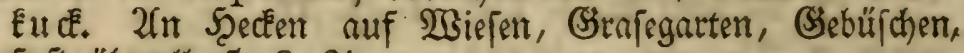
faft iiberall. 5, 6. 24.

H. L. Hb. K. S. Ch. B. O, W.

539. 2. A. alpina, 2ripen= (5). Desgleichen aber feltner. Sajönfeld, Eichberg, -3arthe, Mehrau, Sröbis. 5. 6. 24. H. 2000'. L. Hb. S. Ch. B. O. W.

59. Chamaépitys. 3merggamander.

* 248. Ch. vulgáris, gemeiner 23w. Trodtne, fteinige Brachüdêter. 5, 6. $\odot$.

229. Teúcrium. SGamander.

540. 1. T. Scórdium, R̂noblaudb=(S)., \&adhenEnob=

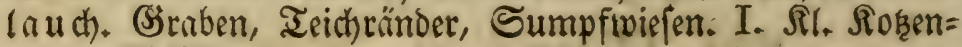
au. $7,8,24$.

B. O. W.

* 24s. T. Botrys, Trauben=\$. 2fecter, ponnige Steintehnen. 7. 8. Hb. (Rişelberg.) K.

230. Oríganum. Doften.

541. O. vulgáre, gemeine $\mathfrak{D}, \mathfrak{W}$ aldo often. Sujchige

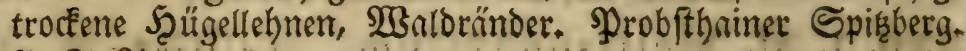
7. 8. 24 .

H. $1400^{\prime}$, L. Hb. K. S. Ch.

231. Méntha. Münze.

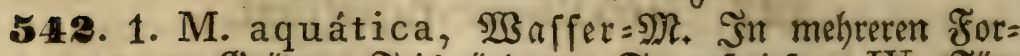
men an Şräben, Teidyrändern, Sumpfwiefen. IV. F̋̈r=

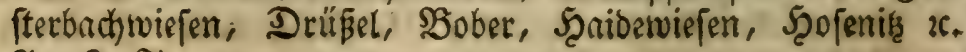
7-9. 4 .

H. $1100^{\prime}$. L. Hb, K. $1480^{\prime}$. S. Ch. B. O. W. 


\section{3}

* 249. M. acutifólia, fpirblättrige M. 2fm WBaffer. 7. 8. 4.

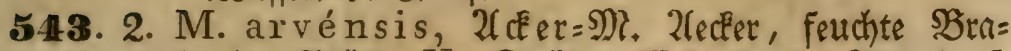

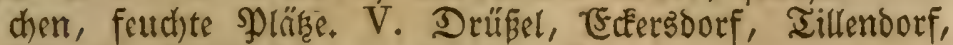

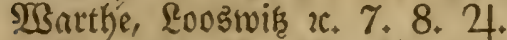

H. 2200' L. Hb. K. S. Ch. B. O. W.

* 250. M. rubra, rotbe $\mathfrak{N}$. Flußufer. Troppau. 7. 4

* 251. M. víridis, grüne M. Dę̧gl. Stober. 7. 8. 2. L.

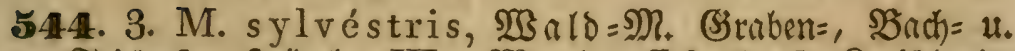

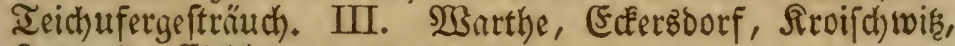

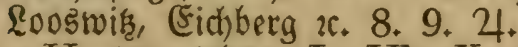

H. 12-1500'. L. Hb. K. S. Ch. B.

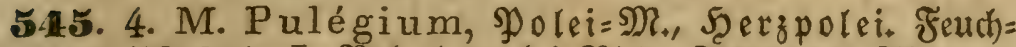

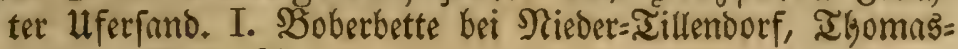
warloau. 7, 8, 24.

H. $10-1300^{\prime}$, L. K. S. B. O. W.

\section{Galeóbdolon. (Siolonefiel.}

546. 1. G. lúteum, Ģoloneffel, gelbe Ianbref=

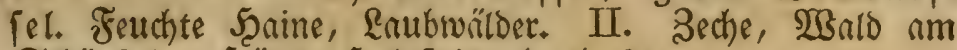
Schönfelder Şü̈gel, Şohlftein. 4. 5. 24.

H. bis 2000'. L. Hb. K. S. Ch. B. O. W.

\section{Gaelópsis. Şanfnefiel.}

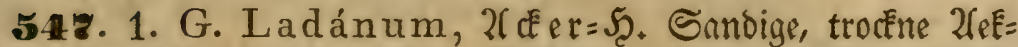

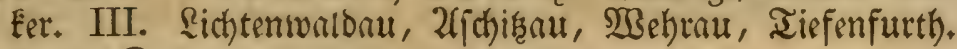
7. 8. $\odot$.

H. $1400^{\prime}$, L. Hb. S. Ch. B. O. W.

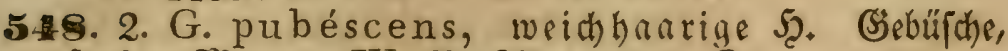

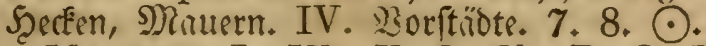

H. $1170^{\prime}$. L. Hb. K. S. Ch. B. O. W.

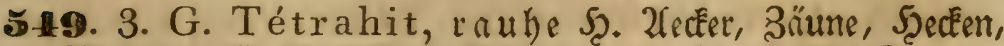

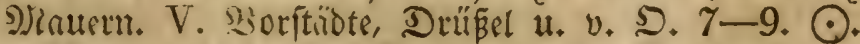

H. bis 2200'. L. Hb. K. S. Ch. B. O. W. 


\section{4}

* 252. G. versícolor, buntblumige Sृ. Fenthte Malloplifice. 7. 8. ○.?

L. S. Ch. O. W.

234. Lámium. Iaubeneffel.

550. 1. L. maculátum, geflecéte $\mathfrak{I}$. Sirabenrionder, fenchte (sebüfabe, Mauern. V. Fraft überall, Strotgraben 2е. 5, 6, 9, 10, 4 .

H. $11-2200^{\prime}$ L. Hb. K. S. Ch. B. O. W.

551. 2. L. album, woeiß̄e I. Sörfer und Borftäbte an Scecken, Maucrn, Scumusboden. II. SBorftïbte hin u. wis= Der, Burglehn, Ilitidjoorf, Richtenwalonu. 5. 6, 8, 9. 24. H. bis 2000'. L. Hb. K. S. B. O. W.

552. 3. L. purpúreum, פ̧urpur= I. 2(edere, Garten= lanb, Schutt, Mauern. Ueberall fehr häuftg, burchs ganze Sึhtr. $\odot$.

H. 2200'. L. Hb. K. S. Ch. B. O. W.

553. 4. L. amplexicaúle, ftengelumfaffende

Zlecker, Ģürten, Srachen. V. Frnf́t überall. 2rnitalt, Drü= Bel zc. Jrühling, Şerbft. 24.

H. 2000'. L. Hb. K. S. Ch. B. O. W.

\section{Glechóma. Ssundermann.}

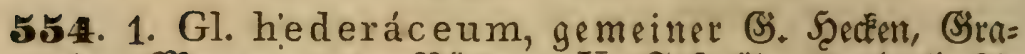
ben, Mauern, unter Bäumen. V. Fraft überill. 4. 5. 24. H. 2200'. L. Hb. K. S. Ch. B. O. W.

\section{Népeta. Raf̧enmünze.}

555. 1. N. Catária, gemeine R. Maucrn, Sdyutthau=

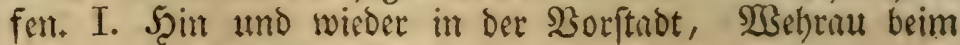
Şammerwerk. 7-9. 4.

H. L. W. Hb. S.

* 253. N. nuda, Eahle $\Re_{+}$Sn Dörferm, nn $\mathfrak{W}_{c=}$ gen. 7. 8. 24. Sheridlefien. 


\section{Stachys. Sieff.}

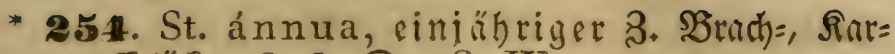
toffelídfer. 8, 9. $\odot$. S. W.

* 255. St. arvénsis, $\mathfrak{X}$ dêter=3. SErachäcter. 7 . 8. ○. Riffa bei Brestau.

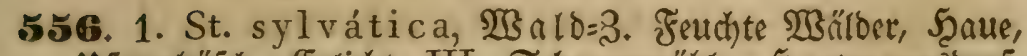
ulfergebuilich, (Erlicht. III. Schautermühle, Scartmannsborf,

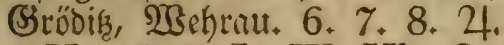

H. 2500'. L. W. Hb. S. Ch. K. B. O.

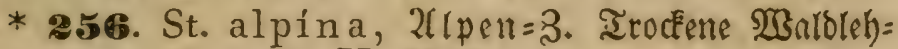
nen. 7. 8, 24, H. 2000'.

* 25\%. St. germánica, beutfder 3., grauer

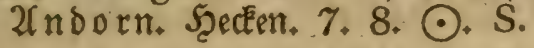

* 258. St. recta, grader 3. Iroctene, fteinige Şü= gel. 7. 8. 2. W. Hb.

558. 2. St. palústris, Sumpf $=3$. Feuchte G̈ebüfhe,

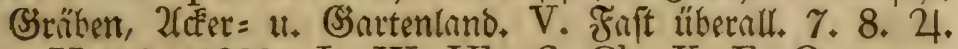
H. $10-1300^{\prime}$. L. W. Hb. S. Ch. K. E. O.

238. Leonúrus. Bärenfdmeif.

558. 1. L. Cardíaca, gemeiner $\mathfrak{B}$. Dorfwege, Seeden,

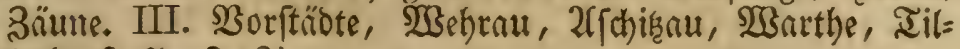
lentorf. 7-9. 4 .

H. $1100^{\prime}$. L. B. O. W. Hb. K. S. Ch.

* 259. L. Marrubiástrum, andornartiger B. Dörfer, Brachen. 7-9. 24 . Ln. B. O. W.

\section{Ballóta. Balllote.}

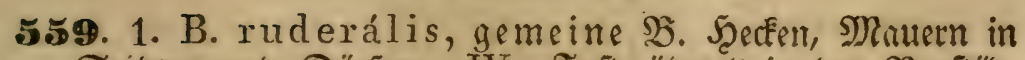
Stibtent und Dörfern. IV. Faft litterall in ben Sorftiob= ten. 233 artlye 2 . $7-10.24$.

H. $11-2200^{\prime}$. I. B. O. W. Hb. Ch. 


\section{Betónica. Setoniéa.}

560. 1. B. stricta, fteife B., 3efreraut. SBurdhige Biefen, grafige lichte Baldolïhe. III. Sthauer= u. B̈old=

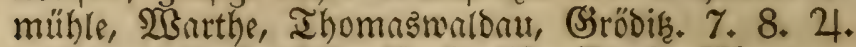

H. 2000'. L. Hb. K. S. Ch. B. O. W.

\section{Marrúbium. 2Tnoorn.}

561. 1. M. vulgáre, gemeiner $\mathfrak{A}$. $\mathfrak{A} \mathfrak{n}$ Seccéen, Săunen,

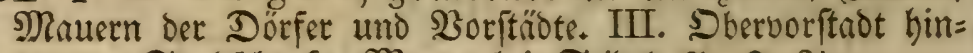
ter der Sandichenee, Mauer bei Seibel. 7-9. 2.

Ln. S. B. O. W.

\section{8weite Sroututg. Angiospérmia. Rapieliantige.}

\section{Verbéna. Eijenf́raut.}

562. 1. V. officinális, arrzneiliches (F. Dörfer uno Borftöbte an Mauern uno $2 B$ egerïndern. V. Sehr bäufig in Den $\mathfrak{B o r f}$ tïbten unt Dörfern. 6-9. $\odot$.

H. $1000^{\prime}$ L. S. B. O. W.

\section{Tózzia. Tozżie.}

* 260. T. alpína, 2 lpen $=$ I. Şoctgebirge. in Sberidhefien. 7. 8. 4.

243. Limosélla. Schlammling.

563. 1. L. aquática, gemeiner Sthl., $\mathfrak{k l}$. Sthlamm=

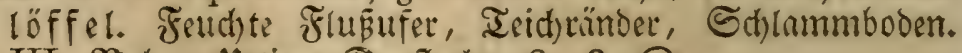

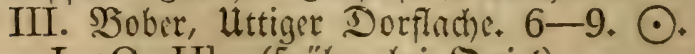

L. O. Hb. (früher bei $2 u i r t)$.

\section{Lindérnia. Rinbernie.}

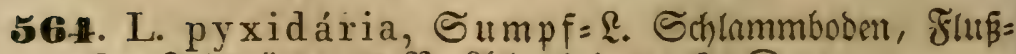
ufer, Ifeichrïnoer. ? 3B. 2ulbertini. 7-9. $\odot$. 


\section{7}

245. Alectorólophus. Şahnentamm.

565. 1. A. minor, Eleiner Sc., $\mathfrak{B}$ iefentapper, Jlaf= fer. Miejen. V. Boberinjel, Schauermüble, Förfterbady, Şartmannzoorf $x, 5,6$. $\odot$.

H. bis 2200'. L. Hb. K. S. Ch. B. O. W.

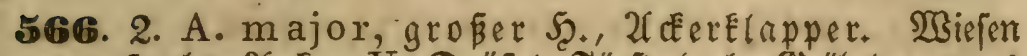
u. feuchte 2lecker. V. Drüßel, Förifterbach), Siröbel ze, etwas ppriter. 5-7. $\odot$.

H. L. Hb. K. S. Ch. B. O. W.

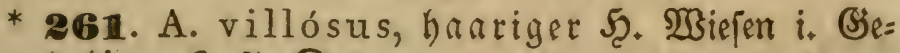
birge. 6. 7. $\odot$.

H. L. Hb. K. 1680-1730'. S. Ch.

60. Bártschia. Baartidje.

* 262. B. alpina, 2(lpen= B. Scochgebirgs $=$ Suel= len. 6. 7. 4 .

\section{Odontítes. 3abntroft.}

568. 1. O. verna, Frühlings= ober gemeiner 3. 2ூ3ie=

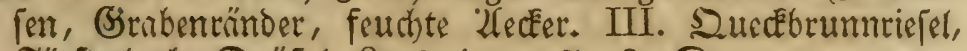
Föriterbnch, Drüsel, Roostwif x. 7-9.

H. bis 2200'. L. Hb. K. S. Ch. B. O. W.

\section{Euphrásia. 2 Uugentroft.}

568. 1. E. officinális, arzmeilicher 22. Grusplïbe in verichiedenen Formen, ie nachoem biefe feucht, trocken oder buichig fino. V. 7-9. $\odot$.

H. bis 2200'. L. Hb. K. S. Ch. B. O. W.

248. Pediculáris. Räujeftraut.

569. 1. P. palústris, $\subseteq u m p f=\Omega . ~ \Re a f f e ~ S u m p f=u n \delta$ Moorwiefen. II. Schönfelder Sümpfe, 2fichigauer Mloor= ftich, Scorenif. 5. 6. बे.

H. $11-1400^{\prime}$, L. Hb. K. S. Ch. B. O.

5ช (1. 2. P. sylvática, $\mathfrak{B a l d}=$, $\mathfrak{B i e f e n =}$. Moorige $\mathfrak{u}_{+}$ 


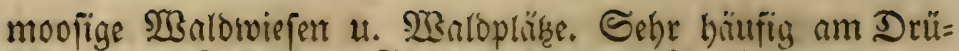
Bel, in ber 3ecthe, am Förfterthed) u. v. פ. 5. 6. 4.

H. 11-2200'. L. Hb. K. S. Ch. B. O. W.

* 263. P. sudética, Subeten=R. Quellenteiche

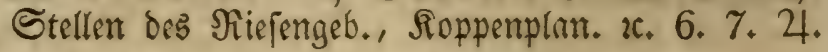
249. Melámpyrum. Rubrweizen.

* 264. M. cristátum, firmmiger $\Re$. 2Barbrïlt= ber. $\mathfrak{B B n}$ rowieren. 6.7. $\odot$.

H. 1500 ' B. O. W.

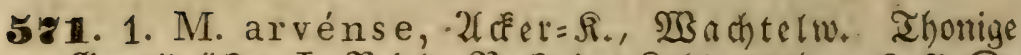

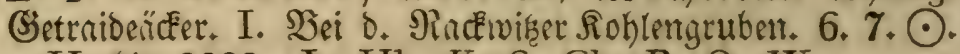
H. bis 2200'. L. Hb. K. S. Ch. B. O. W.

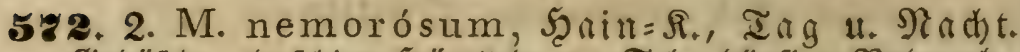

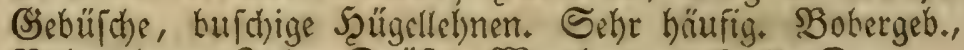

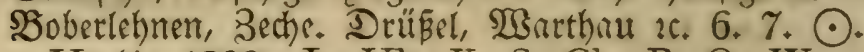

H. bis $1200^{\prime}$. L. Hb. K. S. Ch. B. O. W.

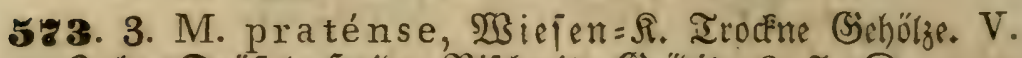

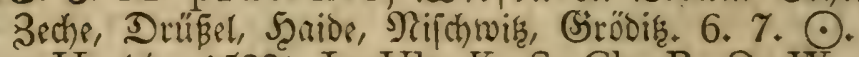

H. $14-1500^{\prime}$. L. Hb. K. S. Ch. B. O. W.

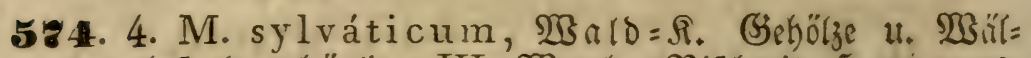

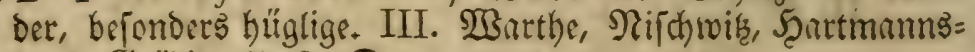

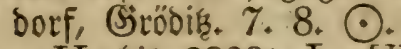

H. bis 2800'. L. Hb. K. S. Ch.

\section{Scrophulária. Rropffraut.}

585. 1. Scr. nodósa, Enotiges fir. Feut)tes 2 Biefen= gebüf(h), utferrïnoer. IV. Bober, Böberte, Bröbir. 6-8. 10. 11. 4 .

H. bis $2200^{\prime}$. L. Hb. K. S. Ch. B. O. W.

586. 2. Scr. aquática, $\mathfrak{B a}[\lceil\in \mathrm{e}=\Re$. WBic und mit 1 . $6-8.4$.

H. B. O. W.

* 265. Scr. glandulosa, orüfiges Mit. Dbet= idblefien. 6-8. 24. Dppe(tn, Iefditen. 
* 266. Scr. vernalis, $\mathfrak{F}$ rühlings = Sir. Schnt=

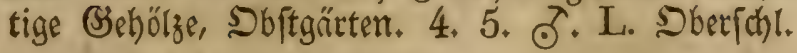

251. Digitális. Fingerfut.

58\%. 1. D. ochroleúca, gelber Fing. Fitüuterteiche,

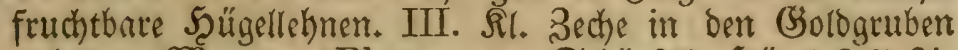
rechts v. Wrese m. Phyteuma, Schönfeld. Sczügel. 6.7. 2. H. 1700'. L. Hb. K. S. Ch.

\section{Linária. Reinktraut.}

588. 1. L. vulgáris, gemeines 导, Łöronmaul,

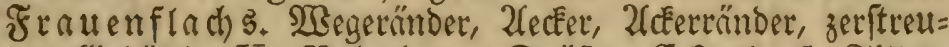
tes (Sebuif Dorf 2 .

H. bis 1200 '. L. Hb. K. S. Ch. B. O. W.

* 268. L. cymbalaria, edeiges 2. 2ulte Mru=

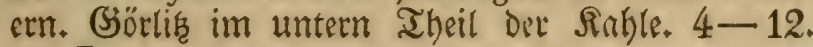
4. L.

* 268. L. Elatine, $\mathfrak{Z}$ dé $=\mathfrak{L}$, Eleine Erowinds. 2tecter. 7-9. ○. L. Şres̆tau.

* 269. L. spuria, unächtes 2 ., grobe E. begght. 6-9. $\odot$. Brezlau.

* 2zo. L. minor, Eleineres \&. 2fefer, Stein= brüct)e. 6-9. $\odot$. Breslau, Neufirch) b. Gsolob. B. O. W.

* 2z1. L. arvénsis, blaues $\mathfrak{Z}$ déer $=$ : Uecter. 6. 7. ○. L. Hb.

61. Antírrhinum. Dorant.

* 2a2. A. majus, groß̧er D., göwentaur. Berglehnen. Fürrtenftein. 6. \%. ఠ. S.

* 283. A. Oróntium, fleiner D. Uecker. 7-9. ๑. Şainau. H. 10-1500', L. B. O. W.

62. Orobánche. Sommermurz.

* 284. O. pallidiflóra, braffe S. 6. 7. 4 . 
* 225. O. caryophyllacéa, meléenartige S. 6. 4. Sprieborn, Sberichlejien.

* 276. O. coeruléa, bıáe S. 6. 24. W.

* 228. O. ramósa, "ritige S. 6. 7. 4. Sïmmtl. nuf trodEnen 2lectern u. Şügeln. 253. Lathraéa. Sduppennurz.

5\%9. 1. L. squamária, gemeine Sđ. Sdymarofere pflanze auf Şajel = uno Şuchentvurzeln im Schatten. I. Şohliteiñ an Der untern Gantenmauer binter bem Schloß SO., Gröbirberg. 3. 4. 4.

H. L. W. Hb. K. S. Ch.

63. Linnaéa. হimna̋a.

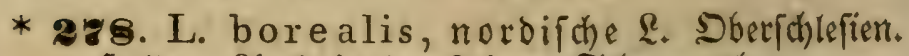
Scaibe. 2fuch in ber Eleiten Sthneegrube.

\section{Funfłebnte אilafie. Tetradynámia. Bier = und Bmeifabige. \\ (Cruciferae. Sireuzblüthige)}

\section{Erite Sroum Synclistae. Schliefêfrürchtige.}

1254. Néslia. Neslie.

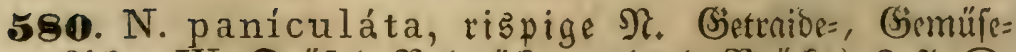

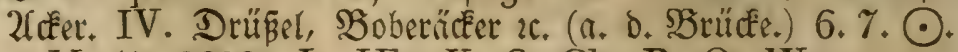
H. bis 2200' L. Hb. K. S. Ch. B. O. W.

64. Coronópus. Samentreffe.

* 2z9. C. Ruéilii, nieberliegende S. $\mathfrak{B}$ egf. 8-10. 21. Brestau, Docrvorfteot. 


\section{2}

\section{Lepídium. Rrefle.}

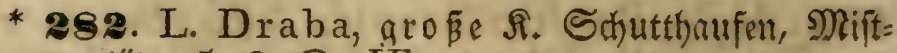
plähe 5. 6. $\odot$. Hb.

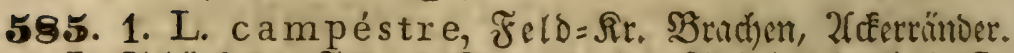

I. Schönfelder Felder? Iunzendorfer Steinbruch. 5.6. $\odot$. H. 2400' L. W. Hb. K. S. Ch.?

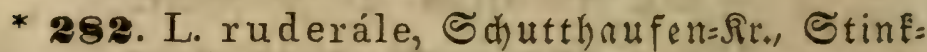

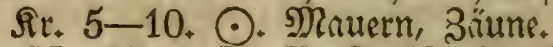

H. 1500', I. K. S. Ch. B. O. W.

\section{Farsétia. Farjetie.}

586. 1. E. incána, weī́grauc F., weiß̄er $\mathfrak{B a u e r n = ~}$ fenf. :

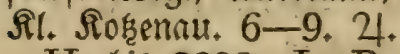

H. bisె 2200'-L. B. O. W.

261. Draba. Scungerbtümdjen.

58\%. 1. Dr. vérna, $\mathfrak{F}$ rühling

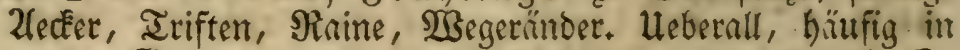
grosen (5)ruppen zufammen, boch aud) einzeltr. $2-5 . \odot$.

H. 2300' L. Hb. K. S. Ch. B. O. W.

\section{Thlaspi. গু fennigftraut.}

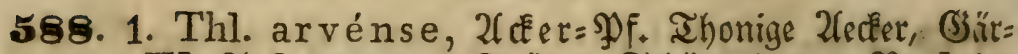

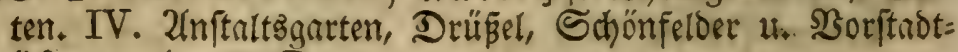
čçer $x .5-7$. $\odot$.

H. bis $2300^{\prime}$. L. Hb. K. S. Ch. B. O. W.

* 284. Thl. montánum, SBcrg= Df. Steinige Berge. 4. 5. 24. H. 2000'.

* 285. Th. perfoliatum, our ch wa h [enes झुf. 2lecter. 4. 5. 24. K.

* 286. Thl. alpéstre, Felfen= DP. Felfige SBü=

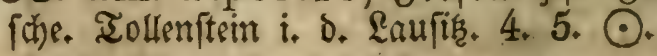

263. Capsélla. Sirtentafide.

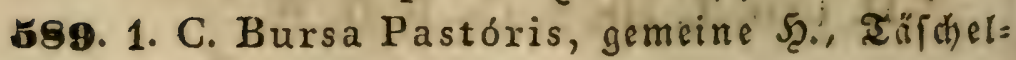




\section{3}

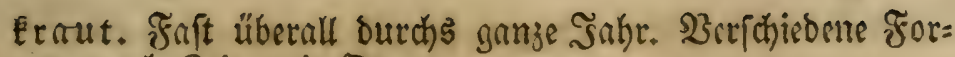
men nach Beit und Sort.

H. 2300'. L. Hb. K. S. Ch. B. O. W.

264. Camelína. Rameline.

590. 1. C. sativa, gefäte $\Omega_{+}$, Reindotter, ginEent=

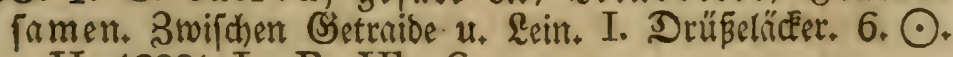
H. $1200^{\prime}$. L. B. Hb. S.

591. 2. C. dentáta, gezăhnte א. W̉ie 1.

L. O. W. Hb. S. Ch.

* 28z. C. austríaca, öltreidif if́e R. Stcinige und fanbige Flufufer. 6. 7. $ఠ$.

\section{Dritte Sronung. Siliquósae. Sibotenfritchtige.}

265. Nastúrtium. Rauke.

592. 1. N. a mphíbium, $\mathfrak{R} \mathfrak{n} \mathbb{\delta}=\mathbb{2 B} \cap[\lceil e r=\Re$., $\mathfrak{B}$ a f

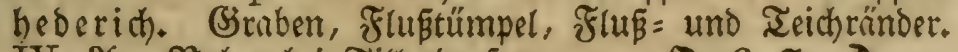
IV. 2Um Sober bei నillenoorf u. v. a. D. 6. 7. ฮ. H. 1050' L. Hb. K. 1430'. S. Ch. B. O. W.

593. 2. N. sylvéstre, wilbe $\Re$. Feuchte $(5 \mathfrak{r a n g}=$ uno

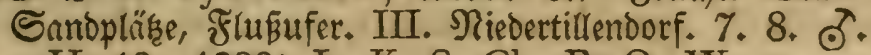
H. $10-1200$ ' L. K. S. Ch. B. O. W.

594. 3. N. palústre, $\subseteq u m p f=\Re$. 2Bie 2. III. (Eich)= berg, Schönfeld, Nothlnch. 6-8. $\odot$.

L. Hb. S. B. O. W.

* 288. N. anceps, Baftard= NR. Flupufer. 6 . 7. 8,2 .

595. 4. N. officinále, arguteiliche $\Re$. Rangfam flie=

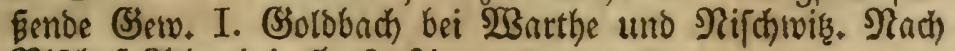
SBifhof Zflbertini. 5. 6. 4 . 


\section{4}

\section{Dentária. Załnnourz.}

596. 1. D. bulbifera, Enospentragende 3.. Schnt=

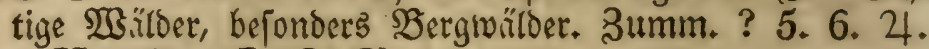
H. $2500^{\prime}$. L. S. Ch.

598. 2. D. enneaphýlla, neunblättrige 3. Desgl. Sprottau im Scochinalde. 4. 5. 4.

H. $2300^{\prime}$. L. Hb. K. S. Ch.

* 289. D. glandulósa, orüfige 3. Bergroälber. 4. 5. 24 .

\section{Cardámine. Sd)aumḱtautt.}

* 290. C. trifólia, oreiblättriges Sth. ऊi:= hirgstwalbiviefen. 6. 7. 24. Bei 23ölfelsborf und Reinerz.

* 291. C. resedifólia, refevablättriges Sd).

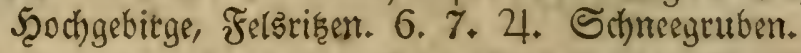

598. 1. C. amára, bitteres S(h)., follefif (he $\mathfrak{B}$ rutt= nenEreffe. $\mathfrak{Z} \mathfrak{n}$ den meiften Quellenftellen, Graben, SB̆̈= chen, Erlichte. 5-7. 4.

H. 2200'. L. Hb. K. S. Ch. B. O. W.

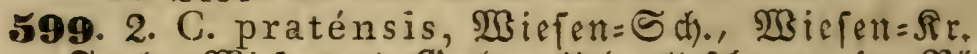

Fenchte Mrsiefen uno Brabern. Heberall fehr gemein. Röth=

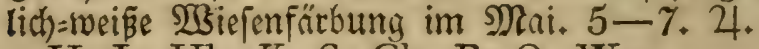

H. L. Hb. K. S. Ch. B. O. W.

600. 3. C. parviflóra, Elsinblumiges Sđ). Enchen= uno Grrabenränder nach Scłönfeld zu. I. 6-9. ๑. O.

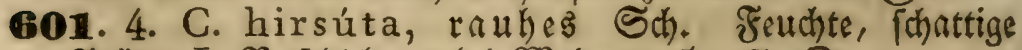

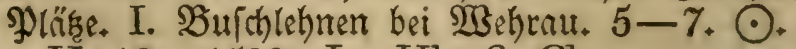

H. $12-1500^{\prime}$. L. Hb. S. Ch.

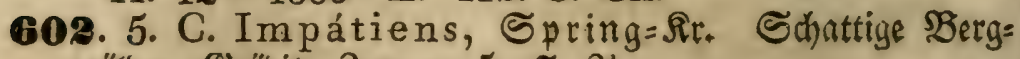
wälber. Srö̉obî, 3umm. 5-7. 24 .

H. $12-1500^{\prime}$. L. Hb. S. Ch. B. O. W.

268. Árabis. ઉ̋annfefraut.

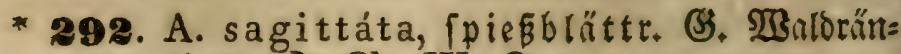
ber. 5. 6. ङ. Ch. W. O. 
* 2D3. A. hirsúta, rauhes (5. Trocene Ëehölze. 5. 6. बे.

H. $12-1500^{\prime}$, L. B. W.

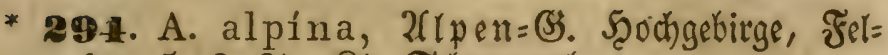
ien. 5. 6. 24. תit. Schneegrube.

* 295. A. Halléri, Şallerg= (5). Girnsplathe uno Ferfentuände ber Şebirge. 6.7. $\odot$.

* 296. A. arenósa, Sand= (5). Sambige 2lecterer,

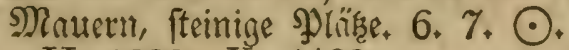

H. $1200^{\prime}$. K. $1480^{\prime}$.

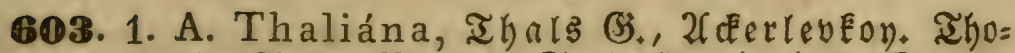
nig=[ambige 2lecter, Brachen, Sandpläke. 4. 5. 9. $\odot$.

H. 2300'. L. Hb. K. $1530^{\prime}$. S. Ch. B. O. W.

269. Barbárea. BarbenÉraut.

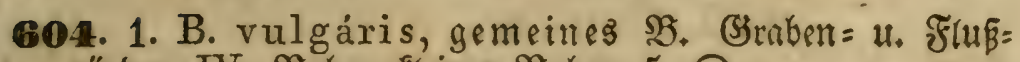
rünoer. IV. Bober, Éleiner $\mathfrak{B o b e r}$. 5 . $\odot$.

H. $2300^{\prime}$, L. Hb. K. S. Ch. B. O. W.

605. 2. B. stricta, fteifes $\mathfrak{B}$. Dిssgl. 5. 6. ఠౌ.

Hb. S. Ch. B. O.

\section{Túrritis. æhurmeraut.}

606. 1. T. glabra, glatteg $\mathfrak{I}$ +, Thurmfenf, $\mathfrak{B a l}$ ald =

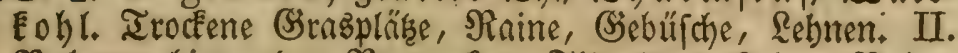
Boberau hinter Den. Sorwerfen, Tillendorf, Eleiner SBober, Schnermühle, 2Barthe $x, 6,7 . \odot$.

H. 1300'. L. Hb. K. S. Ch. B. O. W.

65. Hésperis. গAachtuiole.

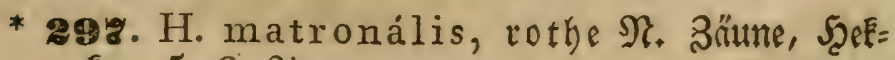
Fen. 5.6. 6 .

Enufitî. H. S.

271. Sisýmbrium. ŞejemÉraut, Rauke.

60\%. 1. S. officinále, arzmeiliches $\mathfrak{Z}, \mathfrak{R} a \mathfrak{u} e$ e. Dört= 
fer und $\mathfrak{B o r f t a ̈ b t e ~ a n ~ \Re a u t e n , ~ S c e c e e n , ~ S d u t t h a u f e n . ~ V . ~}$ Fant überall in ben $\mathfrak{B o r f t i o b t e n . ~ 5 . ~ 6 . ~ 9 . ~ \odot . ~}$

H. L. Hb. K. $1480^{\prime}$. S. Ch. B. O. W.

608. 2. S. Sophia, Sophien = R., 2 urmfamen.

Borftäbte und Dörfer, an und auf MRauern, $\mathfrak{B}$ egeründer, Sdhutthaufer. V. Ueberall, befonders in ber Misbersorftadt. 5-9. $\odot$.

L. Hb. nicht häufig. S. Ch. B. O. W.

\section{Erýsimum. Sibotenootter.}

* 298. E. strictum, fteifer Sth. Sanopläbr. 6. 7. $\odot . \mathrm{K}$.

609. 1. E. cheiranthoides, levÉoienartiger Sđ̆.

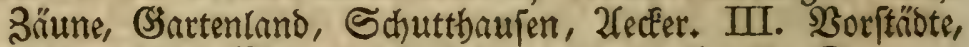

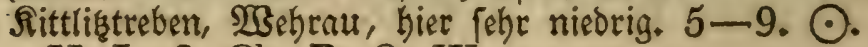

H. L. S. Ch. B. O. W.

\section{Conríngia. Ronringie.}

610. 1. C. perfolíata, burchion hatene $\Omega$. Salkige

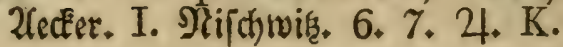

\section{Alliária. \&audjêraut.}

611. 1. A. officinális, gemeines ‥ (Bebülohe und

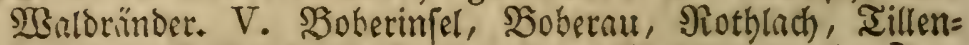

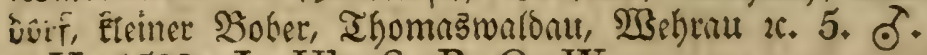

H. $1500^{\prime}$. L. Hb. S. B. O. W.

\section{Brássica. Robl.}

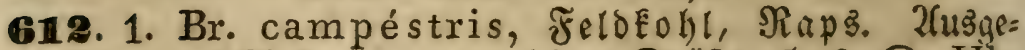

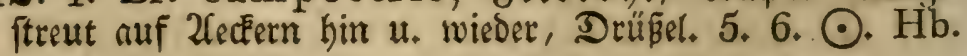

\section{Sinápis. ভenf.}

613. 1. S. arvénsis, $\mathfrak{A}$ dér $=S$. UnEraut unter Dem (Sp: traide, III. 5. 6. $\odot$.

H. $2200^{\prime}$. L. Hb. K. S. Ch.

614. 2. S. alba, weiser S. Desgleichen minoer bäufig. 5. 6. ๑. L. K. 


\section{Gecbszebnte Rlafie, Monadélphia. Esinbündige.}

Evrfte Drouutig. Pentándria. F̌ñnffabige.

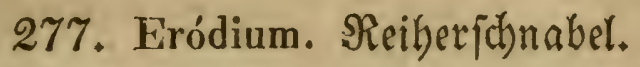

615. E. Cicutárium, fohierlingsartiger $\Re$. Smbige Girajplihé, fanbig=thonigeb 2ldere = und Gartenland. V.

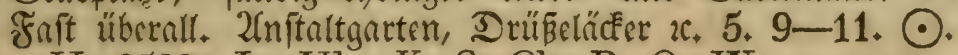
H. 2500'. L. Hb. K. S. Ch. B. O. W.

Bweite Drsutug. Decándria, Behufabiģe.

278. Geránium. Stordj)(d)nabel.

* 299. G. sanguíneum, blutrotber St., SBlutEraut. Irodénes Sieböl. 6-8. 24. B. O. W. K. S.

* 300. G. macrorrhízon, langwurzliger St. Aleine Sthneegrube, 6, 4 .

* 301. G. phaeum, rothbrauner St. Gebirgš= waldungen, 6, 7. 2 .

H. 1150 '. S. Ch.

* 302. G. sylváticum, $23 a l d=$ St. Sebirgb̈= Şrablehnen. 6.7. 4 .

H. $18-2200^{\prime}$, L. S. Ch. Hb.

* 303. G. praténse, $\mathfrak{B}$ iefen $=S_{t}$. $\mathscr{W}$ ald $=$ und

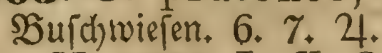

H. 1200' L. K. S. Ch. B. O.

616. 1. G. palústre, Sumpf=St. Sumpfige Miefen 


\section{8}

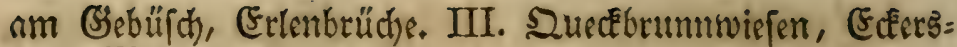
borf, Behrat. 6-8. 24 :

H. $10-1200$ ' L. Hb. K. S. Ch. B. O. W.

* 301. G. pyrenaícum, pyrenäifher St. ऊebüр\%. 6. ఠ. Dels, Iroppau.

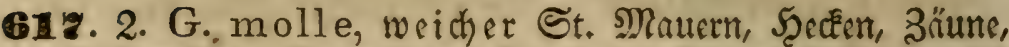

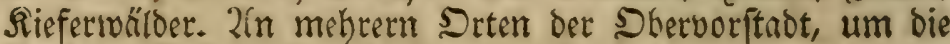
Znittalt, Gröbias. 5-7. $\odot$.

L. K. S. Ch. B. O. W.

* 305. G. bohémicum, böhmifder St.

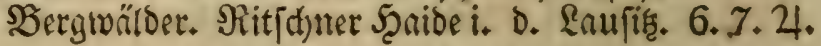

618. 3. G. rotundifólium, runbblättriger St.

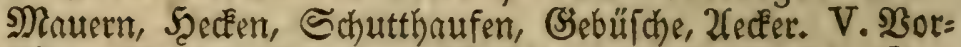

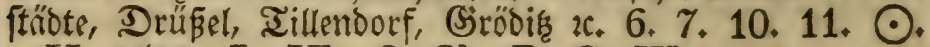
H. $1500^{\prime}$. L. Hb. S. Ch. B. O. W.

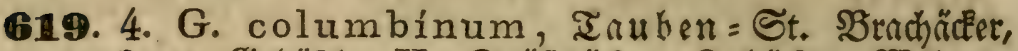

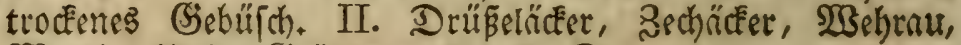

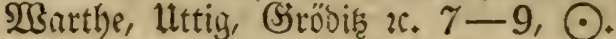

H. L. Hb. K. S. Ch. B. O. W.

620. 5. G. disséctum, zerrechligter St. Setraibe= u.

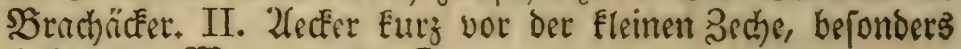

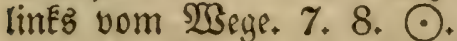

H. L: Hb. K. S. Ch. B. O. W.

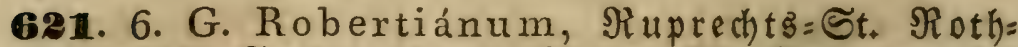
Inufşer. Feuthte, fohattige Stellen. IV. Strobtmauer bei

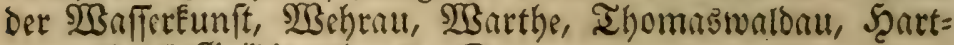
mannsoorf Gróoira. 5-8. $\odot$.

H. 2500'. L. Hb. K. S. Ch. B. O. W.

* 306. 6. G.divaricátum, ausgefpreizte St,

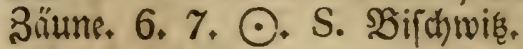




\section{Dritte Stsututy. Polyándria. Saielfónige.}

\section{Malva. Malve.}

622. 1. M. sylvéstris, wilde $\mathfrak{M}$., Roppappet, gr.

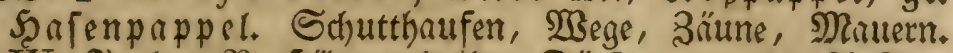
IV. Sn Den Sorftiobten, in ben Dörfern, um bie 2(nftalt. 7-9. $\odot$.

H. L. Hb. K. S. Ch. B. O. W.

623. 2. M. rotundifólia, runbblättrige M., $E_{\text {t. }}$ รุa fenpapper. 2Bie 1. Dod băufiger. 6-9. $\odot$.

H. L. B. O. W. Hb.

624. 3. M. boreális, nöroliche ober Eleinblumige M. Mit 2. untermifcht, boch bei weitem weniger häufig. 6-9. $\odot$.

H. $1500^{\prime}$. K. S. Ch. O.

625. 4. M. Alcéa, fhlifblättrige M., Rofenpap=

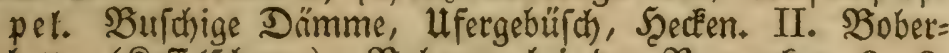
bette, (Ieffelicheuer), Sobernu bei Den Borwerken. 6-8. 24. L. Hb. B. O. W.

66. Lavatéra. \&avatere.

* 30z. L. thuringíaca, thüringifte $\mathfrak{R}$. Dorf= bectên. 7. 8. 21. Um SBres̆tnu.

\section{Siebrénnte Silafie. Diadélphia. 3weibündige.}

Sierte Drbuturg. Hexándria. Sechafabige. 280. Fumária. (Erorauch.

696. 1. F. officinális, arżneilicher E., Felbraute,

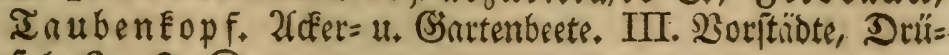
fel. 6-9, $\odot$.

H. bis $2000^{\prime}$, L. Hb. K. S. Ch. B. O. W. 


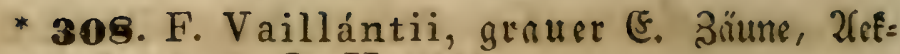

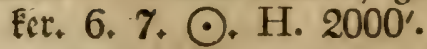

* 309. F. capreoláta, gelblicher E. Şectern. 6-8. ○. Hb. Wratmbrum.

281. Corýdalis. Scoflwulz.

62z. 1. C. fabácea, bohnenartige 5̧. (Sebürjo. I.

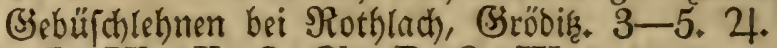

L. Hb. K. S. Ch. B. O. W.

* 310. C. bulbósa, grobe 5̧, Enolliger Ero= rauch. Esehölze uno (sebülche im Norgebirge. 3. 4. 24. Sauer.

H. 1700 ' L. S. Ch. B. O. W.

* 311. C. Halléxi, Şallers Sc. Fethölze im füb= öftichen Schlefien. 3. 4. 24. Seobfhüb.

\section{Fün Ỹte Sronung. Octandria. Yrad)trabige.}

282. Polýgala. Taujendjðőn.

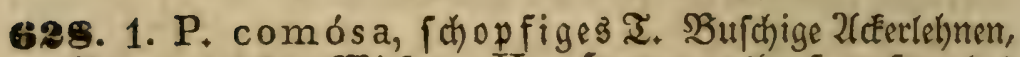

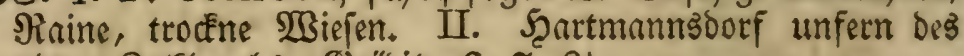
obern SalEbruchs, Srö̈oif. 6. 7. 24.

H. 2000' B. O. W. Hb. K. Eeipe bei Jauer.

629. 2. P. vulgáris, gemeines $\mathfrak{I}$, $\mathfrak{T}$ atte $x=$, Freuz=

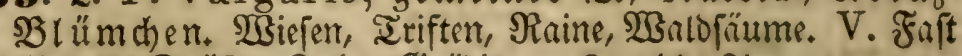

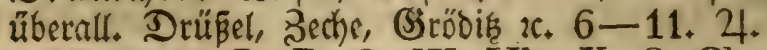
H. 2200' L. B. O. W. Hb. K. S. Ch.

630. 3. P. a mára, bitteres $\mathfrak{T}$. Feuchte $\mathfrak{B}$ iefen, $\mathfrak{B a l}=$

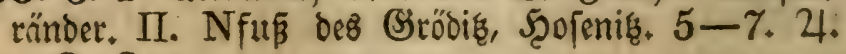
O. S. 


\section{Sechşte Drbnutg. Decándria. Bebufabige.}

283. Onónis. Şauthectel.

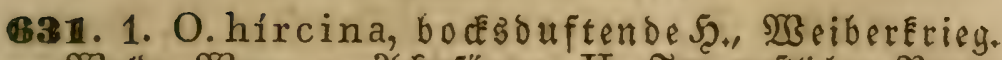

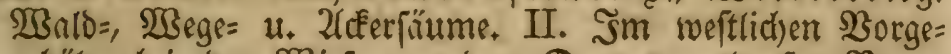
gehölze bei ber $\mathfrak{B}$ siefe vor dem Deutmannsoorfer Serge, Gsoldmühle, am Nabhang bes গleulinder Bergs nach Ref= pelsoorf him. 7-9. 4 .

Hb. K. S. O. W.

* 312. O. spinósa, fta dyliche 5 . 2Cfertünder,

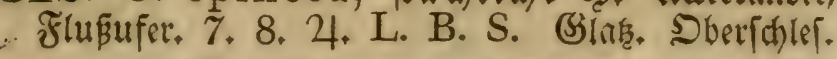
67. Anthýllis. $\mathfrak{B}$ und Elee.

* 313. A. vulnerária, gemeiner 23 . Irockene క̧นügel. 7, 8, 4 .

H. $12-1400^{\prime}$. Hb. K. B. W.

284. Onobrýchis. Ėzparjette.

632. 1. O, satíva, Futter $=$ E. Şügelwiefen, Raine. I. Grö́bín am Fupe der SOabhanges, 6, 7. 24.

B. O. W.

285. Melilótus. Steinflee.

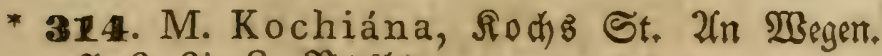
7. 8. 24. S. Brestau.

* 315. M, officinális, arzmeilider St. Dibz= gleichen. $\odot$.

H. 11-1200' L. S. Ch. B. O. W.

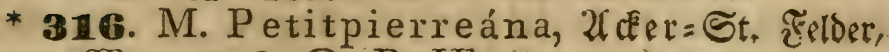
खुege. 7. 8. $\odot$. B. Hb.

633. 1. M. vulgáris, weiner St. W̉ege, 3üme, $2 \mathfrak{k}=$

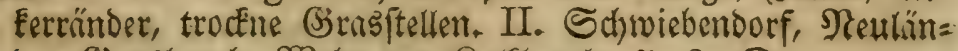

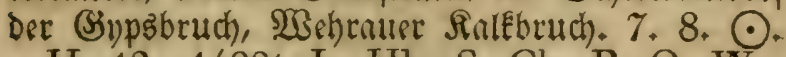

H. $13-1400$ '. L. Hb. S. Ch. B. O. W. 


\section{2}

\section{Trifólium. ふlee.}

634. 1. Tr. arvénse, Bradenelee, Şaafené. B̉ara=

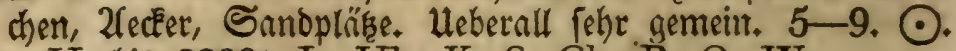
H. bis 2200'. L. Hb. K. S. Ch. B. O. W.

* 318. Tr. striátum, geftreifter $\mathfrak{B}$. Irodene

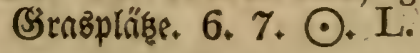

* 318. Tr. rubens, rother 2 ehrenelee, gro= Ber Berger. Bergiviefert. 5, 6. 24.

H. $1200^{\prime} . \mathrm{Hb}$. S. Ch.

635. 2. Tr. alpéstre, Bergfl, rother Spigel. Iroé=

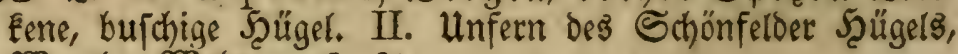
Barthe, $\mathfrak{B}$ rhtau. 6. 4 .

H. bis $2200^{\prime}$. L. Hb. S. Ch. B. O. W.

636. 3. Tr. medium, mittlerer Il. Wällber, (5ehölze, burchige $\mathfrak{E}$ ehnen. III. Sobertegnen bei Uttig, 3ecthe. 5.6.24. H. bis $2200^{\prime}$. L. Hb. K. S. Ch. B. O. W.

638. 4. Tr. montánum, weí̄er Bergkelee, weiß̄er

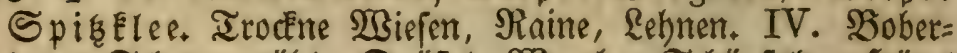
bette, Echauermühle, Drüßel, WBarthe, Echönfelder Şüget H. vielen $5,6,7,4$.

H. 2200' L. Hb. K. $1680^{\prime}$. S. Ch. B. O. W.

* 319. Tr. ochroléucum, reisgelber Iir. Irocéene Giehölze. 7+8. 4 . H. $15-2000^{\prime}$. S. Ch.

638. 5. Tr. praténse, $\mathfrak{B}$ iefenelee. Ulebrrall häufig auf MBiefen. 5. 6. 10\%:4.

H. L. Hb. K. S. Ch. B. O. W.

639. 6. Tr. hýbridum, Baftardel. MBiefen, (E)bülchp.

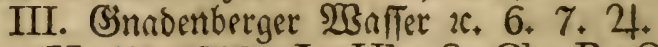

H. bi3 $2300^{\prime}$. L. Hb. S. Ch. B. O. W.

640. 7. Tr. repens, Eriechender, weißer Rl. SGiefen Brachen, feuchte Sanopläze, überall. 5-9. 4.

H. L. Hb. K. S. Ch. B. O. W. 
* 320. Tr. fragiferum, Erbbeer=, Blafen= Iltee. 6. 9. 21. S. B. O. Brestau.

641. 8. Tr. spadicéum, Énftanienbrauner Rl. Bor=

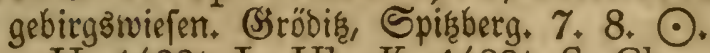

H. $1400^{\prime}$. L. Hb. K. $1480^{\prime}$. S. Ch.

642. 9. Tr. agrárium, ŞopfenEl., GூoloElee. B̈ehölz̧,

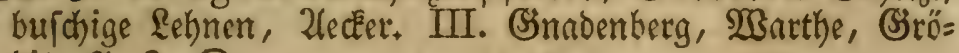
bif. 7. 8. $\odot$.

H. L. Hb. K. S: Ch. B. O.

613. 10. Tr. campéstre, gelber Ferbfl. 2fecter, G̋rng̈=

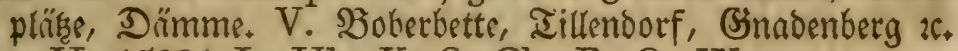
H. $1500^{\prime}$. L. Hb. K. S. Ch. B. O. W.

648. 11. Tr. filifórme, faden itengliger Nit. Trodêne

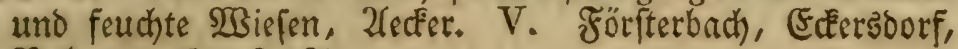
3ober 2. 6-9. 24 .

H. 2300'. L. Hb. K. S. Ch.

\section{Genísta. (sinfter.}

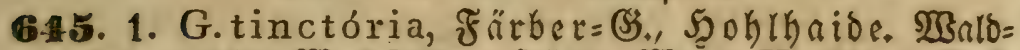

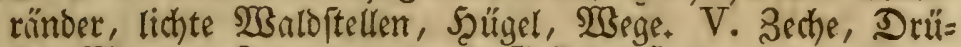

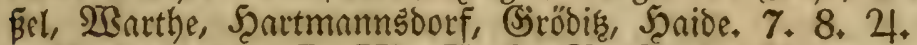

H. biz $1800^{\prime}$. L. Hb. K. S. Ch. B. O. W.

646. 2. G. pilósa, harariger (5., Eropfriemen. Sidyte

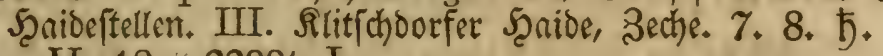

H. $18-2200^{\prime}$. L.

6-18. 3. G. germánica, beutfher ober Strahel=(5).

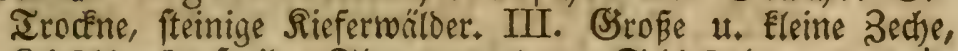
Alitfichoorfer Şaibe, Thomaswaldonuer Edjieferterg. 6.7. 5. H. $12-1800^{\prime}$. L. Hb. K. S. Ch. B. O.

288. Sarothámnus. Befenftrauch.

648. 1. S. vulgáris", gem. B., Befenpfrieme. Sam= bige Stellen, (Mieferwaloränder.) III. Soberbette a. WBehre,

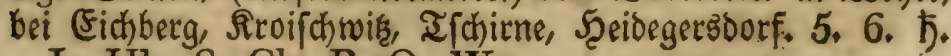
L. Hb. S. Ch. B. O. W. 


\section{Cýtisus. (̧̌eisłlee.}

649. 1. C. nigricans, fđ̆wärzlid)er (5). Rieferwälber, trocenes, lichtes Gebürich. III. Sileine Bedhe, Slitichoorfer Scribe. 6, 7. 5. L. O.

* 321. C. capitátus, Eopfiger (\$). Irodene $\mathrm{GP}^{\circ}=$ hölze. 6-8. Ђ. H. 1800'. O.

* 322. C. supinus, Eriechender (3. Rieferwäl= ber. $6-8,5 . H . \mathrm{O}$.

\section{0. Órobus. WSalberbje.}

650. 1. O. vernus, frühe $\mathfrak{W B}_{.}$Fafankraut. Schat=

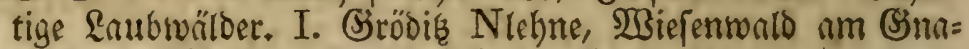
Denberger $23 a f$ Ter, jw. D. Şügeln. 5. 6. 24.

H. 2000'. L. Hb. K. S. Ch. B. O. W.

651. 2. O. tuberósus, Enollige $\mathscr{B}$. Feuchte u. unter=

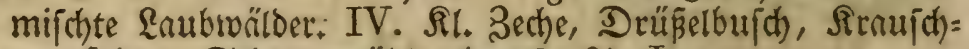
ner Scügel, Schauermühle. 4-6. 24. L.

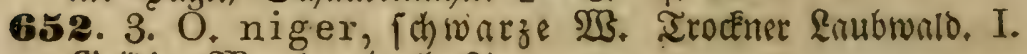
Sröbia, 203arthe. 4.5.24.

L. B. O. W.

291. Láthyrus. গুlatterbje.

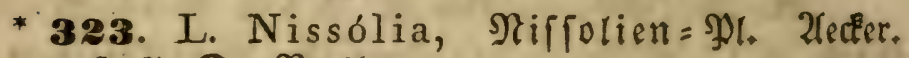
6.7. ๑. Breglıu.

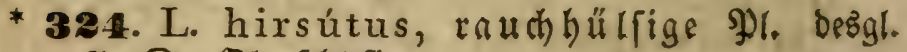
7. $\odot$. Sberichlefien.

* 325. L. tuberósus, Enollige Shr. 2recfer. 6. 7. 4. B. O. W.

653. 1. L. latifólius, breitblättrige गु. Sirafige Şü= gellehnen. Sröbir ? 6.7. ○. Ch.

654. 2. L. sylvéstris, $\mathfrak{B} a l b=\mathfrak{l}$., $\mathfrak{B}$ uhlerḱtaut.

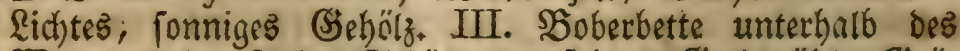

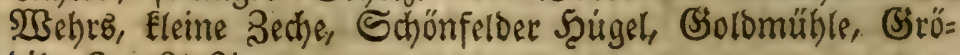
Dik. $6 \div 8.24$.

H. $12-1400^{\prime}$. L. Hb. K. S. Ch. B. O. W. 


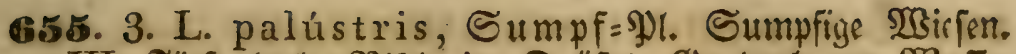

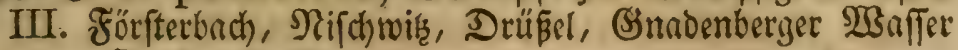
u. v. D. 6-8. 24.

H. $10-1400^{\prime}$. L. B. O. W.

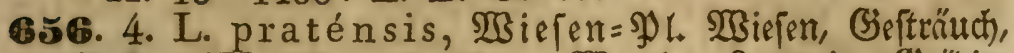

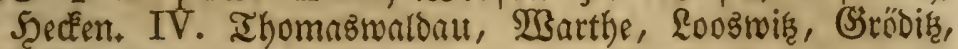

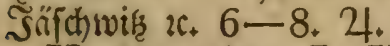

H. $10-1500^{\prime}$. L. Hb. K. S. Ch. B. O. W.

292. Vícia. Widée.

658. 1. V. lathyroides, platterbienartige WB. Trok=

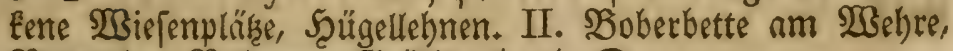
Surglehn, Soberau, (3röbis. 4. 5. $\odot$.

H. $1300^{\prime}$. L. O. W. K.

659. 2. V. angustifólia, f(d)malbiăttrige $\mathfrak{B}$. Sant=

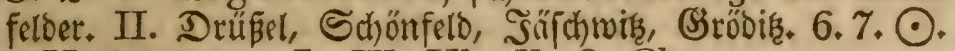
H. bis 2300'. L. W. Hb. K. S. Ch.

659. 3. V. sativa, $\mathfrak{F u t t e r}=\mathscr{W}$. Santfelder. IV. Faft überall. 6.7. $\odot$.

H. bis $1500^{\circ}$ L. Hb. K. S. Ch. B. O. W.

660. 4. V. sépium, $3 \mathfrak{a} u=2 \mathbb{B}$. Gebülche, Scaine, $\mathfrak{B}$ nld $=$ wiejen. II. SRachtigallenbach, Eleiner $\mathfrak{B o b e r}$ bei Trhomaz= maloau, Ërö́iba $5+6 . \odot$.

H. L. Hb. K. S. Ch. B. O. W.

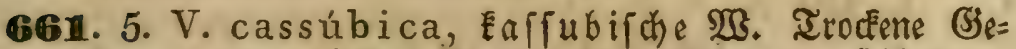

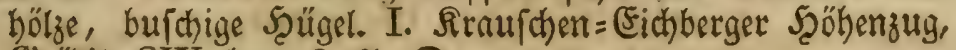
Gróois SWlehne. 6.7. $\odot$.

L. B. O. W. S.

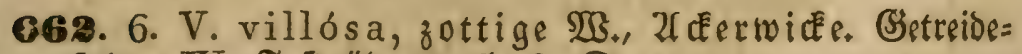
ferber. TV. Faf́t überall. 5, 6. $\odot$.

H. bis $1500^{\prime}$. L. Hb. K. S. Ch. B. O. W.

663. 7. V. Cracca, Rerate = Doer $\mathfrak{B}$ ogel=203. Shectent,

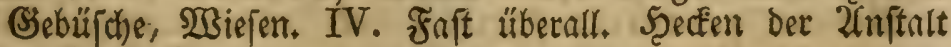
«. 5. 6. $\odot$.

H. biz $2300^{\prime}$. L. Hb. K. S. Ch. B. O. W. 
664. 8. V. tenuifólia, feinblättrige 23 . 2recker, 2Wie=

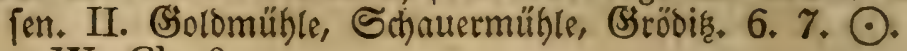
W. Ch. ?

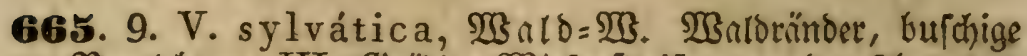

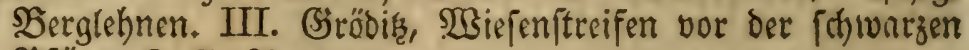
⿰习习 fïke. 6. 7. 24.

H. $10-1200^{\prime}$. L. S.

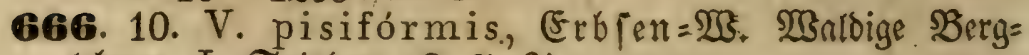
lebnen. I. Spifberg. 6. 7. 4 .

L. K. $1480^{\prime}$. S. Ch.

662. 10. V. dumetórum, $\mathfrak{B} u[(h=\mathfrak{B}$. (Gebüiche, feuthte,

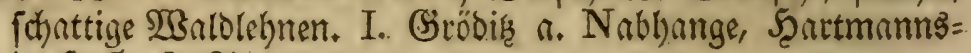
borf. 5. 6. 4 .

S. Ch. B.

293. Ervum. Rinfe.

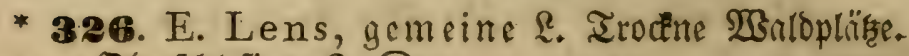
5berichlefien. 6. $\odot$.

668. 1. E. tetraspérmum, vierfamig.e $\mathfrak{R}_{\text {. Sam, }}$

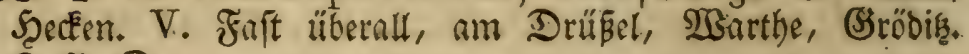
6. 7. $\odot$.

H. bis 1500'. L. Hb. K. S. Ch. B. O. W.

669. 2. E. hirsútum, rauhfrüđtige \&. Wie 1. aber feltener. 6. 7. $\odot$;

L. Hb. K. S. B. O. W.

68. Galéga. (5eizraute.

* 32z. G. officinális, arzneiliđłe (5). Ģebü= bülche, Ratibor. 6. 24.

294. Lotus. Scornflee.

6zo. 1. L. corniculátus, gemeiner Ş. Sehe veridhie= Dene Formen; bie Feldformen auf trodenen Wiefen, Rainen; bie Sumpfformen auf Sumpfwiefen, in Gsrä= ben; bie feinblät trige nuf fruchtbaren $2 B i e j e n .6-8.24$.

H. bis 1800'. L. Hb. K. S. Ch. B. O. W. 
69. Tetragonólobus. Sđjotenêlee.

* 328. T. siliquósus, vierflügliger Sd. Wie=

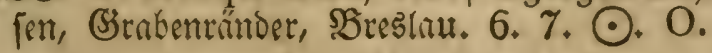

295. Medicágo. Sd)neçenétee.

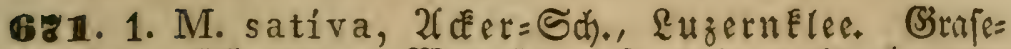
gïrten, Shiligellehnen, Wegeränder. Loostwig, wohl aber ver= willoert. 7.8 .24 .

H. $1100^{\prime}$. B. O. S. Ch.

* 329. M. media, mittlerer Sch. G゙rafepläke. Stezโนa. 6. 7. 2 .

682. 2. M. falcáta, fichelfrühtiger Sd), gelbe $\mathfrak{Q} u=$

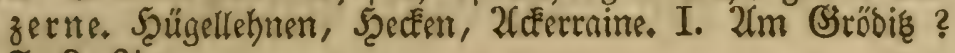
7. 8. 24 .

H. $2200^{\prime}$. L. B. O. W.

683. 3. M. lupulína, hopfenartiger Schn. 2ecker,

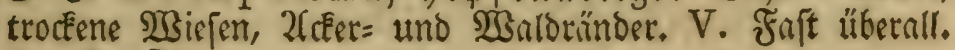
$6-9$.

H. bis 1200 '. L. Hb. S. Ch. B. O. W.

68 4. 4. M. mìnima, Eleinfter Sdyn. ITrocFne Şügel. I. Rähnhausberg am obern Thurm (eigentlich) fdjon nidht mebr im Gebiet, ater an deffen (Brenze)+6.7.

296. Astrágalus. W̉irbelfraut, Iragantb).

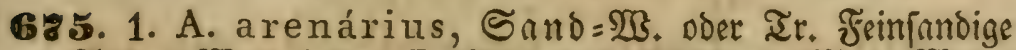

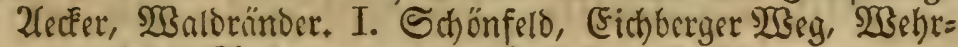
au auf Den Zfectern vor bem Rateofen. 6. 24. Ln. O. W.

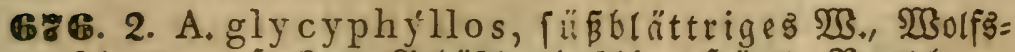

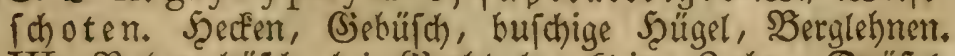
III. Sobcrgebuifich bei Siothlact), Elrime Bcthe, Drübel,

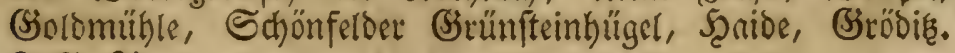
6. 7.24 .

H. bis $1800^{\prime}$. L. Hb. K. 1480'. S. Ch. B. O. W. 
* 331. A. hyppoglóttis, 2Bicfen=Ir. San=

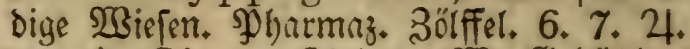

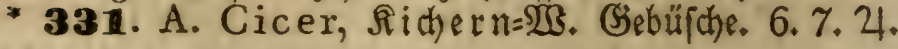
70. Hedýsarum. Süïłlee.

* 332. H. obscúrum, Duneler S. Şodhgebirge. 6. 7.24.

\section{Coronilla. Sronenwidée.}

628. 1. C. vária, bunte $\mathfrak{R r}_{\text {., }} \mathrm{S}_{\text {dh }}$ aflinfen. SBeges,

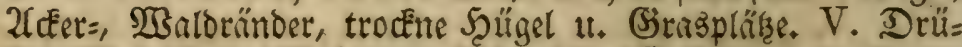

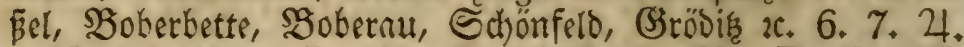
H. bis 2000'. L. Hb. K. 1480'. S. Ch. B. O. W. 298. Ornithopus, গুogelfū̃.

6z8. 1. O. perpusillus, wingiger $\mathfrak{B}_{*}$ Eleime $\mathfrak{B}$ ogels Elaus. Sandige 2lecter und $\mathfrak{B a l b r i n d e r . ~ I I I . ~ D r u ̈ ß e l ~ a u f ~}$

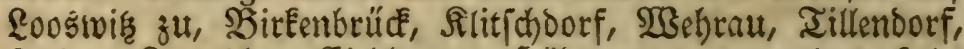
Beche, Rrtaufdhen= (Fid)berger Scöhenzug gegen brä Ealte Borwerk zu am NO $\mathfrak{B a l d j a u m e ~ f e h r ~ g r o ß . ~ 6 . ~ 7 . ~ \odot . ~}$ Ln. B.

\section{2ichtzebnte Rlaffe Polyadélphia. Sielbünolige.}

299. Hypéricum. Šohannizkraut, Scartheut.

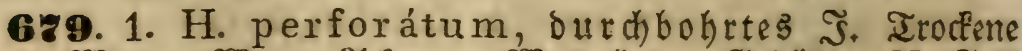

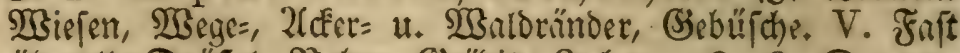

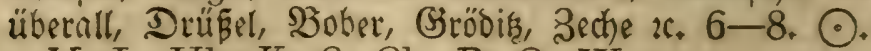
H. L. Hb. K. S. Ch. B. O. W.

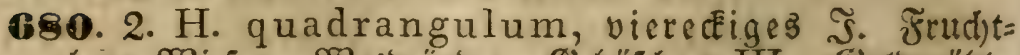

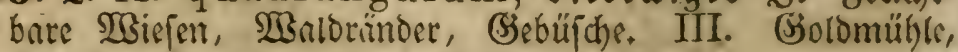

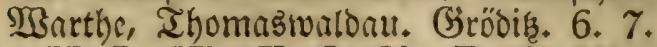

H. L. Hb. K. S. Ch. B. O. W. 
681. 3. H. tetrápterum, vierflügliges Э. Sumpf=

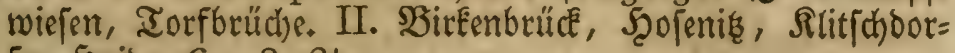
fer Şaibe. 6-8. 24.

H. L. B. O. W. Hb.

682. 4. H. humifúsum, nieberliegendes S. Feuchte thonig=Fandige 2fecter. V. Faft überall. 2fn ber grofen Beche aud) mit aufrectem Stengel. 6-10. $\odot$.

L. Hb. Ch. S. B. O. W.

683. 5. H. montánum, $\mathfrak{B} e r g=\Im$ S. Trockente Gełhölze u.

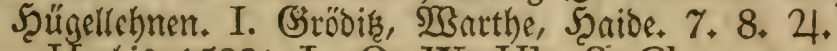

H. bis $1500^{\prime}$. L. O. W. Hb. S. Ch.

* 333. H. hirsútum, rauhhariges S. Schnt= tige 2 Bittort. 7, 8, 24.

L. B. O. W. S. Ch.

\section{SReunzebnte Rlafie. Syngenésia. Staubbutelverwachjene.}

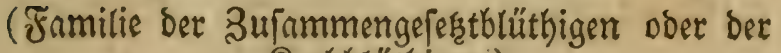
Sorbblütbigen:)

A. Diftelblumige. Cynarocéphalae. \$öhrenbluthige uit baukhigent Relch.

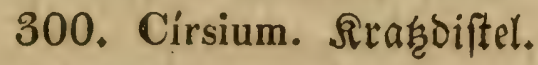

68-1. 1. C. lanceolátum, lanzettblättr. Sir. Sduutt=

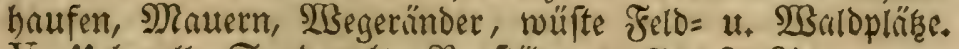
V. Ueberall. Sandgrube, Sorftiobte 2 . $7-9.4$. H. $10-2200^{\prime}$. L. Hb. K.'S. Ch. B. O. W.

685. 2. C. canum, graue Rit. Wanferteiche Moot= und

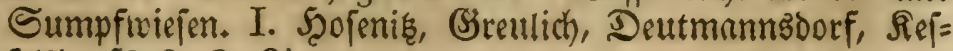
fetsoorf? 8. 9. 4 .

H. L. K. S. Ch. O. 
686. 3. C. palústre, Sumpf=Rr. Sumpf= uno Moors

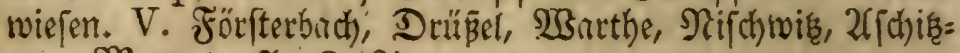
auer Moor $x_{0} 7-9.21$.

H. bis 2200'. L. Hb. S. Ch. B. O. W.

* 334. C. heterophyllum, verfhiedenblät= rige Rit. SBergwiefen, Bebirglebnen. 6. 7. 24. S. L. K. Ch.

* 335. C. serratuloides, fhartertige Sir. Bergwiefen. Dirfhel. 6. 7. 4 .

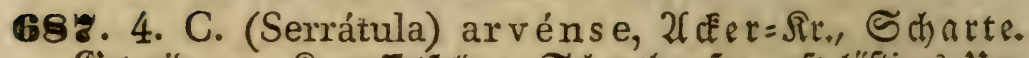

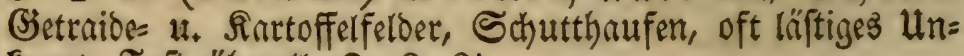
Eraut. Faft überall. 6-8. 4.

H. bis $2200^{\prime}$. L. Hb. K. S. Ch. B. O. W.

* 336. C. acaúle, fiengelofe Nir. utnfututhtbare Iriften mit Sanogruno, 8-10, 2. H. L.

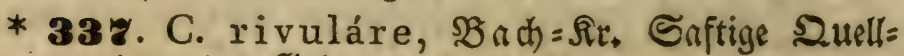
wiejen des Ģebirges. 6. \%. 24. H. L. S. B.

688. 5. C. oleráceum, gelbe art., wirber Saflor,

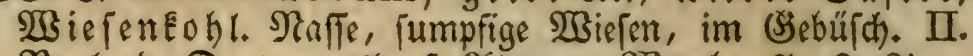
Neutand, Deutmamnsoorf, 2alzenau, 23arthe. 7-9. 4.

H. bis 2000'. L. Hb. K. S. Ch. B. O. W.

* 338. C. tataricum, tatrifiche Ir. Feuchte WBiefen. 7. 8. 24. H. L. S. O.

\section{Carlína. Esberwurz.}

689. 1. C. vulgáris, gemeine (ङ. Irodéne, Eiefigg=fandige STügel, Riefermuittoer. V. Faift überafl, Smogruben, am

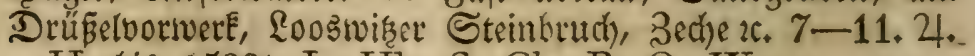
H. biz $1500^{\prime}$. L. Hb. S. Ch. B. O. W.

690. 2. C. ac aúlis, ftiellofe (E. Irodfene, lehmige Reh=

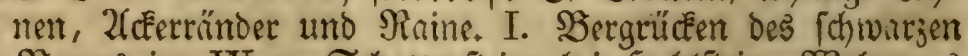

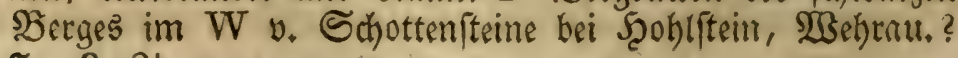
$7-9.24$.

H. bis $1800^{\prime}$. L. Hb. K. S. Ch. W. 


\section{Cárduus. Diftel.}

691. 1. C. nutans, nicéende D. Refhmig=\{andige Sirad)=

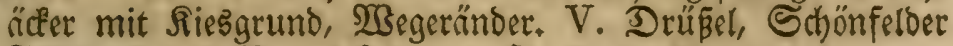
Felder, Enaltes SBortwerk u. m. N, 7. 8. 21.

H. bis $1200^{\prime}$. L. Hb. K. S. Ch. B. O. W.

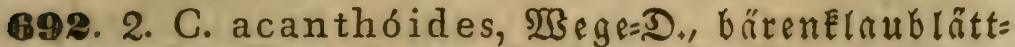
rige D. Wrege, 2fecter, SBrachen. II. WBarther, Scart= mannbDorfer Sinlefteinbrǘche. 6-10. $\odot$.
H. 10-1200' L. Hb. K. S. Ch. B. O. W.

693. 3. C. crispus, Eraufe D. Ufergebüfhe, feuchte Wä́lber. Sthnelle Deichfel am Spif̧berg. 7. 8. 21.

L. K. S. Ch.

*339. C. Personáta, Rletten= D. Betirgsthä= ler. 7. 8. 2\%. H. bis 2200'. L. Hb.

\section{Onopórdon. Rrebzoiftel.}

* 340. O. Acánthium, gemeine Rir. M̉ege, Mauent, Schuttplïhe im fructitb. Boden. 6-8. oे. L. H. $1200^{\prime}$. S. B. O. W.

\section{Arctium. Alette.}

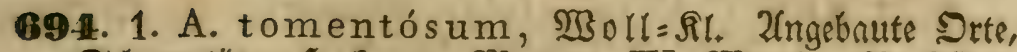

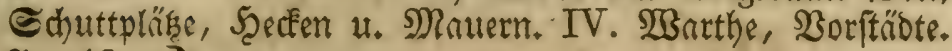
$7-10$. बे.

H. $11-1200^{\prime}$. L. Hb. K. S. Ch. B. O. W.

695. 2. A. minus, Eleine אit. Deß̧gl. besgl. ohne K.

696. 3. A. majus, großse Rit. Desgl, weit feltener, liebt

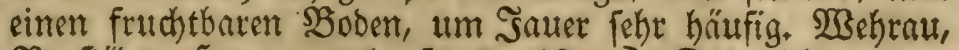
Borftiobte, Scartmannzborf.. 7-10. ఠ). Deşgleichen.

304. Serrátula. Sdbarte, Färberbiftel.

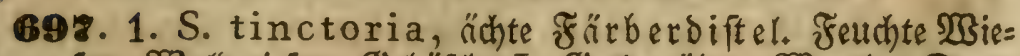

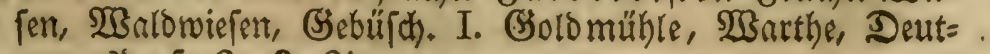
mannzoorf. 6-9, 4 .

H. $1200^{\prime}$. L. K. S. B. O. W. 


\section{Centaúrea. Floctenblume.}

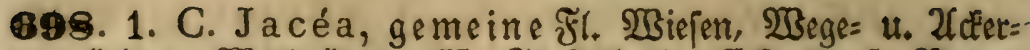

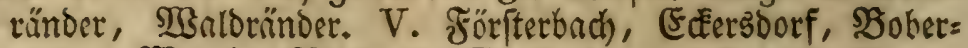
wieim, Marthe, Nifenwig, Sinndenberg 2c.6-10. 4. H. bis 2300'. L. Hb. K. S. Ch. B. O. W.

* 341. C. phrygia, phrygifd)e Fl. Bergwiefen. $6-8.24$.

H. L. Hb. K. $1680^{\prime}$. S. Ch.

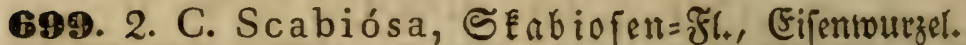

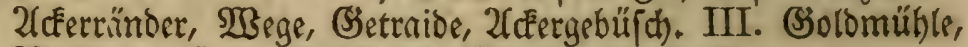

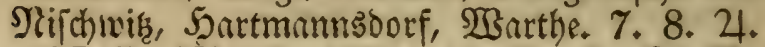

H. L. Hb. K. 1630'. S. Ch. B. O. W.

800. 3. C. paniculáta, rispenblüthige Fl., Rnopf=

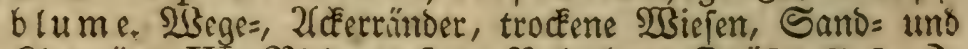

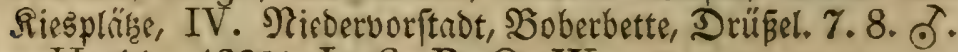

H. $11-1300^{\prime}$. L. S. B. O. W.

801. 4. C. Cýanus, blaue Ffl, Rornblume. Shetrat:= Defelder, Finrtoffelïffer. V. Ueberall, bort 6., hier 9. 10.

(Das Serbacten verzögert die SBlüthezeit. 24.)

H. bis 2000'. L. Hb. K. S. Ch. B. O. W.

* 3月2. C. solstitialis, Sommer = $\mathfrak{F l}$. Staden. I. 7. 8. 24. Brestau bei Rake.

* 313. C. axillaris, armblüthige fl. Jitar= paten. 7. 8. 24. SBarania. Sberfchlefien.

\section{B. Doftenartige.}

Mit ei= oder röhrenförmigem Relch. 306. Eupatórium. Doften.

802. 1. E. cannabínum, Szanf= D., $\mathfrak{B}$ afferbofte.

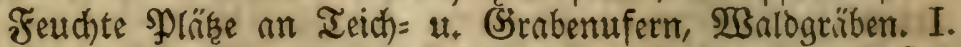
Nifchiser Sumpfroald, Neuland am Nabbang nach Rep= felsoorf zu vom Simonißhaus. 7-9. 24. L. Hb. K. S. B. O. W. 
72. Chrysocóma. Bsolohaar.

* 344. Chr. Linosýris, leinblättriges (5). Burđhige Schügel. 7. 8. 4 .

\section{Cacália. \$ुefftwurzel.}

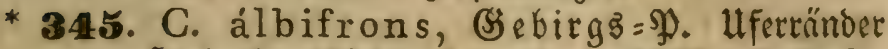

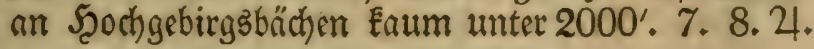
307. Bidens. 3weizalin.

83. 1. B. cérnua, nickender 3w. Gratren, Rachen, feuchte Sanopläß̧e, Moorwiefen, V. Bober $\varkappa_{\text {. auf Den }}$ Torfwiefen Der J̧ofentis feht Eleine cinblüthige Formen. $7-10 . \odot$.

H. L. B. O. W. Hb. K.

804. 2. B. tripartíta, oreiblättriger $2 \mathfrak{w}$, $\mathfrak{B a}$ af hanf. Wie 1. auth mit einer Eletnen und einer zowergigen

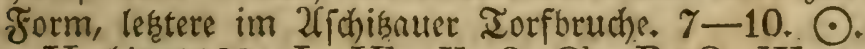

H. bis 1180 ' L. Hb. K. S. Ch. B. O. W.

808. Tussilágo. Scuflattig.

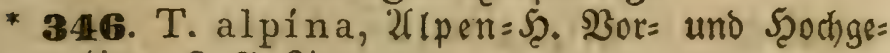
birge. 6. 7. 24.

H. $20-2800^{\prime}$. Lo. Hb. K. 1630'. S. Ch.

ซos. 1. T. Fárfara, gemeiner $\mathfrak{~}$. Feuchte, fette Hecéer, Şohtwege, FalEffteinbrüche. I. WBehrau, Scoblittein. 3. 4. 24. H. L. Hb. K. S. Ch. B. O. W.

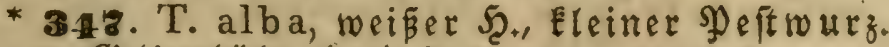
(Gebirgäbriche. 4. 5. 4 .

H. über $1500^{\prime}$. L. Hb. K. $1680^{\prime}$. S. Ch.

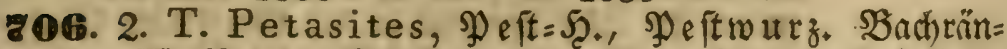
Der. II. SBei Der Schnuermühbre, Nieder= Tillendorf. 4.5.2.

H. $1200^{\prime}$. L. Hb. K. 1530 '. S. Ch. B. O. W.

\section{Gnaphálium. İmmerblume.}

807. 1. Gn. luteo-album, wein=gelbe S. Thonig=

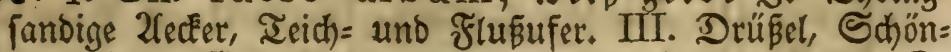
feld, uttig, Esiersoorf, unfern bes Stenbrudys. 7-9. $\odot$.

L. O.W. 


\section{4}

808. 2. Gn. arenárium, $S_{a n b}=$ S., gelbes Raḱcn=

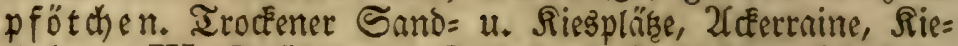
Ferhatue. IV. Drüpeliwald, Tillenoorf, Boberbette, Gichberg,

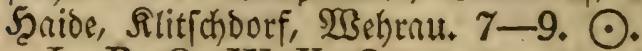

L. B. O. W. K. S.

809. 3. Gn. dióecum, zweihäufige Ș., Rakenpföt=

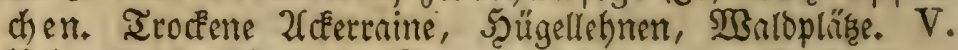
Ueberalt fehr bäufig in Struppen bei cinander. 7. 8. 4. H. L. Hb. K. S. Ch. B. O. W.

810. 4. Gn. rectum, aufrechtes S. Irocente ober mï= Big feuchte Wartoung, bejonders Riefertw., boch audh in trocfenent uno untermirchten (Erlichten. III. 3eche, DrüB̈el,

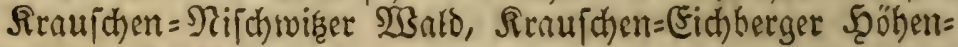
अ. 6.7 .24 .

H. L. B. O. W. Hb. S.

211. 5. Gn. sylváticum, $\mathfrak{B}$ nld = S. Mit bem vorher=

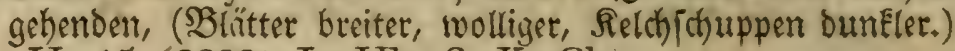
H. 15-2800'. L. Hb. S. K. Ch.

212. 6. Gn. uliginósum, Sump $f=\Im$ S. Feuchte, [um=

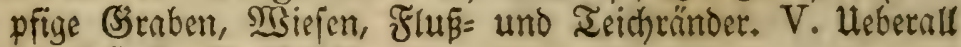
Tehr bäufig. 7-9, 4 .

L. B. O. W. Hb. S.

* 348. Gn. supinum, Eleine Э. Şodgebirgr. 7. 8.24 .

* 349. Gn. margaritaceum, ஒ erlen= Fruthtbare Bjebirgstwirfen. 8. 24. 2(potheker Feu=

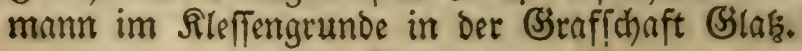

\section{Filágo. Filž́traut.}

213. 1. F. germánica, deutfches F., Sđimmelḱr., Freldenge. Sandige und Eiefige SBradjfelder und andere

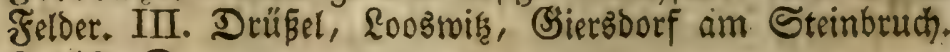
6-10. $\odot$.

L. Hb. S. Ch. B. O. W. 


\section{6 \\ 314. Erigeron. Flőbtraut.}

211. 1. E. acris, blaueş Fl., bIaนe Dürrmünze.

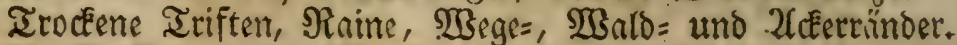

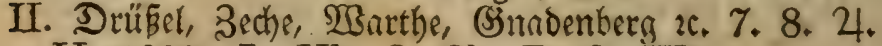
H. 1300'. L. Hb. S. Ch. B. O. W.

222. 2. E. canadéns is, Eanadifhes F̆. Dürre, fan= bige Felder. (sebüiche. V. 2Unlagen, Soberbette. Tillendorf,

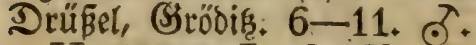

H. $1200^{\prime}$. L. S. Ch. B. O. W.

\section{5. Ínula. 2llant.}

* 350. I. hirta, Eurghariger $\mathcal{A}$. Trodene faub= bügel. Borgebirge. 5. 6. 24. Striegnuer $\mathfrak{B e r g e . ~}$ H. bis $1500^{\prime}$. Hb: K. S. Ch.

823. 1. I. salicina, weibenblätriger 2 . Feubte, bujchige Wiejen. III. Warthe, Nifdyig. 7-9. 4.

H. $10-1300^{\prime}$. L. K. S. Ch. B. O. W.

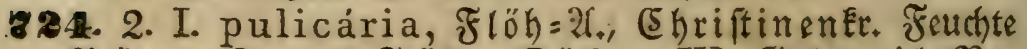

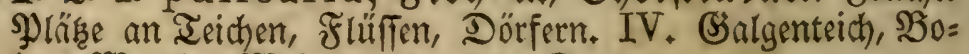
ber, 2 Barthe, 2 Bebrau. $7-10$. $\odot$.

H. $10-1200^{\prime}$. L. B. O. W.

25. 3. I. británnica, brittif́t)er $\mathfrak{X}_{+}$falfither

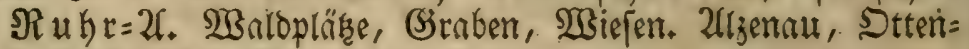
borf. $6-10.24$.

H. $13-1400^{\prime}$. L. B. O. W. Hb. S.

* 351. I. Helenium, wabrer 2 . Walorwie= fer. 7. 8. 2. W. Ch.

\section{Aster. 2ffter.}

* 352. A. salígnus, $\mathfrak{B}$ ei ben $=\mathfrak{U}$. $\mathfrak{B}$ eidengebü= icte. 8. 9. 4 . Hb. S. Ch.

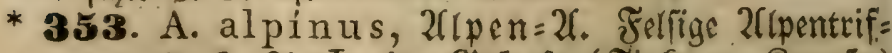
ten. 7. 8. 24. L. im Siefente (Finte u. Siraufe.) 316. Solidágo. Siolorutbe.

826. 1. S. Virgaúrea, gemeine (5., St, Peterfíab,

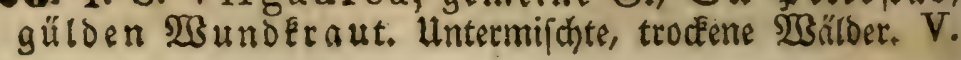




\section{7}

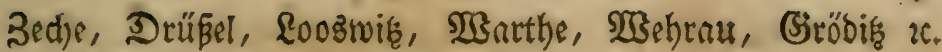
$8-10.24$.

H. 11-1200'. L. Hb. K. S. Ch. O. W.

\section{Cinerária. 2lichenpflanze.}

* 354t. C. crispa, Exaufe 2 . Quelleict)e Stellen beక Ģeלirges. 6. 7: 24.

H. $2200^{\prime}$, L. K. S. Ch.

* 355. C. $p$ alústris, Sumpf=24. Sumpfriejen. 6. 7. ठ․ B. O. W.

\section{Senécio. Îreuzkraut.}

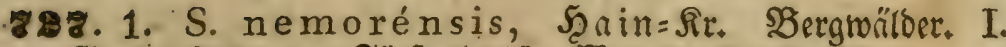
Groke Beche am Förffterhaule, 2 Barthe. 7-9. 4.

H. 2200'. L. Hb. K. S. Ch. O.

* 356. S. saracénicus, Şaiben = Sit. Ufer' Weioengebüfक. 7-9. 4 .. H. $1100^{\prime}$. L. K. S. Ch. B. O.

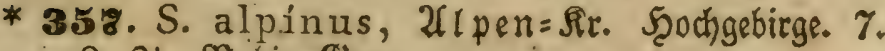
8. 24. Babia Csora.

* 358. S. a quáticus, $\mathfrak{W a f f e r = ~ S i r . ~ . F e u c h t e ~ ( 5 e ~}=$ büfche uno Sräben. $7-9.24$. H. B. O. W.

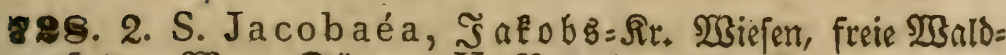
ftetlen, Wege, Dämme. V. Boberbette. u. Boberau $u_{*} a_{*}$ a. $5.7-11 . \sigma^{2}$.

H. 10-1200'. L. B. O. W. Hb. K. S. Ch.

* 359. S. crucifolius, feinblätriges Sir. (Sebüfche. 7. 8. ఠౌ. S. SBei Şrez̆tau.

229. 3. S. viscósus, flébriges Rr. Sanoplähe, fan= bige, offene Sraibeftellen, an Mrauerm. V. Sandgruben, - Sorftiobte, Tillendorf, Rititícho. Scaide. 6-11. $\odot$.

H. biz 2200'. L. S. Ch. B. O. W.

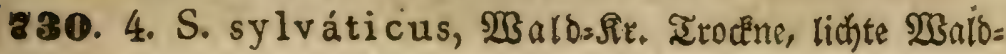




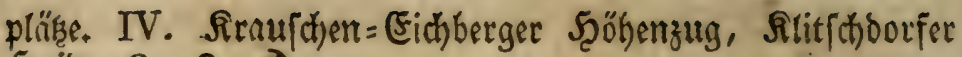
Şaibe. 6-9. है.

H. L. Hb. K. S. Ch. B. O. W

831. 5. S. vulgáris, gemeines R̂r., Bogelfraut. 2fuf humusreichem Boben, Şartenbeeten, Schutthaufen; überall baz gamze Jangr. $\odot$.

- H. L. Hb. K. S. Ch. B. O. W.

* 360. S. vernális, Frühlings= Sit. Soberfdele fien. 4. $\odot$.

318. Árnica. $\mathfrak{B S o h l v e r l e i h . ~}$

32. 1. A. montána, $\mathfrak{B e r} g=2 \mathbb{B}$. Zeuthte, ir fognt moo=

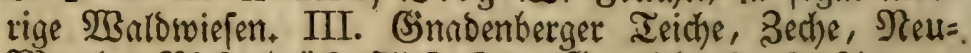
İarthe, Birfenbrüct, Tiefenfurt, (srrulich). 6-8. 4.

H. 13-2300'. L. Hb. K. S. Ch.

76. Dorónicum. (5ెempentwurz.

361. D. scorpioides, $\mathfrak{B}$ erg $=$ (5). Şochgebirg $=$ trifter. 7. 8. 4. Sthmeebery, Z(ltuater.

319. Bellis. Naazlieben.

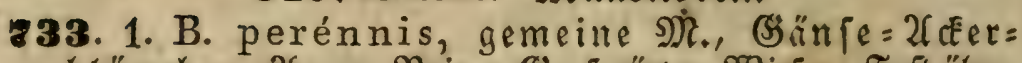

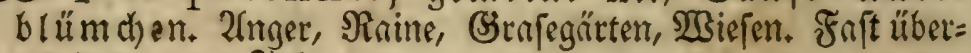
all bas ganze Saht.

H. bis 2500'. L. Hb. K. S. Ch. B. O. W.

320. Matricária. Nutterfraut.

734. 1. M. Chamomilla, Feld = M., Ramille, Rie= merei. Getraibeferder. I. Rooswig. 6-8. ๑.

H. bis $1500^{\prime}$. L. K. S. Ch. B. O. W.

321. Chrysánthemum. (Sold = oder $\mathfrak{S}$ utberblume.

935. Chr. Leucánthemum, weiß̄́trahlige (G), Jo=

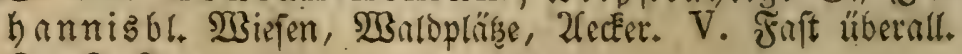
$6-8.24$.

H. L. Hb. K. S. Ch. B. O. W.

* 362. Chr. ségetum, Santen=S. Gielbblühend. 6. 24. K. 


\section{- 322. Pýrethrum. Bertrammurz.}

* 363. P. corymbósum, boldentraubige $\mathfrak{B}$. wilber Bertram. Bewaldete Šerge. 6. \%. 4.

836. 1. P. Parthénium, arzneiliche $\mathfrak{B}_{+}$, römifche Rarmille. $7: 8,24$.

H. $11-1200^{\prime}$. L. Hb. K. S. Ch. B. O.

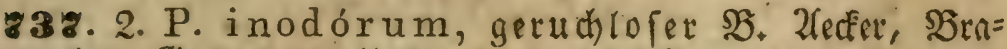
d)en, (S)rttentono. UnErnut. $6-10 . \odot$.

H. $1500^{\prime}$. L. Hb. K. S. Ch. B. O. W.

\section{Anthemis. 21nthemi3.}

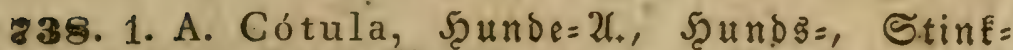
Rrmille. Hecter, SBrachen, ungebrute Stellen. UnEraut. Nieber = Borftrot. $6-8, \odot$.

H. biz 2200', L. S. Ch. B. O. W.

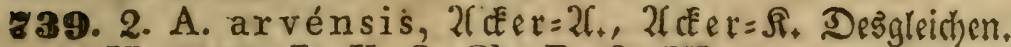
H. $2200^{\prime}$, L. K. S. Ch, B. O. W.

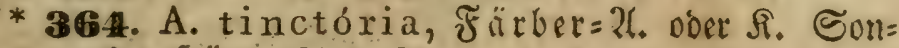
nige Şüget. 6. \%. 4 .

H. 12-1800'. L. Hb. (Eanoshut.) S. Ch.

324. Achilléa. Sdjaafgarbe.

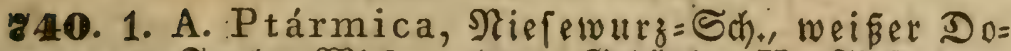
rant. Feuchte WBiefengraben, Beseürfhe. V. Förifterbad), Bober, Goldetach, Eleiner Sober, Drüßrel, Looswis u. m. 5. $6-9,24$.

H. $12-1700$ '. L. Hb. K. S. Ch. B. O. W.

841. 2. A. Millefólium, gemeine Sd), Schaf=

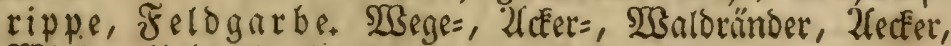
WBiefen. Heberalt, 6-11, 21.

H. biz $2300^{\prime}$, L. Hb. K. S. Ch. B. O. W.

77. Rudbéckia. Ruobeckie.

* 365. R. laciniáta, f̧lígblättrige $\Re$, Ufers gẹbüfch. Böllig verwiloset, wie einhsimifd) am 
Sucisufer b. Friebeberg, Mareliffa, am Baache b. Sdiwerta, im Sdilefierthale u. Deffen Seitenthi"= lern zur Eule hin. 7. 8. 24.

\section{Cichoreae. Cichorienartige, Bungenblutbige.}

325. Crepis. \$ippau.

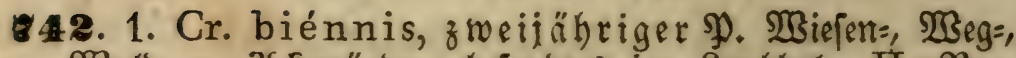

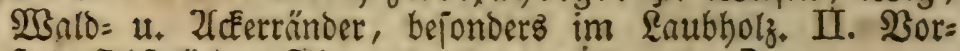

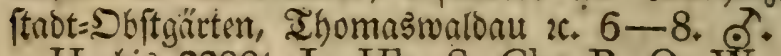

H. bis 2200'. L. Hb. S. Ch. B. O. W.

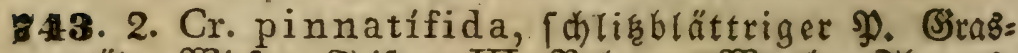
pläke, WBiefen, Triften. III. Soberau, Wratthe, Thyomas= walbau 2c. $6-9 . \odot$.

H. $2200^{\prime}$, L. Hb. K. S. W.

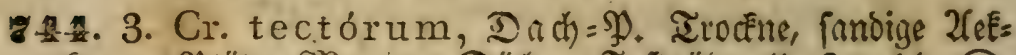

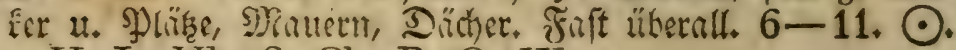
H. L. Hb. S. Ch. B. O.' W.

\section{Borkhausénia. Borḱaulfenie.}

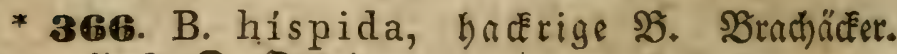
7. 8. ๑. Sppeln. 326. Hierácium. ŞabichtzEtraut.

* 36z. H. alpínum, 2(tpen= Ş. Şochgebirge. 7. 8. Siele Formen. 24. .

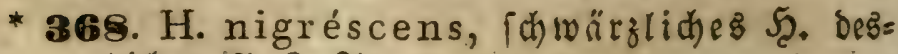
gleichen. 7. 8. 24.

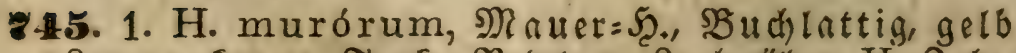

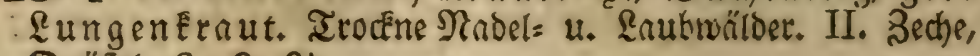
Drüßё. 6-9. 4.

H. bis $2500^{\prime}$. L. Hb. K. S. B. O. W. 


\section{1}

* 369. H. Schmidtii, Sciniots S. Trodene, fteinige Sarge. Prubelberg. 6. 7. 24. Hb.

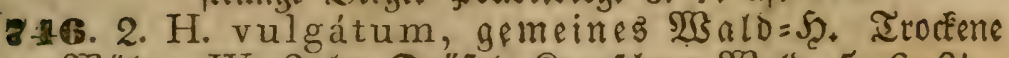

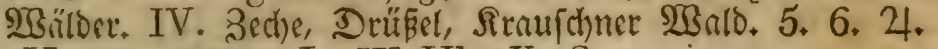
H. 12-2000'. L. W. Hb. K. S.

* 380. H. boreále, nöroliches 5ृ. Rnubwäloer. 7-9. 24. Hb. K. S. Ch.

8. 3. H. umbellátum, fhirmtragendes Sc. WBäl=

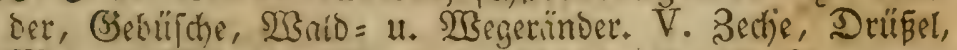

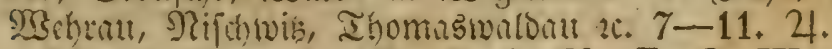

H. bis 2300' L. Hb. K. S. Ch. B. O. W.

*31. H. prenanthoides, ftcinfallatblätt= riges 5. Sochgesinge 7. 8. 24. H. L.

3. 4. H. paludósum, Sumpf $=5$. Sumpfrién,

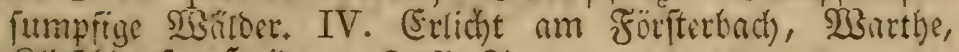
Slitichoorfer Scaide $2,6,7,24$.

H. bis $2200^{\prime}$. L. Hb. K. S. Ch. B. O. W.

* 32. H. succisifólium, abbíblättriges 5. Sumpfige Giantwicjen. 6. 24. II. $1100^{\prime}$ I. K. S. Ch. W.

* 323. H. grandiflórum, groß̧blumiges 5 . Scod)gebirge. $7.8,24$. Hb. (stöberterg.

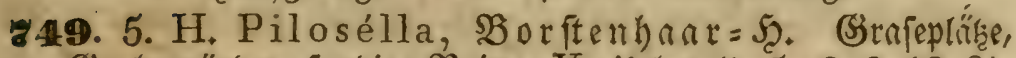

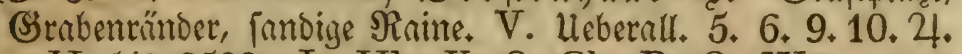
H. bis 2500' L. Hb. K. S. Ch. B. O. W.

850. 6. H. Auricula, Mausohr= S5: Mieren, Iriften,

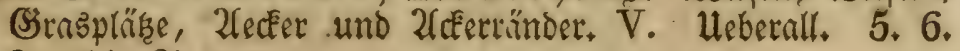
$9-11.24$.

H. $10-1200^{\prime}$. L. Hb. K. S. B. O. W.

* 3. H. floribúndum, blüthenreiches \$ุ. IBicien, 2lckerrïnder, kejond. fruchtbare. 5, 6. 24. $\mathrm{Hb}$. S. Ch.

251. 7. H. praeáltum, (d) lanÉes Ş. Feuthte u. trok=

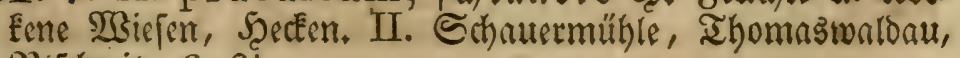
ग)(if () 1 wik. 6. 24.

H. L. K. S. Ch. B. O. W. 


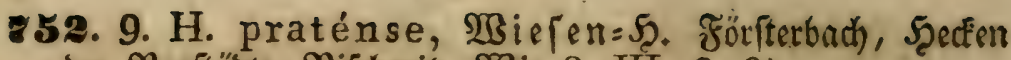

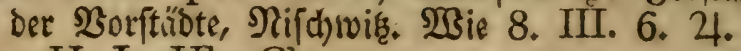

H. L. Hb. Ch.

ซ53. 10. H. cymósum, afterboldiges Ş. Mit 8. u.

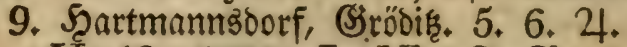

H. 13-2200'. L. Hb. S. Ch.

* 385. H. echioídes, natterkopfartiges Ş. : 2uf Bergen. 6. 24. S.?

* 326. H. villósum, zottiges 5 S. 2(m Reffel im Gejenke. 7. 8. G̈rabowzEn.

* 37z. H. auran tiácum, orangefarbeneż. Ş๐odjgebirge. $6.7 \cdot 24$.

79. Intybus. Sntybuz.

* 3z8. I. praemórsus, abgebiffener I. W̉albs เvie โen. 5. 6. 24. H. 1300'. W.

327. Sonchus. (sänjebiftel.

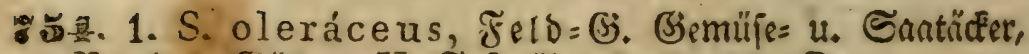

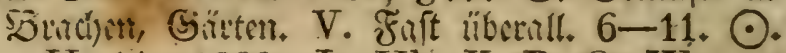

H. $11-1200^{\prime}$, L. Hb. K. B. O. W.

855. 2. S. asper, fot arfe (5. Wie 1. nur feltner. 6-10. 4. L. Hb. K. B. O. W.

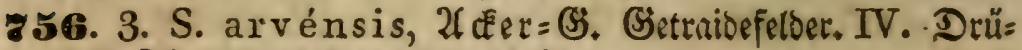
B̧el, Ihommşwaldau. $6-8,24$.

H. L. Hb. K. S. Ch. B. O. W.

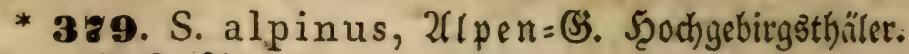
7. 8.4 .

H. $18-2500^{\prime}$. L. Hb. K. $1880^{\prime}$. S. Ch.

328. Prenánthes. Şafenlattig.

258. 1. Pr. purpúrea, purpurblumiger 5., Bergs fallat. Bergrwálder. I. Schtwarzer Berg b. SirEwil über bem Sandfteinbrud). 7. 8. 4 .

H. kis 2500'. L. Hb. K. S. Ch. 


\section{3}

858. 2. Pr. murális, Mauer =5., Mauerfallat.

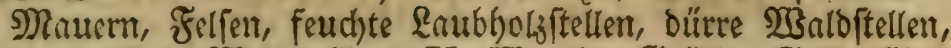

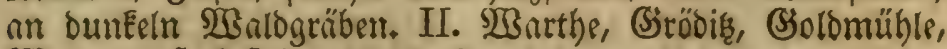
B̧ebrau, Şohlftein. 7-9. 24.

H. $11-2500^{\prime}$. L. Hb. K. S. Ch. B. O. W. 329. Chondrílla. Introrpeljalat.

559. 1. Ch. Júncea, binfenartiger S., gelber Son= nenwirbel. Sandige, fonnige Şügel, Riefertwaldungen. I.

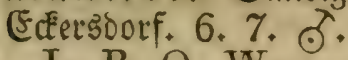

L. B. O. W.

\section{Leóntodon. Römenzabn.}

60. 1. L. Taráxacum, gemeiner R, Maiblume.

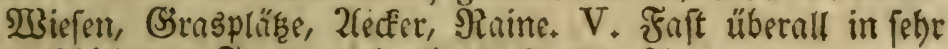
verfdicedenen Formen. 4. 5. 6. 9, 10, 4.

H. bis 2300'. L. Hb. S. Ch. B. O. W.

\section{Lactúca. Sallat.}

861. 1. L. Scariola, wilder S. Wege, Straben, Mau= ern. 6-8, 24. SBifchof 2lbertini.

'H. biz $1500^{\prime}$. L. B. O. W.

332. Tragopógon. Sockzbart.

* 380. Tr. major, gr o. per $\mathfrak{B}$. Şügel, trodene Ziefen, Brachen. 6.7. ఠౌ. S.

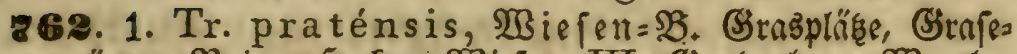

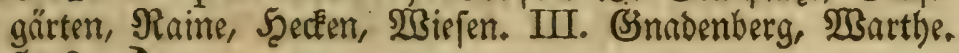
5. 6. बे.

H. $11-1400^{\prime}$. L. K. $1380^{\prime}$. S. Ch. B. O. W.

* 381. Tr. porrifolius, lauchblättriger $\mathfrak{B}$. Sirafeplä̉̆e. 6. 7. 24. Brestaut.

* 382. Tr. orientalis, morgenländifher $\mathfrak{B}$. Fette WBiefen. 5. 6. 24. H. 1100'.

333. Picris. פ̂surmblume.

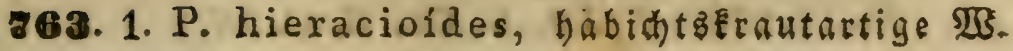




\section{4}

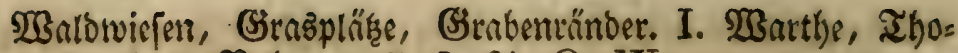
mazmarbau, Boberau. 7. 8. 24. O. W.

334. Apárgia. SPfaffentöbrlein.

264. 1. A. autumnális, F̧erbft = Raime, BBaldrïnder. V. Fant überall.:8-11. 4 . H. L. Hb. S. Ch. B. O. W.

865. 2. A. hispida, fteifhan riges פDF. WWiefen uno

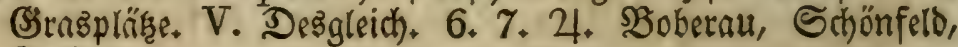
Drüßel 2 .

H. L. Hb. K. S. Ch. B. O. W.

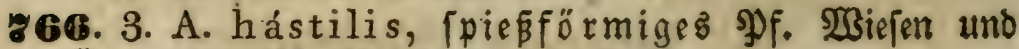
Girnspläge. IV. Des̆gleichen. 6. 7. 2.

H. L. Hb. K. S. Ch. B. O. W.

335. Lápsana. Şafentél.

268. 1. L. commúnis, gemeiner 5ु. Sdhutthaufen,

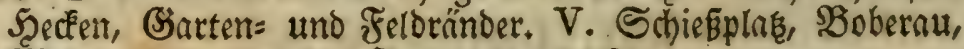
Behrat: u. a. vielen Srten. 6-8, 4 .

H. L. Hb. K. S. Ch. B. O. W.

336. Scorzonéra. Słorzonere.

668. 1. Sc. húmilis, nieorige SE. Moorige, fumpfige

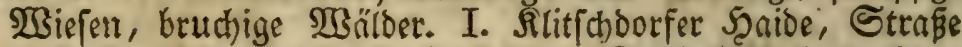
nach Rorenzoorf auf ber Şodjebente, Schönfeld. 5, 6. 24.

L. B. O. W.

ซ69. 2. Sc. laciniáta, f็li

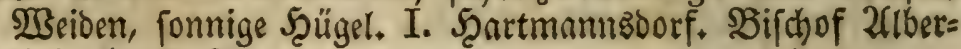
tini. 5-7. 4 .

* 383. Sc. purpúrea, purpurblüthige SE.

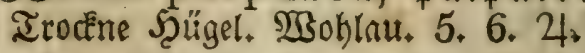

337. Hypochoéris. Ferêeleraut.

80. 1. H. radicáta, gro ß̈ez F. WBiefen, freie, grafige WBalopl, grafige Scaute. IV. Zecthe, Straufhen= Schönfelber 5̧öhenzug 2c. 5-7. 4 .

H. bis $2200^{\prime}$. L. Hb. S. Ch. B. O. W. 
* 384. H. maculáta, gefleceter Fु. Sirafige, betwaldete Scügel, bod)gelegene 2 Biefen, Scoblweg= ränder. 6.7. 4 .

H. $12-1400^{\prime}$. B. Hb. S. Ch.

* 385. H. helvética, beluefifder F. Şochge birge. 7, 8, 4 .

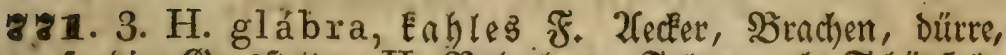
fandige (sirngftellen. II. SBoberbette, Felder nad) Schönfeld. 6. 7. $\odot$. L. S.

\section{Arnóseris. Canowien.}

\%2. 1. A. minima, Eleiner S. Sambige 2fecter. V. Ginnoenberg, Ihomastonldou. $2 \mathrm{~m}$ häufigiten zwilchen $\mathfrak{B}_{0}=$ ber u. হueis, Tillendorf, Sirtenbulü, 2(fchisau, 2Behrau. 6. 7. 9. $\odot$.

Ln. S. B. O. W.

\section{Cichórium. Sichorie.}

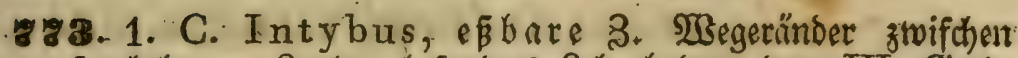
fruchtorem \&ande, befonbers \&efmboden, bort III. Gold= müble, MBatthe, Scartmannsoorf, Mittlau, Siröbis. 6-9. 4 .

H. nicht biz 2000'. L. Hb. K. (Enum im Süben bes: Mettelgebirges, wandett über baffelbe aber alle Sabre meiter ins Scirfdberger Ihal, S. Ch. B. O. W. 


\section{3wanzigite אlafie. Gynándria. Stempelitänoige.}

\section{(Orchidéae: Rutabenfråuter.)}

340, Orchis. Rnabentraut.

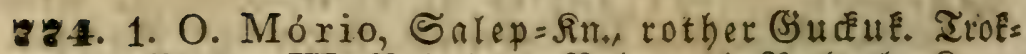

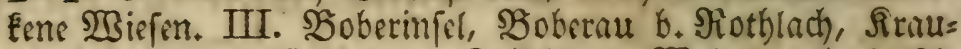

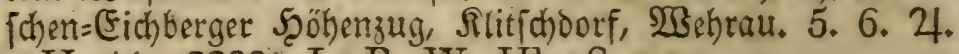
H. $11-2200^{\prime}$. L. B. W. Hb. S.

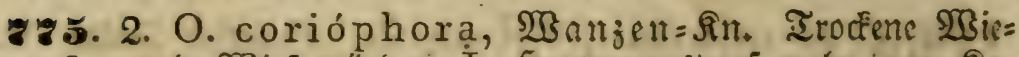
fen und 2 Biejenrinder. $\dot{I}$. Şattmamboorf nathe am Sio= fackengrunde, nach Dem Siröbişberg 34. 6. 24. L. W.

8*6. 3. O. ustuláta, verbranntes In. Trodéne Bie= [en. II. Schauermühle, an beiden Geiten beš El. Boberş, Dort fehr baúfig. 6. 4.

L. W. H. $10-1800^{\prime}$. Hb.

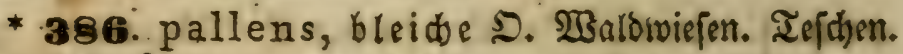
6. 2 .

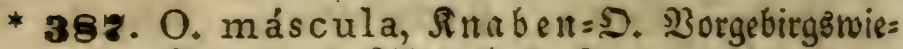
[en. Berbiaborf, Rähn. 5. 6. 24.

H. $18-2000$ ' L. Hb. K. S. Ch.

* 388. O. militáris, geftreifte 5 . Nafie $\mathfrak{W i e =}$ โetr. 5. 24. K.

* 389. O. globósa, Euglige D. Morgebirgşmil= [en. 5. 6. 21.

H. $17-2000$ ' L.

* 390. O.pyramidális, pyramibenblüthigez กีt. Bergmiefen. 5. 4.

* 391. O. sambucína, Şollutiber fir. Şeter. tois bei Sauer. 5.24.

H. 2200. L. Hb. K. S. 


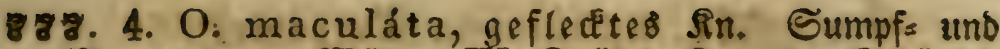

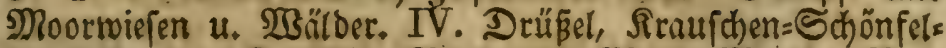

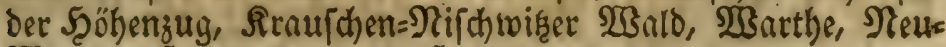
3arthe, Şartmanusoorf, Shofenile. 6. 21.

H. $2200^{\prime}$. L. Hb. K. S. Ch. B. O. W.

888. 5. O. Iatifólia, breitblättriges รin. Sumpfs wiefen. V. Hebetall, mit 4. oft, boct) aud) allein, blüht früber. 5. 6. 24.

H. $12-2000^{\prime}$. L. Hb. K. S. Ch. B. O. W.

ซפ. 6. O. incarnáta, fomalolättriges Sn. Sum=

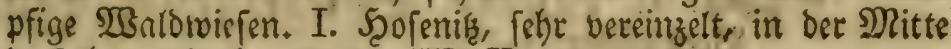
Derjelben mit 4. 6. 24. W. K.

341. Himantoglóssum. Riemenzunge.

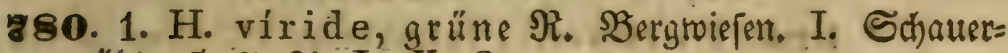
mühle. 5. 7. 24. L. K. S.

881. 2. H. hircina, Bocks=R. Grebirgstvälber. I. Sft vor vieten Sanhern vom Dr. EF(thenbarch sen. in ber Scaibe gefunden roorden. 5. 6.24.

342. Platanthéra. Sglatunthere.

282. 1. Pl. bifólia, zweiblättr. Nh., weisez, wohl=

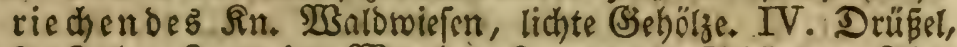

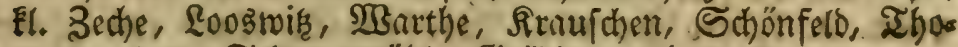

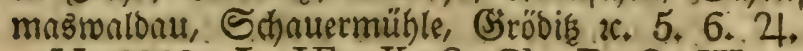

H. 2000' L. Hb. K. S. Ch. B. O. W,

80. Hermínium. Seerminium.

* 393. H. Monórchis, cinEnollige Sุ. 2Roore wiefer. SBoblau. 6. 7, 24.

343. Gymnadénia. Sacetorüuje.

883. 1. G. Conópsea, múdéenartige श. Walbriejen.

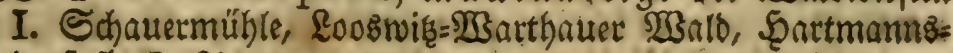
borf 5. 6. 4.

H. $12-2200$, L. Hh. K. S. Ch. 
344. Spiránthes. Drebäbre:

88. 1. Sp. autumnális, herbftlide Dr. B̈rtebhnen. I. Sübtich vom EsröbelworwerE. T. 8. 24. H. $12-1500$ ' L. O. W. Hb.

81. Habenária. 3ügel = Sัrbiz.

* 393. H. álbida, weílidue 3. Scodiggebirge. 6. 7. 4 .

82. Corallórhiza. Rorallentourz.

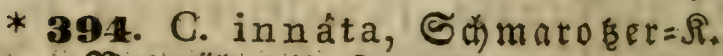
Sergivältoer. 6. 24.

H. 2200'. L. Hb. S. Ch. W.

83. Goodýera. Goodyere.

* 395. G. répens, Eriechende (5). Moofige Site= fermällder. 6.7. 2.

H. $12-1500^{\prime}$. L. B. O. W. Hb. S. Ch. 345. Epipáctis. Sumpfrourz.

785. 1. E. latifólia, breitolättrige S. Sdhattige

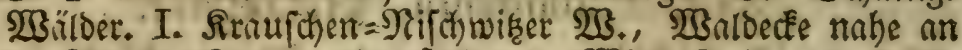
b. Straßie, Rlitjchoorfer Şaide, $a_{*}$ Wiefenftereifen. 6.7.24. H. L. (E. viridiflora). K. S. Ch. B. O. W.

786. 2. E. palústris, Wriefen=S. Torf und Sumpf= swiefen, I. Sumpfwiefe nahe am weftl. Teide) Der (Sold=

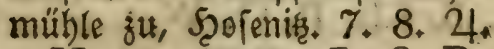

H. $12-2000^{\prime}$. L. S. B. O. W.

* 396. E. atrorúbens, DunEelrothe S.

…... Berge bei Scörnile. 7. 21. L. .

346. Cephalanthéra. Ragnurz.

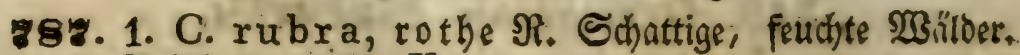
I. Episberg. 6. 4 . K.

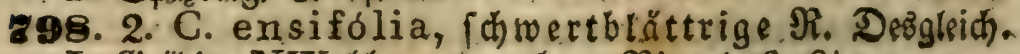
I. Ģröbik, NWabhang im obern $\mathfrak{B i e r t e l . ~ 6 . ~} 24$. H. 1200'. L. Hb. K. 1730'. S. Ch. 


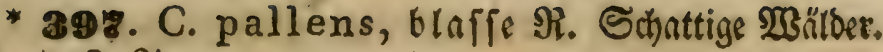
6. 24.

H. $15-1800^{\prime}$, K. $1680^{\prime}$. S. Ch.

347. Neóttia. Nefftwurz:

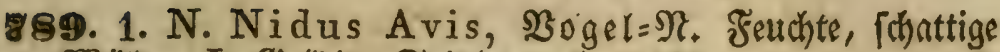

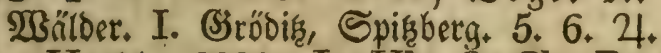

H. 11-2000'. L. Hb. S. Ch. B. O. W.

348. Listera. Riftere.

8פ0. 1. L. ováta, eíflätrige Sd)attige Malbwiefentänder, $\mathfrak{u}$ fergebülch. II. SBoberau bei

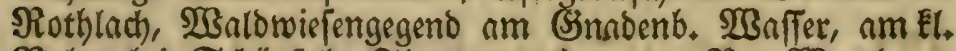
Bober bei Sdjönfeld, İhomaswalbau, zw. Neu=2Barthe u.

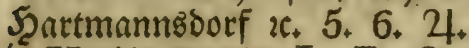

H. bis $2000^{\prime}$. L. B. O. W. Hib.

* 39S. L. cordáta, herzblättrige ̊. Narfe Moorwälloer, Şebirgşwálder. 6-8. 4. L. Hb.

84. Cypripédium. Frauenichuf.

* 399. C. Calcéolus, gelber $\mathfrak{F}$ r. Sdhattige

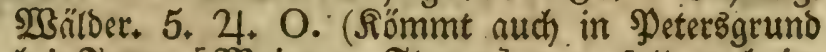
bei Sauter [Mraior y. Flotow] vor, foll aud im Modentwaloe bei Sdjönau [ein.).

85. Maláxis. 3art $=$ Drche.

* 100. M. monophýlla, einbl. 2. Bebirg8mis= fen. 6. 24. Deerichlefien.

86. Stúrmia. Stutmie. .

* 401. St. Loes élii, Löfelz St. Sumpfwiefen. 6. 24. W.

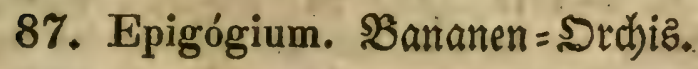

* 402. E. aphýllum, brattlofe SB. Feuchte,

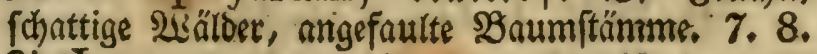
21. L. 
88. A ristolóchia. Sflterluzei.

* 403. A. Clemátitis, gemeine D. T3albrinnder und Şecten. 6. 2\%. H. 1100'.

\section{Einunbzwanzigite Slafie. Monoécia. Esinbäutitg = (šetrenntblütbige.}

349. Calla. Frofdblöffel, Sdblangenwur?.

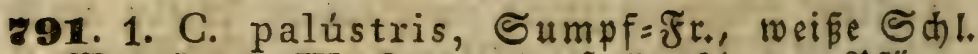

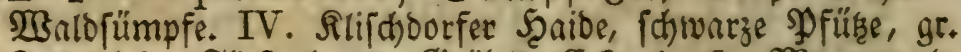
Becte beim Förfterhaus, Ģröbel, Eckersborfer Moorgruno, Grmbenberger $\mathfrak{B a f f e r}$ beim Sdjönfelder Scügel, Şreuliț. 5. 6. 24 .

L. B. O. W. $\mathrm{Hb}$.

350. Euphórbia. $2530 l$ ḟmillḑ.

892. 1. E. Peplus, Graten= 2 . 2uf Brattenbeeten sin UnEraut. V. SBorftióte, 2fnitalt. $6-10 . \odot$.

H. 10-2000'. L. S. Ch. B. O. W.

* 104t. E. exigua, Eleine 2B. Zecter. 7. 8. $\odot$. S. B. O. W. SBrestau, Dberfhlefien.

893. 2. E. helioscópia, fonnentendige 23 . 2déer= und Gartenbeete. III. SBorftíbte. 4. 5, 9, 10. $\odot$.

H. bis $1500^{\prime}$. L. Hb. K. S. Ch. B. O. W.

* 405. E. segetális, Santen=2B. 2fecerer. Şa= belidiwert. $5,6 . \odot$.

894. 3. E. dulcis, füßß̨) $\mathfrak{B}$. Shattige \&aubraäloer, 5.

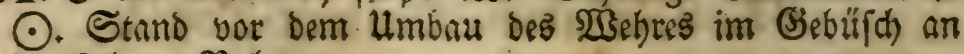
Demielben. Sobernu.

H. 2500' L. Hb. K. S. Ch.

595. 4. E. platyphýllos, breitblăttrige 20 . 2(eder, 


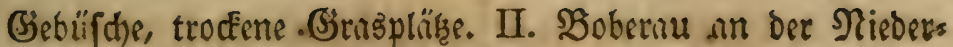
mühle uno abwintts. $6-8 . \odot$.

H. $10-1100^{\prime}$. L. K. $1480^{\prime}$. B. O. W.

* H.66. E. pilósa, han rige $\mathfrak{B}$. Trodéne, fteinige

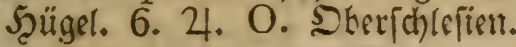

* 408. E. lúcida, glanzblättrige $\mathfrak{W}$. Dorfan= ger, utfergebüín. 7. 8. 2.

B. O. W.

* 108. E. palústris, Sumpf=203. S̈raben; naffe Balopláke. 6. \%. 24. W.

* 109. E. a mygdaloídes, $\mathfrak{B}$ ald $=\mathfrak{B}$. Bebirgs= willoer. 6. 21. H. 10-1200'. Deberfitefien.

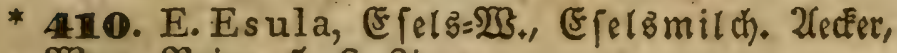
Wege, Raime. 5. 6. 4.

H. $10-1200^{\prime}$. L. B. O. W. Hb. S.

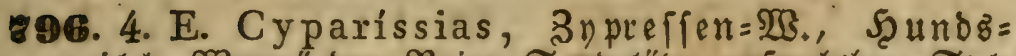

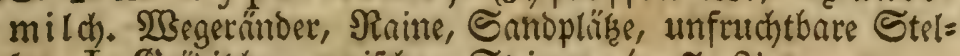

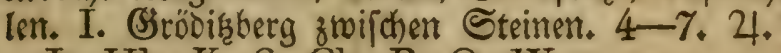

L. Hb. K. S. Ch. B. O. W.

351. Zannichéllia. Zannidbelfie.

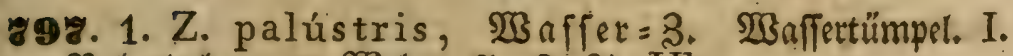
Boberlachen am 23 ehre. 7-9. 24. Hb.

\section{Carex. Rieogras, Segge.}

998. 1. C. dioeca, zweih.n̈ufiges $\Re . ~ \Re o o r=\mathfrak{~}$. Iorf= wiefen. I. Şofenik bei $\mathfrak{W e h t a u}, 5,6.44$. H. W. S. Ch.

* 411. C. Davalliana, Davalls R. Sumpf= wiefer. H. Hb. Şofenif?

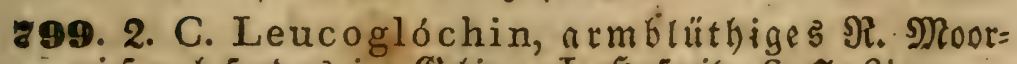

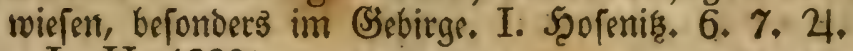
L. H. $1200^{\prime}$. 
800. 3. C. pulicáris, $\mathfrak{F} 10 h=\Re$. Sumpfwiefen. I. Şos [enis. 6-8. 2 .

H. L. O. W.

* 112. C. rupéstris, Felfen= $=\Re$. Felfen im (Se= fente. 6.44 .

* 13. C. chordorhiza, fadenturzliges ঐ. Torfboden. 5, 6. 24 .

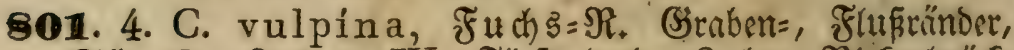

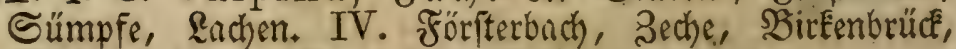
Schönfeld u. m. Sorte. 5. 6. 24. H. 11-1400' L. Hb. K. S. Ch. B. O. W.

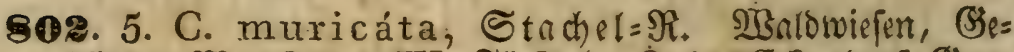

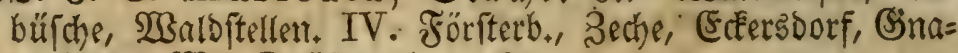
Denberger $2 B_{*}$, Drlíbel. 5. 6. 24 .

H. 11-2200'. L. Hb. K. S. Ch. B. O. W.

* 14. C. intermédia, mittleres̊. Strnan= graben, 23iefer. 5. 6. 24. H. $1400^{\prime}$. S, Ch.

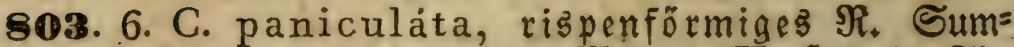

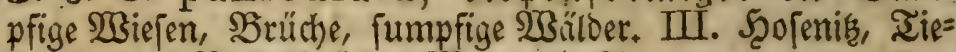
fenfurther, BirEenbrücerer Moor. 5. 4 .

H. $1200^{\prime}$, L. S. Ch. B. O. W.

804. 7. C. teretiúscula, runohalmiges $\Re$. Sumpf= wiefen. III. Bech)e, (Eckersborf, Sirfenbrücê, 5. 6. 24. H. $1200^{\prime}$. L. W.

805. 8. C. paradóxa, [onderbares $\Re$. Moorboben. 5 . 6. 21. Sุof

806. 9. C. cyperoídes, 3ypergrasartiges $\Re$. (Sras fige Teichränder, Sümpfe. II. (Sindonberger Ieiche. 6-8. 4. H. 1000'. L. K. O. W

808. 10. C. Schrebéri, Shrebers $\Re$. Trockene Şü= gellehnen, Gsrabentïnder, SBaldfüume. III. Boberimfel,

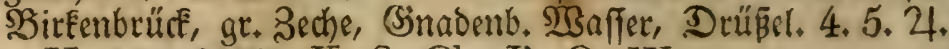
H. $11-2200^{\prime}$. K. S. Ch. B. O. W. 


\section{3}

808. 11. C. brizoídes, zittergrnbartiges $\Re$. 2 Balbs

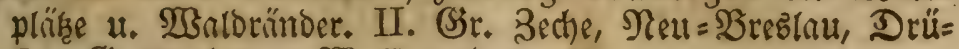

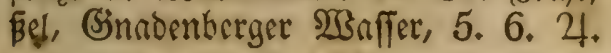

L. Hb. K. S. Ch.

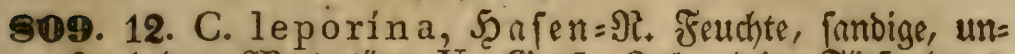
fruchtbare Wraldplïhe. V. Giroße 3eche beim Förfterhaus, ङrröbel, Birfenbrǘce. 5. 6. 2\%.

H. L. Hb. K. S. Ch. B. O. W.

810. 13. C. canéscens, weiß̈graués $\Re$. Sumpf= und

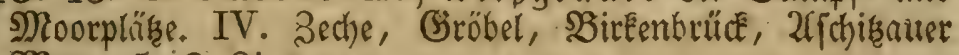
Noor, 5.6. 4 .

H. L. Hb. S. Ch. B. O: W.

8111. 14. C. a renária, $\subseteq_{n n d}=\Re$. Fłtugfunbferber. IV. İiefenfurth, Schönfeld, Nhühlbod. (Sonjt nicht in Der Sprovinz.) $5-7.24$. L.

812. 15. C. elongáta, langähriges $\Re$. Feuchte $\mathfrak{B a l b =}$ ftellen. IV. (Srope 3eche am Förtferbach, Sinndenberger 23. 5. 6. 24 .

H. L. Hb. S. B. O. W.

813. 16, C. stelluláta, fternförmiges $\Re$. Sumpf=u.

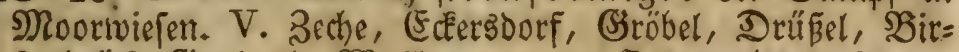
Eenbrǘc, Sinndenb. SBaffer u. v. n. Drten, 5-7. 24.

H. bis $2200^{\prime}$. L. Hb. K. S. Ch. B. O. W.

814. 17. C. remóta, entferntblüthiges $\Re$. そeuchte $u$.

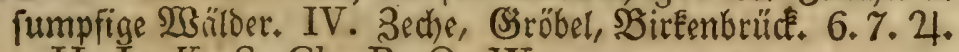
H. L. K. S. Ch. B. O. W.

* 45. C. atrata, gef $6-8.4$.

815. 18. C. Buxbaúmii, $\mathfrak{B} u \mathfrak{r b a u m} \xi=\Re$. Bsraben= rünber, Moortwiefen. III. Förfterbady), Edétersborf 2 . H. $11-1200^{\prime}$. L. W. Hb.

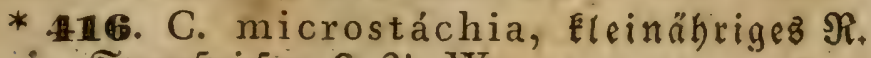
Sumpfriefen. 6, 24. W. 


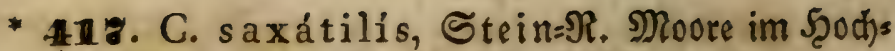
gebirge. 6-8. 24. H. 20-2600'.

816. 19. C. ericetórum, Şaiben= S. Sandige, 2 Bäl=

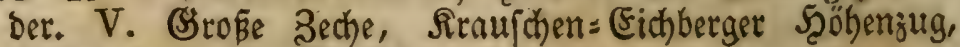
Şaibe. 4. 5. 24. Drüßel.

L. S. Ch. B. O. W.

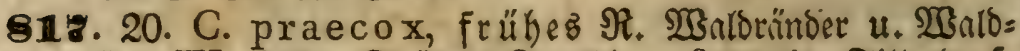
pläks. IV. Seche, Drüßel, Siraujhen, Roostwik, Iillendorf. 4. 5. 24.

H. L. Hb. K. S. Ch. B. O. W.

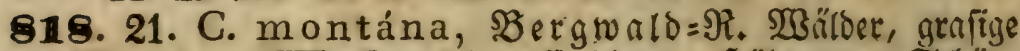
SBerglefnen. III. Iraufden= Eichberger Scöbenjug, Echön= felo, Ihomasmaldau. 4. 5. 24.

L. S. Ch. O. W.

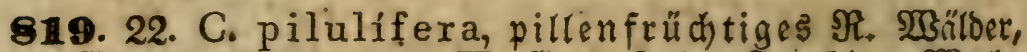

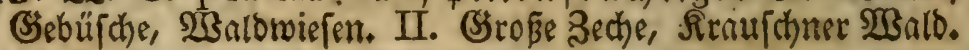
4. 5. 2 .

H. $1200^{\prime}$. L. Hb. S. Ch. B. O. W.

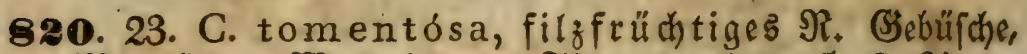
Walbrïnber. Maldwiefen. ? Thomastwalbat. 5, 6. 4 . S. Ch. O. W.

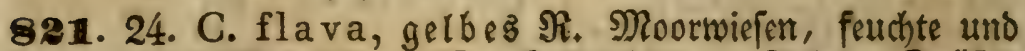

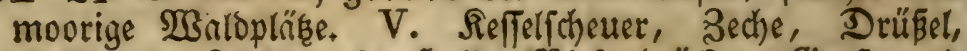

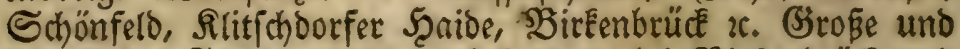
febr Eleine Formen, leģtere befonderg bei Sirfenbrüce und auf oen. Mooren. 5, 6. 24 .

H. $12-2200^{\prime}$ L. Hb. K. S. Ch. B. O. W.

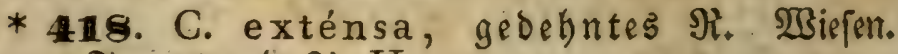
Iroppriu. 4. $24 . \mathrm{H}$.

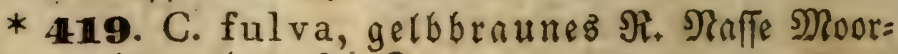
เviejen. 5. 6. 4.0 .

822. 25. C: distans, abftehendes $\Re$. Unfruchtbure,

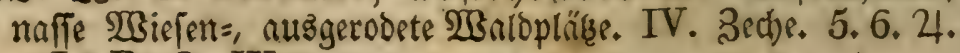
L. B. O. W.

823. 26. C. panicéa, hirfenfrüđtiges $\Re$. Feudte u. 


\section{5}

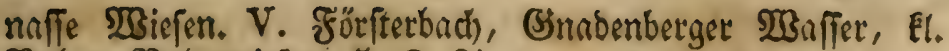
Bober, SBoberwiefen. 5. 6. 4 .

H. $1300^{\prime}$. L. Hb.. K. S. Ch. B. O. W.

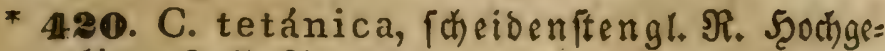
birge. 6. 7. 24.

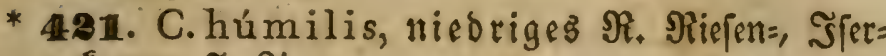
Enmm, \%, 4 .

* 42. C. ornithópoda, vogelfúpörmiges R. Feuthte $\mathfrak{W i e f e n ~ u . ~ M o o r e . ~}$

824. 27. C. digitáta, fingeröhriges $\Re$. Sdunttige (Se=

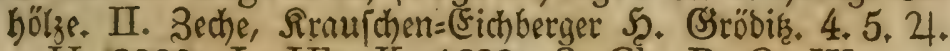

H. '2000'. L. Hb. K. 1680'. S. Ch. B. O. W.

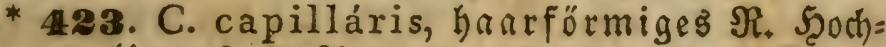
gebirge. 6. 7.24.

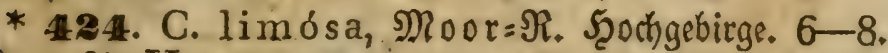
24. $\mathrm{H}$.

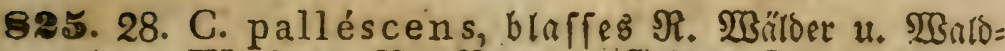

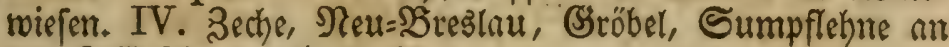
Der Reffelfdieuer. 5, 6. 2 .

H. $1200^{\prime}$. L. Hb. K. S. Ch. B. O. W.

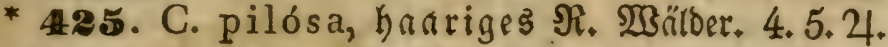

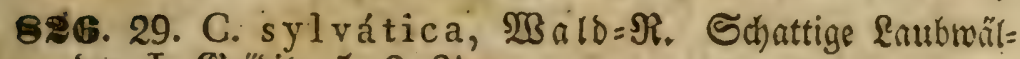
Der. I. Gröobir. 5. 6. 21.

H. $12-2000^{\prime}$. L. Hb. K. S. Ch. B. O. W.

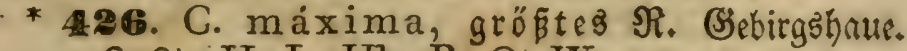
6. 24. H. L. Hb. B. O. W.

822. 30. C. Pseudo-Cyperus, 3yper $=\mathfrak{I}$ ruggras. Sumpfige 2 nnlogegenden. 6. 24.

828. 31. C. ampulácea, Flafden $=\Re$. Sumpfige (5) ben, Moore. II. Förffterbach, 5. 6. 4 .

H. $11-2200^{\prime}$. L. Hb. S. Ch. O..W.

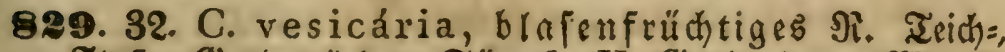

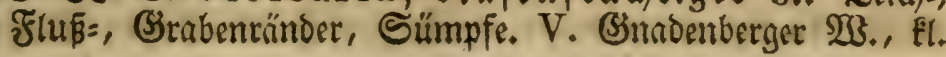




\section{6}

SBober, Bober, Förifterbach, Moorgruno am (Bröbel nach Eckersoorf. 5. 6.24.

H. L. Hb. K. $1480^{\prime}$. S. Ch.. B. O. W.

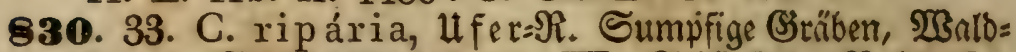

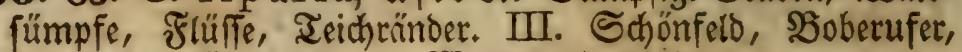
El. Bober, Ginabenberger $\mathfrak{B a f f e r}$. 5. 6. 24. H. $10-1400^{\prime}$. L. B. O. W.

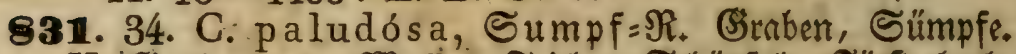

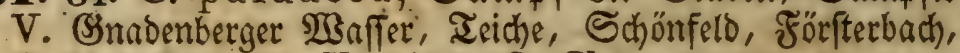
Drǘel. 5. 6. 24. K. 1490'. S. Ch.

832. 35. C. stricta, fteifes $\Re$. Graben, Sümpfe. II. Becthe, Srröbel, Ecferersoorf. 5. 6. 4.

H. bis $2200^{\prime}$. W. Hb. S. Ch.

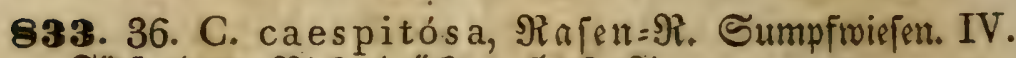
Fôrfterbach), Birfenbrüđe 2c. 5. 6. 4.

H. L. Hb. K. S. Ch. B. O.:W.

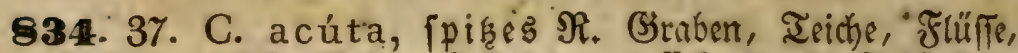
ulfer ano. IV. Bober, Refferfheuer, Eckerboorf, Schönfelo, Gnndenberger $\mathfrak{2 B a f f e r} 2$ 2. 5. 6. 24.

H. L. Hb. K. $1430^{\prime}$. S. Ch. B. O. W.

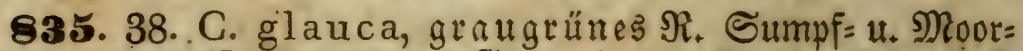

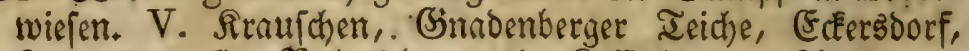

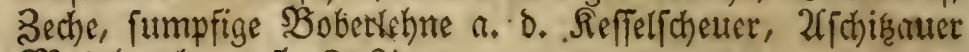
Moorbruch 2 . $5,6,4$.

H. $10-1500^{\prime}$. Hb. K. 1430 ' S. Ch. B. O. W.

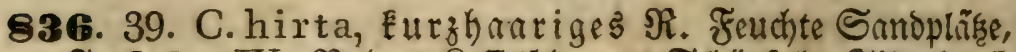
JitußuFer. IV. Bober, Reffelicheuer, Schönferd, IIllentorf, EdFersoorf, DrüBel 2c. 5.6. 4 .

H. $10-1200^{\prime}$. L. Hb. K. S. Ch. B. O. W:

838. 40. C. filifórmis, fadenförmiges $\Re$. Iorfige WBaldwiefent. Bifchof 2frbertini. 5. 6. 4 . H. L.

353. Spargánium. S̄gelênozppe.

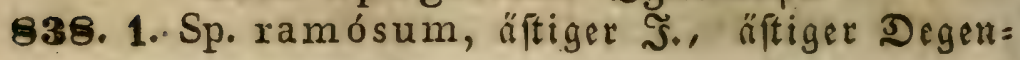




\section{7}

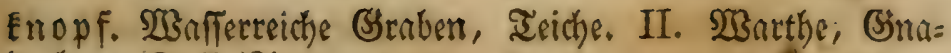
benberg. 6.7. 4.

H. $10-1100^{\prime}$. L. Hb. S. B. O. W.

839. 2. Sp. simplex, einfacher $\Im$. (Graben=, Teith=u.

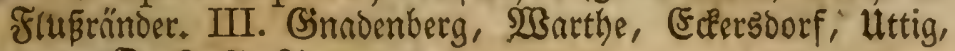
u. ส. פ. 6.7. 4 .

H. L. B. O. W. Hb. K. Ch.

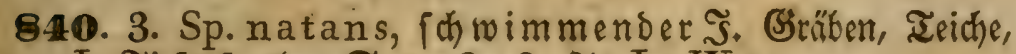

I. Tiefenfurther Seen. 6-8. 24. L. W.

\section{Urtíca. Neffel.}

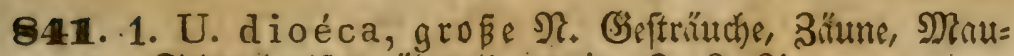
ern, Sd)utthaufen, überall gemein. 6-8. 21.

H. L. Hb. K. S. Ch. B. O.'W.

842. 2. U. urens, Eleine গ., Brenneffel. Desgleith. (3nttenbeete. 6-10. $\odot$.

H. L. Hb. K. S. Ch. B. O. W.

\section{Alnus. Erte.}

843. 1. A. glutínósa; flebrige, $\Re$ oth=

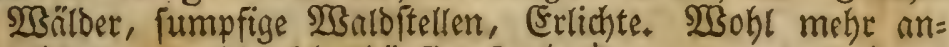
gebrut als wilb, fehr häufig. 3. 4. 万.

H. 10-2200'. L. Hb. K. S. Ch. B. O. W.

814. 2. A. incána, (Sீrau=

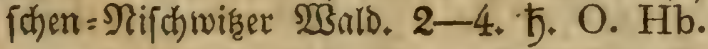

89. Xánthium. Spirflette.

* 4z. X. strumárium, gemeine Sp. W3ege, Schutthaufen, ungebaute SDrte. 7-9. $\odot$.

H. Li. O.

356. Amaránthus. $\mathfrak{2}$ marantf).

845. 1. A. adscéndens, auff́teigender 2 . Ungebaute Nläke, Mifthaufen, Şartentbeete. 7-9. $\odot$.

H. $11-1200$ ' L. B. O. W. 
846. 2. A. retrofléxus, übergebeugter 2 . Ungebaute

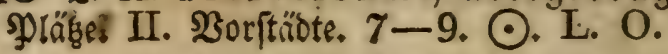

\section{Arum. 2ron.}

* A. maculátum, gemeiner $\mathfrak{H}$. Sdattige TBäl= Der, feuchte Şecten. 6. 7. 24. Scjönbrunner : Berg in ber $\mathfrak{R}$ urf

\section{Ceratophýllum. Şornblatt.}

848. 1. C. demérsum, verfenftes Sh., fpiffrüdti= ger Binken. Langfam fleeßende.u. ftillítehende Setwäfler. V. $\mathfrak{B}$ ober u. Soberlachen am 2 Bebre fehr bäufig, Siroifd)= wis $7,8.24$.

\section{S. Ch. B. O.}

358. Myriophýllum. Teidfendhel, Federtraut.

848. 1. M. spicátum, ähriger $\mathfrak{I}$. Stehenbe $\mathfrak{B}$ nffer,

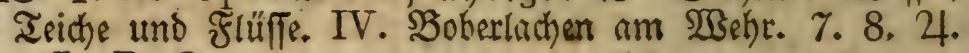
L. B. O.

849. 2. M. verticillátum, quirlblättriges $\mathfrak{T}$. Des= . gleidjen. II. Sobarlachen. 7. 8. 24. L. B. O.

\section{Sagittária. Pfeilf́raut.}

850. 1. S. sagittifóliả, gemeines গুF. Stehenoe (b)=

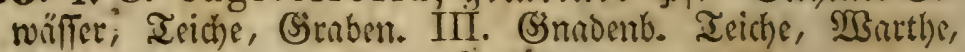

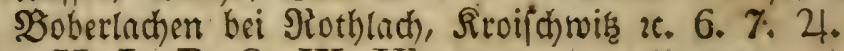

H. L. B. O. W. Hb.

\section{Potérium. Secherblume.}

851. 1. P. Sanguisórba, gemeine. $\mathfrak{B}_{\ldots}$ weliche $\mathfrak{B} i$ bernell. Irodene Şüget, Şerglehnert. I. Greödis, Sab= hang. $5-7.4$.

H. bis $1500^{\prime}$. L. Hb. K. $1430^{\prime}$. S. Ch.

\section{Córylus. Scajelftrauto).}

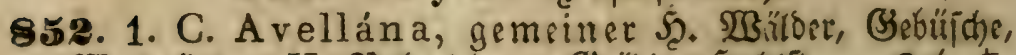

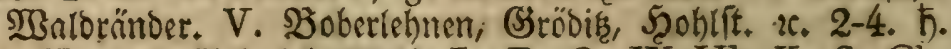
H. nod) über 15-2000' L. B. O. W. Hb. K. S. Ch. 


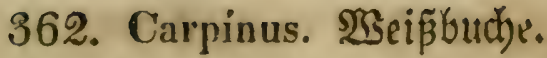

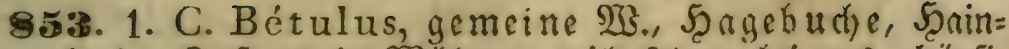
bud)s. Serfit:eut in 2 billdern, will feltnet bei unt, bruffi= get als Secfen, j. 2B. Rebrergarten, Sinnodnbery ac. 5. 5. H. bis 1100'. angebaut L. B. O. W. Hb. S. Ch.

\section{Fagus. SBuche.}

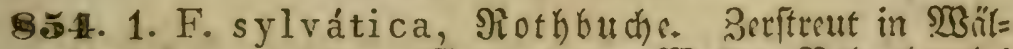

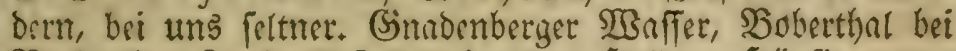
Siotblich, Raubwaldftellen in Der Şaibe. Scäufiger um Eprottau, 5. 6. 5 .

H. bis 2300'. vollffommen, höhar frünêtno. L. B. O. W. Hb. K. S. Ch.

\section{Quercus. Eiche.}

855. 1. Q. Robur, Stein=, $\mathfrak{Z}$ inter $=F_{.}, \mathfrak{B a l b b a u m . ~}$

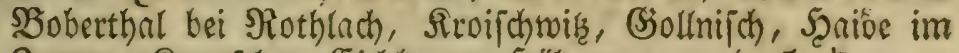
3umm, Rraufchen=(Fichterger Scöhenzug 2c.4. 5. 5.

H. bis $1500^{\prime}$ von ba bis $2300^{\prime}$ nur ftraudjartig. L. B. O. W. K. S.

856. 2. Q. pedunculáta, ftielfrüdtige Sommer= (Eiche. Mit 1. untermicht. 4.5. 5.

L. B. O. W. Hb. K. S. Ch.

\section{Bétula. SBirke.}

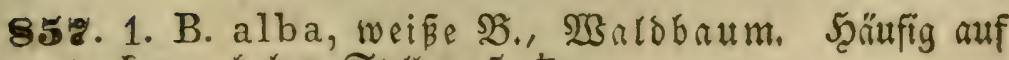
trodentrin, hohen Stellen. 5. ち.

H. bis 2800'. L. Hb. K. S. Ch. B. O. W.

858. 2. B. pubéscens, weichyarige B. Desgleiden. Mehe auf fumpfigem, moorigem SBooen. Seche, Şaide. 4. 5. 5 .

$\mathrm{H}$. biร $2800^{\prime}, \mathrm{Hb}$.

* 428. B. nana, 3werg=\$2. Şoke Torfmoore. 5. 6.1 . 


\section{Pinus. Fichte.}

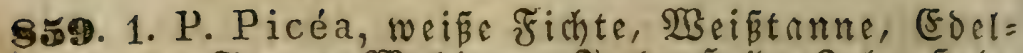
tanne, Ianne. Tुaldbaum. Sn ber J̧aibe, 3eche, Şoh! ftein, vereingelt oder gruppentweife mit Sicfern und Fich)= tert. 5. i.

H. bis 2300'. L. Hb. K. S. Ch. O. W.

869. 2. P. Abies, Nothe Fid)te, Iannen= Mie 1.

H. bis 2800'. im Sumpfboden ftcht fie bis 2700', L. Hb. K. S. Ch. B. O. W.

861. 3. P. sylvéstris, fiefer $\mathscr{J}_{*}$ Riefer, Föhre.

Simeinfter scroelwald baum bei uns. 5. ந.

H. 10-2800'. L. Hb. K. $1430^{\prime}$. S. Ch. B. O. W.

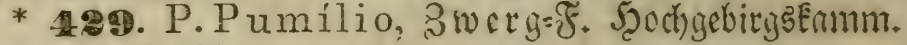

6. 7.24.

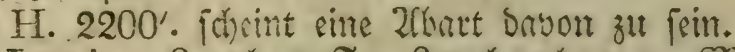

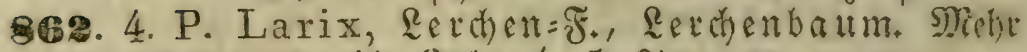
ingepflimyt ats willo. 3ed) s. 4. 5. 21.

H. bis 2300' L. Hb. K. S. Ch. B. O. W.

367. Typha. Robtkolben.

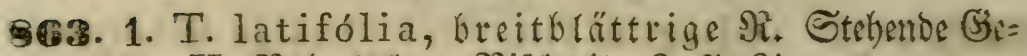

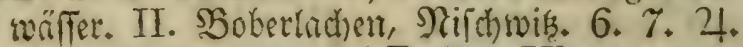

H. $10-1100^{\prime}$. L. B. W. Hb.

56. 2. T. angustifólia, fhmalblättrige গi. Des= gleichert. Siolsmilt)le? 6.7.24.

L. O. W. S. Ch.

\section{Bryónia. Saunrübe.}

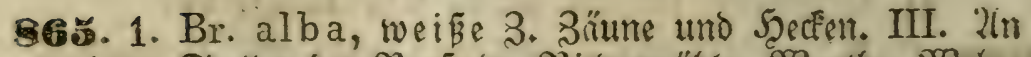

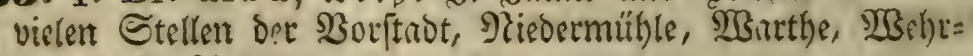
๙u. $7,8,2$.

H. bis $1200^{\prime}$. L. B. O. W. Hb. 


\section{3weiund zwanzigfte RTaffe. \\ Dioécia. 3̂weifäufige Sy flanzen.}

\section{Crite Dronutut. Diándria. Bweifabige.}

\section{Salix. 25seibe.}

\section{a. Srudisweiber.}

866. 1. S. pentándra, fünffadige $2 \mathbb{B}_{+}$, Rbrbeer $=\mathbb{M}$.

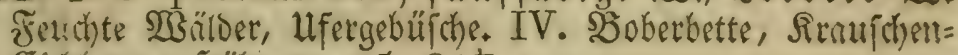
Eichterger 5̧öhengug. 5, 6, 万.
H. 12-1300'. L. Hb. K. 1430'. S. Ch. O. W.

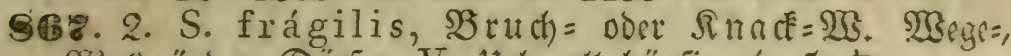

glandotänder, Dorfer. V. Hcberall häufig. 4. 5. 5.

H. bis 2200' L. Hb. K. 1430'. S. Ch. B. O. W.

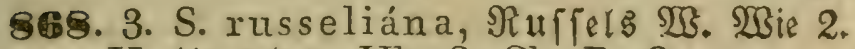

H. bis $1500^{\prime}$. Hb. S. Ch. B. O.

869. 4. S. alba, wei ß̊ $2 \mathfrak{B G}_{+}$Wie 3 .

L. Hb. S. Ch. B. O. W.

\section{b. Mandertreiben.}

880. 5. S. amygdalína, mandelblättrige $2 \mathfrak{B}$. Fluß $=$ ufer, Bachränoer. V. Boberbette 2.

H. 12-2200' L. Hb. K. S. Ch. B. O. W.

* 430. S. hippophaefólia, fandoornblät = rige $\mathfrak{2 B}$. 4. Breglau.

\section{c. Reifiveiber.}

* 131. S. daphnoides, lorbectättrige $\mathfrak{W}_{\text {. }}$. Sberidylefien. 3.

\section{d. Spurpurweiden.}

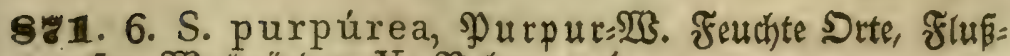
ufer, $\mathfrak{2 a}$ altorñnder. V. Sober $2 c^{4} 4$.

H. 1300'. L. Hb. K. S. Ch. B. O. W.

8ร2. 7. S. rubra, rothe $\mathfrak{B}$. Deøigleiden. Hb. S. 
e. Sorbweiben. .

823. 8. S. viminális, $\mathfrak{T}$ orb $=, \mathfrak{B a n} \delta=\mathfrak{B}$. Desgleidhen. H. 2000' L. Hb. B. O. W. K.

* 3:. S. stipuláris, afterblätrige:2B. Dber= fdilefiett. 4.

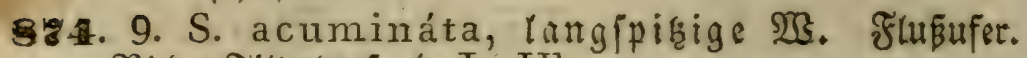
Nieder=Iillentoorf. 4. L. Hb.

f. Saativeiben.

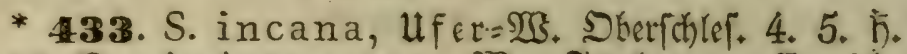
825. 10. S. cinérea, graue 203 . Feuthte, walterteiche

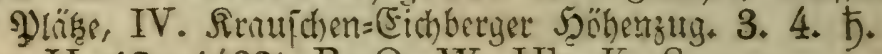

H. $12-1400^{\prime}$. B. O. W. Hb. K. S.

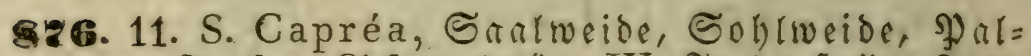

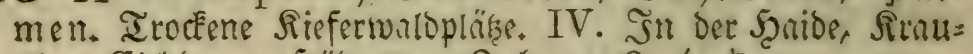

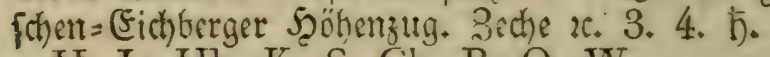

H. L. Hb. K. S. Ch. B. O. W.

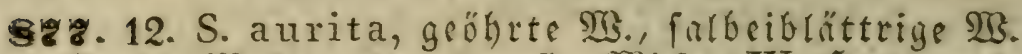

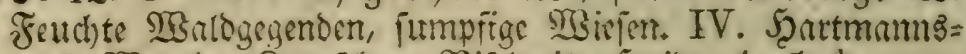

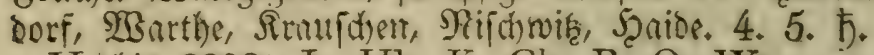

H. bis 2200' L. Hb. K. Ch. B. O. W.

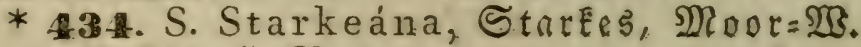
Sitüche. 4. Ch.?

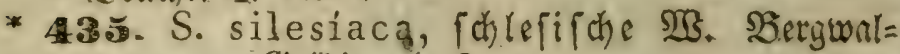
Dungen.? Gröbif. 5. 6.

H. $2400^{\prime}$. Hb. S. Ch.

* 36. S. hastáta, pontonträtrige 23 . SFe= ferte. 5. 6 .

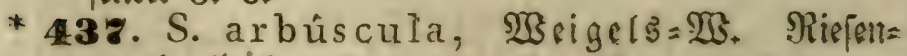
gruno. 5,6 .

g. Silberuveibent.

8z9. 13. S. repens, Eriedsende 213., Eleine $\mathfrak{Z}$ iefen = MB. Baldige Torf

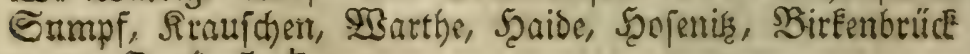
a. m. S. 4. 5. ந.

H. $1200^{\prime}$, L. Hb. K. $1380^{\prime}$. Ch. B. O. W. 
* 139. S. ambigua, zweifelhaftige 2 . Sum prige Şaiden. 4. 5. H. 11-1300'.

* 139. S. finnmárchica, finnmarkifore $\mathfrak{W}$. Degsgleichen. Sppeln. 5, 6.

* Ado. S. myrtilloídes, heiderblättrige $\mathscr{B}$.

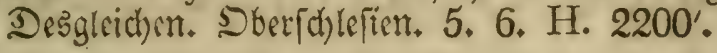

\section{h. 2rrpenweiben.}

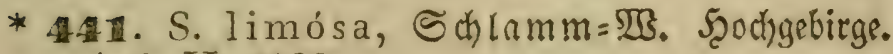
5. 6. H. 2200'。

\section{i. (STlet fdjertweiber.}

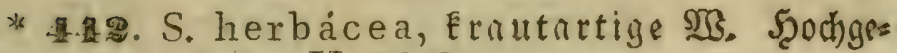
birge. 5. 6. H. 1800'.

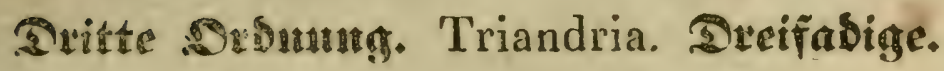

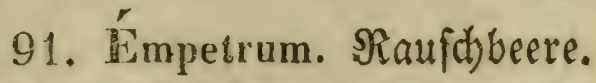

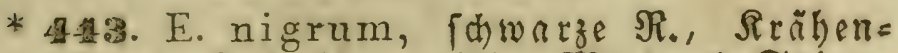
berte. Jenochgebirge zivifat)ent Moos unt Steimen. 5. 6. 5. H. 22-2800'. Soll nuf Dem Gröbis= Eerge vorEommen. SBiz iff́t von mir nid)t aufgef.

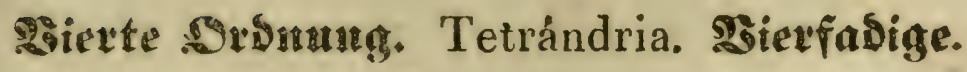

\section{Viscum. Miftel.}

829. 1. V. album, weí̄er $\mathfrak{R}_{+}$Bogelleimpflange.

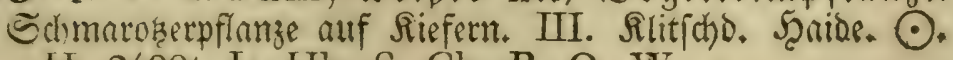
H. $2400^{\prime}$. L. Hb. S. Ch. B. O. W. 
Fünfte Dronutug. Pentándria. Frunffadige. 371. Húmulus. Şopfen.

880. 1. H. Lúpulus, gemeiner 5 . Utfergebüifoh, Şecten, Bantbrïnber. IV. Bober, Eleiner Bober, Ģröbel, Sueis 2 . 7. 8. 24.

H. $10-1150^{\prime}$. L. B. O. W. Hb. K. S. Ch.

Sedofte Sronumg. Octándria. Ildrtrabige. 372. Pópulus. Pुappel.

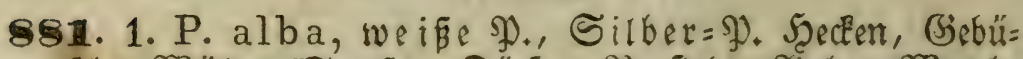

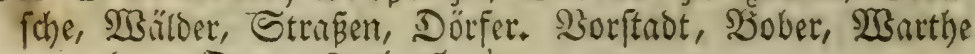
u. andern Srter. 3. 4. 5. h.

H. 1190'. L. B. W. Hb. S. Ch.

852. 2. P. trémula, 3itter= \$. Desgleichen befonders

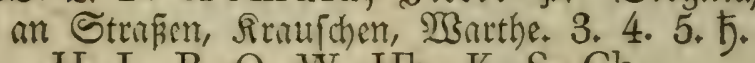

- H. L. B. O. W. Hb. K. S. Ch.

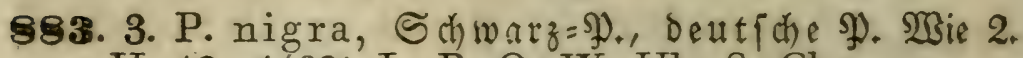
H. $12-1400$ '. L. B. O. W. Hb. S. Ch.

\section{Siebente Sorsunuty. Enneándria. शeunfabige.}

373. Mercuriális. SBingelfraut.

884. 1. M. perénnis, auร̧duerndes \$B. Feuchte

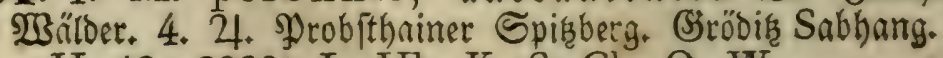
H. 12-2000'. L. Hb. K. S. Ch. O. W. 
585. 2. M. ånnua, ciniấhriges $\mathfrak{B}$. Rräutereien, $\mathfrak{B}$ ein= berge. 7. 8. $\odot$. Nieberborftaot an ben Băunen. L.

374. Hydrocháris. そ̛rof(hloffel.

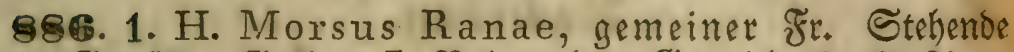

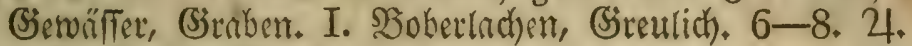
L. B. O. W. S.

Belunte Seśnunto. Dodecándria. Bülffradige.

92. Stratiótes. SBafferfdyeere.

* Str. aloides, aloentige 263 . Stehentie Gerwaiffer. 7. 8. 24. L. B. O. W.

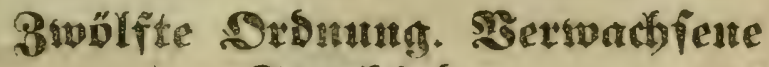
Strubfasen.

375. Juniperus. S3achbolderftrautch. 858. 1. J. commúnis, gemeiner $\mathfrak{2}$. Siemein in ber

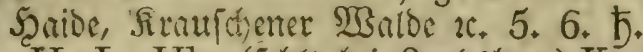

H. L. Hb. (fehit hei $\mathfrak{a}$ moshut.) K. S. Ch. B. O. W. * Jor nana, 2merg=20. Géenke. 5. 6. 5. 376. Taxus. Eibenbaum.

888. 1. T. baccáta, (Fibenbrum, Taxus. TBälber, befonders Gebirgswillber. I. Spifberg bei Probfthain, im Stwalde bei Ihommentorf, wo früher pehr viele gewejent,

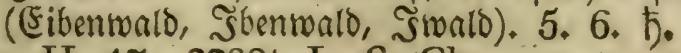

H. 17-2200'. L. S. Ch. 


\section{Nadd trag.}

3u Seite 81. Erica herbacea, Erautige \$ृaibe. Bergwither. Sberichlefien, Gejente bei Einfiedel. 5. ந.

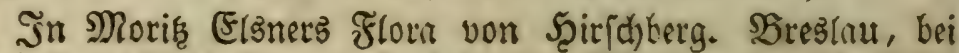
2roerthols, 183\%. find nod) folgende SPflanjen als fdblefifche Bürger rufgeführt:

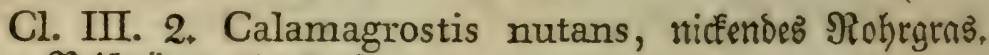
Reiftrïger. 7-9. 24.

Cl. V. 1. Gentiana chloraefolia, d) lorablättriger Enjint, zroifden Bärnoorf uno Den Friefeniteinen. 8, 9. 24.

Gentiana pyramidalis, pyramibenblütbigrt $\mathfrak{F}_{\text {. }} \mathrm{Rn}_{\mathrm{n}}$ pellenberg, Riselberg. 7-9. 24.

Cl. V. 2. Heracleum longifolium, langblättriges $\mathfrak{B}$ ä= renElau. Sdnregruben. 7-9. 24.

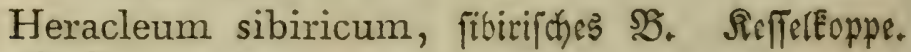
7-9. 24.

Cl. VIII. 3. Alisma natans, fohtrimmenoer Frofhlöfel. STergebirge um bie Tafelfichte. 6-8. 24.

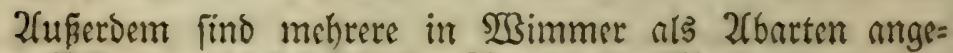
gebene PSflanzen als felbftïnoige 2frten nufgefühtt. 
Acer......... 82 Achillen ....... 149 Aconitum ....... 105 Acorus ......... 75 Actaea . . . . . . 104 Adonis ........... 106 Adloxa .......... 85 Aegopodium ....... 67 Aethusa ....... 66 Agraphis ...... 74 Agrimonia ....... 96 Agropyrum ...... 39 Agrostemma . . .... 94 A prostis ....... 30 Aira........... 33 Ajuga.......... 112 Alchemilla....... 45 Alectorolophus ..... 117 Alisma ........ 80 Alliaria ...... 126 Allium ........ 72 Alnus.......... 167 Alopecurus ...... 29 Alyssum ........ 121 Amaranthus ....... 167 Anagallis ...... 51 Anchusa ....... 48 Andromeda ...... 86 Androsace ....... 49 Anemagrostis ..... 30 Anemone ....... 109 Angelica ........ 64 Anthemis ...... 149 Anthericum ...... 75 Anthoxanthum ......24 Anthriscus ...... 68 Anthyllis ....... 131
Antirrhinum ..... 119 Apargia. ........ 154 Aquilegia .......... 105 Arabis .........124 Archangelica ...... 64 Arctium ........ 141 Aretostaphylus ..... 86 Arenaria ........ 91 Aristolochia ...... 160 Armeria ....... 71 Arnica ......... 148 Arnoseris ....... 155 Arrhenatherum .... 32 Artemisia ....... 145 Arum ........... 168 Asarum ....... 96 Asparagus ....... 75 Asperugo ...... 49 Asperula ........ 42 Aster . . . . . . . 146 Astragalus ........ 137 Astrantia........ 68 Atriplex ........6 61 Atropa ........ 54 Avena ......... 33

Ballota ........ 115

Barbaxea. . . . . . 125 Bartschia ...... 117 Beclimannia ....... 34 Bellis ......... 148 Berberis ........ 78 Berula ....... 66 Betonica ........ 116 Betula . . . . . . 169 Bidens .......... 143 Biscutella ....... 121 
Blitum .......... 19

Blysmus . . . . . . . 28

Borkliausenia . . . . . 150

Brachypodium ...... 38

Brassica . . . . . . 126

Briza . . . . ..... 36

Bromus ... . . . . . 38

Bryonia ....... 170

Bupleurum ......66 66

Butomus .........85

Cacalia $\ldots \ldots \ldots .443$

Calamagrostis ..... 31

Calla .......... 160

Callitriche ...... 19

Calluna ....... 81

Caltha ......... 109

Camelina ....... 123

Campanula ...... 52

Capsella ........ 122

Cardamine.......124

Carduus . . . . . . 141

Carex ......... 161

Carlina ....... 140

Carpinus . . . . . 169

Carum .......... 67

Caucalis .........64 64

Centaurea ....... 142

Centunculus ....... 44

Cephalanthera .... 158

Cerastium ....... 92

Ceratophyllum ..... 168

Cerinthe . . . . . . . 49

Chaerophyllum .....667

Chamaepitys ...... 112

Chelidonium ...... 104

Chenopodium ......60 60

Chimophla ....... 87

Ghondrilla ....... 153

Chrysanthemum . . . 148

Clurysocona ......143
Chrysosplenium .... 88

Cichorium ........ 155

Cicuta ........67 67

Cineraria ...... 147

Circaea .......223

Cirsium . . . . . . 139

Cladium .......26

Clinopodium ......111

Cnidium ........ 65

Colchium .......8 80

Comarum ....... 102

Conioselinum ...... 64

Conium ........668

Conringia ...... 126

Convallaria .......74

Convolvulus ...... 51

Conyza ........ 145

Corallorhiza ..... 158

Cornus ......... 44

Coronilla ....... 138

Coronopus ...... 120

Corrigiola .......70

Corydalis . . . . . 130

Corylus ......... 168

Corynephorus ..... 33

Crepis ......... 150

Critamus........66 67

Crocus ........2 25

Cucubalus........99 91

Cuscuta .......6.62

Cynanchum .......660

Cynoglossum ...... 48

Cynosurus ........ 36

Cyperus . . . . . . 28

Cypripedium ...... 159

Gytisus ........ 134

Dactylis ........ 36

Daphine ....... 80

Datura . . . . . . . 54

Daucus ........ 64

Delphinium ......... 105 
Dentariat ....... 121

Dianthus

Dictamnus...... 87

Digitalis 119

Digitaria

Dipsacus

Dondia

Doronicum

Draba

Drosera

Echinochloa

Echinosperum

Echium

Elatine

Elymus

Empetrum ....... 173

Epilobium

Epipactis ...... 158

Epigogium.

Exica.

Evigeron 146

Eriophorum

Erodium

Ervurn ......... 136

Eryngium

Erysimum

Erythraea ....... 55

Eupatorium ...... 142

Euphorbia . . . . . 160

Euphrasia ....... 117

Erongmus ....... 57

Fagus ........ 169

Farsetia ....... 122

Fedia $\ldots \ldots \ldots . . .25$

Festuca ....... 37

Ficaria .........108

Filago ......... 144

Fragaria ....... 100

Fraxinus ........20

Fumaria ........ 129
Galanthus....... 72

Galega .......... 136

Galeobdolon ......113

Galeopsis ....... 113

Galium ........ 42

Genista .............. 133

Gentiana....... 55

Geranium ...... 126

Geum.......... 102

Gladiolus ........ 25

Glaux.......... 57

Glechoma .........114

Globularia........ 40

Glyceria ........ 35

Gnaphalium...... 143

Goodyera ........ 158

Gratiola ........ 22

Gymnadenia...... 157

Gypsophila ...... 89

Habenaria ....... 158

Hedera ......... 57

Hedysarum ....... 138

Helianthemum ..... 10/4

Helleborus ....... 108

Hepatica........ 109

Heracleum ....... 63

Herminium ...... 157

Herniaria ...... 60

Hesperis ........ 125

Hieracium ....... 150

Hierochloa ...... 32

Himantoglossum .... 157

Hippuris . . . ..... 19

Holeus ......... 32

Holoschoenus ..... 28

Holosteum ....... 40

Hordeum ....... 40

Hottonia ........ 50

Humulus ........ 174

Hydrocharis...... 175

Hydrocotyle ....... 69 
Hyoscyamus ....... 54 Hypericum ....... 138 IIypochaeris ....... 154

Jasione......... 51 Illecebrum ...... 57 Impatiens ....... 58 Imperatoria ....... 63 Intybus ....... 152 Inula . . . . . . . 146 Iris ......... 25 Isopyrum ........ 108 Juncus . . . . . . 76 Juniperus . . . . . . 175

Koeleria ........ 34

Lactuca ........ 153

Lamium ........ 114

Lapsana ........... 154

Laserpitium .......62

Lathraca ........ 120

Lathyrus ......... 134

Lavatera . . . . . . . 129

Ledum ......... 86

Leersia .......... 29

Lemna .........20

Leonuxus ......... 115

Leontadon ....... 153

Lepidium ....... 122

Leucojum ....... 72

Ligustrum ....... 20

Lilium ........ 73

Limosella ........ 116

Linaria ....... 119

Lindernia .......116

Linnaea ....... 120

Linum ......... 71

Listera ......... 159

Lithospermum ..... 47

Lolium ......... 39
Lonicera ...... 56

Lotus . . . . . . . 136

Lunaria ......... 121

Luzula ......... 77

Lychnis ........ 93

Lycopsis ........ 48

Lycopus ........ 23

Lysimachia ..... 50

Lythrum ....... 96

Majanthemum ..... 44

Malachium ...... 92

Malaxis ....... 159

Malva ........ 129

Marrubium ...... 116

Matricaria ....... 148

Medicago ........ 137

Melampyrum ...... 118

Melica ......... 34

Melilotus ........ 131

Melittis ..........111

Mentha ........ 112

Menyanthes ....... 51

Mercurialis .......174

Mespilus .........998

Meum ....... 65

Milium ........ 31

Molinia ......... 35

Monotropa ...... 87

Montia ........ 26

Muscari ....... 74

Myosotis ........ 46

Myosurus ......... 108

Myriophyllum .... 168

Myruhis ....... 68

Narcissus ....... 72

Nardus ........ 29

Nasturtium .......... 123

Neottia........ 159

Nepeta ........ 114

Neslia ....... 120 
Nigella ................ 106

Nuphar 104

Nymphaea 104

Odontites ....... 117

Oenanthe

Oenothera ....... 82

Omphalodes....... 48

Onobrychis ........ 131

Ononis ............131

Onopordon .......141

Orclis ......... 156

Origanum ....... 112

Ornithogalum .... 73

Ornithopus ....... 138

Orobanche....... 119

Orobus ......... 134

Oxalis ........... 95

Panicum ......... 31

Papaver........ 103

Parietaria ....... 45

Paris ......... 85

Parnassia ...... 70

Passerina........ 81

Pastinaca ....... 63

Pedicularis ...... 117

Peplis .......... 78

Peucedanum. ..... 63

Phalaris ....... 30

Phleum ........ 29

Phragmites. ..... 32

Phyteuma ....... 52

Picris ......... 153

Pimpinella ...... 66

Pinguicula ....... 23

Pinus ........... 170

Plantago ........ 43

Platanthera ...... 157

Pleurospermum ..... 68

Poa.......... 35
Polycarpum .......40

Polyenemum ..... 26

Polygala ........ 130

Polygonatum ...... 75 .

Polygonum ...... 83

Populus ........ 174

Portulaca ....... 96

Potamogeton ..... 45

Potentilla ....... 100

Poterium ....... 168

Prenanthes ...... 152

Primula ........ 49

Prunella ........ 110

Prunus ........ 97

Pulmonaria ....... 48

Pyrethrum ....... 149

Pyrola ........ 86

Pyrus ........... 99

Quercus ........ 169

Radiola ....... 46

Ranunculus ............ 106

Raplianus ....... 121

Reseda......... 97

Phamnus ........ 56

Rhynchospora ...... 26 .

Rhytispermum. ..... 48

Ribes......... 57

Rosa .............. 102

Rubus ............. 99

Rudbechia...... 149

Rumex ........ 78

Sagittaria ....... 168

Salix ......... 171

Salvia ....... 23

Sambucus ........ 69

Sanguisorba ....... 44

Sanicula . . . . . . 6 69

Saponaria ........ 89 
Sarothamnus ......133

Saxifraga ....... 88

Scabiosa........ 41

Sehenchzeria ...... 79

Scilla .........74

Scirpus ......2.20

Sceleranthus ......990

Scorzonera ........154

Scrophuiaria ...... 118

Scutellaria .......111

Sedum ........ 95

Selinum . . . . . . 64

Senpervivum ......997

Senecio ........ 147

Serratula ........ 141

Seseli...........65

Setaria ......... 32

Sherardia ........4 4

Silaus ........65

Silene . . . . . . . 99

Sinapis ........ 126

Sisymbrium . . . . 125

Sium : : : : : : . . 66

Solanum....... . 54

Soldanella . . . . . . 50

Solidago ......... 146

Sonchus . . . . . . 152

Sparganium . . . . . 166

Spergula.........994

Spiraea.......... 98

Spiranthes....... 158

Stachys . . . . . . 115

Staphylea ...... 70

Stellaria ........990

Stratiotes ....... 175

Streptopus........75

Sturmia ........ 159

Swertia ....... 56

Symphitum . . . . . 49

Tamarix ......... 70

Tanacetum
Taxus ......... 175

Teesdalia ....... 121

Tetragonolobus ..... 137

Teucrium ....... 112

Thalictrum ....... 120

Thesium . . . ..... 58

Thlaspi ....... 122

Thymus .......... 111

Tilda........ 104

Tofieldia. . . . . 79

Tozzia ........116

Tragopogon ....... 153

Trapa $\therefore \ldots \ldots .44$

Trientalis ....... 80

Trifolium. ........ 132

Triglochin ....... 79

Triodia ....... 38

Trollius ....... . 108

Tulipa ......... 73

Turritis ....... 125

Tussilago ....... 143

Typha ....... 170

Ulmus . . . . . . . 62

Urtica . . . . . . . 167

Utricularia . . . . . 23

Vaccinium ...... 81

Valeriana ........ 24

Veratrum ...... 79

Verbascum ...... 53

Verbena ........ 116

Veronica ........20

Viburnum . . . . . . 69

Vicia........... 135

Villarsia ........ 51

Vinca .......... 58

Viola ........... 58

Viscum ........... 173

Xanthium ........ 167

Zannichellia $\ldots \ldots \ldots 161$ 


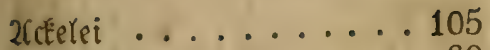

2reterweizen ......... 39

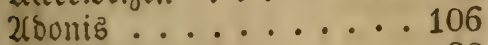

2thorn.......... 82

2riant .................. 146

2rlpengrocklein ...... 50

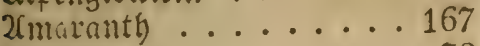

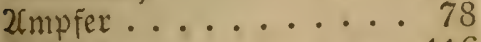

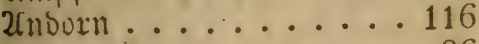

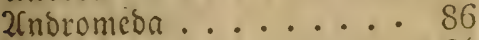

2ungetifa ....... 64

Xnthemis ....... 149

2rum ........... 168

2ffdenpflanze ..... 146

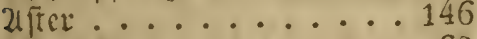

2firtantie....... 68

2!ugentroft ...... 117

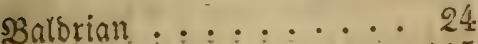

SBrllotte $\ldots 115$

Balfamine ...... 58

Sananenordis ...... 159

Sarbentraut ...... 125

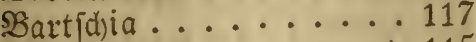

SBárenfdulveif . . . . . 115

Sárentrauke ...... 86

SBárentvurz...... 65

Bedjerblume ...... 168

Bectemannia ....... 34

Senebiftenuturz .....102

Berberife ......... 78

Serte ........... 66

Befenfitraudi) ..... 133

Bertram ....... 149

Setonifa ........... 116

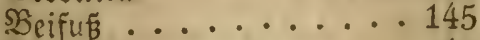

SBeintuurz ...... 49

Bibernell....... 66

Bilfenklaut ...... 54

sienenEraut ...... 111
SingelEraut ..... 174

SBinje......... 76

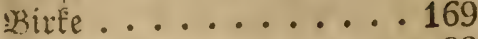

Sirne ......... 99

sBifamtraut ...... 85

Blápnnús...... 70

Slumenbinje...... 85

slutauge....... 102

Buctsbart .................... 153

SBoithanfenia ..... 150

SBorftoolde ...... 64

SBorftengras ...... 29

Straundle .................. 110

Stemndolde ....... 65

Brombere ....... 99

S3rillenidjote ....... 121

sgucie............ 169

Shimophile...... 87

Darrgras ....... 26

Diptam ......... 87

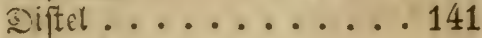

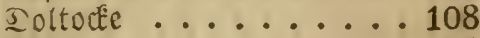

Dondia ................. 64

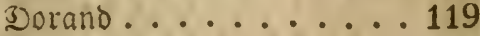

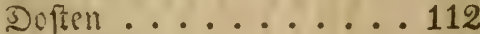

Drehâtre ........ 158

Sreizad .......... 79

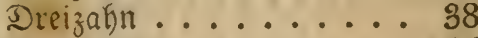

Durrtourz . . . . . . 145

Eberwurz $\ldots \ldots 140$

Efrenpleiż..... 20

Eibenbaum ........ 175

Sithe ............. 169

Einbeere ....... 65

Eifenbut................ 105

Eifeneraut ........ 116

Engetwurz ....... 64 
Enzian ........ 55 Eppeu ........ 57 Srobeere ........ 100 Erobeeripinat $\ldots \ldots 19$ Eroraud) ........ 129 Erle .......... 167 Eruthree ....... 55

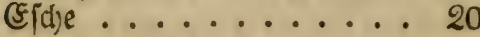

Farietie ........ 122

Feberkraut ......... 168 Fennid)..................... 32 Fettfraut ........ 23 Fid te......... 170 Fid)tenfpargel ..... 87 Filjkraut ........ 144 Fingergras ....... 131 Fingerhut ...... 118 Fingerkraut ...... 100 Flad)Eleibe........ 62

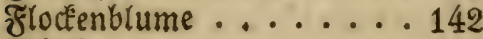
Fróberaut $\ldots \ldots \ldots 146$ Fraueniduth ....... 159 Froichlóffet....8 80160175

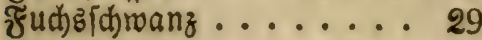

(samander $\ldots \ldots \ldots 112$ (saudbbeil ........ 51 Gánfediftet ....... 152

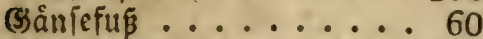
હånié̂́raut $\ldots \ldots \ldots 124$ (3emburm ....... 148

(sermer ........ . 79 Bseisflee $\ldots \ldots \ldots 134$ Bseisraute . . . . . 136 (sierfd) .......6.67 (sinfter ......... 133 (3)anzgraz ....... 30 (stabtraut $\ldots \ldots \ldots 45$ (slatthafer ....... 32 હ)eiß́.........6 66 BSlockenblume . ...... 52 ङnabenḱkaut .......22
Bolbbanar ............. 141

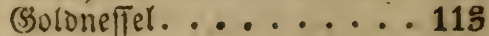
(Soldruthe........ 146 (S)rasnelfe........ 71 Sraufilge........ 64 Sunbermann ...... 235 (Sinnfel ............. 112 Sinpelrat ...... 89

Saargras ...... 39 Saarftrang ....... 63 Şabid tह̄Eraut...... 150 Safer.......... 32 Saftbolde........ 64 Šahnenfuß ...... 106 5ahnentamm .......... 117 Saibe ............... 81 Şainfimie........ 77 Sanfneffel ....... 113 Salelftraud) ....... 168 Şafelrourz ...... 96 Sajentoht ....... 154 5ૃafenlattig ...... 152 sajenobi ........66 66 Scaunedyel ......... 131

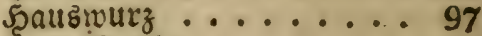
sectenkirichle $\ldots \ldots \ldots 56$ seibelbeere $\ldots \ldots .88$ Sheilfraut . . . . . . 63 Shelmeraut $\ldots \ldots \ldots \ldots 111$ serminium.$\ldots \ldots \ldots 157$ ŞerenÉraut $\ldots \ldots \ldots 2$ Siriengrab ....... 31 sirtentafde $\ldots \ldots \ldots 122$ 5ohtruturz........ 130 sholder.......... 69 šoniggras ....... 32 Sopfen . . . . . . 174 5ुornblatt . . . . . . . 168 5horntraut ....... 92 5ुorntlee ......... 136 కุ้นflattig . . . . . . . 143 
Scunbszange $\ldots \ldots \ldots 48$ 5unostourtrger . . . . . . 61 Ssungerblume ........ 122

sgetenoekpe . . . . . . 166 Igelfame . . . . . . 47 smmerbtume ....... 143 ฐntybub......... 152 Jafione ........ 51 Jothannizbeere ....... 57 ฐolanniafraut . . . . . 138

salmus 75 Sameline . . . . . . . . 123 Rammgrą ........ 36 fiarbe. . . . . . . 67 §arben ..........4 41 Лaf̧enmunge ....... 114 SálberÉropf........667 Reulengrab ........ 32 Ilee . . . . . . . 132 Sleinling ........... 44 Rlette ........... 141 Rlettenér rbet . . . . . 68 Snabenfraut . . . . . 154 Sinaulgraz ....... 36 Snopfgras ........ 26 Inorperblume..$\ldots \ldots 57$ Inotpelfraut . . . . . 26 sinorpelialat ........ 153 Snotenblume . . . . . 72 \&notenfuß . . . . . . 75 Soht ......... 126 Ronringie. . . . . . 126 Sorallemivurz. . . . . . 158 Sornrathe ........ 94 Sôterie ......... 34

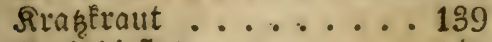
srebssieftet . . . . . . 141 Sretie ......... 122 నieuzfraut ......... 147 sriftophth traut . . . . 104
Sronentwicke ...... 138 Sropfleraut ........ 118 Rigelblume ....... 40 Sugelranunfel ...... 108 sugelriet ........ 28 Sillbreizen ......... 118

\&abtraut ....... 42 Eaid) Eraut ...... 45 Eajerfraut ....... 62 \&aud)........... 72 Eauchtraut ........ 126 Eaufekraut ....... 117 ¿avatera ........ 129

Reberbrume ........ 109 Eeerfia ........ 29 \&eimfraut ........ 91 sein ........... 71 \&einfraut ........ 118 Ridtblume ....... 93 Rieid)gras........ 29 filic ............. 73

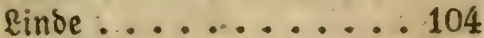
Rindernia ........... 116 Rinnåa ............ 120 凤inie............ 136

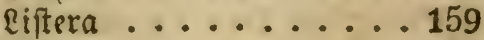
Eold ................. 39

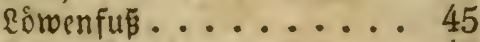
\&ungentraut ...... 48 \&nfimachia ....... 50

Maaslieben ....... 148 פalve ........ 129

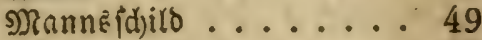
9) ?annztret....... 69 Matterpfeffer ....... 95 9) Ratizobr .......... 46

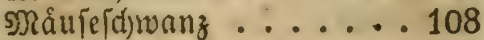
গMeerziviebel ...... 75 sketbe ......... 61 geifterwurz...... 63 
פMi(d̆) fraut . . . . . 57 9)ildftern ...... 73 MilgEraut ....... 88 פriftel ............ 173

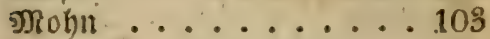
S) Rolinia ........... 35 9) Tunoviole ........ 121 Moónre ......... 103 Mlubfari ....... 74 Mutterf́nut .......... 148 Miunze ......... 112

Sabeleraut ....... 48 raditkerze ...... 82 SRaditidjatten ...... 54 Radituide ...... 82 Sacetorite ........ 157 Nagelfraut ...... 40 গarzille......... 72 Ratterf́upf ....... 49 Relke......... 89 Reslia ......... 120

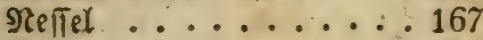
গReftrourz .......... 150 פliejerurz ....... 108

Sd) fenzunge ....... 48 Soermennig ...... 96 spterluzei........ 160

Spappel............... 174 sparnaltia ....... 70 Salferine ....... 81 Soaltinat ........ 63 Sperlgras .............. 34 Djeftruurz........... 143 Эु faffentófrlein...... 154 Dpfeiltraut ....... 168 Spfennigfraut :..... 122 Dflaume ....... 97 Spippalt ........ 150 sylanthatbera ...... 157
Jolatterb/e ...... 134 Vorf̂. ......... 86 sjortulat ....... 96 sjprimel ........ 49

Duellriet ......... 28 Duendel ...........111

アragtwurz........ 158

ఇainfarrn ....... 145

Ruplourz ....... 52

Ralute ........ 123

Ralijbere ...... 173

Mibentoide ....... 65

SRefeda ........... 97

Rettig ........... 121

Sarilyeridynabel ..... 127

«ibbenjame ..... 68

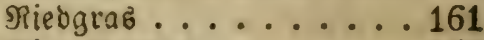

Riemenzunge ...... 157

Ris̄pengraz ...... 35

Ritterfporn ....... 105

Rohrgras ...... 31

Rohrtolben ....... 170

Ropfendiel ....... 65

Rudjgros . . . . . . . 24

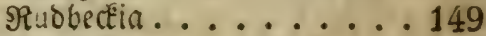

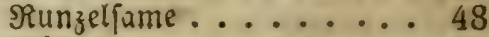

Rutter ........ 62

Safrran ........ 25 Salbei ........... 23

Sallat ............ 153

Samentrefle ...... 120

Sandfraut ....... 91

Sanbrien ........ 155

Sunitel......... 69

Sauerklee......... 95

Edjafyarbe...... 149

Sd)arbodezEraut ..... 108

Sd)arfigrub....... 49

Sd)attenblume ...... 44 
Edjaumeraut ........ 124 Ed)eudizeria ... . . . . 79 Eidjiff........... 32 Edierting ........68 68 Edrlammling . . . . . . 116 Edimiele ......... . 33 Ed)mirget.. . . . . . . . 109 Ednneeball ......... 69 Edjnergloctiden...... 72 Ed)nnctenflee. . . . . . . 137 Sthoblffraut ........ 104 Ed)orenootter ...... 126 Edotentlee ......... 137

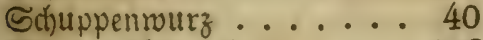
Echłvarztummel ...... 106 Scherertitie......... 25 Edhwingel ........ 37 GeeEandet... . . . . . . 104 Seerofe . . . . . . . . 104 Segge ... . . . . . 161 ভenf .......... 126 Seibelbaft ........ 80 Seifentraut .......889 Sefer ........... 65 Eiderbolde ........667 Silau .......... 65 Sitge............ 64 Simre .......... 26 Sinngruin ........ 58 Efabiofe ........4 41 Eforzonere........ 154 Gommerwu:z. ....... 119 Gonnenróbdjen ....... 104 Sonnenthau .......71 Epargel ......... . 75 Epart .......... 94 Epinbelbaum ........ 57 Spigklette. . . . . . . . 167 Epierftaube......... 98 Spurre ........... . 40 बtadjetgrab ....... 31 Sted)apfet
Gternblúmdien . . . . . 8 80 Sternbyazinthe...... 74 Sterntraut ........ 90 Steinbred..... .888 SteinElee . . . . . . . . . 131 EteinEraut . . . . . . 121 Steinkerfie ......... 121 Steinfame ......... . . 47 Stord)rdinaber . . . . . 127 Etranoling........ 70 Straußrgras ....... 30 Sturmia . . . . . . . . 159 Sumpfaraz .......2 26 Sumpfrutr ........ 158 Sinsootbe .........6 68 ๔ü Sreertia . . . . . . . 56

Tamarizle ........70 Tannenweder ....... 19. Taubenfropf .......991 Ianbneffel ........ 114 Zaunet.......... 85 Iauiendidjón ....... 130 Inallitie ....... 74 Ihefium ........ 58 Th)urmÉraut . . . . . 125 Iofietdie ........7 79 Iolteir[the ...... 54 Iozzie ......... 116 Iraganth $\ldots \ldots \ldots 137$ Izrespe $\ldots \ldots \ldots .38$ Iutpe . . . . . . . . 73

ulme . . . . . . . 62

Reilden . . . . . . 58 Bergi(smeinnid)t . . . . 46 Billarfie ........ 51 Bogetfup . . . . . 138

शşacbyolber. . . . . . 175 


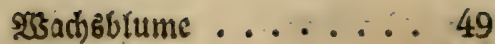
Walberbfe ........ 134 Maltmeifter $\ldots \ldots \ldots .42$ खsanterfeber . . . . . . 5 59 刃ुaiferlinfe ....... 20 Waafiermere . . ....66 66 şanfernabel ........ 69

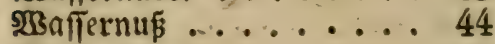
Wafferjalat ....... 26

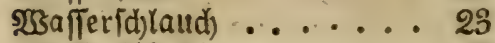
खुafferfdierling ..... 67 $\mathfrak{B}_{3 e g}$ born $\ldots \ldots \ldots \ldots \ldots 66$ rssegerid $\ldots \ldots \ldots \ldots 43$ $\mathfrak{S}_{3}$ ideteraut . . . . . . 92 20seibe. .......... 171 \$Beibenróscten . . . . . 82 श3siorich .......... 96 शुsei

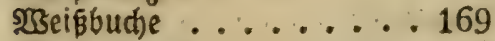

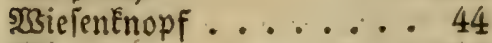
Ssiécuraute ...... . 110 ख̋sicfenjdjwertel . . . . 35

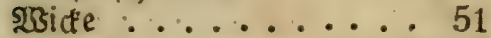
פsinoblume ....... 109 खzinbe .......... 51 פæsinobalm . . . . . 30 खintergruin $\ldots \ldots \ldots 86$ wsirbeldooften . . . . . . 111 श्2irbeltraut. ...... 197 WJoblverleih) ....... 148

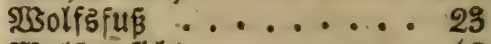

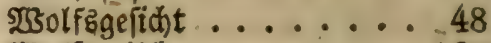
$2330 l$ fmild $\ldots \ldots . . .160$ গુకollgrą ....... 28 গ⿺辶万lléraut ....... 55 İollriet ........ 28 IB undfler.......... 131 श्wsurmblume ....... 153

3abntroft...... 117 3ahnnurz ....... 124 3annid)ellie....... 161 3artorchis ....... 159 3aunblume....... 75 3aunrube ....... 170 3ెeitlole ....... 80 Sidjorie ........ 155 Sieft .................. 115 3ipfelfraut ...... 78 3ittergras ......... 36 3ivedfe ........... 38 3rwergflad)s ....... 48 3nergamander ..... 112 3tweizahn............ 141

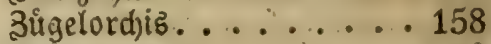
3ıpergras ......... 28

\section{2ter Nachtrag, Neut aufgefunbene গ̧flanzen:}

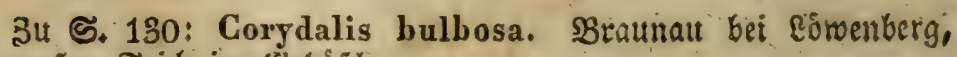
am gropen Ieid) im (sebuifd.

3u ङ. 143: Tussilago alba. গgrobfthainer Spif̧berg, in ber Mitte bes STordabbangeb. 


\section{Dritter 2 Cfichnit.}

\section{Sergleid)ungen Der Gebiete und Der अfinten.}

Dutch) bie mir von mefrem Seiten geworbenen, gütigen Mit= theilungen unt Unterftürsungen ift es mir möglid') getworden,

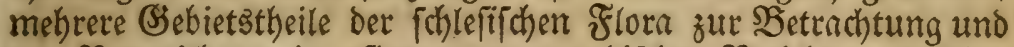
zur Sergleichung in pflanzen=geographifcher $\mathfrak{B}$ eziehung vorfüh=

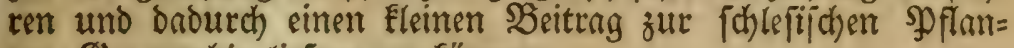
zen = Seographie liefern zu Eönnen.

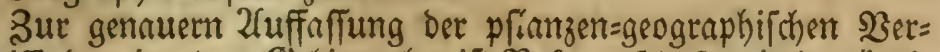

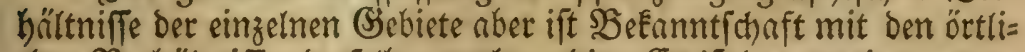
d)en SBerbältniffen berfelben notbrwendig. (Es ift Darum eine, wemt auch nur in Eurzen Ulmriffen entworfene Schilderung Der BPe=

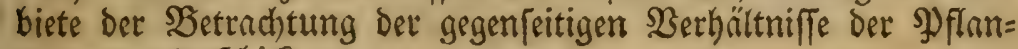
zen vorausgejojictet.

\section{Die örtlichen Berbälniñe Der \$flatzen: gebiete.}

a. Die einzelnen Ssebiete.

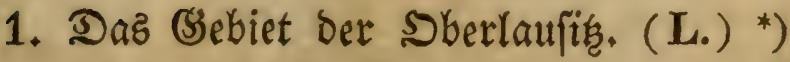

(E5 umfät c. 70 2. M. und bildet bie nöroliche $\mathfrak{2}(b=$

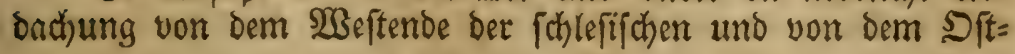

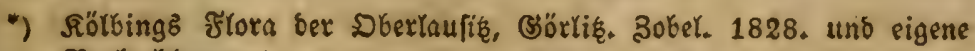
\$Beobañtungen: 


\section{0}

ende ber laufifiger Subeten. Son ber 3379' hohen Iafelfidte im O, Dem 2900' h. Säji kenberge in ber Mitte (Bittru 763',

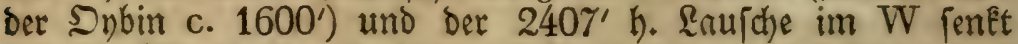

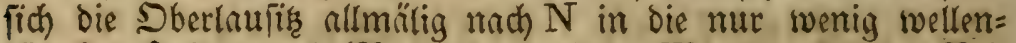

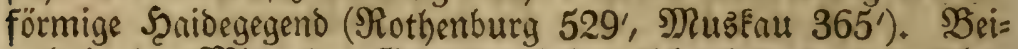
nath in ocr Mitte des (S̈ebiets ertheben fich, befonders zrwifchen Scerrnhut 1054' uno (Sörlif 664', einzelne Berge und 'Berg=

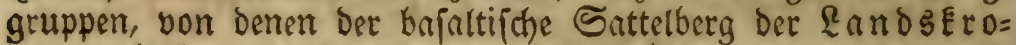
the $1304^{\prime}$ h. und die Sönigshainer Berge c. 1200' h. Die

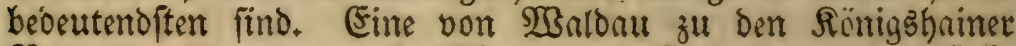

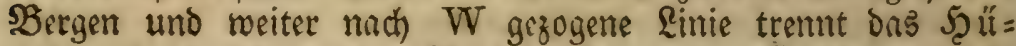
gelland oder bie niedere Berggegeno von der Szaibegegend, welche jebodi) an mehrern Stellen nod) etwas füblicher eintritt. Dar:

So ftellt fich uns bas (sebiet in brei (sefichtspuneten

1) als höhere $\mathfrak{B}$ erg= oder Montanregion im $S$ (Lo.)

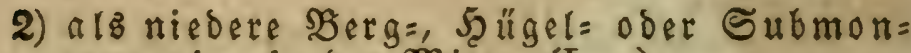

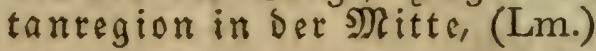

3) alb bie Scaibeebene im N. (Ln.)

Die vorwaltenden Şebitgsmaffen ber böbern und aud ber fubmontanen Region wiro burch bie v. Raumeriche nöroliche (S) ranit= SBneusformation gebildet, welche in Den Sionigs: bainer Bergen am nörolichften auftritt uno im $\mathrm{N}$ burch) Den bon Iauban nörblich Gsörliş vorüberziehenden, roeftlichen $\mathfrak{U} r=$ (d)iefer begrengt wird. Im SW zeigt fid) Scornfteinpor= phyt und aling ftein; an vielen Dunten, befonders zwifhen Görtis, Rauban und Scertnbut tritt $\mathfrak{B a f a l t ~ a u s ~ b e m ~ U t g e = ~}$

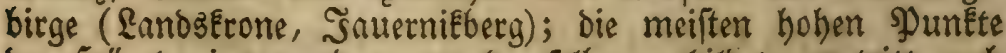
ber Şügelregion tverden von bempelben gebilbet; et tritt auth noch an einigen Stellen ber Şaibe auf uno ift bie pflanzen= reid)ite Unterlage bes (S)ebiets. SilEftein Eömmt nirgenos

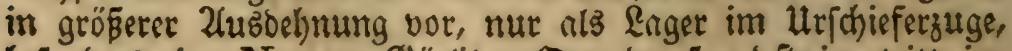
beronderz im $N$ von Börlif. Sunderfanditein tritt in

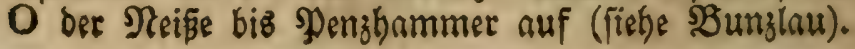




\section{1}

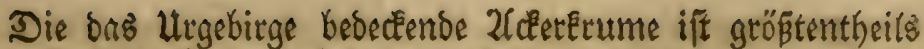
fruchtbarer Şumusboden, befonders in ben umgebun= gen bes SBajalts. S Sempeits bes nörolid)en Utrichiefers aber fängt ber Sand ber Scaide an, ber an mehreen Stellen Flugfano, größtentheils aber ein torfiger Siesfand ift uno zum Theil mit Sineneifenerz, zum groben Theil aber mit Iorfmooren wechfelt und mur von einer bïmen,

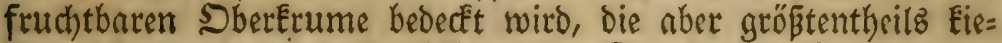
fig troden und renig fruchtbar ift. Der feuchte Scumus bat überall Scinneigung zur Jorfbiloung. Srope Streden fino fumpfbedecft. Der übrige Şaided)arakter ift bei Sumzlau ge= fchilloett.

Der Reichthum an fließenden (S) äffern ift midt un= bedeutend; fie find Ssebirgs= oder Scaiberwilfer; erftere mit Ela= rem, leştere mit Moorwaffer. Sie gehören Den Ssebieten Des

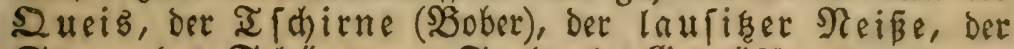
Spree, des Schops an. Stehende Geroüffer fino in ber Şaide viele. Fruditbare $\mathfrak{X}_{3}$ iefen find im Sberland büufig, bie ber Scaide find meiftens moorig uno moofitg.

Der vorwaltende $2 B a l b b a u m$ der Scaibe ift bis Fie= fer, unter und neben berfelben alles überdecfent bas Sุaibe= Eraut oder Fledten; mur an einzelnen Stellen oder verein= zelt Tannen uno Fidjten, an wenigen Stellen $\mathfrak{B} u$ d)en uno (Fichen. Mit ber Sunahme ber Şöhe und des fruchtbaren 20odens nimmt die Menge ber Riefern $a b$, bie der Iannen, fid)ten, SBudyen zu, bis biefe in Der höhern Region faft allein berrfchen. (Eichentwilloer fino nirgends von groker SEedeutung, viele (Eidhen fino im Neis̄thale bei Mušau.

Nach ben burch bie gütige Bermittelung Des Seerm $5 \mathrm{r}=$

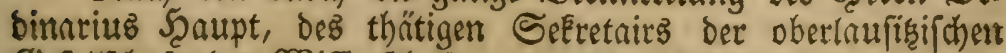

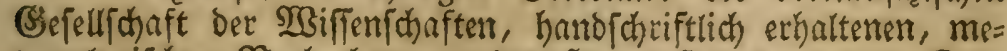
teorologifchen Seobachtungen des Seertm Scauptmann v. Dre= verhof ift in Bittau nadh 5jährigen SBeobachtungen ber mitt=

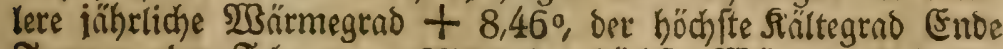
Samuar oder Februar - 27,70, Der höchfte 20 ärmegrad in ber Mitte bes Suli $+32,5^{\circ}$ bes hunderttheiligen Ihermometerz. 
Die Şauptwindrichtunt ift SW, bie Menge bes meteo= rifken 2 Bnffers $17-26$ parifer Soll; 3ahl ber Gewitter $11-19$.

Das Sebiet tremnt und verfindet die Flora der Mare von ber Böhmens, bie von Sachjen von ber fehlefifchen, bilbet Den Hetergang Der Soerflora zu Der Elbflora uno ift im 2Ull=

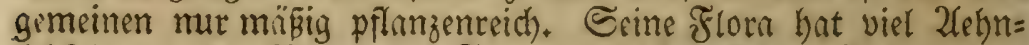

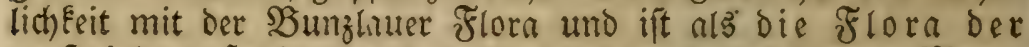
weftlichen Şaide und der niedern und böhern Mon= tan= oder Bergregion des Siergebirges und ber $\mathfrak{L} a$ u= firer Sudeten zubetradien.

Sie enthillt, wenn wir bie zweifelfaften 2trten abrechnen, 922 offenblüthige Pflamzenarten in 404 Battungen uno 91 Familien; 29 Pflanjenarten find bem Gebiet vor den andern sigenthümlich.

\section{Das (3̧ebiet ber Sceufcheuer (H.) *)}

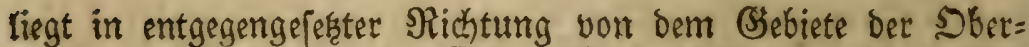

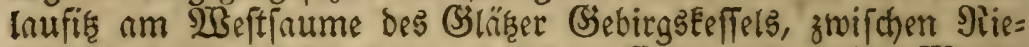
jengebirge und Schneeberg, ztwifhen Eule uno hoher Menje,

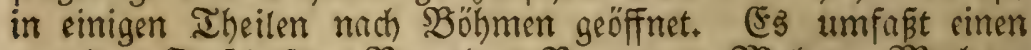
yon Den Srtichaften Reurode, Braunau, Meiden, Machau, Straufenen, Dernifau, Sitifhnen, Rieinerz, RücFerts, 2 Balliz= furt, Samnik, Schwenz, EdFerzborf und Sd)legel umgrenzten,

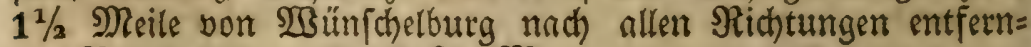

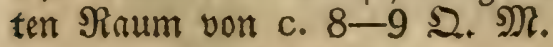

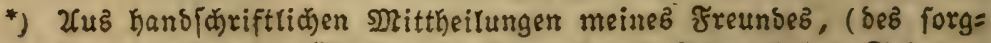
famen und unermüblid)en ?(npfanzerz unb गुflegerz ber Subeten= flor in bem bockgelegenen, botanifden (Sarten ber Sceufdeuer),

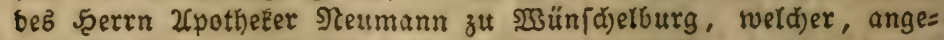
regt ourch Scerrn SBeilformies in Shlau, fleipig und umfichtig

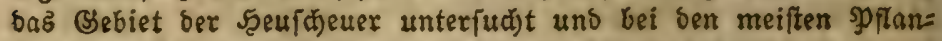

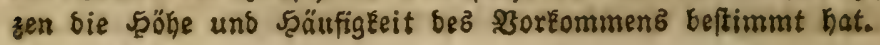




\section{3}

Diefrs ganze vielgeftaltete (Gebiet erhebt fich von 968' bis zu 2831' Meereşböhe, nus Dem Ihal Der Steine bei Nieders

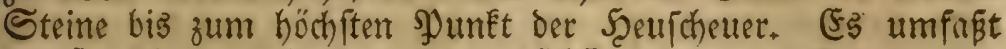
bie Sceurcheuer mit den iht zuniehit gelegenen und fie stmge= benoen Ifailern und ftellt fich) uns nur als SBerg und I That zivifhen Eule und hoher Menfe liegend bar.

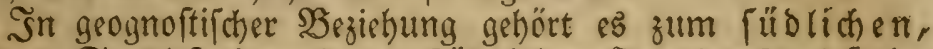
rothen Sandftein und zum fild lichen Suaderfand fein yon v. Raumer, an welches fich im $S$ bei Reimerz bas füb= liche Urgebirge und im $\mathrm{O}$ bei Steine ons fübliche bergangagebirge anichließst.

Die Ibäler und bie nicbern Scöhen, befonders in ber Ulm= gegend von $\mathscr{B}$ ünfchelburg. (Der Scopfenberg 1565') rwerden von einem rothen, grobeornigen Ronglommerat gebiloet, welcher alth) im $S$ Der Scrulfheuer im Ihale yon Rewin er= [cheint und fich bis zum rothen Berge bei (3)Int fortgieht. 2(n baffelbe lehnt fict) I

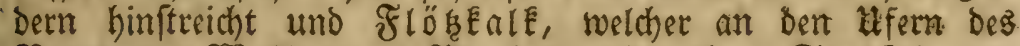
Braumauer $\mathscr{B}$ affers von Sorphne und rothem Sanditein un= terbrochen, in müchtigen $\mathbb{E}$ agern und mit vielen organifd)en

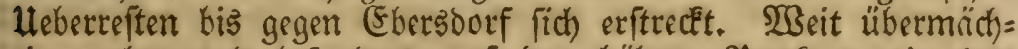
tiger aber und bejonders auf ben höhern SDunEten tritt Das 2uaberfanditeingebirge auf, welches bas Seurcheuerge= birge mit feinen ziemlich fteilem 2(bbängen und feiner äuserft fhroffen, felfigen, viclfach zerêlüfteten Sammböhe auf weite 2lus= Dehmunz bildet. SBei DerniEau ift die mähtige SBuchenlehne

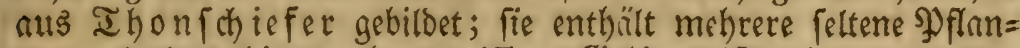
zen und bns hier mehr zerriffene Bebirge ift reich an engen,

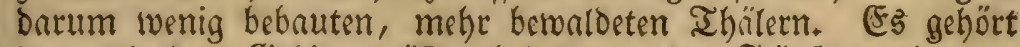
Demnach) Das (şebiet gróstentheils nur Der Flö́zformation an

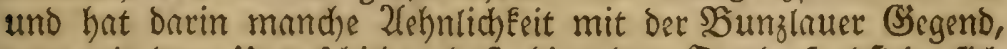

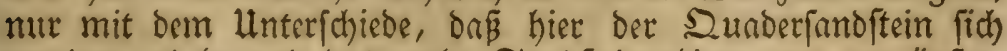
weniger erkebt und das rothe Sanditeingebirge nut am äuвer= ften Sïbfaum füdlich von \&örenberg beginnt.

Die 2 dererume ift über bem rothen Songlommerat, alfo in ber nördlicken Şälfte, tho onig und rothgefärbt, längs bez Sceufcheuerguges mehr lehmig uno weisfandig, boch 


\section{4}

nuch) mit vielem Şumus bin und roieber vermirat, thonig und EalEig find bie 2lecker von Neichenforft uno Niederrathen. Die Şochebene vor Rarlaberg, welche Den weiken Suader[and=

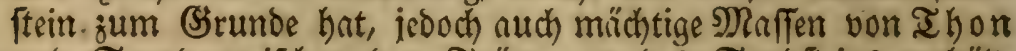
uno Sand zwifchen den Irümmen bes Sanditeins enthält, wiro größtentheils von cinem burch bie Fäulnis ber üppigen

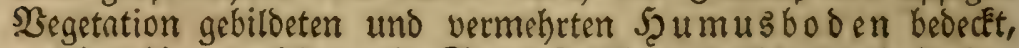
welcher hin $u_{+}$wieder mit $\varsigma_{a n d}$ ftridhen wechfelt und in ben Sertiefungen in fauern Şumusboden, befonders in ber märtigen Iorflagern bes gro Ben uno Eleinen Sees bei Friebrichsgrumo, ronhrfheinlid) mit Ihonunterlage, übergeht

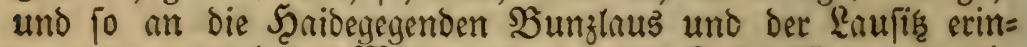

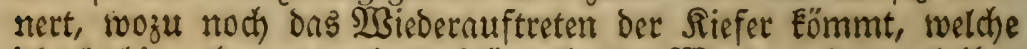
jeboch bier oben nur einen Erïppelhaften $13 u$ dhs zeigt, und ifre

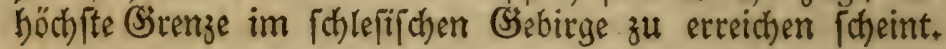

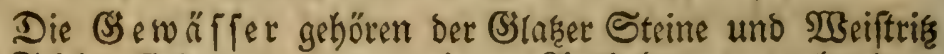
an; Ieiche find nur von geringer 2(usbehnung vorhanben; ) Xoore hin und wieder mehrere Eleinere auser Den fohon ange= führten, größ̈ern Mooren.

Die $\mathfrak{B}$ aldungen weroen gröBtentheils von Innnen uno Fichten auf ben 2abhïngen gebildet, bei DerniEau von $\mathfrak{B}$ uchen, auf ben mehr ebenen Iheilen won אiefern, foft überall find Sirên untermengt; die Steineiche Eömmt nur fparfam in den niebern Siegenoen bis 1000', bei 1500' und baruber nur ftratchartig, bie Sommereidye gar nicht vor.

$2{ }^{3}$ iefen werden an vielen Stellen getroffen und burch ben 2Baffertrichthum begünftigt felbft auf ber Şod)flich) von Sarlš berg.

(5)enauere Flimatifche Berbältniffe liegen nicht vor vout ber Sceufcheuer. Nach ben mir aber burch bie folefifthe (Sefell= fafaft freundlichit mitgetheilten, handrchriftlich aufgereichneten Beobachtungen des Scerrn Schichtmeifter Rhode von 18261834 ift in Neurode (1207' Srehöhe) Der mittlere 2 Bärmegrno bes Snhres $+5,26^{\circ} \mathrm{R}$., Der föchite 23 irrmegrad in ber lef̧=

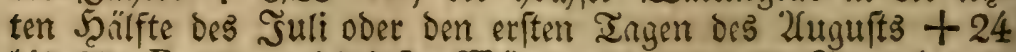

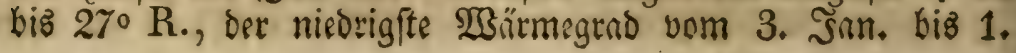




\section{5}

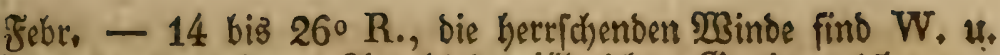
Nrwinde, bie mittlere 2fnzahl ber iübrlichen Ssewitter 15.

Dns Sebiet bilbet sin Uteberganggglied zwifhen Schlefien

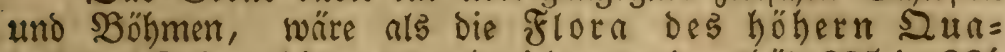
berfandfeingebirges zu bejeichnen und enthält 825 in 364 Banttungen uno 84 Familien vertheilte SPflanzenarten, von Denen 21 2lrten Dem Gebiete yor Den nnoern Şebieten eigen= thümlid) find.

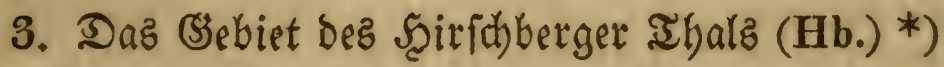

ift ber ohere Ihail bes Ihals, welches im $S$ vom Scochgebir= ge, in $\mathrm{N}$ vom Mittelgebirge, im $\mathrm{O}$ vom Sdhmiedeberger und Lanoshuter framm, im W von ber fiemnirs begrenzt uno burch mehrere Bergreihen in mehrere Ihaltheile (bas 5 d) mie= Deberger Ihal, bie gró̧e Scirichberger Ihalebene, das Maimaloer Thal) getheilt wiro. Die Ihallohle des Sd)mie= Deberger Ihats ift c. 13-1400', Die Deš Scirfchberger 10$1100^{\prime}$ und die bes Maiwalder Thats 12-1300' hoch anzu= nehmen. 2lus Demielben iteigen mehrere vereinzelte $\mathfrak{B}_{\text {erge }}$ wie Die Fralkenberge (2099', die (S3ruppe Der Şirfhberger Berge,

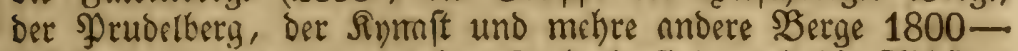
$2000^{\prime}$ hod empor, unt eben fo hoch find auch bie 2rbhänge Dez Riefengebirges zu verfolgen; bieferbe Scöhe erreichen auch

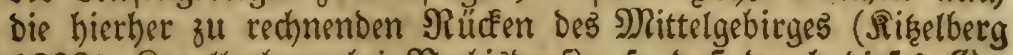
1993', Japellerberg bei Berbizborf), fo baß burds biefes (s) =

*) Die 2rngabe ber in Siefem Bebiete enthaltenen झुflanjen verbanke

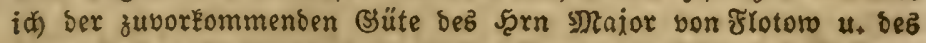

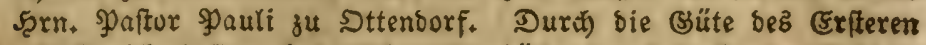
erbielt id befien botanifobe Ingebücher aub mehrern Jabren, ser Reştere ftellte mix ouf meine sitte die von inm gefunbenen

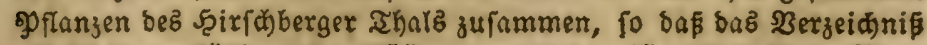
alb fehr zuberläffig uno volrftänoig anzufeben ift. (Fine Eleine flota

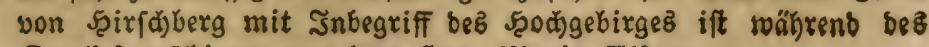

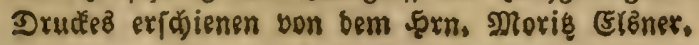




\section{6}

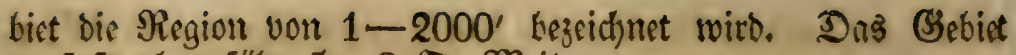
umfnß̧t ohngeföhr 5-6 2. Meilen.

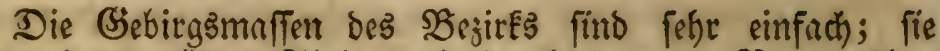

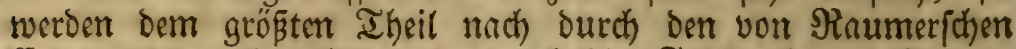
Sentralgranit gebildet, Der burch feime Feelofpathe uno Nuarze ausgegeichnet ift, uno bejonders in Der umgegeno von Filch=

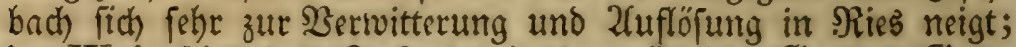

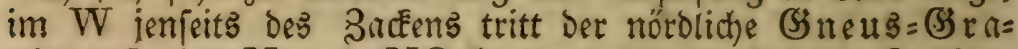
nit auf, im $N$ und NO begrenzt ber burd) feime Raléla=

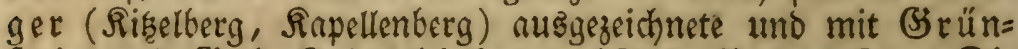

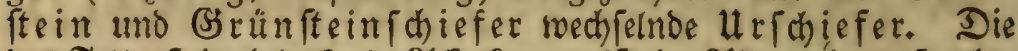

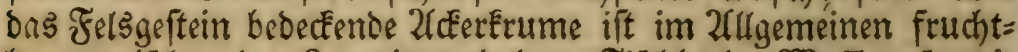

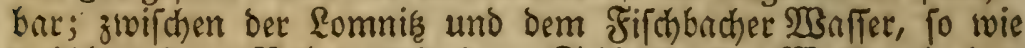
zrwifhen Dem 30 ber und Dem Seifersborfer $\mathfrak{B a n f f e r}$ breiten fich nicht unbebeutende Torfmoore, befonders bei Lomnif uno Rohrlach aus; bei $\mathfrak{B}$ uchwald treten uns mehrere Teiche ent= gegen, bie meiften Ieiche fo wie nuth) Sümpfe finden fid aber

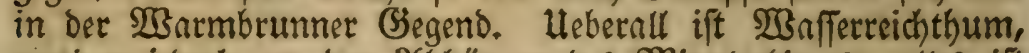
wentiger jeboch an ben 2(b)ängen Des Mittelgebirges; alles ift Bobergebiet.

TBarbungen mäBig, Feine von bedeutender 2fusbehnung;

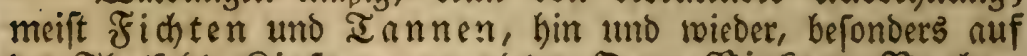
Det IIbnlfohle Riefern, an vielen Drten SBitken; $\mathfrak{B}$ ud dyen

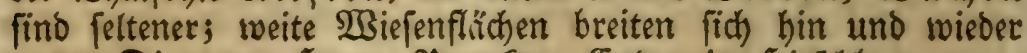
aus. Die vom Sherrm Sroreftor (Ender in Scirjhberg ange= ftellten meteorologif(hen Seobachtungen find zu Eurze Seit an= geftellt, um aus Denfelben fhon allgemeine Repultate ziehen zu Eönnen.

Das Bsebiet, Das obere, weite Boberthal, die Flora des Eentralgranits, entfält 699 arten in 333 Gattungen und 81 Familien. 3 פुflanzenatten find ifm vor ben übrigen Şebieten eigenthümlich. 


\section{Das (Sebiet von Supferberg (K.)*)}

umfäpt nur ben Eleinen SRaum von einigen Sieviertmeilen unঠ

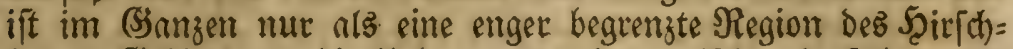
berger (S)biets als bie Hebergangstegion zrwifchen Darfelben uno Dem des Mittelgebirges in Dem. J̧öhenraume von 13-2500', 200 ' unterbatb uno oberhalb Supferberg anzufehen, Der böchfte

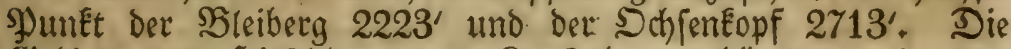
Sebiete von Şirfoberg uno Supferberg gebören zu cintander und ergünzen eimander als von bem Riefengebirge abhängige, niebere (stieber, als bie Bergregion des Rielengebirges.

Die ben Untergrund bildenden Felfenmaffen werben

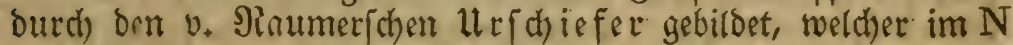
Dns Şirfdjberger Thal begrenzt uno Durd) Die SupferEies =

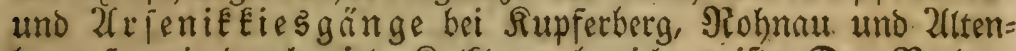
berg, jo roie Durch, viele Sallelager bezeichnet ift. Der: Boden ift gröstentheils fruchtbar uno mit vielen einzelnen. Steinen un=

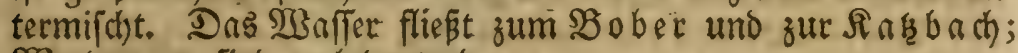
Waloungen fino unbedeutend.

Das Giebiet enthält 636. Spflanzenarten in 309. (S)attun= gen und 77 Familien; 7 Pflanzenarten und 2 Battungen fino: Dem Sebiet vor ben andern Ssebieten eigenthümlich.

\section{Das (Şebiet von Salzbrum (S.) **).}

Die Sröße beffelben läßt fich wie feine SBegrenzung nicht: befitimmt angeben, indem die Funoorte. Der Pffrngen nicht ver:

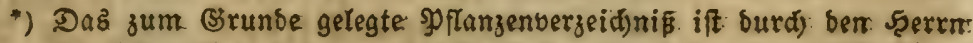

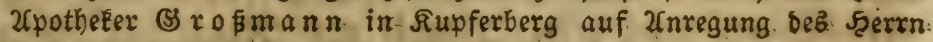

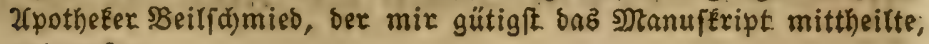
entworfen.

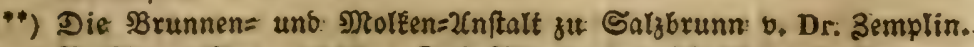

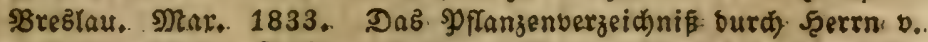

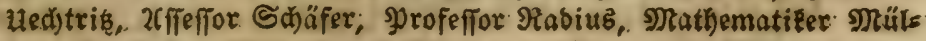

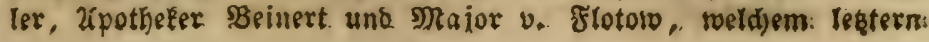




\section{8}

merft find. 2(m natürlich) ften und wahrfcheinlichfifen förmen wir bie beiden Flukthäler Deb Salz= und Şellenbaths an=

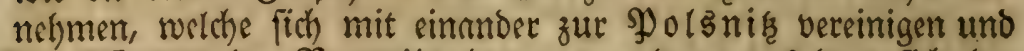
im W von ber Sergreibe begrenzt werden, aus bem fich ber Şo ch wald 2588' und ber Şo ch berg 2166' erheben unt dem

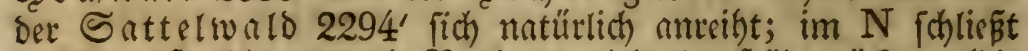

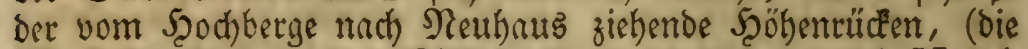

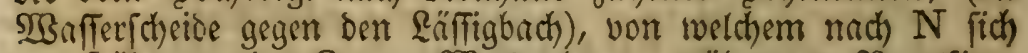
cin Scöhenzug im $O$ von $\mathfrak{B a l b e n b u r g}$ vorüber zur $\mathfrak{B}$ ogelfippe atmo weiter zieft und bie Ogrenze bildet; im N fenEen fich bie vor

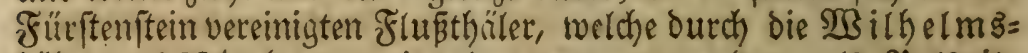

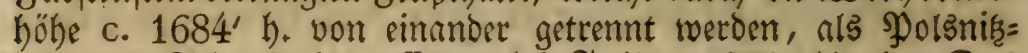
that zur ভahweionifer (Ekene bei Freiburg 870' himab. Das Gebbiet ber Flora würde Demmach aus ber c. 870' hohen Efbene in Den böd)ften \$ounften bis gegen 2600' amiteigen und po Die

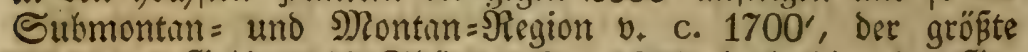

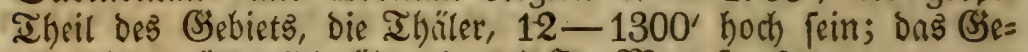
biet aber wiltroe fid) über $4-5 \mathfrak{2}$. M. erftecten.

Die Felsెmaffen des Gebiets fino fehr mannigfaltig. Die fübliche Schilfte wiro bis gegen 2fitwaffer und Neu=Salzbrumn burch bas Steinélylengebirge gebildet, an welches fich im NW das nördliche Uebergangsgebirge, hefonders Rons glommerat und Ssrautwade, im NO eitue Eleime Santhie bes nörolichen ungebirges von ber (Eule her anreiht. Șm lekptern liegen RalÉluger, aus dem erftern f́teigen bie fteilen Sูorphyr

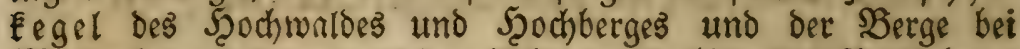

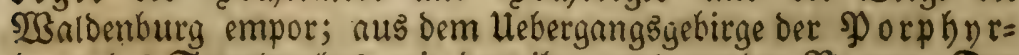
berg bes Satteltroaldes mit den ihn umlagernden Bergen. Der Boden meift fruchtbar; die Berge find alle berwaldet aber zum Iheil auch bis hoch binnuf bebaut. Fichten und $\mathfrak{T}$ nnnen,

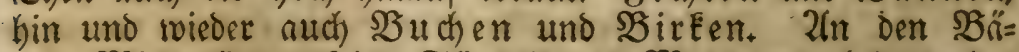
d)en 2 Biefenfin̈ume, Eeine Sümpfe und Moore von bebeutenber

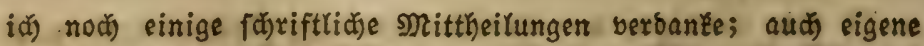

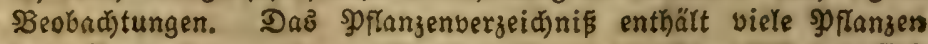
ser nähern Lungegend von હ̧arlottenbrunn; mehrexe \$g fanzen find 3tweifertyaft. 


\section{9}

2utsbehmung, bie Riefer felten. Die flimatifhen $\mathfrak{B}$ erbältniffe

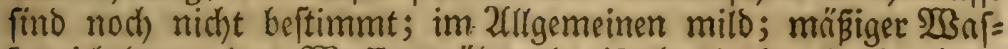

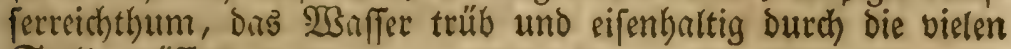
Stollenwäffer.

Das Siebiet, bie bergumfäumten, weiten Borthäler bes 2Ba a loenburger Bjebirgs umfaffend, enthöilt 796 pplanzen= atten in 351 Sinttungent und 82 Familien. 12 spflanzenar= ten find Dem (sebiete vor ben übrigen eigenthümlich, mehrere angeführte 2 (rten fraglict) ooer aus einem ertweiterten S̈ebiete.

\section{Das Ģebiet von (5Garlottenbrum (Ch.)*)}

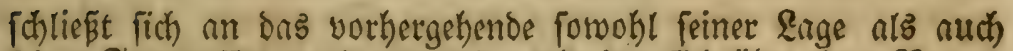
feimer Felsmaffen nach an und verbreitet fíd über einen Raum,

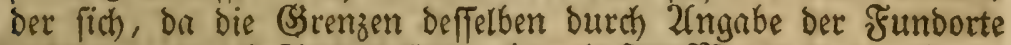
nicht genauer beftimmt fino, 4-5 2. M. groß annehmen

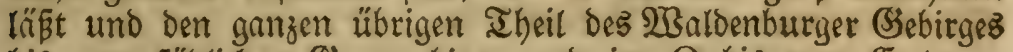
bis zum rüblichen Sistenzgebirge und im O bis zum Eulenge=

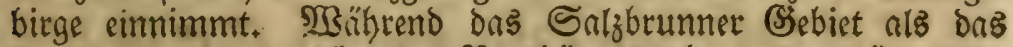
Der weiten, bergumfäumten $\mathfrak{B o r t b}$ ăler zu benemnen toäre, ließße fich bas (Sharlottenbrummer als bas Sesebiet bes von vielen

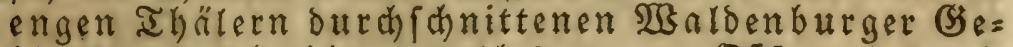
birgslandes bezeidynetr, welches nur am SPftaum bas wei=

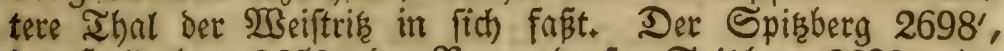

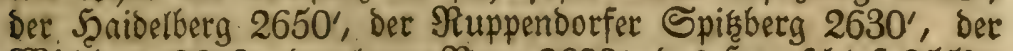

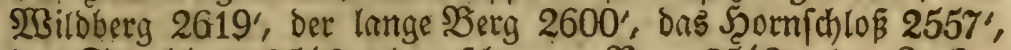
Der: Storchberg 2546', Der fotworize Berg 2543', Der 3udfer=

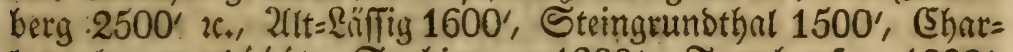
lottenbrum 1444', Sophienau 1300', Innnfaufen 1200', Sทnin 1128'.

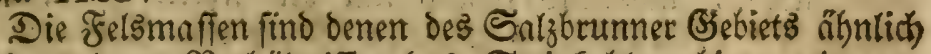
nur in anderm SBerbültniffe; Dả SteinEoblengebirge tritt zu=

") Stady cinem vom. Seerrn 2(pot). SBeinert für ben Serrn 2(poth. Beilfdimied angefertigten uns yon biefem mir gütigft mitgetbeit=

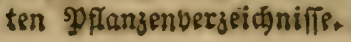




\section{0}

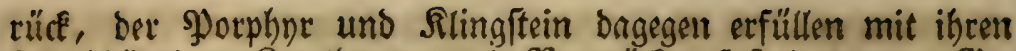

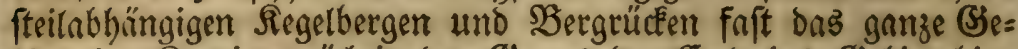
biet, im $\mathrm{O}$ tritt mäd)tig Der (S)neus Der Fule ins SSebiet hitt= cin, befonders im $N$ bei Annnu. Die 2reÉerf́rume gröpten= theilz fruchtbar aber fteinig, bin und wieber ourch ben rothen Eantitein rothgefärbter SBoden, an vielen Stellen nackte Fel=

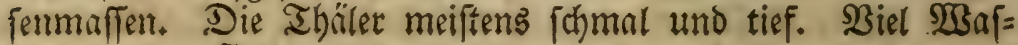
jer an allen Drten.

Die Berglehnen meift mit Fichten und בannen bicht be= wachfen, an ben untern 2abhängen mit Raubholz untermifht, bie engen Flufthäler mit $\mathscr{B}$ iefen, die minber fteilen Scöhen mit 2fectern bedecet; an einzelmen Stellen moorige 2 Befen, nir= gends ftefende Serwäifer von einiger Sebeutung. Flusgebiet

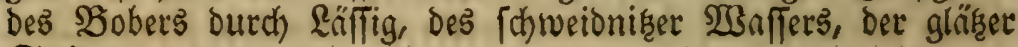
Steine.

Das Ssebiet enthält 750 offenblïthige Pflangenarten in 331 (3attungen uno 79 Familient.

\section{Das (šebiet von Beutben an ber $\operatorname{Dber}(B .)^{*}$ )}

ift bas Bsebiet ber an bie weftliche Scaibe grenzenden, von bem Soerthale burchfurchten, von den Şügeln bei DalEau unter= brochenen Ebene, von abrwech felnoem, aber mehr mittelmäBigem

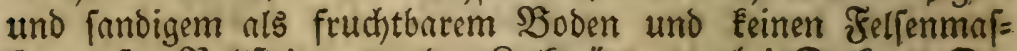
fen außer Roufteinen $u$. Den RalEtrümmern bei DalEau. Der JalEauer Şöhenzug 1/2 פ⿹ im $S, 1$ M. im $S O$ und $1 \frac{1}{2}$ M. im SW von Seuthen, alfo von $O$ nach W ziebend, beu=

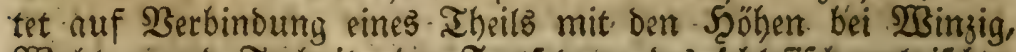
MBohlau uno Irebnifs, ber Fortfercung bes ichlefifh = polnifd)en Eanorückens, nnoerfeits füblich mit ben leçten Scöhenverbrei=

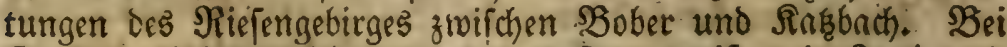
Dalfau erhebt ec fich am bebeutendften, meift mit $\mathfrak{a}$ aub uno

-) Nach einem yon meinem um SPflanzengeographie bodjuetoienten Freunbe Srrn. Beilfdimieb in 5hlau. angefertigten. Pflanzenver=

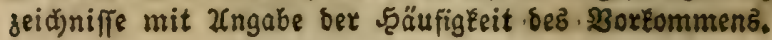


Riefertwald bewathfen; bort wact)fen Sanicula, Asperula, auf Eanounterlage Ornithopus, Genista germanica, Astragalus arenarius, Lycopodium complanatum. $23 e i t e r$ [üb=

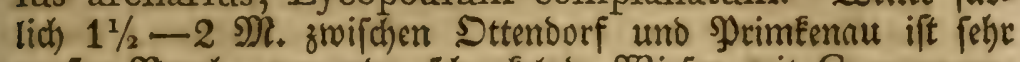
grober SBruch; wogende, ¡chaufertnde $23 i e$ ent mit Comarum; nate ber Sprotte $1 / 2$ M. v. Norimfenmu ift Utricularia intermedia. 2(uf ber rech)ten Soerfeite ift mehr (Fbente (nur Sarolath fteht auf einem Şügel); weite SBSiefen, viel $\mathfrak{L a u b}=$ wato, bejonders (Fichen, Dod) aud bei Rölmchen $1 \frac{1 / 2}{M}$. im NW breitent fich SBucten aus nabe Der SDer; zwifthen Şo=

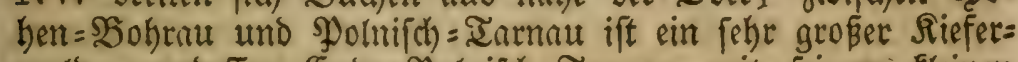
wald, an beffen. Endo spolnif See liegt; bier wachjen Cyperus flavus, Stellaria uliginosa, Eriophorum angustifolium, Carex flava, intermedia, Utricularia minor, Epilobium palustre; nörolich bavon

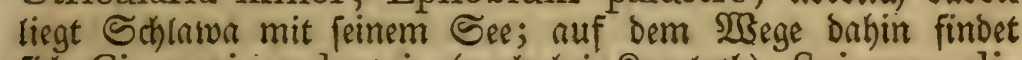
(id) Cineraria palustris (audt) bei Sinro(nth), Spiraea salicifolia, Berberis, Spartium scoparium faft walbweife nebft Corynephorus canescens sveftlich $\mathfrak{B}$ euthen nad) $\Re$ Reufalz hin im fantigen Riefertualde. Das Sebiet ift.c. 62 . 2 .

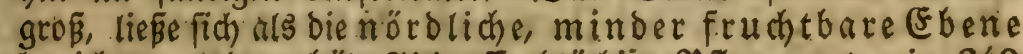
begeidjnen - und entgält 711 offenblüthige \$flanzenarten in 340 (Sattungen uno 84 . Familien.

\section{Daz Ssebiet yon Sollau (O.)*)}

ift bas Ssebiet ber fruditbaren Doerebene mit faft gar kei= nen 2unböhen, bejonbers in ber Richtung nach Strehlen bin, hinter roeld)er Striot fich erft Die erften 2inböben bie Strehl= net Serge mit iften Erranitmaffer ergebent Doer= und Sh = laugebiet mit gröBtentheils fruchtbatem, hin uno roieber von Sand unterbrochenem Boben; him uno vieder ftefende Genoúl= fer und Sümpfe, feltener Noors am redjen ufer ragt bie

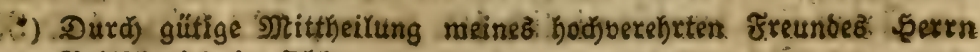
Beilfamies in SJ) Iau. 
oberiftlefithe Staibegegent in bas c. 10 2. M. grope G̈ebiet; fonft (Eichentorloungen uno \&aubgebüfche.

Das Gebiet hat auf bem linken Soerufer renig $2 B a l b$, um bie Stabt weithin faft nur Tabaffelder, weiterhin Geettei= Debau. Die Berge bei Strehten baben oben nur Actaea, Asperula odorata, einige $\Re u b u s$ formen uno Trientalis; nuf ber rechten Doerfeite vire (Eichenwald an ber Doer, weiters=

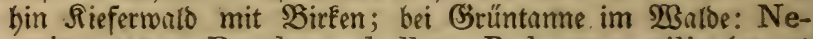
ottia repens, Pyrola umbellata, Rubus saxatilis (nur 1 (Ex.), Circaea alpina, Calla, ferner Mercurialis perennis nur 400-430' über oem MRere; $1 / 2 \mathfrak{2}$. Ginter Brüntanne, noch mehr nörolich bei Giarfuche mebrere Teiche und fumpfige $\mathfrak{B}_{\mathfrak{r}}$ fer, no Utricularia intermedia, Cardamine parviflora, Carex paradoxa, im Errenwaloe baran Lycopodium anotinum u. Selago, unter Riefern Hydrocotyle u. Lycopodium inudat., Chara flexuosa, vulgaris $\mathfrak{u}$. pulchella, Eriophorum vaginatum, Pyrola uniflora, minør, rotundifolia; im Schatten, wo fich \&aubroald einmiicht, mehrere Jungermanniae. 2(uf Dem rechten SDerufer übethaupt überall Frtiche, fehr wenig 2anböhe, auch auf ber linken Seite nur bei (S)on nadte Şügel, bei Bedlis mit Rieferwalb bebedt. 13/4 (M. nad) WNW von Dhthu bei Fottroik Biscutella, Phleum Boshmeri, Iris nudicaulis, Arabis sagittata;, Salix acuminata, alleb in mit $\mathfrak{R} a u b h o l z_{\text {gemifathem }}$ Rieferwalbe, in bem

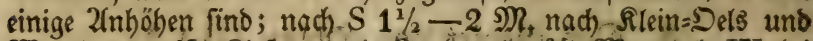

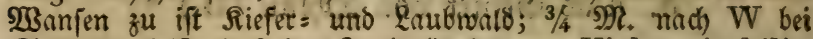
Seifersoorf ift ein Eleines \&aubwäldochen, wo Viola mirabilis, Isopyrum, Asarum, Ligustrum (viêlecicht bon früberm $\mathfrak{I} \mathfrak{n}=$ bau?2), Eưphorbia pilosa, Polygala amará, var. uliginosa gans ftach obne 2Inböbe, ebenfo Spiranthes, Gentiana Pneumonantha, Salix repens uno Aconitum variegatum.

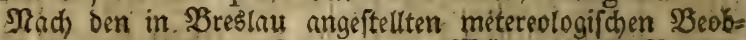
aditungen v. 1820-31 ift der mittlere. 2 Bärmegrab in Brezlaus $+5,75^{\circ} \mathrm{R}$., der böchife $+21,8$ bis $25^{\circ}$ faft in Der Mitte bez Juli, Der nieoriggfte -6 bis $22,5^{\circ} \mathrm{R}$. im leẹten Drittel bes Jamuar;

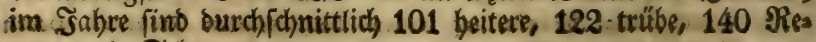
gen= und Sdneetage. 


\section{3}

Das B̧ebiet enthält 819 offenblütbige Yflanzenarten in 369 Ëattungen und 88 Familien; 9 Sp flanzenatten eigen= thümlidh vor ben andern Eebieten.

\section{Daz (şebiet von $\mathfrak{s}$ oblau (W) *)}

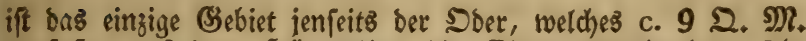
umfäst, auf Dem Scügeltreihen bie Ebene unterbrechen, fehr fruchtbarer 2 Boden mit mittlerm und mit Sand, Raubroaldun=

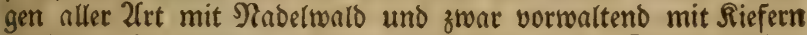
wechfeln; in welchem einzelne Raltetrümmer bei Şerrnmotfchel= nif auftreten, fhlammige Sümpfe und Moore an trodennen Sand fich anreiben, viele Eleine PBafferbetälter fich vorfinben.

Das Gebiet, welches im $S$ von Den Eichentwaldungen bex Sober, im $\mathrm{N}$ von ber Fortfę̧ung ber Irebnizer Şüget begrenzt und zum Theil erfüllt wito, ließęe fich als Das Şebiet ber hüg=

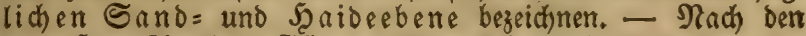
vom Şrn. 2(potheker Şünzel $1830-35$ angefítellten meteorolo= gifchen $\mathfrak{B}$ eobachtungen ift ber mittlere 20 ärmegrno $+7,18$, ber böchfte +22 bis $28^{\circ}$ im Iuli und 2(uguft, ber nieorigfte im samuar und Februar -20 biz $23^{\circ}$ R. $10-25$ Ěeroitter iährlich.

(5z enthält 789 offenblïthige- \$pllanzenarten in 268 Sat= tungen uno 83 framilien; 8 sp flanzenarten fino eigenthümlid).

\section{b. Die einzelnen Esebiete vereinigt.}

Fraffén wit alle biefe Bebiete zufammen; fo ftellen fie uns

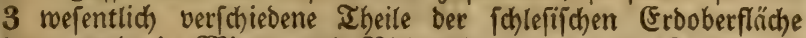

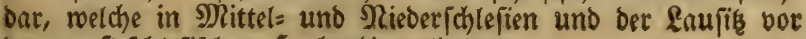
Dem weft=[chlefif chen Scodogebirge liegen:

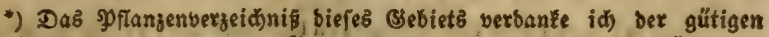

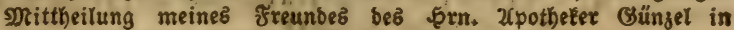

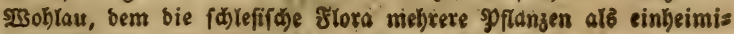
fäe ßürger verbankt, 


\section{4}

I. als Fbene,

II. als Şügelland,

III. als $\mathcal{S}^{2}$ or $=$ und $\mathfrak{M}$ ittelgebirg.

I. Die Ebene, die Region ber Ebene it:

1) ihrer $\mathfrak{L} a g e$ nady

a) Die bem Ģebirge nähere, brrum höhere (Ebene $365-700^{\prime}$

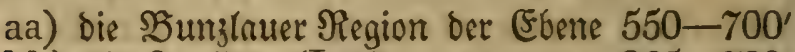

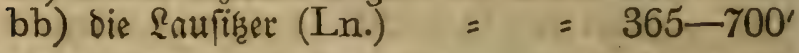

b) bie bem Bsebirge ferme ober bie tiefe sober= ebere $190-500^{\prime}$

aa) Die (übliche, obere, bie Shltauer (5)bene 4-500!

bb) bie mittlere, bie 230 hlauer (5bene 250-400'

cc) bie nörolidfe ober niedere, Besuthner (Ebene $190-250$.

2) \$hrem Boden nach:

a) Die frudtbare Ebene, Dhlau mit 2 sohlau zum Theil.

b) Die mäsig fruchtbare Ebene, SBeutben uno Wohlau zum Theil:

c) Die fandige ober Şaibegegend, Bumzlau (Bz.) unb bie \&aufith (Ln.)

3) ihree D berflä the nad):

a) Die eigentlidhe Ebbene, Beutben u. Şblau uno. Laufie zum I I heil,

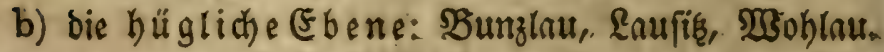

II. Dิaร รૃügelland, bie regio submontana,

1) nad ber Erhebung und ber Maffe

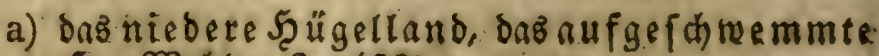
Sc., 20 ohlau $3-400^{\prime}$

b) bis höhere Sุügetland 


\section{5}

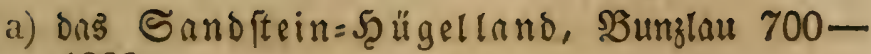
$1000^{\prime}$

b) Dab granitifhe und bafaltifhe f̧ägelland 7-1200' h. Der $\mathfrak{E a u l f i g}$

c) Dass Şügellatid des Steinéblengebirges, Sallbrunn.

III. Das Ģebirgsland die regio montana. Das $\mathfrak{B o r =}$ und Mittelgebirge Des weftlichen ober böbmifch = [chlefi=

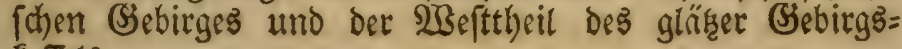
Eeffels.

1) $\mathfrak{R}$ a d) $\mathfrak{E a g e ~ u n d ~ ( E r b e b u n g : ~}$

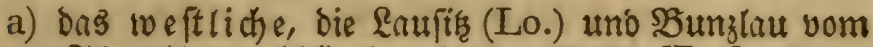
STergebirge abbängige $\left(10-2788^{\prime}\right.$ [Bo.], $13-$ 3379' Lo.)

b) Drs mittlere, Şirfd)berg, Jupferberg 10-2099'

c) Das öftliçe, Ealzbrunm u. Sharlottenbrunn 11$2698^{\prime}$

d) Dnร füböftliche, bie Şeufcheute 968-2831',

2) nach ben Maffen:

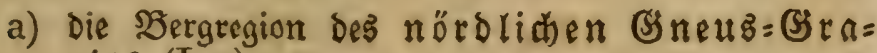
nits. (Lo.)

b) Die ber vereinzelten, bafaltifhen Bergeup= pen, (Bz.)

c) Die bes Sentralgranits (Hb.)

d) Die bes nörblichen $\mathfrak{u}$ ridhieferzuges (K.)

e u. f) Der öftlidyen গoorphrekegel (S. u. Ch.)

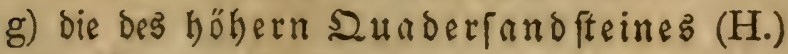

3) $\mathfrak{R a c h}$ ifrer $\mathfrak{F}$ usbreitung

a) Die Serge Der Şügelregion, bie untere Bergregion

aa) Der bem Şauptgebirge näherm, bie Salz= 


\section{6}

brunner (verbältnismäsig reicher an Sub= alpinpflanzen),

bb) Der Dem Şauptgebirge fernetn, bie $\mathfrak{B} u n z=$ Iauer (Êne Subalpimpflanzen) -

b) bie İhäler ber Bergregion, Şirfhberg, Sulz= brunn, Sharlottenbrunn, (fie enthalten verhä́ltnis= mäßig mehr Sergpflamzen ats bie böhern Berge Der Şüge(region),

c) Die eigentliche ooer höhpre Bergregion ber bicht bei einander ftehenden $\mathfrak{B e r g e}$ ober der von weni= gen Thïlern unterbrochemen böheen Berge, Supfer=

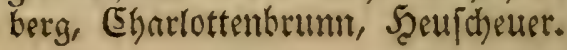

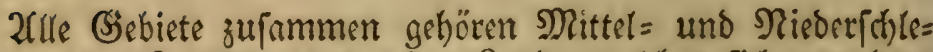

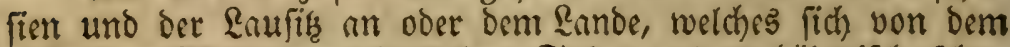

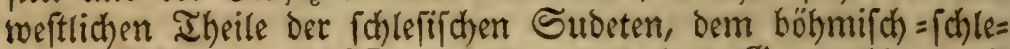
fidden Gebirge nach N ausbreitet und bas Ssrenzgebirge mit

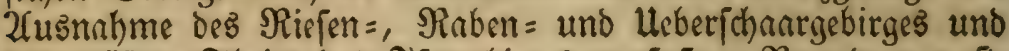

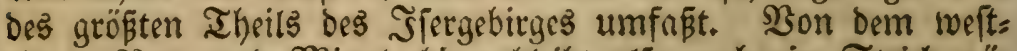
lichen S3or = und Mittelgebirge bleibt alio noch ein Strid) nä= her zu beftimmen übrig, Der fich vom STergebirge zwif đen Remnit uno Sueis nach $N$ zum Mitteigebirge und zur Süb= grenze bes Sumzlauer Siebiets uno von hier die Mittel = und

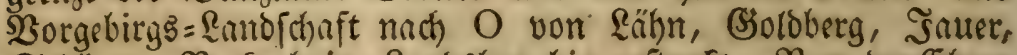
Schonau, Bolfent,ain, Ranoshut hin erftrect't. Son ber Ebene blieben aber noch bie meiften Theile berielten näher zu beftim= men und zu vergleichert, wozu mir aber Eeine Belegenteit ge= worden ift. Diefe Unterfucthungen aber find eten fo wie bie über Die pflamzen=geographifhen Serhälltniffe ber sinzernen ver=

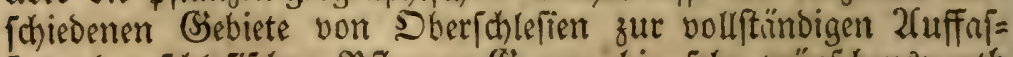
fung ber foblefiidhen SPflamzen=(S3eographie fehr wünichenswerth und liesen fich bei bem in Sallefien weit verbreiteten Eifer

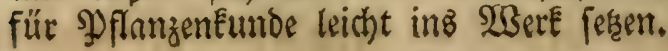




\section{Die \$sflangen in Den werichiebenter Gebieten.}

Die in ben verifhiedenen singelnen SGebieten und in allen Giebieten zufammen vorkommenden Pflangen laffen fid aus verfohiedenen (SefichtşpunEten auf= uno zufammenfaffen.

Suntidifit wollen wir nut 1) ihre $3 a \mathfrak{l} l, 2)$ ibre $\mathfrak{B e r}=$ breitung, 3) ifre Dertheilungsweife näber ins 2luge faffen uno aus eimanoer zu fegen verfuchen.

\section{Die Babl ber Şflanzenarten in ben (s)bieten.}

Die 3ahl ber SPflanzenarten in einem beftimmten Gebiet ift von ber größern uno geringern Nanniģaltigeeit ber örtli=

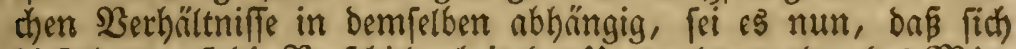

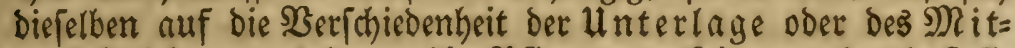
tels bezieben, aus benen die झूflntzen entEeimen, oder baß̧ fie von ben umgebungen, unter benen fich bie DPflanzen entwideln, beftimmt werden. Den großen Einflü ber Unterlinge bat $\$ D B=$ wald Sceer in feinen 2 egetationšverbältniffen bes fïböftlichen

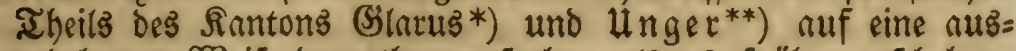

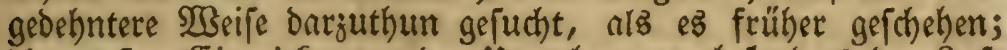
Die großsen Finwirkungen der umgebungen, befonders ber \&uft

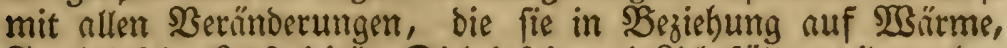
Feuchtigkeit, Eleftrizitüit, Didytigkesit uno Richtfülle erleidet, eben fo ben (Finflus des Sodens, wenn auch) nicht in ber 2 (ušbeh)= nung wie Sceer, bat fobon früber Schour im 3ufammentange

*) SBeiträge zur Şfanzen=(S̈eographie von \$zిwals sceex ober aud)

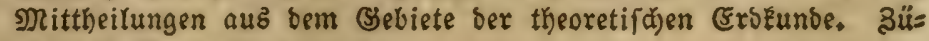
rich. Strell. 1836.

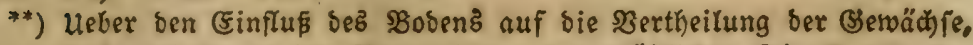

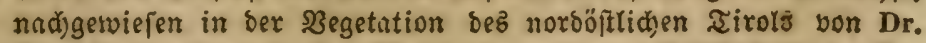

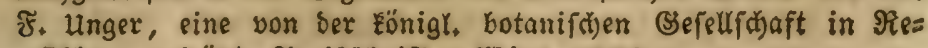

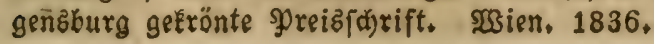




\section{8}

nachgewiejen. *) (Fine vollftintoige 3ufammenftellung und 2(n= oronung biefer $\mathfrak{B e r b a ̈ l t n i f f e ~ b e h a l t e ~ i c h ~ m i r ~ f u ̈ r ~ e i m e ~ a n d e r e ~}$ (S)elegentheit vor; 2fnoeutungen bazu finden fich in meinem Reitfaden ber Elementar = Erofunde oder die (Frofunde in it)ren Girunobegriffen uno in ifren Bejiefungen zur Natur = unt MenichenEunde. SBunzlan, 1836.

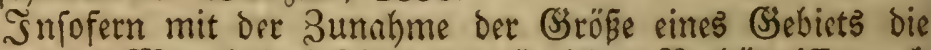
Menge uno Mannigfaltigkeit biefer örtlichen $\mathfrak{B}_{\text {erbältniffe aud }}$ zunehmen Eam, ift aud mit berfelben bie MöglithEeit ciner Sunahme ber SPflanzenartenzahl in größern (S)ebisten gegeben. $\$ b$ aber, wie Sibation annimmt**) oder folgert, citte ber $z u t=$ nef)menden (S)röße dor (Sebiete entiprechende Sunathme der DPflam= zenartent ftatt findet, tonge ich nid)t zu entid)eiden. MSenigitent? habe ich bei ben sinzelnen, vorliegenden fohlefird)en (Bebieten

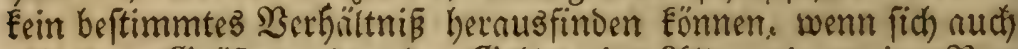

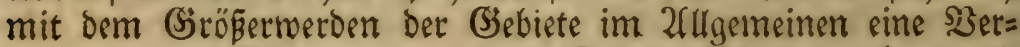
mekrung ber SDflanzenarten herausftellte. (5s fino aber auch bie borliegenden (Sebiete zu Elrin, um Sunabme ber 2(rtenzahl

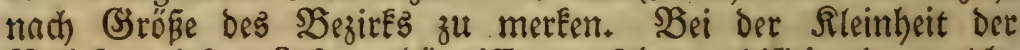
Sezirte roirten Rokalverbältmiffe zu fehr modifizirend, weldhe Regestere in grosen (s̈pbieten fich ausgleichen. Der 2uffaffung beftimmter 3nhlenverhältniffe ber 2reten in verfhiedenen Bsebie= ten ftellen fich mehrere erft zu bereitigende Scinderniffe entge= gen, wenn man nicht in (S)fafhe Eommen will, falfche Rejul= tate und $\mathfrak{B e r h a ̈ l t n i f f e ~ z u ~ e r h a l t e n , ~ i n d e m ~ i n ~ u n j e r e r ~ S e i t ~ b e r ~}$ Sertrennung und Berfpaltung biefe fich aud) in bem (Sebiete Der DPflanjenEunde geltend gemacht und fo eine oft unnöthige Serfpaltung einzelner Pflanzenarten in eine größere oder ge= ringere 2(nzahl vou felbitftündigen 2(rten herbeigeführt haben, welche nach genauerer SBetrachtung im lebenden 3uftanoe, und nicht nach getrodtneten eimzenen Snoiviouen, fith beffer und

*) (Srunbjüge einer nllgemeinen Sgfanzen = Spograplie von Sdoutv.

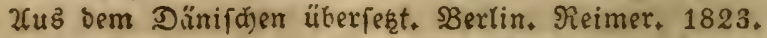

**) Sosmere. über bie geographifde \$Berbreitung ber britifden (Spe

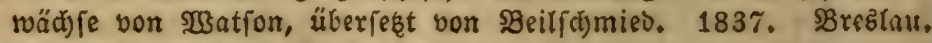
XX. uno 262 . 8te $\subseteq . ~ 31,32$. 


\section{9}

natürlicher als 2(bänderungen uno 2(barten barftellen. Um ben baraus entftehenden Srrthümern auszuweid)en, ift es nothwen=

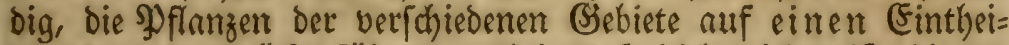

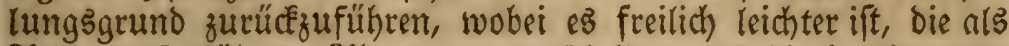
2(rten aufgeführten 2lbarten nuzzufcheiben, als bie in sinjelnen Zrten wirflidy vorbandenen, verichiedenen 21rten herauşufin= Den. STm 2ullgemeinen wurbe mir bie 3u[ammen|tellung ber Utrtenzahlen aus den verichiedenen (d)lefird)en (S)ebieten leicht, indem biefelben grö̈tenthsils nach ber trefflichen, fhlefif chen Flora von 2 Bimmer uno GrabowsEn) entworfen waren, nur hin und wieder, bejonders aber bei ben SPlanzen ber Raufizer Flora war eine Eritifche Unterjuchung nothroendig. Ulm für allgemeinere $\mathfrak{B e r g l e i d j u n g e t t}$ ber folefifchen Flora mit ber ge= fammten beutichen und mit ben einzelnen Iheilen bes prenki=

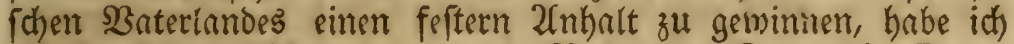
Die fdlefift)en SPflanzen auf bie Norm Der Synopsis florae germanicae et helveticae auctore Koch, fo roeit fie er= idhienen, in Framilien, Şattungen und 2rten gebracht.

STiernad) ftellt fich Das 3athlenverbältnis der SPflanzenarten in ganz Shlefien uno in ben einjelnen Siebieten in nach folgender $\mathfrak{I} a$ belle bar. WBenn bie hier angegebene 2rtenzahl ber in ganz Sehle= fien wildwach fenden, offenblüthigen PD flanzen von ber Gejammtjum= me berfelben in ber flora Silesiae v. 2WB. uno (Sir. abreid)t, fo hat Diefes feinen Scauptgrund vorzüglich Darim, baß in ber lefętern ourch einen 3̈̈hlungsfebler (von 1031 an) Die Summe um 100 zuviel angegeben, überbaupt mehrere Spflanzenabarten, be jonders die G̈at= tung Rubus als 2(rten aufgefít)rt find. 2(ud) bie von mir in mei=

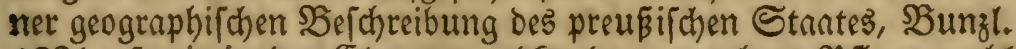
1831, fowie in ber Elementarerofunde angegebene PPflanzenzahl weicht bavon ab, indem auch bier bie 2(rten nicht nach Foch geord= net und auch bie neu aufgefundenen Bürger unferer Flora, wor= unter auch einige Bunzlauer, nicht mitgezaihlt worden find. Das Љerzeichnis ber anderwäts in Sd)lefien reu aufgefundenen, in

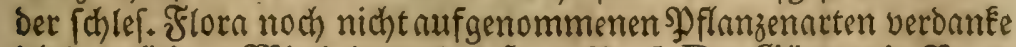

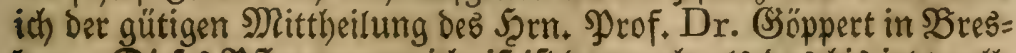
lau. Diefes Sp flanzenverzeidjniß ift bemnach alb bas bis jefst voll= ftändigite ber falefifchen Flora anzufehen. 


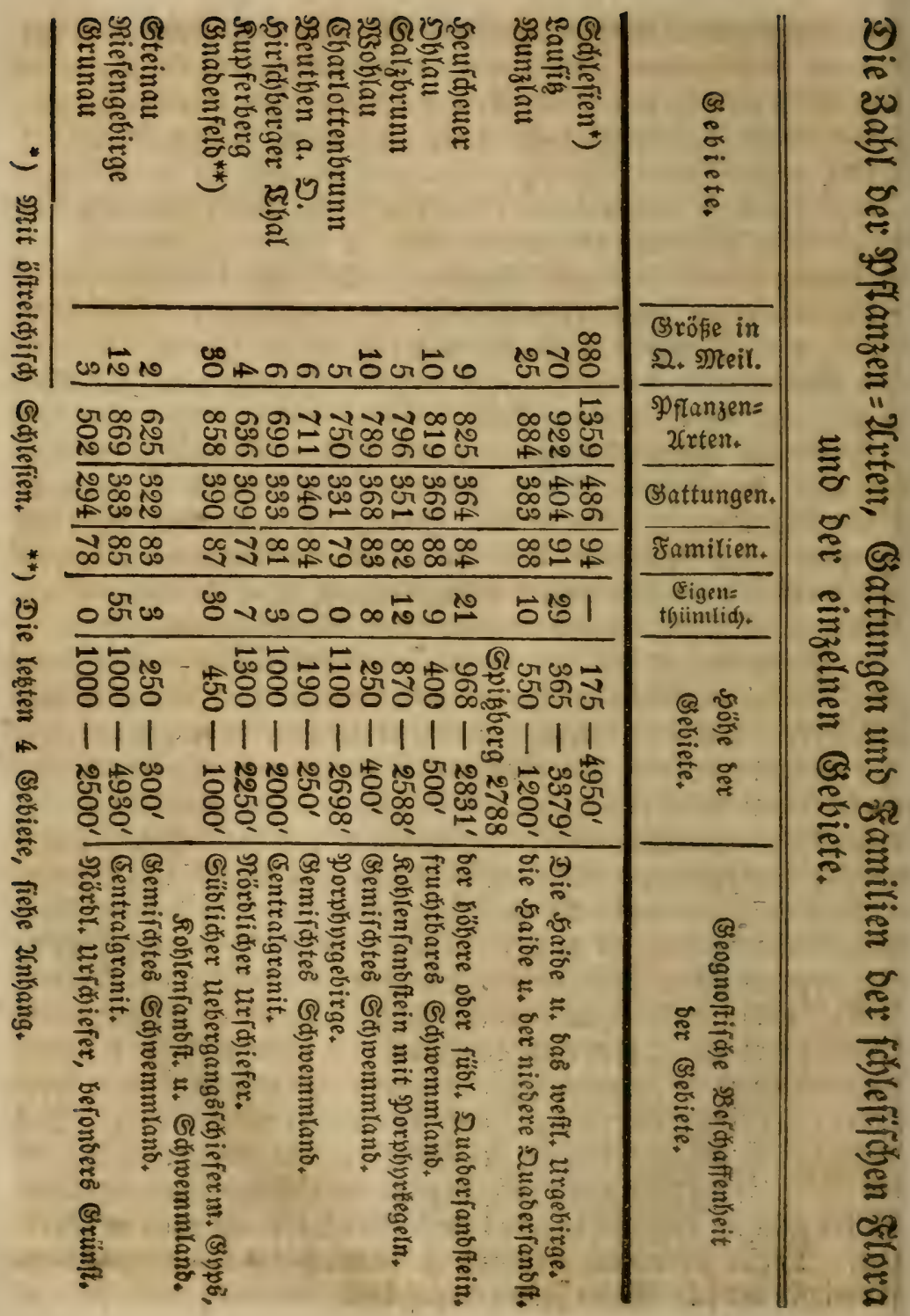




\section{1}

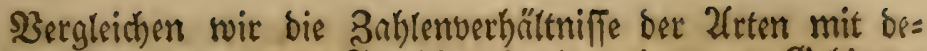
nen ber Ënttungen und Familien in Den einzelnen SBebieten,

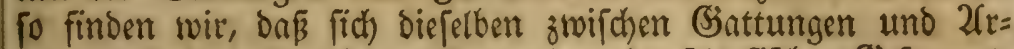

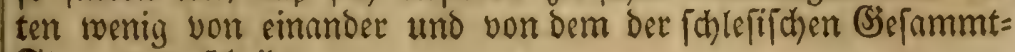
Flora unterfheiben.

Stellen mir für bie Ganttungszahl 1, fo ergiebt fich) für bie 2 rtenzahl in ben sinzelnen Gebbieten:

Sdhlefien Bz. L. H. Ch. S. O. W. Hb. Bt. $\begin{array}{lllllllllllllll}2,79 & 2,31 & 2,28 & 2,27 & 2,27 & 2,24 & 2,23 & 2,17 & 2,10 & 2,10\end{array}$ $\begin{array}{cc}\text { K. } & 211 l e \\ 2,06 & 2,56\end{array}$

voer es betragen in ben einzelnen (s)bieten im Mittel bie 2fr= ten $2^{1 / 4}$ ber (S)attungen; Diefe enthalten im Mittel $2^{1 / 4}$, in

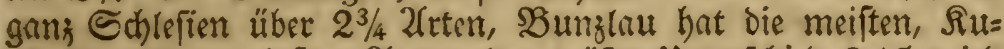

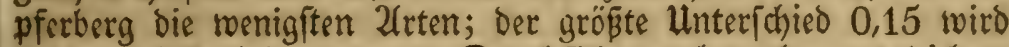
(ich) vielleicht bei genauerer Durdhficht noch mehr ausgleidjen, gewis trïgt aber bie höhere Rage von K. Fhon viel zur Ber= minderung ber 2(rten bei; bei oen übrigen SBerggebieten tritt bie Fiegion ber Ebene uno Der Shügel fajon mit eim. İn al=

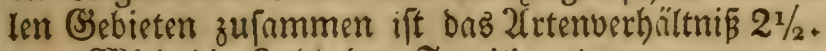

W3ito bie Zahl ber Familien 1 angenommen, fo ift bie 3abt Der 2(rten in

Sd)leften L. Bz. H. S. W. Ch. O. Hb. Bt. $\begin{array}{lllllllllllll}14,40 & 10,09 & 10,00 & 9,77 & 9,66 & 9,46 & 9,44 & 9,28 & 8,58 & 8,29\end{array}$

K. 2ule

$8,21 \quad 12,82$

ober es fommen in ganz Sd)lefien burchfonnittlich 14,40, in allen 10 Sebieten 12,82, als Marimum Der einzelnen Sebiete über 10, als Mebium $9 \frac{1}{2}$, nls Minimum $8^{1 / 5}$ शflanzenart auf eime গুflamzenfamilic. Es verfteht fich von felbft, bas ber Unterfdbied Der Berhilltniffe bei ben einzelnen S̈ebieten zwifchen Framilien und 2frten (wie fie jef̧t fiffematif umgrenzt werben) größer als zwifhen (S)attungen und 2(rten ift; boch) nähetn fich) beibe Differenzzahlen, wenn wir fie mit ben ihnen entfprectien= ben 2(rtenzahlen im Marimo 2,31 uno 10,09 vergleichen. 


\section{2}

Dons $\mathfrak{B e r h a ̈ l t n i}$ ber Santtungen zu ben Familien ftellt (iid) aber alfo, bie Familie burdh 1 bezeichnet:

$\begin{array}{cccccccccc}\text { Schleften } & \text { W. } & \text { L. Bz. } & \text { H. } & \text { S. } & \text { Ch. } & \text { O. } & \text { Hb. } & \text { K. } \\ 5,15 & 4,42 & 4,41 & 4,33 & 4,31 & 4,25 & 4,16 & 4,10 & 4,8 & 4 \\ \text { Bt. } & \text { 2alke } \\ 3,95 & 5\end{array}$

Es Eommen alfo butchichnittlidh in Sd)lefien auf 1 Familie 5,15 Bsattungen, in allen (Setieten zufammen 5, in ben ein= zelnen Sebieten als Marimum 4,42, als Minimum 3,95 Santtunger. Uunterforied barum 0,47 ober c. 1/5 bes Mari= mums; aljo faft baffelbe Berbältnis roie bei dem Unterfojiede

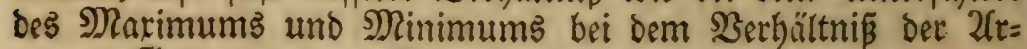
ten zu Ģattungen und Familien.

2fm günifigften ftellen fich biefe 3ahlenverhältniffe bei den

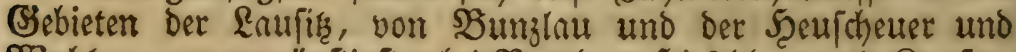
2Boblau, am ungünftigften bei Beuthen, Scirfebberg und Rupfer= berg bar. Es nimmt bemnnd) im 2arlgemeinen bie 2(teten= und

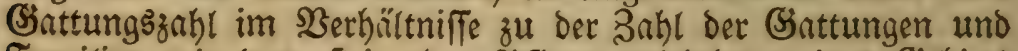

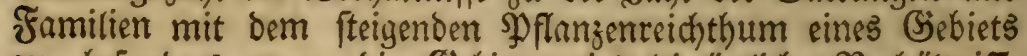
zu, bejonders wenn bie Gebiete vielertei ortliche Serbältniffe zeigen.

- Unter allen Ģebieten fat das der Raufí ben gröBten abjotuten Pflanzenreidsthum. Bei ithm vereinigen fich aber auch alle

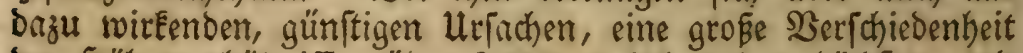

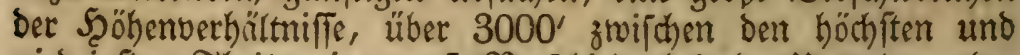

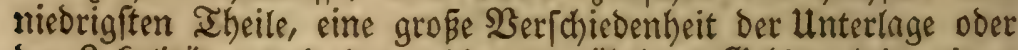
Der $\mathcal{L}$ Eaklitïten und Dann bie alle übrigen Şebiete bei weitem

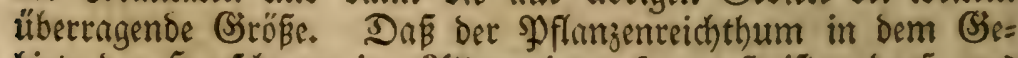

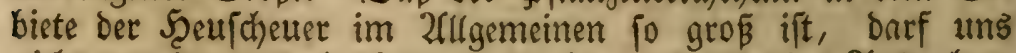
nicht wundern, noch vielweniger aber uns als eine 2(usnahme

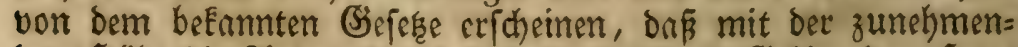

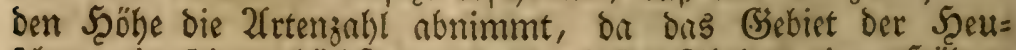
fcheuer in feimen böchften uno niebrigiten Iheilen einen Scöhen= unterichied von faft $2000^{\prime}$ zrigt, wemm auch ber gröste I Iheil

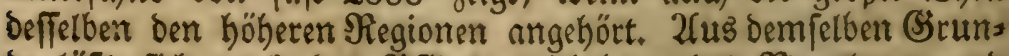

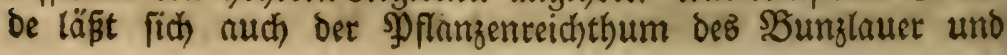




\section{4}

trachtumg wenig zu beachten, weil Die Entfermung Der (Sebiete fowohl von $\mathrm{N}$ nat) $\mathrm{S}$, als yon $\mathrm{O}$ nath $\mathrm{W}$ zu unbedeuteno ift,

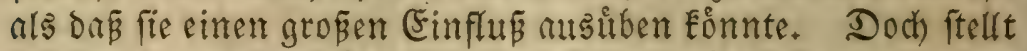
fich bas lestere Berbåltnis in der I hat noch wichtiger als das

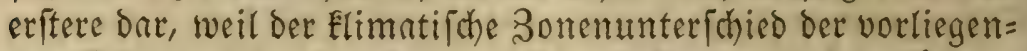
Den Gsebiete, Der ofnedies mur fehr unbedeutend fein Eonnte,

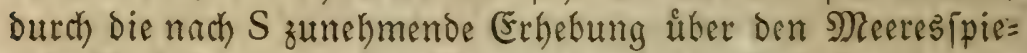
gel aufgehoben wird, butch bie geographifhe 2fušdehmung von $\mathrm{O}$ nech W aber, biejer fich fethon mehr ber mittlern norboent=

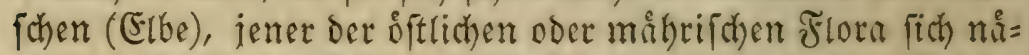
hert; bod) find and Diefe Einfluffe von nur geringer SBedeu= tung.

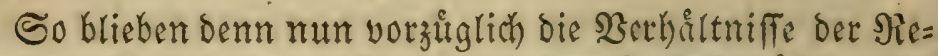
gionen uno ber Unterlage nder ber Rofalititen alb Scruptmomente bei Der Berbreitung ber Pflamzen Durch die einzelten (S)ebiete librig. Beibe haben wir, wenn aud) mur in wenigen Umeiffen bei ben cinzelnen Gebieten Eenten ge= lernt, auth haben wir nath Den Siegionen mehrere sinzelne (S) biete mit einander zu verbinden gelucht. Die unterite (sirenze ber von uns zu betrachtenden (Sebiete ift uns mit 190', bie oberite Grenze mit 3379' gegeben, alfo ein Raum von mefr als 3000' nuffereigentor Şơhe, in welchem bie cigentliche (5bene nicht bis 600' miteigt, die Sgügelesgion 121300', die niedere SEergregion 13-2000', die hóhere Bergregion uber 2000' anjumehmen wire. SEeftimmte Grenzen lallen fich nicht leicht zichen, on theils die verid)iede= nen Berbiltniffe ber Unterlage, theits die grónere oder gerin= gere Entfermung oer sinjelnen (sicbiete und (sebietstheile von

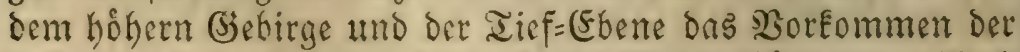

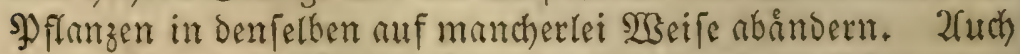
liegen über Das ŞokenvorEmmen Der einzelnen DPflamzenar= ten in ben verichiedenen Giebieten, mit 2(usnathme bes bet 
Seeufheuer, in Dem Dnffelbe, bei allert, und beb von Supfer= berg, in Dem bei vielen PJflanzen Die obere oder untere Brenze, bei Den íbrigen fich Die Seehobe Rupferbergs verfteht, ange= geben ift, zu wenig befimmte 2(ngaben vor; auch find in ben einzelnen Gsebieten nut felten die Standorter angegeben, ob

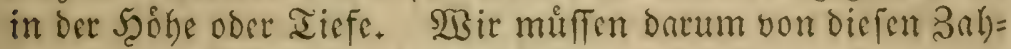
lenangaben abfoken und es fecheint mir biefes auch weniger wichtig zu feit, vielmehr wichtig bagegen bas 3ufammenfar = fen ber Pffanzen nach ifrem gemeinfamen 30 rhandenfein in ben verichicdenen cinzelnen (Sebieten. (Es wurde bies zu noch) gentureren Ergebniffen führen, wenn eine noch gro̊Bere Babl Der Gebiete, befonders ber (Siebiete der (5bene zut Bergleichung vorbanden twire, indem nun leicht nus dem Maximum und Minimum ihres 2 orkommens in ben (S)ebieten der Ebene

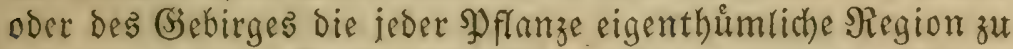
erkennen wire. Dod) laffen fich aus den vorliegenden Ihat fachen nuth fhon ziemlich beftimmte (Ergetniffe ziehen, indem wir zwar mut 3 reine Siebiete Der (5bene habert, die mir aber

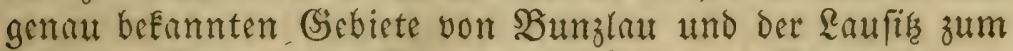
Theil ber (5bene, zum Ibeil bem Scuget= und Berglande an= geboren und nuf biefe 2 beife leicht zut genauern Beftimmung gebraucht werden Eonnen. Noch genauer wurden biefe $\mathfrak{B} e=$ fitimmungen werden, wenn bei jeder Şflamzenart Der (5)rad

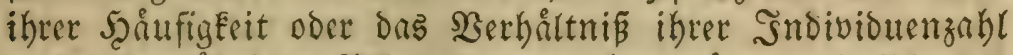
zu ber ber ủbrigen Sflamzen angegeben wåre, weil fich aus bem Maximum oder Minimum Der einzelnen Sndividuen einer 21rt, bejonders in \$erbindung mit den vorgenannten Berbåltniffen genau das (Sebiet ber ßegion und fomit die

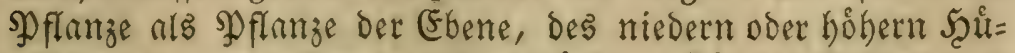
gellandes zc. zu erEennen geben wirtoe. Fur bie (3)biete von Beuthen, Shlau, Bunzlau und ber Şeuicheuer ift bieies 3ath=

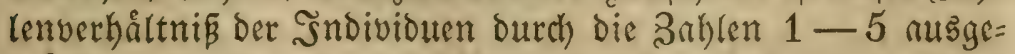
brudef, woburch auch wieder 2(nhaltspunete gegeben fint. Soch) 
fheinen mir biefe (S) renzen zu enge zu fein; es ift zu genauern Seftimmungen berfelben sine 2lusbefnung ber 3nhlen noth=

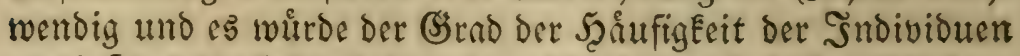
wenigftens burch bie 3ahlen 1 - 10 ober nod) beffer von 1-

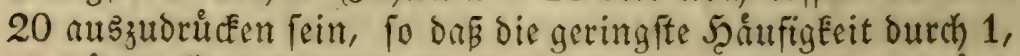

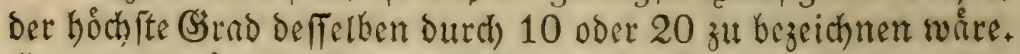

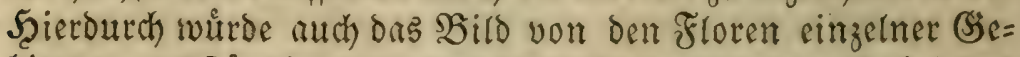
biete vervollftandigt und um fo mekr, wein man babei bas vereingelte, zerftreute, genåherte, gruppentweife ober georaingte Borkommen ber einzelmen Sindiviouen nach dem $\mathfrak{B o r g a n g e}$

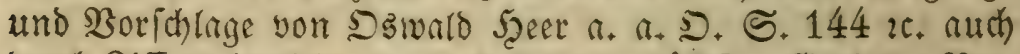

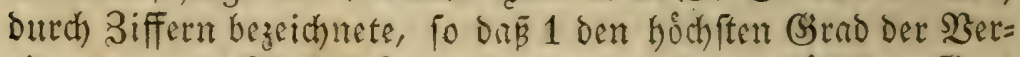
einzelnung f. $\mathfrak{B}$. bei Listera ovata, 10 ben hóchifen Girad bes Bereinigt Teing der Sndiviouen z. B. bei Calluna angeben

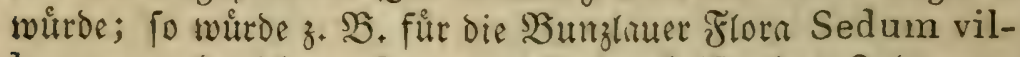
losum zu bezeidnneu fein $1 \times 8$, bas heipt, oas 3ahtenver= håltnis ber Sndiviouen von S. villosum zur Gefanmtzahl aller Syflamzenarten = Sndividuen ber Sumzlauer Flora ift ein fehr geringes, es ifit bas Sedum nut auf eine Roealitát be= fohrinet, Eommt aber bort in groper Menge neben einander vor; brs 3ahkenverhåttnis von Calluna würoe bagegen burch

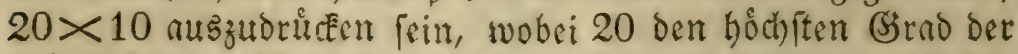

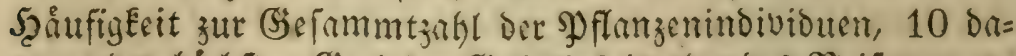

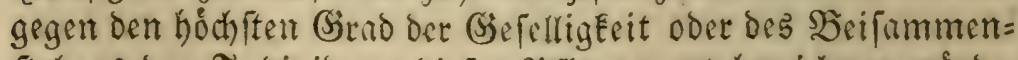

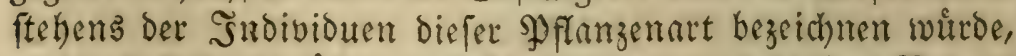

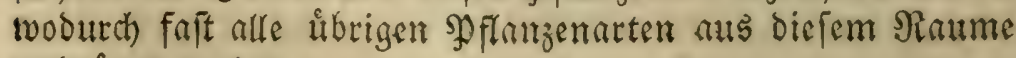
veroringt werben.

B̧enden wir uns mun zu ber Şetrechtung ber Berbrei=

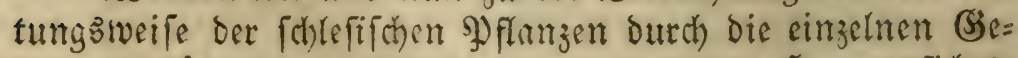
- biete, fo fonnen wir biefelben anter eimzelnen Scauptgefithts= punEten zufammen foffer. 


\section{7}

1) פflanzen, die in Eeinem ber vorliegenden B̈ebiete vor= Eommen.

2) Wflanzen, bie allen vorliegenden Gesebieten gemeinjhaft lich find: Gsanz allgemein und gemifot oer= breitete Splanzen.

3) PJ flanzen, die nut einem der vorliegenden Ģebiete eigen= thimlid) find: SPfanzen mit vereinzelter $\mathfrak{B} e r=$ breitung.

4) Эyflanzen, Die nur 2-3 Ģebieten eigenthümlich fino: D) flanzen mit fehr befcheneter Berbreitung.

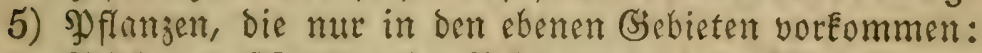

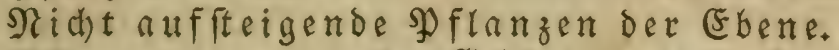

6) ઝ̧flanzen, bie mur in Den Sebirgşgebieten vorkommen: Sicht abfteigenbe Bergpflanzen.

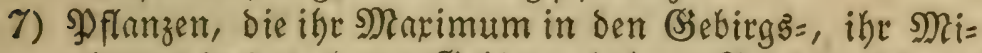
nimum in den ebenen Gebieten Gaben: Sn bie Ebene herabfeigende Bergpflanzen.

8) Mfflanzen, Die ihr Marimum in Der Ebene, ihe Mini=

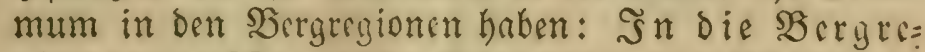

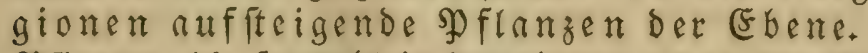

9) $\mathfrak{P}$ flamzen, bie fo woht in ben ebenen als in ben $\mathfrak{b e r g}=$ gebieten verbreitet find: $\mathfrak{D}$ flanzen gemifd) ter $\mathfrak{B}$ er= breitung;

a) mit vorwaltendem $\mathfrak{B e r g} \mathfrak{k} a r a \mathfrak{E} t e r$,

b) mit vorwaltendem Rarafter ber Ëbene.

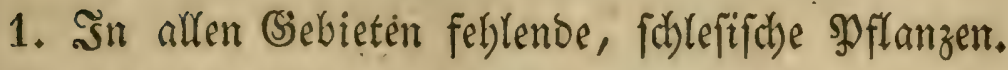

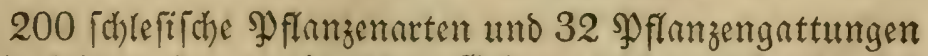

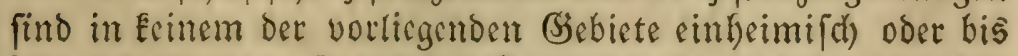

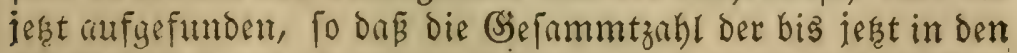

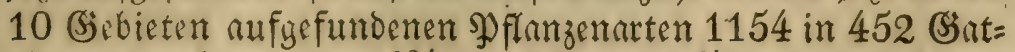
tumgen betrigt, alfo c. ${ }^{13} / 14$ aller 2(rten, $15 / 16$ aller fohlefifohen Bantungen: 


\section{8}

Diefe in ben (Gebieten fehtenden গু flanzen laffen fidd) fĭg= lict) in 3 2(btheilungen bringen

a) Zlpenpfanzen oder Subalpinpfanzen, wel= dye in Eeines Der Gebicte herabgeftiegen fino, alfo in

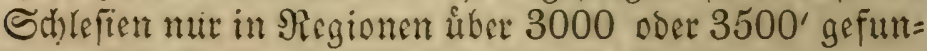
Den werben.

b) SPfanzen der fudoftricten ober oberfhlefi= fden Flora, welche theits aus firbocut (d)en, theils

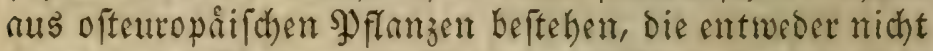
fo weit nach $\mathrm{N}$ oder mach $\mathrm{W}$ geographifd verbrcitet fint.

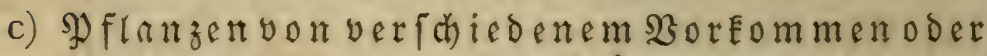

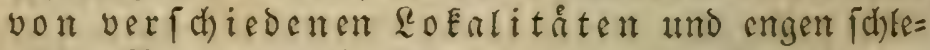
fiichen Segrenzungabezirfen, von denen visle Der frutht=

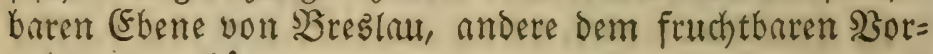
gebirge angehoiren.

a) Subalpin= und 2thin = Dpflanzen.

89 sigenthumliche 2 reten in 65 Giattungen, won denen

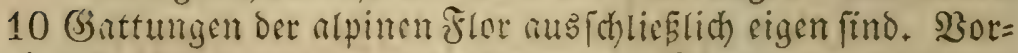
juglich Compositae, Saxifrageae, Rosaceae, Gramineae, mur eine Leguminosa.

Diefe alpinen uno fubalpinen SPflamgen fino auf cine Dreifache 2 Beife verbreitet:

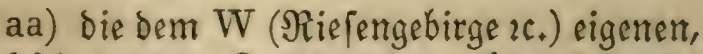

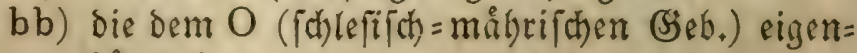
thutmlichen,

cc) die bem ganzen Şochgebirge angehơrigen.

aa. Die weitlict)en 2(lpit= uno Subalpin=Pflanzen.

25 2frten in 19 Grittungen, von Denen die geppert ge= orucken Gattunigen in Feinem andern Gebiet:

Arabis alpina; Alsine rerna; Geranium macrorrhizon; Geum pyrenaicum, montanum; Rubus Chamaemorus; Alche- 


\section{9}

milla fissa; Pyrus Axia (am Elbgrunbe, weniger zu ben fubalpis nen গPflanzen zu red)nen); Saxifraga oppositifolia, bryoides, muscoides; Veronica alpina, bellidioides; Pedicularis sudetica; Androsace Chamaejasme; Primula minima; Salix Arbuscula'keffer Weiggeliana (fie fângt an zt ver jurwirben Durd) bab (Einfammeln bofmifder Botanifer), Betula nana; Swerti a perennis; Pinguicula alpina?; Luzula spicata; Carexva ginata, humilis; Eriophorum alpinum; Agrostis rupestris.

\section{bb) Die öftlichen 2lpin= und Subalpin = YPflanzen.}

Aconitum Stoerhianum; Stellaria Friesiana seu longifolia; Cerastium alpinum; Potentilla salisburgensis; Sedum Fabaria seu purpureum; Saxifraga Aizoon, umbrosa, nivalis; Meum mutellina; Laserpitium Archangelica; Valeriana Tripteris?; Senecio alpinus; Doronicum scorpioides; Centaurea axillaris; Hieracium villosum; Crepis sibirica; Campanula barbata; Gentiana rerna, punctata; Echinospermum deflexum; Tozzia alpina; Sold an ella alpina; Plantago montana; Salix hastata; Juniperus nana; Pinus austriaca - Orchis pallens; Luzula spadicea; Carex rupestris; Agrostis alpina; Poa aspera, alpina; Avena planiculmis; bie oftlidje Flora ift Demnad) reid)er: 34 2rrten in 29 (Sat= tungen.

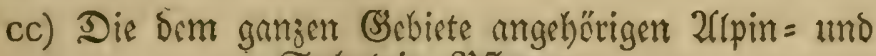
Subalpin = I) flanzen.

30 21rten in 26 Sinttungen, von Denen die mit gepperter Schrift in Ecinem moern Bebicte vor Eommen:

Anemone alpina; Delphinium elatum (fteigt bis 2fgneten=

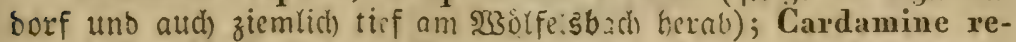
scdifolia; Viola lutea; Spergula saginoides; Hedy sarum olscurum; Potentilla aurea (jerigt bis $1600^{\prime}$ an simzelnen Stel=

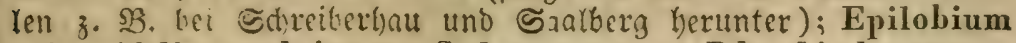
origanifolium, alpinum; Sedum repens; Rhodiola rosea; Sempervivum montanum; Bupleurum longifolium (Stbaipin= und s)rontanpflanje); Scabiosa lucida; Pyrethrum corymbosum (milyr in bie subalpingegenden auffteigende s) sontanpflanze); Aden ostyles albifrons (Cacalia), Gnaphalium supinum, Hieracium aurantiacum, alpinum, Hypochoeris helvetica;

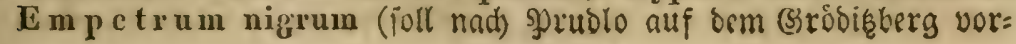




\section{0}

fommen?); Bartsia alpina, Rumex alpinus; Habenaria albida, Allium Schoenoprasum, Juncus trifidus, Carex atrata; Phleum alpinum, Poa laxa, Festuca varia.

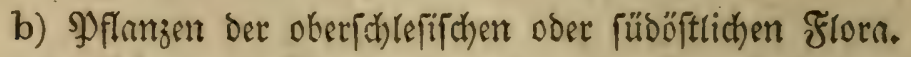
56 2frter, 50 Battungen, bie mit gepperrter ভdyrift 15. cigenthümliche (Gattungen. Theils PJflanjen ber (Ebone, theils Şügel= tmo Bergpflanzen, vicle Labiatae uno Liliaceae, viele Bsattungen eigenthümlich.

Aconitum Lycoctonum, Ranunculus illyricus, Nuphar minimum; Dentaria glandulosa; Saponaria Vaccária, Silene nemoralis; Spergula subulata; Staph y le a pinnata ; Corydalis solida (IIalleri); Galega officinalis, Lathyrus hirsutus; Epilobium Dodonaei (angustissimum); M y ricaria germanica; Sedum album, Saxifraga Hireulus, (aud) in פreu= Een, מommern); H a c q u e ti a (Dondia) Epipactis, Bupleurum rotundifolium, Conioselinum Fischeri, Caucal is daucoides; Seabiosa suaveolens; Senecio vernalis, Cirsium serratuloides, Carduus eriophorus; Campanula bononiensis, sibiriea; Linnaea borealis (aud) in ber Edtheearube jest gef $n=$ Den !; Asperula cynanchica; Erica herbacea; Lycops is pulla; Serophularia glandulosa; Salvia glutinosa; Chama e pitys vulgaris, Te u eri um montanum, Nepeta nuda, Mentha rubra; Androsace elongata; Globularia cordifolia, vulgaris; Thesium ebracteatum; Passerina annua; Salix daphnoides, stipularis, incana, finnmarchica. - Orchis pyramidalis, Malaxis monophyllos; Iris graminea, Ornithogalum bohemicum, Cxocus vernus, Scilla amoena, bifolia; Juncus obtusifolius; Carex pilosa; Alopecurus agrestis, Lolium multillorum.

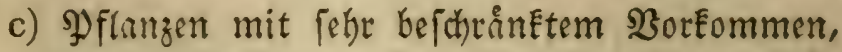
(wegen nur feiten. recht zufagender Unterlage.)

56 2ren in 43 G5attungen, von berten die 7 Giattungen mit gefperter Schrift in feinem andern Bebiete vorfommen. Anemone Pulsatilla, sylvestris, patens; Cardamine trifolia; Nasturtium austriacum, Barbarea arcuata, S e neb i cra Coronopus; Resedea lutea; Dianthus arcuarius; Moench i a crecia, Stellaria viscida, Cerastium glomeratum?, ano. 
malum; Lavatera thuringiaca; Geranium pyrenaicum; Evonymus verucosus; Astragalus hypoglottis, Cicer, Lathyrus Nissolia; Geum intermedium, Potentilla alba, patula, Pyrus torminalis; Epilobium virgatum, Laserpitium latifolium; Sambucus Ebulus; Dipsacus laciniatus, pilosus; Chry ocoma Linosyris, Inula germanica, Centaurea solstitialis, Tragopogon porrifolius; Campanula lilifolia; Linaria spuria; Salvia sylvestris, Prunella grandiflora; Veroniea peregrina, prostrata; Orobanche pallidiflora, caryophyllacea, ramosa; Plantago maritima; Chenopodium opulifolium, ficifolium; Euphorbia segetalis; Xanthium spinosum, Salix hippophaëfolia. - Orchis militaris, Tulipa sylvestris; Triglochin maritimum, Cladium germanicum; Scirpus Tabernaemontani, Michelianus; Glyceria distans, Festuca montana, Beckma n $n$ i a eruciformis.

\section{2fAgemein verbreitete Pflanzen.}

Bon ben 1171 Shflumzennten, welde in 453 Sinttur= gen für alle vorliegende Bscbiete ubrig bleiben, finden wir 511 21rten in 272 33attumgen uno 70 Familien in jedem ber ein= jelnen Biebiete vorkomment oder fie fehlen mur in einem oder Dem andern (Sebiete. Sie zeigen fich aljo von ben verfhis=

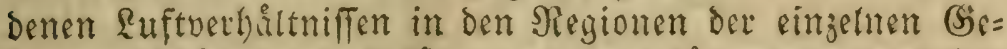
biete unabhingig; Doch Eonnen wir nut fü wenige berfelben eine Berbreitungstegion von 190 bis 3379 oder 2800', woht aber wenigitens bis 1500 uno $2000^{\prime}$ alfo beinahe burch 2000'

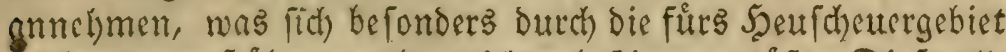
vorbanoenen Scơhenangaben leichter beftimmen liẽt. Diefe all= gemein verbreiteten $\$$ flanzen bilden mehr als $1 / 3$ aller fdi)left fdyen Şflanzenarten und mehs als $\frac{\mathrm{T}}{2}$ aller $\mathrm{D}$ flanzengattungen; fie verbalten fich zu ben in allen (sicbieten zu fammen vorke mmenden Sf flangen in Den 2rten wie 2:5, in Den Ssittungen c. 13:23. Sei vielen Derfelben liest fith ibr 2lufferigen aus ber Ebene in bie Sargregion, bei mebrern bas 5cerabiteigen aus ber Derg= region in bie (5tome nnchweifen, noch genmer ließ̧e fith diefes befitimmen, wemn von allen in allem Gebieten ber Grad ber 
Jृåufigfeit angrgeben wåre; Dann Eơnte man als wirflich all= gemein verbreitete, wenig von biefom Scobenverbiltniffe ab=

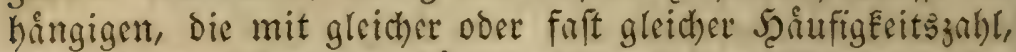
als mehr ber (Ebene angehorend, die mit Dem Marimum ber Sndiviouen in ber (Ebene, als Sergpflanzen mit bem Mari= mum in ber SBergrigion anfehen. (5.z. Fe)eiment aus dem (S)e= biete Der (5bene mehr \flanzen in bie Submontan = ঐegion zu fteigen, als umgetétet aus biefer in iene. Nachfolgendes $\mathfrak{B e r}=$

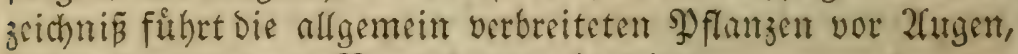
bin uno wicoer mit Semerkungen begleitet.

Ranunculaceae: An emone nemorosa, ranunculoides; Ranunculus aquatilis, Flammula, Ficaria, auricomus, lanuginosus, arvensis, acris; C al tha; A quil e gi a vulgaris (fehit W., woht mehr sebiraspflanke, in oer (5bene mefo verwibert); Delphinium Consolida (ffert $\mathrm{k}$.)

Papaveraceae: Paparer Argemone, Rhoeas (feglt Ch.); Che lid o n i um majus.

Fumariaceae; Corydalis fabacea; Fumaria officinalis. Cruciferae: Nasturtium amphibium, sylvestre (figlt Hb.); B a r b a r a e a vulgaris, Turritis; Cardam in e Impatiens (affer K.), pratensis, amara; $S$ i s y m bri um officinale,

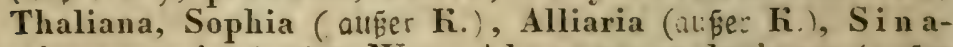

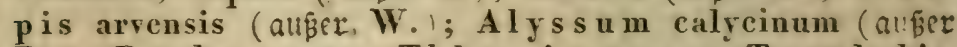
Bt.), Draba verna; Thlaspi arrense; Teesdalia nudicaulis; Lepidum campestre; Caps ella; Neslia paniculata; Raphanus.

Violariae: Viol a palustris, hirta (anper Hb.), odorata, sylvestris, canina, stagnina (uhne L. uno K.), trieolor.

Droseraceae: Drosera rotundifolia, Parna ssia palustris. Polygaleae: Polygala vulgaris, comosa (añer $\mathrm{Ch}$.)

Sileneae: Gypsophil a muralis (aver H.); Dianthus deltoides; Silene nutans (auper Bt.), inflata; Ly ch$\mathrm{n}$ is alle 5 urten.

Alsineae: Sagina procumbens; Spergula nodosa ( ( K.), arvensis, Alsine rubra, Mo ehringia trinervia; Arenaria serpyllifolia; Holosteum; Stellaria media, holostea, glauca (anfper Hb.), graminea, uliginosa; I alachium aquaticum; Cerastium semidecandrum (auber K.), triviale, arvense. 


\section{3}

Lineae: Linu m catharticum, (usitatissimum). Malvaceae: M a lv a sylvestris, rotundifolia.'

Tiliaceae: Til i a parvifolia.

Hypericinae: Hypericum perforatum, humifusum (auper K.), quadrangulum.

Acerinae: A cer Pseudo-Platanus (auger W.), platanoides

(auper K.), campestre (außer L. B. ?).

Geraniaceae: Geranium palustre, dissectum ( außrev Bt.), columbinum, rotundifolium ( atıper Ii.), Robertianum;

Erodi u m.

Balsamineae: Impa ti ens noli tangere.

Oxalideae: Oxal is Acetosella, stricta (aupir Hb.).

Celastrinae: Evon y m us europaeus.

Rhamneae: R h a m u s cathartica, Frangula.

Papilionaceae: Sarothamnus (auger H.); Genista tinetoria, germanica (anker W.); Me dicagyo lupulina (au= fer Ki.); Melil otus vulgaris ( außer Kr.); Trifolium pratense, medium, alp̧restre (auser $K_{\text {. }}$ ), arvense, montanum, repens, hybridum, agrarium, procumbens, filiforme (auper W.); Lotus; Astragalus. glyeyphyllos; C oronill a raria; V i c ia hirsuta, tetrasperma, Cracca, villosa, sepium, sativa, angustifolia; Lathyrus pratensis, sylvestris; Orobus vernus.

Amygdaleae: $\mathbf{P}$ r u n $\mathrm{s}$ spinosa, avium, Padus.

Rosaceae: Spirae a Ulmaria; Geum urbanum, rivale; Rubus idaeus, fruticosus, caesius ( (аһ tilis (amie: Hb.); Fragaria vesca; Potentilla Anserina, argentea (auß̧er Ch.), reptans, opaca (auß̄er L.); Tormentilla erecta; reptans (auper Hb.); Rosa canina.

Sanguisorbeae; Alchemilla vulgaris, arvensis; Sanguisorba; Poterium (außßer O. und Bt.)

Pomaceue: Crataegus Oxyacantha; Pyrus communis,

Malus; S.orbus Aucuparia.

Onagrariae: Epilobium angustifolium, montanum, palustre, roseum; $O$ en othera biennis, Circa a alpina. (auser W.)

Halorageae: Callitrich e 4 ?

Lythrariae: Ly th $\mathbf{r}$ m Saliearia. Paronychieae: Herniaria glabra. Scelerarthée: Sceleranthus perennis, annuus. 


\section{4}

Crassulaceae: Sc du m Telephium, acre, sexangulare (auper IIb.), S e m pervirum hirtum (аһвеr $K_{\text {.) }}$

nibesinae: Rib es rubrum, nigrum.

Saxifrageae: Saxifraga granulata; Chrysosplenium alternifolium.

Umbelliferae: Astrantia major (B.?); Aegopodium; Carum; Pimpinella magna (h. nidgt), Saxifraga ; Oenanthe Phellandrium (H. ni(d)t); Aethusa; Selinum; Angelica; Pastinaca; Heracleum; Daucus-(a'fer K.); Torilis; Anthriscus sylvestris; Chacroph yll um bulbosum (auker K.); Conium (biefe mehr פpflanje opr (Ebene als allgemein verbreitet, im (sebirge nur fehr felten und vidlleid)t mit (setreicejaamen auzgeftreut.) Araliaceoe: He dera.

Corneae: Cor n us sanguinea.

Loranthaceae: Vis cum (auper K.).

Caprifoliaceae: Adoxa; Sambucus nigra; Viburnum Opulus.

Stellatae: Sherardia (F. nid)t); Asperula odorata; Gatli m Aparine, uliginosum, palustre, boreale (S. nid)t), verum sylvaticum, Mollugo.

Valerianéae: Valeriana officinalis; Valerianella olitoria, Auricula (Hb. nidj), dentata.

Dipsaceae: Fin a thia (Scabiosa) arvensis; S u c c is a pratensis.

Compositae: Tussilago Farfara; Petasites; Inula salicina (Hb. ni(d)t); Solidago; Gnaphali um dioecum. sylvaticum; Filago montana, arvensis (K. nid)t); Erigeron acre $\boldsymbol{K}$ midir), canadense (Hb. nidt); Senecio Jacobaca, vulgaris, sylvaticus, Chrysanth emum; Pyre thr um Parthenium (S. und W. nddt), inodorum; Matricaria (mob) eigentlid) spflange ber (5bone uno zwar Der frudtbaren, im (3)etinge faft nut voribergeheno ourd) 20ub= fuat); Bellis; Tanacetum; Artemisia rulgaris; Anth em is arvensis; A chille a Ptarmica, Millefolium; Bidens tripartita; Arctium tomentosum, majus; Cardu us nutans, acanthoides; Centaurea Jacea, Scabiosa, Cyanus; Cirs ium lanceolatum, palustre (F. ni(d)t), arvense, oleraccum; Carlina vulgaris; $L$ a psana communis; Prenanthes muralis; Sonehus oleraceus, arvensis; Hieracium murorum, umbella- 


\section{5}

tım, paludosum, Pilosella, Auricula, praealtum (außer Hb.); Crep is biennis (auper K.), pinnatifida, tectorum (K. nid)t); Leontodon; Apargia hispida, hastilis; Trago pogon pratensis; Cich orium.

Campanulaceae: Jasione; Phyteuma spicatum; Camp a nul a rotundifolia, patula, persicifolia, Trachelium, rapunculoides, glommerata (K. nid)t).

Ericaceae: Call u n a, Pyrol a uniflora (Bt. nidt); secunda, minor $(H$. nid)t ), rotundifolia (Bt. m(d)t); Vace in i u m Myrtillus, Vitis idaca, Oxyccoccos.

Ascle piadeae: Cy nan ch u m (H. ni(d)t).

Gentianeae: Menyanthes, Erythrae a Centaur., ramos. (K. nicht).

Convolvulaceae: Convolvulus arrensis, sepium (K. nidt);

Cus cut a europaea.

Borragineae: Echium; Rhytis permum; Pulmonaria officinalis; Symphitum officinale;. Myosotis palustris, sylvatica (W. nid)t, arvensis versicolor; Anchu$\mathrm{s}$ a officinalis, arvensis.

Solaneae: Verbascum nigrum, Hyos cyamus, Solan u m Dulcamara.

Scrofularinae: S crophularia nodosa; Linaria vulgaris. Labiatae: Lycopus; Ajuga reptans, alpina (K. nid)t); Menth a aquatica, arrensis, sylvestris (Bt. nid)t); Glechoma; Lamium 4; Galeobdolon; Galeopsis Ladanum (K. nicht), pubcscens, Tetrahit: Betonica; Stachys palustris, sylratica; Ballota; Leonurus Cardiaca; Clinopodium: Thymus Serpyllum, Acinos, Prunclla vulgaris; $S$ cutellaria galerieulata.

Rhinanthaceae: Veronica agrestis, Anagallis, arvensis, Beccabunga, Chamaedrys, hederifolia, officinalis, scutelata (H. nid)t), serpyllifolia; Euphrasia, Odontites, Alectorolophus major, minor; Pedicularis sylvatica (Bt. nid)t), palustris (W. nidt); M e la mp yru m arvense, pratense, nemorosum; Lath ra e Squamaria. Jasmineae: Fraxinus, Ligustrum. Primulaccae: Ly s im a ch i a numularia, vulgaris, thyrsiflora (K. und H. ni(d)t); Trientalis (W. nid)t); Primula officinalis; Anagallis.

Plantagineae: Plantago lanceolata, major, media. 
Chenopodiaceae: Che nopodium bonus Henricus, murale u. album (ofine $H_{\text {.) }}$; Atriplex patula (ohme L.)

Polygoneae; Polygonum alle aufer laxillorum u. minus, amphibium u. dumetorum in li. nicht; $R$ umex crispus, Acetosa, Acetosella, obtusifolius.

Thymeleae: D a p he Mezercum, Aristolochiae: As a rum europaeum.

Euphorbiaceae: Euphorbia helioscopea, Mercurialis perennis (ofine Bt.)

Urticeae: Humulus, Urtica urens, diceca.

Amentaceae: Salix pentandra (ofne Bt.), fragilis, anygdalina, purpurca, cincrea, Caprea, aurita, repens (ohne K.); P p p lus alba, tremula (ohme H.), nigra; A I nus glutinosa; Betula alba; Carpinus; Fagus; CoryI us; $Q$ uercus pedunculata; Ulmus campestris ( $\mathrm{l}$. liicit)

Coniferae: Pinus Picea (Bt.?), Abies, sylrestris, Larix; Juniperus communis.

\section{Monocotyledones.}

Orchideae: Orchis maeulata, latifolia; Platanthera;

Ne ottia nidus avis; Listera ovata.

Irideae: Ir is Pseud acorus.

Liliaceae: Lilium Martagon (ohne Bt.); Ornithogalum

luteum, stenopetalum, Le u c ojum vernum (ohme $B t$.)

Alismaccae: Potamogeton natans (F. nidit); Alisma; Triglochin.

Asparageae: Paris, Conrallaria, Polygonatum anceps u. multiflorum, M a j a n th e mum.

Juncaceae: Acorus, Luzula pilosa, campestris; Ju nc us conglommeratus, effusus, bulbosus ( $(\boldsymbol{r}$. nich)t), bufonius, lamprocarpus.

Typhaceae: S pargan i um ramosum (fi. nid)t).

Cyperaceae; Care $\mathbf{x}$ vulpina, muricata, Schreberi, leporina, elongata, stellulata, praecox, pilulifera, extensa, panicea, digitata, pallescens, sylvatica, ampulacea, vesica ria, caespitosa Hb. ni(t)t), acuta, glauca (L. nicht), hirta; Eriophorum vaginatum, angustifolium, latifolium; Sc ir pus palustris, sylvaticus. 


\section{7}

Gramineae: Anthoxanthum; Alopecurus pratensis, geniculatus, fulvus; Phle um pratense; Phal aris; Set a ria glauca, riridis (K. nid)t, Agrostis rubra, stolonifera, canina (?), Anemagrostis, Spica Venti, Calamagrostis Epigeios, Melica nutans; Aira caespitosa; Foeleria cristata (auper L.) Molinia; Phragmites; Glyceria lluitans: Poa anña, trivialis (K. nict)t, pratensis, nemoralis (K. nid)t), compressa; $\mathbf{B}$ riza; $\mathbf{C}$ yn osurus; Dactylis; Festuc a ovina (h nicht), duriuscula (K. ni(t)t), pratensis (Ch. ni(d)t); Bracleypodiu m sylraticum; Triodia (li. nid)t); Bromus tectorum; Agropyrum repens (im thonig=fandigen Booen báufiger aib im ftud)tbaren); L oli u m perenne, temulentum; Horde um murinum; Holcus lanatus; Nardus stricta (fi. nid(j)

Najades: L e mn a minor, polyrrhiza; Monotropa Hypopitys.

$\mathscr{2 B a s ~ b i e ~}$ Berbreitung Der Familien betrifft, fo find bie artenreichen Familien der Alsineae, Labiatae, Rhinanthaceae, Papilionaceae, Amentaceae, Borragineae, Cyperaceae, Compositae, Rosaceae, Gramineae mit $4 / 12$ bis $5 / 12$ ifrer ichlefifden 2 rten, Dic Cruciferae mit $1 / 3$, die Ranunculaceae mit $1 / 4$, die Orchideae nur mit I/ Derferben in alfen Gebieten verbreitet, von den Personatae aber nut ${ }^{\mathrm{T}} / 10^{-}$

\section{106 Sfflanzen mit vereinzelter Serbreitung.}

Sie bilden jene (Struppen von PJflanzen, welche nut in einem der 10 gentanten (Sebiete oder fogar nur in einem (S) = biet in ganz Schlefien gefunden werden, oder bis jest gefun= dent worden find. Sie erfcheinen weniger abjingig von ben

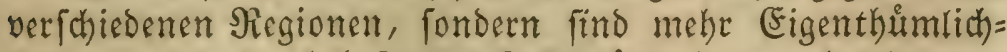
Eeiten, welche butch befondere SoEalitaten hervorgebrad)t wor= Den find. Shre 3ahl ift im 2ulgemeinen nut Elein; bie bis jest nut einem Ssebiete in ganz Schleften eigenthumlichen 


\section{8}

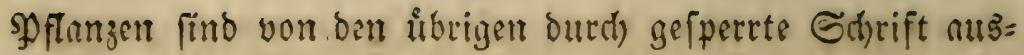
gezeidhent.

a) 14 Bereimzelte गुflanzen ber Şumglnuer Florn. *)

Rhamnus saxatilis, M e di c a go m in ima (weld)e id) 1826 in grofer Jjerge cm Meel eहftrant tri yeizia nefunden, 1828 auf bem Eábngaus̆berue), Scorzonera laciniata, Himantoglossum hircinum, Allium senescens, Agrostis stricta, Melittis Melissophyllum, Sagina apetala?, Lathyrus latifolius?, Zannichellia palustris, Juncus melanthos, Scirpus caespitosus, Lindernia Pyxidaria?, Lonicera Periclymenum (ję̧t aud) bei Sfarline gefunoen ron Dem um Gdiefitenz FFlora veroienten Scrn. Eegrer Sd)ummel in SErsblau.) 1837 aud) Caucalis grandiflora.

b) 28 vereingelte Pflanzen ber \&nufir.

Thlaspi alpestre, Viola uliginosa, Silen e erastoides, Gerani um boh emicum, Trifolium striatum, Trapa natans, Herniaria hirsuta, Bupleurum falcatum, Meum athamanticum, Eryngium campestre, Galium saxatile, Aster alpinus, Rudbeclia laciniata (ift zrour Éeine ein= heimiidje spflanze, fimmt aber in mehreren Iateilen ber sberlau=

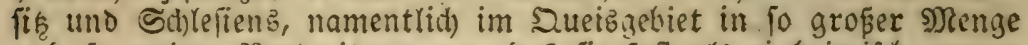
und fo weiter Sier ireitung vor, baf fie faft alb einf)eimiich geroor= ben zil betra(j)ten ift), Epipactis viridiflora?, Gladiolus communis?, Muscari comosum, Potamogeton heterophyllus, Juncus capitatus, Tenageja, Agrostis arenaria, Elymus arenarius, Arum maculatum, Monotropa glabra ? ( wohl Eeine eigene $2(r t)$, Cardamine sylvatica, Scrophularia vernalis, Linaria $\mathrm{Cy}_{\mathrm{y}} \mathrm{b}$ a laria, Mentha viridis, Stachis arvensis.

\section{c) 21 vereingelte Sুflanzen ber Sceupheuer.}

Helloborus viridis, Thlaspi montanum, Saxifraga s p on h emic a, Fumaria Vaillantii, Asperula Aparine, Borlihausenia hispida; Salix ambigua, myrtilloides, limosa, her-

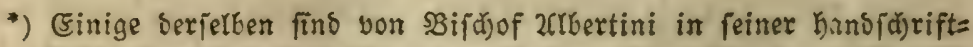
liden flora montis gratiarum angegeben, von mir aber nod) nidat nufgefunben worben. 


\section{9}

bacea; Pinus Pumilio seu Mughus; Allium Scorodoprasum; Carex capillaris, limosa, chordorhiza, filiformis, ornithopoda; Dianthus caesius, Verbascum phoeniceum; Stachys alpina; Euphorbia anygdaloides.

\section{d) 5 vereinzelte Şflanzen ber Scirfd berger Flora.}

Lepidium Draba, Hieracium Sehmidtii, Fumaria capreolata, Ribes petraeum, Festuca heterophylla.

e) 5 vereinzelte Pुflanzen Der Supferberger Florn.

Erysimum strictum, Brassica orientalis, Asperula arrensis, Campanula Rapunculus; $\mathbf{A}$ gra $\mathbf{p}$ h is $\operatorname{cernu}$ a (Reipe), Cypripedium (besgleident u. Miod)au); bic letgtern beiden fïn mebr bem smittergebirge angehorig.

f) 14 vereinzelte Pflanzen ber Salzbrunner Flora.

Helleborus niger?, Nasturtium anceps, Ervum Lens?, Valeriana Phu?, Kna uthia hybrida, sylvatica?, Tragopogon major, Selix Starkeana?, Allium carinatum, Melilotus dentatus, Antirrhinum majus, Stachys germanica, Chenopodium Botrys, Mentha acutifolia.

g) 9 vereinzelte SPflanzen ber Shlauer Flora.

Isopyrum, Cardamine parviflora, Biscutella, Polycarpon, Cerinthe minor, Iris nudicaulis, Potamogeton trich odes; Carex fulva; (Cypripedium Bernftaot), Valerianella exiocarpa.

h) 8 vereinzelte $\mathfrak{P f l a n z e n ~ b e r ~} \mathfrak{B}$ ohlauer Flora.

Silene chlorantha, Scorzonera purpurea, Herminium Monorchis, Sturmia Loesellii, Carex microstac hya, Holoschoenus filiformis, Orobanche coerulca, Androsace septentrionalis.

\section{Nflanzen mit befdränfter ober unterbrodener Berbreitung.}

Sie umfaffen jene Ifflanzenarten, weldye nur 2 bis 3

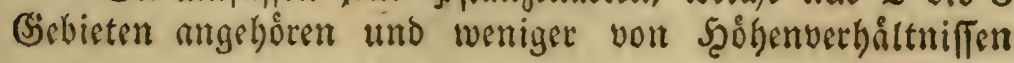




\section{0}

als vielmebr von befondern Rokntititen in ifrem $\mathfrak{B o r f o m =}$ men kedingt find. Sie laffen fid in 4 (Stuppent kringen

a) SPflanzen der Şaibeebene.

b) PY flumzen ber niedern Ebene.

c) Sergpilanjent.

d) \$flanjen mit gemifhter Berbreitung.

a) Dftanzen ber Şaidecbene.

aа) Scaibe = Moorpflanzer.

Bz. uno L.: Drosera intermedia (aud) auf ber Jierrviefé). Rynchospora fus ca, Elatine Alsinastrum. Bu. $u_{+} W_{0}$ : Tofieldia. B., L. u. H. : Scheuchzeria palustris, Drosera Iongifolia, Erica Tetralix, Carex Leucoglochin. B., L. u. W.: Sparganium natans.

\section{bb) Scaide = Sandopflanzen.}

Bz. uno L. : Illecebrum verticillatum (aud) in Stherid) (e= fien), Anemone vernalis, Carex arenaria, Poa bulbosa, Festuca bromoides, Cytisus nigricans, (autd) in ber oberid)le: fifden şaibe und fitt) bent (Sebiete von Shlau náberno), Corrigiola litoralis?. Bz., L. u. H. : Genista pilosa, Aretostaphylos, Avena strigosa.

$$
\text { cc) Şaide = Şügelpffanzent }
$$

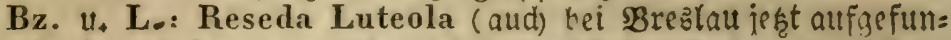
ien), Orobus tuberosus (ïdeint bie Sanofteinunterlage zu lieben,

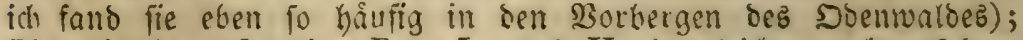
Pinguicula vulgaris (Bz., L. uno H. beggleid)en aud) auf bem

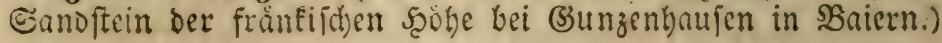

\section{b) Sૈ flanzen der niebern Ebene.}

\section{aa) nur in 2 (siebieten.}

Verbascum Blattaria, Cardamine parviflora; Hicracium grandiflorum, Blitum, Falcaria Rivini; Cnidium venosum, Potamogeton rufescens, perfoliatum, pectinatum, Parictaria, Alsine tenuifolia, Linaria arvensis. 


\section{bb) in 3 Gebieten.}

Bt., O. W. Adonis, Anemone pratensis, Cineraria palustris, Scutellaria hastifolia, Euphorbia lucida, palustris, Polygala amara, Atriplex rosea, Avena praecox, Leonurus Marrubiastrum; Elatine Hydropiper, Limosella, Centunculus, Amarantus retroflexus, Potentilla norweģica, Picris.

c) Sergpfinzen mitbefbranter Berbreitung.

aa) Şügelpflanzen.

Omphalodes, Potentilla recta, rupestris, Avena flavescens, Orchis coriophora, Ornithogalum umbellatum, Dianthus prolifer. (Omphalodes ald) nuf bcm (sicibis 1837.)

\section{bb) in 2 (siebieten.}

Hesperis matronalis, Arabis arenosa, Thlaspi perfoliatum, Carduus personata, Epilobium trigonum, Cirsium acaule, Hicracium prenanthoides, echioides, Tragopogon orientale, Archangelica officinalis, Chacrophyllum aureum; Gentiana asclepiadea, Asperula tinctoria; Salvia verticillata, Teucrium Botrys Thesium alpinum, Symphitum tuberosum, Salix silesiaca, Orchis gुlobosa, Listera cordata, Epigogium aphyllum, Asparagus, Allium Victorialis. - Carex maxima, Davalliana; Luzula maxima, Bromus arrensis, pratensis.

\section{cc) in 3 (5)ebieten.}

H., S. u. Ch. Geranium phaeum, Trifolium ochroleucum, Anthemis tinctoria, Phyteuma orbiculare, Lithospermum offieinale, Carex intermedia, saxatilis (rookt mef)r actpin= pitanze, rreldye in bie J)ontonregion herabzeftiegen), Chrysosplenium oppositifolium, Galium vernum, Pulmonaria angustifolia, Cephalanthera pallens, rubra; Imperatoria Ostruthium; Streptopus, Aconitum Napellus, lektere 3 subalpine. Narcissus.

d) SDfanzen gemifhten SorEommens.

Die 32 hierker gebörigen 1 flanzen zeigen Eeinen beftimm= ten Fintheilungsgrund ibrer gemifhten Berbeitung; Eommen

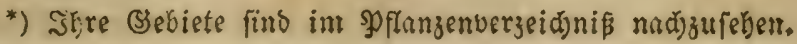




\section{2}

fowohl in ber Ebene, als in fubmontanen und montanen Re= gionen vor, und find zum Iheil Pflanzen auf gebrutem $\mathbb{R}_{\text {ande }}$ oder feudfer unterlage.

a) Sinapis alba, Brassica; Chenopodium rubrum, Portulaca, Echinospermum Lappula, Hypochoeris glabra, Trifolium fragiferum.

b) Senceio aquaticus, Cirsium tataricum, Intybus pracmorsus, Salix rubra, acuminata, Alnus incana; Orchis incarnata, Carex paradoxa.

c) Ranunculus cassubicus, Nigella; Arabis Gerardi; Inula Helenium: Vicia tenuifolia, Ononis spinosa, Melilotus Petitpierreana, Tetragonolobus, Cytisus capitatus,- supinus, Senecio erucifolius, Stachys annua, recta; Geranium divaricatum (Cypripedium), Villarsia, Allium acutangulum.

\section{Ueber 4 Ģebiete verbreitete Şflanzen mit befdränfter Serbreitung.}

Sie ftellen fich in 4 (5rtuppen bar, je nachdem fie a) nut in

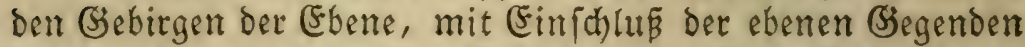
des bunzlauer und laufízer (sebietes, oder b) in 3 der ebenen unt in einem $\mathfrak{B e r g g e b i e t e}$ ober c) in 3 ber $\mathfrak{B e r g}=$ und in sinem der ebenen Giebieten, doer mur in 4 der 7 Beriggebiete vorEommen, und bilden faft bie an 2(rten irmfte 2(btheilung.

a) Nicht auffeigende befdréneter Berbreitung 4 u. 0 .

aa) mit feuchter, thonig= Fandiger unterhge.

Radiola, Gnaphalium luteo-album, Myosotis, Cyperus fuscus.

\section{bb) $\mathscr{B} a f \mathfrak{e r}=$ und Eumpfpflanzen.}

Teucrium Scordium, Stratiotes (1837 aud) in Bz.), Myriophyllum spicatum, verticillatum, Potamogeton compressus, obtusifolius, Scirpus maritimus, Eriophorum gracile. 


\section{3}

cc) Siemifate unterlinge.

Potentilla supina, Euphorbia platyphyllos, Ornithopus, Onobrychis, Linaria elatine, Veronica spicata, longifolia; Plantago arenaria.

b) $\mathfrak{s}$ die Şügel = und SBergregion aufiteigende Pftanzen ber Ebene 3 und 1 .

Thalictrum flarum, Arabis hirsuta; Viola arenaria; Medicago; Lathyrus palustris: Hippuris; Rosa cinamomea, Potentilla norweggica; Veronica Teucrium, Melampyrum cristatum, Linaria; Herniaria glabra, Artiplex; Anthriscus cerefolius, Eryngium planum; Euphorbia exigua, AsperugoCuscuta; Iris sibirica: Gladiolus imbricatus; Scirpus uni; glumis, ovatus, acieularis, Blysmus compressus; Digitaria sanguinalis; Koeleria glauca.

c) Sี bie Şưgefregion und bie tebene abftei= gende Bergpflanzen 1 und 3.

Alyssum montanum, Veronica polita; Aster salignus, Cirsium rivulare; Himantoglossum, Orchis ustulata, Carex paludosa; Festuca Myurus.

d) Nicht abfeigende Bergpflanzen mit be= 何råneter Berbreitung 0 uno 4 .

Cotoneaster; Libanotis montana, Pleurospermum; Geranium sylvaticum; Cuscuta Epithymum; Veronica montana; Galium cruciatum; Thesium montanum; Trifolium rubens; Carduus crispus, Hypochoeris maculata; Inula hirta; Poa sudetica.

\section{Heber 5 Ģebiete verbreitete SDflanzen.}

Sie ftellen fid unter fehr mancherlei Berbåtniffen bar und find theils allgemein verbreitete Plflanzen ber Ebene oder faft allgemein verbreitete Bergpflanzen, theils zeigen fie eine mehr in ber Ebene und ben $\mathfrak{B e r g}=$ und Schigelgebieten ge= mifhte Berbreitung, boch tritt bei innen immer ber Sharak = 


\section{4}

ter bez 2ingeforoene ciner beftimmten Siegion hervor. Sie laffen fich am beften in folgente fechs Scauptgruppen bringen, bei denen die erfte ber hinten ftehenden 3ahlen Die 3ahl ber ebenen, Die Kintere bie ber \$Berggebiete, in denen fie getroffen werden, angiebt.

a) Nicht aufiteigende aber allgemein verbreitete Pflanzen oer Efene 5 at. 0 .

Malva Alcea; Astragalus arenarius, Orobus vernus; Butomus: Sedum reflexum; Hydrocotyle, Berula, Oenanthe fistulosa; Chondrilla, Scorzonera humilis; Gentiana Pneumonanthe, Polygonum lapathifolium; Polyenemum arvense; Calla; Anthericuin; Scirpus radicans; Carex distans, Pseudo-Cyperus, Phleum Boihmeri, Digitaria humifusa.

b) DDfIanzen der (5bene mit Neigung zum $\mathfrak{2} u f=$ fteige n. 4 und 1.

Paparer dubium; Cerastium brachypetalum; Lathyrus tuberosus; Lythrum Hyssopifolia; Potamogeton acutifolius, pusillus; Utricularia minor, intermedia, vulgaris, Scrophularia aquatica, Anthirrhinum Oronthium; Xanthium strumarium; Scirpus setaceus; Carex pulicaris; Rhynchospora alba; Leersia oryzoides, Setaria verticillata.

c) Ḑ finnzen ber (5ene mit zunehmender গRei= gung zum 2lufiteigen. 3 u. 2 .

$$
\text { a) Şügelpflanzer. }
$$

Rosa tomentosa; Medicago media; Galeopsis versicolor; Polygonum laxiflorum; Silene Otites; Betula pubescens; Muscari racemosum, Spiranthes.

$$
\text { bb) MOor = uno Mafferpflanjen. }
$$

Andromeda, Glyceria spectabilis, Lemna gibla, Carex Buxbaumii u. tomentosa, Hypericum tetrapterum.

d) Sृugel= แno Montanpflanzen. 2 u. 3 .

Thalictrum minus; Silene gallica; Anthyllis, Avenafatua. 


\section{5}

e) Sergpfinzen mit geringer Neigung zum ab fteigen+ $1 \mathfrak{u}+4$;

Dentaria bulbifera. Seseli, Avena pratensis, Hieracium boreale.

f) Nicht abfeigende Bergpflanzen mit 2 feh = lenden sebieten. 5 .

aа) Niedere mit Bz.

Cameline dentata; Ribes alpinum; Vicia pisiformis, sylratica; Galium sylvestre; Poa hybrida.

bb) Jૃg̊here Sierg= und Supalpinpflanzen ohme Bz.

Arabis Halleri; Viola biflora; Lonicera nigra; Atropa; Myrrhis; Campanula latifolia; Gentiana ciliata, germanica; Petasites allus, Cirsium heterophyllum, Cineraria crispa. Veratrum; Juncus filiformis, Elymus europaeus.

\section{Dutch 6 (3ebiete verbreitete Sুflanzen.}

Zudu) fie zeigen eine große Mammigfaltigkeit in iheer $\mathfrak{B e r}=$ breitung bei einem grósern 2 (retenreichthum, bie allgemein ver= breiteten, nidyt aufferigenden Wflanzen ber (Ebene verichwin= Den; bei allen গyflanzen ber CEbene findet Neigung zum 2luf= fteigen in Gơhere Regionen ftatt, bei einigen berferben tritt

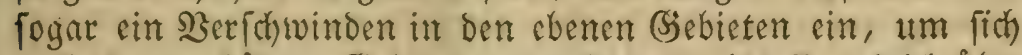
mehr in ben hóhern Gebieten zu verbreiten, bis fie endich über

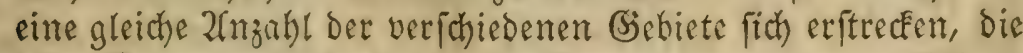
leste Grappe twird von nut Den Berggebieten eigenen Dflan= zen gebildet. 2futd) Gier treten uns 6 Şat

a) Zulgemein verbreitete ঐु flanzen Der Esbenemit

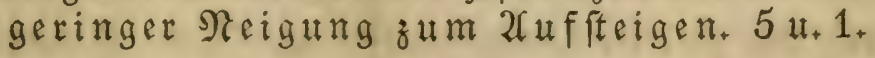

\section{aa) alle ebenen Grebiete mit $\mathrm{H}$.}

Myosurus, Ranunculus Philonothis, sceleratus, Nymphaea, Nuphar luteum; Farsetia; Comarum, Agrimonia; 
Epilobium hirsutum; Artemisia campestris, Lactuca; Datura; Veronica triphyllos; Amaranthus adscendens; Rumex maritimus; Allium arenarium; Festuca inermis. bb) mit Hb. Ranunculus Lingua (aber fehr felten Hb.), Bryonia (mehr angebaut Hb.), Cicuta, Hottonia, Potamogeton lucens. cc). mit Ch. Gratiola, Carex montana. dd) mit I. Peplis, Sium. ee) mit S. Arnoseris, Hydrocharis, Scabiosa columbaria, Marrubium, Corynephorus.

b) Spfanzen ber Ebene mit fteigender Berbrei= tung in ber ŞD he 4 u. 2 .

Cucubalus, Spergula pentandra, Viola pratensis, Corydalis cava, Vicia lathyroides, Medieago falcata, Dipsacus sylvetris, Onopordon (nid; in bir Sandebene), Gnaphalium uliginosum, Ceratophyllum, Circaea intermedia (menn ite be(ondere $2(\mathrm{rt})$, Thymus angustifolius, Chenopodium hybridum, Hypericum hirsutum, Carex cyperoides, pulicaris, teretiuscula, Melica uniflora.

c) Bsemeinfortliche Pffanzen ber Ebene und ber Sergregion mit befthranter Berbreitung úber die einzelnen (s)ebiete.

Trollius, Barbarea stricta, Silene noctiflora, Viola mirabilis, Ononis hircina, Vieia dumetorum, Geranium sallguineum,") Rosa gallica, Scabiosa ochroleura, Myosotis sparsiflora, hispida, Armeria, Chenopodium urbicum. Ornithogalum arvense, Scirpus Baeothryon, Juncus acutiflorus, Festuca elatior, inermis. Galanthus (ood) nur einmal bei Hb. gejunten; fdeint im W. Der siaf̧bach nicht vorzufommen, zwifhen ing und ber wuthenden seiffe bei sauer baufti, liebt frudbtbaren Sgoden mit \&aubholzbeid)attung, im (Sebirge in (Bárten angebaut). ${ }^{*}$ ) 1837 aud) bei $\mathfrak{B}$ Bunzlau.

d) Zuteigende Bergpflanzen.

aa) mit gróserer $\mathfrak{B e r b r e i t u n g ~ i n ~ b e r ~ F b e n e ~} 2 u .4$.

Euphorbia pilosa, Sedum villosum, Inula britanica

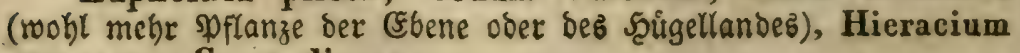
cymosum, Carex dioeca. 


\section{8}

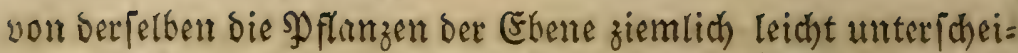
ben unb berausfinden. WBir Eonnen fie in 4 Sruppen ver= einigen.

a) Int bas $\mathfrak{B o r g e b i r g e ~ h e r n b i t e i g e n d e ~ B e r g p f l a n z e n ~ o b e r ~ S u b = ~}$ montan=Pf flanzen. 0 u. 7 .

Actaea (aud) in 0 , sodh nut am Rummeleberge); Dentaria enneaphylla, Lunaria; Stellaria nemórum; Trifolium spadiceum; Potentilla rerna; Ribes grossularia; Valeriana dioeca (audi) Riemberg W); Conyza, Prenanthes purpurea, Arnica montana; Campanula Cervicaria; Lysimachia nemorum, Primula elatior; Gentiana campestris; Verbascum Thapsus; Digitalis; Origanum ( nur am Episherg, alio mehr stuntan= SDflanze), Melampyrum sylraticum, Nepeta Cataria; Euphorbia Cyparissias. Gymnadenia, Cephalanthera ensifolia; Luzula albida; Carex brizoides; Arena flexuosa, Festuca sylvatica.

b) $\mathfrak{B e r g}=$ und $\mathfrak{B o r g e b i t g s p f l n m z e n ~ m i t ~ N e i g u n g ~ z u ! m ~ 2 t b = ~}$

$$
\text { fteigen. } 1 \text { u. } 6 \text {. }
$$

Ranunculns nemorosus; Cardamine hirsuta; Tilia grandifolia; Anthriscus rulgaris; Hieracium pratense; Malva horealis, Pyrola media; Colchicum (mit O.); Avena carrophyllea, Bromus secalinus.

c) 2rofteigende und nicht allgemein verbreitete Serg= pflanjen. 2 u. 5 .

Cerastium ovatum; Geranium molle, pratense; Hicracium floribundum, Senecio saracenicus; Valeriana sambucifolia; Vinca minor; Pyrola chlorantha; Potentilla Guentheri, Salix Russeliana, Orchis Morio; Juncus uligginosus, squarrosus; Carex ericetorum; Brachypodium pinnatum, Glyceria aquatica; Arena pubescens, Agropyrum caninum.

\section{d) Siemifd)te, ůber 7. (Sebiete verberetete Şflutzen.}

Sn einigen SBerggebieten fehlend, in allen ebenen vor. banden; die geiperrt gebrucken wohl mehr

Ranunculus bulbosus, Spiraea Filipendula; Erysimum cheiranthoides, Lepidium ruderale, Di- 
anthus Armeria, Carthusianorum, superbus, Saponaria; Berberis; Helianthemum; Melilothus officinalis, Vicia cassubica; Sagittaxia, Thys elinum, Peucedanum Orcoselinum, Silaus; Rosa rubiginosa, Agrimonia; Gnaphali um arenarium, Centaurea panicuI ata, Inula pulicaria, Eupatorium, Sonchus asper; Che nopodium gla cum, polyspermum, olidum; Atriplex latifolia; Verbena, Verouicarerna; Salviapratens is (nicht im reftlidien (sebiet); Rumex couglommeratus; Cynoglossum, Euphorbia Esula; Salix viminalis, Ulmus suberosa; Scirpus lacustris ; Carex paniculata, riparia, Bromus sterilis, Typha angustifolia, Lemua trisulca.

\section{Ditrd) 8 (Gebiete verbreitete, faft allgemein ver= breitete şflamzen.}

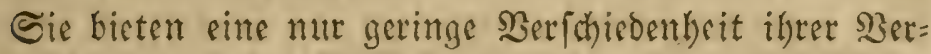
ureitung bar, auch bei ihnen ift biefelbe mehr anf Die Serg=

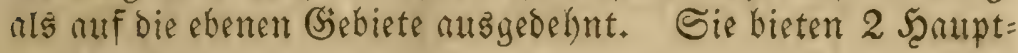
gruppen bar.

a) Zugrmein verbreitete, etwas abfteigende $\mathfrak{B e r g}=$ und Borgebirgs=:Pflanzen. 1 u. 7 .

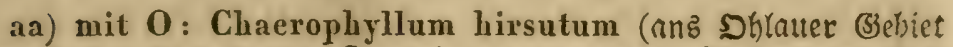

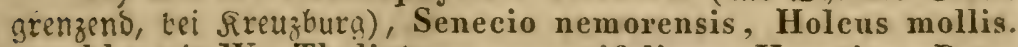

bb) mit W : Thalictrum angustifolium, Hepatica; Poterium; Galium rotundifolium; Sanicula, Sambucus racemosa; Epilobium parviflorum, Montia fontana; Carlina acaulis, Hieracium vulgatum, Milium effusum, Festuca aspera. Iab

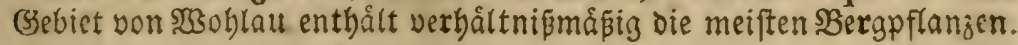

b) Jaft allgemein verbreitete, abfteigende 20rgebirgs = P) flanzen. 2 u. 6 .

Spiraea salicifolia (ift gewis mehr verwilderte als einfeimi=

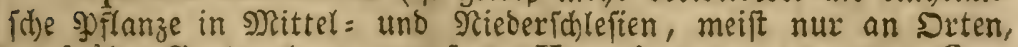
wofritfer (3artenalagen gewejen); Hypericum montanum; Cuscuta Epilinum; Calamagrostis lanceolata, sylyatica, Festu. ca gigantea, Arrhenatherum. 


\section{0}

c) Şflnmzen gemifdt in 8 (sebieten verbreitet.

Sie tragen mehr ben Sarakter ber Ebene mit Şinmei= gung zum 2ufiteigen in bie Şóhe, boch mehr in bie Submon= tan= als in bie Montan=Region, befonders a, weniger $b$.

a) Nasturtium palustre, Sisymbrium Sophia; Fragaria collina; Chimophila, Vaccinium uliginosum; Ledum palustre, (bie ihr S) Rarimum in ber Saibe bat); Verbaseum Lychnitis, Solanum nigrum; Circaea lutetiana; Senceio riscosus, Arctium minus, Artemisia Absinthium (im (Sebirge vooht mehr anaebaut), Anthemis Cotula; Euphorbia Peplus; Ulmus effusa, Epipactis palustris; Cyperus flavescens; Bromus mollis; Echinochloa, Festuca rubra.

b) Nasturtium amphibium, Camelina sativa; Sempervivum tectorum, Verbascum thapsiforme, phlomoides; Bidens cernua, Serratula tinctoria, Apargia autumnalis, Gnaphalium rectum, Filago germanica; Charophyllum temulum, Myosotis stricta; Mentha Pulegium, Rumex Nemolapathum; Potamogeton crispus; Quercus Robur; Salix alba. Allium oleraceum; Epipactis latifolia, Sparganium simplex, Typha latifolia; Juncus glaucus; Carex canescens, remota.

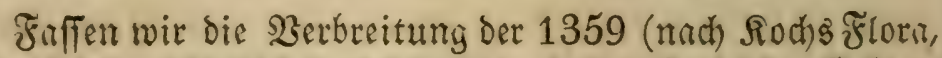
sectio I, ) Der folefifhen Pflanzen nach Der 3 ahl ber Sebiete auf, ilber welche fie verbreitet find, fo ergiebt fich folgende ùberfichtliche 3ufammenftellung.

Die folefifden Pflangen in ibret Derbreitung nach
Dabl ber Sebiete.

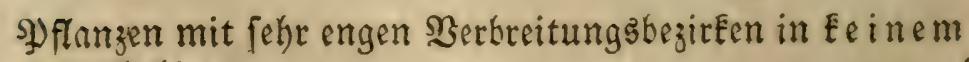

(S)ebiet. ..., . . . . . 201

Gehr vereinzelte Pflanzen mur in einem sebiet . 106 Sebr beforinft verbreitete in 2-3 Gebieten. . . 131 Sefchronft verbreitete in 4 Siebieten . . . . . 67 Må̄ig verbreitete in 5 Gebieten. . . . . . . 80

Desgl. 'in 6 =...., .., 101 


\section{1}

Fał alfgemein verbeitete \$flanzen in 7 (Sebieten . . 96 Desgl. in 8

66 2urgemein verbereitete \$ुflanzen in 9 und in 10 Gebieten 511

Betrachten wir bagegen bie $2(r t$ ber (Siebiete nach $\mathbb{R}$ age uno Erhebung, fo ftellt fich ung bie Serbreitung ber Şflin= zenarten auf folgende 2 beife entgegen.

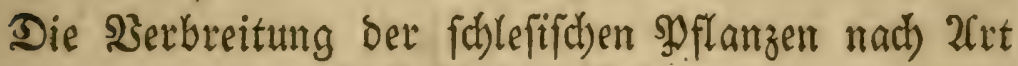
und Babl Der Ģebiete.

1) Nicht unter $3000^{\prime}$ abfeigende 2 tpin= und Subalpin=s flanzen.

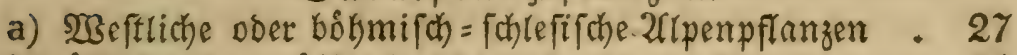

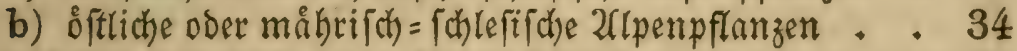

c) allgemein verbreitete 2ripenpflanzen . . . . 30 zufammen in Sd)lefien ......... . 91

2) 2Cbfeigende 2 lpin= und Subalpinpflangen bis $17-18-2200^{\prime}$.

a) Sereinzelte , , , , , , , , , 3

b) Tehr befarånte , .......... 8

c) verbreitete............... 16 zu[ammen ilt Shlefien . . . . . . . . 27

3) Nicht ab feigende Bergpflanzen, bobere Serg= und Montanpflanzen.

Seftliche Sergpflanzen in 1 (S)ebiete . . . . . 13 vereinzelte beggl. in besgl. . . . . . . 36 berdurinte deggl. in 2 u. 3 sebrieten..... 37 faft allgemeine besgl. in 4 u. 5 besgl. . ..... 23 allgemein verbr, Sergpfl. in 6 u. 7 beggl. . . . . 34 zufammen in Sdylefien, ..., ..., ... 143 


\section{2}

4) Sัn bie Şůgelregionabfeigende Sergpflanzen. Niebere Berg= und Submontanpflangell.

Seftlicte Submontanpflanzen .. vereingelte $==$ in 1 G̈sbiete .... ... 26 berchrånet verbreitete in 2 u. 3 kjebieten . . . . 7 fait allgemein verbreitete in 4 ut. $5==\ldots . .16$ allgemein verbreitete in $6,7 \mathfrak{u}, 8==. .+.42$ zufammen in Salefien. . . . . . . . . 100

5) Sn die Ebene abiteigende Bergpflanzen Doer Şïgelpflanzen bis unter 1000'.

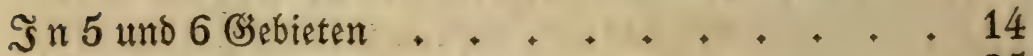
in 7 uno 8 Siebieten ............ 25 in allen zufammen ............ . 39 Summe der $\mathfrak{B e r g p f l a n z e n ~ . ~ + ~ . ~ . ~ . ~ . ~ . ~ . ~} 282$

6) Şfangen der Ebene.

a) Nicht nuffiteigende

in 1 Bsebiete.............. 97 in $2 \mathfrak{u}_{+} 3$ sebieten . . . . . . . . 28 in $4 \mathfrak{u}_{+} 5==+,+\ldots+,+, 41$ in $6===+.+\ldots+,+., 31$

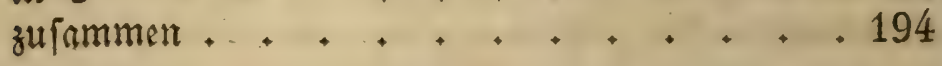

b) aufíteigende

in 4 Bsebieten.......... 26 in $5==\ldots .+\ldots, 21$ in $6==\ldots .+. . ., 18$ zufammen +........... 61

c) bie Scaide = (5)bene

in $2 \mathfrak{u}_{+} 3$ Bsebieten , $,+, \ldots, ., 22$

d) Der offtichen Ebene . . . . . . . . . 34 afke Pflanzen der Ebene. . . . . . . . . 315 


\section{3}

7) Dुflangen gemifdter Berbreitung.

2ulgemein verbreitete in nllen Bebieten

506

befchrånet verbrcitete

in $2 \mathfrak{u}_{+} 3$ Gebieten .......... 30

in 6 in $=$ = . . ... 19

in $7:=\ldots+\ldots+\ldots 1$

in $8==\ldots . . . .44$

Summe ............ 134

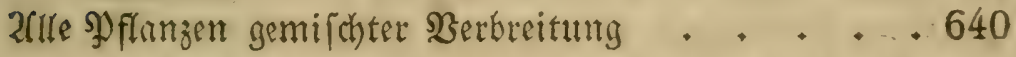

argemeine Uebericht ber sertbeilung.

\section{2lipenpflanzen}

Subalpinpflanzen , . . , . , , . . 27

Bergpflanzen +.....................

Submontanpflanzen , . . . , , , , 100

Şügelpflanzen + . . . . . . . 39

פDflanzen Der Ebene . . . . . . . . 315

(S)mifhte ⿰מ丨flanzen . . . . . . . . 640

Die 2llpen= uno Subalpinpflanzen zufammen verhalten fich zut ben SEerg = und Şügelpflimzen, den SPflanzen Der Ebene uno denen gemifcter Serbreitung wie 117:282:315:640 ober faft twie $2: 5: 6: 12$, ober es find uber noch einmal fo viel sergpflanzen, faft breimal fo viel SPflanzen ber Ebene und fait fectsmal fo viel gemifhter Serbreitung als alpine und fubalpine Spflanzen. Fine großße 3ahl der Sfflanzen ge= mifhter 2 Berbreitung gehoirt zu Den aus der Ebene in bie SBerg=

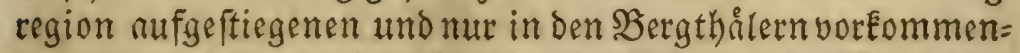
ben, cine geringere zu ben abgeftiegenen DPflinzen ber Bergregion fehr viele jeboch fint ziemlich gleichmåzig ůber alle (siebiete ver=

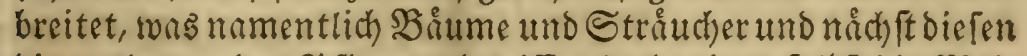
Die ausbauernden DPflanzen betrifft: Dod) zeigen felbft bie $\mathfrak{2 B e i =}$ Den und Rieferarten cine beftimmte ßorneigung für eine ober

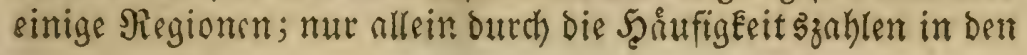




\section{4}

einzelnen Bebieten laffen fich diefe Pl flanzen gentu von e:nan= Der nach ifrer Berbreitung uber bie verfhiedenen Segionen foreiber.

3. Die Sertheilung Der \$flanzen in ifren Urten in Den einzelnen Gsebieten.

Unter $D$ flanzenvertheilung verftehen wir bas ge=

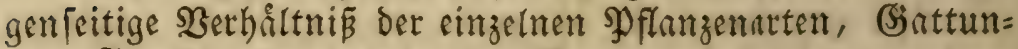
gen, Familien zu einander im Ssanzen wie in ben einzelnen

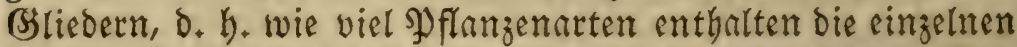
Şflanzenfamilien in diefer und jener (S̈egent, in welchem $\mathfrak{B e r}=$

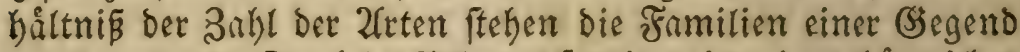
zu einander. Da iede SPflanzenfamilie ein eigenthumliches Gepråge an fid) tri̊gt, fo wirb baffelbe auch jener (segend auf=

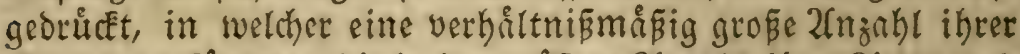

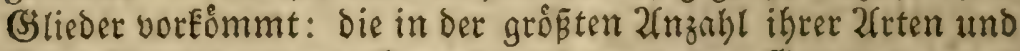
Sndividuen in eirter Giegend vorEommenden Sattungen und Familien beftimmen bemnach Den गुflanzenEarater berfelben, bas 2fusfeben bes über biefelbe gebreiteten SPflanzenteppichs. (Elementargeogr. S. 81 u. f.) Diefe Berbåltniffe laffen fich auf eine zwiefache 20 eife auffaffen: 1) bie 3ahl ber jeber einzel= nen Pflanzenfamilie in einer (3)egeno angehórigen (3)attungen und 2frten im 2flgemeinen ohne weitere Bergleichung und

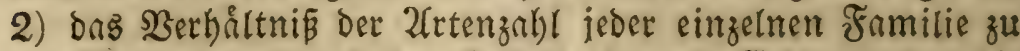
Der Sefammtartenzahl ber siegend oder des Gebiets, oder die Beftimmung der Iheilzahl, welche die 2frtenmenge einer Fn= milie zur Sefammtartenmenge des ganzen Sebiets billot. Das zreite Berbialtniß ift fur die Beftimmung bes botani= ichen Rarakters einer (S̈egeno bas wichtigite, bod) gehoren zur Bervolfítindigung aud bie .Şåufigkeitszablen. Es folgen fur beide Berbåltniffe Uleberfidytştabellen. 


\section{Die PfInustramilien}

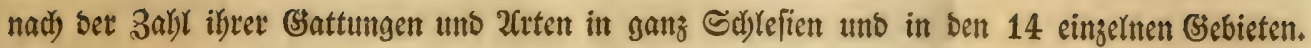

(3u Seite 244.)

\begin{tabular}{|c|c|c|c|c|c|c|c|c|c|c|c|c|c|c|c|c|c|c|c|c|c|c|c|c|c|c|c|c|c|c|c|}
\hline $\begin{array}{r}\text { \$rto } \\
\text { Fa m }\end{array}$ & & & & & & & & & & & & & $\mathrm{K}$ & & S. & & & & & & & & $\begin{array}{c}V_{0} \\
x_{4}\end{array}$ & (5) & & & & & & & r. \\
\hline & & & 42 & & & $10^{2}$ & 40 & & $9 \pm 1$ & 37 & & & 75 & 40 & 991 & 137 & i) & 40 & 14 & 41 & 6 & 43 & $87 \mid$ & 4 & & 120 & & $1+2$ & 00 & 10 & \\
\hline & & & 134 & & & $\overline{80}$ & 0134 & & 7213 & 31 & & 28 & 461 & 133 & 81 & 132 & 268 & 132 & 55 & 34 & $6 \pm 1$ & 30 & 5915 & 32 & & 29 & & 31 & 7218 & 125 & $\overline{11}$ \\
\hline & 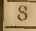 & 821 & 16 & $56 !$ & 16 & 53 & 316 & & $5+1$ & 3 & 2) & 3 & 291 & 14 & (3) & 4 & 361 & 4 & 371 & 5 & 501 & 17 & $51 !$ & 6 & 81 & 3 & 19? & 4 & 491 & 31 & $\overline{18 ?}$ \\
\hline ae . & 20 & 691 & 15 & क) & & 47 & 112 & & 4111 & 13 & 31 & 12 & 31 & 13 & & 11 & & 15 & $4 \pm 11$ & & & 14 & & 16 & & 12 & 3311 & 15 & & 9 & 21 \\
\hline$\ldots \ldots$ & $2 \pm$ & 7 & 20 & iा & & 27 & 121 & & 311 & & 11 & $1 \mathrm{~s}$ & 261 & 120 & 331 & 19 & & 17 & 61 & 19 & & 17 & 2712 & 120 & 11 & 16 & & 121 & & 16 & 22 \\
\hline c & 38 & $5+1$ & 125 & 21 & & & & & & 23 & 1 & 20 & & 24 & 11 & 23 & 3012 & 24 & & 26 & & 25 & & 27 & & 27 & & 29 & & 15 & 17 \\
\hline-3 & 22 & 53 & 20 & 331 & & +1 & & & 8111 & 18 & & 18 & 1 & 19 & 31 & 17 & 301 & 18 & 1 & 18 & & 18 & 512 & 21 & 11 & 19 & & 15 & & 4 & 24 \\
\hline ie & 14 & 81 & 10 & 321 & 9 & 81 & 11 & & 271 & 8 & & 3 & & 11 & 31 & 8 & 211 & 8 & & 11 & & 10 & $25 \mid 1$ & 12 & 30 & 8 & $2 \pm 11$ & 110 & - & 9 & 20 \\
\hline$\cdots$ & 9 & & 19 & 281 & 9 & 293 & 19 & & 251 & 8 & ए। & 7 & 31 & 17 & 261 & 7 & 231 & 9 & & 9 & 271 & 9 & 281 & 9 & 301 & 8 & 201 & 9 & 321 & 8 & 15 \\
\hline & 9 & & $y$ & $2 \pi 1$ & $y$ & $\ddot{2}+1$ & 19 & & 271 & $y$ & 231 & 9 & 01 & 9 & 231 & 9 & $2+1$ & 9 & 41 & 9 & 271 & 9 & $23 !$ & 9 & 26 & 9 & 15 & 9 & 30 & 8 & \\
\hline ceae.. & 10 & & 7 & $2+1$ & 7 & 27 & 17 & & 271 & 7 & 091 & 7 & 1 & 7 & $2+1$ & 7 & 24 & \pm & 201 & 5 & 231 & 8 & 261 & 8 & 21 & 6 & & 8 & 261 & 7 & 20 \\
\hline $16 \ldots$ & 17 & \pm 1 & 9 & 171 & 12 & $2 \pm 1$ & & & $17 / 1$ & 10 & $1 i$ & 7 & 4 & 112 & 171 & 9 & $1 \pm 1$ & 5 & 71 & 6 & 9 & 10 & & 12 & 20 & \pm & & 14 & & S & $E$ \\
\hline 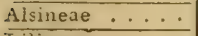 & 19 & ग1 & 9 & 211 & $y$ & 201 & 13 & & 15. & 9 & 161 & 9 & 171 & 9 & 181 & 9 & 171 & 9 & 17 , & 9 & 19] & 9 & 201 & 9 & 19 & 9 & & 9 & & 8 & 16 \\
\hline e.... & 11 & & 6 & 111 & 6 & $1+1$ & 17 & & $11 !$ & 7 & 81 & 5 & 71 & 6 & 111 & 6 & 121 & 5 & 71 & 6 & $9 !$ & $\pi$ & $1:$ & 8 & & 5 & & 7 & & \pm & 6 \\
\hline$\ldots$ & 6 & 51 & 6 & 131 & 6 & 18 & 5 & & $1 \geq 1$ & 6 & 121 & 6 & 121 & $1 \pm$ & 1 & 4 & - & 5 & 12 & 6 & $17 !$ & 6 & 15 & 6 & & 5 & 1. & 5 & प्रा & 5 & \\
\hline 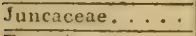 & 3 & $\div 31$ & 3 & 151 & 3 & 17! & 3 & & 13 & 3 & $1+1$ & 3 & 1 & 13 & 111 & 3 & 121 & 3 & 91 & 3 & 111 & 3 & 131 & 3 & 15 & 3 & & 3 & 16 & 3 & 7 \\
\hline- & 13 & $2+1$ & 7 & 151 & 10 & 181 & 19 & & 151 & 6 & 111 & 6 & 121 & 19 & 171 & 9 & $1 \tau \mid$ & 7 & $11 \mid 1$ & 10 & 171 & 8 & $15 i 1$ & 10 & 20 & 8 & 15 & 6 & 13| & 5 & 6 \\
\hline 0 & 9 & 1) & 18 & 111 & 18 & $1 \pm 1$ & 16 & & 7 & \pm & $6 !$ & 3 & 31 & 4 & 41 & 4 & 41 & 5 & & 6 & - & 5 & 81 & 6 & & 4 & & 4 & 51 & 2 & $\underline{2}$ \\
\hline$\cdots$ & 2 & 0) & 12 & 191 & 2 & 181 & 12 & & 161 & 2 & $1 \pm 1$ & 2 & $13:$ & 2 & 141 & 2 & 151 & 2 & 181 & $\ddot{2}$ & 181 & 2 & 17 & 2 & 1. & 2 & 10 & 2 & 181 & 2 & 10 \\
\hline$\cdots$ & 7 & 91 & 7 & 131 & 16 & $15 !$ & 15 & & $\pi 1$ & 4 & 61 & 2 & 91 & 13 & 41 & 3 & 41 & 5 & 81 & 5 & 14 & 5 & 12 & 6 & & 3 & 7 & 5 & 101 & 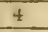 & \\
\hline & 4 & 181 & 14 & 161 & 3 & 11 & 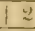 & & 31 & 2 & 51 & 2 & i) & 2 & 101 & 2 & & 3 & 131 & 3 & 1 & 4 & 101 & 2 & & 2 & 111 & 3 & 8, & 2 & \\
\hline- & 4 & 181 & 13 & 111 & 14 & 19! & 13 & & 101 & 3 & 8, & 3 & 81 & 3 & 101 & 3 & 9) & 3 & 81 & 3 & 101 & 3 & 9 & 4 & & 3 & 1 & 3 & 151 & 3 & \\
\hline & 3 & 81 & 3 & 111 & 13 & 121 & 13 & & 14 & 3 & 111 & 2 & $1+1$ & 3 & 111 & 3 & 121 & 3 & 9 & 3 & 1 & 3 & $10 \mid$ & 3 & 15 & 2 & 51 & 3 & $13 !$ & 3 & \\
\hline & 8 & 71 & 8 & 161 & 17 & 13 & 17 & & 14 & 4 & 1 & 3 & 91 & 14 & 21 & 4 & $1:$ & 4 & - & 5 & 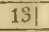 & 4 & 11 & 4 & 4 & 3 & & $=$ & 1 & 3 & \\
\hline & 3 & 161 & 3 & 91 & 13 & 10 & 13 & & 101 & 3 & 101 & 3 & 101 & 3 & 11 & 3 & 111 & 3 & 8 & 3 & & 3 & 8 & 3 & & 2 & & 3 & 21 & 3 & \\
\hline & 8 & 51 & 6 & 101 & 6 & 10 & 4 & & 6 & 5 & 91 & 4 & 71 & 4 & 8) & - & & 5 & & 5 & $\ldots$ & $\ldots$ & & 6 & & 4 & & 7 & 12 & $=$ & \\
\hline & 2 & 51 & 2 & & 2 & 101 & 12 & & 10 & 2 & 61 & 2 & S1 & 12 & 121 & 2 & 101 & 2 & & 2 & प1 & 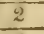 & 9 & 2 & 10 & 2 & & 2 & 101 & 2 & \\
\hline & 2 & \pm 1 & 2 & & 12 & 81 & 12 & & 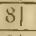 & 2 & 51 & 2 & 5 & 2 & 81 & 2 & 51 & 2 & 71 & 2 & 91 & - & $\mathrm{S}$ & 2 & 9 & 1 & & 2 & 4 & 2 & 7 \\
\hline$\cdots$ & 1 & & 1 & 101 & 1 & 81 & 11 & 11 & 10 & 1 & 81 & 1 & 6 & 1 & - & 1 & 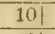 & 1 & & 1 & 91 & -1 & 91 & 1 & 6 & 1 & & T & 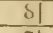 & 1 & \\
\hline$\cdots \ldots$ & 5 & ए। & 4 & 101 & 5 & 91 & 15 & & 10 & 4 & & 3 & & 4 & 81 & - & & 4 & & 4 & & 4 & 8 & 5 & 12 & 4 & & 4 & 71 & 5 & 7 \\
\hline$\ldots$ & 4 & 2 & 3 & 31 & 4 & 51 & 13 & & & 2 & 21 & 2 & & 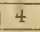 & 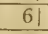 & 4 & 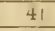 & 4 & 1 & 4 & 51 & 4 & & 1 & 6 & 1 & & 1 & 4 & 1 & 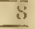 \\
\hline$\because \ldots$ & 3 & i & 2 & & 2 & 7 & 12 & & & 2 & 41 & 2 & & 2 & 51 & 2 & & 2 & 61 & 2 & 61 & 2 & 61 & 4 & 71 & 3 & & 3 & 4 & 2 & $\overrightarrow{0}$ \\
\hline & 2 & 121 & 2 & & 2 & 41 & 2 & & & 2 & & 2 & & 2 & $2 !$ & 2 & $3 !$ & 2 & 31 & 2 & 31 & 2 & 31 & 2 & $4 !$ & 2 & 21 & 3 & 81 & 2 & 3 \\
\hline & 5 & & 4 & & 4 & & 14 & & & 4 & $6 i$ & 4 & & 4 & 61 & 4 & 61 & 3 & उ। & 3 & 31 & 3 & 41 & + & 61 & 3 & - & 5 & 61 & 3 & 4 \\
\hline & 2 & & 2 & & 2 & 5 & 12 & & & 2 & 51 & 2 & 51 & 2 & 71 & 12 & 61 & 2 & 51 & 2 & 61 & 2 & 51 & 2 & 51 & 2 & 21 & 2 & 5) & 2 & 1 \\
\hline 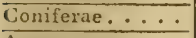 & 3 & & 3 & & 3 & 6 & 3 & & & 3 & 6) & 2 & 51 & 3 & 61 & 3 & 61 & 2 & 41 & 2 & 51 & 2 & $5 !$ & 2 & 51 & 2 & 1 & 3 & 71 & 2 & \pm \\
\hline 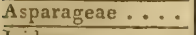 & 6 & 81 & 4 & & 5 & 1 & 15 & & 7 & 4 & 61 & 5 & & 5 & 7 & 5 & $\pi$ & 4 & 5 & 4 & 51 & 4 & 51 & 4 & 51 & $=$ & & 5 & 71 & 4 & - \\
\hline$\cdots$ & 3 & 71 & 2 & i. & 2 & & 4 & & & 1 & & 2 & & 2 & & 2 & & - & -1 & & & & - & 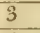 & & & & 1 & & - & $=$ \\
\hline$\ldots \ldots$ & - & $-\tilde{n}$ & 5 & & 4 & 51 & 14 & & & 3 & 4 & 3 & & 4 & & 13 & $i$ & 3 & 41 & $\ddot{3}$ & & - & : & 3 & \pm 1 & & & -1 & 5 & 3 & 4 \\
\hline$\cdots$ & 4 & 61 & 2 & & 4 & & 13 & & & 2 & & 2 & & 2 & & 2 & & 3 & $A 1$ & 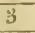 & - & - & & & & & & 2 & . & 9 & \\
\hline$\cdots$ & 1 & & 1 & & 1 & & 11 & & & 1 & - & & & 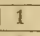 & & 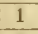 & & 1 & 4 & 1 & 41 & - & 41 & 1 & & & & 1 & 31 & 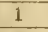 & $=$ \\
\hline . & 2 & & 2 & & - & & 12 & & & 2 & & 2 & & 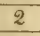 & & 2 & & 2 & $4 \mid$ & -1 & 41 & 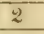 & 41 & 2 & & 2 & & 2 & 41 & 2 & 3 \\
\hline - & 1 & & 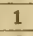 & & 1 & & 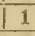 & & & 1 & & 1 & 2 & 1 & & - & & 1 & 41 & 1 & 51 & 1 & 61 & 1 & & 1 & 3 & 1 & 5 & 1 & 3 \\
\hline & 2 & & -2 & & 12 & & 12 & & & 2 & 31 & 2 & & 2 & 31 & 12 & 31 & 2 & 31 & 2 & 31 & 2 & 31 & 2 & 1 & 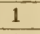 & - & 2 & 3 & 2 & $\underline{y}$ \\
\hline & 2 & & 2 & & 2 & & 12 & & & 2 & 31 & 2 & 3 & 2 & 31 & 2 & 31 & 2 & 31 & 2 & 41 & 2 & 31 & 2 & 41 & 1 & 31 & 1 & $3 !$ & 1 & 1 \\
\hline$\cdots$ & 3 & & 3 & & 3 & 4 & 10 & & & 3 & $=1$ & 3 & 4 & 3 & 41 & 3 & 1 & 2 & 31 & 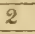 & $3 !$ & 3 & 41 & 3 & $4 !$ & 2 & - & 3 & 51 & 3 & 3 \\
\hline$\cdots$ & 4 & 51 & 3 & & 3 & & 11 & & & 1 & 1) & 1 & 11 & 1 & 11 & 1 & 11 & 1 & 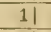 & 2 & 21 & 1 & 11 & 1 & 11 & 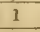 & 1 & 2 & 2 & 1 & 1 \\
\hline$\because \cdots$ & 1 & & 1 & & 1 & $=1$ & 11 & & & 1 & & 1 & 3 & 1 & 41 & 1 & & 1 & 2 & & 21. & 1 & 2 & 1 & 3 & $\ldots$ & 1 & 1 & 4 & 2 & 3 \\
\hline$\cdots$ & 2 & 51 & 2 & & ? & 1 & 12 & & & 2 & & 2 & & 2 & 21 & 12 & & 2 & 4 & - & $4 \mid$ & 2 & 5 & & & 2 & 2 & 2 & 31 & 1 & 2 \\
\hline & 1 & & 1 & & 1 & & 1 & & & 1 & 1 & -1 & 21 & 1 & 1) & 1 & - & 1 & 3 & 1 & 3i & 1 & 3 & 1 & & 1 & 2 & 1 & & - & $E$ \\
\hline & -7 & 41 & 2 & & 2 & 1 & 12 & & & - & -1 & 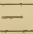 & 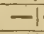 & - & -1 & $1-$ & - & 1 & - & -5 & 1) & 1 & 1 & 2 & 31 & 2 & 2 & 1 & 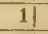 & 1 & 1 \\
\hline & 1 & & 1 & & 1 & 4 & 1 & & & 1 & 4 & & 4 & 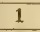 & 41 & 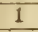 & & 1 & 4 & & & & 4 & & & 1 & & 1 & & & \\
\hline & 2 & 2 & 2 & & 2 & & 12 & & & 2 & $\approx$ & & 2 & 2 & -1 & $\sim$ & & 2 & $\approx$ & -1 & 4 & 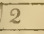 & 2 & & & 1 & 2 & & & 2 & \\
\hline & 2 & & 2 & & 2 & 4 & 12 & & 3 & 2 & -1 & 2 & ग1 & 2 & 31 & 12 & 31 & 2 & 41 & 2 & $4 !$ & 2 & 3 & 2 & $4 !$ & 2 & 3 & 2 & 31 & 2 & 2 \\
\hline & 2 & 31 & 2 & 21 & 2 & 21 & 12 & & $21-$ & $=$ & -1 & - & 1 & - & -1 & - & -1 & 2 & 21 & 2 & 21 & 2 & 21 & 1 & 21 & 2 & 21 & 1 & & - & $=$ \\
\hline$\cdots$ & 1 & 1 & 11 & & $=$ & & 1 & & 21 & 1 & 21 & 1 & 21 & 1 & 21 & 11 & 21 & 1 & 31 & - & 31 & -2 & 21 & 1 & 31 & 1 & i & 1 & 41 & 1 & $\overline{1}$ \\
\hline$\cdots$ & 1 & & 1 & & 1 & 2 & 1 & & 31 & 1 & 31 & 1 & 21 & 1 & 31 & 11 & 31 & 1 & 31 & 1 & 3 & 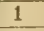 & 21 & 1 & 31 & 1 & 1) & 1 & 31 & 1 & 1 \\
\hline 0 & 2 & & 1 & & 11 & 1 & 11 & & 11 & 1 & 1) & 1 & 1) & 1 & 11 & 1 & 1) & 1 & 11 & 3 & 1) & 1 & 1) & 2 & 21 & 1 & 1 & 2 & 21 & 1 & 1 \\
\hline . & 1 & & 1 & & 1 & & 11 & & & 1 & 31 & 1 & 3 & 1 & 31 & 1 & 31 & 1 & 31 & 1 & $3 \mid$ & 1 & 31 & 1 & 21 & 1 & 1 & 1 & 21 & 1 & $\overline{1}$ \\
\hline ae & 1 & & 1 & & 1 & 1 & 1 & & & 1 & 5 & & & 1 & 31 & 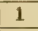 & & 1 & 31 & - & 3) & $x$ & 31 & 1 & ग1 & 1 & 3 & 1 & 21 & 1 & 3 \\
\hline - & 2 & 31 & 2 & & 2 & 31 & 1 & & 2 & 1 & 1 & 4 & & 1 & & 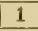 & & 1 & 1) & 2 & 3) & 2 & 31 & 2 & 31. & 1 & 11 & 1 & 1) & 1 & \\
\hline 0. & - & 31 & & 1 & & 1 & $1-$ & & $=$ & 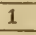 & & & & & & & & - & $-1-$ & - & & $=$ & -1 & - & -1 & - & - & & 11 & & \\
\hline eae ... & & & & & & 2 & 2 & & & 1 & & & & an & 21 & 12 & & & & & 1) & 1 & & 3 & & - & & 2 & 21 & 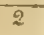 & \\
\hline
\end{tabular}

") $\mathbf{G n}_{0}=$ (snabenfetb, St. = Steinau, $\mathbf{R} .=$ Riefengebirge, Gr. = Brunau. 



\section{6}

Framilien in bie Giefammtnatenzahl ber Flota sines Biebiets

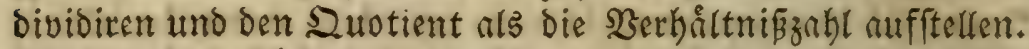
So bilderi bie 143 2rten ber Compositae in gamz Echlefien

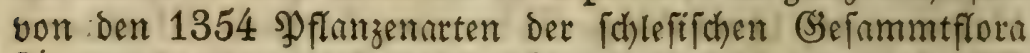

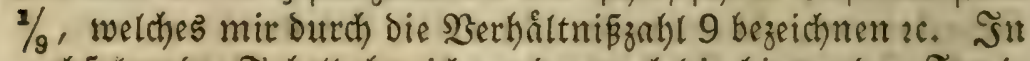
nachfolgender Tabelle bezeifhnen bemnad) bie hinter. Den Fami= liennamen und unter ben Gebietsnamen ftebenden 3ahlen den wievielteften Theil die Framilienarten von ben Gef fammtarten in einem Gebiet bitben und bienen fo zur Ereennung der (5i=

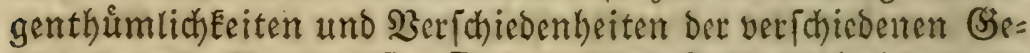
bietsfloren. Die mit Gr. B. bezeidynete 3aflenteife brzeichnet

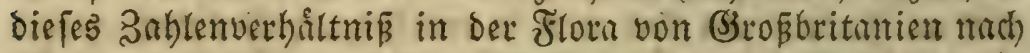

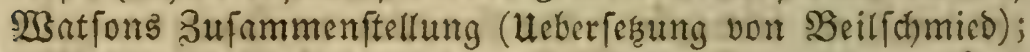
fie ift zur Bergleidyung mit ber fchlefifchen Flori binzugefügt. (20ergleithe bie Iabclle).

Sei den mur 1 2fet enthaltenden 14 Familien ift bis Berbåltnişahl 1/1354 Der Ģefammtarten.

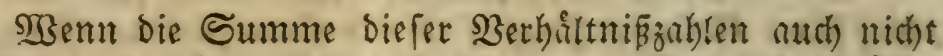

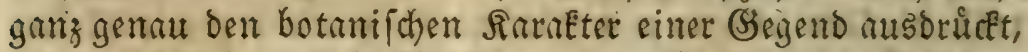

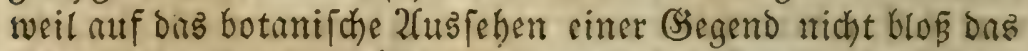

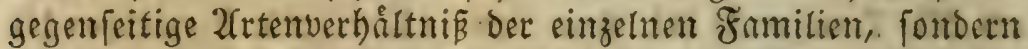

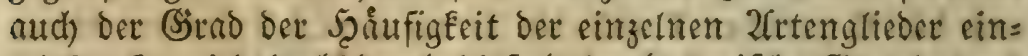
wilet, fo wird boch' Durch biefelbe ber botanifa)e (Grundtypus

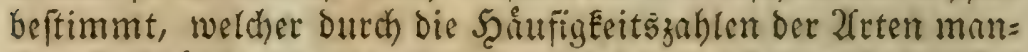
cherlei 2fbinderungen erleibet. Hnwefentlicher-and weniger auf ben alfgemeinen $\mathfrak{P f l a n z e n t n p u s ~ c i n e r ~ G e g e n d , ~ b e f o n d e r s ~}$ in oer gemåâigten 3one, eintwirkend find bie 2fbonderungen bei

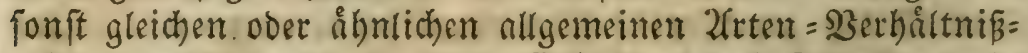

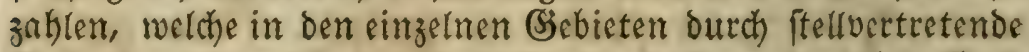
Zrten bewiret werben, bas heißst burd) 2frten Derfelben Ģat= tung, welde in einem Bsebiete an bie Stelle einer andern hier fehlenden, in einem andern Bsebiete aber vorÉommenden 2frt 


\section{Die 2utengal ser Framilien}

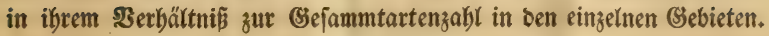

(3u Seite 246.)

\begin{tabular}{|c|c|c|c|c|c|c|c|c|c|c|c|c|}
\hline Familien. & Schl. & $\begin{array}{c}\text { Gr. } \\
\text { B. }\end{array}$ & Bz. & L. & H. & $\mathrm{Hb}$ & K. & S. & Ch. & B. & O. & W. \\
\hline Compositae . . & 9 & 11 & $9 \cdot 10$ & 9 & $8-9$ & $8-9$ & 8 & 8 & 8 & 8 & 9 & 9 \\
\hline Gramineae .... & 13 & 13 & $11-12$ & 12 & 12 & 12 & 15 & 12 & 11 & 13 & 13 & $14^{2}$ \\
\hline Cyperaceae.... & 16 & 16 & 16 & 17 & 15 & 22 & 22 & 22 & 21 & 19 & 16 & 15 \\
\hline Papilionaceae ... & 19 & 22 & 19 & 19 & 20 & 21 & 20 & 20 & 19 & 16 & 17 & 20 \\
\hline Cruciferae ... & 24 & 21 & 25 & 34 & 25 & 33 & 28 & 24 & 24 & 27 & 26 & 32 \\
\hline Umbellatae.... & 25 & 23 & 28 & 25 & 83 & 26 & 29 & 25 & 24 & 25 & 25 & 26 \\
\hline Labiatae ..... & 25 & 29 & 27 & 27 & 27 & 24 & 23 & 22 & 24 & 25 & 26 & 22 \\
\hline Ranunculacese . & 28 & 421 & 28 & 139 & 30 & 28 & 31 & 28 & 35 & 28 & 29 & 31 \\
\hline Rosaceae ..... & 32 & $19^{2}$ & 31 & 31 & 33 & 31 & 27 & 30 & 32 & 29 & 30 & 28 \\
\hline Amentaceae ... & 32 & $18^{3}$ & 32 & 38 & 30 & 30 & 31 & 35 & 31 & 29 & S0 & 30 \\
\hline Rhinanthaceae - & 33 & $88^{4}$ & 96 & 34 & 30 & 30 & .30 & 32 & 31 & 35 & 35 & 30 \\
\hline Orchideae .... & 40 & 41 & 50 & 38 & 48 & 41 & 45 & 46 & 53 & 95 & 89 & 51 \\
\hline Alsineae ..... & 47 & -5 & 42 & 46 & 46 & 43 & 37 & 44 & 44 & 41 & 42 & 39 \\
\hline Liliaceae .... & 48 & 66 & 80 & 65 & 75 & $87 \quad$ & 90 & 72 & 62 & 99 & 89 & 65 \\
\hline Juncaceae .... & 54 & 54 & 58 & 54 & 63 & 531 & 57 & 72 & 62 & 75 & 78 & 60 \\
\hline Sileneae...... & 54 & -5 & 67 & 51 & 68 & 58 & 54 & 46 & 44 & 58 & 47 & 41 \\
\hline Borragineae ... & 56 & 63 & 58 & 51 & 55 & 68 & 59 & 46 & 44 & 63 & 47 & 52 \\
\hline Scrofulerinae .. & 64 & 76 & 80 & 65 & 117 & 116 & 211 & 198 & 174 & 70 & 73 & 98 \\
\hline Polygoneae.... & 68 & 66 & 51 & 51 & 51 & 49 & 48 & 57 & 49 & 39 & 45 & 46 \\
\hline Alismaceae.... & 71 & 89 & 67 & 61 & 117 & 116 & 316 & 198 & 186 & 84 & 58 & 65 \\
\hline Chenopodiaceae. & 75 & 61 & 55 & 83 & 273 & 139 & 90 & 79 & 81 & 53 & 67 & 78 \\
\hline Onagrariae.... & 75 & 115 & 80 & 76 & 82 & 87 & 79 & 79 & 82 & 87 & 80 & 87 \\
\hline Stellatae .... & 75 & 72 & 80 & 76 & 59 & 63 & 45 & 72 & 62 & 71 & 80 & 78 \\
\hline Ericaceas.... & 79 & 76 & 55 & 70 & 59 & 58 & 70 & 66 & 62 & 99 & 62 & 71 \\
\hline Campanulaceae & 85 & 115 & 98 & 91 & 82 & 69 & 63 & 71 & 68 & 81 & 101 & 98 \\
\hline Primulaceas ... & 90 & 80 & 88 & 91 & 137 & 77 & 90 & 97 & 93 & 99 & 115 & 112 \\
\hline Geraniaceae... & 90 & 95 & 125 & 91 & 82 & 116 & 79 & 66 & 74 & 99 & 89 & 87 \\
\hline Gentianeae.... & 97 & 101 & 146 & 114 & 82 & 87 & 90 & 99 & 93 & 174 & 202 & 196 \\
\hline Euphorbiaceae. . & 97 & 89 & 125 & 114 & 103 & 139 & 126 & 99 & 149 & 99 & 90 & 98 \\
\hline Violariae .... & 105 & 190 & 88 & 114 & 82 & 87 & 105 & 87 & 74 & 74 & 81 & 87 \\
\hline Solaneae .... & 113 & 126 & 58 & 102 & 82 & 87 & 126 & 99 & 198 & 83 & 89 & 98 \\
\hline Dipsaceae.... & 113 & 253 & 293 & 183 & 273 & 347 & 816 & 132 & 186 & 174 & 176 & 157 \\
\hline Crassulaceae... & 113 & 95 & 125 & 131 & 137 & 174 & 126 & 158 & 186 & $3+8$ & 404 & 262 \\
\hline Saxifrageae.... & 113 & $80^{6} 1$ & 220 & 229 & 205 & $17 \pm$ & 316 & 296 & 248 & 232 & 269 & 262 \\
\hline Caprifoliaceae... & 150 & 138 & 146 & 153 & 137 & 116 & 126 & 132 & 124 & 232 & 269 & 196 \\
\hline Valerinneae ... & 150 & 190 & 146 & 153 & 137 & 139 & 126 & 113 & 124 & 139 & 134 & 157 \\
\hline Coniferae..... & 150 & $\$ 79$ & 146 & 158 & 117 & 116 & 126 & 132 & 124 & 174 & 161 & 157 \\
\hline Asparageae.... & 169 & 190 & 176 & 131 & 117 & 99 & 90 & 113 & 107 & 139 & 161 & 157 \\
\hline Irideae ....... & 193 & 217 & 440 & 906 & 273 & 231 & 632 & 792 & 746 & 348 & 202 & 392 \\
\hline Pomaceae..... & 193 & - & 176 & 188 & 164 & 174 & 158 & 158 & 186 & 174 & 202 & 196 \\
\hline Urticeae $\ldots \ldots$ & 226 & 305 & 293 & 183 & 235 & 231 & 211 & 264 & $2 \pm 8$ & 174 & 202 & 157 \\
\hline Plantagineae... & 226 & 305 & 293 & 229 & 273 & 231 & 211 & $26 t$ & 248 & 174 & 202 & 196 \\
\hline Convolvulaceae. & 226 & 305 & 176 & 185 & 164 & 281 & 158 & 132 & 124 & 174 & 202 & 196 \\
\hline Hypericinae ... & 226 & 138 & 176 & 153 & 164 & 139 & 316 & 158 & 149 & 174 & 161 & 131 \\
\hline Fumariaceae... & 226 & 253 & 440 & 306 & 273 & 231 & 316 & $26 \pm$ & 248 & 292 & 269 & 262 \\
\hline Malvaceae .... & 271 & 253 & 220 & 306 & 273 & 231 & 211 & 264 & 248 & 232 & 202 & 262 \\
\hline Sanguisorbeae... & 271 & - & 220 & 229 & 273 & 174 & 158 & 198 & 186 & 232 & 269 & 196 \\
\hline Paronychiae ... & 271 & 217 & 293 & 229 & 205 & 695 & 632 & 792 & 746 & 697 & 404 & 785 \\
\hline Ribesinae..... & 271 & 253 & 220 & 229 & 273 & 139 & 211 & 198 & 186 & 232 & 404 & 392 \\
\hline Typhaceae .... & 271 & 253 & 176 & 183 & 164 & 231 & 516 & 364 & 373 & 174 & 202 & 157 \\
\hline Najades ....... & 339 & 379 & 220 & 306 & 273 & 231 & 316 & 792 & 1248 & 232 & 269 & 196 \\
\hline Amaranthaceae. & 399 & $1517^{7}$ & 298 & 229 & 410 & 一 & - & - & $1-$ & 697 & 404 & 392 \\
\hline Callitrichinae .. & 339 & 305 & 220 & 229 & 235 & 174 & 158 & 198 & 186 & 174 & 202 & 196 \\
\hline Droseraceae ... & 339 & 506 & 220 & 229 & 273 & 347 & 316 & 396 & 373 & 348 & 404 & 392 \\
\hline Papaveraceae .. & 839 & 138 & 220 & 229 & 273 & 174 & 211 & 264 & 248 & 174 & 202 & 262 \\
\hline
\end{tabular}

Nymphaceae, Polygaleae, Acerinae, Celastrinae, Rhamneae, Amygdaleae, Lythrariae, Eleagneae, Colchiaceap, jebe mit 3 2rten ober mit $1 / 452$ ber Befammtarten in Sditefien.

Globulariae, Aroideae, Monotropeae, Hyprocharides, Aristolechiae, Thymeleae,

Apocyneae, Scelerantheae, Portulàcaceae, Halorrhageae, Oxalideae, Tiliaceae, Lineae, Elatineae, Resedeaceae, Jasmineae, jebe mit 2 2 frten ober 1/678 ber Gejammiarten.

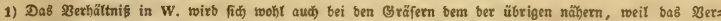
geiđ̆nib berietben von $\mathbf{W}$, niळht volffünbig ift.

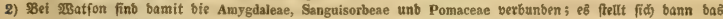
Z3erhältni in Sditefien 22.

3) SThne Ulmacese $z$ Xrten.

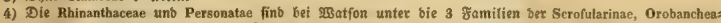

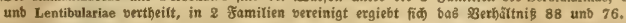

5) श्3ation vereinigt bie Alsineae unb Elatineae mit ben Sileneae unb erhalt bie \$erbältnifzaht 26 für

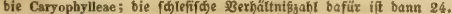

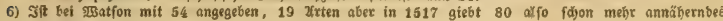
刃erbättnif.

7) \$Bei Tgation if Iferniaria mit 2 zrten babon getrennt. 



\section{7}

auftritt. (Ein 2efhnliches, went auch nicht in biefer 2(usbef)= mung, gilt von ben fterlyertretemben Barattungen.

W3endent wir uns zut Setrachtung der \$ertheilungşweife Der Familien und 2frten in ben 10 einzelmen fchlefif Then Gse= bieten, fo finden wir bei vielen \$gfanzenfamilien oaffelbe

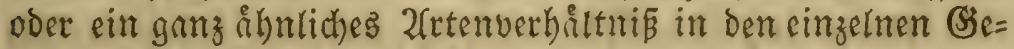
bieten, wie in ben Bebietsgruppen (Der (Ebene ze.) mit Dem Der fohlefif fhen (Sefammtflora, namentlich gilt biefes von dent artenreid)en Familien im 2argemeinen, weniger bei ben arten= armen, weil in benfelben der 2frtenunterfchied und barum suth)

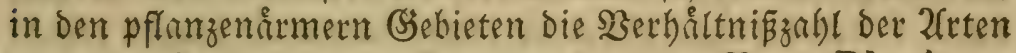
geringer ift ats in bent pflamzenteichern, z. BB. Die Rhoderaceae enthalten in Schlefien nur 1 2rt, bie ßethåltniszaht für biefelbe ift für ganz Schlefien 1354, firt Das Ëebiet von אupferterg mit 632 2(rten aber 632.

WBir wollen nicht auf Die Eleinere Berfchiedenbeiten in Den cinzelnen B̈ebieten und Giebietsgruppen eingehen, weil fie zu fegr ins (Einzelne und bennoch) zu Eeinen wichtigen Er= gebniffen ůber die \$flanzenvertheilung fưfren witrben, fon= Dern nur vorzigglich bie Serfchiedentheiten auffaffen, welche Den cinzelnen Gebietsgruppen ober Den verfchiebenen Regio=

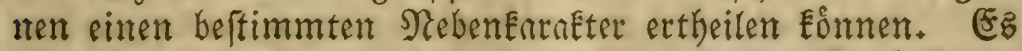
Iaffen fich in diefer Beziehung die \$flanzenfamilien in 4 Ģrup= pen bringen:

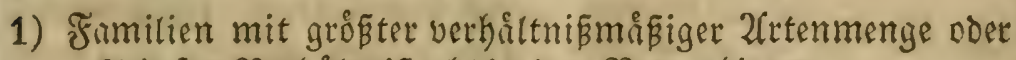
Eleinfter Berfi̊ltni Bzagl in Den Berggebieten.

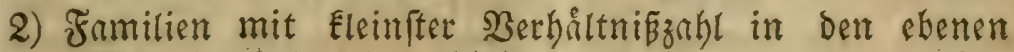
Gebieten.

3) Familien mit gleicher ober faft gleither Werhåltnişabl in beiben, Sebietsgruppen.

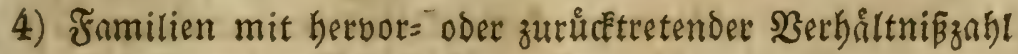
in einem ber Bsebiete. 


\section{8}

Die beiben erften (Simppen find bie wichtigften, ourch fie treten uns die EigenthủmlichEeiten und $\mathfrak{B e r}$ fhiebenheiten ent= gegen, burd) welde bie burch die britte (Sruppe hervorgehrachte (šleid)förmigkeit, wenn auch nicht ginnzlich aufgehohen, Dod) wenig/tens megr oder wentiger abgeandert wird.

um zu gewiffen Refultaten zu gelangen, faffen wir bie 5 eigentlichen SBerggebiete-H., Hb., K., S., Ch. als eine

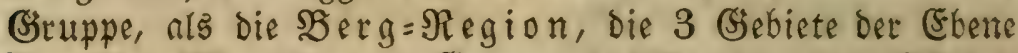
Bt., O., W. als ziveite Siruppe, die Region ber Efbene zufammen und laffen Bz. uno L. als gemifhte (Sebicte ats

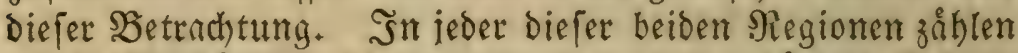
wir bie Serhåltniß̈zahlen der einzelnen ihr zugehorigen (sebiete zufammen und nefmen die mittlere Durchichnittşahl als die

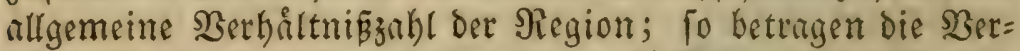

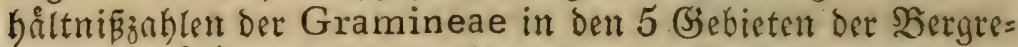
gion $62,62 / 5=12,4\left(12 \frac{4}{10}\right)$, in den 3 Siebieten Der ebe= nen Region $40,4 \%=13,3\left(13 \frac{3}{10}\right) ; 12,4$ und 13,3 be $=$

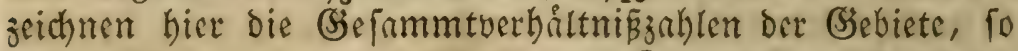

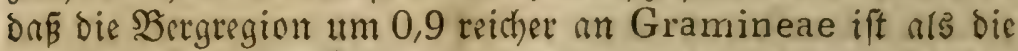
ber Ebene. 0,9 wutrde alfo ben botanifhen unterfded bei= Der Siegionen hinfichtlich Der Gramineae ausbruden. Dieje Unterichieosjaflen fino aber nicht gleichwerthig bei ben an $2(r=$ tengahl verfdicoenen Familien, on bie allgemeinen \$erbåltniß = zahlen mit ber 2(bnal)me ber 2(rtenzahlen junehmen und bar: um fich grópere unterichiedszahlen heraus/tellen, burch welche jeboth Der botanijche Rarafter einer Siegent wentiger verindert

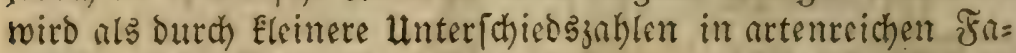
milien. Es find aus biefer Urfache in nachfolgender Ueber=

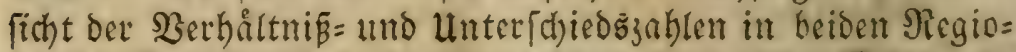
nen bie Frmilien nach ifrem 2(rtenteichthum in ber (Sefammt= flora und nicht nach ibern Unterichiedszahlen an sinander $g e=$ oronet. M. bezeichnet die Serbåltniß̈zahlen ber Şrig=, Pl. der ebenen Region, D. bie Unterichiedszathlen. 


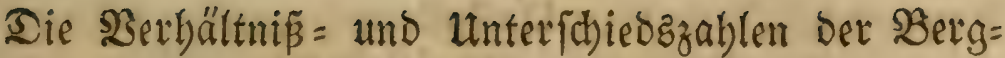 und ebenen Region.}

ఇalanzenfamilien mit dem Marimum.

a. in Der ミergregion. Fumilien. M. Pl. D. Frmilien. M. Pl. D.

\section{ůber 100 2reten}

Compositae $\quad 8,3 \quad 8,6 \quad 0,3$

Gramineae $\quad 12,4,13,3 \quad 0,9$

zwi chen 50 und 100 2frten

$\begin{array}{lllllllll}\text { Cruciferae } & 24,8 & 28,3 & 3,5 & \text { Cyperaceae } & 20,4 & 16,6 & 3,8\end{array}$ Labiatae $\quad 24,0 \quad 24,3 \quad 0,3$ Papiliona-

\begin{tabular}{|cccc} 
ceac & 20,0 & 17,6 & $\mathbf{2 , 4}$ \\
Umbellatac & $\mathbf{2 7 , 4}$ & $\mathbf{2 5 , 3}$ & $\mathbf{2 , 1}$
\end{tabular}

givifacen 40 und 50 2rten

Rhinanthaceae

$30,633,32,7 \mid \begin{array}{llll}\text { Ranuncula- } & & & \\ \text { ceae } & 30,4 & 29,3 & 1,1 \\ \text { Rosaceac } & 30,6 & 29,0 & 1,6 \\ \text { Amentaceae } & 31,4 & 29,6 & 1,8\end{array}$

zwifhen 20 und 40 2(rten

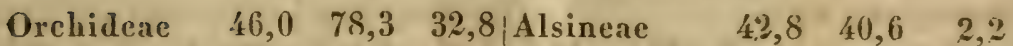
$\begin{array}{llllllll}\text { Liliaceae } & 77,2 & 8,3,3 & 7,1 & \text { Sileneae } & 54,0 & 48,6 & 5,4\end{array}$

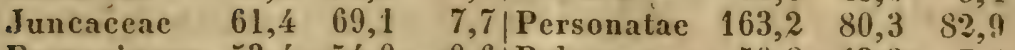

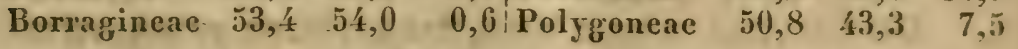
3mifden 15 und 20 2frten

$\begin{array}{lllllll}\text { Onagrariac } & 82,2 & 84,6 & 2,4 \\ \end{array}$ Stellatae $\quad 60,2 \quad 78,3 \quad 18,1$ ChenopodiEricaceae $\quad 63,0 \quad 77,3 \quad 14,3$, aceae $132,6 \quad 66,0 \quad 66,6$ Campanulaceae $\begin{array}{lllll}70,6 & 93,3 & 22,7\end{array}$ Primulaceae $\quad 98,8 \quad 105,6 \quad 6,8$ Gentianeae $\quad 90,2: 190,6: 100,4$ Geraniaceáe $83,4 \quad 91,6 \quad 8,2$ 


\section{0}

Frmilien. M. PI. D. Familien. M. PI. D. zwi Then 10 und 15 2frten

Crassulaceae 158,2 $338 \quad 179,8$ Euphorbia

$\begin{array}{lrrr}\text { ceae } & 123,2 & 95,3 & 27,9 \\ \text { Violariae } & 87,0 & 80,6 & 6,4 \\ \text { Solaneae } & 97,0 & 90,0 & 7 \\ \text { Dipsaceae } & 251,6 & 164,0 & 87,6 \\ \text { Saxifrageae } & 267,8 & 254,3 & 13,5\end{array}$

zwifchen 7 und 10 2(rten

Caprifoliaceae

$127,8 \quad 232,3 \quad 105,3$

Irideae

$534,4314 \quad 220,4$

Valerianeae $127,8 \quad 143,3 \quad 15,5$

Coniferae $\quad 123,0 \quad 164,0 \quad 41,0$

Asparageae $105,2,152,10 \quad 46,8$

Pomaceac $168,0190,6 \quad 22,6$

$6 \cdot 2$ rten

Convolvulaceate

$161,8 \cdot 190,6$

Urticeac $\quad 231,8 \quad 177,6 \quad 54,2$ $28,8 \mid \begin{array}{lrrr}\text { Plantagi- } & & & \\ \text { neac } & 244,6 & 190,6 & 54,0 \\ \text { Hypericinae } & 185.2 & 155,3 & 29,9 \\ \text { Fumariaceae } 266,4 & 254,3 & 12,1\end{array}$

52 frten

Malvaceac $2450,8 \quad 254,3 \quad 8,5$ Typhaceac $\quad 291,6 \quad 177,6 \quad 114$

Sanguisor beae $199,8 \quad 254,3 \quad 54,5$

Paronychi. eae

$614,0 \quad 628,6 \quad 14,6$

Ribesinae. 201,6 342,6, 141

Die Framilien unter 5 arten find bei ihrem geringen Ein: flus auf ons (sefammtausfelen ber Flora nicht in Sbetracht gezogen worden.

Zlts Die Bergregion butd) iffr Marimum bezeichnente $\mathfrak{F}$ : milien wurben bemmach feitt: Die Gramineae, Cruciferae, Rhinanthaceae, Orchideae, Liliaceae, Iuncaceae, Stellatae, Ericaceae, Campanulaceae, Gentianeae, (Primulaceae, Geraniaceae), Crassulaceae, Caprifoliaceae, Asparageae. 


\section{1}

S̈n Der Region Der Ebene Dagegent witroen bie Cyperaceae, Papilionaceae, Umbellatae, (Ranunculaceae, Rosaceae, Amentaceae), Sileneae, Personatae, Alismaceae, Chenopodiaceae, Dipsaceae, Irideae, Urticeae, Plantagineae, Typhaceae den NebenEntateter ks: zeichnen und beftimmen.

Die Frmilien ber Amarantaceae, Aroideae, Elatineae, Cucurbitaceae, Berberideae, Halorageae fehten gant oder zum I Theil Den Berggebieten, Die Elaeagneae der Ebene; alle biefe Familien fint jedoch fo artenarm, das fie

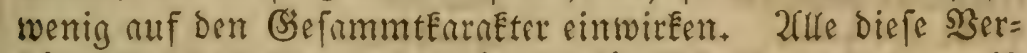

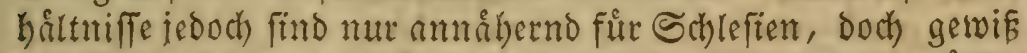

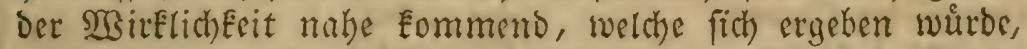
went man alle Berggebiete von Schlefien mit benen ber Ébene Itatt ber wenigen, Die zut \$ergleichuntg vorlagen, vergleichen Econnte. Einige Fumilisn, als bie Cyperaceae $\mathfrak{k}$. Gramineae, bic Rhinanthaceae $\mathfrak{u}$. Personatae, Dic Stellatae $u$. Caryophylleae (Alsineae $\mathfrak{~ S i l e n e a e ) , ~ b i e ~ G e n t i a n e a e ~}$ u. Alismaceae, Dic Campanulaceae $\mathfrak{~}$. Gentianeae forci=

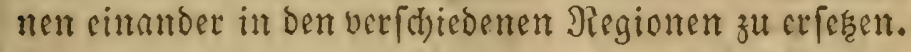

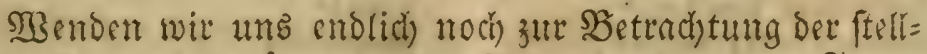
vertretenden und ergånzendenten 2reten Der einzelnen Frami= lien in ben briben Regionen, fo weit nuch für biefe Rerglci= d)ung bie vorlisgenden ahat [achen ausreichen, fo finden wir, oas bei vielent Framilien fich biefelben nachweifen laffen, ji

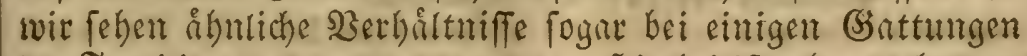
Der Familien uns entgegentreten. Sezerbei ift aber zu bemer= Een, Daßs viele biefer 2(rten, befonder's die nit Fragezeich)en be= zeichneten, nicht im ftrengften Sinne nls ftelluertretento anju= nehmen find, intom fie fich durch sin anderes $\mathfrak{B}$ orfommen und

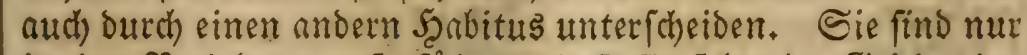
in ber Beziehung aufüufuhren, daß fie fehlende Bstieder ber (B)attungen und ramitien erg̊ng̊n. 


\section{2}

\section{Stellvertretende Irten}

Der $\mathfrak{B e r g}=$

Anemone alpina

Ranunculus aconitifolius

\section{nemorosus}

Delphinium elatum (A. \&. i. Consolida 5.0(i)gebirge.)

Arabis Halleri

Viola biflora

Dianthus prolifer

Vicia sylvatica

pisiformis

Astragalus Cicer

Spiraca Aruncus

Fragaria elatior

Potentilla verna

Rosa alpina

$$
\text { aurea (A.) }
$$

Epilobium origanifolium

Sedum villosum

Aster alpinus (A.)

Cineraria crispa

Anthemis tinctoria

Autirrhinum majus

Teucrium Botrys

Androsace Chamaejasme

Plantago montana

Veronica bellidioides (A.) montana (alpina $A$.)

Gentiana cruciata

Lysimachia nemorum

Allium ursinum?

Gerardi

tricọlor

Armeria

dumetorum

cassubica

arenarius

Filipendula

collina?

supina

rubiginosa

(A.) hirsutum

reflexum

salignus

palustris

Cotula

Orontium

Scordium

arenaria?

triphyllos

Numularia

arcnarium der ebenen Region. Spratensis (fruchtbare (Fbene) vernalis (5aibe, গRiefengebirge)

Lingua?

\{bulbosus

septentrionalis

serpyllifolia?

Pneumonanthe

Stelluertratende Gsattungen

\section{ber Berg=}

Aquilegia

Helleborus

Aconitum

Actaca
Nigella Adonis ?

Isopyrum

Myosurus? 
Lunaria

Hedysarum

Sanicula

Imperatoria

Pleurospermum

Chaerophyllum aureum

Homogyne

Arnica

Carduus crispus

Prenanthes purpurea

Conyza

Omphalodes

Atropa

Digitalis

Nepeta

Leucojum

Streptopus

Lilium Martagon

Veratrum

Axum
Farsetia

Onobirychis

Hydrocotyle ?

Berula

Sium ?

Cicuta

Eupatorium

Matricaria

Onopordon

Chondrilla

Artemisia campestris ?

Asperugo

Datura.

Gratiola

Marrubium

Galanthus

Sayiltaria ?

Anthericum

Tofieldia

Calla. 


\section{2f it a it $I$.}

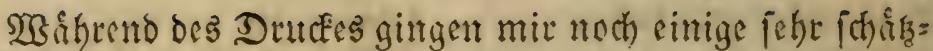
bare Seitrige outh gutige Mittheilung einiger Ro Ealfloren ju. Reiber fonnte ich ben in Denfelben enthaltenen Stoff nicht vollfạndig benůgen und bem ůbrigen Stoff gemås verarbei= teit, weil ber Drute bercits zu weit vorgefd)ritten war. J(t) muste mich begnitgen, bis Bablenverbaltniffe in ben beiben Uteberfichtstabellen mit anzufügen, und mur einige Şauptre fultate in biefem Nachtrng zufammen zu ftellen. Durch bis Bermittelung des Şerrn SProf. Dr. GS Dop pert erbielt ich bie

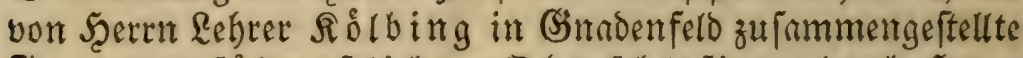
Flora vom fúb weftlichen Sber fhlefien, duth Şetrn

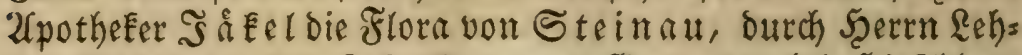
rer Scellwig die Rokalflora von Gsunau bei Şirfhberg und butch Scerrn Morif Els ner feine neu im Druce er: fohientene Flora von Şirfaberg und dem Riefengebirge, soobutch ich mid ben gentanten Scerren zum gro̊sten DanE verpflichtet fühle.

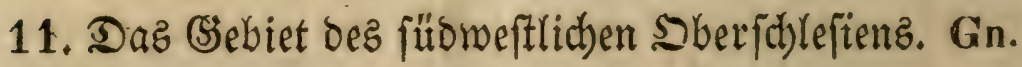

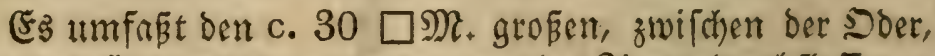
Sppa und Seokenplof gelegenten, von ber Binna burchfloffenen fübeftlichen Theil von Sberfdefefen unb bildet eine nach $O$ und $S$ geneigte, wellenformige, von mut geringen Erhebungen,

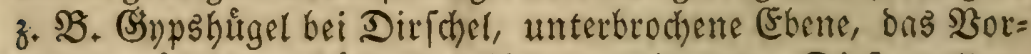
lano ber offliden Scálfte bes 2fltuatergebirges. Dieje wellen: 


\section{5}

formige (Ebene erbebt fich) von c. 444' (Şorenplof̧můnoung bei Irrappis) bis c. 1000' (Dppabette bei Şigernoorf 959'). Soerberg 605', Ratibor 552', Fofel 506', (sinpsberg bei

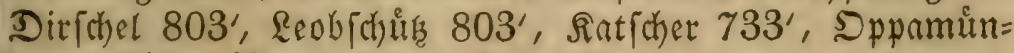
bung 656', Bsmadenfeld 650'. Das hin und wieder zu Iage liegende, grofistentbeils aber mit fruchtbarer 2(deerErume

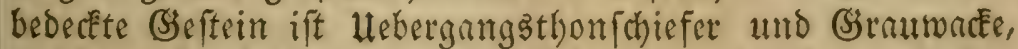

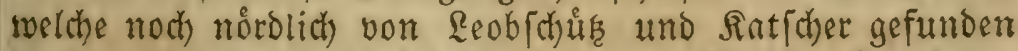
werden, nus benen fich bei NeuEirch und Dirfhel Sinpsberge,

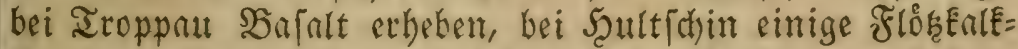

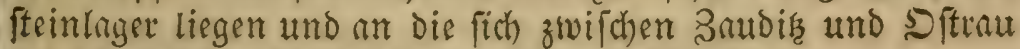
ein fohmales Sohlenfandfreingebirge anfhliekt.

Dic Flora bes (siebiets exicheint im 2lllgemeinen reidy;

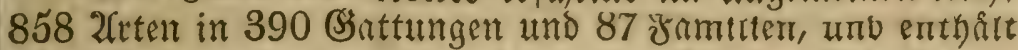
29 Pflanzenarten, welche in feimem ber ubrigen Gebiete vor= Eommen, und gróftentheils zu ben 56 şflamzen der oberfchle= fifchen Flora gehoren. Der Eharafter ber Flora ift in bee Iabelle ausgefprochen; fie enthåt noch manche Sergpflanze, befonders in ber Nente ber $\$$ ppr, welche an biefer in bie Ebene

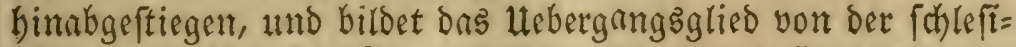

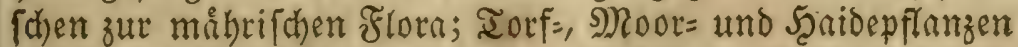
fehlen faft gånzlich, bie SPflanzen der fruchtbaren Ebene find mei= f́tens vorkanden, das (Ssebiet alfo als die Flora der fruthtbren,

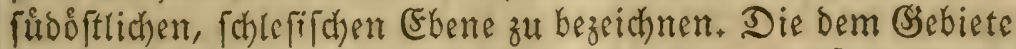
vor ben übrigen eigenen Pflanzen fint: Ranunculus illyricus, Nasturtium austriacum, Dentaria glandulosa, Silene nemoralis, Saponaria Vaccaria, Lavatera thuringiaca, Geranium pyrenaicum, Galega officinalis, Potentilla alba, Epilobium Dodonaei, Myricaria, Saxifraga Hirculus, Hacquetia Epipactis, Sambucus Ebulus, Laserpitium latifolium, Asperula cynanchica, Dipsasus pilosus, laciniatus, Scabiosa suaveolens, Cirsium serratuloides, Campanula bononiensis, Salvia 
glutinosa, Nepeta nuda, Prunella grandiflora, Salix daphnoides, Veronica prostrata, Malaxis monophylla, Crocus vernus, Scilla bifolia, Festuca montana.

\section{Dab Sebiet von Steinau}

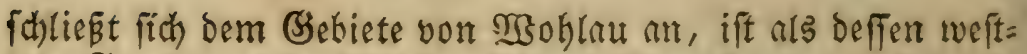
liche Fortfergung jenfeits der Soer zu betrachtert, umfant ei=

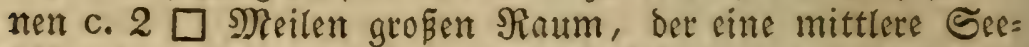
Gỏhe von 250-300' hat und 625 9y flanzenarten, 322 (3at= tungen und 83 Framilien enthålt, weld) gróß̄tentheils nuch ir

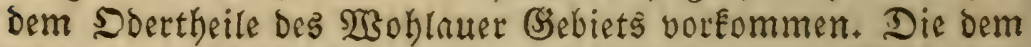
leşterm angehơrigen Bergpflanzen fehlen ihm ganz. Barbarea arcuata, Buplourum rotundifolium, Chenopodium opulifolium bat es yor ben ưbrigen Şebirten voraus. Lepidium perfoliatum, welches fonft in Ungarn, Deftreich, Sie= benbưrgen vorEęmmt, ift einmal gefunden worden und fomit

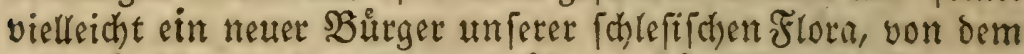
wir wünfhen, bas er fid noch offerer móge finden laffen.

\section{Das Giebiet von (Strunau}

ift nur ein Theil dez B̧ebiets Der Şirfhberger Florn und zivar Der weftlichfte, $1 / 2 \square$ Meile großße, bem Mittelgebirge zuge= wendete Iheil, weldher wentiger Branit, mehr Ssrunftein zur

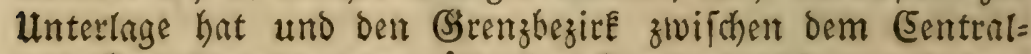
unt Şneußgranit, Dem nórolichen Quaderfandftein und bem

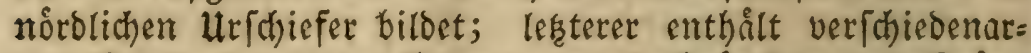

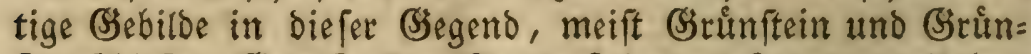

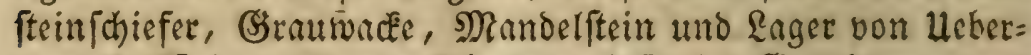
gangzEalEftein, alfo eine fehr mannigfaltige Gstundlage, uno fteigt in feinen hoofften \$ounten, in Der hohen Sulie, im.

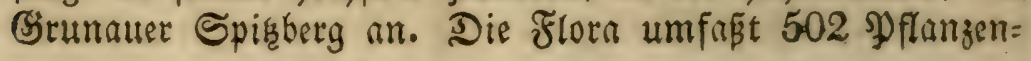




\section{7}

arten in 294 Sattungen und 79 Familien; feine Pflanze ift bem Ģebiet vor ben ůbrigen eigenthümlidy.

\section{Das Shirfhberger Ihal mit bem Riejen= gebirge*)}

umfañt bar Ģebiet des Scirihberger Thats und Die ganze

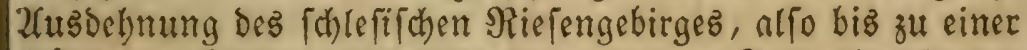

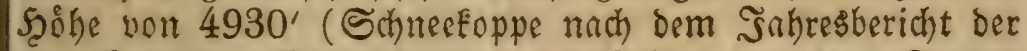
vaterlindifa)en (sefell fonaft von 1836), an einigen Stellen greift es nod) ůber bas Mittelgebirge binnus und ernåhrt

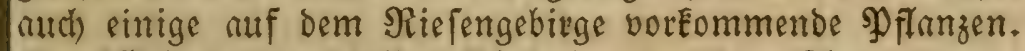

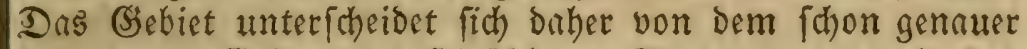

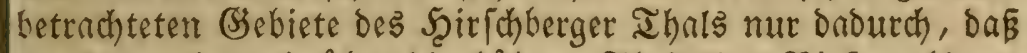

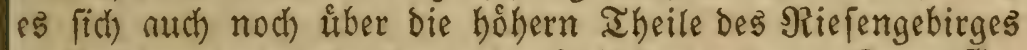
ausbreitet, und babutch zu ber 3ahl ber in bem erfteren Gese= biete enthaltenen Pflanzen noch die bes STochgebirges hinzu= gefugt merden, die in ben vorbergehenden Betradtungen fojon angefingt und angegeben worden find, bie eigentlichen Şoch=

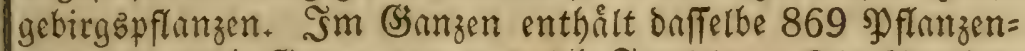
arten in 384 Sinttungen uno 84 Familien. Die Berthei= lung biefer SPflanzenarten in den einzelnen Familien, bas

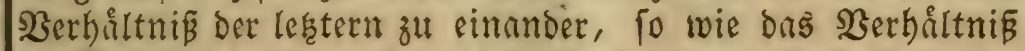
Der Familien zu Dem ber andern Grebiete und namentlich zu

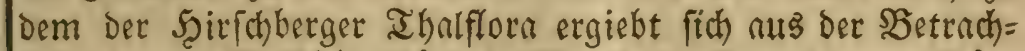
tung ber zu S. 244 gehórigen Iabelle 1. Die als neue Birt=

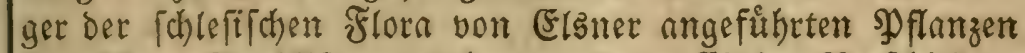

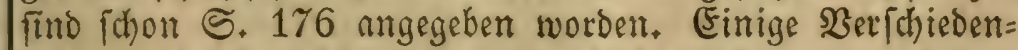
beiten und 2lbweichungen, welche biefe wadfer ausgentbeitete

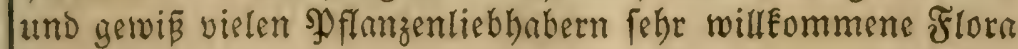

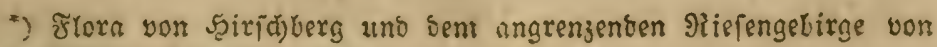

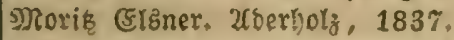




\section{8}

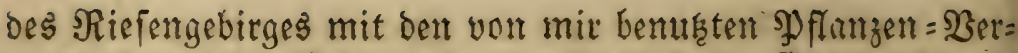
zeichniffen biefes (sebietes barbictet, find im Ganzen fo we=

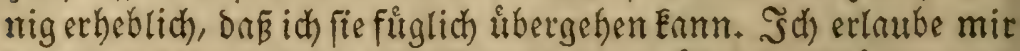
bagegen, hiet einige Eurze BemetEuttgen über die $2 B$ irme= und

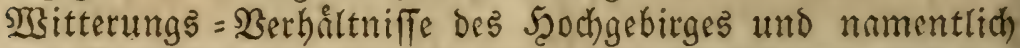
Der Riefenteppe nieder zu legen, wie fie fich mir aus den SBeobadtungstabelfen rrgeten, weld)e der (S3nftwirth Sieben=

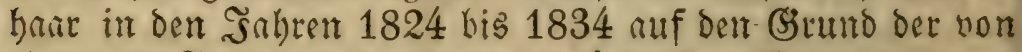

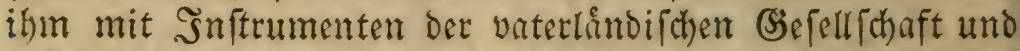
auf Deren $\mathfrak{B e r a n l a f f u n g ~ a u f ~ d e r ~ \Re i e f e n t e p p e ~ a n g e f t e l l t e n ~} \mathfrak{S}_{e}=$ obuchtungen angefertigt. Şierbei fuhle id mich georungen,

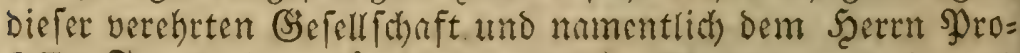
feflor Fronfenteim fü Die frentudiche Mittheilung biefer und ihnlicher Tabellen meinen immigften DanE offentlich auszu= fprechen. Diefe burch einen 11 iâhrigen șcitrnum angeftellten Beobachtungen umfarfen nur Die Monate Mai bis DEtober, und zwar im erftern Monate mur Durd) 5 Sahre, 1829 1832, in Feinem leften Sorittel vom 18ten ober 23/ten all,

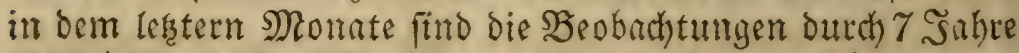
(1825-29, 1832-33) nber mur bis zum 6ten u. 14ten ange= itellt worben, weil der zu lange liegenbleibende Sunec dus fru=

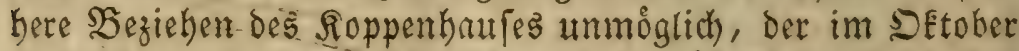
wicoer daternd erid)einende Schnee ein fo frühes Berlaffen deflel= ben nothig madte. Die Seobachtungen. umfaffen bacum

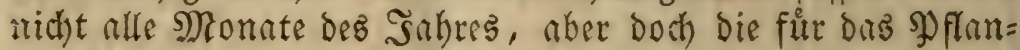
zenleben, vom bleibenden Schnee begrinzte, barum aud fur umpern Betracht mur widtige Sahreßzeit. Bom Sahre 1834 liegen mur bie Seobachtungen vom 21ugut und September vor, von 1831 mur vom Mrni, S̈uni und 2(ugutt, es find ba= rum nur bie SBeobad)tungen vom 2uguit 10 jịhrig, bie ůbri= gen 5 bis 9 jåhrig.

Bei ineer Fintvirkung auf das Pfflangenleben find be=

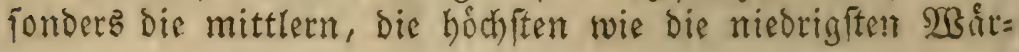




\section{9}

megrabe von 2 Bichtigfeit; fie find aus folgenben Tabellent $\mathrm{ft}=$ fitchtlid):

\section{Sitttlere monatl. Thernometerf́t}

\begin{tabular}{|c|c|c|c|c|c|c|}
\hline & Mai. & Sun & șuli & $2 \mathfrak{u g g u f t}$ & Sept. & DEtober. \\
\hline 1824 & & & 6,2 & 6,8 & 6,7 & \\
\hline 25 & $"$ & & & & 5,3 & $3,3 b, z+12 \mathrm{ten}$ \\
\hline 1826 & & 6 & 9,5 & 9,5 & 5,6 & $3,6-9$ \\
\hline 1827: & & 8,4 & 8,1 & 6,9 & 4,6 & $3,6-12$ \\
\hline 1828: & 21 an 5,5 & 5,4 & 7,7 & 4,9 & 2,9 & $1,2-14$ \\
\hline $329: 8$ & 1) $21,2,4$ & 4,6 & 7,1 & 5,6 & 4,5 & $4,2-6$ \\
\hline $330: x$ & . $21,4,7$ & 6,1 & 7 & 6,3 & 3,3 & \\
\hline & 18 , 4,4 & 3,7 & & 5,9 & & \\
\hline $\mathrm{t}$ & $.23,2,3$ & 4,2 & 4,1 & $\tau$ & 2,7 & $4,7-12$. \\
\hline 33 & 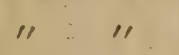 & 7,2 & 4,9 & 3,3 & 3,9 & $3,1-7$ \\
\hline & & & 10,2 & 8,5 & & \\
\hline
\end{tabular}

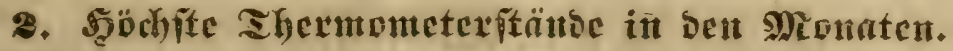

\begin{tabular}{|c|c|c|c|c|c|c|}
\hline & Mni. & Iunt. & Sulli. & faugut & Sept. & $D$ Etbr. \\
\hline 1824 & " & "I & 15 & 13,7 & 12 & \\
\hline 32 & & & & & 12 & 6 \\
\hline 1 & & 14 & 15 & & 11,5 & 6 \\
\hline 1827: & & 14 & 15 & 15 & 11 & 8 \\
\hline 1828: & 10 & 11,5 & 13,5 & 8 & 9 & 6 \\
\hline 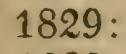 & 7,5 & 12 & 14 & 13 & 11 & 8 \\
\hline 183 & 10,5 & 15,5 & 14 & 1 & 8 & \\
\hline & 10 & 11 & & 1 & & \\
\hline 1832: & 7 & 12,8 & 15 & 13 & 8 & 9 \\
\hline & " & 14 & 15 & 7,5 & 8 & 6 \\
\hline . & & & 17 & 17,7 & & II \\
\hline
\end{tabular}




\section{0}

3. Niebrigfte Therntontertertünbe in ben Mionaten

\begin{tabular}{|c|c|c|c|c|c|c|}
\hline & Mai & รันni. & suli. & 2 (uguft. & Sept. & $D \mathfrak{E} t \in \mathfrak{R}$. \\
\hline $\begin{array}{l}1824 \\
1825\end{array}$ & $"$ & & +1 & +2 & {$\left[\begin{array}{l}-0,2 \\
-4\end{array}\right.$} & -4 \\
\hline $1826:$ & " " & -1 & $\begin{array}{r} \\
+3,5\end{array}$ & +3 & $\begin{array}{l}-4 \\
-5\end{array}$ & $\begin{array}{l}-4 \\
+1\end{array}$ \\
\hline 1827: & & +3 & +1 & -4 & -2 & $+0,5$ \\
\hline 18 & +1 & 0 & 0 & +1 & $-3,5$ & -2 \\
\hline 1825 & -3 & -4 & -2 & $+1,5$ & 0 & 0 \\
\hline 18 & -5 & -2 & -1 & +1 & -5 & \\
\hline 1831 & -1 & -1 & " & +2 & & \\
\hline 1832 & 2 & $-1,5$ & -3 & +1 & $-4,5$ & +1 \\
\hline 1833 & " & -2 & +1 & $-1,5$ & -4 & $-0,1$ \\
\hline & & & & & 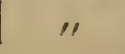 & \\
\hline
\end{tabular}

1. Tage mit Trojt it beu cinzelnen Mronnten.

\begin{tabular}{|c|c|c|c|c|c|c|}
\hline & Mai. & Šni. & $\mathfrak{\Im} u l i$. & 2luguft. & Sipt. & Setter. \\
\hline 824 & " " & & 0 & 0 & 28.29 & \\
\hline $\begin{array}{l}1825: \\
1826:\end{array}$ & " " " & 17. 18.20 & 0 & 0 & $\begin{array}{l}28-30 \\
21.22\end{array}$ & 0 \\
\hline 1827: & & & 0 & 24. $28-30$ & 26 & 13 \\
\hline 1829: & ${ }_{0}{ }^{\prime}$ & $\begin{array}{c}9.10 \\
1-7.12\end{array}$ & $\begin{array}{r}29 \\
0\end{array}$ & $\begin{array}{l}0 \\
0\end{array}$ & {$\left[\begin{array}{l}3.16-25 \\
16.27\end{array}\right.$} & $\mid \begin{array}{l}7-14 \\
5-6\end{array}$ \\
\hline 1850: & 23. 31 & $21-23$ & 6 & 0 & 1.6.26 & " \\
\hline 1832: & 18. & 18.21 .27 & 13. 19.21 & 0 & 15.16 .20 & " \\
\hline $\begin{array}{l}1839 \\
1834\end{array}$ & 27 & & $\begin{array}{l}0 \\
0\end{array}$ & $\begin{array}{c}0 \\
4-7.13 .14\end{array}$ & 2.3 .4 .14 & 5.7 \\
\hline
\end{tabular}




\section{1}

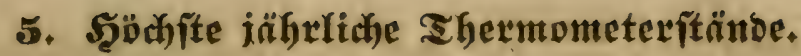

1824: $15^{\circ}$ Den 15 . Suli.

1826: 16 " 4. 2fuguft.

1827: 15 "3. Juli und 2. 2(ugutt.

1828: 13,5" 5., 6., 9. Suuli.

1829: 14 " 15. ฐuli.

1830: $16^{\circ} "$ "5, 6. 2(uguft.

1831: 11,9 " 1. 2fuğuft.

1832: 15 " 13,14 . Suli.

1833: 15 1. Sัuli.

1834: 17,7" 1. und 2. 2fuguft.

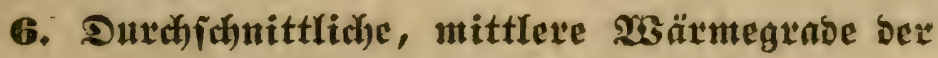
Mtonate nach 5-10 Iahren.

Mni 3,8 ; Sumi 5,8 ; Suli 7,2 ; 2uguft 6,5 ; Sep= tember 4,4 ; SEtober 3,4 .

2fus biefen Iabellen ergiebt fich, baß bie mittlern aher= mometerftånte, aus brei tỏglichen Beobachtungen, im lesten

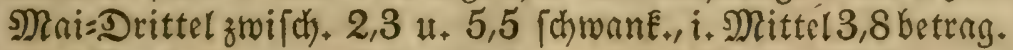

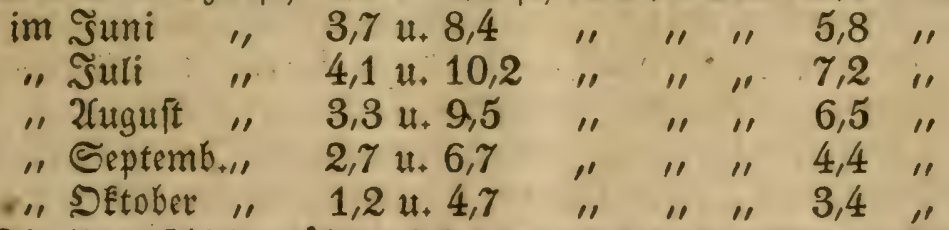

Die Unterfohiede nåhern fich bemnnch fehr Den Mittelzahlen. Die bơchften Stinde fhwanken

im Mni zrwifthen 7 und 10,5, im Suni zwifden 11 uno 15,5, im Suli zwifhen 13,5 und 17 , im 2fuguft zwifhen 7 uno 17,7, im September zrwifhen 8 und 12, im SEto= ber zrwifchen 6 und 9; Der gróste ulnterichied findet beinnach im 2fuguft, Der fleinfte im erften Drittel bes \$્ktobers ftatt. 


\section{2}

Die niebrigften 23 \& im Mni zwi (d)en -5 uno +1 , im Suni zwi chen -4 uno +3 , im suli zrwifhen -4 uno +3 , im 2fuguft besgleichen, im September zwifhen 0 und -5 , im Setober zwi chen -4 und +1. Die Unteridiede fint im Suni, Suli uno

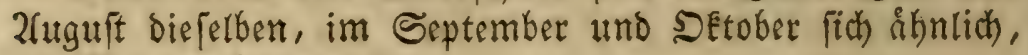
im Mni am bedeutenften.

Die Unterfhiebe der hochiten und niedrigften 2 såmegrabe in ben verfobiedenen Monaten find

im Mai 15,5 aนร -5 uno $+10,5$, im Sัแni 19,5 สนร -4 und $+15,5$, im Suli 21 ณuร -4 uno +17 , im $2 \mathfrak{U}=$ gut 21,7 aus -4 und $+17,7$, im September $17 \mathfrak{n t s}-5$ uno +12 , im 5 Etober 13 ans -4 und +9 .

Betrachten twir bie cinzelnen Monte in Besziefung auf bie in ifnen vorÉommenden Frofttrige, fo ift Eeiner abjolut von benfelben frei. Son bem lesten Maidrittel war nur bas von 1828 froftfrei, 1831 uno 1832 batten 1,1829 aber 2 und 1830 fogar 3 Frofttage, alfo Dutch fd mittlich mit $12 / 5$ Frofttig.

Der Ș ni war in ben 8 Șabren auth nur einmal ohne, 7 Sahre bagegen mit Froft; im Maximo 1829 mit 8, 1826 und 32 mit 4, 1830 mit 3, 1828 und 31 mit 2, 1833 mit 1 Frofttrige, nlfo burch/chnittlich 3 Frofttnge im Suni.

Der Suli batte in ben 9 beobaditeten Sabren nur 9 Frofttrige in 3 sinheen $1830-32$ mit 7,28 uno 30 mit 1 Frofttrige, alfo burch fdinittlich 1 Frofttag.

Der 2fuguft jeigte fide) int ben 10 beobachteten Sabren 8 mal froftfrei, 2 mal mit $\mathfrak{F r o f t}, 1827$ mit 4,1833 mit 6

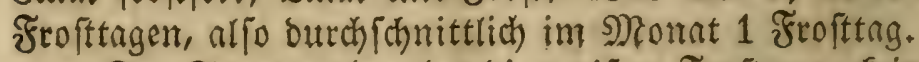

Der September kat bie meiften Frofttange, Eeines der beobachteten Sahre war frofffrei, alle batten 34 Frofttage; 1828 mit 11, 1832 mit 6, 1833 mit 4, 1825 uno 1830 


\section{3}

mit 3, 1824, 1826, 1829 mit 2 Frofttagen, burch f(t) nittlid) mit 3,77 Frofttingen.

Der Detober war in den 7 Sabren zweimal froftfrei, hatte im Gianzen 14 Frofttnge und zlvar 1828 mit 8, 1829 und 33 mit 2, 1825 und 1827 mit 1, burch [dyittlich mit 2 Frofttrigen.

Die hoobften Thermometeritande im Sahre fetwanken

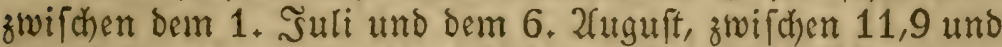
$17,7^{\circ}$, Die mittlere ober burchidnittliche Sommermitte

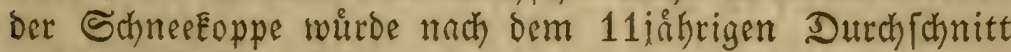
auf ben 22. Sulti fallen.

Die mittlern 23 årmegrade erreichen in Suli die gróßste

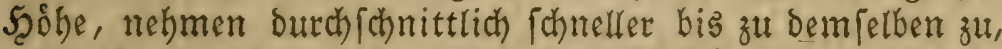
Damn aber langfanter ab, fait in bem $\mathfrak{B e r b a ́ l t m i}$ son 2:1; Die 3nhl Der Frofttage ift in den lesetern Mennten gróker als

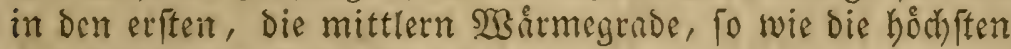
und niedrigften find niedriger.

Benn wir alle biefe Berbalttniffe zufammenfaften, fo treten uns eigentlich) auf ber Soppe und faft auf allen obern Theilen bes Şochgebirges mur ztwei Sathreszeiten entgegen,

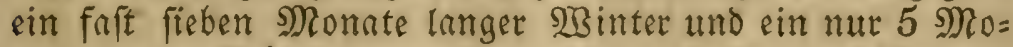
nate langer Frůfling, weldher abfolut in Eeinem Monate ganz froftfrei ift, ieboch in manchen Sahren einzelne Monate

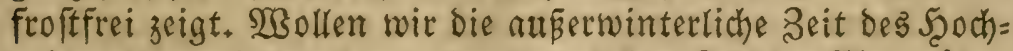
gebirges in mit ben Der außerwinterlicyen 3eit ber (Ebene ăhn= liche Beitabjadnitte zertheilen, fo Eornnten wir bent zweimonat=

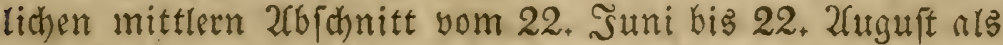
ben Scod) gebirgs Seit von ber Maimitte an ben Scochgebirgsfruhling, Die Seit bis zur Mitte bes SDEtobers Den Scod)gebirgs= herb ft bezeichnen, und es wưrden bie mittlern 23 a rmegrade

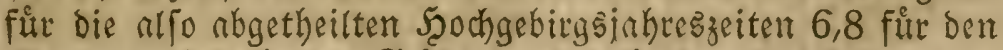
Sommer, 4,8 fưt Das Frühjahr, 3,9 fưr Den Jृerbft fein. Uum 


\section{4}

noch gentuer biefe Sabreszeiten abzutheilen, müsten zu ben

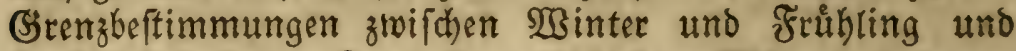
Şerbft bie mittlern 3eiten der zeitlichen Sđunegrenzen bes liegengebliebenen und liegenbleibenden Sdhnees angetven= bet werben, wozu mir aber bie nothrwendigen auf vieliåhrige

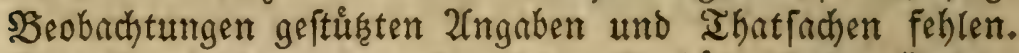
Eine Bergleid)ung ber pflanzlichen $\mathfrak{B e r h a ̊ l t n i f f e ~ b e r ~ S c o c h g e = ~}$ birgsflora mit biefer ver[uthten 2(btheilung ber Şochgebirgs=

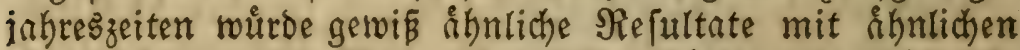

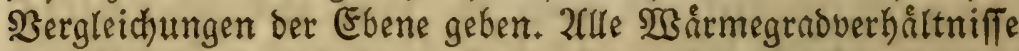

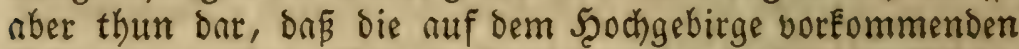

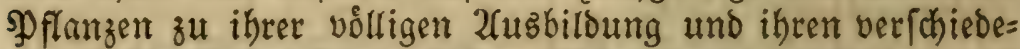
nen Rebensftufen Éeiner hohen 233 åmegrnde bedưrfen, und im 2ellgemeinen auch felbft voribbergehende Frófte ertragen Eonnen, ofne von benfelben getootet zu werben; einige 2fus= nahmen finden allerdings ftatt; modhte in biefer Segiehung bas Riefengebirge fo genaue und ausbauernde Beobachter Des Sy flanzentebens finden, als Introl in Unger und bie Schweiz in Sceer gefunden hat.

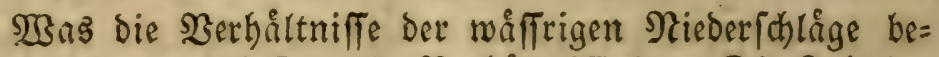
trifft, fo bieten fich folgende Berbåltniffe bar. Die 3ahl ber Regentage fdwantet

im Maibrittel zwi jochen 1 uno 9, betri̊gt im Mittel 3,8

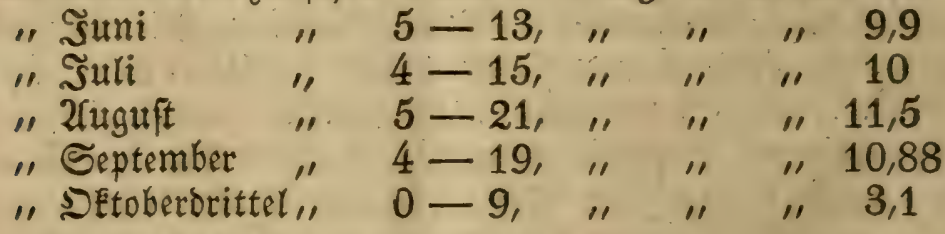

Die 3nhl der Riegentage ift fich in ben verfohiedenen Monaten ziemlich) gleieh, erreicht im 2(uguft ifr Marimum und nimmt zu beiden Seiten ziemlidh gleid)mą̧ig ab, ift in Den veridhiedenen Sathen fehr verihieben. 


\section{5}

Die Sabl ber Schneetage forwanet

im Maibrittel zwifhen 0 und 4, betriggt im Mittel 1,6

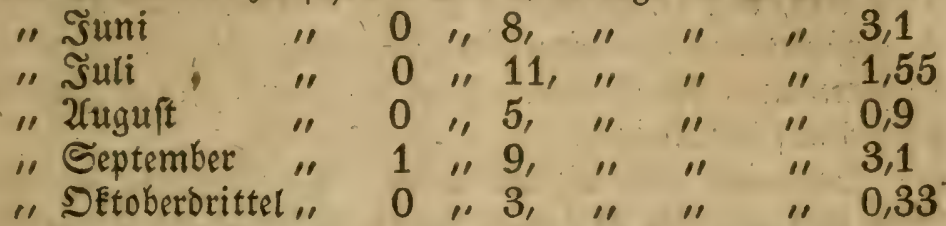

Das leste Maibrittel ift in 5 Sahten nut zweimal, ber Juni $2 \mathrm{mal}$ in 9 Sahren, der Sull $6 \mathrm{mal}$ in 9 Sahren, ber 2uguft 7 mal in 10 Sahten, Der September Eeinmal in 9 Sahren, bas erite SEtoberbrittel ift $6 \mathrm{mal}$ in 7 Sahren obne Sdnee getwefen. Der 21uguit ift ber fhneefreifte Nonnt, bet September und Șuni find einander gleid).

(5) eroitter Eamen

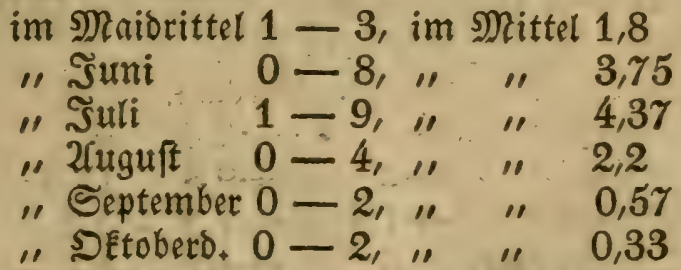

vor. Der Suli ift bemnad) Der gewitterreichfte Monat, Eein Salhr ohne (s)ewitter, ber Suni ift reicher als ber 2fugutt, boch hat Diefer mehr (semitteriahre, ber SEtober ift am årmiten barnt.

Bergleichen wir endlich die 2 Barmegraboerhiltniffe nuf Der foppe mit benen von Brestau, lef̧tere nach einem 12jåh = rigen Durdjidnitt von 1820-1831, fo finden wir

Mittlere Wåmegrabe

Roppe. Breslau. Differenz. Sunnhme

im Mniorittel $3,8-9,65-5,85\} 0,67$ Des

"Suni

" Sัuli

$5,8-12,42-6,52$

"2uguit

$7,2-13,87-6,67$

0,15 un $=$

$6,5-13,63-7,13$

0,46 ter

"I

September

4,4

1,08 [นh)ieds 


\section{6}

Der mittlere Temperaturunterfhied zwifchen ber Soppe und SBeşlau betrigt bemmath gegen $6,5^{\circ}$, ex nimmt bis zum 2fuguit zu, von dem elben an ab; biejer unterfaled wird burch die verfhiedenen Scóhenverhåltniffe beider Srte bedingt. Die Sommermitte für SBeslnu ift ber 17. Suli, alfo 5 Inge frü= ber als auf ber Foppe.

Bergleichen wir die monatlichen 23 årmegrade im Sahte 1829 mit einander in ben 4 Monaten Suni bis September, fo ergeben (id folgende Rejultate :

Mittlere.

Sृóch fte.

Soppe. Strshlu. Diff. Soppe. Breslau. Diff:

\begin{tabular}{|c|c|c|c|c|}
\hline Sันni & $4,6-11,6$ & -7 & 11,5 & 5 \\
\hline șuli & $7,7-14,1$ & -6 & 13,5 & \\
\hline Zugurt & $6,3-12,3$ & -6 & 8 & \\
\hline$m b$. & $3,3-10,0$ & $6-6$ & 9 & \\
\hline & & & gite. & \\
\hline & Sันni & & $+2,0$ & \\
\hline & Șuli & -2 & $+9,3$ & \\
\hline & 2uguit & $+1,5$ & $+7,3$ & \\
\hline & Septemiber & 0 & $+4,7$ & \\
\hline
\end{tabular}

Die Unterichiebe zeigen fich bier bei ben mittlern 23 ar megraden åbnlid) den burch (d) nittlichen aller Sabre, jeboch mit 2rbweid)ungen, bedeutent ift aber bie Berfchiedentheit bei ben hóchiten und niebrigften $\mathfrak{B}$ rirmegraben, befonders in ben Sommermonaten ber Foppe.

(5in weiteres (Fingehen in bie meteorologifchen Berhålt= niffe würde bie hier gezogenen Bsenzen überfdreiten; es wird (ith Dafur an sinem andern Drte eine beffere (5ielegen= heit finden; bier mógen biefe 21ndeutungen genügen, welche bod) einiges \&icht, befondres auf bie fur bas \$flanzenleben widhtigften meteorologifhen $\mathfrak{B}$ erbåltniffe werfen. 


\section{7}

\section{2( i b a a}

Bergleidung ber fdefifchen Flora mit ber

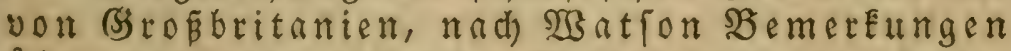
úber bie geographifhe Berbreitung ber briti= (d) en (s)ewaed 1837.

Wahtend ber Searbeitung ber voritehenden, pflanzen=

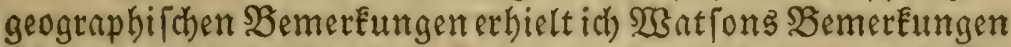

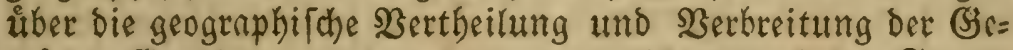
twåch fe (s)ropbritaniens u. F+ wo. in meines verebrten Freun= des Seilfchmied umfichtiger und bereicherter Heberferang. Der fo reiche Sinkalt bes $\mathfrak{B u c h e s , ~ b i e ~ v i e l e n ~ i n t e r e f f a n t e n ~}$ Ihatfachen in bemfelben veranlasten mich, biefelben mit ben

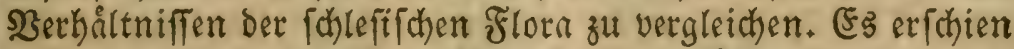
mir intereffant, zwei fo verfchiedenartige gróbere N3ebiete, das noroweftiche, meerumgebene Ssrobbritanien mit feinen fo

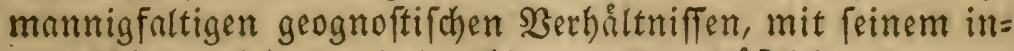
fularifhen Rlimn, uno bas binnengelegene offtiche, ber gro= Ben ofteuropaif d)en Ebene zugerwentete Schlefien mit feinem im 2(Ifgemeinen einfachern geognoftifoch SBau, rücefichtlich iher pflanzengeographifden Berhåltniffe nåher zu betrachten. Nachfitebende 2(ngnben find (Ergebniffe biefer Bergleid)ung; vielleid)t find biefelben ben zathleid)en Freunden ber fdyleft= f(hen \$flangenEunde nicht unwilleommen, ich erlaube mir bar= um, biefelben hier bei ber Betrachtung âhnlidher Berbåltniffe alb an einem nicht unpaffentorn Srte anzufügen. 


\section{8}

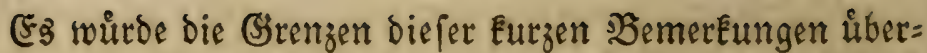
fareiten, went in benfelben bie grographifche $\mathbb{R} a g e$, die oro= und hybrographifhen, fo wie die Elimatifhen Serhåltniffe Schle= fiens im $\mathfrak{B e r g l e i c h}$ zu ben britifhen bier genauer angegeben würden; es tvird fich bafür eime paffende, anderweitige Ssele= genbeit finden; bier mógen die Ihatfachen genugen, weldje bie baburch bedingten 9 flanzen barbieten.

Betrachten wir 1) die gegenfeitigen 3ahlenver= båltniffe beiber Floren mit einander, to ftellen fich uns

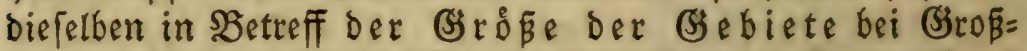
britanien mit $3900 \square$ M., bei Schlefien mit Integriff des Diftreidhifhen 2(ntheils mit $888 \square$ M. Dar.

Die ak foluten 3ahlenverhåltniffe der Sp flan=

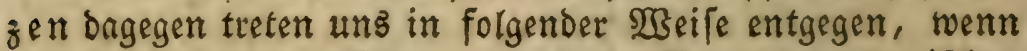
wir bie fdhlefifhen Slflanzen auf bie Norm ber englifhen Splanzenarten, Gsattungen und Familien, wie fie von $\mathfrak{B a t =}$ Fon aufgeltellt find, bringen*)

(S). SBritanien: 1469 2(rten,**) 485 (s)attungen, 95 Famil. Shlefien : 1375 " 453 " 93 "

Daraus ergiebt fich, baß bie follefifhe Flora um $942(\mathrm{t}=$ ten, 32 హ゙rttungen irmer ift; bie 3nhl ber Familien ift in Britanien um 2 Familien gróßer. Diefer 3ahlenunterfchied

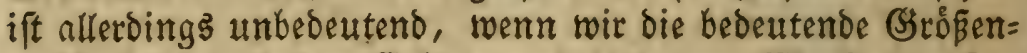
verichiedenteit beiber Bsebiete und bas von 20 ation aufge=

*) Şierburd ftellen fit) నిahlenverbältnifle heraub, tweld)e von ben

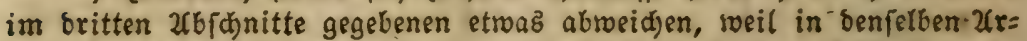
ten und (sattungen auf bie నorm ber Synopsis von Лod) gebraळt worben find; basి నె fonb Eintheilung fdien mir aker zu vortiegenber Bergleidung noth= roendig.

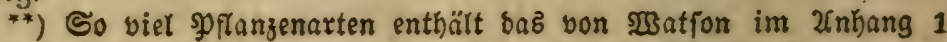

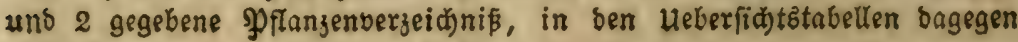
1517 2irter. 


\section{9}

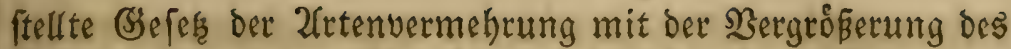

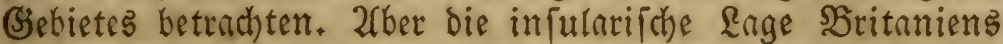
einerfeits, fo mie bie Stellumg Sdhlefiens zwifhen dem offt= lichen und weftlichen Europa anderfeits, mogen bierbei bie bedingenten urfachen fein, da bie mehr nóroliche $\mathfrak{a}$ nge Bri= taniens, burch weldhe alleroings nach Den befannten pflanzen=

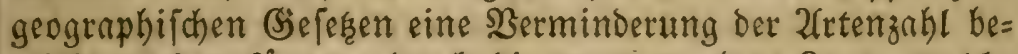
wirkt werDen Énnte, Durdh Die meerumgebene Lnge, welche eine ber nórolichern $\mathfrak{E} a g e$ ent Euftwo̊rmegrades verhindert, unwirefam gemacht wird. Diefe Bablenverhåltniffe witrden fich vielleicht auch noch anders ge= ftalten, wenn fich) genau bie von manchen BotaniEern als 2rbarten angenommenen, aber in ber fallefifchen Ftora als be=

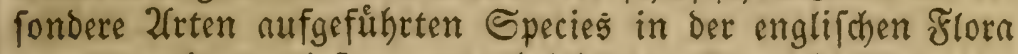
genau ermitteln ließen, was bei bloßen ßerzeichniffen nicht mogglich ift. SBetrachten wir aber bie auth mur als fraglich

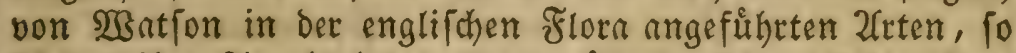
foheint ihre 2fnzabl bei weitem gróser zu fein, als bie allen= falls als fraglich zu betrachtenden ber fohlefichen Frora. Bu oem tretent uns in bem Berzeichniffe oer englifchen Flora nod viele \$Pflanzen entgegen, Deren wirkliches engliches \$Burger= thum felbft von 23 at fon als ein fragliches bezeichnet ift. 233 at= fon ferbit bezeichnet 54 Sy flanzen als eingefühtete \$planzen, welche jeboch gewiffermanen einheimif geworden, 10 sh flan= zen, welche Enum ats wilbwach fend angentommen werden E⿱on: nen, 14, welthe zwar willowedjend gefunden werben, Deren wahres Boirgerthum nber fraglich ift, 69 laffen fich als |rin=

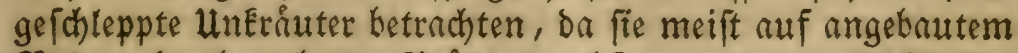
SSoden ober bewohnten झুązen wach fen, und gegen 100 2fr= ten find vielleid)t mehr 2 (barten als wirêliche 2(rten. Siehen wir bierbei die Menge ber Salices (52 2(rten) und bie gróß̄te 2(nzanhl Der $2(r t e n$ von Rosa (16 2(rten) in Betracht, yon Denen fich mandi) 2(rten auch als 2(barten in ber follefifchen 


\section{0}

Flora muffinden laffen, fo wơrden fid die Gefammtzahlen ber

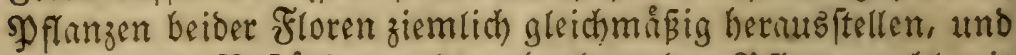
baburch bie Seftátigung Der abnefmenden \$flanzenzahl mit Der zumehmenden SBreite geben. "פBir muiffen uns bei biefen

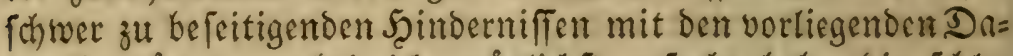
ten begnigen, wobei idh moglidsit gefucht babe, bie foble= fifchen

Faffen wir die 3ahlenverbaltniffe der naturlichen $\mathfrak{F} \Omega$ milien muf, fo crgeben fie fich aus ber im 2(nthange befind=. lichen $\mathfrak{I} a b e l l e ~ 1$.

Zuts biefen Bnhlenverhiltniffen ergiebt fich:

2) Der botanifhe Eharakter ber beiden Floren, wenn wir mit ber 3aht Der 2reten jeder Familie in bie Gies fammtartenzahl des betreffenden Bsebirtes dividiren. Diefer

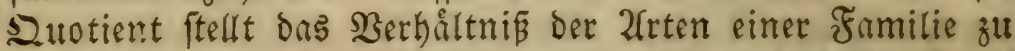
Den 2frten ber Gefammtfora Dar, oder ben wiebielten Theil bie 2(rten eitner Familie von allen গfllanzenarten bitben; bie Quotienten aller Familien aber bezeichnen ben botantifhen

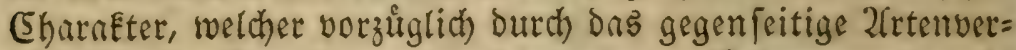

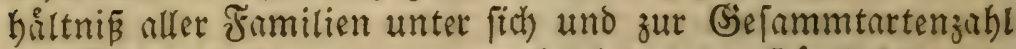

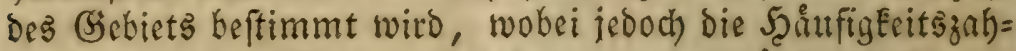
len ber einzelnen 2 lrten mehr oder weniger verånderno cinwir= Een. Diefer botanifhe (Sharneter wird burdh nachfolgende

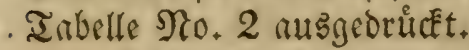

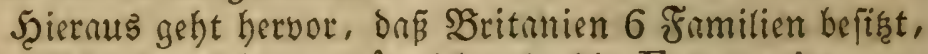
welche Shlefien fehlen, numlid): 1. Die Franceniaceae, 2. Lobeliaceae, 3. Tameae, 4. Elaeagneae, 5. Polemoniaceae, 6. Eriocauleae; alle bie fe Familien entbalten nur eime Grattung und cine 2(rt, nur bie Lobeliaceae bat

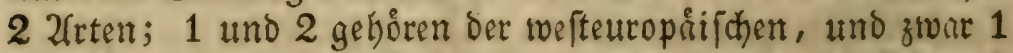
Der Rífitenflora an; Lobelia Dortmanna verbeeitet fich auth

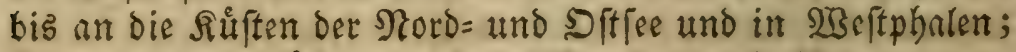
Polemonium frimmt atch in \$nreusen uno ungarm vor. 


\section{1}

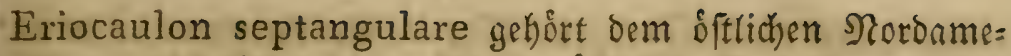

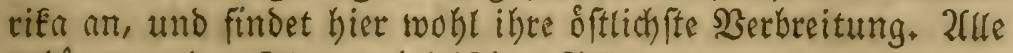

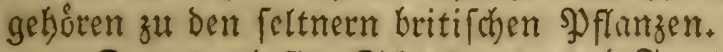

Dagegen befibt Schlefien auth 4 Familien, welche nicht in Sritanien gefunden werben, Die Rhodoraceae, Globulariae, Asclepiadeae und Rutaceae, auch lebe mit einer Siattung und einer 2frt, nur bie Globulariae mit 2 2frten. Die Rhodoraceae fcheinen Dem $\delta$ fttichen Mittel=Europa, in ifrer [d)lefichen (Sattung Ledum, die Globulariae und Rutaceae bem fúblichen und gemånigten Europa (Dictamnus Eommt jeboch auch noch in \$reuren vor) anzugeforen, Cynanchum fakeint fich nicht fo weit nach) Sorben zu-ver= breitent. 2flte biefe (Slieder find auth) mit 2(usnahme von Le-

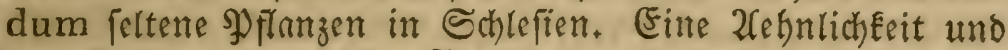
ein Stelluertreten biefer Framilient mit eimander findet nicht ftatt.

Bergleichen wir bie artenreichen Familien nach ber Zngl ibrer 2frten mit cinander, fo finden twir eine im 2utugemeinen

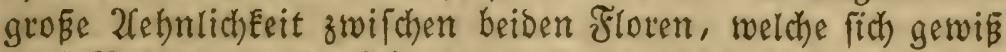
nach) Befeitigung ber 2rbarten und ber nicht wilbwach fenden

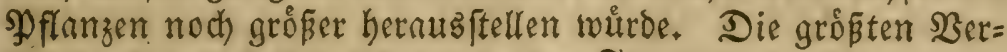
fohiedenfeiten fellen fich bar in den Familien ber Compositae, Leguminosae, Caryophylleae, Labiatae, Ranunculaceae, Borragineae, Scrofularinae, Liliaceae, Geraniaceae, Gentianeae, Onagrariae, Campanulaceae, Solaneae, Violareae, Dipsaceae, Paronychiae, Irideae, Coniferae, an welthen Echlefien reicher ift, wogegen bie bri= tifche Florn ein ůberwiegendes 2frtenverhåltnis in Den Amentaceae, Rosaceae, Cruciferae, Umbelliferae, Saxifrageae, Chenopodieae, Polygoneae, Ericinae, Primulaceae, Euphorbiaceae, Potameae, Crassulaceae, $\mathrm{Pa}$ paveraceae, Hypericinae, Alismaceaè, Valerianeãe, Orobancheae, Asparageae, Cistinae, Lineae, Plumba- 


\section{2}

gineae, Amaryllideae zeigt. Diefe ßerfhbiedentheiten brúk= Een den ver[chiedenten botani jchen (Sharakter beiber \&ånder aus, und werben theils burch bie Siriftengegenden Britaniens, theils ourd) bie mehe noorolict)e Rnge bedingt. Befonders groß ift ber unterfhied bei ben Amentaceis, Rosaceis und Saxifrageis; bei ben erftern vielleicht aus ben fchon angegebenen unfachen; auffallent ift bie 2frtenarmuth Sritaniens an Coniferae, befonders an ben baumartigen, von benen nut eime 2frt Pinus sylvestris und diefe vielleidht jefst gat nicht in Englano heimifch, fondern mut angefiebelt, nur in Styottlano fino noch einige bebeutende $\mathfrak{B g}_{3}$ aldungen, welche iebocth auch

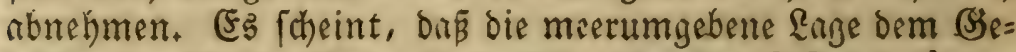
Deifen ber Pinus = 2(rten Ginderlich), Dem Der Salices fórber= (id) iff, bas iene von biefen bier verteeten werben; muth auf Şรland und Bstonland finden fidh Eeine zapfentragenden B̊a ume vor. Diefe Berfhiedenteit bildet cinen Scraptun= ter[chied in Dem botanifchen (E)arakter beiber Floren.

Die gegenfeitigen anhlen= Berbåltniffe ber Ssattungen Der beiben Floren zeigen für bie britifhe Flora einen gróBern

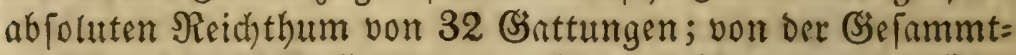
zahl Der britifchen Gonttungen nber bat Sritanien 88 Gant= tungen, weldhe Schlefien fehlen, biefes aber befít 56 nicht

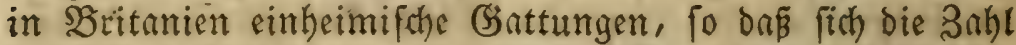
Der beiben Floren gemeinf(huftlithen (Sattungen auf 397 be= låuft, bie nicht f(h)lefif (h)en Ginttungen verbalten fich zu allen britifchen wie $1: 5 \frac{1}{2}$, bie utur fohlefifchent betragen $1 / 8$ aller

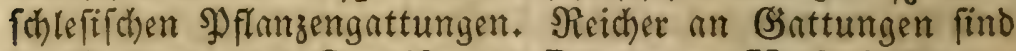
in Stitanien Die Cruciferae, Rosaceae, Umbelliferae, Papaveraceae, Malvaceae, Celastrinae, Crassulaceae, Ericaceae, Gentianeae, Labiatae:, Plantagineae, Chenopodiaceae, Polygoneae, Euphorbiaceae, Amentaceae, Alismaceae, Orchideae, Fluviales, Cyperaceae und Gramineae, in Sđlefien dic Papiliona- 
ceae, Borragineae, Scrofularinae, Urticeae, Portulaceae, Thymelaceae; bie ubrigen Frmilien haben gleiche (Sattungszahl, bie britifhen झflanzenfamilien fint bemmach

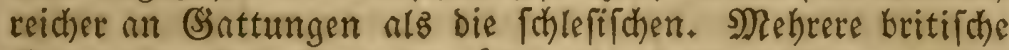
(Sattungen find Strinbgewåd) fe ober baben bod) vorwalten=

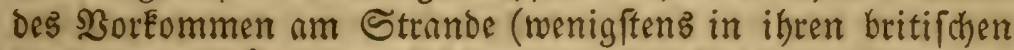
2(rten), twie Glaucium, Meconopsis, Cakile, Crambe, Cochlearia, Konigia, Matthiola, Litorella, Salsola, Beta, Salicornia, Ruppia, Zostera.

Zfnbere fheinen fich in ben gegenfeitigen Floren zut ver= treten, そ. B.

Isatis

Hutchinsia

Cherleria

Ulex

Hippocrepis

Oxytropis

Tamarix

Menziesia

Chlora

Borrago

Salsola

Beta

Aceras

Trichonema

Ruscus

Fritillaria

$\mathrm{Gr}$.

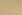




\section{4}

entichicden ber weftlid)en unt furbweftlichen ober atlantifden, andere, aber mur twenige, der borenten, noch) andere ber 2flpen= flora mit.

Bon Den fohlefifhen \$flanzengattungen foheinen einige cine mehr offtiche SBerbreitung zu haben, ba fie im Botanicon gallicum von De (Endolle uno Duby nidht aufgefúht find, aljo Ecine fo weftliche Serberitung haten als: Conioselinum, Hacquetia, Beckmannia ; bie ůbrigen (5)attum= gen, zum Eleinften IYheil ber Zltpenflora angehórig, find nicht

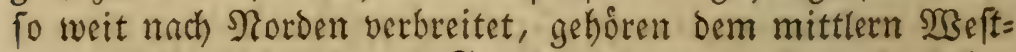
europa (Deutjhland und Franfreich) an, mehreve berfritben find im D ften håufiger als im 2 Beften und umgeêefrt.

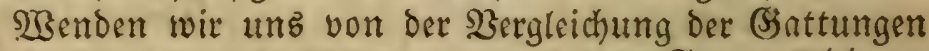
zu benen der 2frten, fo finden wir in heiden Floren 944 ge= meinfdhaftliche 2(rten; 525 hat bie englirche Flora, weld)e ber

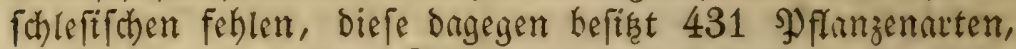
welche in ber englifochen Flora fich nicht vorfinden. Son ben in Britanien fehlenden 431 fohlefifd)en Pflanzenarten geho: ren 69 ben 57 eben bafelbft nicht vorbandenen Ssittungen an, von ben 525 britifhen, in Schlejïn fehlenden brgegen 107 den gleichfalts fehlenden 88 Şattungen an. Daraus ergiebt fid), Das bie meiften ber fehlenden Grattungen faft auf gleithe 233 cife artenarm finto. Sn ber britichen Fुtora baben unter Dent in Schlefien fehlenden (Sattungen Cochlearia uns Ophrys 5, Heliosciadium 3, Glaucium, Althaea, Ulex, Oxytropis, Petroselinum, Calamintha, Salicornia, Polypogon, iede 2 2frten, in Der fhlefif(c)en Cytisus, Laserpitium, Androsace 3, Grpsophila, Echinospermum, Globularia, Xanthium, Gladiolus, Köhleria 2 2frten.

Die in Schlefien fehlenten britifhen \$ flanzenarten bil= Den mehe alz $1 / 3$ Der britifahen Gefammtarten, Die in Brita= nien fehlenden idslefifden bagegen c. $3 / 10$ Der ibrigen. Der unterfhies deş 2(rtenreichthums in den einzelnen Familien 


\section{5}

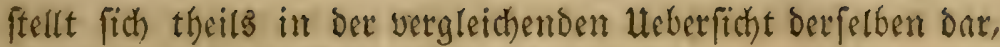
theils ift er fochon Seite 271 nusggefprochen.

Bertheilen wir bis in Schlefien fehtenten britifhen

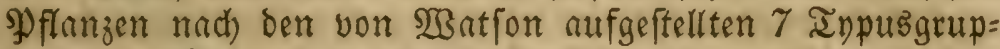

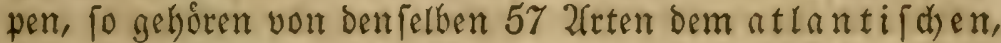
69 dem deutfden, 149 dem englifaren, 103 dem bri=

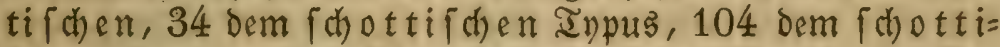

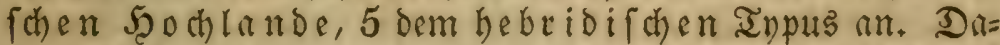
mit ift ieboch) feineswegs misgefprochen, bas bie in biefe $2(b=$ theilungen gebrechten Sy flanzen nicht auth in andern (Gebie=

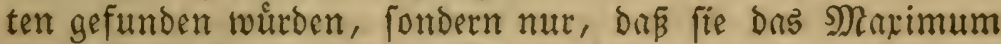
ihrer Snoividuen in bem Seftiete haben, nach weldyem ber Typus benament ift. So finden wir unter den 520 in Schle= fien fehlenden britifchen 2(rten mur 56 \$flanzenarten, welche

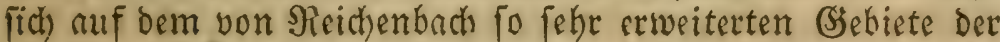
oeut fhen Floren nidht vorfinden; die ubrigen 464 werben in Demfelben und zum grósten Theile in Dem furbweftlichen Theile biefes (sechietes angetroffen, woourch fich allerdings bie britifche Flora mehr ber fild = und wefteuropeisichen Flora an=

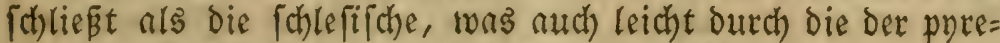
naifchen Şarbinfel zugetwendete lenge zu erElåren ift.

Son diefen 56 in ber beutfhen Flora fehlenden \$flan= zen geforen dem atlantiforen Inpus an: Paeonia corallina, Meconopsis cambrica, Brassica monensis, Vicia laevigata, Trinia glaberrima, Carum verticillatum, Physospermum cornubiense, Lobelia urens, Erica ciliaris, Sibthorpia europaea, Ulmus stricta, Scirpus Savii.

Dem fdottifchen Scod)lande: Stellaria scapigera, Potentilla tridentata, Saxifraga denudata, elongella, laetevirens, pedatifida, Hieracium denticulatum, Potamageton praelongum, Salix petiolaris, Doniana, petraea, propinqua, tenuior, laxiflora, Borre- 


\section{6}

riana, phyllireifolia, vaccinifolia, procumbens, $\mathrm{Ca}$ rex rariflora, phaeostachya, stictocarpa, angustifolia, Alopecurus alpinus. Die Dem atlantichen Inpus angeho $0^{\circ}=$ rigen find meift aus artenarmen, bie bes fohottifhen Şoth= landes aus artenreichen (S)attungen, biet 8 Garttungen uno 23 2rten, bort 12 Gantungen mit eben fo viel 2 reten; bier

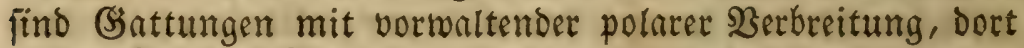
mehr füblichere Formen, welche nach Norden zu an 2(rten= reichthum verlieren.

Dem hebribifden Inpus geforen bavon an Orobanche rubra, Primula scotica, Eriocaulon, (und nuß̃er= dem Ajuga pyramidalis, Avena planiculmis); Dem bri= tifden Lepidium Smithii, Hypericum calycinum, Trifolium ornithopoides, Oenanthe apiifolia, Erythraea latifolia, Heleocharis, multicaulis; Dem englifđen Ulex nanus, Lotus angustissimus, Helminthia echioides, Linaria repens, Statice spathulata, Salix ferruginea; bem [hottifden Fragaria cal ycina, Sanguisorba media, Ligusticum scoticum, Valeriana pyrenaica, Salix damascena, Davalliana,

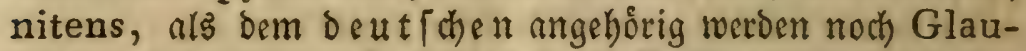
cium violaceum, Actinocarpus Damasonium, Salix Woolgariana grnannt, bie aber bie flora excursoria nicht alb beutfche Bỏrger auffíthet.

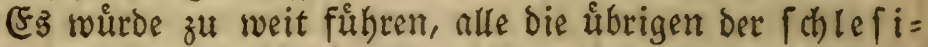
(d) en Flora nidht angehoorigen, aber als beut the Bărger in ber flora excursoria nufgeführten $\mathfrak{P}$ flanzen hier anzuge= ben, und wir begnủgen uns mit 2(nfübeung der bem at 1 an=

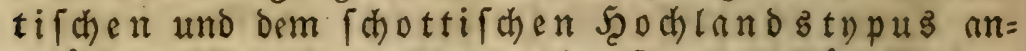

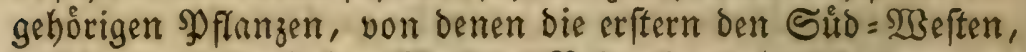
Die legtern Den hohen Storden Sritaniens bezcidinen, uno

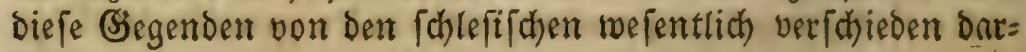
ftellen. 


\section{7}

Dem atlantifhen anpus gehoiren meer ben fdjon vorgenannten $\mathfrak{P} f(a n z e n$ Coronopus didymus, Hudchinsia petraea, Draba aizoides, Arabis stricta, Matthiola, Reseda alba und fruticulosa, Helianthemum guttatum, polifolium canum, Elatine hexandra, Oxalis corniculata, Trifolium resupinatum, Vicia hybrida, Rosa sepium, Wilsonii, Cotyledon, Sedum anglicum rupestre, Forsterianum, Bupleurum, Odontites, Daucus maritimus, Gnaphalium margaritaceum, ${ }^{*}$ ) Senecio squalidus, Campanula hederacea, Erica vagans, Exacum filiforme, Bartsia viscosa, Pinquicula lusitanica, Polygonum litorale, Euphorbia Paralias, Peplis, Alisma natans, Trichonema Columnae, Scilla verna, autumnalis, Allium Ampeloprasum, Agrostis setacea, Briza minor, Cynodon Dactylon, Carex clandestina. Meiftens fübliche Formen.

Dem fottifden Şodylande geforen auser ben fachon genannten noch folgende beut fohe $\mathfrak{B u r g e r}$ an: Thalictrum alpinum, Ranunculus alpestris, Subularia a quat ica, Draba rupestris, incana, Arabis petraea, ciliata, Silene acaulis, Lychnis alpina, Stellaria cerastioides, Cherleria sedoides, Oxytropis uralensis, campestris, Astragalus alpinus, Dryas octopetala, Potentilla alpestris, Sibbaldia, Alchemilla alpina, Rosa caesia, Epilobium alsinifolium, Saxifraga stellaris, aizoides, cernua, rivularis, hypnoides, Cornus suecica, Prenanthes hieracifolia, Hieracium pulmonarium, cerinthoides, amplexicaule, Saussurea

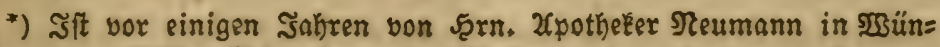

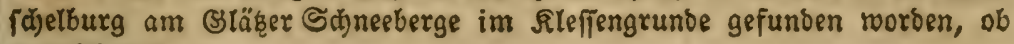
verwitbert? 


\section{8}

alpina, Erigeron alpinum, Lobelia Dortmanna, Arbutus alpina, Menziesia coerulea, Azalea procumbens, Gentiana nivalis, Myosotis alpestris, Veronica fruticulosa, saxatilis, Polygonum viviparum, Oxyria reniformis, Tofieldia palustris, Juncus balticus, castaneus, tenuis? biglumis, triglumis, El rna caricina, 28 Salices uno 13 Carices, Phleum Michelii, Sessleria coerulea mit ben fothon genannten. 2ll po meiftens 2lipen= pflanzen und zmar ber bo̊kern 2(tpenregion, bis in welche un= fer fohlefifches SEebirge bei feiner mehr füolichen Enge nicht

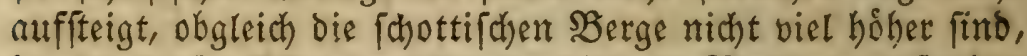
in viele biefer $\mathfrak{g} f l a n z e n$ fdyon in niederen Regionen gefunden

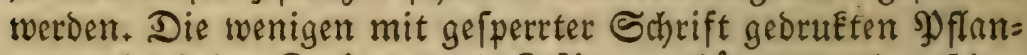
zen und einige Carices uno Salices gehoren ber bettichen Ebene an, fteigen aber ins fohottifche Şochland empor.

Bon Den in Britanien fehlenden fchlefifchen Pflanzen=

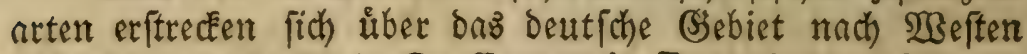
nicht hinnus, ba fie in De Enndolle's Botanicon gallicum. von Duby nicht mufgezeichnet find: Anemone patens, Ranunculus illyricus, cassubicus, Delphinium elatum, Aconitum variegatum, Arabis Halleri, Barbarea arcuata, Nasturtium anceps, Viola uliginosa, sylvestris, pratensis? Dianthus arenarius, Silene nemoralis, chlorantha, Stellaria Friseana, Malva borealis, Lavathera thuringiaca, Geranium macrorrhizon, bohemicum, divaricatum, Evonymus verrucosus, Cytisus nigricans, Melilotus dentatus, Petitpierreana, Astragatus arenarius, Geum intermedium ? Potentilla norvegica, Güntheri, patula, salisburgensis, Alchemilla fissa, Epilobium virgatum, Sempervivum hirtum, Cnidium venosum, Conioselinum Fischeri, Laserpitium, latifolium, Archangelica, Hacquetia Epipactis, Asperula Aparine, Tragopagon orientale, Hieracium 


\section{9}

vulgatum ? boreale, echioides, Scorzonera laciniata, Chondrilla Juncea, Carlina acaulis, Gnaphalium rectum? Senecio alpinus, vernalis, Inula germanica, Cineraria crispa, Centaurea, axillaris, Campanula liliifolia, Erica herbacea, Pyrola chlorantha, Ledum palustre, Lycopsis pulla, Echinospermum deflexum, Verbascum phöniceum, Scrophularia glandulosa, Pedicularis sudetica, Stachis recta; Orobanche pallidiflora, Primula minima, Androsace elongata, Amarantus adscendens, Atriplex nitens, Polygonum laxiflorum, Rumex conglommeratus, Thesium, montanum, Pinus austriaca, Epigogium aphyllum, Orchis incarnata, Malaxis monophyllos, Gladiolus imbricatus? Iris sibirica, nudicaulis, Ornithogalum stenopetalum, Tofieldia calyculata, Veratrum lobelianum, Scirpus radicans. Sie formen eime mebr offliche Berbrei= tung zu haben und barum ber britifden Flora zu fehlen; bei

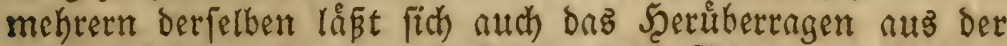
ofteuropaifchen Flora nachweifen. Das Fehlen ber übrigen fdhlefifhen SDflangen in ber britifhen Flora bagegen ftheint reniger von ber Rången= nls vielmehr burch bie SBreitenver= breitung biefer SPflanzen bebingt zu fein.

Finige SPflanzentarten ber beiben Floren fcheinen fich gegenfeitig zu vertreten ober zu repre̊fentiren. Die wichtigften rürden folgende fein.

Britania.

Thalictrum alpinum " majus Anemone apenina Adonis autumnalis Helleborus foetidus Ranunculus alpestris
Silesia.

Thalictrum aquilegifol. $"$ angustifolium Anemone sylvestris Adonis aestivalis Helleborus niger Ranunculus aconitifolius? 
Britania.

Papaver hybridum

Corydalis lutea

" claviculata

1satis tinctoria.

Hutchinsia petraea

Lepidium latifolium

29

Smithii

Arabis stricta

" petraea

¿ ciliata

,Turrita

Barbaraea praecox

Silene anglica

" italica

Elatine hexandra

Stellaria scapigera

" cerastoides

Cerastium tetrandrum

Malva moschata

Lavatera arborea

Geranium nodosum

, lucidum

Oxalis corniculata

Genista anglica

Ulex europaeus

„nanus?

Medicago denticulata " maculata

Trifolium subterraneum
Silesia.

Papaver Argemone

Corydalis cava

fabacea

Biscutella laevigata

Thlaspi montanum

Farsetia incana

Alyssum montanum

Arabis alpina

, Halleri

"Gerardi

, arenosa

Barbaraea arcuata

Silene gallica

$"$ nemoralis

" chlorantha

") cerastoides

- Elatine Alsinastrum ?

Stellaria viscida

" Friseana

Cerastium brachypetalum

Malva Alcea

Lavatera thuringiaca

Geranium palustre

,9 bohemicum

Oxalis stricta

Genista germanica

Cytisus nigricans

" capitatus

" supinus

Melilotus dentatus

$" \quad$ Petitpierreana

Trifolium fragiferum 


\section{1}

Britania.

Trifolium glomeratum Oxytropis campestris Vicia lutea

" hybrida? ?

Lathyrus pisiformis

Orobus sylvaticus

Dryas octopetala

Fragaria calycina

Potentilla alpestris

" Fragariastrum

Alchemilla alpina

Epilobium alsinifolium

Tamarix gallica

Bryonia alba

Sedum dasy phyllum

" rupestre

Saxifraga aizoides

$"$

\section{hypnoides}

Bupleurum tenuissimum Peucedanum officinale Eryngium maritimum Ligusticum scoticum Lonicera Caprifolium Galium aristatum ") pusillum Rubia peregrina Sonchus palustrịis Prenanthes hieracifolia Apargia Taraxaci Barkhausia foetida Carduus tenuiflorus Cnicus pratensis
Silesia.

Trifolium striatum Astragalus arenarius Vicia pisiformis , cassubica Lathyrus tuberosus Orobus vernus Geum pyrenaicum Fragaria collina Potentilla aurea

Alchemilla fissa Ep. origanifolium Myricaria germanica Bryonia dioeca Sedum Fabaria " repens
Saxifraga Aizoon
Brioides Bupleurum longifolium Peucedan. Oreoselinum Eryngium planum Laeserpitium Archangel. Lonicera nigra

Galium silvaticum ") silvestre Asperula arvensis Sonchus asper? Prenanthes purpurea Apargia hastilis Barkhausia hispida . Carduus crispus Cnicus rivularis 
Britania.

Gnaphalium margaritaceum

Gnaphalium gallicum

3)

minimum

Senecio tenuifolius

Aster Tripolium

Limbarda crithmoides

Cineraria campestris

Doronicum Pardalianches

Pyrethrum maritimum

Centaurea nigra

Erica vagans

Chlora perfoliata

Cynoglossum silvaticum

Verbascum pulverulentum

Linaria repens

Scrophularia Scorodonia

Digitalis purpurea

Veronica saxatilis

Teucrium Chamaedrys.

Betonica officinalis

Calamintha Nepeta

Scutellaria minor

Orobanche rubra

Cyclamen hederifolium

Primula scotica

", farinosa

Statice reticulata

Plantago Coronopus

Amarantus Blitum
Silesia.

Gnaphalium arenarium

Gn. montanum

Gn. arvense

Senecio crucifolius

,9

vernalis

Aster salignus

Inula germanica

Cineraria crispa

Doronicum scorpioides

Pyr. corymbosum

Cent. phrygia

Erica herbacea

Sivertia perennis

Omphalodes scorpioides

Verbasc. phlomoides

Linaria arvensis

Scrophul. glandulosa

Digitalis chroleuca

Ver. bellidioides

Teucrium montanum

Bet. stricta.

Nepeta nuda

Scut. hastifolia

Orob. pallidiflora

Soldanella alpina

Primula minima

Androsace Chamaejasme

Globularia vulgaris ?

Plantago arenaria

Am. adscendens 
Britania.

Atriplex litoralis

$"$

laciniata

Beta maritima

Salicornja herbacea?

Euphorbia hiberna

Ulmus montana

Orchis fusca

Epipactis grandiflora

Malaxis paludosa

Iris foetidissima

Leucojum aestivum

Ruscus aculeatus

Anthericum serotinum

Scilla verna

autumnalis

Allium Ampeloprasum

Fritillaria Meleagris

Tofieldia palustris

Potamogeton oblongus?

Luzula arcuata

Juncus compressus.

Cyperus longus

Heleocharis multicaulis

')

fluitans

Eriophorum pubescens

Agrostis setacea

Poa maritima

Bromus diandrus

"velutinus
Silesia.

Atriplex nitens

Blitum virgatum

Polycnemum arvense?

Euphorbia dulcis

Ulmus effusa

Orchis coriophora

Epipactis pallens

Malaxis monophyllos

Iris graminea

Leucojum vernum

Streptopus amplexifol.?

Anth. Liliago

Scilla amoena

, bifolia

Allium Scorodoprasum

Lilium Martagon

Tofieldia calyculata

Potam. trichodes

Luzula spadicea

Juncus Tenageja

Cyperus flavus

Hel, uniglumis

" ovata?

Eriophorum alpinum

Agrostis rupestris

Poa spectabilis

Bromus tectorum

11. patulus

BBerden zu biejen 128 einnonder in beiben (s)ebieten ent=

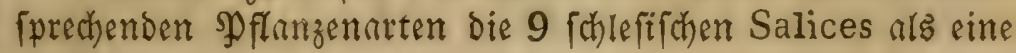




\section{4}

gleich groß̧e 3ahl ber britifhen Salices vertretent, ferner bie 23 fdhleftichen Carices alz Stellyertreter einer gleich grosen Sakl ber 25 britifhen Carices angenommen, weil bie 2lrten biefer beiden Bsattungen in ihrem sejammtausfehen mit einander fehr ůbereinfimmen, fo erfalten wir im Ssanzen 160 Pflanzenarten in beiben Floren, welche fich fehr åthnlich

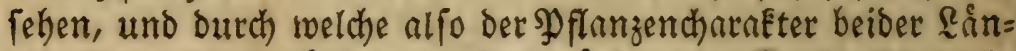
ber nur wenig vernindert werden wuirde; in, eš liểe fich leicht die 3abl ber åfnlichen Pflanzenarten noch vermebren. (5z blieben al po nur 365 Ş flanzenarten für Britanien und 271

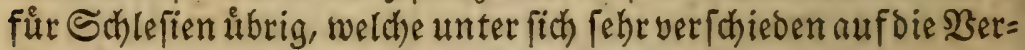

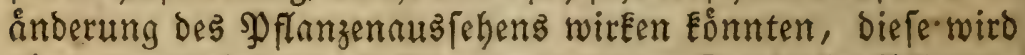
aber nur unbedeutend fein, weil bie mei/ten biefer Pflanzen nid)t zahlreich in ihren Individuen fino. Die Ssattungen Anemone, Ranunculus, Aconitum, Papaver, Cardamine, Viola, Dianthus, Geranium, Potentilla, Epilobium, Asperula, Dipsacus, Hieracium, Scorzonera, Cnicus, Gnaphalium, Senecio, Campanula, Gentiana, Veronica, Salvia, Pinus, Orchis, Iris, Ornithogalum, Allium, Scirpus, Festuca, Poa find in Schleften, Die (Sat: tungen Lepidium, Draba, Brassica, Sinapis, Helianthemum, Silene, Arenaria, Hypericum, Rosa? Pyrus', Sedum, Saxifraga, Oenanthe, Galium, Lactuca, Anthemis, Lithospermum, Mentha? Teucrium, Primula, Statice, Chenopodium, Atriplex, Rumex? Euphorbia, Ulmus, Salix, Ophrys, Croous, Narcissus, Potamogeton, Juncus, Carex, Phleum, Triticum, Bromus Dagegen in Britanien artenteicher. Das $\mathfrak{B e r h a ̊ l t n i ß ̄ ~ D e r ~ S ̧ a t = ~}$ tungen und 2frten zu ben Familien ift oben fhon ange= geben.

Es fheint, onß die गुflanzen mit mehr ausgebildeten Blumen in Sritanien zurukfs= in Shlefien mehr berbortreten (Ranunculaceae, Compositae, Campanulaceae, As- 


\section{5}

phodelae, Violariae), die mit mintor ausgebildeten bage: gen bort vorkerrichender wirtbent (Amentaceae, Chenopodiaceae, Polygoneae, Euphorbiaceae Potameae zum Theit die Gramineae utmb Cyperaceae), twas trobl aus

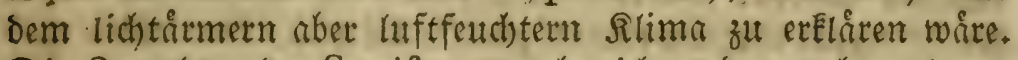
Die Bunafme Der Saxifrageae brzeichnet ben mehr polaten, Die Der Crucifereae den nlpinen und maritimen (Sharakter; Die Coniferae werben butch bie Salices vertreten, weil für jente die hochften 233 R rmegrade des Sommers zu gering, die Ruft zu feucht ift; merEwirbig ift das geringere 2(rtenverbailt= nís der Ranunculaceae in ber britifđen gegen bas in ber Fohlefifchen Flori, weil bie Ranunculaceae in ber relativen 2(rtenzahl nadh Den গুolen zunehmen; vielleicht ift es erelirt=

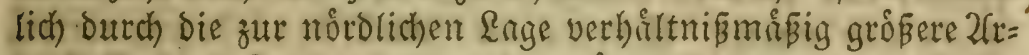
temmenge des \&andes, woourch naturtlich die relative Jahl Der Ranunculaceae zuricktreten mus; ifr foheint die 3unahme Der Rosaceae in Sritanien zu entiprect)en; die grósere Menge der Umbelliferae wirt woht butd bie maritime Enge bedingt, bafferbe gilt von den Plumbagineae.

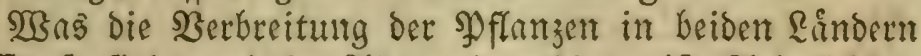
betrifft, fo finden twir im 2urgemeinen bie meifte 2(ehnlicheeit zwifchen ber fallefif

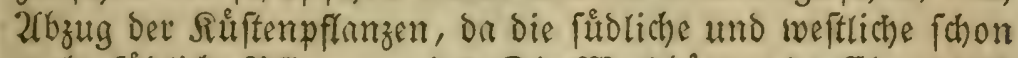

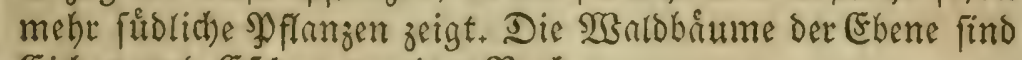
Eidhen und Efchen, weniger Butchen.

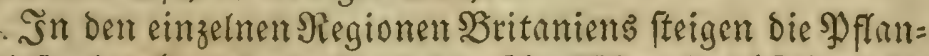
zen tiefer berab, als wir.fie in ben foblefif fon herabfteigen fe= hen, uno es treten uns fajon in den britichen Șugelrigionen,

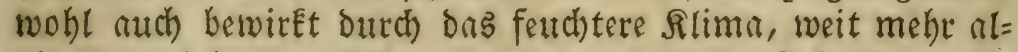

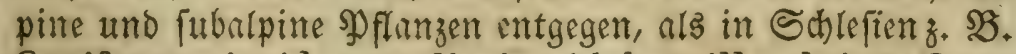
Saxifraga aizoides, stellaris, Alchemilla alpina, Oxyria reniformis $u, a+m$. SEefonders bezeichnet ift bie Şü= geltegion buth Ericinae und Cyperaceae, weldhe nlfe hibri= 


\section{6}

gen \$flanzen verbrången; Sirken und Siefeen fino die vot:

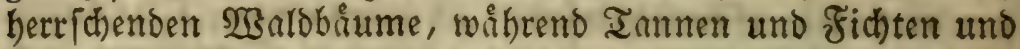
zum Iheil Buchen in Schleften in Diefer Region vorbert= fohender werden. Calluna fteigt nod) in Britanien bis in die fubalpine Region empor, wo fie bei uns faton früher nuf=

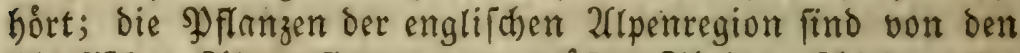

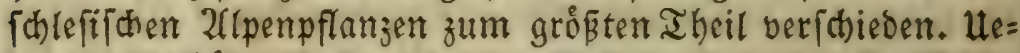

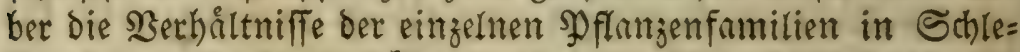
fren bei verichiedener Şöhe liegen Fcine Bornerbeiten vor; ein Fpezielleres Eingehen in bie Bergleichung ber einzelnen $\Re_{R}=$ gionen ber beiben Floren nach ben ifnen eigenthumlichen

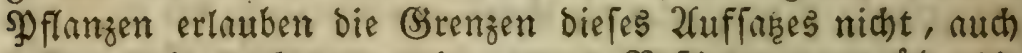
befigen wir noch zu wenig genture Beftimmungen ůber bie obern und untern Gsenzen Der foleffichen Pflanzenarten. Modthten fid Dazu, wie auth zur unterfuchung und Beftim= mung ber gegenfeitigen \$erhåltniffe ber \$flanzen in ben ver= (c)iedenen Regionen für viele Bsebirgsgegenden Schlefiens 2rbeiter finden. 


\section{7}

Anbang $B$.

\section{Bablentberbältnifie der britiphen unb fable. fifothen Flora.}

1) Die Sablen Der Şattungen uno 2rter.

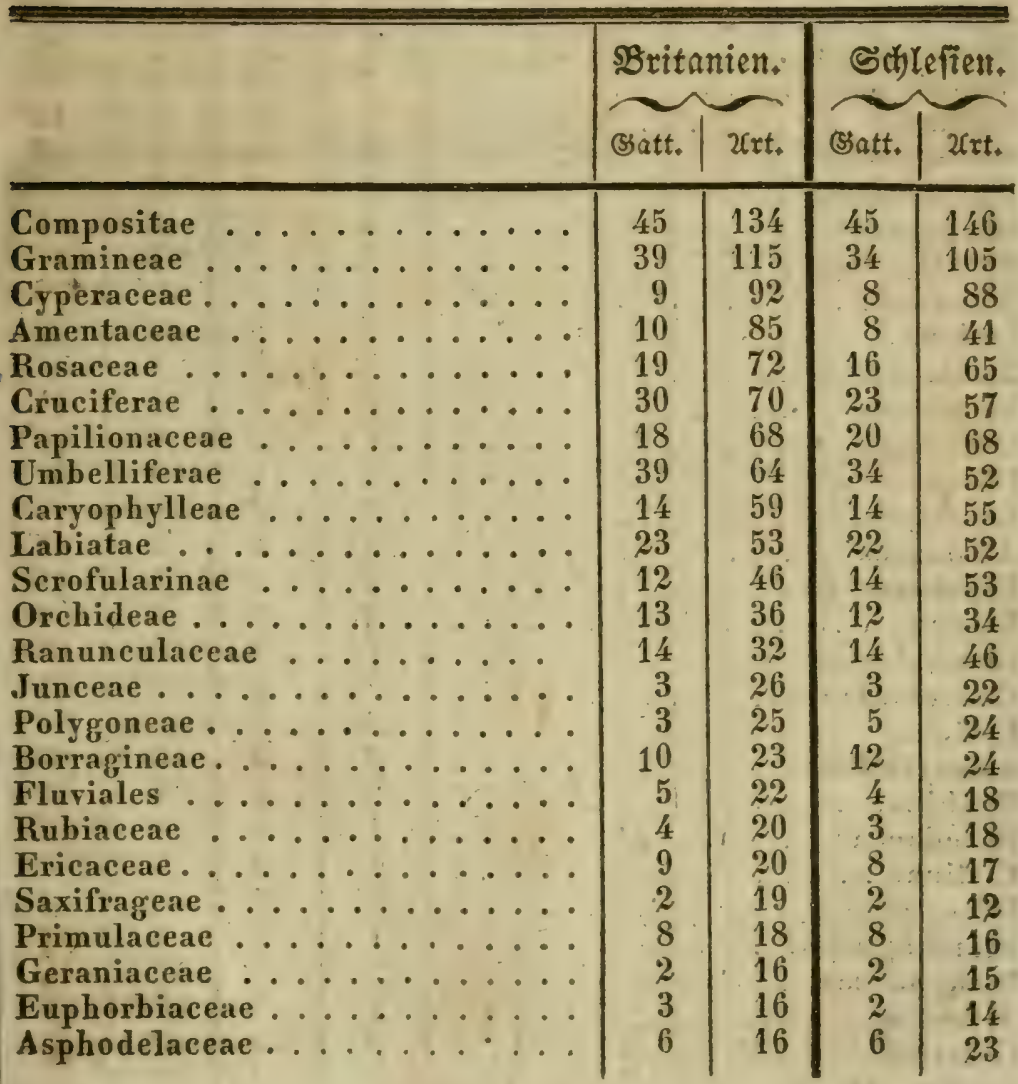




\section{8}

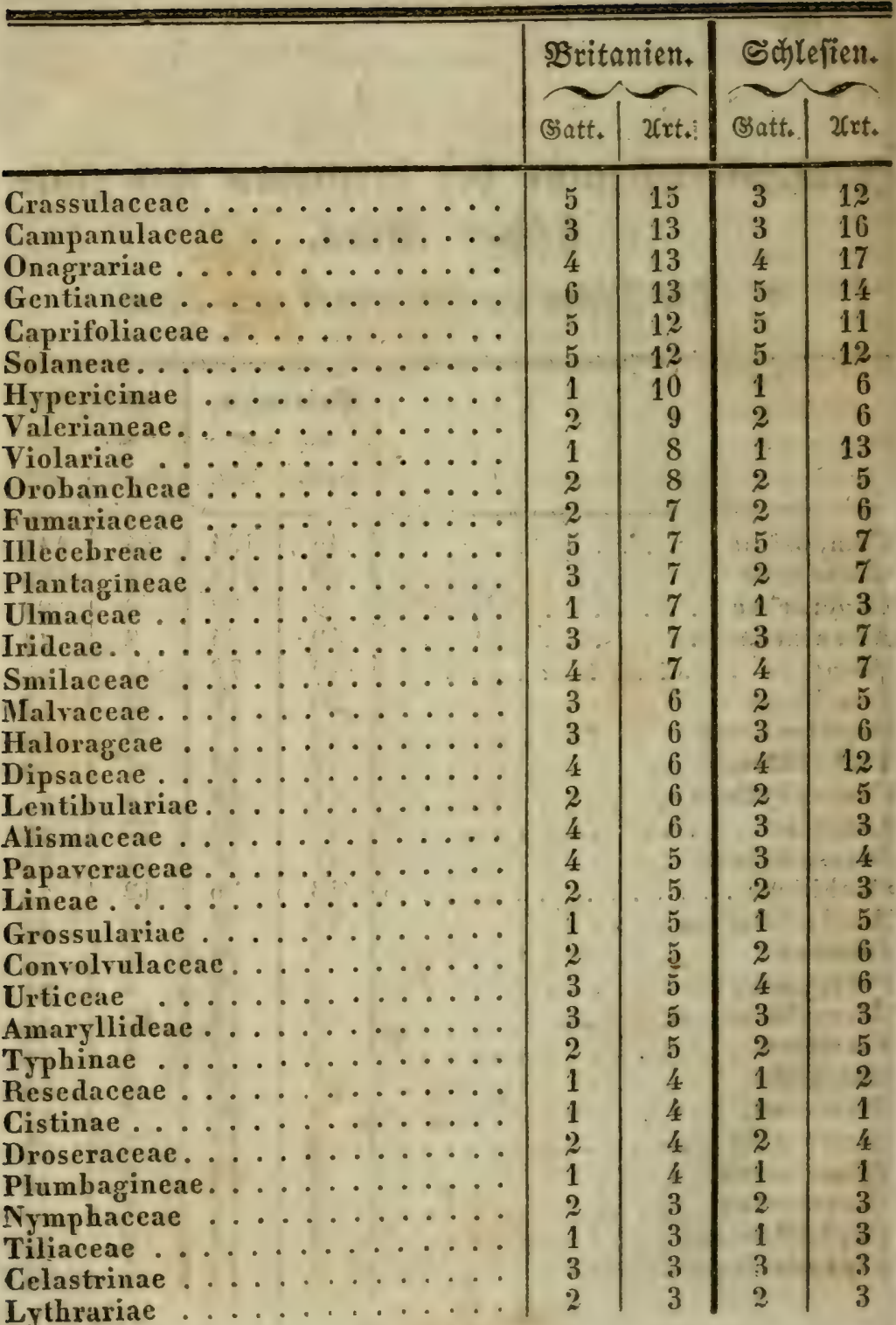




\begin{tabular}{|c|c|c|c|c|}
\hline 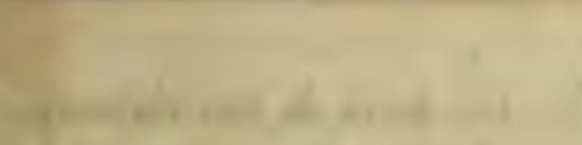 & $\overbrace{\text { Batt. }}^{\text {Srit }}$ & $\underbrace{\text { nien. }}_{2 \text { rrt. }}$ & & efien. \\
\hline Coniferae . . . . . . . . & 3 & 3 & 3 & 8 \\
\hline Juncagineae $\ldots \ldots \ldots \ldots$ & 2 & 3 & 2 & 3 \\
\hline Accrinae $\ldots \ldots \ldots \ldots$ & 1 & 2 & 1 & 3 \\
\hline Oxalideae $\ldots \ldots \ldots \ldots \ldots$ & 1 & 2 & 1 & 2 \\
\hline Rhamneae $\ldots \ldots \ldots \ldots$ & 2 & 2 & 2 & 3 \\
\hline Ceratophylleae $\ldots \ldots \ldots \ldots$ & 1 & 2 & 1 & 2 \\
\hline Arialiaceae $\ldots \ldots \ldots \ldots \ldots$ & 2 & 2 & 2 & 2 \\
\hline 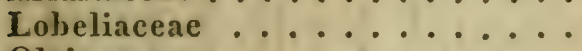 & 1 & 2 & 0 & 0 \\
\hline Oleinae $\ldots \ldots \ldots \ldots \ldots$ & 2 & 2 & 2 & 2 \\
\hline Apocyneae $\ldots \ldots \ldots$ & 1 & 2 & 1 & 2 \\
\hline Thymelaceac ......... & 1 & 2 & 2 & 2 \\
\hline Asarinae $\ldots \ldots \ldots \ldots$ & 2 & 2 & 2 & 2 \\
\hline Hydrocharides . . . . . . . & 2 & 2 & 2 & 2 \\
\hline Tulipaceae $\ldots \ldots \ldots \ldots$ & 2 & 2 & 2 & 2 \\
\hline Melanthiaceae $\ldots \ldots \ldots \ldots$ & 2 & 2 & 2 & 3 \\
\hline Aroideae $\ldots \ldots \ldots \ldots \ldots$ & 2 & 2 & 2 & 3 \\
\hline Polygaleae $\ldots \ldots \ldots \ldots \ldots \ldots$ & 1 & 1 & 1 & 3 \\
\hline Frankeniaceae $\ldots \ldots \ldots \ldots$. & 1 & 1 & 0 & 0 \\
\hline Berberideae $\ldots \ldots \ldots \ldots \ldots$ & 1 & 1 & 1 & 1 \\
\hline 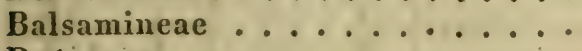 & 1 & 1 & 1 & 1 \\
\hline Rutaceae $\ldots \ldots \ldots \ldots \ldots$ & 0 & 0 & 1 & 1 \\
\hline Tamariscinae $\ldots \ldots \ldots \ldots$ & 1 & 1 & 1 & 1 \\
\hline Cucurbitaceae $\ldots \ldots \ldots \ldots$ & 1 & 1 & 1 & 1 \\
\hline Portulaceae . . . . . . . . . . & 1 & 1 & 2 & 2 \\
\hline Loranthaceae . . . . . . . . & 1 & 1 & 1 & 1 \\
\hline Asclepiadeae $\ldots \ldots \ldots \ldots$ & 0 & 0 & $\overline{1}$ & 1 \\
\hline Rhodoreae $\ldots \ldots \ldots \ldots$ & 0 & 0 & 1 & 1 \\
\hline Polcmoniaceae $\ldots \ldots \ldots \ldots \ldots$ & 1 & 1 & 0 & 0 \\
\hline Verbenaceae .......... & 1 & 1 & 1 & 1 \\
\hline Globulariae $\ldots \ldots \ldots \ldots$ & 0 & 0 & 1 & 2 \\
\hline Amaranthaceae ........ & 1 & 1 & 1 & 2 \\
\hline Santalaceae $\ldots \ldots \ldots \ldots$ & 1 & 1 & 1 & 3 \\
\hline Elaeagneae . . . . . . . . . & 1 & 1 & 0 & 0 \\
\hline Empetrae $\ldots \ldots \ldots \ldots \ldots$ & 1 & 1 & 1 & $\mathbf{I}$ \\
\hline Tameae $\ldots \ldots \ldots \ldots$ & 1 & 1 & 0 & 0 \\
\hline Eriocauleae. . . $\ldots \ldots \ldots$ & 1 & 1 & 0 & 0 \\
\hline
\end{tabular}




\section{0}

2) Die Bablenverbältniffe Der $\mathfrak{A r t e n}$ in ben einzelnen Familien zur Gejammtartenzabl Der Flora.

\begin{tabular}{|c|c|c|}
\hline & Britanien. & Schleften. \\
\hline 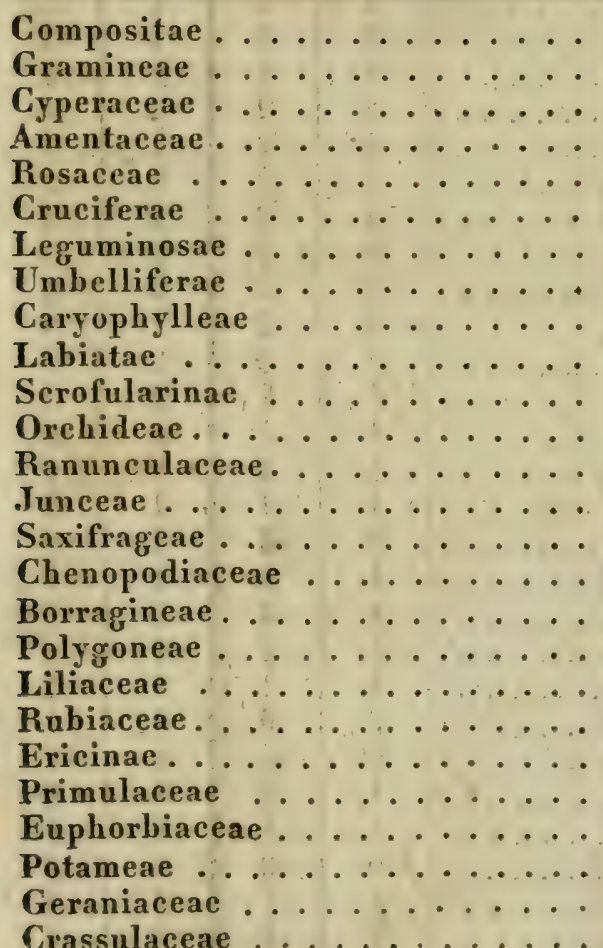 & $\begin{array}{l:c}1 & 11 \\
1 & 13 \\
1 & 16 \\
1 & 18 \\
1 & : 19 \\
1 & : 21 \\
1 & : 22 \\
1 & : 23 \\
1 & \vdots 26 \\
1 & \vdots 29 \\
1 & \vdots 33 \\
1 & \vdots 41 \\
1 & \vdots 42 \\
1 & \vdots 54 \\
1 & : 80 \\
1 & : 61 \\
1 & \vdots 63 \\
1 & \vdots 66 \\
1 & \vdots 66 \\
1 & : 72 \\
1 & \vdots 76 \\
1 & : 80 \\
1 & \vdots 89 \\
1 & \vdots 94 \\
1 & : 95 \\
1 & : 95\end{array}$ & $\begin{array}{l:}\left.1: 9^{*}\right) \\
1: 13 \\
1: 16 \\
1: 32 \\
1: 22 \\
1: 24 \\
1: 19 \\
1: 25 \\
1: 24 \\
1: 25 \\
1: 26 \\
1: 40 \\
1: 28 \\
1: 54 \\
1: 113 \\
1: 75 \\
1: 56 \\
1: 08 \\
1: 48 \\
1: 75 \\
1: 79 \\
1: 90 \\
1: 97 \\
1: 96 \\
1: 90 \\
1: 113\end{array}$ \\
\hline
\end{tabular}

*) Die Compositae bitoen in Sritanien $1 / 11$, in Sdilefien $1 / 9$ ber Befamuntartenjaht u. F, w. 


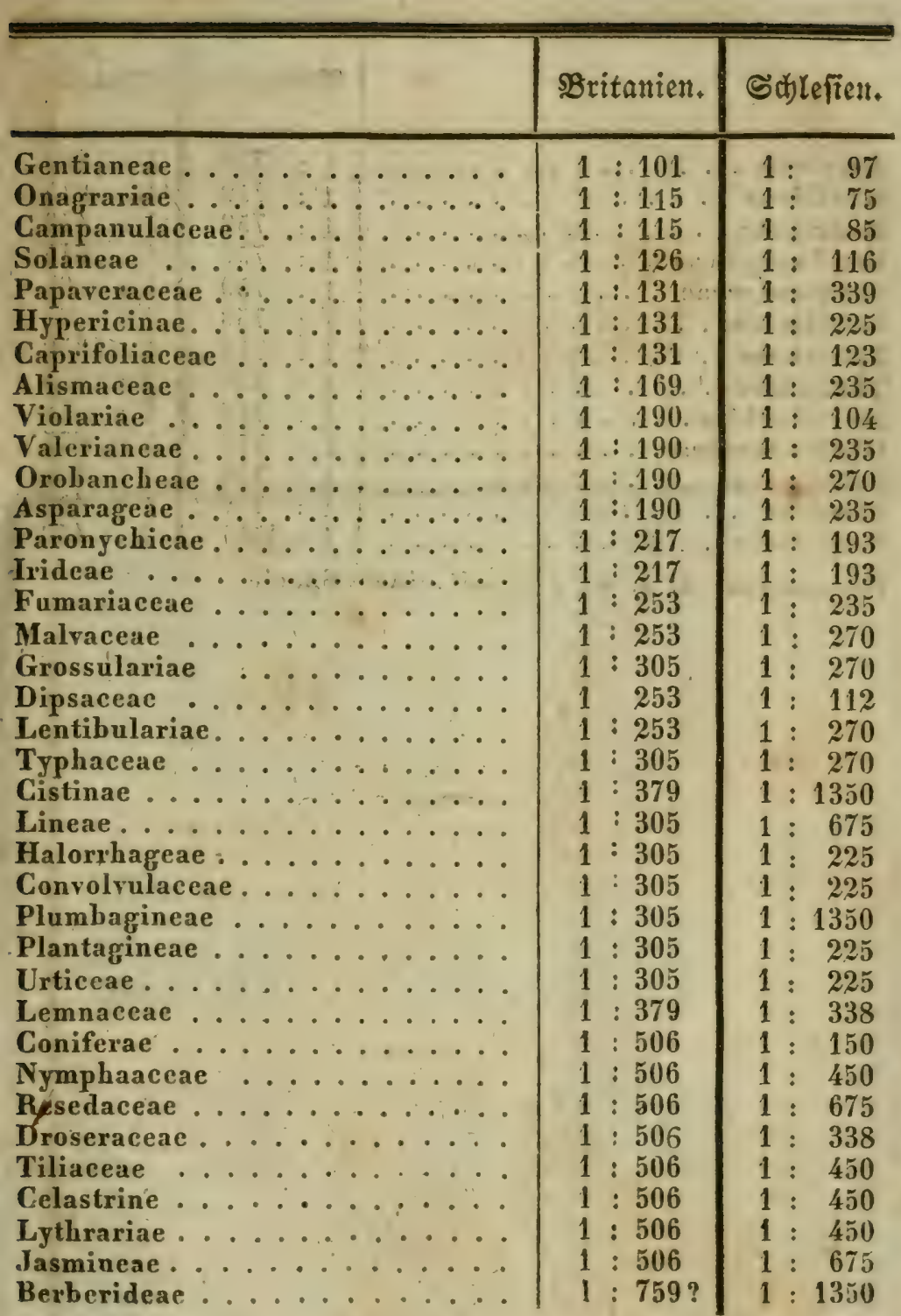




\section{2}

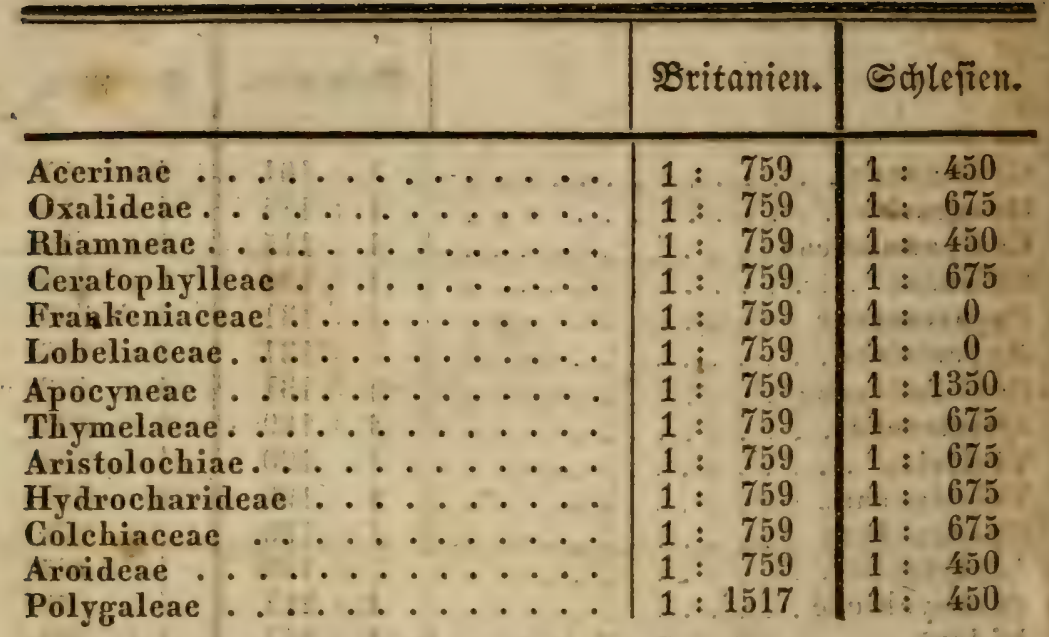

Balsamineac u. s, w. alle mit einer Species. 


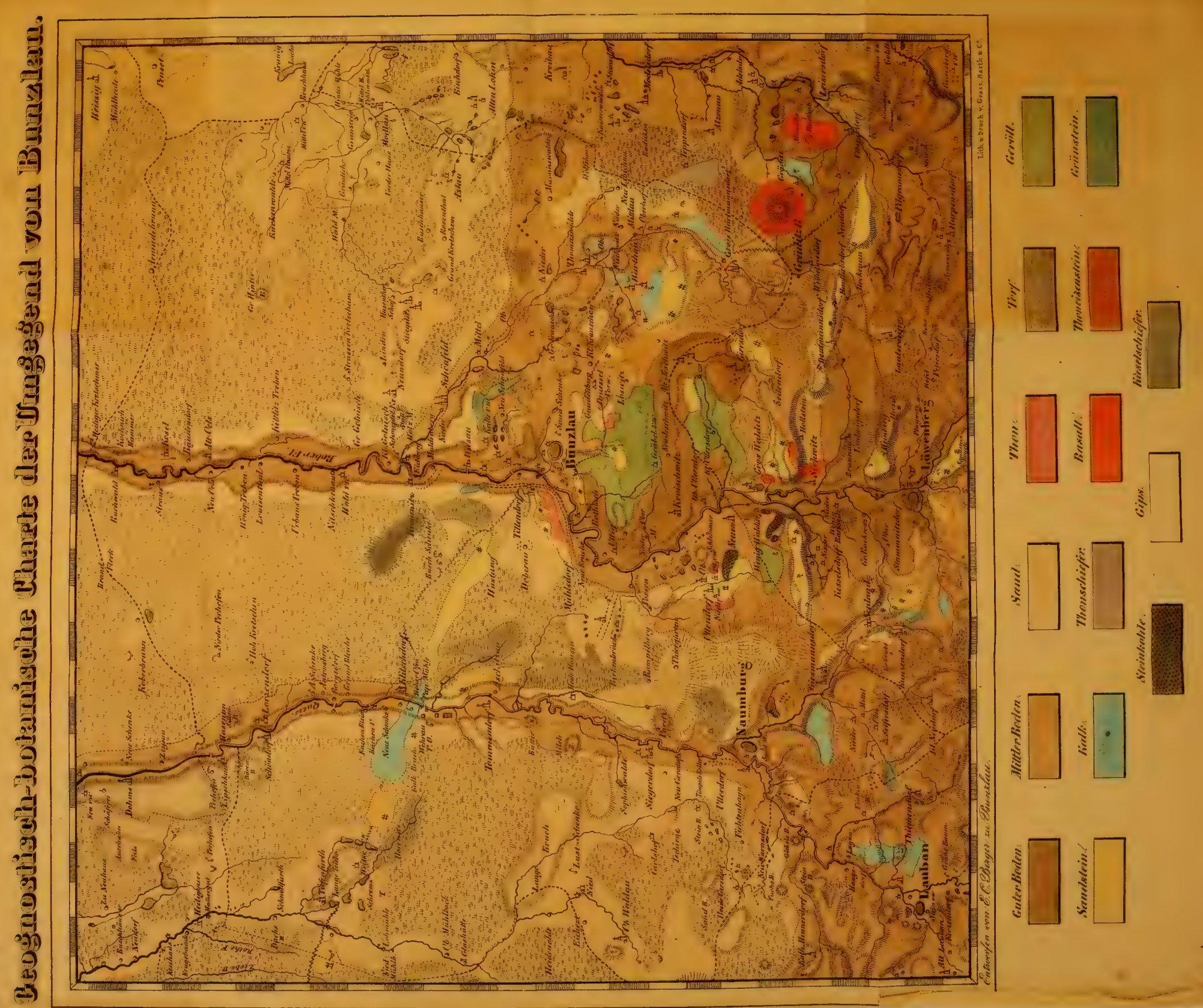




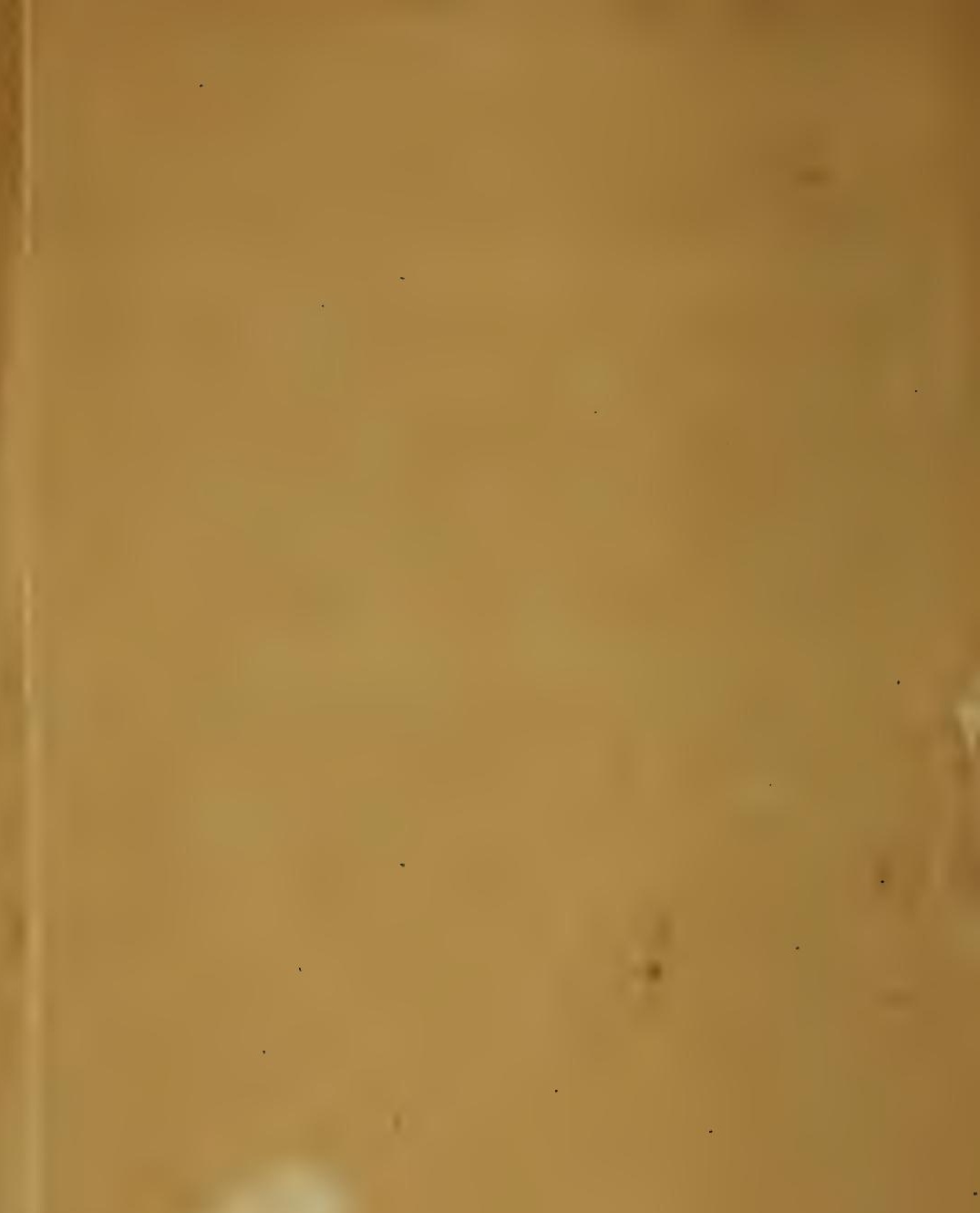







